\title{
Liquid Effluent Study Characterization Data
}

Prepared for the U.S. Department of Energy Office of Environmental Restoration and Waste Management

\section{We Westinghouse \\ Hanford Company Richland, Washington}

Hanford Ope ations and Engineering Contractor for the

U.S. Defartmint of Energy under Contract DE-AC06-87RL10930

Approved for Public Release 
LECAL DuClanen

This report we prepated as in account of work sponsorod by en eqency of the Unitod States Government. Metther the Uniled Sulas Oovernment nor any soency thereol, nol any of their ecmptoyees, nor any of their contraciors, subcontractors of their employees. makes any warranty. expreses or implied, * - asumes any logal liability or responsibility for the eceuracy, completenese, or eny third party's use or the results of such use of any inlormation. apparatus, product, or procese diucloesed, of represente that its ure would not intringe privateh owned rights. Relerence herein to any specific commercid product. process, or service by irade name. indemart, manuleclurer. of otherwise. doen nol mocesearity constifure or imply its endorsement, recommendation, of lavering by the Unitod Siates Government of any apency thereol of its contraclors of eubcontractors the viom and opinions of suthors expresed herein vo nol necceserily otale a reflect those of the Uniled Sules Government of eny egency thercol.

This repent has been roproduced liom the best aveilable cops Avelable in paper copy and microliche.

The U.S. Department of Energy and lle contractore cen obuin copion of the report from:

Otives of Scientilic and Technical Information

P. O. Dor 62

Oat Rinoe. TN 37091

(615) $5 \pi .401$

This repon is putticty avaitable from:

Malional Technical Inlormabon Servica

5205 Port Royd Rasd

Bpringfield, VA 22161

(tos) 407 ieso

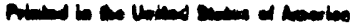

man ion $110 \mathrm{~cm}$ 


\begin{abstract}
During the development of the Hanford Federal Facility Agreement and Consent Order (Tri-Party Agreement), public comments were received regarding reduction of the discharge of liquid effluents into the soil column. As a result, the U.S. Department of Energy (DOE), with concurrence of the Washington State Department of Ecology (Ecology) and the U.S. Environmental Protection Agency (EPA), committed to a special project designed to document the discharge history and the character of Hanford Site liquid discharges. The results of this project will be used in determining the need for additional waste stream analysis, and/or to negotiate additional milestones pertaining to such discharges in the Tri-Party Agreement.

Wastestream sampling data collected prior to October 1989 were reported the Waste Stream Characterization Report. Prel iminary Stream-specific Reports were prepared which evaluated that data and proposed dangerous waste designations for each stream. This document contains the wastestream sampling and analysis data collected as part of the liquid effluent study. Data contained in this report were obtained from samples collected from October 1989 through March 1990. Information is presented on the wastestreams that have been sampled, the parameters analyzed, and the dates and times at which the samples were collected. This information will be evaluated in the final Stream-specific Reports.
\end{abstract}




\section{CONTENTS}

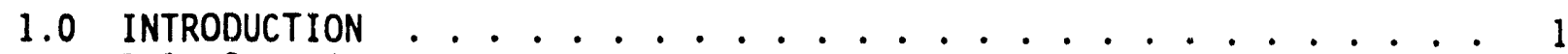

1.1 Overview .......................... 1

1.2 Purpose .................... 1

1.3 Scope ........................... 1

2.0 SAMPLING SCHEDULE ................. 3

2.1 Tentative Sampling Schedule ............. 3

2.2 Actual Sampling Schedule ............... 3

2.3 Waste Generation Configuration ............. 4

3.0 SAMPLE COLLECTION .................. 6

4.0 SAMPLE ANALYSIS .................... 8

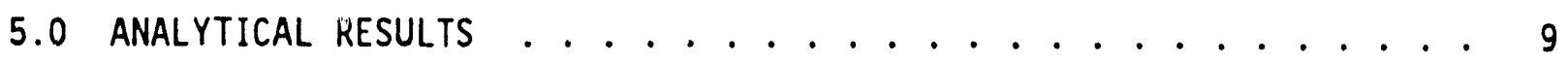

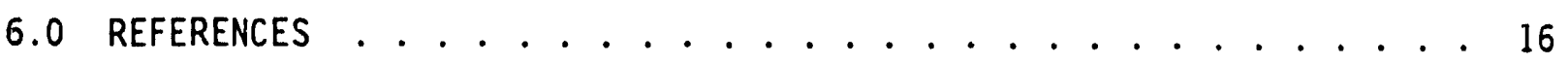

APPENDIX A $\ldots$ Sample Numbers .................. . . 1

APPENDIX B -. Sampled Wastestreams and Sampling Locations ...... B - 1

A.PPENDIX C -. Analytical Sample Specifications . . . . . . . . . C - 1

APPENDIX D -. Analytical Results (Data Sheets) .......... D - 1

APPENDIX E .. Analytical Results (Microfiche) .......... E - I

APPENDIX F .- List of common names .............. . . 1

\section{TABLES}

Table 1.1 Liquid Effluent Wastestreams ............ 2

Table $2-1$ Wastestreams and Alternative Configurations . . . . . 5

Table 5-1 Analytes Detected At Least Once............. 11

Table 5-2 Analytes Reported But Never Detected .......... 13 
WHC-EP-0355

\subsection{INTRODUCTION}

\subsection{Overview}

During the public comment period of the Hanford Federal Facility Agreement and Consent Order (Ecology/EPA/DOE 1989), several comments were received concerning the disposal of liquid effluents to the ground. In response to these comments and at the request of the Washington State Department of Ecology (Ecology) and the U.S. Environmental Protection Agency (EPA), the U.S. Department of Energy (DOE) initiated a program to report the history and characteristics of these effluents.

This program is described in the document entitied Draft Liquid Effluent Study Project Plan (WHC 1990a). The results of this program will be utilized in determining the need for revising the current pian for eliminating discharges of contaminated liquid effluents into the soil column. A portion of this study consists of characterizing 33 key liquid effluent streams on the Hanford Site. Individual, preliminary reports for each effluent were prepared as part of this stream characterization effort (WHC 1990b). These addressed data collected prior to October 1989, with the exception of the reports for the $N$ Reactor effluent and the 300 Area process wastewater, which factored in more recent data.

\subsection{Purpose}

This document contains the waste stream sampling and analysis data from effluent samples collected between October 2, 1989, and March 29, 1990. Information is presented on the wastestreams that have been sampled, the parameters analyzed, and the dates and times at which the samples were collected. Additional stream-specific information was presented in the individual, preliminary reports for each effluent (WHC 1990b). The final stream-specific reports will address the recent data presented herein.

\subsection{Scope}

The characterization effort addresses the 33 liquid effluent streams identified in the document Annual Status Report of the Plan and Schedule to Discont inue Disposal of Contaminated Liquid into the Soil Column at the Hanford Site (Millikin 1989). These effluent streams are listed in Table 1-1. This document provides data for 24 of these streams. The remaining nine streams were not sampled for reasons explained in Section 2.2. This document does not address other sections of the Draft Liquid Effluent Study Plan (KHC 1990a), such as groundwater characterization or waste disposal site assessment. 
MHC-EP-0355

Table 1-1 Liquid Effluent Wastestreams

\begin{tabular}{|c|c|}
\hline Area & Wastestream \\
\hline 100 Areas & $\begin{array}{l}N \text { Reactor effluent } * \\
163-N \text { Demineralizer Plant wastewater * } \\
183-D \text { Filter backflush wastewater } \star\end{array}$ \\
\hline 200 Arezs & 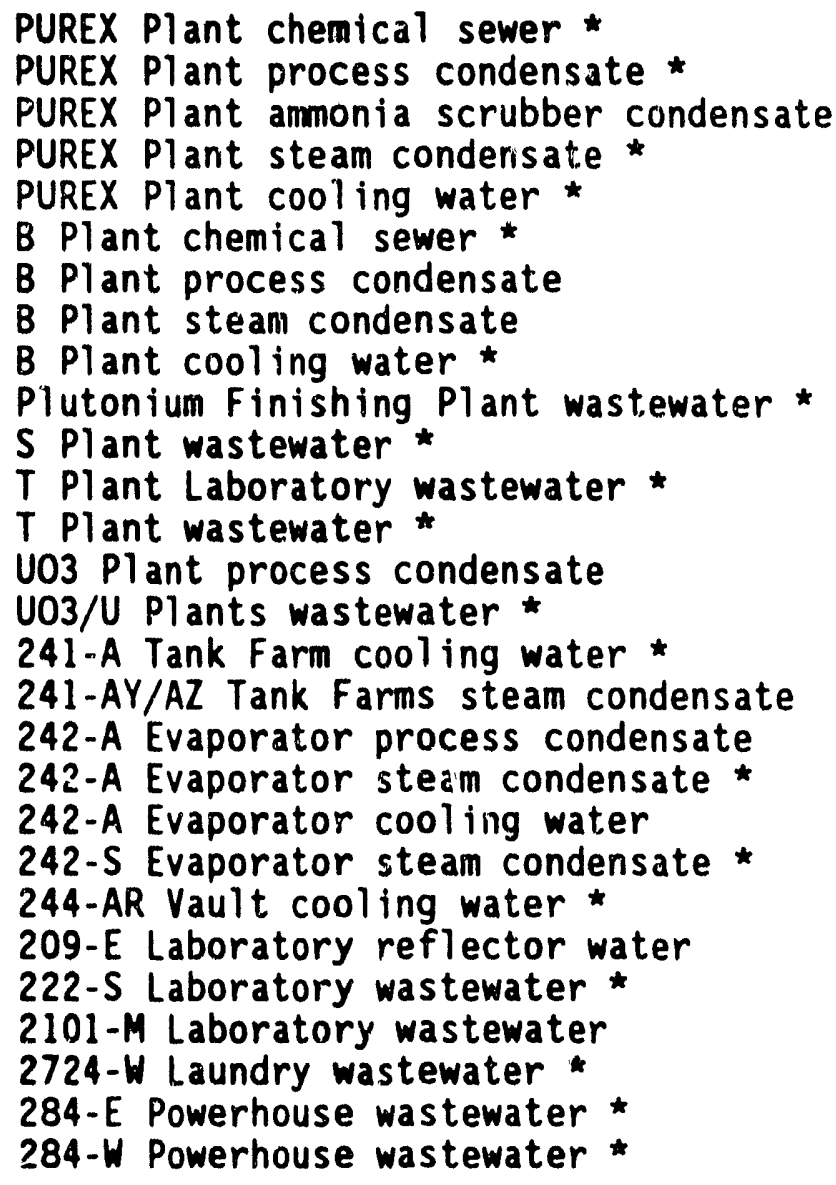 \\
\hline 300 Area & 300 Area wastewater * \\
\hline 400 Area & 400 Area secondary cooling water * \\
\hline
\end{tabular}




\subsection{SAMPLING SCHEDULE}

\subsection{Tentative Sampling Schedule}

The tentative sampling schedule was presented in the Waste Stream Characterization Report (WHC 1989). This schedule was based on the collection of four random samples from each of the active wastestreams between October 2, 1989, and January 31, 1990. When the schedule was prepared, it was recognized that modifications might be necessary depending on manpower, equipment availability, changing sampling priorities, and the status of the various facilities.

The tentative schedule called for the sampling of 27 wastestreains. During the preparation of this oriçinal sampling schedule, it was anticipated that the following six streams would be inactive during the sampling period:
- PUREX Plant Process Condensate
- PUREX Plant ammonia scrubber condensate
- 242-A Evaporator process condensate
- 242-A Evaporator cooling water
- 241-AY/AZ Tank Farms steam condensate
o 209-E Laboratory

\subsection{Actual Sampling Schedule}

The actual sampling schedule reflects the sampling of 24 wastestreams. The list of active streams experienced a net decrease of two over those identified above. Another stream, the 2101-M Laboratory wastewater, was not sampled because of standing water at the only available sampling location. The actual list of inactive streams includes (* additions):
O B Plant process condensate *
- B Plant steam condensate *
- $\mathrm{UO}_{3} \mathrm{Pl}$ an $\imath$ process condensate *
- PUREX Plant ammonia scrubber condensate
- 242-A Evaporator process condensate
- 242-A Evaporator cooling water
- 241-AY/AZ Tank Farms steam condensate
o 209-E Laboratory

The PUREX Plant process condensate was taken off the inactive list as a consequence of the stabilization or "clean-out" run at PUREX. It is also worth noting that the 2101-M stream, mentioned above, currently consists only of effluent related to heating and cooling the building, since the laboratory is not in operation. 
The tentative sampling schedule was also modified to allow the collection of additional samples during the PUREX Plant clean-out run. Additional samples (more than the 4 originally planned) were collected from the PUREX Plant cooling water, steam condensate, and process condensate.

In summary, 9 of the 33 streams were not sampled during the sampling period from October 2, 1989, to March 29, 1990. Of the sampled streams, 17 were sampled 4 times each; 3 were sampled 6 times each; one was sampled 3 times; one was sampled 5 times; one was sampled 9 times; and one was sampled 27 times. Appendix A contains a listing of the date and time each sample was collected.

\subsection{Waste Generation Configuration}

The operating configuration of the waste generator can influence wastestream properties. A total of 24 alternative waste generation configurations have been identified for 9 of the 33 wastestreams. The 9 wastestreams and their alternative process configurations are listed in Table 2-1. These configurations are explained in more detail within the individual stream-specific reports (WHC 1990b). 
WHC-EP-0355

Table 2-1 Wastestreams and Alternative Configurations

Wastestream subset

163-N Demineralizer Plant wastewater-1 163-N Demineralizer Plant wastewater-2

PUREX chemical sewage-1

PUREX chemical sewage-2

$B$ Plant chemical sewage-1A

B Plant chemical sewage-1B

B Plant chemical sewage-2

Plutonium Finishing $\mathrm{Pl}$ lant wastewater-1 Plutonium Finishing Plant wastewater-2

Plutorium Finishing Plant wastewater-3

T Plant Laboratory wastewater-1

$T$ Plant Laborator'y wastewater-2

U03 Plant process condensate-1

U03 Plant process condensate-2

242-A Evaporator process condensate-1 242-A Evaporator process condensate-2 242-A Evaporator process condensate-3 242-A Evaporator process condensate-4

284-E Powerhouse wastewater-1 284-E Powerhouse wastewater-2 284-E Powerhouse wastewater-3

284-W Powerhouse wastewater-1 284-W Powerhouse wastewater-2 284-W Powerhouse wastewater-3
Alternative Configuration

Demineralizer bypass

Ion exchanger regeneration

Ion exchanger regenerate Routine operation

Anion exchanger regenerate Cation exchanger regenerate Routine operation

Plutonium Recla stion Facility (PRF) Remote Mechanical C Line (RMC)

Not during PRF or RMC

Plasma torch operation

Plasma torch standby

Uranium calcination

Standby

Clad-removal waste feed

Linked feed

Ammonia scrubber feed

Saltwe!l feed

Blowdown

Water softener regenerate

Routine operation

Blowdown

Water softener regenerate

Routine operation

\footnotetext{
Note: The configuration of the generator was recorded when one of these 9 wastestreams was sampled. Appendix A identifies the configuration of the waste generator during the collection of each of the samples collected as part of this study.
} 


\subsection{SAMPLE COLLECTION}

Procedures for sample collection, preservation, transport, documentation, and maintaining chain of custody are consistent with U.S. Environmental Protection Agency procedures (EPA 1986). Appendix B ioentifies the locations that were used to collect the Wistestream samples. These sampling locations are consistent with the locations presented in the Waste Stream Characterization Report (WHC 1989), with one exception, the PUREX Plant process condensate. Since flow to the 216-A-45 crib was discontinued, the former sampling location for this stream is no longer in line, and another appropriate location was selected.

Appendix $C$ identifies 28 sample containers that are filled in the course of collecting a wastestream sample. The analytical methods that are performed on the contents of each container; the container volume; the composition of the container, the lid, and the lid liner; the preservatives that are employed; and whether any headspace can exist in the container after sampling are also identified.

The contents of each sample container supports one or more analytical methods. These method samples can be primary wastewater samples, duplicate wastewater samples, sample blanks, or transfer blanks. Duplicates provide the opportunity for the laboratory to repeat analyses. The methods that require duplicates are selected based on the experience of the analytical 1 aboratory.

Four sample numbers are routinely assigned by the analytical laboratory to various containers, or partial analyses thereof, within each complete sample set:

0 The first is the primary sample number that is assigned to waste analyses associated with most of the laboratory procedures.

- The second is a blank sample number for volatile organic analys is (VOA). The blank is prepared from high-quality water and sealed in the Westinghouse Hanford Company Process Development Laboratory before the samples are collected. The blank remains sealed until it is opened in the analytical laboratory where it is processed and analyzed with the VOA sample.

0 The third is a VOA transfer blank sample number. The transfer blank is handled exactly like the blank with one exception -- the transfer blank is opened in the field while the VOA sample is being collected and resealed when the VOA sample is sealed.

- The fourth sample number has no unique label. It is assigned to that portion of the sample that is analyzed by the Extraction Procedure Toxicity Test, the Ignitability Test, the Reactivity Tests, and laboratory measurements of $\mathrm{pH}$ and conductivity. 
WHC-EP-0355

Appendix $A$ ists the sample numbers assigned to samplos collected during this study. Section 5 discusses a modification of this fo:mat which results in a more easily recognizable numbering system. The alternate (or abbreviated) format is used in the data appendices. 


\section{WHC-EP-0355}

\subsection{SAMPLE ANALYSIS}

Appendix C lists the analytical methods that were employed during this study. In all cases, preference is given to EPA methods described in Test Methods for Evaluating Solid Waste Physical/Chemical Merhods, SW-846 (EPA 1986). When an SW-846 method was not available, alternative methods were selected in the following order: 1) other EPA methods, 2) standard methods (APHA/AWWA/WPCF 1985 or ASTM 1986), or 3) local methods. These methods are discussed in greater detail in the Waste Stream Characterization Report (WHC 1989).

All analyses 1 isted in Appendix $C$ were requested for all samples except for the $N$ Reactor effluent; only 4 of the $27 \mathrm{~N}$ Reactor effluent samples received the full suite of analyses. This was due to the incorporation of additional, routine data (obtained using the same sampling protocol as the Site-wide program) being collected by $N$ Reactor operations, into the available data pool. The preliminary stream specific report for the $N$ Reactor effluent (WHC 1990b, Addendum 3) explains these additional samples in detail.

All samples were analyzed by the U. S. Testing Company, Incorporated, of Richland, Washington. 


\section{WHC-EP-0355}

\subsection{AMLLTICAL RESULTS}

The results of the sampling and analysis study are reported in Appendices $D$ and $E$. Appendix $D$, a printed report, is a subset of the complete listing of resis ts on microfiche that appear in Appendix $E$.

Appendix $D$ excludes some of the "censored" data that are reported within Appendix E. Censored data consist of less-than detection limit or greaterthan detection limit data. For example, typical censored data include ranges such as $<5 \mathrm{ppb}$ or $>208{ }^{\circ} \mathrm{F}$. The first example, $<5 \mathrm{ppb}$, indicates that the concentration of the analyte is in the range trat extends from $0 \mathrm{ppb}$ to less than the decection limit of the method (i.e. $5 \mathrm{ppb}$ ). The second example indicates that the flash poir: of the sample is greater than 208 OF. The data provided in Appendix $D: s$ similar to the subset employed in the streamspecific reports (WHC 1990b) as nart of the designation process according to WA. 173-303 (Ecology 1989).

Except for the following two cases, censored values are not included in the 0 . ia reports contained in Appendix D:

1. Analyte data sets that contain at least one detected (uncensored) value. For example, if the results of analytical procedures for four samples were: $<10 \mathrm{ppb}, 20 \mathrm{ppb},<10 \mathrm{ppb}$, and $50 \mathrm{ppb}$, all four concentrations would be reported in Appendix $D$. If all four values were <10 ppb, they would not appear in Appendix $D$. For example, if no sample-based evidence exists that zinc is present in the wastewater, it is not reported in Appendix $D$.

2. Characteristic data for ignitability, reactivity and extraction procedure toxicity. Results for characteristics are reported by the analytical laboratory as censored values when they are less than the limits of WAC 173-303-090 (Ecology 1989). These censored data are included in Appendix $D$.

Data reported by the laboratory under the primary sample number (see Section 3.0) are listed in the daia reports with the primary sample number. Data for VOA blanks ai"e 1 isted in the data reports by the primary sample number and the suffix "B". Data for VOA transfer blanks are listed in the data reports by the primary sample number and the suffix " $T$ ". Data from extraction procedure toxicity (EP TOX) testing, ignitability testing, reactivity testing and laboratory measurements of $\mathrm{pH}$ and conductivity are listed in the data reports by the primary sample number with the suffix " $E$ " (for "extract", a term only applicable to the EP Tox portion).

The use of sample numbers and suffixes facilitates the computer sort of all data for a wastestream sample that was collected from a single location at a specific time, without losing track of appropriate distinctions. This format does not impact traceability to any relevant laboratory records. 
Table 5-1 lists each analyte that has been detected at least once during the study. Table 5-2 lists each analyte that has consistently been reported below the detection limit. That is the analyte was looked for, according to a specific requirement in the analytical laboratory's contract, but never detected.

Appendix F lists the common names of the molecular analytes in this study with sufficient information to unamb:guously identify them. The handbook name and formula can be used with any stalidard chemical handbook to develop the compound's structure. The Chemical Abstracts Service name and associated number can be used to access the chemical literature. 
Table 5-1 Analytes Detected At Least Once. (Sheet 1 of 2)

\section{INORGANICS}

Aluminum

Arsenic

Barium

Barium (EP Toxic)

Beryllium

Boron

Cadmium

Calcum

Chloride

Chromium

Cobalt

Copper

Cyanide

Fluoride

Iron

Lead

Lithium

Magnesium

Mangarrese

Mercury

Nickel

Nitrate

Nitrite

Phosphate

Potassium

Silicon

Sodium

Strontium

Sulfate

Sulfide

Thallium

Titanium

Uranium

Vanadium

Zinc

\section{ORGANICS}

\section{Acetone}

Ammonia

Benzoic acid

Benzyl alcohol

Bis(2-ethylhexyl) phthalate
ORGANICS (Cont'd.)

Bromodichloromethane

1-Butanol

2-Butanone

1-Butoxy-2-propanol

Butylated hydroxy toluene

Butylbenzyl phthalate

Cafteine

Decane

Dibutylphosphate

Dichloromethane

Di-n-octyl phthalate

Dodecane

Hydrazine

MIBK (Hexone)

Phthalic anhydride

Tetrachloromethane

Tetradecane

Tetrahydrofuran

Toluene

Tributylphosphate

Trichloromethane

Tridecane

Undecane

Unidentified constituent

Unknown

Unknown acid

Unknown aliphatic alcohol

Unknown al iphatic $H C$

Unknown aliphatic nitric acid ester

Unknown aliphatic phosphate ester

Unknown amide

Unknown amine

Unknown compound

Unknown cyclic or unsaturated $H C$

Unknown ester

Unknown fatty acid

Unknown glycol

Unknown hydrocarbon

Unknown oxygenated PAH

Unknown phthalate

Unknown polycyclic $\mathrm{HC}$

Unknown sulfur compound 
WHC-EP-0355

Tabie 5-1 mialytes Dettcted At Least Once. (Sheet 2 of 2)

MISCELLANEOUS

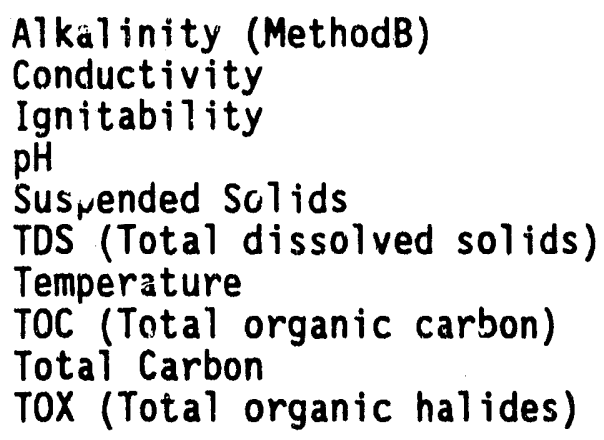

RADIONUCLIDES

Alpha Activity

Beta Activity

Radium Total
Am-241
$\mathrm{Cm}-242$
$\mathrm{Cm}-244$
Co-60
Cs -134
Cs -137
C -14
$\mathrm{H}-3$
I -129
$\mathrm{Mn}-54$
$\mathrm{Na}-22$
$\mathrm{Pb}-210$
$\mathrm{Pu}-238$
$\mathrm{Pu}-239 / 240$
$\mathrm{Ra}-228$
$R u-106$
$\mathrm{Sr}-90$
$U-234$
$U-235$
$U-238$ 
Table 5-2 Analytes Reported But Never Detected (Sheet 1 of 3)

\section{INORGANICS}

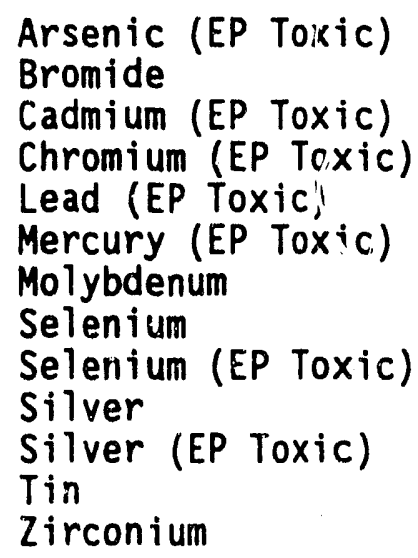

ORGANICS (Cont'd.)

Benzo[ghi]perylene

Benzo[a]pyrene

4-Benzoquinone Benzyl chloride

Bis (2-chloroethoxy)methane Bis(2-chloroethyl) ether Bis (chloroisopropyl) ether Bis (chioromethyl) ether Bischioromethylethyl ethe: Bromomethane 4-Bromophenylphenyl ether 1-Butynol

Carbon disulfide

Chloroalky? ethers 4-Chloroaniline Chiorobenzene 4-Chiloro-3-cresol Chloroethana Chloroethene 2-Chlorcathylvinyl ether Chloromethane

Chloromethylmethyl ether

Chloronaphazine

2-Chlorophenol

3-Chloropropionitrile

Chrysene

Cresol

Crotonaldehyde

2,4-DCP

Diallate

Dibenz [a,h]acridine

Dibenz $[a, j]$ acridine

Dibenz $[a, h]$ anthracene

7H-Dibenzo[C,g]carbazole

Dibenzofuran

Dibenzo[a,e]pyrene

Dibenzo[a,h]pyrene

Dibromochloromethane

1,2-Dibromo-3-chloropropane

1,2-Dibromoethane

Dibromomethane

Di-n-butyl phthalate

1,2-Dichlorobenzene

1,3-Dichlorobenzene 
WHC-EP-0355

Table 5-2 Analytes Reported But Never Detected (Sheet 2 of 3)

ORGANICS (Cont'd.)

1,4-Dichlorobenzene

3,3'-Dichiorobenzidine

1,4-Dichloro-2-butene

Dichlorodifluoromethane

I,1-Dichloroethane

1,1-Dichloroethene

1,2-Dichloroethene

Dichloromethylbenzene

2,6-Dichlorophenol

1,2-Dichloropropane

1,3-Dichloropropane

$N, N-D i e t h y l h y d r a z i n e$

Diethyl phthalate

Dihydrosafrole

3, 3'-Dimethoxybenzidine

4-Dimethyl aminoazobenzene

Dimethyibenz [a] anthracene

3, 3'-Dimethylbenzidine

$N, N$-Dimethylhydrazine

$N, N^{\prime}$ - Dimethylhydrazine

Dimethy phthalate

Dinitrobenzene

Dinitrocresol and salts

Dinitro-2-cyclohexylphenol

2,4-Dinitrophenol

2,4-Dinitrotoluene

2,6-Dinitrotoluene

1,4-Dioxane

Diphenyl amine

$N, N^{\prime}$ - Dipheny 7 hydrazine

Epichlorohydrin

Ethanol

Ethenyl acetate

Ethylbenzine

Ethyl cyanide

Ethylene glycol

Ethylene oxide

Ethylmethacrylate

Ethylmethanesulfonate

Fluoranthene

Fluorene

Formaldehyde

Hexachl orobenzene

Hexachlorobutadiene
ORGANICS (Cont'd.)

Hexachl orocycl ope'itadiene He achloroethane

Hexachl orophene

Hexach loropropene

2-Hexanone

Indeno $(1,2,3-\mathrm{cd})$ pyrene

Iodomethane

Isodrin

Isophorone

Isosafrole

Kerosene

Maleichydrazide

Malononitrile

Melphalan

Methacrylonitrile

Methapyrilene

2-Methyl aziridine

3-Methylchol anthrene

Methylenebischloroaniline

2-Methyllactonitrile

Methyl mercaptan

Methylmethacrylate

Methylnitrosourethane

2-Methylpropan-1-01

2-Methylpyridine

Methyl thiouracil

Monobutylphosphate

Naphthalene

1,4-Naphthal enedione

Nicotinic acid

2-Nitroaniline

3-Nitroaniline

4-Nitroaniline

Nitrobenzene

2-Nitrophenol

4-Nitrophenol

4-Nitroquinol ine-1-oxide

$N$-Nitroso-n-butylamine

$\mathrm{N}$-Nitrosodiethanol amine

$\mathrm{N}$-Nitrosodimethyl amine

$\mathrm{N}$-Nitrosodiphenyl amine

$\mathrm{N}-\mathrm{N}$ itrosomethylethyl amine

$\mathrm{N}$-Nitrosomethylvinylamine $\mathrm{N}$-Nitrosomorphol ine 
Table 5-2 Analytes Reported But Never Detected (Sheet 3 of 3)

ORGANICS (Cont'd.)

N-Nitrosonornicotine Nitrosopiperidine

$N$-Nitroso-n-propylamine

Nitrosopyrrolidine

Paraldehyde

Pentachlorobenzene

Pentachloroethane

Phenacet in

Phenol

2-Pheny1-tert-butylamine

Phthalic acid esters

Pronamide

1-Propanamine

1-Propanol

2-Propyn-1-o1

Pyridine

Reserpine

Resorcinol

Safrol

Strychnine

Styrene

1,2,3,4-Tetrach 1orobenzene

$1,2,3,5$-Tetrachlorobenzene

$1,1,1,2$-Tetrachloroethane

$1,1,2,2-$ Tetrach 1 oroethane

Tetrachloroethene

$2,3,4,6$-Tetrachlorophenol

Tín i of anox

Thionazin

Thiophenol

Thiuram

Toluened iamine

2-Toluidine hydrochloride

Tribromomethane

1,2,3-Trichlorobenzene

1,2,4-Trichlorobenzene

1,3,5-Trichlorobenzene

1,1,1-Trichloroethane

1,1,2-Trichloroethane

Trichloroethene

Trichiorofluoromethane

Trichloromethanethicl

2,4,5-Trichlorophenol

1,2,3-Trichloropropane
ORGANICS (Cont'd.)

Triethylphosphorothioate

1,3,5-Trinitrobenzene

TRIS

Unknown aliphatic nitric acid ester

Unknown aliphatic phosphate ester

Unknown cyclic or unsaturated hydrocarbon

Urethane

Warfarin

$1,2-x y l$ ene \& $1,4-x y l$ ene

$1,3-x y l$ ene

RADIONUCLIDES

$k-40$ 


\subsection{REFERENCES}

APHA/AWWA/WPCF 1985

ASTM 1986

Ecology 1989

EPA 1986

Millikin, E.J. 1989

Ecology/'PA/DOE 1989

candard Methods for the Examination of Water and Wastewater, 16th Ed., American Public Health

Association, American Water Works Association, and Water Pollution Control Federation, Washington, D.C.

1986 Pinnual Book of ASTM Standards, American Society for Testing and Materials, Philadelphia, Pennsylvania

Dangerous Haste Regulations, Washington [State] Administrative Code (WAC) 173-303, State of Hashington Department of Ecology, niympia, Washington.

Hanford Federal Fa: ility Agreement and Consent Order, Washington State Department of Ecology, U.S. Environmental Protection Agency, U.S. Department of Energy, Olympia, Washington

Iest Methods for Evaluating Solid Waste Physical/Chemical Methods, Third Edition, SW-846, U.S. Environmental Protection Agency, Washington, D.C.

Annual Status Report of the Plan and Schedule to Discont inue Disposal of Contaminated Liquids into the Soil Column at the Hanford Site - Fiscal Year 1989, WHC-EP-0196-2, September 1989, Hestinghouse Hanford Company, Richland, Washington.

WHC 1989

WHC 1990a

WHC $1990 \mathrm{~b}$
Waste Stream Characterization Report, WHC-EP-0287, Volume 1, August 1989, Westinghouse Hanford Company, Richland, Washington.

Draft Liquid Effluent Study Project Plan, WHC-EP-0275, Rev. 1, September 1989, WHC, Richland, WA.

Hanford Site Prel iminary Stream-Specific Reports, WHCEP-0342, May 1990, WHC, Richland, WA. 
WHC-EP-0355

APPENDIX A

\section{SAMPLE NIMBERS}

A- $1 / 2$ 
Appendix A--Sample Numbers. (sheet 1 of 5)

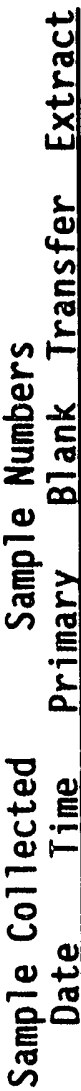

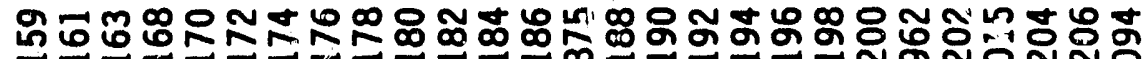

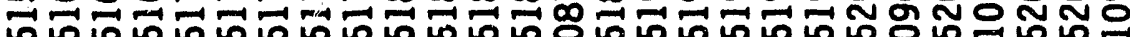

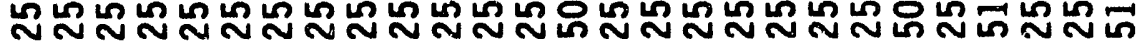

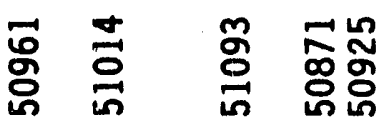

$\underset{\infty}{\mathbb{\infty}}$

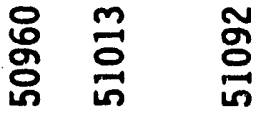

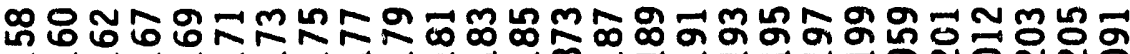

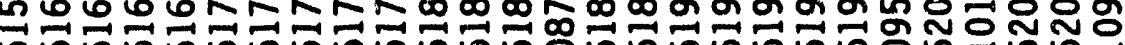

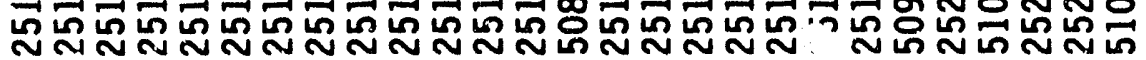

:̊ํㅛ

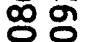
내요

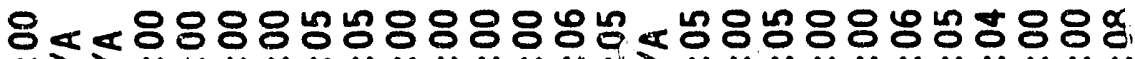

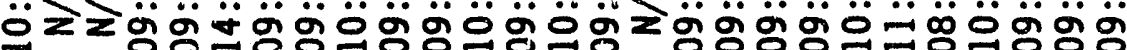

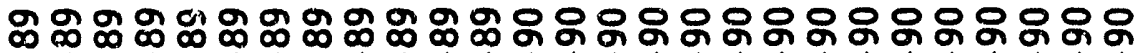

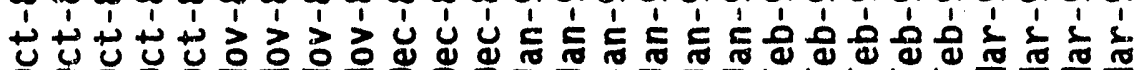

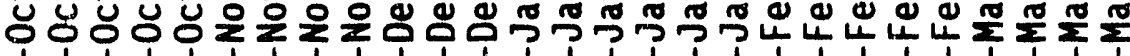

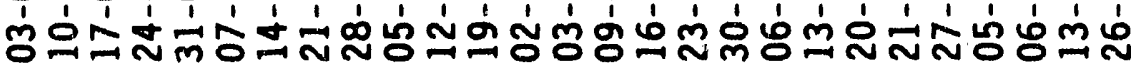

No 疋 은요

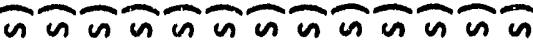

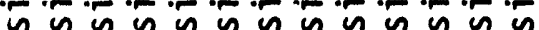

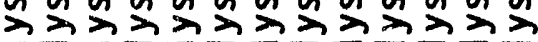

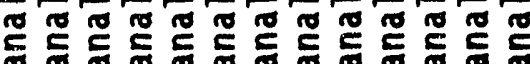

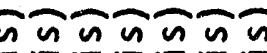

$\bar{n} \cdot \bar{n} \cdot \bar{n} \cdot \bar{n}$

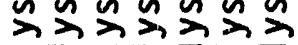

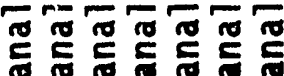

$\sum_{\frac{n}{n}}^{\frac{n}{n}} \frac{n}{n} \frac{n}{n}$

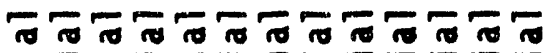

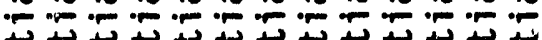

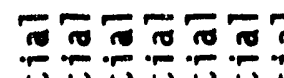

$\pi \pi$

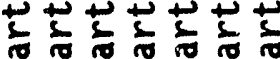

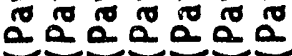

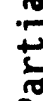

$\stackrel{+}{2}$

$\mp \mp$

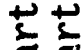

$\pi$

?

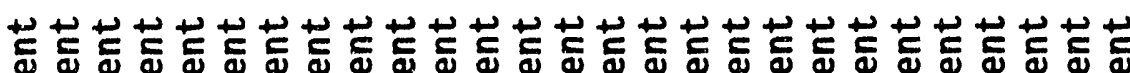

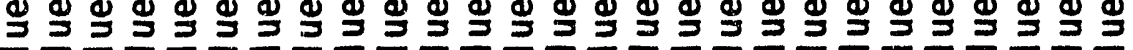

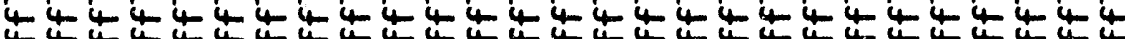

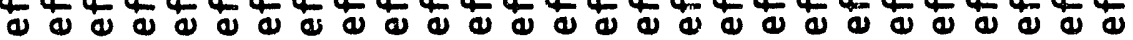

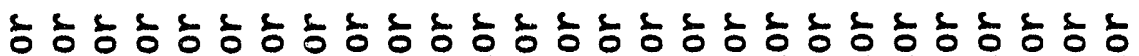
بي

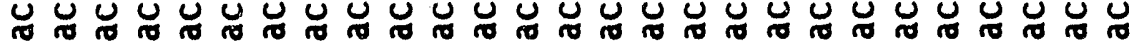

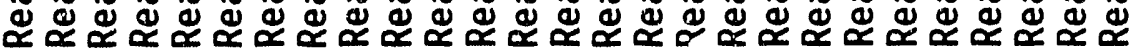
zizzzzzzzzzzzzzzzzzzzzzzzzzz
ชัㅇㅇㅇ $\ddot{m} \ddot{\Xi}$ 용요 它毩 $?_{1}^{2}$ ติ 
Appendix A--Sample Numbers. (sheet 2 of 5 )

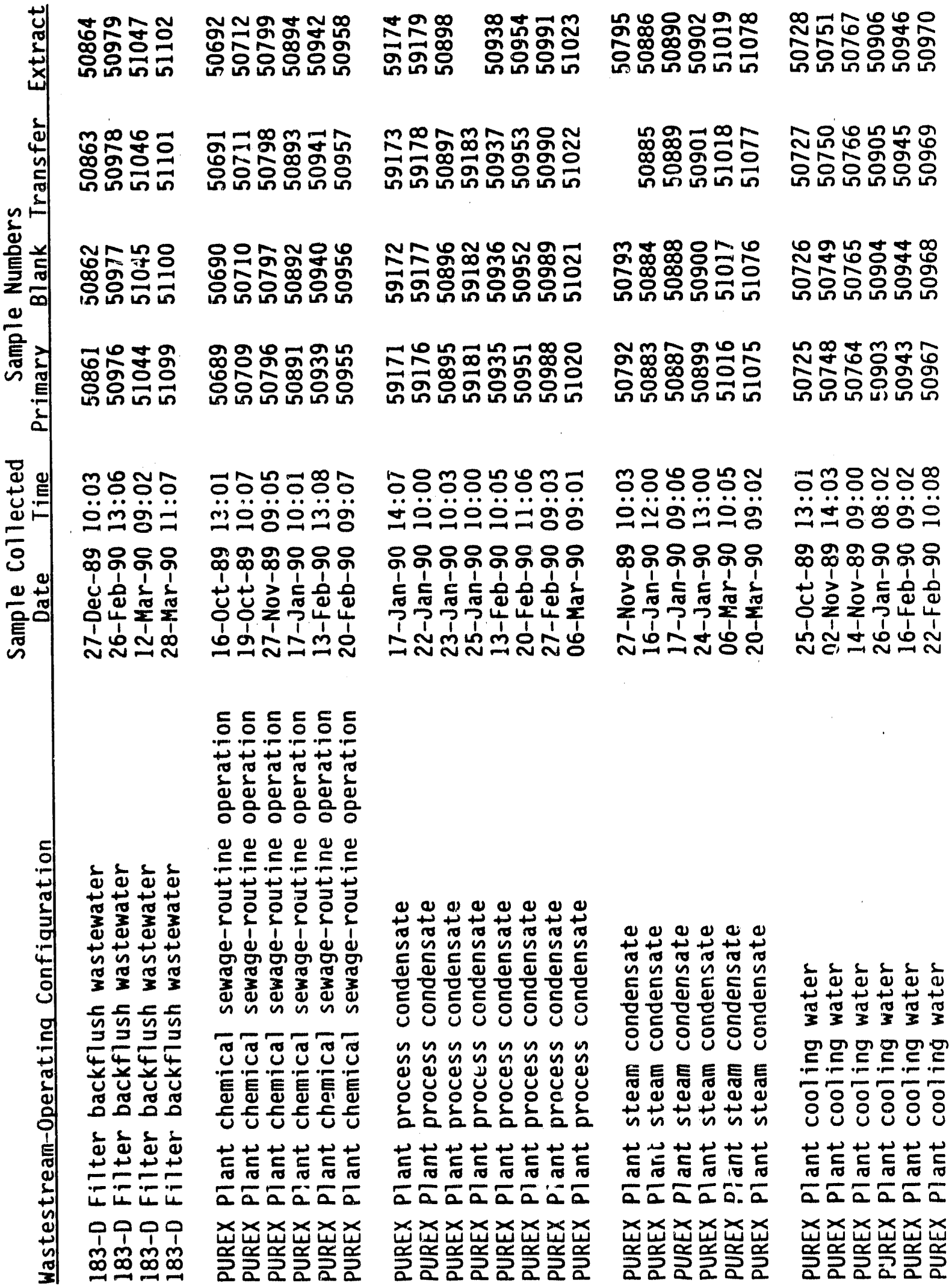


Appendix A--Sample Numbers. (sheet 3 of 5)

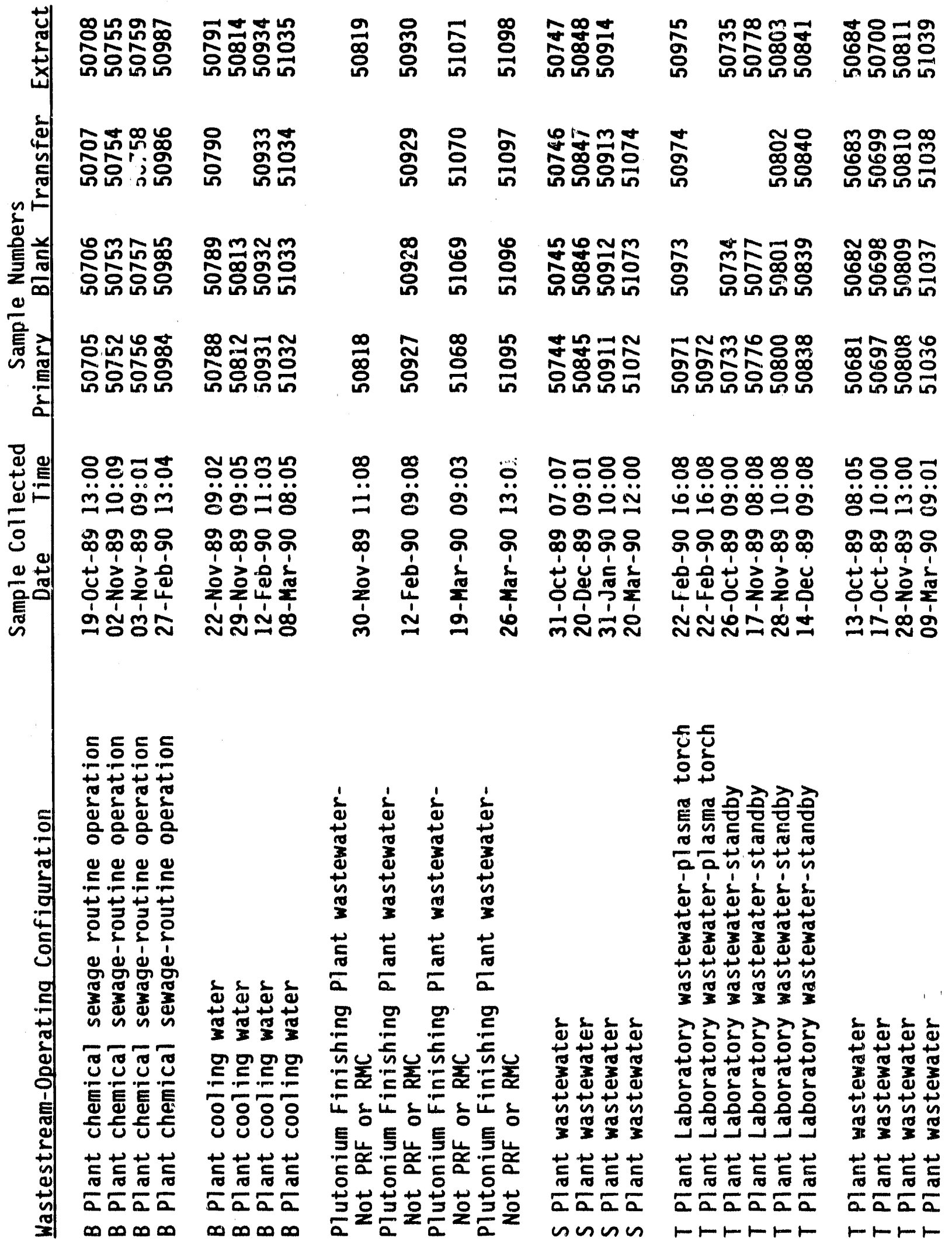


Appendix A--Sample Numbers. (sheet 4 of 5 )

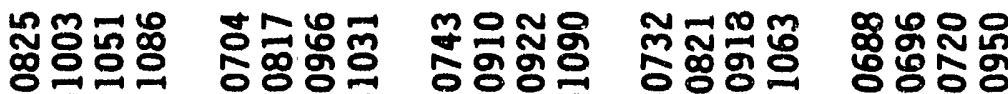

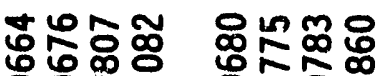
든

융유융유

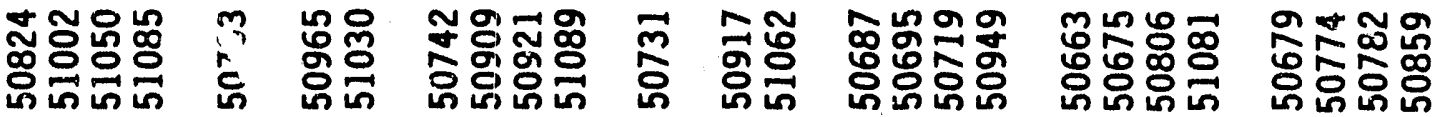

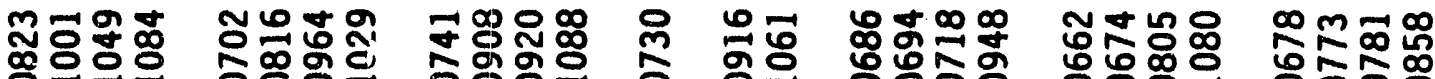

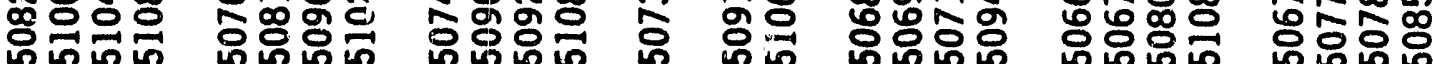

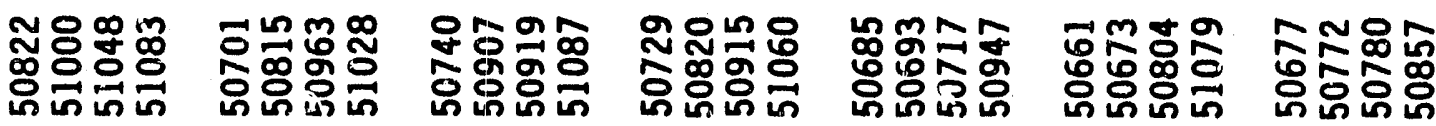

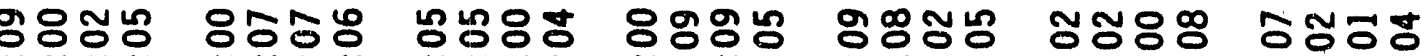

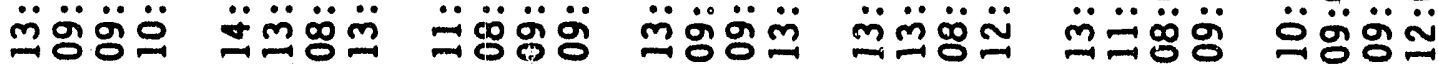

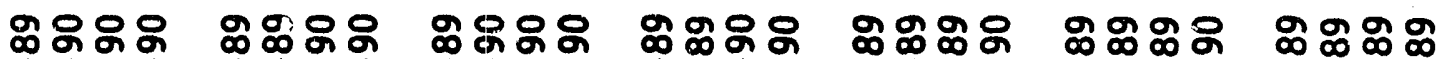

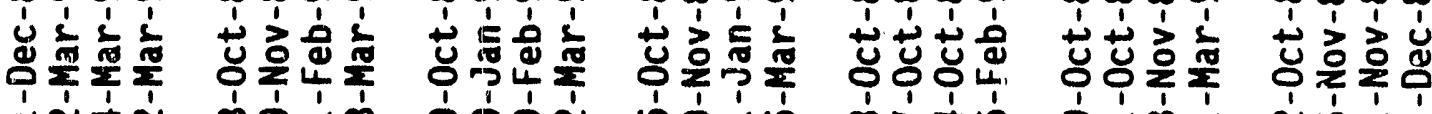

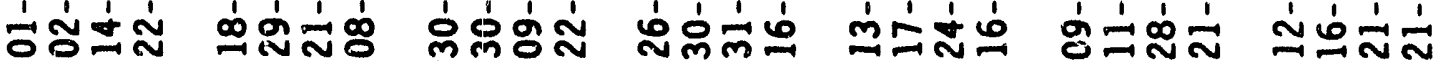

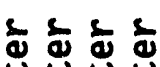
苋芯茎范

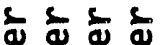

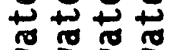

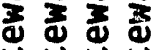
$+$

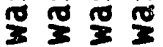
an nu

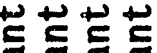
$\frac{\pi}{2} \frac{\pi}{2}$ 가류 뚠

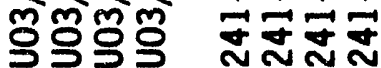

잉요

EEE

的完市

兰兰兰兰

可分

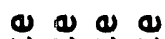

$\stackrel{4}{0}$

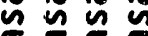

ฮิ ธ్ ธิ

응응흥

…… 틍

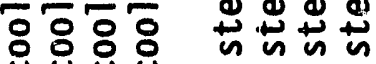

放斿

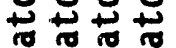

능ㅇㅇㅇㅇㅇㅇㅇㅇㅇ

응융융은

شا شا

$\varangle<\ll<$

บับับ

岕导导
Q

必萬 出 ฮิ ฮ 응헝홍엉 통튜

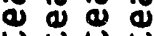
芯芯芯菂 京京京 $+\frac{\omega}{\omega}+\infty$ 형ㅇㅇㅇㅇㅇㅇㅇㅇㅇ 융유융 它 unun

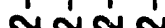
㐫灾恋

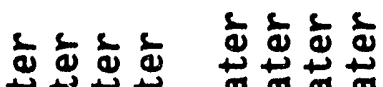
芯芯芯范范节 के के 웅망 드. = รัตำ 858

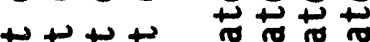
需要需 $>>>$

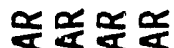
$\frac{1}{d} \dot{d} \frac{1}{d} d$ 离峦恋

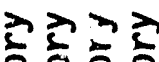
岕芯萬 ㄴㅇㅇㅇㅇㅇㅇㅇㅇㅇㅇㅇ त्ठ unenes ฉั்่่ NNNN

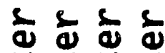
若范范

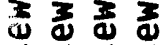

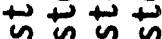

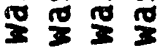
전진준잔 ㅎํㅇㅎㅎ웡ㅎํ ร5ํํㄱ

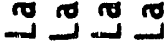

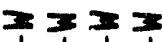
華志交 ลล̃ก 
Appendix A--Sample Numbers. (sheet 5 of 5 )

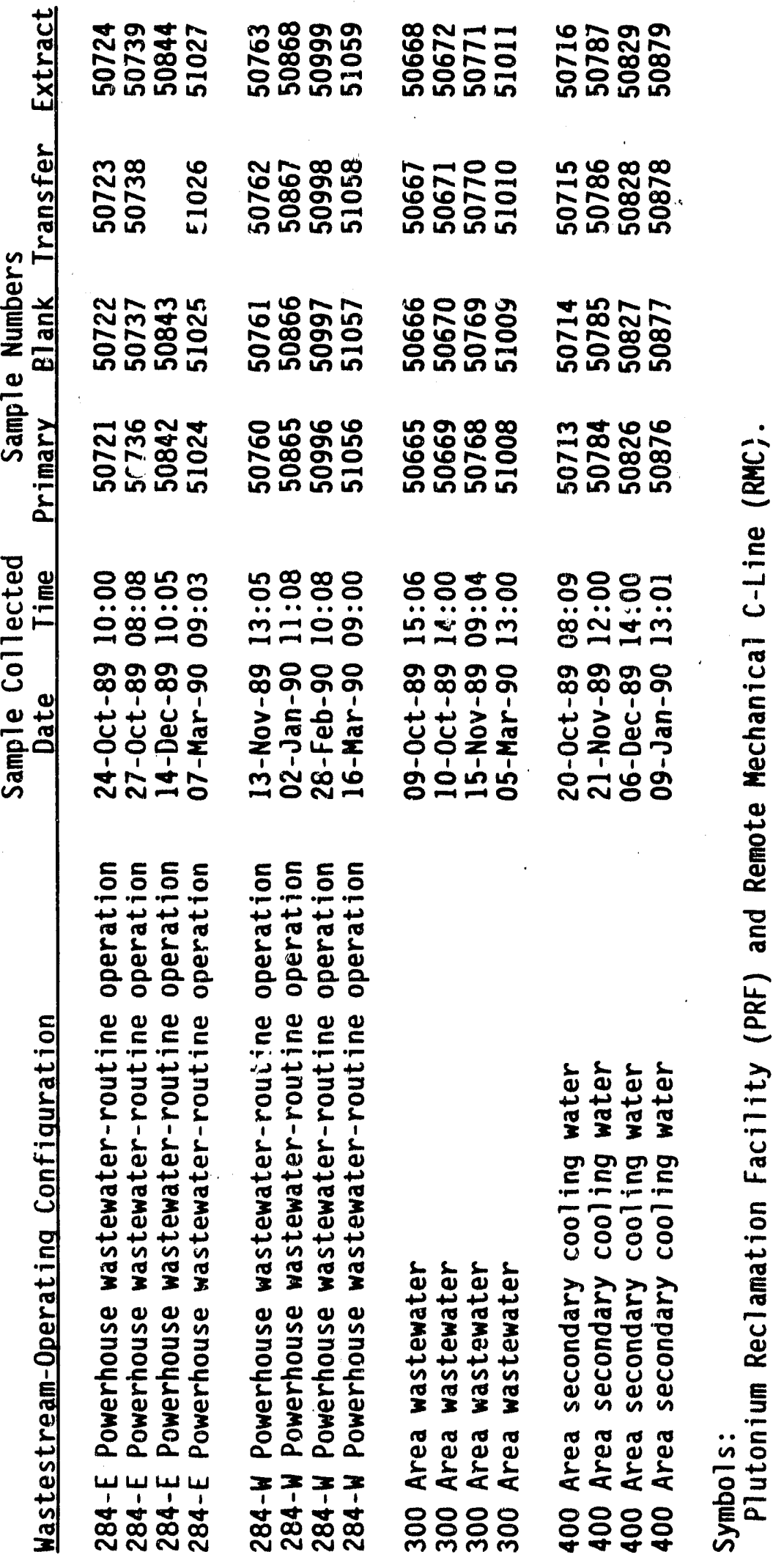


WHC-EP-0355

\section{APPENDIX B}

SAMPLED WASTESTREAMS AND SÄMPLING LOCATIONS 
Appendix B--Sampled Wastestreams and Sampling Location.

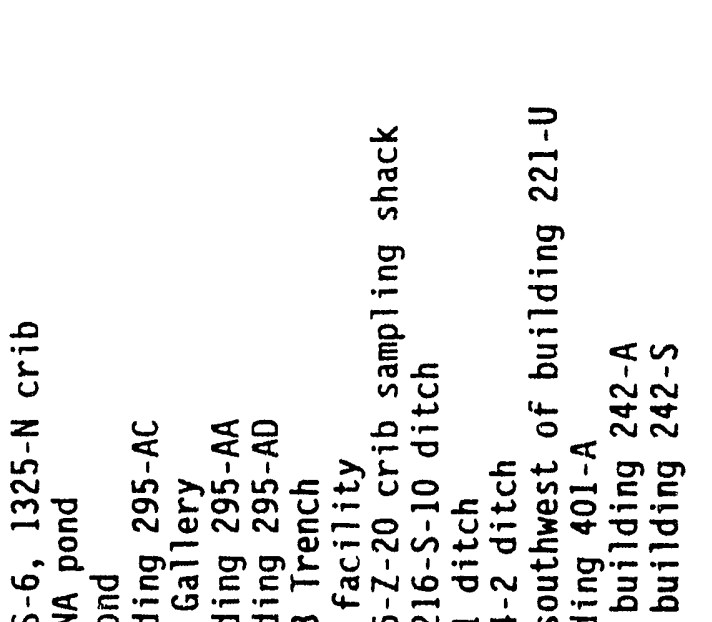

片

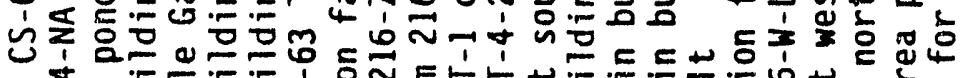

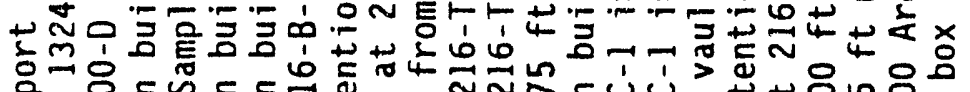

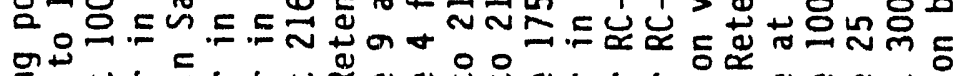

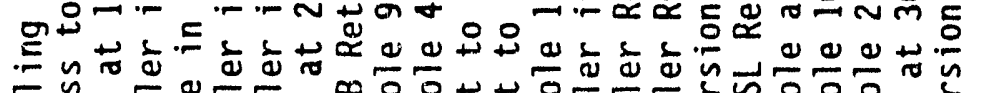

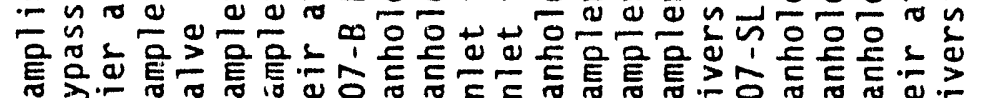

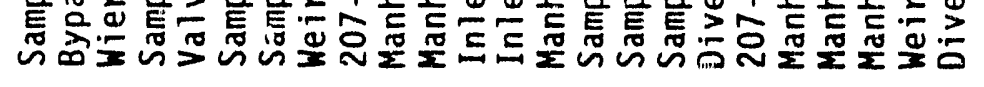

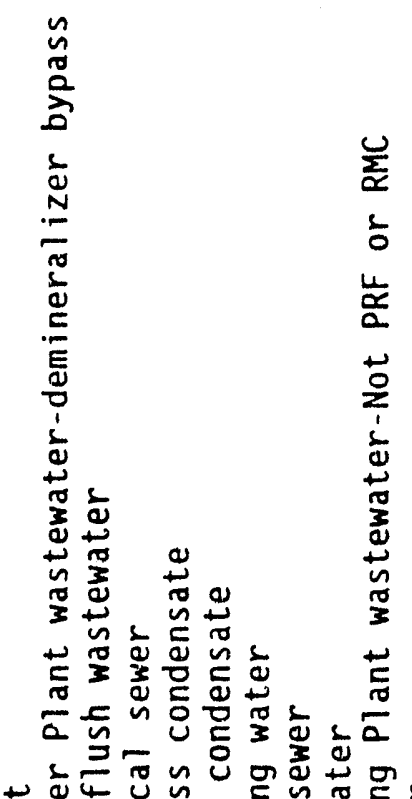

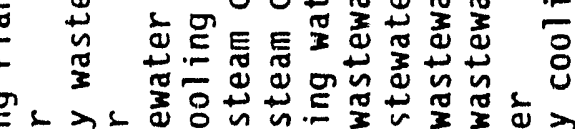

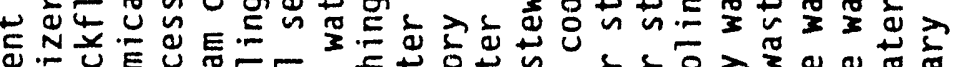

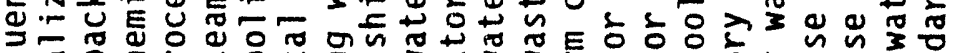

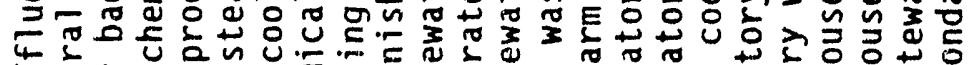

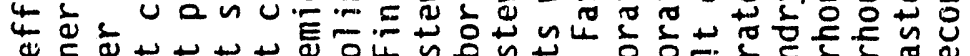

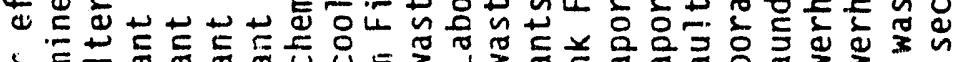

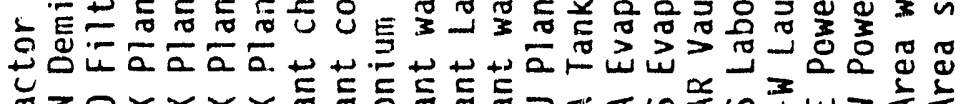

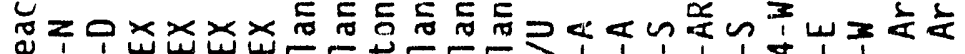
z 
WHC-EP-0355

\section{APPENDIX C}

ANALYTICAL SAMPIEE SPECIFICATIONS 
Appendix C--Analytical Sample Specifications. (sheet 1 of 2)

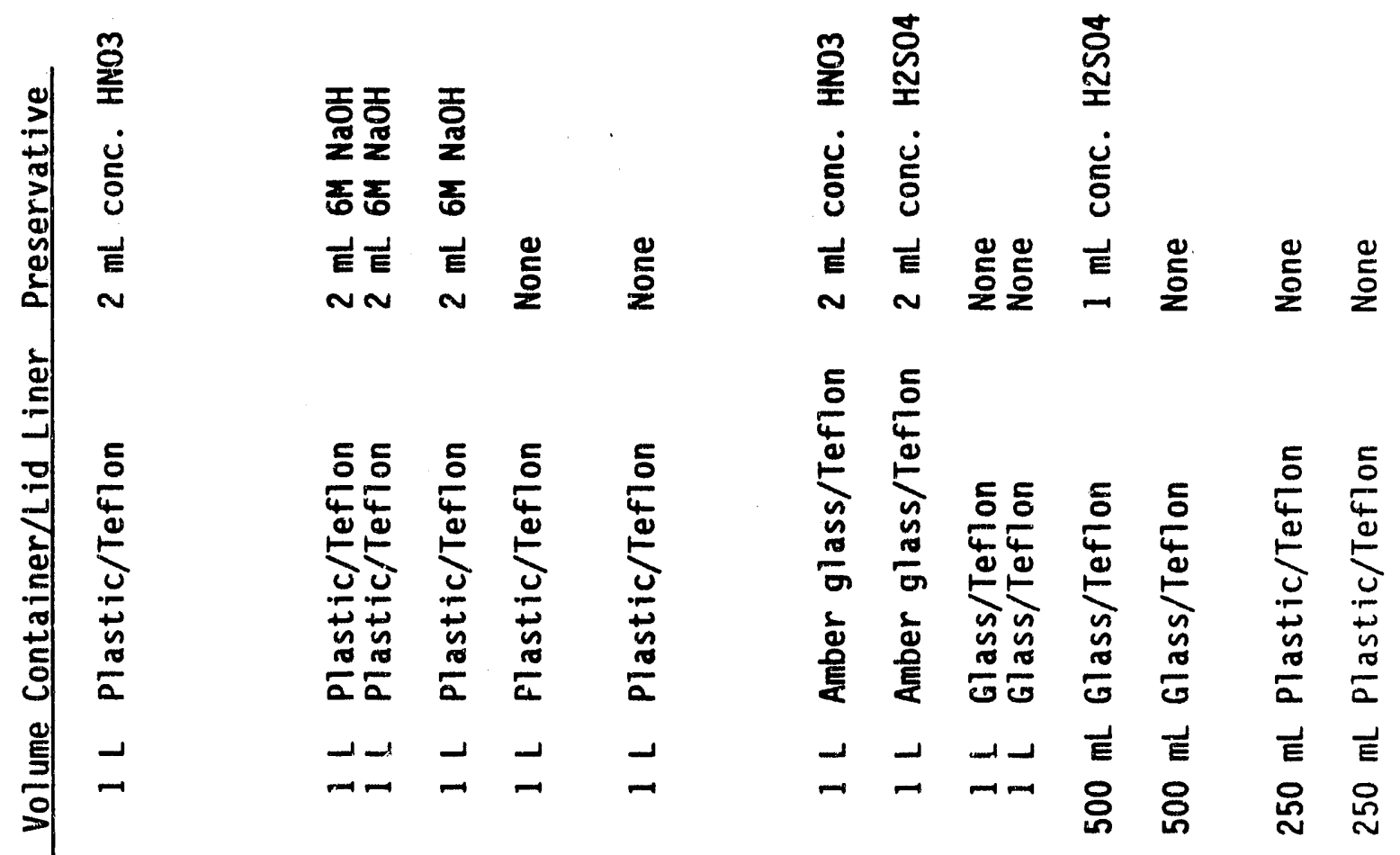

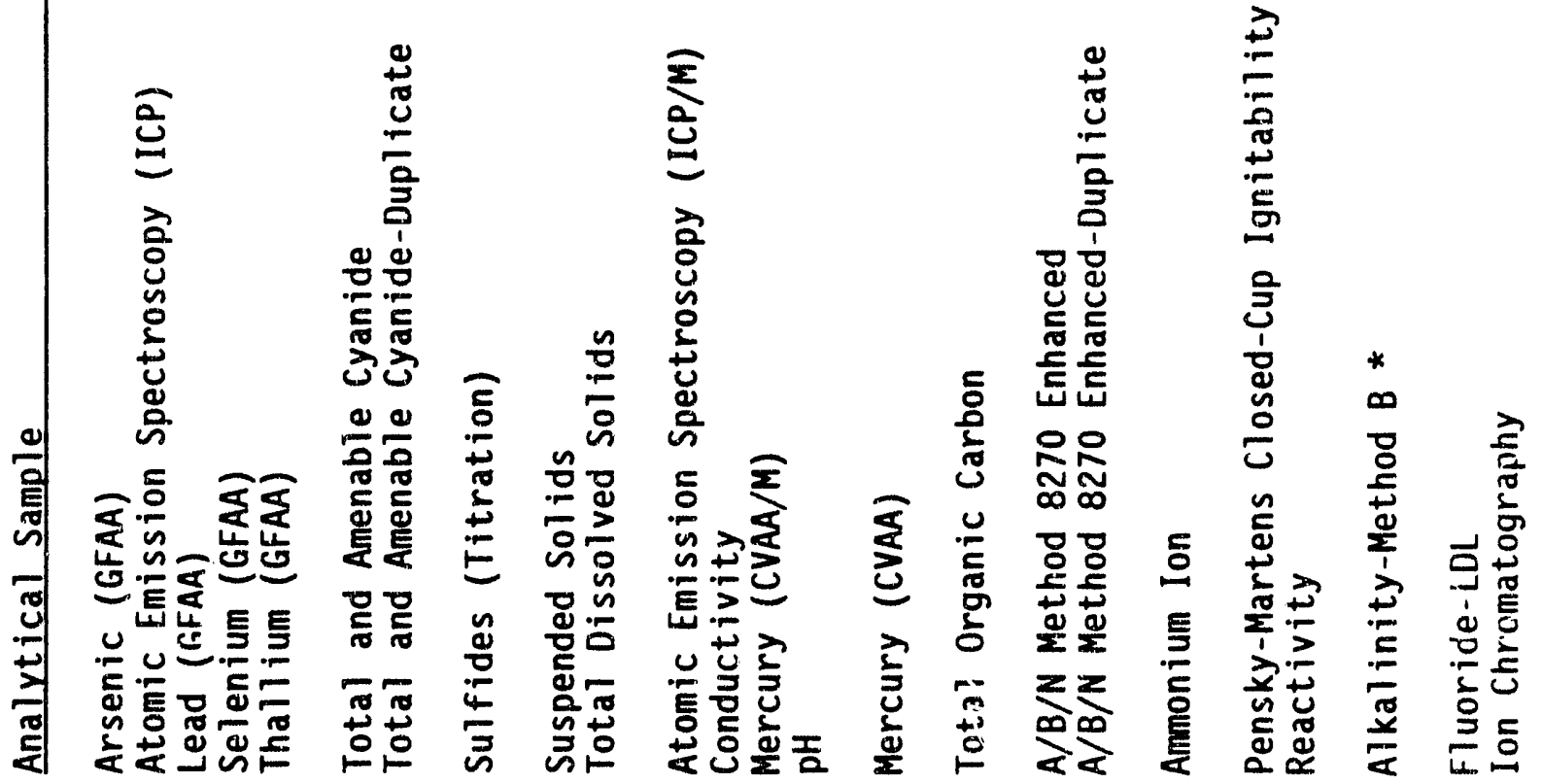


Appendix C--Analytical Sample Specifications. (sheet 2 of 2)

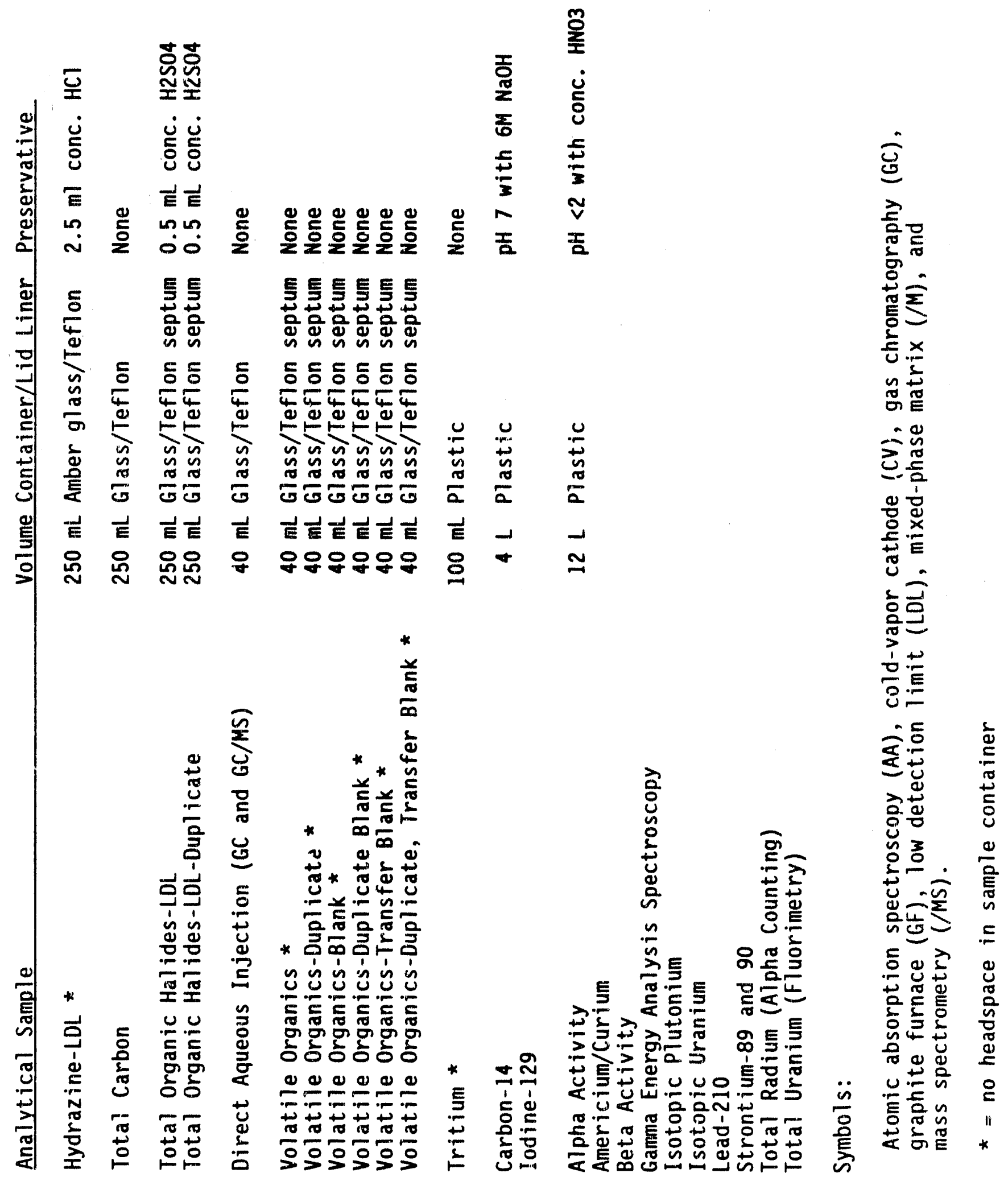


WHC-EP-0355

APPENDIX D

ANALYTICAL RESULTS (DATA SHEETS)

$0-1 / 2$ 


\section{WHC-EP-0355}

\section{DATA REPORT LEGEND}

Sample\# is the number of the sample.

Sample\# suffixes are: B (blank), E (extract) and T (transfer blank). Date is the sampling date.

Results are in ppb (parts per billion) unless otherwise indicated.

The following table lists the methods that are coded in the method column.

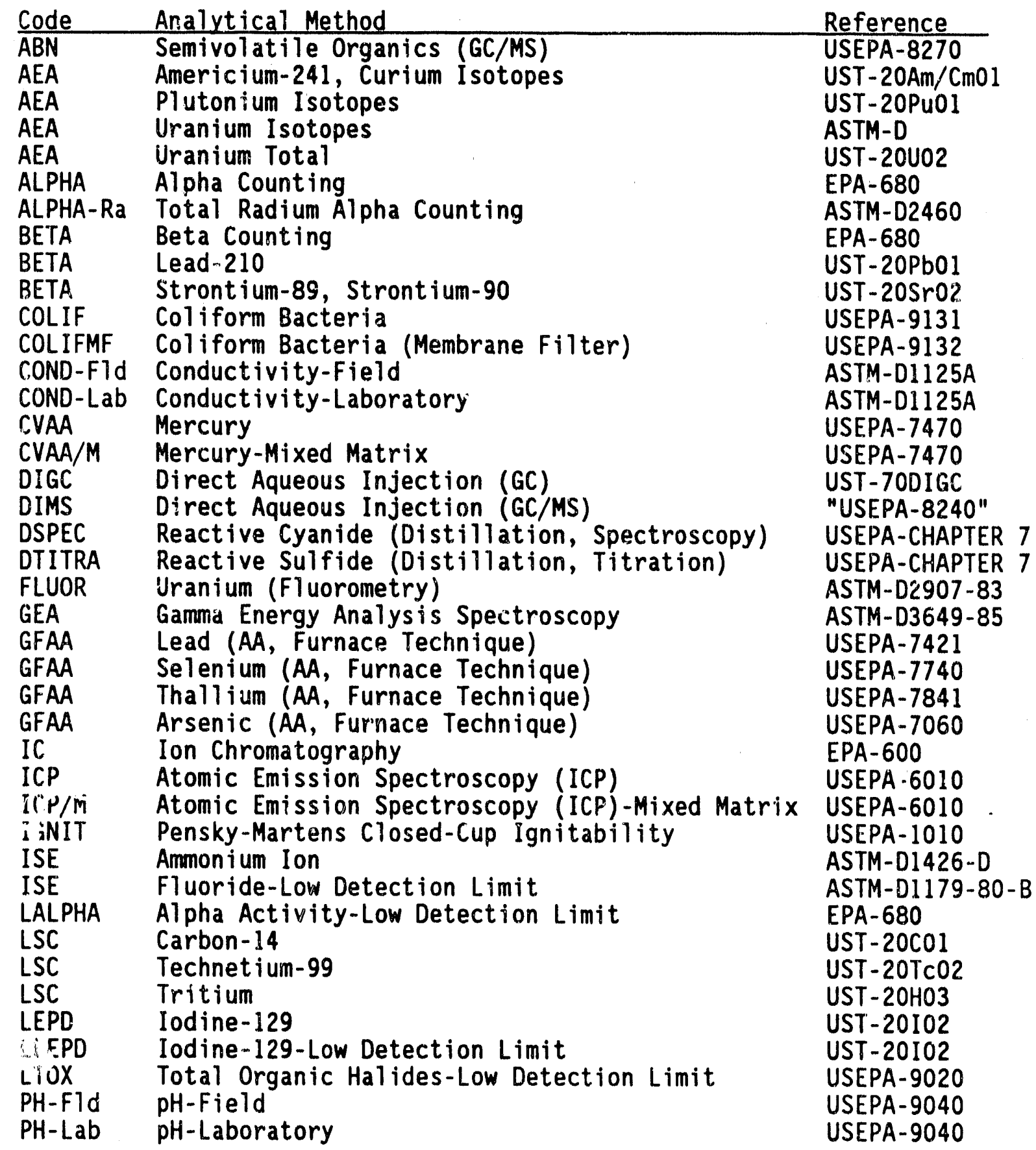


WHC-EP-0355

\section{DATA REPORT LEGEND (Cont'd)}

\begin{tabular}{lll} 
Code & Analytical Method & Reference \\
\hline SPEC & Total and Amenable Cyanide (Spectroscopy) & USEPA-9010 \\
SPEC & Hydrazine-Low Detection Limit (Spectroscopy) & ASTM-D1385 \\
SSOLID & Suspended Solids & SM-208D \\
TC & Total Carbon & USEPA-9060 \\
TDS & Total Dissolved Solids & SM-208B \\
TEMP-FId & Temperature-Field & LOCal \\
TITRA & Alkalinity-Method B (Titration) & ASTM-D1067B \\
TITRA & Sulfides (Titration) & USEPA-9030 \\
TOC & Total Organic Carbon & USEPA-9060 \\
TOX & Total Organic Halides & USEPA-9020 \\
VOA & Volatile Organics (GC/MS) & USEPA-8240
\end{tabular}

Symbols:

Atomic absorption spectroscopy (AA), base/acid-neutral extract analysis (ABN), alpha energy analysis (AEA), cold-vapor cathode (CV), gas chromatography (GC), graphite furnace (GF), low-energy proportional detection (LEPD), liquid scintillation counting (LSC), inductivelycoupled plasma spectroscopy (ICP), volatile organics analysis (VOA), mixed-phase matrix (/M), and mass spectrometry (/MS).

References:

ASTM - "1986 Annual Book of ASTM Standards", American Society for Testing and Materials, Philadelphia, Pennsylvania.

EPA680 "Handbook of Radiochemical Analytical Methods, EPA-680/4-75/001, National Environmental Research Center, Office of Research and Development, U.S. Environmental Protection Agency, Las Vegas, Nevada.

EPA600 "Test Methods for Determination of Inorganic Ions in Water by Ion Chromatography, EPA-600/4-84/017, Environmental Monitoring and Support Laboratory, U.S. Environmental Protection Agency, Cincinnati, Ohio.

SM - "Standard Methods for the Examination of Water and Wastewater", 16th ed., American Public Health Association, American Water Works Association and Water Pollution Control Federation, Washington, D.C.

USEPA- "Test Methods for Evaluating Solid Waste Physical/Chemical Methods", 3rd ed., SW-846, U.S. Environmental Protection Agency, Washington, D.C.

UST - "Procedure Manual", UST-RD-PM, Section 10, U.S. Testing Company, Incorporated, Richiand, Washington. 
DATA FOR 300 Area Wastewater-Sample Point 1

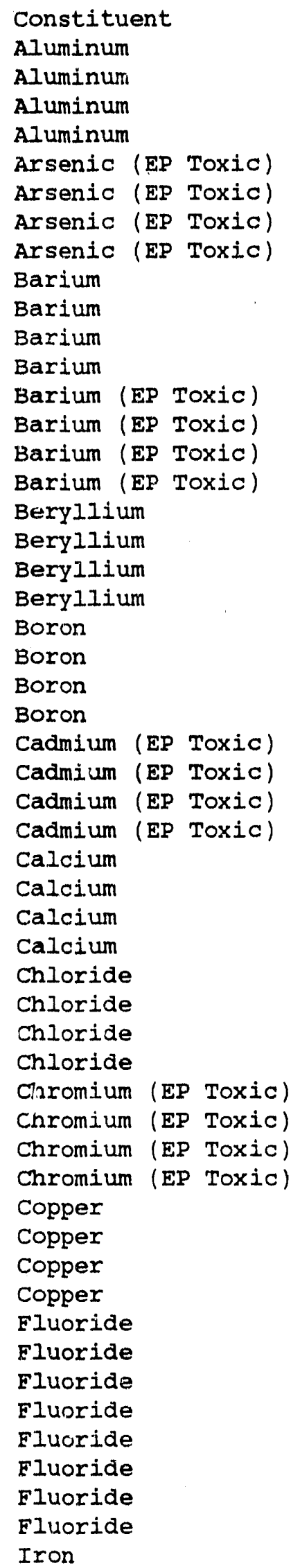

\begin{tabular}{|c|c|c|c|}
\hline Sample \# & Date & Method & Result \\
\hline 50665 & $10 / 09 / 89$ & ICP & $1.83 E+02$ \\
\hline 50669 & $10 / 10 / 89$ & ICP & $<1.50 \mathrm{E}+02$ \\
\hline 50768 & $11 / 15 / 89$ & ICP & $4.48 E+02$ \\
\hline 51008 & $3 / 05 / 90$ & ICP & $<1,50 \mathrm{E}+02$ \\
\hline $50665 \mathrm{E}$ & $10 / 09 / 89$ & ICP & $<5.00 E+02$ \\
\hline 50669E & $10 / 10 / 89$ & ICP & $<5.00 E+02$ \\
\hline $50768 \mathrm{E}$ & $11 / 15 / 89$ & ICP & $<5.00 E+02$ \\
\hline $51008 \mathrm{E}$ & $3 / 05 / 90$ & ICP & $<5.00 E+02$ \\
\hline 50665 & $10 / 09 / 89$ & ICP & $3.60 E+01$ \\
\hline 50669 & $10 / 10 / 89$ & ICP & 3. $30 \mathrm{E}+01$ \\
\hline 50768 & $11 / 15 / 89$ & ICP & $2.90 E+01$ \\
\hline 51008 & $3 / 05 / 90$ & ICP & $3.00 E+01$ \\
\hline $50665 \mathrm{E}$ & $10 / 09 / 89$ & ICP & $<1.00 \mathrm{E}+03$ \\
\hline $50669 \mathrm{E}$ & $10 / 10 / 89$ & ICP & $<1.00 \mathrm{E}+03$ \\
\hline $50768 \mathrm{E}$ & $11 / 15 / 89$ & ICP & $<1.00 E+03$ \\
\hline $51008 \mathrm{E}$ & $3 / 05 / 90$ & ICP & $<1.00 \mathrm{E}+03$ \\
\hline 50665 & $10 / 09 / 89$ & ICP & $<5.00 E+00$ \\
\hline 50669 & $10 / 10 / 89$ & ICP & $<5.00 E+00$ \\
\hline 50768 & $11 / 15 / 89$ & ICP & $8.00 E+00$ \\
\hline 51008 & $3 / 05 / 90$ & ICP & $<5.00 E+00$ \\
\hline 50665 & $10 / 09 / 89$ & ICP & $2.80 \mathrm{E}+01$ \\
\hline 50669 & $10 / 10 / 89$ & ICP & 1. $40 \mathrm{E}+01$ \\
\hline 50768 & $11 / 15 / 89$ & ICP & 1. 10E+01 \\
\hline 51008 & $3 / 05 / 90$ & ICP & $2.50 \mathrm{E}+01$ \\
\hline $50665 \mathrm{E}$ & $10 / 09 / 89$ & ICP & $<1.00 E+02$ \\
\hline 50669E & $10 / 10 / 89$ & ICP & $<1 ., 0 \mathrm{E}+02$ \\
\hline $50768 \mathrm{E}$ & $11 / 15 / 89$ & ICP & $<1.00 \mathrm{E}+02$ \\
\hline $51008 \mathrm{E}$ & $3 / 05 / 90$ & ICP & $<1.00 \mathrm{E}+02$ \\
\hline 50665 & $10 / 09 / 89$ & ICP & $2.04 \mathrm{E}+04$ \\
\hline 50669 & $10 / 10 / 89$ & ICP & $1.82 \mathrm{E}+04$ \\
\hline 50768 & $11 / 15 / 89$ & ICP & $1.91 \mathbf{E}+04$ \\
\hline 51008 & $3 / 05 / 90$ & ICP & $2.02 \mathrm{E}+04$ \\
\hline 50665 & $10 / 09 / 89$ & $I C$ & $4.30 E+03$ \\
\hline 50669 & $10 / 10 / 89$ & IC & $3.90 \mathrm{E}+03$ \\
\hline 50768 & $11 / 15 / 89$ & IC & $3.70 \mathrm{E}+03$ \\
\hline 51008 & $3 / 05 / 90$ & $I C$ & $4.40 E+03$ \\
\hline $50665 E$ & $10 / 09 / 89$ & ICP & $<5.00 \mathrm{E}+02$ \\
\hline $50669 \mathrm{E}$ & $10 / 10 / 89$ & $I C P$ & $<5.00 \Sigma+02$ \\
\hline $50768 \mathrm{E}$ & $11 / 15 / 89$ & ICP & $<5.00 \mathrm{E}+02$ \\
\hline $51008 \mathrm{E}$ & $3 / 05 / 90$ & ICP & $<5.00 \mathrm{E}+02$ \\
\hline 50665 & $10 / 09 / 89$ & ICP & $2.20 \mathrm{E}+01$ \\
\hline 50669 & $10 / 10 / 89$ & ICP & $<1.00 \mathrm{E}+01$ \\
\hline 50768 & $11 / 15 / 89$ & ICP & $1.90 \mathrm{E}+01$ \\
\hline 51008 & $3 / 05 / 90$ & ICP & 1. $30 \mathrm{E}+01$ \\
\hline 50665 & $10 / 09 / 89$ & IC & $<5.00 E+02$ \\
\hline 50665 & $10 / 09 / 89$ & ISE & $2.13 \mathrm{E}+02$ \\
\hline 50669 & $10 / 10 / 89$ & $I C$ & $<5.00 E+02$ \\
\hline 50669 & $10 / 10 / 89$ & ISE & $2.18 \mathrm{E}+02$ \\
\hline 50768 & $11 / 15 / 89$ & IC & $<5.00 E+02$ \\
\hline 50768 & $11 / 15 / 89$ & ISE & $2.05 \mathrm{E}+02$ \\
\hline 51008 & $3 / 05 / 90$ & IC & $<5.00 E+02$ \\
\hline 51008 & $3 / 05 / 90$ & ISE & $1.59 \mathrm{E}+02$ \\
\hline 50665 & $10 / 09 / 89$ & ICP & $7.30 E+01$ \\
\hline
\end{tabular}


WHC-EP-0355, Appendix D

DATA FOR 300 Area Wastewater-Sample Point 1 Continued

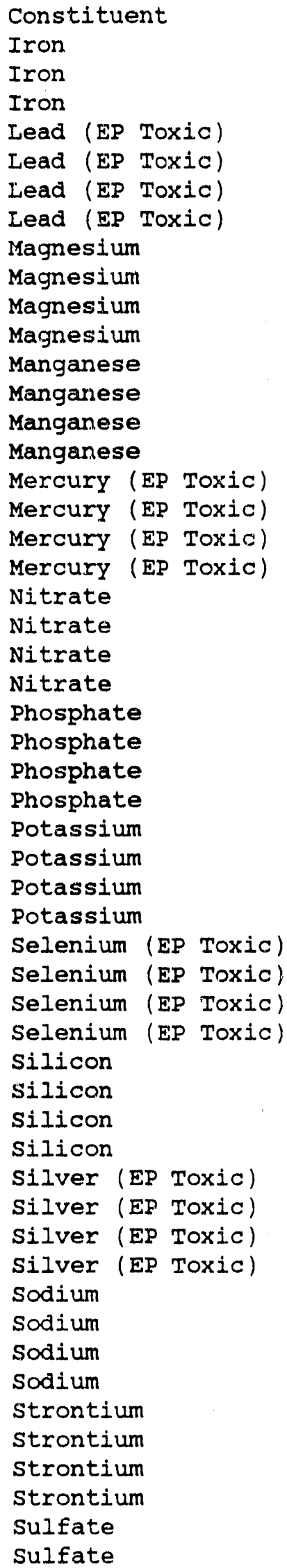

\begin{tabular}{|c|c|c|c|}
\hline Sample \# & Date & Method & Result \\
\hline 50669 & $10 / 10 / 89$ & ICP & 4. $70 E+01$ \\
\hline 50768 & $11 / 15 / 89$ & ICP & $1.25 \mathrm{E}+02$ \\
\hline 51008 & $3 / 05 / 90$ & ICP & 4. $40 \mathrm{E}+01$ \\
\hline $50665 \mathrm{E}$ & $10 / 09 / 89$ & ICP & $<5.00 \mathrm{E}+02$ \\
\hline $50669 \mathrm{E}$ & $10 / 10 / 89$ & ICP & $<5.00 \mathrm{E}+02$ \\
\hline $50768 \mathrm{E}$ & $11 / 15 / 89$ & ICP & $<5.00 E+02$ \\
\hline $51008 \mathrm{E}$ & $3 / 05 / 90$ & ICP & $<5.00 E+02$ \\
\hline 50665 & $10 / 09 / 89$ & ICP & $4.92 \mathrm{E}+03$ \\
\hline 50669 & $10 / 10 / 89$ & ICP & $4.46 \mathrm{E}+03$ \\
\hline 50768 & $11 / 15 / 89$ & ICP & $4.24 E+03$ \\
\hline 51008 & $3 / 05 / 90$ & ICP & $4.68 E+03$ \\
\hline 50665 & $10 / 09 / 89$ & ICP & $6.00 \mathrm{E}+00$ \\
\hline 50669 & $10 / 10 / 89$ & ICP & $<5.00 \mathrm{E}+00$ \\
\hline 50768 & $11 / 15 / 89$ & ICP & $6.00 E+00$ \\
\hline 51008 & $3 / 05 / 90$ & ICP & $<5.00 \mathrm{E}+00$ \\
\hline $50665 \mathrm{E}$ & $10 / 09 / 89$ & CVAA/M & $<2.00 E+01$ \\
\hline $50669 \mathrm{E}$ & $10 / 10 / 89$ & $\mathrm{CVAA} / \mathrm{M}$ & $<2.00 E+01$ \\
\hline $50768 \mathrm{E}$ & $11 / 15 / 89$ & CVAA/M & $<2.00 E+01$ \\
\hline $51008 \mathrm{E}$ & $3 / 05 / 90$ & $\mathrm{CVAA} / \mathrm{M}$ & $<2.00 \mathrm{E}+01$ \\
\hline 50665 & $10 / 09 / 89$ & IC & $1.00 E+03$ \\
\hline 50669 & $10 / 10 / 89$ & IC & $1.30 \mathrm{E}+03$ \\
\hline 50768 & $11 / 15 / 89$ & IC & $9.00 E+02$ \\
\hline 51008 & $3 / 05 / 90$ & IC & $2.10 E+03$ \\
\hline 50665 & $10 / 09 / 89$ & IC & $<1.00 \mathrm{E}+03$ \\
\hline 50669 & $10 / 10 / 89$ & IC & $1.40 \mathrm{E}+\mathrm{C} 3$ \\
\hline 50768 & $11 / 15 / 89$ & IC & $<1.00 E+03$ \\
\hline 51008 & $3 / 05 / 90$ & $I C$ & $<1.00 \mathrm{E}+03$ \\
\hline 50665 & $10 / 09 / 89$ & ICP & $1.06 \mathrm{E}+03$ \\
\hline 50669 & $10 / 10 / 89$ & ICP & $9.42 \mathrm{E}+02$ \\
\hline 50768 & $11 / 15 / 89$ & ICP & $7.64 \mathrm{E}+02$ \\
\hline 51008 & $3 / 05 / 90$ & ICP & $9.73 E+02$ \\
\hline $50665 \mathrm{E}$ & $10 / 09 / 89$ & ICP & $<5.00 \mathrm{E}+02$ \\
\hline $50669 \mathrm{E}$ & $10 / 10 / 89$ & ICP & $<5.00 E+02$ \\
\hline $50768 \mathrm{E}$ & $1.1 / 15 / 89$ & ICP & $<5.00 E+02$ \\
\hline $51008 \mathrm{E}$ & $3 / 05 / 90$ & ICP & $<5.00 E+02$ \\
\hline 50665 & $10 / 09 / 89$ & ICP & $2.68 \mathrm{E}+03$ \\
\hline 50669 & $10 / 10 / 89$ & ICP & $2.25 E+03$ \\
\hline $50 \% 68$ & $11 / 15 / 89$ & ICP & $2.41 \mathrm{E}+03$ \\
\hline 51008 & $3 / 05 / 90$ & J.CP & $2.90 E+03$ \\
\hline $50665 \mathrm{E}$ & $10 / 09 / 89$ & ICP & $<5.00 E .02$ \\
\hline $50669 \mathrm{E}$ & $10 / 10 / 89$ & ICP & $<5.00 E+02$ \\
\hline $50768 \mathrm{E}$ & $11 / 15 / 89$ & ICP & $<5.00 E+02$ \\
\hline $51008 E$ & $3 / 05 / 90$ & ICP & $<5.00 E+02$ \\
\hline 50665 & $10 / 09 / 89$ & ICP & 4. $99 \mathrm{E}+03$ \\
\hline 50669 & $10 / 10 / 89$ & ICP & $3.90 \mathrm{E}+03$ \\
\hline 50768 & $11 / 15 / 89$ & ICP & $2.74 E+03$ \\
\hline 51008 & $3 / 05 / 90$ & ICP & $6.77 E+\cup 3$ \\
\hline 50665 & $10 / 09 / 89$ & ICP & $1.16 \mathrm{E}+02$ \\
\hline 50669 & $10 / 10 / 89$ & ICP & $1.02 \mathrm{E}+02$ \\
\hline 50768 & $11 / 15 / 89$ & ICP & $9.60 \mathrm{E}+01$ \\
\hline 51008 & $3 / 05 / 90$ & ICP & $1.01 \mathrm{E}+02$ \\
\hline 50665 & $10 / 09 / 89$ & IC & $1.82 \mathrm{E}+04$ \\
\hline 50669 & $10 / 10 / 89$ & IC & $1.74 \mathrm{E}+04$ \\
\hline
\end{tabular}


DATA FOR 300 Area Wastewater-Sample Point 1 Continued

\begin{tabular}{|c|c|c|c|c|}
\hline Constituent & Sample \# & Date & Method & Result \\
\hline Sulfate & 50768 & $1.1 / 15 / 89$ & IC & $1.76 \mathrm{E}+04$ \\
\hline Sulfate & 51008 & $3 / 05 / 90$ & IC & $1.51 \mathrm{E}+04$ \\
\hline Uranium & 50665 & $16 / 09 / 89$ & GEA & $1.45 \mathrm{E}+00$ \\
\hline Uranium & 50768 & $11 / 15 / 89$ & GEA & 1. $45 \mathrm{E}+00$ \\
\hline Uranium & 51008 & $3 / 05 / 90$ & GEA & $1.41 \mathrm{E}+00$ \\
\hline Zinc & 50665 & $10 / 09 / 89$ & ICP & $2.70 \mathrm{E}+01$ \\
\hline Zinc & 50669 & $10 / 10 / 89$ & ICP & $1.00 \mathrm{E}+01$ \\
\hline Zinc & 50768 & $11 / 15 / 89$ & ICP & $2.50 \mathrm{E}+01$ \\
\hline Zinc & 51008 & $3 / 05 / 90$ & ICP & $1.30 \mathrm{E}+01$ \\
\hline Acetone & 50665 & $10 / 09 / 89$ & VOA & $<1.00 E+01$ \\
\hline Acetone & 50665 & $10 / 09 / 89$ & $\mathrm{ABN}$ & $<1.00 E+01$ \\
\hline Acetone & $50665 \mathrm{~B}$ & $10 / 09 / 89$ & VOA & $<1.00 E+01$ \\
\hline Acetone & $50665 \mathrm{~T}$ & $10 / 09 / 89$ & VOA & $<1.00 \mathrm{E}+01$ \\
\hline Acetone & 50669 & $10 / 10 / 89$ & VOA & $<1.00 \mathrm{E}+01$ \\
\hline Acetone & 50669 & $10 / 10 / 89$ & $A B N$ & $<1.00 \mathrm{E}+01$ \\
\hline Acetone & $50669 \mathrm{~B}$ & $10 / 10 / 89$ & VOA & $<1.00 E+01$ \\
\hline Acetone & $50669 \mathrm{~T}$ & $10 / 10 / 89$ & VOA & $<1.00 E+01$ \\
\hline Acetone & 50768 & $11 / 15 / 89$ & VOA & $<1.00 E+01$ \\
\hline Acetone & 50768 & $11 / 15 / 89$ & $\mathrm{ABN}$ & $<1.00 \mathrm{E}+01$ \\
\hline Acetone & $50768 B$ & $11 / 15 / 89$ & VOA & $<1.00 \mathrm{E}+01$ \\
\hline Acetone & $50768 \mathrm{~T}$ & $11 / 15 / 89$ & VOA & $<1.00 E+01$ \\
\hline Acetone & 51008 & $3 / 05 / 90$ & VOA & $2.00 \mathrm{E}+01$ \\
\hline Acetone & 51008 & $3 / 05 / 90$ & $\mathrm{ABN}$ & $<1.00 \mathrm{E}+01$ \\
\hline Acetone & $51008 \mathrm{~B}$ & $3 / 05 / 90$ & VOA & $<1.00 \mathrm{E}+01$ \\
\hline Acetone & $51008 \mathrm{~T}$ & $3 / 05 / 90$ & VOA & $<1.00 E+01$ \\
\hline Ammonia & 50665 & $10 / 09 / 89$ & ISE & 8. $80 \mathrm{E}+01$ \\
\hline Immonia & 50669 & $10 / 10 / 89$ & ISE & $6.10 \mathrm{E}+01$ \\
\hline Ammonia & 50768 & $11 / 15 / 89$ & ISE & $2.53 \mathrm{E}+02$ \\
\hline Ammonia & 51008 & $3 / 05 / 90$ & ISE & $<5.00 \mathrm{E}+01$ \\
\hline Bis(2-ethylhexyl) phthalate & 50665 & $10 / 09 / 89$ & $A B N$ & $<1.00 E+01$ \\
\hline Bis(2-ethylhexyl) phthalate & 50669 & $10 / 10 / 89$ & $A B N$ & $<1.00 \mathrm{E}+01$ \\
\hline Bis(2-ethylhexyl) phthalate & 50768 & $11 / 15 / 89$ & $\mathrm{ABN}$ & $1.40 \mathrm{E}+01$ \\
\hline Bis(2-ethylhexyl) phthalate & 51008 & $3 / 05 / 90$ & $\mathrm{ABN}$ & $<1.00 \mathrm{E}+01$ \\
\hline 1 -Butanol & 50665 & $10 / 09 / 89$ & DIGC & $<1.00 \mathrm{E}+04$ \\
\hline 1-Butanol & 50669 & $10 / 10 / 89$ & DIGC & $<1.00 E+04$ \\
\hline 1-Butanol & 50768 & $11 / 15 / 89$ & DIGC & $<1.00 \mathrm{E}+04$ \\
\hline 1-Butanol & 51008 & $3 / 05 / 90$ & VOA & 3. 10E+01 \\
\hline 1-Butano1 & 51008 & $3 / 05 / 90$ & DIGC & $<1.00 \mathrm{E}+04$ \\
\hline Dichloromethane & 50665 & $10 / 09 / 89$ & VOA & $<2.60 \mathrm{E}+00$ \\
\hline Dichloromethane & $50665 B$ & $10 / 09 / 89$ & VOA & $5.50 E+02$ \\
\hline Dichloromethane & $50665 \mathrm{~T}$ & $10 / 09 / 89$ & VOA & $5.80 \mathrm{E}+01$ \\
\hline DichJoromethane & 50669 & $10 / 10 / 89$ & VOA & $<5.00 \mathrm{E}+00$ \\
\hline Dichloromethane & $50669 B$ & $10 / 10 / 89$ & VOA & $5.30 \mathrm{E}+02$ \\
\hline Dichloromethane & $50669 \mathrm{~T}$ & $10 / 10 / 89$ & VOA & $4.90 \mathrm{E}+02$ \\
\hline Dichloromethane & 50768 & $11 / 15 / 89$ & VOA & $<5.00 \mathrm{E}+00$ \\
\hline Dichloromethane & $50768 \mathrm{~B}$ & $11 / 15 / 89$ & VOA & $<5.00 \mathrm{E}+00$ \\
\hline Dichloromethane & $50768 \mathrm{~T}$ & $11 / 15 / 89$ & VOA & $<5.00 \mathrm{E}+00$ \\
\hline Dichloromethane & 51008 & $3 / 05 / 90$ & VOA & $<5.00 \mathrm{E}+00$ \\
\hline Dichloromethane & $51008 \mathrm{~B}$ & $3 / 05 / 90$ & VOA & $<5.00 \mathrm{E}+00$ \\
\hline Dichloromethane & $51008 \mathrm{~T}$ & $3 / 05 / 90$ & VOA & $<5.00 \mathrm{E}+00$ \\
\hline Trichloromethane & 50665 & $10 / 09 / 89$ & VOA & I. $90 \mathrm{E}+01$ \\
\hline Trichloromethane & $50665 B$ & $10 / 09 / 89$ & VOA & $<5.00 \mathrm{E}+00$ \\
\hline Trichloromethane & $50665 \mathrm{~T}$ & $10 / 09 / 89$ & VOA & $<5.00 E+00$ \\
\hline
\end{tabular}


WHC-EP-0355, Appendix D

DATA FOR 300 Area Wastewater-Sample Point 1

Continued

Constituent

Trichloromethane

Trichloromethane

Trichloromethane

Trichloromethane

Trichloromethane

Trichloromethane

Trichloromethane

Trichloromethane

Trichloromethane

Unknown

Alkalinity (Method B)

Alkalinity (Method B)

Alkalinity (Method B)

Alkalinity (Method B)

Alpha Activity (pCi/L)

Alpha Activity (pCi/L)

Alpha Activity (pCi/L)

Condu tivity (us)

Conductivity (us)

Conductivity (us)

Conductivity (uS)

Ingitability (degrees F)

Ingitability (degrees F)

Ingitability (degrees F)

Ingitability (degrees F)

$\mathrm{pH}$ (dimensionless)

$\mathrm{pH}$ (dimensionless)

$\mathrm{pH}$ (dimensionless)

pH (dimensionless)

Reactivity Cyanide ( $\mathrm{mg} / \mathrm{kg}$ )

Reactivity Cyanide ( $\mathrm{mg} / \mathrm{kg}$ )

Reactivity Cyanide $(\mathrm{mg} / \mathrm{kg})$

Reactivity Cyanide (mg/kg)

Reactivity sulfide (mg/ $\mathrm{kg}$ )

Reactivity sulfide ( $\mathrm{mg} / \mathrm{kg}$ )

Reactivity Sulfide (mg/ $\mathrm{kg})$

Reactivity Sulfide ( $\mathrm{mg} / \mathrm{kg}$ )

$\operatorname{TDS}(\mathrm{m} g / \mathrm{L})$

$\operatorname{TDS}(\mathrm{mg} / \mathrm{L})$

$\operatorname{TDS}(\mathrm{mg} / \mathrm{L}$ )

TDS ( $\mathrm{mg} / \mathrm{L}$ )

Temperature (degrees C)

Temperature (degrees $C$ )

Temperature (degrees C)

Temperature (degrees $C$ )

$\operatorname{TOC}(u g / g)$

TOC $(u g / g)$

TOC (ug/g)

TOC (ug/g)

Total Carbon (ug/g)

Total Carbon (ug/g)

Total Carbon (ug/g)

Total Carbon (ug/g)

\begin{tabular}{|c|c|c|c|}
\hline Sample \# & Date & Method & Result \\
\hline & & & \\
\hline $50669 \mathrm{~B}$ & $10 / 10 / 89$ & VOA & $<5.00 \mathrm{E}+00$ \\
\hline $50669 \mathrm{~T}$ & $10 / 10 / 89$ & VOA & $<5.00 \mathrm{E}+00$ \\
\hline 50768 & $11 / 15 / 89$ & VOA & $1.10 \mathrm{E}+01$ \\
\hline $50768 B$ & $11 / 15 / 89$ & VOA & $<5.00 \mathrm{E}+00$ \\
\hline $50768 \mathrm{~T}$ & $11 / 15 / 89$ & VOA & $<4.00 \mathrm{E}+00$ \\
\hline 51008 & $3 / 05 / 90$ & VOA & $2.60 \mathrm{E}+01$ \\
\hline $51008 B$ & $3 / 05 / 90$ & VOA & $<5.00 \mathrm{E}+00$ \\
\hline $51008 \mathrm{~T}$ & $3 / 05 / 90$ & VOA & $<5.00 \mathrm{E}+00$ \\
\hline 51008 & $3 / 05 / 90$ & $A B N$ & $8.00 \mathrm{E}+00$ \\
\hline 50665 & $10 / 09 / 89$ & TITRA & $5.40 \mathrm{E}+04$ \\
\hline 50669 & $10 / 10 / 89$ & TITRA & $5.30 \mathrm{E}+04$ \\
\hline 50768 & $11 / 15 / 89$ & TITRA & $5.40 \mathrm{E}+04$ \\
\hline 51008 & $3 / 05 / 90$ & TITRA & $6.00 \mathrm{E}+04$ \\
\hline 50665 & $10 / 09 / 89$ & A].pha & $2.58 \mathrm{E}+00$ \\
\hline 50768 & $11 / 15 / 89$ & Alpha & $<5.31 \mathrm{E}-01$ \\
\hline 51008 & $3 / 05 / 90$ & Alpha & 1. $14 \mathrm{E}+00$ \\
\hline 50665 & $10 / 09 / 89$ & COND-FId & 1. $56 \mathrm{E}+02$ \\
\hline 50669 & $10 / 10 / 89$ & COND-EId & $1.70 \mathrm{E}+02$ \\
\hline 50768 & $11 / 15 / 89$ & COND-FId & $1.62 \mathrm{E}+02$ \\
\hline 51008 & $3 / 05 / 90$ & COND-FId & 1. $48 \mathrm{E}+02$ \\
\hline $50665 \mathrm{E}$ & $10 / 09 / 89$ & IGNIT & $2.10 \mathrm{E}+02$ \\
\hline 50669E & $10 / 10 / 89$ & IGNIT & $2.08 \mathrm{E}+02$ \\
\hline $50768 \mathrm{E}$ & $11 / 15 / 89$ & IGNIT & 2. $12 \mathrm{E}+02$ \\
\hline $51008 \mathrm{E}$ & $3 / 05 / 90$ & IGNIT & 2. $12 \mathrm{E}+02$ \\
\hline 50665 & $10 / 09 / 89$ & PH-FId & $6.70 E+00$ \\
\hline 50669 & $10 / 10 / 89$ & PH - FId & $7.10 \mathrm{E}+00$ \\
\hline 50768 & $11 / 15 / 89$ & PH-FId & $7.37 \mathrm{E}+00$ \\
\hline 51.008 & $3 / 05 / 90$ & PH-FId & $8.04 \mathrm{E}+00$ \\
\hline $50665 \mathrm{E}$ & $10 / 09 / 89$ & DSPEC & $<1.00 \mathrm{E}+02$ \\
\hline 50669E & $10 / 10 / 89$ & DSPEC & $<1.00 E+02$ \\
\hline $50768 \mathrm{E}$ & $11 / 15 / 89$ & DSPEC & $<1.00 \mathrm{E}+02$ \\
\hline $51008 E$ & $3 / 05 / 90$ & DSPEC & $<1.00 \mathrm{E}+02$ \\
\hline $50665 \mathrm{E}$ & $10 / 09 / 89$ & DTITRA & $<1.00 \mathrm{E}+02$ \\
\hline $50669 \mathrm{E}$ & $10 / 10 / 89$ & DTITRA & $<1.00 \mathrm{E}+02$ \\
\hline $50768 \mathrm{E}$ & $11 / 15 / 89$ & DTITRA & $<1.00 \mathrm{E}+02$ \\
\hline $51008 \mathrm{E}$ & $3 / 05 / 90$ & DTITRA & $<1.00 \mathrm{E}+02$ \\
\hline 50665 & $10 / 09 / 89$ & TDS & $8.60 \mathrm{E}+04$ \\
\hline 50669 & $10 / 10 / 89$ & TDS & 8. $30 E+04$ \\
\hline 50768 & $11 / 15 / 89$ & TDS & $6.90 \mathrm{E}+04$ \\
\hline 51008 & $3 / 05 / 90$ & $\operatorname{TDS}$ & $1.00 E+04$ \\
\hline 50665 & $10 / 09 / 89$ & TEMP-FId & $2.40 E+01$ \\
\hline 50669 & $10 / 10 / 89$ & TEMP-FId & $2.40 \mathbb{E}+01$ \\
\hline 50768 & $11 / 15 / 89$ & TEMP-FId & $1.50 \mathrm{E}+01$ \\
\hline 51008 & $3 / 05 / 90$ & TEMP-FId & 1. $29 E+01$ \\
\hline 50665 & $10 / 09 / 89$ & TOC & $<1.50 \mathrm{E}+03$ \\
\hline 50669 & $10 / 10 / 89$ & TOC & $<1.50 \mathrm{E}+03$ \\
\hline 50768 & $11 / 15 / 89$ & TOC & 2. $10 \mathrm{E}+03$ \\
\hline 51008 & $3 / 05 / 90$ & TOC & 1. $40 \mathrm{E}+03$ \\
\hline 50665 & $10 / 09 / 89$ & $\mathrm{TC}$ & 1. $40 E+04$ \\
\hline 50669 & $10 / 10 / 89$ & $T C$ & 1. $40 \mathrm{E}+04$ \\
\hline 50768 & $1.1 / 15 / 89$ & TC & 1. $40 \mathrm{E}+04$ \\
\hline 51008 & $3 / 05 / 90$ & $\mathrm{TC}$ & $1.67 E+04$ \\
\hline
\end{tabular}


DATA FOR 300 Area Wastewater-Sample Boint I Continued

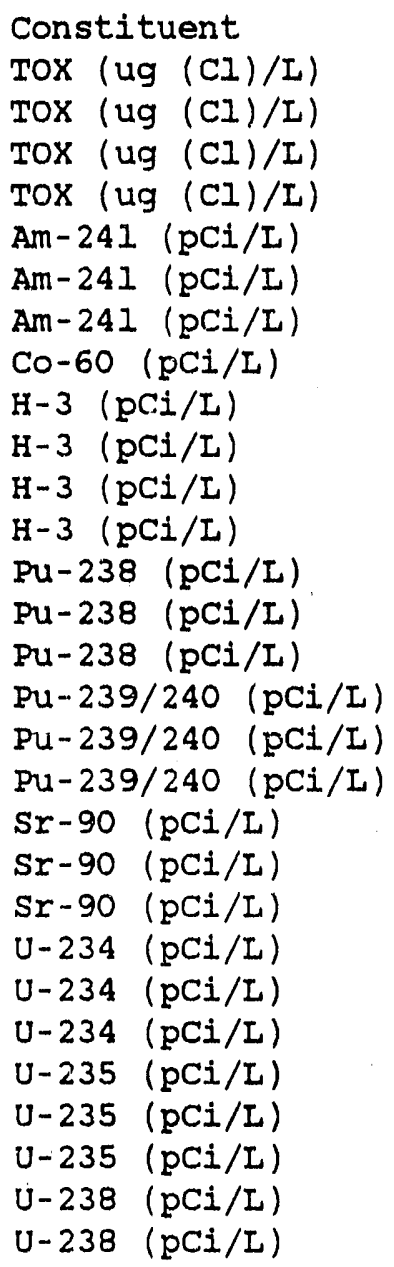

\begin{tabular}{crlr} 
Sample \# & \multicolumn{1}{c}{ Date } & Method & \multicolumn{1}{l}{ Result } \\
50665 & $10 / 09 / 89$ LTOX & $1.77 \mathrm{E}+02$ \\
50669 & $10 / 10 / 89$ LTOX & $1.71 \mathrm{E}+02$ \\
50768 & $11 / 15 / 89$ LTOX & $1.33 \mathrm{E}+02$ \\
51008 & $3 / 05 / 90$ LTOX & $1.34 \mathrm{E}+02$ \\
50665 & $10 / 09 / 89$ AEA & $4.54 \mathrm{E}-02$ \\
50768 & $11 / 15 / 89$ AEA & $<2.99 \mathrm{E}-03$ \\
51008 & $3 / 05 / 90$ AEA & $4.46 \mathrm{E}-02$ \\
51008 & $3 / 05 / 90$ GEA & $6.11 \mathrm{E}-01$ \\
50665 & $10 / 09 / 89$ LSC & $2.92 \mathrm{E}+02$ \\
50669 & $10 / 10 / 89$ LSC & $<2.09 \mathrm{E}+02$ \\
50768 & $11 / 15 / 89$ LSC & $<6.46 \mathrm{E}+01$ \\
51008 & $3 / 05 / 90$ LSC & $.3 .58 \mathrm{E}+02$ \\
50665 & $10 / 09 / 89$ AEA & $4.72 \mathrm{E}-03$ \\
50768 & $11 / 15 / 89$ AEA & $<1.75 \mathrm{E}-04$ \\
51008 & $3 / 05 / 90$ AEA & $6.74 \mathrm{E}-02$ \\
50665 & $10 / 09 / 89$ AEA & $8.18 \mathrm{E}-03$ \\
50768 & $11 / 15 / 89$ AEA & $<1.35 \mathrm{E}-03$ \\
51008 & $3 / 05 / 90$ AEA & $4.39 \mathrm{E}-02$ \\
50665 & $10 / 09 / 89$ Beta & $<1.03 \mathrm{E}-01$ \\
50768 & $11 / 15 / 89$ BEta & $<1.27 \mathrm{E}-01$ \\
51008 & $3 / 05 / 90$ BEta & $5.05 \mathrm{E}-01$ \\
50665 & $10 / 09 / 89$ AEA & $7.81 \mathrm{E}-01$ \\
50768 & $11 / 15 / 89$ AEA & $7.12 \mathrm{E}-01$ \\
51008 & $3 / 05 / 90$ AEA & $7.29 \mathrm{E}-01$ \\
50665 & $10 / 09 / 89$ AEA & $2.58 \mathrm{E}-02$ \\
50768 & $11 / 15 / 89$ AEA & $<1.94 \mathrm{E}-02$ \\
51008 & $3 / 05 / 90$ AEA & $<1.11 \mathrm{E}-01$ \\
50665 & $10 / 09 / 89$ AEA & $6.81 \mathrm{E}-01$ \\
51008 & $3 / 05 / 90$ AEA & $6.23 \mathrm{E}-01$ \\
& & &
\end{tabular}


WHC-EP-0355, Appendix D

DATA FOR 400 Area Secondary Cooling Water

\begin{tabular}{|c|c|c|}
\hline Constitue & dent & \\
\hline Arsenic & (EP : & Toxic) \\
\hline Arsenic & (EP : & Toxic) \\
\hline Arsenic & (EP : & Toxic) \\
\hline Arsenic & (EP: & Toxic) \\
\hline Barium & & \\
\hline Barium & & \\
\hline Barium & & \\
\hline Barium & & \\
\hline Barium ( & (EP $T$ & Coxic) \\
\hline Barium ( & (EP T & Coxic) \\
\hline Barium & $\operatorname{EP} T$ & (oxic) \\
\hline Barium & EP $T$ & Coxic) \\
\hline Boron & & \\
\hline Boron & & \\
\hline Boron & & \\
\hline Boron & & \\
\hline Cadmium & (EP : & Toxic) \\
\hline Cadmium & (EP : & Toxic) \\
\hline Cadmium & (EP & Toxic) \\
\hline Cadmium & (EP : & Toxic) \\
\hline Calcium & & \\
\hline Calcium & & \\
\hline Calcium & & \\
\hline Calcium & & \\
\hline Chloride & & \\
\hline Chloride & & \\
\hline Chloride & & \\
\hline Chloride & & \\
\hline Chromium & $n$ (EP & Toxic) \\
\hline Chromium & $n$ (EP & Toxic) \\
\hline Chromium & n (EP & Toxic) \\
\hline Chromium & $n$ (EP & Toxic) \\
\hline Copper & & \\
\hline Copper & & \\
\hline Copper & & \\
\hline Copper & & \\
\hline Fluoride & & \\
\hline Fluoride & & \\
\hline Fluoride & & \\
\hline Fluoride & & \\
\hline Fluoride & & \\
\hline Fluoride & & \\
\hline Fluoride & & \\
\hline Fluoride & & \\
\hline Iron & & \\
\hline Iron & & \\
\hline Iron & & \\
\hline Iron & & \\
\hline Lead (EP & Tox & (ic) \\
\hline Lead (EP & Tox & (ic) \\
\hline Lead (EP & Tox & (ic) \\
\hline Lead (EP & Tox. & ic) \\
\hline
\end{tabular}

\begin{tabular}{|c|c|c|c|}
\hline Sample \# & Date & Method & Result \\
\hline $50713 \mathrm{E}$ & $10 / 20 / 89$ & ICP & $<5.00 \mathrm{E}+02$ \\
\hline $50784 \mathrm{E}$ & $11 / 21 / 89$ & ICP & $<5.00 E+02$ \\
\hline $50826 \mathrm{E}$ & $12 / 06 / 89$ & ICP & $<5.00 \mathrm{E}+02$ \\
\hline $50876 \mathrm{E}$ & $1 / 09 / 90$ & ICP & $<5.00 \mathrm{E}+02$ \\
\hline 50713 & $10 / 20 / 89$ & ICP & $3.00 \mathrm{E}+01$ \\
\hline 50784 & $11 / 21 / 89$ & ICP & $4.00 E+01$ \\
\hline 50926 & $12 / 06 / 89$ & ICP & $3.80 E+01$ \\
\hline 50876 & $1 / 09 / 90$ & ICP & $3.00 \mathrm{E}+01$ \\
\hline $507.13 E$ & $10 / 20 / 89$ & ICP & $<1.00 E+03$ \\
\hline $50784 \mathrm{E}$ & $11 / 21 / 89$ & ICP & $<1.00 E+03$ \\
\hline $50826 \mathrm{E}$ & $12 / 06 / 89$ & ICP & $<1.00 \mathrm{E}+03$ \\
\hline $50876 \mathrm{E}$ & $1 / 09 / 90$ & ICP & $<1.00 \mathrm{E}+03$ \\
\hline 50713 & $10 / 20 / 89$ & ICP & 4. $60 \mathrm{E}+01$ \\
\hline 50784 & $11 / 21 / 89$ & ICP & $5.50 E+O 1$ \\
\hline 50826 & $12 / 06 / 89$ & ICP & 5. 20E+01 \\
\hline 50876 & $1 / 09 / 90$ & ICP & 4. 10E+01 \\
\hline $50713 E$ & $10 / 20 / 89$ & ICP & $<1.00 \mathrm{E}+02$ \\
\hline $50784 \mathrm{E}$ & $11 / 21 / 89$ & ICP & $<1.00 \mathrm{E}+02$ \\
\hline $50826 \mathrm{E}$ & $12 / 06 / 89$ & ICP & $<1.00 E+02$ \\
\hline $50876 \mathrm{E}$ & $1 / 09 / 90$ & ICP & $<1.00 \mathrm{E}+02$ \\
\hline 50713 & $10 / 20 / 89$ & ICP & $4.89 E+04$ \\
\hline 50784 & $11 / 21 / 89$ & ICP & $6.79 \mathrm{E}+04$ \\
\hline 50826 & $12 / 06 / 89$ & ICP & $5.47 \mathrm{E}+04$ \\
\hline 50876 & $1 / 09 / 90$ & ICP & $4.89 E+04$ \\
\hline 50713 & $10 / 20 / 89$ & IC & $1.90 \mathrm{E}+04$ \\
\hline 50784 & $11 / 21 / 89$ & IC & $1.51 E+04$ \\
\hline 50826 & $12 / 06 / 89$ & IC & $2.72 E+04$ \\
\hline 50876 & $1 / 09 / 90$ & IC & $2.10 \mathrm{E}+04$ \\
\hline 50713E & $10 / 20 / 89$ & ICP & $<5.00 E+02$ \\
\hline $50784 \mathrm{E}$ & $11 / 21 / 89$ & ICP & $<5.00 \mathrm{E}+02$ \\
\hline $50826 \mathrm{E}$ & $12 / 06 / 89$ & ICP & $<5.00 \mathrm{E}+02$ \\
\hline $50876 \mathrm{E}$ & $1 / 09 / 90$ & ICP & $<5.00 \mathrm{E}+02$ \\
\hline 50713 & $10 / 20 / 89$ & ICP & $<1.00 \mathrm{E}+01$ \\
\hline 50784 & $11 / 21 / 89$ & ICP & $<1.00 E+01$ \\
\hline 50826 & $12 / 06 / 89$ & ICP & $<1.00 \mathrm{E}+01$ \\
\hline 50876 & $1 / 09 / 90$ & ICP & 1. 10E+01 \\
\hline 50713 & $10 / 20 / 89$ & IC & $7.00 \mathrm{E}+02$ \\
\hline 50713 & $10 / 20 / 89$ & ISE & $6.22 \mathrm{E}+02$ \\
\hline 50784 & $11 / 21 / 89$ & IC & $5.35 \mathrm{E}+02$ \\
\hline 50784 & $11 / 21 / 89$ & ISE & $7.50 \mathrm{E}+02$ \\
\hline 50826 & $12 / 06 / 89$ & IC & $7.00 \mathrm{E}+02$ \\
\hline 50826 & $12 / 06 / 89$ & ISE & $6.88 \mathrm{E}+02$ \\
\hline 50876 & $1 / 09 / 90$ & IC & $7.00 \mathrm{E}+02$ \\
\hline 50876 & $1 / 09 / 90$ & ISE & $6.55 \mathrm{E}+02$ \\
\hline 50713 & $10 / 20 / 89$ & ICP & $3.40 E+01$ \\
\hline 50784 & $11 / 21 / 89$ & ICP & $<3.00 E+01$ \\
\hline 50826 & $12 / 06 / 89$ & ICP & $7.90 \mathrm{E}+01$ \\
\hline 50876 & $1 / 09 / 90$ & ICP & $7.20 \mathrm{E}+01$ \\
\hline $50713 F$ & $10 / 20 / 89$ & ICP & $<5.00 \mathrm{E}+02$ \\
\hline $50784 \mathrm{E}$ & $11 / 21 / 89$ & ICP & $<5.00 \mathrm{E}+02$ \\
\hline $50826 \mathrm{E}$ & $12 / 06 / 89$ & ICP & $<5.00 \mathrm{E}+02$ \\
\hline $50876 \mathrm{E}$ & $1 / 09 / 90$ & ICP & $<5.00 \mathrm{E}+02$ \\
\hline 50713 & $10 / 20 / 89$ & ICP & $2.50 \mathrm{E}+01$ \\
\hline
\end{tabular}


DATA FOR 400 Area Secondary Cooling Water Continued

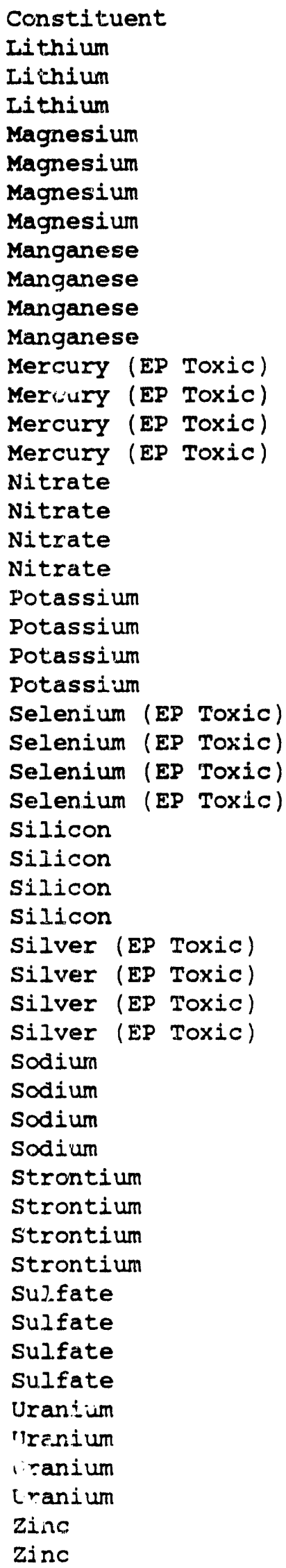

\begin{tabular}{|c|c|c|c|}
\hline Sample \# & Date & Method & Result \\
\hline 50784 & $11 / 21 / 89$ & $I C P$ & $3.40 \mathrm{E}+01$ \\
\hline 50826 & $12 / 06 / 89$ & ICP & $3.00 E+01$ \\
\hline 50876 & $1 / 09 / 90$ & ICP & $2.60 \mathrm{E}+01$ \\
\hline 50713 & $10 / 20 / 89$ & ICP & $1.45 \mathrm{E}+04$ \\
\hline 50784 & $11 / 21 / 89$ & ICP & $1.83 \mathrm{E}+04$ \\
\hline 50826 & $12 / 06 / 89$ & ICP & $1.64 \mathrm{E}+04$ \\
\hline 50876 & $1 / 09 / 90$ & ICP & 1. $38 \mathrm{E}+04$ \\
\hline 50713 & $10 / 20 / 89$ & ICP & $1.80 \mathrm{E}+01$ \\
\hline 50784 & $11 / 21 / 89$ & ICP & $8.00 E+00$ \\
\hline 50826 & $12 / 06 / 89$ & ICP & $1.50 \mathrm{E}+01$ \\
\hline 50876 & $1 / 09 / 90$ & ICP & 1. $20 \mathrm{E}+01$ \\
\hline $50713 \mathrm{E}$ & $10 / 20 / 89$ & CVAA/M & $<2.00 \mathrm{E}+01$ \\
\hline $50784 \mathrm{E}$ & $11 / 21 / 89$ & CVAA/M & $<2.00 E+01$ \\
\hline $50826 \mathrm{E}$ & $12 / 06 / 89$ & CVAA/M & $<2.00 E+01$ \\
\hline $50876 \mathrm{E}$ & $1 / 09 / 90$ & CVAA $/ M$ & $<2.00 E+01$ \\
\hline 50713 & $10 / 20 / 89$ & IC & $1.10 E+03$ \\
\hline 50784 & $11 / 21 / 89$ & IC & $9.37 \mathrm{E}+02$ \\
\hline 50826 & $12 / 06 / 89$ & IC & 1. $40 \mathrm{E}+03$ \\
\hline 50876 & $1 / 09 / 90$ & $I C$ & $1.00 E+03$ \\
\hline 50713 & $10 / 20 / 89$ & $I C P$ & 1. $43 E+04$ \\
\hline 50784 & $11 / 21 / 89$ & ICP & $1.95 E+04$ \\
\hline 50826 & $12 / 06 / 89$ & ICP & $1.71 \mathrm{E}+04$ \\
\hline 50876 & $1 / 09 / 90$ & ICP & $1.41 \mathrm{E}+04$ \\
\hline $50713 \mathrm{E}$ & $10 / 20 / 89$ & ICP & $<5.00 \mathrm{E}+02$ \\
\hline $50784 \mathrm{E}$ & $11 / 21 / 89$ & ICP & $<5.00 E+02$ \\
\hline $50826 \mathrm{E}$ & $12 / 06 / 89$ & ICP & $<5.00 \mathrm{E}+02$ \\
\hline $50876 \mathrm{E}$ & $1 / 09 / 90$ & ICP & $<5.00 E+02$ \\
\hline 50713 & $10 / 20 / 89$ & ICP & 3. $46 \mathrm{E}+04$ \\
\hline 50784 & $11 / 21 / 89$ & ICP & $4.41 E+04$ \\
\hline 50826 & $12 / 06 / 89$ & ICP & $3.87 E_{1}+04$ \\
\hline 50876 & $1 / 09 / 90$ & ICP & 3. $21 \mathrm{E}+04$ \\
\hline $50713 \mathrm{E}$ & $10 / 20 / 89$ & $I C P$ & $<5.00 \mathrm{E}+02$ \\
\hline $50784 \mathrm{E}$ & $11 / 21 / 89$ & $I C P$ & $<5.00 \mathrm{E}+02$ \\
\hline $50826 \mathrm{E}$ & $12 / 06 / 89$ & ICP & $<5.00 \mathrm{E}+02$ \\
\hline $50876 \mathrm{E}$ & $1 / 09 / 90$ & $I C P$ & $<5.00 \mathrm{ES}+02$ \\
\hline 50713 & $10 / 20 / 89$ & ICP & $5.911 \mathrm{H}+04$ \\
\hline 50784 & $11 / 21 / 89$ & ICP & $7.61 \mathrm{I}+04$ \\
\hline 50826 & $12 / 06 / 89$ & $I C P$ & $6.75 \mathrm{E}+04$ \\
\hline 50876 & $1 / 09 / 90$ & ICP & $5.58 \mathrm{E}+04$ \\
\hline 50713 & $10 / 20 / 89$ & ICP & $2.57 \mathrm{E}+02$ \\
\hline 50784 & $11 / 21 / 89$ & $I C P$ & $3.42 E+02$ \\
\hline 50826 & $12 / 06 / 89$ & ICP & 2. $94 \mathrm{E}+02$ \\
\hline 50876 & $1 / 09 / 90$ & ICP & $2.49 \mathrm{E}+02$ \\
\hline 50713 & $10 / 20 / 89$ & IC & $6.40 E+04$ \\
\hline 50784 & $11 / 21 / 89$ & IC & $5.12 E+04$ \\
\hline 50826 & $12 / 06 / 89$ & IC & $6.88 E+04$ \\
\hline 50876 & $1 / 09 / 90$ & IC & $7.19 \mathrm{E}+04$ \\
\hline 50713 & $10 / 20 / 89$ & GEA & ]. $85 \mathrm{E}-01$ \\
\hline 50784 & $11 / 21 / 89$ & GEA & $2.14 E-0 I$ \\
\hline 50826 & $12 / 06 / 89$ & GEA & $5.68 \mathrm{E}-01$ \\
\hline 50876 & $1 / 09 / 90$ & GEA & $3.08 \mathrm{E}-01$ \\
\hline 50713 & $10 / 20 / 89$ & ICP & 4. $2 C E+01$ \\
\hline 50784 & $11 / 21 / 89$ & ICP & 2.OCE+OI \\
\hline
\end{tabular}


WHC-EP-0355, Appendix D

DATA FOR 400 Area secondary Cooling water

Continued

Constituent
Zinc
zinc
Acetone
Acetone
Acetone
Acetone
Acetone
Acetone
Acetone
Acetone
Acetone
Acetone
Acetone
Acetone
Acetone
Acetone
Acetone
Acetone
Ammonia
Ammonia
Ammonia
Ammonia
Bromodichloromethane
Bromodichloromethane
Eromodichloromethane
Bromodichloromethane
Bromodichloromethane
Bromodichloromethane
Bromodichloromethane
Bromodichloromethane
Bromodichloromethane
Bromodichloromethane
Bromodichloromethane
Bromodichloromethane
2-Butanone
2-Butanone
2-Butanone
2-Butanone
2-Butanone
2-Butanone
2-Butanone
2-Butanone
2-Butanone
2-Butanone
2-Butanone
2-Butanone
Dichloromethane
Dichloromethane
Dichloromethane
Dichloromethane
Dichloromethane
Dichloromethane
Dichloromcthanc

\begin{tabular}{|c|c|c|c|}
\hline $\begin{array}{c}\text { Sample \# } \\
50826\end{array}$ & $\begin{array}{c}\text { Date } \\
12 / 06 / 89\end{array}$ & $\begin{array}{l}\text { Method } \\
\text { ICP }\end{array}$ & $\begin{array}{l}\text { Result } \\
2.60 \mathrm{E}+01\end{array}$ \\
\hline 50876 & $1 / 09 / 90$ & ICP & 4. $.80 E+01$ \\
\hline 50713 & $10 / 20 / 89$ & VOA & $2.70 \mathrm{E}+01$ \\
\hline 50713 & $10 / 20 / 89$ & $A B N$ & $<1.00 \mathrm{E}+01$ \\
\hline $50713 B$ & $10 / 20 / 89$ & VOA & $<1.00 \mathrm{E}+01$ \\
\hline $50713 \mathrm{~T}$ & $10 / 20 / 89$ & VOA & $<1.00 \mathrm{E}+01$ \\
\hline 50784 & $11 / 21 / 89$ & VOA & $<1.00 \mathrm{E}+01$ \\
\hline 50784 & $11 / 21 / 89$ & $A B N$ & $<1.00 \mathrm{E}+0.1$ \\
\hline $50784 \mathrm{~B}$ & $11 / 21 / 89$ & VOA & $<1.00 E+01$ \\
\hline $50784 \mathrm{~T}$ & $11 / 21 / 89$ & VOA & $<1.00 \mathrm{E}+01$ \\
\hline 50826 & $12 / 06 / 89$ & VOA & $<1.00 \mathrm{E}+01$ \\
\hline 50826 & $12 / 06 / 89$ & $A B N$ & $<1.00 \mathrm{E}+01$ \\
\hline $50826 B$ & $12 / 06 / 89$ & VOA & $<1.00 \mathrm{E}+01$ \\
\hline $50826 \mathrm{~T}$ & $12 / 06 / 89$ & VOA & $<1.00 \mathrm{E}+01$ \\
\hline 50876 & $1 / 09 / 90$ & VOA & $<1.00 \mathrm{E}+01$ \\
\hline 50876 & $1 / 09 / 90$ & $A B N$ & $<1.00 \mathrm{E}+01$ \\
\hline $50876 B$ & $1 / 09 / 90$ & VOA & $<6.00 \mathrm{E}+00$ \\
\hline $50876 \mathrm{~T}$ & $1 / 09 / 90$ & VOA & $<6.00 E+00$ \\
\hline 50713 & $10 / 20 / 89$ & ISE & $1.00 \mathrm{E}+02$ \\
\hline 50784 & $11 / 21 / 89$ & ISE & $<5.00 E+01$ \\
\hline 50826 & $12 / 06 / 89$ & ISE & $<5.00 E+01$ \\
\hline 50876 & $1 / 09 / 90$ & ISE & 1. $20 \mathrm{E}+02$ \\
\hline 50713 & $10 / 20 / 89$ & VOA & $<5.00 E+00$ \\
\hline 507138 & $10 / 20 / 89$ & VOA & $<5.00 \mathrm{E}+00$ \\
\hline $50713 \mathrm{~T}$ & $10 / 20 / 89$ & VOA & $<5.00 E+00$ \\
\hline 50784 & $11 / 21 / 89$ & VOA & $<5.00 \mathrm{E}+00$ \\
\hline $50784 B$ & $11 / 21 / 89$ & VOA & $<5.00 E+00$ \\
\hline $50784 \mathrm{~T}$ & $11 / 21 / 89$ & VOA & $-5.00 \mathrm{E}+00$ \\
\hline 50826 & $12 / 06 / 89$ & VOA & $5.00 \mathrm{E}+00$ \\
\hline $50826 B$ & $12 / 06 / 89$ & VOA & $<5.0 O E+00$ \\
\hline $50826 \mathrm{~T}$ & $12 / 06 / 89$ & VOA & $<5.00 E+00$ \\
\hline 50876 & $1 / 09 / 90$ & VOA & $<5.00 \mathrm{E}+00$ \\
\hline $50876 \mathrm{~B}$ & $1 / 09 / 90$ & VOA & $<5.00 \mathrm{E}+00$ \\
\hline $50876 \mathrm{~T}$ & $1 / 09 / 90$ & VOA & $<5.00 \mathrm{E}+00$ \\
\hline 50713 & $10 / 20 / 89$ & VOA & $1.20 \mathrm{E}+01$ \\
\hline $50713 B$ & $10 / 20 / 89$ & VOA & $2.30 \mathrm{E}+01$ \\
\hline $50713 T$ & $10 / 20 / 89$ & VOA & $1.90 \mathrm{E}+01$ \\
\hline 50784 & $11 / 21 / 89$ & VOA & $<1.00 \mathrm{E}+01$ \\
\hline $50784 \mathrm{~B}$ & $11 / 21 / 89$ & VOA & $<1.00 \mathrm{E}+01$ \\
\hline $50784 T$ & $11 / 21 / 89$ & VOA & $<1.00 \mathrm{E}+01$ \\
\hline 50826 & $12 / 06 / 89$ & VOA & $<6.00 \mathrm{E}+00$ \\
\hline $50826 \mathrm{~B}$ & $12 / 06 / 89$ & VOA & $<7.00 E+00$ \\
\hline $50826 \mathrm{~T}$ & $12 / 06 / 89$ & VOA & $<1.00 E+01$ \\
\hline 50876 & $1 / 09 / 90$ & VOA & $<1.00 \mathrm{E}+01$ \\
\hline $50876 \mathrm{~B}$ & $1 / 09 / 90$ & VOA & $<1.00 \mathrm{E}+01$ \\
\hline $50876 \mathrm{~T}$ & $1 / 09 / 90$ & VOA & $<1.00 \mathrm{E}+01$ \\
\hline 50713 & $10 / 20 / 89$ & VOA & $<5.00 E+0 O$ \\
\hline $50713 \mathrm{~B}$ & $10 / 20 / 89$ & VOA & $1.00 \mathrm{E}+01$ \\
\hline $50713 \mathrm{~T}$ & $1.0 / 20 / 89$ & VOA & $7.90 \mathrm{E}+01$ \\
\hline 50784 & $11 / 21 / 89$ & VOA & $<5.00 \mathrm{E}+00$ \\
\hline $50784 \mathrm{~B}$ & $11 / 21 / 89$ & VOA & $<3.00 E+00$ \\
\hline $50784 \mathrm{~T}$ & $11 / 21 / 89$ & VOA & $<3.00 F+00$ \\
\hline 50926 & 12,06, & YOA & $=5.0 O E: 00$ \\
\hline
\end{tabular}


DATA FOR 400 Area Secondary Cooling Water Continued

Constituent

Dichloromethane

Dichloromethane

Dichloromethane

Dichloromethane

Dichloromethane

Tetrahydrofuran

Tetrahydrofuran

Tetrahydrofuran

Tetrahydrofuran

Tetrahydrofuran

Tetrahydrofuran

Tetrahydrofuran

Tetrahydrofuran

Tetrahydrofuran

Tetrahydrofuran

Tetrahydrofuran

Tetrahydrofuran

Trichloromethane

Trichloromethane

Trichloromethane

Trichloromethane

Trichloromethane

Trichloromethane

Trichloromethane

Trichloromethane

Trichloromethane

irichloromethane

Trichloromethane

Triciloromethane

Alkalinity (Method B)

Alkalinity (Method B)

Alkalinity (Method B)

Alkalinity (Method B)

Beta Activity (pCi/L)

Beta Ictivity (pCi/L)

Beta Activity (pCi/L)

Beta Activity (pCi/L)

Conductivity (us)

Conductivity (us)

Conductivity (us)

Conductivity (us)

Ingitability (degrees F)

Ingitability (degrees F)

Ingitability (degrees F)

Ingitability (degrees F)

$\mathrm{pH}$ (dimensionless)

$\mathrm{pH}$ (dimensionless)

$\mathrm{pH}$ (dimensionless)

$\mathrm{pH}$ (dimensionless)

Reactivity Cyanide ( $\mathrm{mg} / \mathrm{kg}$ )

Reactivity Cyanide ( $\mathrm{mg} / \mathrm{kg}$ )

Reactivity Cyanide (mg/kg)

peactivity Cyanide (mg/kg)

\begin{tabular}{|c|c|c|c|}
\hline ample \# & & & Result \\
\hline $50826 \mathrm{~B}$ & $12 / 06 / 89$ & VOA & $<5.00 E+00$ \\
\hline $50826 \mathrm{~T}$ & $12 / 06 / 89$ & VOA & $<5.00 E+00$ \\
\hline 50876 & $1 / 09 / 90$ & VOA & $<5.00 E+00$ \\
\hline $50876 \mathrm{~B}$ & $1 / 09 / 90$ & VOA & $<5.00 \mathrm{E}+00$ \\
\hline $50876 \mathrm{~T}$ & $1 / 09 / 90$ & VOA & $<5.00 \mathrm{E}+00$ \\
\hline 50713 & $10 / 20 / 89$ & VOA & $<1.00 \mathrm{E}+01$ \\
\hline $50713 B$ & $10 / 20 / 89$ & VOA & $2.00 E+01$ \\
\hline $50713 \mathrm{~T}$ & $10 / 20 / 89$ & VOA & $<1.00 \mathrm{E}+01$ \\
\hline 50784 & $11 / 21 / 89$ & VOA & $<1.00 \mathrm{E}+01$ \\
\hline $50784 B$ & $11 / 21 / 89$ & VOA & $<1.00 \mathrm{E}+01$ \\
\hline $50784 \mathrm{~T}$ & $11 / 21 / 89$ & VOA & $<1.00 E+01$ \\
\hline 50826 & $12 / 06 / 89$ & VOA & $<1.00 E+01$ \\
\hline $50826 B$ & $12 / 06 / 89$ & VOA & $<1.00 E+01$ \\
\hline $50826 \mathrm{~T}$ & $12 / 06 / 89$ & VOA & $<1.00 E+01$ \\
\hline 50876 & $1 / 09 / 90$ & VOA & $<1.00 E+01$ \\
\hline $50876 \mathrm{~B}$ & $1 / 09 / 90$ & VOA & $<1 \quad O O E+0 Z$ \\
\hline $50876 \mathrm{~T}$ & $1 / 09 / 90$ & VOA & $<6.00 E+00$ \\
\hline 50713 & $10 / 20 / 89$ & VOA & $<5.00 E+00$ \\
\hline $50713 B$ & $10 / 20 / 89$ & VOA & $<5.00 \mathrm{E}+00$ \\
\hline $50713 \mathrm{~T}$ & $10 / 20 / 89$ & VOA & $<5.00 \mathrm{E}+00$ \\
\hline 50784 & $11 / 21 / 89$ & VOA & $<5.00 E+00$ \\
\hline $50784 B$ & $11 / 21 / 89$ & VOA & $<4.00 \mathrm{E}+00$ \\
\hline $50784 \mathrm{~T}$ & $11 / 21 / 89$ & VOA & 1. 1OE+01 \\
\hline 50826 & $12 / 06 / 89$ & VOA & $8.00 \mathrm{E}+00$ \\
\hline $50826 B$ & $12 / 06 / 89$ & VOA & $<4.00 E+00$ \\
\hline $50826 \mathrm{~T}$ & $12 / 06 / 89$ & VOA & $<4.00 E+00$ \\
\hline 50876 & $1 / 09 / 90$ & VOA & $<5.00 E+00$ \\
\hline $50876 B$ & $1 / 09 / 90$ & VOA & $<3.00 \mathrm{E}+00$ \\
\hline $50876 \mathrm{~T}$ & $1 / 09 / 90$ & VOA & $<3.00 \mathrm{E}+00$ \\
\hline 50713 & $10 / 20 / 89$ & TITRA & $2.42 \mathrm{E}+05$ \\
\hline 50784 & $11 / 21 / 89$ & TITRA & $3.09 \mathrm{E}+05$ \\
\hline 50826 & $12 / 06 / 89$ & TITRA & $2.73 E+05$ \\
\hline 50876 & $1 / 09 / 90$ & TITRA & $2.86 \mathrm{E}+05$ \\
\hline 50713 & $10 / 20 / 89$ & Beta & 1. $22 \mathrm{E}+01$ \\
\hline 50784 & $11 / 21 / 89$ & Beta & $2.02 E+01$ \\
\hline 50826 & $12 / 06 / 89$ & Beta & $3.12 \mathrm{E}+01$ \\
\hline 50876 & $1 / 09 / 90$ & Beta & 1. $26 \mathrm{E}+01$ \\
\hline 50713 & $10 / 20 / 89$ & COND-FId & $5.27 \mathrm{E}+02$ \\
\hline 50784 & $11 / 21 / 89$ & COND-FId & $7.53 E+02$ \\
\hline 50826 & $12 / 06 / 89$ & COND-FId & $6.85 E+02$ \\
\hline 50876 & $1 / 09 / 90$ & COND-FId & $6.98 E+02$ \\
\hline $50713 E$ & $10 / 20 / 89$ & IGNIT & $2.12 E+02$ \\
\hline $50784 \mathrm{E}$ & $11 / 21 / 89$ & IGNIT & $2.14 \mathrm{E}+02$ \\
\hline $50826 \mathrm{E}$ & $12 / 06 / 89$ & IGNIT & 2. $10 \mathrm{E}+02$ \\
\hline $50876 E$ & $1 / 09 / 90$ & IGNIT & 2. $12 \mathrm{E}+02$ \\
\hline 50713 & $10 / 20 / 89$ & PH-FId & $8.85 \mathrm{E}+00$ \\
\hline 50784 & $11 / 21 / 89$ & PH-FId & $8.80 E+00$ \\
\hline 50826 & $12 / 06 / 89$ & PH-FId & $7.25 \mathrm{E}+00$ \\
\hline 50876 & $1 / 09 / 90$ & PH-FId & $8.80 E+00$ \\
\hline $50713 \mathrm{E}$ & $10 / 20 / 89$ & DSPEC & $<1.00 E+02$ \\
\hline $50784 \mathrm{E}$ & $11 / 21 / 89$ & DSPEC & $<1.00 E+02$ \\
\hline $50826 \mathrm{E}$ & $12 / 06 / 89$ & DSPEC & $<1.00 E+02$ \\
\hline 5О8? & $1 / 09 / 90$ & DSPEC & $<1, \cap \cap E+\cap 2$ \\
\hline
\end{tabular}


DATA FOR 400 Area Secondary Cooling Water

Continued

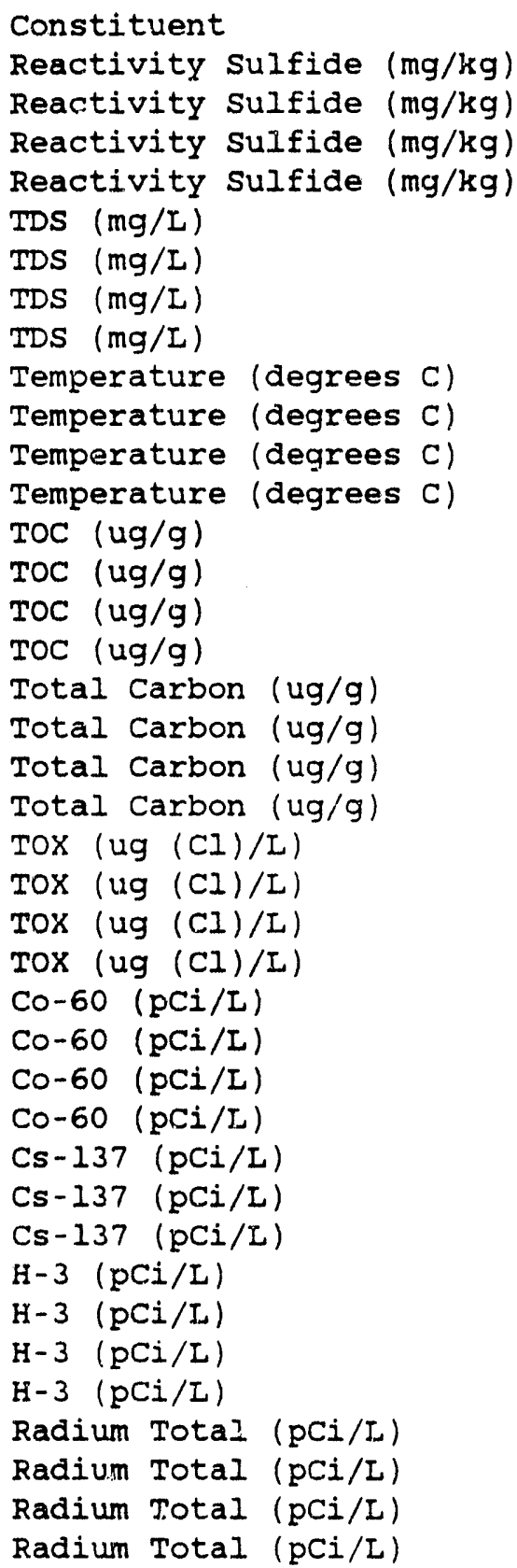

\begin{tabular}{crlr} 
Sample \# & \multicolumn{1}{c}{ Date } & Method & \multicolumn{1}{l}{ Result } \\
$50713 \mathrm{E}$ & $10 / 20 / 89$ DTITRA & $<1.00 \mathrm{E}+02$ \\
$50784 \mathrm{E}$ & $11 / 21 / 89$ DTITRA & $<1.00 \mathrm{E}+02$ \\
$50826 \mathrm{E}$ & $12 / 06 / 89$ & DTITRA & $<1.00 \mathrm{E}+02$ \\
$50876 \mathrm{E}$ & $1 / 09 / 90$ DTITRA & $<1.00 \mathrm{E}+02$ \\
50713 & $10 / 20 / 89$ TDS & $4.01 \mathrm{E}+05$ \\
50784 & $11 / 21 / 89$ TDS & $5.53 \mathrm{E}+05$ \\
50826 & $12 / 06 / 89$ TDS & $4.65 \mathrm{E}+05$ \\
50876 & $1 / 09 / 90$ TDS & $4.11 \mathrm{E}+05$ \\
50713 & $10 / 20 / 89$ TEMP-EId & $1.93 \mathrm{E}+01$ \\
50784 & $11 / 21 / 89$ TEMP-FId & $1.86 \mathrm{E}+01$ \\
50826 & $12 / 06 / 89$ TEMP-FId & $1.63 \mathrm{E}+01$ \\
50876 & $1 / 09 / 90$ TEMP-EId & $1.77 \mathrm{E}+01$ \\
50713 & $10 / 20 / 89$ TOC & $2.40 \mathrm{E}+03$ \\
50784 & $11 / 21 / 89$ TOC & $<1.90 \mathrm{E}+03$ \\
50826 & $12 / 06 / 89$ TOC & $2.30 \mathrm{E}+03$ \\
50876 & $1 / 09 / 90$ TOC & $2.10 \mathrm{E}+03$ \\
50713 & $10 / 20 / 89$ TC & $6.00 \mathrm{E}+04$ \\
50784 & $11 / 21 / 89$ TC & $6.95 \mathrm{E}+04$ \\
50826 & $12 / 06 / 89$ TC & $6.28 \mathrm{E}+04$ \\
50876 & $1 / 09 / 90$ TC & $5.14 \mathrm{E}+04$ \\
50713 & $10 / 20 / 89$ LTOX & $7.30 \mathrm{E}+01$ \\
50784 & $11 / 21 / 89$ LTOX & $1.54 \mathrm{E}+02$ \\
50826 & $12 / 06 / 89$ LTOX & $2.61 \mathrm{E}+02$ \\
50876 & $1 / 09 / 90$ LTOX & $7.90 \mathrm{E}+01$ \\
50713 & $10 / 20 / 89$ GEA & $<4.02 \mathrm{E}-01$ \\
50784 & $11 / 21 / 89$ GEA & $9.16 \mathrm{E}+01$ \\
50826 & $12 / 06 / 89$ GEA & $<2.36 \mathrm{E}-01$ \\
50876 & $1 / 09 / 90$ GEA & $<5.94 \mathrm{E}-01$ \\
50713 & $10 / 20 / 89$ GEA & $<3.48 \mathrm{E}-01$ \\
50784 & $11 / 21 / 89$ GEA & $9.48 \mathrm{E}+01$ \\
50826 & $12 / 06 / 89$ GEA & $<6.67 \mathrm{E}-01$ \\
50713 & $10 / 20 / 89$ LSC & $6.65 \mathrm{E}+03$ \\
50784 & $11 / 21 / 89$ LSC & $6.31 \mathrm{E}+03$ \\
50826 & $12 / 06 / 89$ LSC & $6.89 \mathrm{E}+03$ \\
50876 & $1 / 09 / 90$ LSC & $6.31 \mathrm{E}+03$ \\
50713 & $10 / 20 / 89$ Alpha-Ra & $1.82 \mathrm{E}-01$ \\
50784 & $11 / 21 / 89$ Alpha-Ra & $1.70 \mathrm{E}-01$ \\
50826 & $12 / 06 / 89$ Alpha-Ra & $<1.32 \mathrm{E}-01$ \\
50876 & $1 / 09 / 90$ Alpha-Ra & $<7.35 \mathrm{E}-02$
\end{tabular}


DATA FOR 242-A Evaporator Steam Condensate

\begin{tabular}{|c|c|c|}
\hline \\
\hline \multicolumn{3}{|c|}{$\begin{array}{l}\text { Constituent } \\
\text { Arsenic (EP Toxic) }\end{array}$} \\
\hline Arsenic & (EP I & Toxic) \\
\hline Arsenic & (EP I & Toxic) \\
\hline Arsenic & (EP T & Toxic) \\
\hline Barium & & \\
\hline Barium & & \\
\hline Barium & & \\
\hline Barium & & \\
\hline Barium & (EP TC & (oxi.c) \\
\hline Barium & (EP TC & Soxic) \\
\hline Barium & (EP TC & (oxic) \\
\hline Barium & (EP TC & (oxic) \\
\hline Boron & & \\
\hline Boron & & \\
\hline Boron & & \\
\hline Boron & & \\
\hline Cadmium & (EP I & Toxic) \\
\hline Cadmium & (EP I & Toxic) \\
\hline Cadmium & (EP I & Toxic) \\
\hline Cadmium & (EP I & Toxic) \\
\hline Calcium & & \\
\hline Calcium & & \\
\hline Calciumi & & \\
\hline Calcium & & \\
\hline Chloride & & \\
\hline Chloride & & \\
\hline Chloride & & \\
\hline Chloride & & \\
\hline Chromium & m (EP & Toxic) \\
\hline Chromium & m (EP & Toxic) \\
\hline Chromium & m (EP & Toxic) \\
\hline Chromium & $m$ (EP & Toxic) \\
\hline Copper & & \\
\hline Copper & & \\
\hline Copper & & \\
\hline Copper & & \\
\hline Fluoride & & \\
\hline Fluoride & & \\
\hline Fluoride & & \\
\hline Fluoride & & \\
\hline Fluoride & & \\
\hline Fluoride & & \\
\hline Fluoride & & \\
\hline Fluoride & & \\
\hline Iron & & \\
\hline Iron & & \\
\hline Iron & & \\
\hline Iron & & \\
\hline Lead & & \\
\hline Lead & & \\
\hline Lead & & \\
\hline Lead & & \\
\hline Lead & $\mathrm{P}$ & $11 C$ \\
\hline
\end{tabular}

\begin{tabular}{|c|c|c|c|}
\hline ample \# & & & Result \\
\hline $50740 \mathrm{E}$ & $10 / 30 / 89$ & ICP & $<5.00 \mathrm{E}+02$ \\
\hline $50907 \mathrm{E}$ & $1 / 30 / 90$ & $I C P$ & $<5.00 E+02$ \\
\hline $50919 \mathrm{E}$ & $2 / 09 / 90$ & ICP & $<5.00 E+02$ \\
\hline $51087 \mathrm{E}$ & $3 / 22 / 90$ & ICP & $<5.00 E+02$ \\
\hline 50740 & $10 / 30 / 89$ & ICP & $3.30 \mathrm{E}+01$ \\
\hline 50907 & $1 / 30 / 90$ & $I C P$ & $3.20 E+01$ \\
\hline 50919 & $2 / 09 / 90$ & ICP & $3.10 \mathrm{E}+01$ \\
\hline 51087 & $3 / 22 / 90$ & ICP & 2. $90 \mathrm{E}+01$ \\
\hline $50740 \mathrm{E}$ & $10 / 30 / 89$ & ICP & $<1.00 \mathrm{E}+03$ \\
\hline $50907 \mathrm{E}$ & $1 / 30 / 90$ & ICP & $<1.00 E+03$ \\
\hline $50919 E$ & $2 / 09 / 90$ & ICP & $<1.00 \mathrm{E}+03$ \\
\hline $51087 \mathrm{E}$ & $3 / 22 / 90$ & ICP & $<1.00 \mathrm{E}+03$ \\
\hline 50740 & $10 / 30 / 89$ & ICP & $2.30 E+O 1$ \\
\hline 50907 & $1 / 30 / 90$ & ICP & $<1.00 \mathrm{E}+01$ \\
\hline 50919 & $2 / 09 / 90$ & ICP & $2.00 \mathrm{E}+01$ \\
\hline 51087 & $3 / 22 / 90$ & ICP & $2.10 E+01$ \\
\hline $50740 \mathrm{E}$ & $10 / 30 / 89$ & ICP & $<1.00 E+02$ \\
\hline $50907 \mathrm{E}$ & $1 / 30 / 90$ & ICP & $<1.00 \mathrm{E}+02$ \\
\hline $50919 \mathrm{E}$ & $2 / 09 / 90$ & ICP & $<1.00 \mathrm{E}+02$ \\
\hline $51087 \mathrm{E}$ & $3 / 22 / 90$ & ICP & $<1.00 E+02$ \\
\hline 50740 & $10 / 30 / 89$ & ICP & $1.87 \mathrm{E}+04$ \\
\hline 50907 & $1 / 30 / 90$ & ICP & $2.07 \mathrm{E}+04$ \\
\hline 50919 & $2 / 09 / 90$ & ICP & $1.99 E+04$ \\
\hline 51087 & $3 / 22 / 90$ & ICP & $1.78 \mathrm{E}+04$ \\
\hline 50740 & $10 / 30 / 89$ & IC & $1.30 \mathrm{E}+03$ \\
\hline 50907 & $1 / 30 / 90$ & IC & $1.10 \mathrm{E}+03$ \\
\hline 50919 & $2 / 09 / 90$ & IC & $1.10 \mathrm{E}+03$ \\
\hline 51087 & $3 / 22 / 90$ & IC & $9.00 E+02$ \\
\hline 50740E & $10 / 30 / 89$ & ICP & $<5.00 E+02$ \\
\hline $50907 \mathrm{E}$ & $1 / 30 / 90$ & ICP & $<5.00 \mathrm{E}+02$ \\
\hline 50919E & $2 / 09 / 90$ & ICP & $<5.00 E+02$ \\
\hline $51087 \mathrm{E}$ & $3 / 22 / 90$ & ICP & $<5.00 \mathrm{E}+02$ \\
\hline 50740 & $10 / 30 / 89$ & ICP & $1.10 \mathrm{E}+01$ \\
\hline 50907 & $1 / 30 / 90$ & ICP & $1.00 \mathrm{E}+01$ \\
\hline 50919 & $2 / 09 / 90$ & ICP & $1.30 \mathrm{E}+01$ \\
\hline 51087 & $3 / 22 / 90$ & ICP & $<1.00 \mathrm{E}+01$ \\
\hline 50740 & $10 / 30 / 89$ & IC & $<5.00 E+02$ \\
\hline 50740 & $10 / 30 / 89$ & ISE & 1. $32 \mathrm{E}+02$ \\
\hline 50907 & $1 / 30 / 90$ & $I C$ & $<5.00 E+02$ \\
\hline 50907 & $1 / 30 / 90$ & ISE & 1. $28 \mathrm{E}+02$ \\
\hline 50919 & $2 / 09 / 90$ & IC & $<5.00 E+02$ \\
\hline 50919 & $2 / 09 / 90$ & ISE & 1. $30 \mathrm{E}+02$ \\
\hline 51087 & $3 / 22 / 90$ & IC & $<5.00 E+02$ \\
\hline 51087 & $3 / 22 / 90$ & ISE & 1. $25 \mathrm{E}+02$ \\
\hline 50740 & $10 / 30 / 89$ & ICP & $2.11 E+02$ \\
\hline 50907 & $1 / 30 / 90$ & ICP & $4.90 E+01$ \\
\hline 50919 & $2 / 09 / 90$ & ICP & $3.70 E+01$ \\
\hline 51087 & $3 / 22 / 90$ & ICP & $3.90 E+01$ \\
\hline 50740 & $10 / 30 / 89$ & GFAA & $7.00 \mathrm{E}+00$ \\
\hline 50907 & $1 / 30 / 90$ & GFAA & $<5.00 \mathrm{E}+00$ \\
\hline 50919 & $2 / 09 / 90$ & GFAA & $<5.00 E+00$ \\
\hline 51087 & $3 / 22 / 90$ & GFAA & $<5.00 E+00$ \\
\hline $50740 \mathrm{E}$ & $10 / 30 / 89$ & ICP & $<5.00 \mathrm{E}+02$ \\
\hline
\end{tabular}


WHC-EP-0355, Appendix D

DATA FOR 242-A Evaporator Steam Condensate Continued

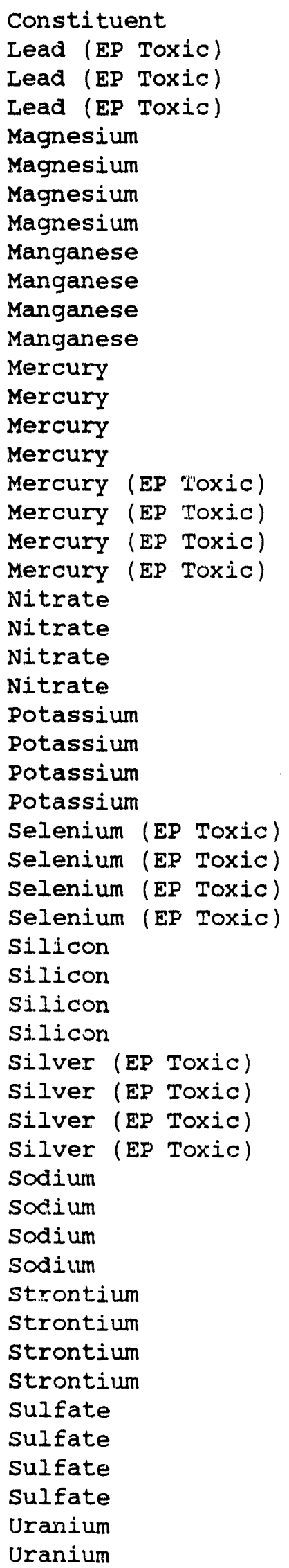

\begin{tabular}{|c|c|c|c|}
\hline Sample \# & Date & Method & Result \\
\hline $50907 \mathrm{E}$ & $1 / 30 / 90$ & ICP & $<5.00 E+02$ \\
\hline $50919 \mathrm{E}$ & $2 / 09 / 90$ & ICP & $<5.00 \mathrm{E}+02$ \\
\hline 51087E & $3 / 22 / 90$ & ICP & $<5.00 \mathrm{E}+02$ \\
\hline 50740 & $10 / 30 / 89$ & ICP & $4.43 E+03$ \\
\hline 50907 & $1 / 30 / 90$ & ICP & $4.71 E+03$ \\
\hline 50919 & $2 / 09 / 90$ & ICP & $4.52 \mathrm{E}+03$ \\
\hline 51087 & $3 / 22 / 90$ & ICP & $4.49 E+03$ \\
\hline 50740 & $10 / 30 / 89$ & ICP & 4. $20 E+01$ \\
\hline 50907 & $1 / 30 / 90$ & ICP & $<5.00 \mathrm{E}+00$ \\
\hline 50919 & $2 / 09 / 90$ & ICP & $<5.00 E+00$ \\
\hline 51087 & $3 / 22 / 90$ & ICP & $<5.00 E+00$ \\
\hline 50740 & $10 / 30 / 89$ & CVAA & $<1.00 \mathrm{E}-01$ \\
\hline 50907 & $1 / 30 / 90$ & CVAA & $<1.00 E-01$ \\
\hline 50919 & $2 / 09 / 90$ & CVAA & $<1.00 \mathrm{E}-01$ \\
\hline 51087 & $3 / 22 / 90$ & CVAA & 1.20E-01 \\
\hline $50740 \mathrm{E}$ & $10 / 30 / 89$ & CVAA/M & $<2.00 \mathrm{E}+01$ \\
\hline $50907 \mathrm{E}$ & $1 / 30 / 90$ & CVAA/M & $<2.00 \mathrm{E}+01$ \\
\hline $50919 \mathrm{E}$ & $2 / 09 / 90$ & CVAA/M & $<2.00 \mathrm{E}+01$ \\
\hline $51087 \mathrm{E}$ & $3 / 22 / 90$ & $C V A A / M$ & $<2.00 \mathrm{E}+01$ \\
\hline 50740 & $10 / 30 / 89$ & IC & $6.00 \mathbf{E}+02$ \\
\hline 50907 & $1 / 30 / 90$ & IC & $6.00 E+02$ \\
\hline 50919 & $2 / 09 / 90$ & $I C$ & $5.00 \mathrm{E}+02$ \\
\hline 51087 & $3 / 22 / 90$ & IC & $<5.00 \mathrm{E}+02$ \\
\hline 50740 & $10 / 30 / 89$ & ICP & $8.27 \mathrm{E}+02$ \\
\hline 50907 & $1 / 30 / 90$ & ICP & $7.37 \mathrm{E}+02$ \\
\hline 50919 & $2 / 09 / 90$ & ICP & 7. 11E+02 \\
\hline 51087 & $3 / 22 / 90$ & $I C P$ & $7.26 \mathrm{E}+02$ \\
\hline $50740 E$ & $10 / 30 / 89$ & ICP & $<5.00 E+02$ \\
\hline $50907 \mathrm{E}$ & $1 / 30 / 90$ & ICP & $<5.00 \mathrm{E}+02$ \\
\hline $50919 \mathrm{E}$ & $2 / 09 / 90$ & ICP & $<5.00 \mathrm{E}+02$ \\
\hline $51087 \mathrm{E}$ & $3 / 22 / 90$ & ICP & $<5.00 \mathrm{E}+02$ \\
\hline 50740 & $10 / 30 / 89$ & ICP & $2.69 \mathrm{E}+03$ \\
\hline 50907 & $1 / 30 / 90$ & ICP & $2.65 \mathrm{E}+03$ \\
\hline 50919 & $2 / 09 / 90$ & ICP & $2.50 E+03$ \\
\hline 51087 & $3 / 22 / 90$ & ICP & $2.16 \mathrm{E}+03$ \\
\hline $50740 \mathrm{E}$ & $10 / 30 / 89$ & $\pi C P$ & $<5.00 E+02$ \\
\hline $50907 \mathrm{E}$ & $1 / 30 / 90$ & ICP & $<5.00 E+02$ \\
\hline 50919E & $2 / 09 / 90$ & ICP & $<5.00 E+02$ \\
\hline $51087 \mathrm{E}$ & $3 / 22 / 90$ & ICP & $<5.00 \mathrm{E}+02$ \\
\hline 50740 & $10 / 30 / 89$ & ICP & $2.34 E+03$ \\
\hline 50907 & $1 / 30 / 90$ & ICP & 2. $22 \mathrm{E}+03$ \\
\hline 50919 & $2 / 09 / 90$ & ICP & $2.23 E+03$ \\
\hline 51087 & $3 / 22 / 90$ & ICP & $2.03 E+03$ \\
\hline 50740 & $10 / 30 / 89$ & $I C P$ & $1.02 \mathrm{E}+02$ \\
\hline 50907 & $1 / 30 / 90$ & ICP & $9.30 E+O 1$ \\
\hline 50919 & $2 / 09 / 90$ & ICP & $9.40 \mathrm{E}+01$. \\
\hline 51087 & $3 / 22 / 90$ & ICP & $9.60 E+01$ \\
\hline 50740 & $10 / 30 / 89$ & $I C$ & $1.07 \mathrm{E}+04$ \\
\hline 50907 & $1 / 30 / 90$ & IC & $1.04 \mathrm{E}+04$ \\
\hline 50919 & $2 / 09 / 90$ & IC & $1.08 E+04$ \\
\hline 51087 & $3 / 22 / 90$ & IC & $1.03 E+04$ \\
\hline 50740 & $10 / 30 / 89$ & GEA & $6.21 E-01$ \\
\hline 51087 & $3 / 22 / 90$ & GEA & 4. 19E-OI \\
\hline
\end{tabular}


DATA FOR 242-A Evaporator steam Condensate Continued

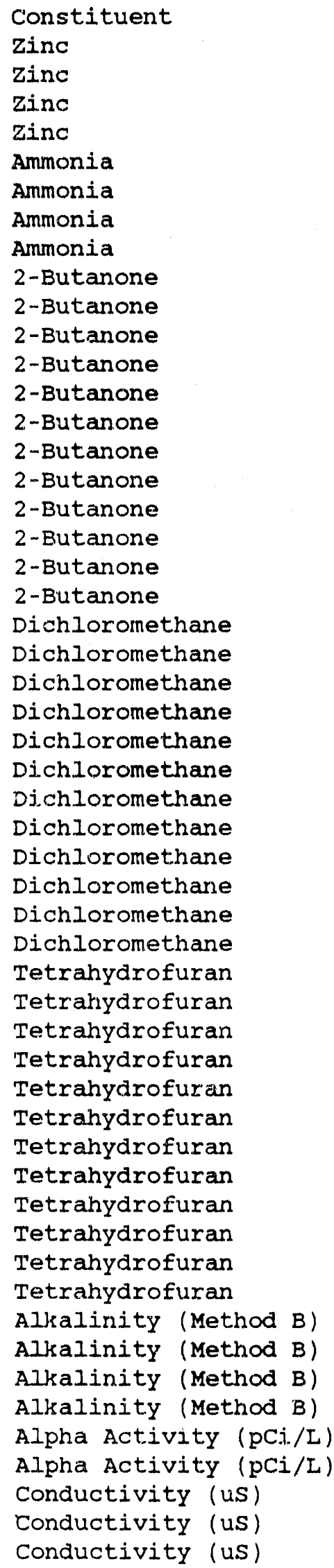

\begin{tabular}{|c|c|c|c|}
\hline Sample \# & Date & Method & Result \\
\hline 50740 & $10 / 30 / 89$ & ICP & $2.90 \mathrm{E}+01$ \\
\hline 50907 & $1 / 30 / 90$ & ICP & $1.00 E+01$ \\
\hline 50919 & $2 / 09 / 90$ & ICP & $2.90 \mathrm{E}+0.1$ \\
\hline 51087 & $3 / 22 / 90$ & ICP & $7.00 \mathrm{E}+00$ \\
\hline 50740 & $10 / 30 / 89$ & ISE & $7.10 \mathrm{E}+01$ \\
\hline 50907 & $1 / 30 / 90$ & ISE & $<5.00 \mathrm{E}+01$ \\
\hline 50919 & $2 / 09 / 90$ & ISE & $<5.00 \mathrm{E}+01$ \\
\hline 51087 & $3 / 22 / 90$ & ISE & 8. 10E+01 \\
\hline 50740 & $10 / 30 / 89$ & VOA & $<1.00 \mathrm{E}+01$ \\
\hline $50740 B$ & $10 / 30 / 89$ & VOA & $<6.00 \mathrm{E}+00$ \\
\hline $50740 \mathrm{~T}$ & $10 / 30 / 89$ & VOA & $1.80 \mathrm{E}+01$ \\
\hline 50907 & $1 / 30 / 90$ & VOA & $<1.00 E+01$ \\
\hline $50907 \mathrm{~B}$ & $1 / 30 / 90$ & VOA & $<6.00 \mathrm{E}+00$ \\
\hline $50907 \mathrm{~T}$ & $1 / 30 / 90$ & VOA & $<8.00 E+00$ \\
\hline 50919 & $2 / 09 / 90$ & VOA & $<1.00 E+01$ \\
\hline $50919 B$ & $2 / 09 / 90$ & VOA & $<5.00 E+00$ \\
\hline $50919 \mathrm{~T}$ & $2 / 09 / 90$ & VOA & $<6.00 \mathrm{E}+00$ \\
\hline 51087 & $3 / 22 / 90$ & VOA & $<1.00 \mathrm{E}+01$ \\
\hline $51087 \mathrm{~B}$ & $3 / 22 / 90$ & VOA & $<1.00 E+01$ \\
\hline $51087 \mathrm{~T}$ & $3 / 22 / 90$ & VOA & $<1.00 \mathrm{E}+01$ \\
\hline 50740 & $10 / 30 / 89$ & VOA & $<5.00 \mathrm{E}+00$ \\
\hline $50740 \mathrm{~B}$ & $10 / 30 / 89$ & VOA & $<3.00 \mathrm{E}+00$ \\
\hline $50740 \mathrm{~T}$ & $10 / 30 / 89$ & VOA & 2.30E+01 \\
\hline 50907 & $1 / 30 / 90$ & VOA & $<5.00 \mathrm{E}+00$ \\
\hline $50907 \mathrm{~B}$ & $1 / 30 / 90$ & VOA & $<4.00 E+00$ \\
\hline $50907 \mathrm{~T}$ & $1 / 30 / 90$ & VOA & $<4.00 \mathrm{E}+00$ \\
\hline 50919 & $2 / 09 / 90$ & VOA & $<5.00 E+00$ \\
\hline $50919 B$ & $2 / 09 / 90$ & VOA & $<5.00 \mathrm{E}+00$ \\
\hline $50919 \mathrm{~T}$ & $2 / 09 / 90$ & VOA & $<5.00 E+00$ \\
\hline 51087 & $3 / 22 / 90$ & VOA & $<5.00 E+00$ \\
\hline $51087 \mathrm{~B}$ & $3 / 22 / 90$ & VOA & $<5.00 \mathrm{E}+00$ \\
\hline $51087 T$ & $3 / 22 / 90$ & VOA & $<5.00 E+00$ \\
\hline 50740 & $10 / 30 / 89$ & VOA & $<1.00 \mathrm{E}+01$ \\
\hline $50740 \mathrm{~B}$ & $10 / 30 / 89$ & VOA & $1.70 \mathrm{E}+01$ \\
\hline $50740 \mathrm{~T}$ & $10 / 30 / 89$ & VOA & $<1.00 \mathrm{E}+01$ \\
\hline 50907 & $1 / 30 / 90$ & VOA & $<1.00 E+01$ \\
\hline $50907 \mathrm{~B}$ & $1 / 30 / 90$ & VOA & $<1.00 E+01$ \\
\hline $50907 \mathrm{~T}$ & $1 / 30 / 90$ & VOA & $<8.00 \mathrm{E}+00$ \\
\hline 50919 & $2 / 09 / 90$ & VOA & $<1.00 E+01$ \\
\hline $50919 \mathrm{~B}$ & $2 / 09 / 90$ & VOA & $<1.00 E+01$ \\
\hline $50919 \mathrm{~T}$ & $2 / 09 / 90$ & VOA & $<1.00 E+01$ \\
\hline 51087 & $3 / 22 / 90$ & VOA & $<1.00 \mathrm{E}+01$ \\
\hline $51087 \mathrm{~B}$ & $3 / 22 / 90$ & VOA & $<9.00 E+00$ \\
\hline $51087 \mathrm{~T}$ & $3 / 22 / 90$ & VOA & $<6.00 \mathrm{E}+00$ \\
\hline 50740 & $10 / 30 / 89$ & TITRA & $6.0 O E+04$ \\
\hline 50907 & $1 / 30 / 90$ & TITRA & $6.60 \mathrm{E}+04$ \\
\hline 50919 & $2 / 09 / 90$ & TITRA & $6.30 \mathrm{E}+04$ \\
\hline 51087 & $3 / 22 / 90$ & TITRA & $6.00 E+04$ \\
\hline 50740 & $10 / 30 / 89$ & Alpha & $<3.48 \mathrm{E}-01$ \\
\hline 51087 & $3 / 22 / 90$ & Alpha & $9.44 E-01$ \\
\hline 50740 & $10 / 30 / 89$ & COND-FId & 1. $48 \mathrm{EE}+02$ \\
\hline 50907 & $1 / 30 / 90$ & COND-FId & $1.77 \mathrm{E}+02$ \\
\hline 50919 & $2 / 09 / 90$ & COND-EId & 1. $82 \mathrm{E}+02$ \\
\hline
\end{tabular}


DATA FOR 242-A Evaporator steam Condensate Continued

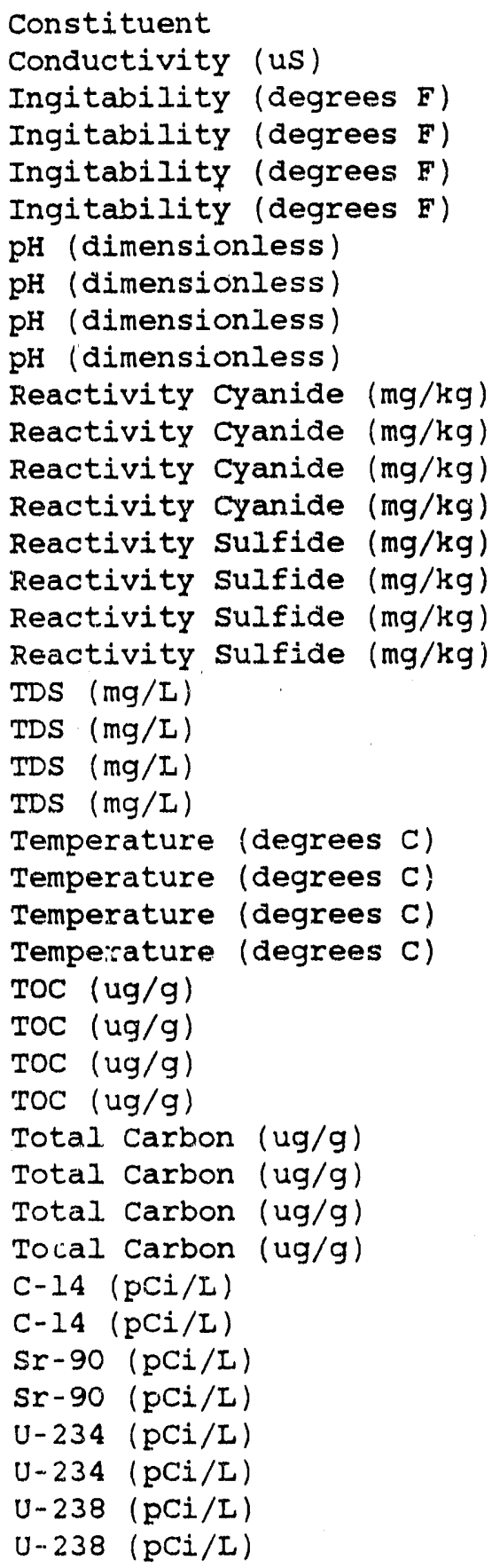

\begin{tabular}{|c|c|c|c|}
\hline ample \# & Date & & \\
\hline 51087 & $3 / 22 / 90$ & COND-FId & $1.52 \mathrm{E}+02$ \\
\hline $50740 \mathrm{E}$ & $10 / 30 / 89$ & IGNIT & 2. $10 E+02$ \\
\hline $50907 \mathrm{E}$ & $1 / 30 / 90$ & IGNIT & $2.10 \mathrm{E}+02$ \\
\hline 50919E & $2 / 09 / 90$ & IGNIT & $2.04 E+02$ \\
\hline $51087 \mathrm{E}$ & $3 / 22 / 90$ & IGNIT & $2.04 \mathrm{E}+02$ \\
\hline 50740 & $10 / 30 / 89$ & $\mathrm{PH}-\mathrm{F} I \mathrm{~d}$ & $7.98 E+00$ \\
\hline 50907 & $1 / 30 / 90$ & $\mathrm{PH}-\mathrm{Fld}$ & $8.05 \mathrm{E}+00$ \\
\hline 50919 & $2 / 09 / 90$ & PH-FId & $7.96 \mathrm{E}+00$ \\
\hline 51087 & $3 / 22 / 90$ & PH - FId & $8.18 E+00$ \\
\hline $50740 \mathrm{E}$ & $10 / 30 / 89$ & DSPEC & $<1.00 \mathrm{E}+02$ \\
\hline $50907 \mathrm{E}$ & $1 / 30 / 90$ & DSPEC & $<1.00 \mathrm{E}+102$ \\
\hline $50919 \mathrm{E}$ & $2 / 09 / 90$ & DSPEC & $<1.00 \mathrm{E}+02$ \\
\hline $51087 \mathrm{E}$ & $3 / 22 / 90$ & DSPEC & $<1.00 \mathrm{E}+02$ \\
\hline 50740E & $10 / 30 / 89$ & DTITRA & $<1.00 E+02$ \\
\hline $50907 \mathrm{E}$ & $1 / 30 / 90$ & DTITRA & $<1.00 \mathrm{E}+02$ \\
\hline 50919E & $2 / 09 / 90$ & DTITRA & $<1.00 \mathrm{E}+02$ \\
\hline $51087 \mathrm{E}$ & $3 / 22 / 90$ & DTITRA & $<1.00 \mathrm{E}+02$ \\
\hline 50740 & $10 / 30 / 89$ & TDS & $7.50 E+04$ \\
\hline 50907 & $1 / 30 / 90$ & TDS & $7.30 \mathrm{E}+04$ \\
\hline 50919 & $2 / 09 / 90$ & TDS & $7.20 \mathrm{E}+04$ \\
\hline 51087 & $3 / 22 / 90$ & TDS & $8.40 E+04$ \\
\hline 50740 & $10 / 30 / 89$ & TEMP-FId & $2.05 E+01$ \\
\hline 50907 & $1 / 30 / 90$ & TEMP-FId & 2. $37 \mathrm{E}+01$ \\
\hline 50919 & $2 / 09 / 90$ & TEMP-EId & $2.88 \mathrm{E}+01$ \\
\hline 51087 & $3 / 22 / 90$ & TEMP-FId & $2.19 \mathrm{E}+01$ \\
\hline 50740 & $10 / 30 / 89$ & TOC & $<1.70 \mathrm{E}+03$ \\
\hline 50907 & $1 / 30 / 90$ & TOC & $<1.10 \mathrm{E}+03$ \\
\hline 50919 & $2 / 09 / 90$ & TOC & $<1.10 \mathrm{E}+03$ \\
\hline 51087 & $3 / 22 / 90$ & TOC & $1.10 \mathrm{E}+03$ \\
\hline 50740 & $10 / 30 / 89$ & TC & 1. $44 \mathrm{E}+04$ \\
\hline 50907 & $1 / 30 / 90$ & TC & $1.35 E+04$ \\
\hline 50919 & $2 / 09 / 90$ & $T C$ & $1.32 E+04$ \\
\hline 51087 & $3 / 22 / 90$ & $\mathrm{TC}$ & $1.61 \mathrm{E}+04$ \\
\hline 50740 & $10 / 30 / 89$ & ISC & $4.50 E+00$ \\
\hline 51087 & $3 / 22 / 90$ & LSC & $3.58 \mathrm{E}+00$ \\
\hline 50740 & $10 / 30 / 89$ & Beta. & $3.08 \mathrm{E}-01$ \\
\hline 51087 & $3 / 22 / 90$ & Beta & $6.88 \mathrm{E}-01$ \\
\hline 50740 & $10 / 30 / 89$ & $A E A$ & $2.01 E-01$ \\
\hline 51087 & $3 / 22 / 90$ & $A E A$ & $1.84 \mathrm{E}-01$ \\
\hline 50740 & $10 / 30 / 89$ & AEA & $1.61 \mathrm{E}-01$ \\
\hline 51087 & $3 / 22 / 90$ & $A E A$ & $1.78 \mathrm{E}-01$ \\
\hline
\end{tabular}




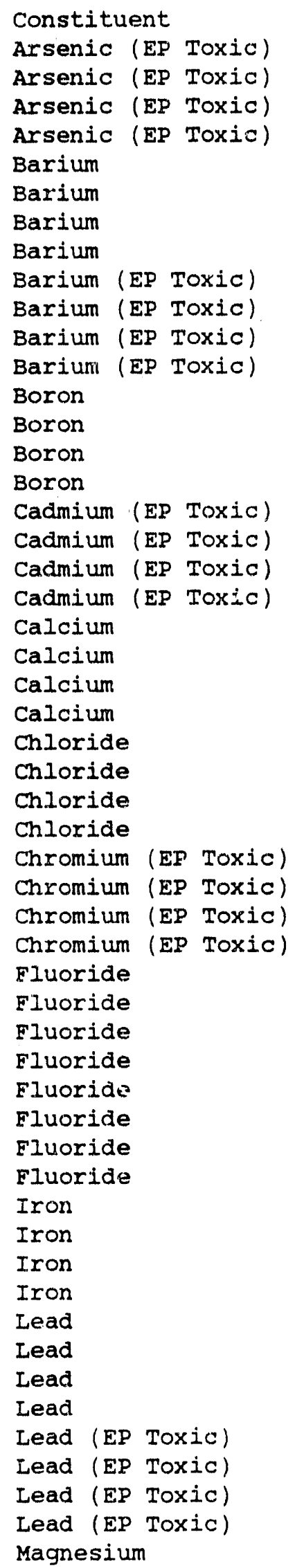

\begin{tabular}{|c|c|c|c|}
\hline Sample \# & Date & Method & Result \\
\hline $50788 E$ & $11 / 22 / 89$ & ICP & $<5.00 E+02$ \\
\hline $50812 \mathrm{E}$ & $11 / 29 / 89$ & ICP & $<5.00 \mathrm{E}+02$ \\
\hline $50931 \mathrm{E}$ & $2 / 12 / 90$ & ICP & $<5.00 \mathrm{E}+02$ \\
\hline $51032 E$ & $3 / 08 / 90$ & ICP & $<5.00 E+02$ \\
\hline 50788 & $11 / 22 / 89$ & ICP & 3.10E+01 \\
\hline 50812 & $11 / 29 / 89$ & ICP & 3. $30 \mathrm{E}+01$ \\
\hline 50931 & $2 / 12 / 90$ & ICP & 3. $20 \mathrm{E}+01$ \\
\hline 5.1032 & $3 / 08 / 90$ & ICP & $3.00 \mathrm{E}+01$ \\
\hline $50788 \mathrm{E}$ & $11 / 22 / 89$ & ICP & $<1.00 \mathrm{E}+03$ \\
\hline $50812 \mathrm{E}$ & $11 / 29 / 89$ & ICP & $<1.00 \mathrm{E}+03$ \\
\hline $50931 \mathrm{E}$ & $2 / 12 / 90$ & ICP & $<1.00 \mathrm{E}+03$ \\
\hline $51032 \mathrm{E}$ & $3 / 08 / 90$ & ICP & $<1.00 \mathrm{E}+03$ \\
\hline 50788 & $11 / 22 / 89$ & ICP & $<1.00 \mathrm{E}+01$ \\
\hline 50812 & $11 / 29 / 89$ & ICP & $<1.00 \mathrm{E}+01$ \\
\hline 50931 & $2 / 12 / 90$ & ICP & 1. $20 \mathrm{E}+01$ \\
\hline 51032 & $3 / 08 / 90$ & ICP & 3. $10 \mathrm{E}+01$ \\
\hline $50788 \mathrm{E}$ & $11 / 22 / 89$ & ICP & $<1.00 E+02$ \\
\hline $50812 \mathrm{E}$ & $11 / 29 / 89$ & ICP & $<1.00 E+02$ \\
\hline $50931 E$ & $2 / 12 / 90$ & ICP & $<1.00 \mathrm{E}+02$ \\
\hline $51032 \mathrm{E}$ & $3 / 08 / 90$ & ICP & $<1.00 \mathrm{E}+02$ \\
\hline 50788 & $11 / 22 / 89$ & ICP & $1.82 \mathrm{E}+04$ \\
\hline 50812 & $11 / 29 / 89$ & ICP & $1.95 \mathrm{E}+04$ \\
\hline 50931 & $2 / 12 / 90$ & ICP & $1.90 \mathbf{E}+04$ \\
\hline 51032 & $3 / 08 / 90$ & ICP & $1.86 \mathrm{E}+04$ \\
\hline 50788 & $11 / 22 / 89$ & $I C$ & $<5.00 \mathrm{E}+02$ \\
\hline 50812 & $11 / 29 / 89$ & IC & $9.00 \mathrm{E}+02$ \\
\hline 50931 & $2 / 12 / 90$ & IC & $1.10 \mathrm{E}+03$ \\
\hline 51032 & $3 / 08 / 90$ & $I C$ & $1.00 \mathrm{E}+03$ \\
\hline $50788 \mathrm{E}$ & $11 / 22 / 89$ & ICP & $<5.00 \mathrm{E}+02$ \\
\hline $50812 \mathrm{E}$ & $11 / 29 / 89$ & ICP & $<5.00 \mathrm{E}+02$ \\
\hline $50931 \mathrm{E}$ & $2 / 12 / 90$ & ICP & $<5.00 \mathrm{E}+02$ \\
\hline $51032 \mathrm{E}$ & $3 / 08 / 90$ & ICP & $<5.00 \mathrm{E}+02$ \\
\hline 50788 & $11 / 22 / 89$ & IC & $<5.00 \mathrm{E}+02$ \\
\hline 50788 & $11 / 22 / 89$ & ISE & $1.38 \mathrm{E}+02$ \\
\hline 50812 & $11 / 29 / 89$ & IC & $<5.00 \mathrm{E}+02$ \\
\hline 50812 & $11 / 29 / 89$ & ISE & 1.31E+02 \\
\hline 50931 & $2 / 12 / 90$ & IC & $<5.00 \mathrm{E}+02$ \\
\hline 50931 & $2 / 12 / 90$ & ISE & $1.27 \mathrm{E}+02$ \\
\hline 51032 & $3 / 08 / 90$ & IC & $<5.00 E+02$ \\
\hline 51032 & $3 / 08 / 90$ & ISE & 1. $32 \mathrm{E}+02$ \\
\hline 50788 & $11 / 22 / 89$ & ICP & $3.80 \mathrm{E}+0.1$ \\
\hline 50812 & $11 / 29 / 89$ & ICP & $3.90 \mathrm{E}+01$ \\
\hline 50931 & $2 / 12 / 90$ & ICP & $<3.00 E+01$ \\
\hline 51032 & $3 / 08 / 90$ & ICP & 3. 30E+OI \\
\hline 50788 & $11 / 22 / 89$ & GFAA & $<5.00 \mathrm{E}+00$ \\
\hline 50812 & $11 / 29 / 89$ & GFAA & $<5.00 E+00$ \\
\hline 50931 & $2 / 12 / 90$ & GFAA & $<5.00 E+00$ \\
\hline 51032 & $3 / 08 / 90$ & GEAA & $7.00 \mathrm{E}+00$ \\
\hline $50788 \mathrm{E}$ & $11 / 22 / 89$ & ICP & $<5.00 E+02$ \\
\hline $50812 \mathrm{E}$ & $11 / 29 / 89$ & ICP & $<5.00 E+02$ \\
\hline $50931 \mathrm{E}$ & $2 / 12 / 90$ & ICP & $<5.00 E+02$ \\
\hline $51032 \mathrm{E}$ & $3 / 08 / 90$ & ICP & $<5.00 \mathbf{E}+02$ \\
\hline 50788 & $11 / 22 / 89$ & ICP & $4.05 \mathrm{E}+03$ \\
\hline
\end{tabular}


WHC-EP-0355, Appendix D

DATA FOR B Plant Cooling water

Continued

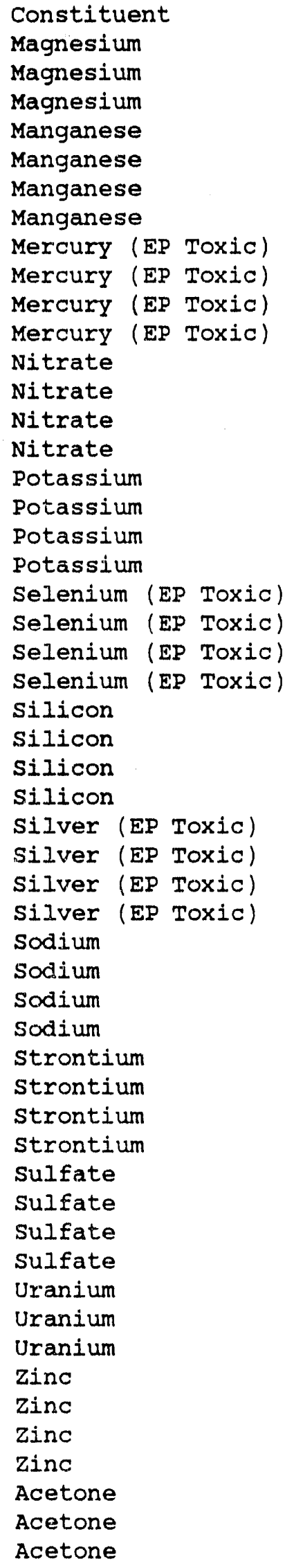

\begin{tabular}{|c|c|c|c|}
\hline Sample \# & Date & Method & Result \\
\hline 50812 & $11 / 29 / 89$ & ICP & $4.46 \mathrm{E}+03$ \\
\hline 50931 & $2 / 12 / 90$ & ICP & $4.36 E+03$ \\
\hline 51032 & $3 / 08 / 90$ & ICP & $4.57 \mathrm{E}+03$ \\
\hline 50788 & $11 / 22 / 89$ & ICP & $<5.00 \mathrm{E}+00$ \\
\hline 50812 & $11 / 29 / 89$ & ICP & $5.00 \mathrm{E}+00$ \\
\hline 50931 & $2 / 12 / 90$ & ICP & $<5.00 \mathrm{E}+00$ \\
\hline 51032 & $3 / 08 / 90$ & ICP & $<5.00 E+00$ \\
\hline $50788 \mathrm{E}$ & $11 / 22 / 89$ & $C V A A / M$ & $<2.00 E+01$ \\
\hline $50812 \mathrm{E}$ & $11 / 29 / 89$ & $C V A A / M$ & $<2.00 \mathrm{E}+01$ \\
\hline $50931 \mathrm{E}$ & $2 / 12 / 90$ & $C V A A / M$ & $<2.00 \mathrm{E}+01$ \\
\hline $51032 \mathrm{E}$ & $3 / 08 / 90$ & CVAA/M & $<2.00 \mathrm{E}+01$ \\
\hline 50788 & $11 / 22 / 89$ & IC & $5.00 \mathrm{E}+02$ \\
\hline 50812 & $11 / 29 / 89$ & IC & $5.00 \mathrm{E}+02$ \\
\hline 50931 & $2 / 12 / 90$ & IC & $5.00 \mathrm{E}+02$ \\
\hline 51032 & $3 / 08 / 90$ & IC & $<5.00 E+02$ \\
\hline 50788 & $11 / 22 / 89$ & ICP & $7.33 E+02$ \\
\hline 50812 & $11 / 29 / 89$ & ICP & $7.68 \mathrm{E}+02$ \\
\hline 50931 & $2 / 12 / 90$ & ICP & $7.01 \mathrm{E}+02$ \\
\hline 51032 & $3 / 08 / 90$ & ICP & $7.61 \mathrm{E}+02$ \\
\hline $50788 \mathrm{E}$ & $11 / 22 / 89$ & ICP & $<5.00 E+02$ \\
\hline $50812 \mathrm{E}$ & $11 / 29 / 89$ & ICP & $<5.00 E+02$ \\
\hline $50931 \mathrm{E}$ & $2 / 12 / 90$ & ICP & $<5.00 E+02$ \\
\hline $51032 \mathrm{E}$ & $3 / 08 / 90$ & ICP & $<5.00 \mathrm{E}+02$ \\
\hline 50788 & $11 / 22 / 89$ & ICP & $2.36 \mathrm{E}+03$ \\
\hline 50812 & $11 / 29 / 89$ & ICP & $2.66 E+03$ \\
\hline 50931 & $2 / 12 / 90$ & ICP & $2.44 \mathrm{E}+03$ \\
\hline 51032 & $3 / 08 / 90$ & ICP & $2.39 E+03$ \\
\hline $50788 \mathrm{E}$ & $11 / 22 / 89$ & ICP & $<5.00 E+02$ \\
\hline $50812 \mathrm{E}$ & $11 / 29 / 89$ & ICP & $<5.00 E+02$ \\
\hline $50931 \mathrm{E}$ & $2 / 12 / 90$ & ICP & $<5.00 E+02$ \\
\hline $51032 \mathrm{E}$ & $3 / 08 / 90$ & ICP & $<5.00 E+02$ \\
\hline 50788 & $11 / 22 / 89$ & ICP & $2.01 E+03$ \\
\hline 50812 & $11 / 29 / 89$ & ICP & $2.13 E+03$ \\
\hline 50931 & $2 / 12 / 90$ & ICP & $2.00 \mathrm{E}+03$ \\
\hline 51032 & $3 / 08 / 90$ & ICP & $2.09 E+03$ \\
\hline 50788 & $11 / 22 / 89$ & ICP & $9.30 E+0 I$ \\
\hline 50812 & $11 / 29 / 89$ & ICP & $9.90 \mathrm{E}+01$ \\
\hline 50931 & $2 / 12 / 90$ & ICP & $8.70 \mathrm{E}+01$ \\
\hline 51032 & $3 / 08 / 90$ & $I C P$ & $9.70 \mathrm{E}+01$ \\
\hline 50788 & $11 / 22 / 89$ & IC & $9.30 \mathrm{E}+03$ \\
\hline 50812 & $11 / 29 / 89$ & IC & $9.90 E+03$ \\
\hline 50931 & $2 / 12 / 90$ & IC & $1.13 E+04$ \\
\hline 51032 & $3 / 08 / 90$ & IC & $1.08 E+04$ \\
\hline 50788 & $11 / 22 / 89$ & GEA & $3.95 E-01$ \\
\hline 50812 & $11 / 29 / 89$ & GEA & 1. $42 E+00$ \\
\hline 51032 & $3 / 08 / 90$ & GEA & $5.58 \mathrm{E}-01$ \\
\hline 50788 & $11 / 22 / 89$ & ICP & $<5.00 \mathrm{E}+00$ \\
\hline 50812 & $11 / 29 / 89$ & ICP & $<5.00 E+00$ \\
\hline 50931 & $2 / 12 / 90$ & ICP & $8.00 \mathrm{E}+00$ \\
\hline 51032 & $3 / 08 / 90$ & $I C P$ & $6.00 E+00$ \\
\hline 50788 & $11 / 22 / 89$ & VOA & $<1.00 \mathrm{E}+01$ \\
\hline 50788 & $11 / 22 / 89$ & $A B N$ & $<1.00 E+01$ \\
\hline $50788 B$ & $11 / 22 / 89$ & VOA & $<7.00 \mathrm{E}+00$ \\
\hline
\end{tabular}


DATA FOR B Plant Cooling water

Continued

\begin{tabular}{|c|c|c|c|c|}
\hline $\begin{array}{l}\text { Constituent } \\
\text { Acetone }\end{array}$ & $\begin{array}{l}\text { Sample \# } \\
50788 \mathrm{~T}\end{array}$ & $\begin{array}{c}\text { Date } \\
11 / 22 / 89\end{array}$ & $\begin{array}{l}\text { Method } \\
\text { VOA }\end{array}$ & $\begin{array}{l}\text { Result } \\
<1.00 \mathrm{E}+01\end{array}$ \\
\hline Acetone & 50812 & $11 / 29 / 89$ & VOA & $<1,00 E+01$ \\
\hline Acetone & 50812 & $11 / 29 / 89$ & ABN & $<1.00 \mathrm{E}+01$ \\
\hline Acetone & $50812 B$ & $11 / 29 / 89$ & VOA & $<1.00 \mathrm{E}+01$ \\
\hline Acetone & 50931 & $2 / 12 / 90$ & VOA & $1.60 \mathrm{E}+01$ \\
\hline Acetone & 50931 & $2 / 12 / 90$ & $A B N$ & $<1.00 E+01$ \\
\hline Acetone & $50931 B$ & $2 / 12 / 90$ & VOA & $5.00 E+01$ \\
\hline Acetone & $50931 \mathrm{~T}$ & $2 / 12 / 90$ & VOA & $2.50 E+01$ \\
\hline Acetone & 51032 & $3 / 08 / 90$ & VOA & $<1.00 E+01$ \\
\hline Acetone & 51032 & $3 / 08 / 90$ & $A B N$ & $<1.00 E+01$ \\
\hline Acetone & $51032 \mathrm{~B}$ & $3 / 08 / 90$ & VOA & $<1.00 E+01$ \\
\hline Acetone & $51032 \mathrm{~T}$ & $3 / 08 / 90$ & VOA & $<5.00 \mathrm{E}+00$ \\
\hline 1-Butanol & 50788 & $11 / 22 / 89$ & DIGC & $<1.00 E+04$ \\
\hline 1-Butanol & 50812 & $11 / 29 / 89$ & DIGC & $<1.00 E+04$ \\
\hline 1-Butanol & 50931 & $2 / 12 / 90$ & DIGC & $<1.00 E+04$ \\
\hline 1-Butanol & 51032 & $3 / 08 / 90$ & VOA & $1.20 \mathrm{E}+01$ \\
\hline 1-Butanol & 51032 & $3 / 08 / 90$ & DIGC & $<1.00 E+04$ \\
\hline I-Butanol & $51032 \mathrm{~B}$ & $3 / 08 / 90$ & VOA & $1.30 E+01$ \\
\hline 1-Butanol & $51032 \mathrm{~T}$ & $3 / 08 / 90$ & VOA & $1.40 E+01$ \\
\hline 2-Butanone & 50788 & $11 / 22 / 89$ & VOA & $<1.00 \mathrm{E}+01$ \\
\hline 2-Butanone & $50788 B$ & $11 / 22 / 89$ & VOA & $<1.00 \mathrm{E}+01$ \\
\hline 2-Butanone & $50788 \mathrm{~T}$ & $11 / 22 / 89$ & VOA & $<1.00 E+01$ \\
\hline 2-Butanone & 50812 & $11 / 29 / 89$ & VOA & $<1.00 E+01$ \\
\hline 2-Butanone & $50812 \mathrm{~B}$ & $11 / 29 / 89$ & VOA & $<1.00 \mathrm{E}+01$ \\
\hline 2-Butanone & 50931 & $2 / 12 / 90$ & VOA & $1.00 \mathrm{E}+01$ \\
\hline 2-Butanone & $50931 \mathrm{~B}$ & $2 / 12 / 90$ & VOA & $1.03 \mathrm{E}+02$ \\
\hline 2-Butanone & $50931 \mathrm{~T}$ & $2 / 12 / 90$ & VOA & $9.10 E+01$ \\
\hline 2-Butanone & 51032 & $3 / 08 / 90$ & VOA & $<1.00 \mathrm{E}+01$ \\
\hline 2-Butanone & $51032 \mathrm{~B}$ & $3 / 08 / 90$ & Vun & $<1.00 E+01$ \\
\hline 2-Butanone & $51032 \mathrm{~T}$ & $3 / 08 / 90$ & VOA & $<1.00 E+01$ \\
\hline Trichloromethane & 50788 & $11 / 22 / 89$ & VOA & $<5.00 \mathrm{E}+00$ \\
\hline Trichloromethane & $50788 \mathrm{~B}$ & $11 / 22 / 89$ & VOA & $<3.00 E+00$ \\
\hline Trichloromethane & $50788 \mathrm{~T}$ & $11 / 22 / 89$ & VOA & $1.10 \mathrm{E}+01$ \\
\hline Trichloromethane & 50812 & $11 / 29 / 89$ & VOA & $8.00 \mathrm{E}+00$ \\
\hline Trichloromethane & $50812 B$ & $11 / 29 / 89$ & VOA & $<5.00 \mathrm{E}+00$ \\
\hline Trichloromethane & 50931 & $2 / 12 / 90$ & VOA & $<5.00 E+00$ \\
\hline Trichloromethane & 509318 & $2 / 12 / 90$ & VOA & $<4.00 E+00$ \\
\hline Trichloromethane & $50931 \mathrm{~T}$ & $2 / 12 / 90$ & VOA & $<3.00 \mathrm{E}+00$ \\
\hline Trichloromethane & 51032 & $3 / 08 / 90$ & VOA & $<5.00 E+00$ \\
\hline Trichloromethane & $51032 B$ & $3 / 08 / 90$ & VOA & $<5.00 \mathrm{E}+00$ \\
\hline Trichloromethane & $51032 T$ & $3 / 08 / 90$ & VOA & $<5.00 \mathrm{E}+00$ \\
\hline Alkalinity (Method B) & 50788 & $11 / 22 / 89$ & TITRA & $5.80 E+04$ \\
\hline Alkalinity (Method B) & 50812 & $11 / 29 / 89$ & TITRA & $5.90 \mathrm{E}+04$ \\
\hline Alkalinity (Method B) & 50931 & $2 / 12 / 90$ & TITRA & $6.20 \mathrm{E}+04$ \\
\hline Alkalinity (Method B) & 51032 & $3 / 08 / 90$ & TITRA & $6.00 E+04$ \\
\hline Beta Activity (pCi/L) & 50788 & $11 / 22 / 89$ & Beta & $2.69 \mathrm{E}+00$ \\
\hline Beta Activity (pCi/L) & 50812 & $11 / 29 / 89$ & Beta & $2.29 E+00$ \\
\hline Conductivity (uS) & 50788 & $11 / 22 / 89$ & COND-Fld & $1.44 E+02$ \\
\hline Conductivity (uS) & 50812 & $11 / 29 / 89$ & COND-Fld & $1.40 E+02$ \\
\hline Conductivity (us) & 50931 & $2 / 12 / 90$ & COND-Fld & 1. $26 \mathrm{E}+02$ \\
\hline Conductivity (US) & 51032 & $3 / 08 / 90$ & COND-Fld & 1. $32 \mathrm{E}+02$ \\
\hline Ingitability (degrees F) & $50788 \mathrm{E}$ & $11 / 22 / 89$ & IGNIT & $2.08 \mathrm{E}+02$ \\
\hline Ingitability (degrees F) & $50812 \mathrm{E}$ & $11 / 29 / 89$ & IGNIT & $2.12 \mathrm{E}+02$ \\
\hline
\end{tabular}


DATA FOR B Plant Cooling water

Continued

Constituent

Ingitability (degrees F) Ingitability (degrees F) $\mathrm{pH}$ (dimensionless)

$\mathrm{pH}$ (dimensionless)

$\mathrm{pH}$ (dimensionless)

$\mathrm{pH}$ (dimensionless)

Reactivity Cyanide (mg/kg)

Reactivity Cyanide (mg/kg)

Reactivity Cyanide (mg/kg)

Reactivity Cyanide ( $\mathrm{mg} / \mathrm{kg}$ )

Reactivity sulfide ( $\mathrm{mg} / \mathrm{kg}$ )

Reactivity sulfide ( $\mathrm{mg} / \mathrm{kg}$ )

Reactivity Sulfide ( $\mathrm{mg} / \mathrm{kg}$ )

Reactivity sulfide (mg/rg)

$\operatorname{TDS}(\mathrm{mg} / \mathrm{L}$ )

$\operatorname{TDS}(\mathrm{mg} / \mathrm{I})$

$\operatorname{TDS}(\mathrm{mg} / \mathrm{I})$

$\operatorname{TDS}(\mathrm{mg} / \mathrm{I})$

Temperature (degrees C)

Temperature (degrees C)

Temperature (degrees C)

Temperature (degrees $\mathrm{C}$ )

TOC (ug/g)

TOC (ug/g)

TOC (ug/g)

TOC (ug/g)

Total Carbon ( $\mathrm{ug} / \mathrm{g}$ )

Total Carbon (ug/g)

Total Carbon ( $u g / g$ )

Total Carbon (ug/g)

TOX (ug ( Cl)/L)

$\operatorname{TOX}(\mathrm{ug}(\mathrm{Cl}) / \mathrm{L})$

$\operatorname{TOX}(\operatorname{ug}(\mathrm{Cl}) / \mathrm{L})$

$\operatorname{TOX}(\mathrm{ug}(\mathrm{CI}) / \mathrm{L})$

Co-60 (pCi/L)

Co-60 (pCi/L)

Co-60 (pCi/L)

$\mathrm{Pu}-239 / 240(\mathrm{pCi} / \mathrm{L})$

$\mathrm{Pu}-239 / 240$ (pCi/L)

Radium Total ( $\mathrm{pCi} / \mathrm{L}$ )

Radium Total ( $\mathrm{pCi} / \mathrm{I}$ )

Radium Total ( $\mathrm{pCi} / \mathrm{L}$ )

Sr-90 (pCi/L)

$\mathrm{Sr}-90(\mathrm{pCi} / \mathrm{L})$

Sr-90 (pCi/L)

$\mathrm{U}-234(\mathrm{pCi} / \mathrm{L})$

$\mathrm{U}-234(\mathrm{pCi} / \mathrm{L})$

$\mathrm{U}-234(\mathrm{pCi} / \mathrm{L})$

$\mathrm{U}-238(\mathrm{pCi} / \mathrm{L})$

$\mathrm{U}-238(\mathrm{pCi} / \mathrm{L})$

$\mathrm{U}-238(\mathrm{pCi} / \mathrm{L})$

\begin{tabular}{|c|c|c|c|}
\hline & & & \\
\hline $50931 \mathrm{E}$ & $2 / 12 / 90$ & IGNIT & $2.08 \mathrm{E}+02$ \\
\hline $51032 \mathrm{E}$ & $3 / 08 / 90$ & IGNIT & $2.02 E+02$ \\
\hline 50788 & $11 / 22 / 89$ & PH-Fld & $7.50 \mathrm{E}+00$ \\
\hline 50812 & $11 / 29 / 89$ & $P H-F I d$ & $7.70 E+00$ \\
\hline 50931 & $2 / 12 / 90$ & $P H-F l d$ & $7.25 \mathrm{E}+00$ \\
\hline 51032 & $8 / 90$ & $\mathrm{PH}-\mathrm{F} \perp \mathrm{d}$ & $8+\infty$ \\
\hline $50788 \mathrm{E}$ & $11 / 22 / 89$ & DSPEC & $<1.00 E+02$ \\
\hline $50812 \mathrm{E}$ & $11 / 29 / 89$ & DSPEC & $<1.00 E+02$ \\
\hline $50931 \mathrm{E}$ & $2 / 12 / 90$ & DSPEC & $<1.00 \mathrm{E}+02$ \\
\hline $51032 E$ & $3 / 08 / 90$ & DSPEC & $<1.00 E+02$ \\
\hline $50788 \mathrm{E}$ & $11 / 22 / 89$ & DTITRA & $<1.00 \mathrm{E}+02$ \\
\hline $50812 \mathrm{E}$ & $11 / 29 / 89$ & DTITRA & $<1.00 \mathrm{E}+02$ \\
\hline $50931 \mathrm{E}$ & $2 / 12 / 90$ & DTITRA & $<1.00 \mathrm{E}+02$ \\
\hline $51032 \mathrm{E}$ & $3 / 08 / 90$ & DTITRA & $<1.00 \mathrm{E}+02$ \\
\hline 50788 & $11 / 22 / 89$ & TDS & $8+04$ \\
\hline 50812 & $11 / 29 / 89$ & TDS & $20 E+04$ \\
\hline 50931 & $2 / 12 / 90$ & TDS & $6.30 E+04$ \\
\hline 51032 & $3 / 08 / 90$ & TDS & $6.30 \mathrm{E}+04$ \\
\hline 50788 & $11 / 22 / 89$ & TEMP-FId & $1.39 \mathrm{E}+01$ \\
\hline 50812 & $11 / 29 / 89$ & TEMP-FId & 1. $13 \mathrm{E}+01$ \\
\hline 50931 & $2 / 12 / 90$ & TEMP-FId & $8.60 \mathrm{E}+00$ \\
\hline 51032 & $3 / 08 / 90$ & TEMP-FId & $8.80 E+00$ \\
\hline 50788 & $11 / 2$ & TOC & $<1.30 \mathrm{E}+03$ \\
\hline 50812 & $11 / 2$ & TOC & $8+03$ \\
\hline 50931 & $2 / 12 / 90$ & TOC & $<1.00 E+03$ \\
\hline 51032 & $3 / 08 / 90$ & TOC & $1.10 \mathrm{E}+03$ \\
\hline 50788 & $11 / 22 / 89$ & TC & $1.53 E+04$ \\
\hline 50812 & $11 / 29 / 89$ & TC & $1.58 \mathrm{E}+04$ \\
\hline 50931 & $2 / 12 / 90$ & TC & $1.31 E+04$ \\
\hline 51032 & $3 / 0$ & TC & $1.59 \mathrm{E}+04$ \\
\hline 50788 & $11 /$ & LTOX & $1.00 \mathrm{E}+01$ \\
\hline & & LTOX & \\
\hline 50931 & 190 & ITOX & $<8.00 E+00$ \\
\hline 51032 & $3 / 08 / 90$ & LTOX & $<7.00 \mathrm{E}+00$ \\
\hline 50788 & $11 / 22 / 89$ & GEA & $<9.40 \mathrm{E}-02$ \\
\hline 50812 & $11 / 29 / 89$ & GEA & $<1.75 \mathrm{E}-01$ \\
\hline 51032 & $3 / 08 / 90$ & GEA & $5.86 E-01$ \\
\hline 50788 & $11 / 22 / 89$ & $A E A$ & $3.19 \mathrm{E}-03$ \\
\hline 50812 & $11 / 29 / 89$ & AEA & $<1.56 \mathrm{E}-03$ \\
\hline 50788 & $11 / 2$ & $A \perp p h \equiv-R a$ & $2.04 \mathrm{E}-01$ \\
\hline 50812 & $11 / 2$ & Alpha & -02 \\
\hline & & Alpha- & \\
\hline 50788 & $11 / 22 / 89$ & Beta & $2.39 \mathrm{E}-01$ \\
\hline 50812 & $11 / 29 / 89$ & Beta & $<1.29 E-01$ \\
\hline 51032 & $3 / 08 / 90$ & Beta & $1.78 E-01$ \\
\hline 50788 & $11 / 22 / 89$ & AEA & $2.43 \mathrm{E}-01$ \\
\hline 50812 & $11 / 29 / 89$ & $A E A$ & $2.81 \mathrm{E}-01$ \\
\hline 51032 & $3 / 08 / 90$ & AEA & $2.28 \mathrm{E}-01$ \\
\hline 50788 & $11 / 22 / 89$ & AEA & $1.77 \mathrm{E}-01$ \\
\hline 50812 & $11 / 29 / 89$ & $A E A$ & $2.02 \mathrm{E}-01$ \\
\hline 51032 & $3 / 08 / 90$ & AEA & $1.98 \mathrm{E}-01$ \\
\hline
\end{tabular}


DATA FOR B Plant Chemical Sewage-Routine Operation

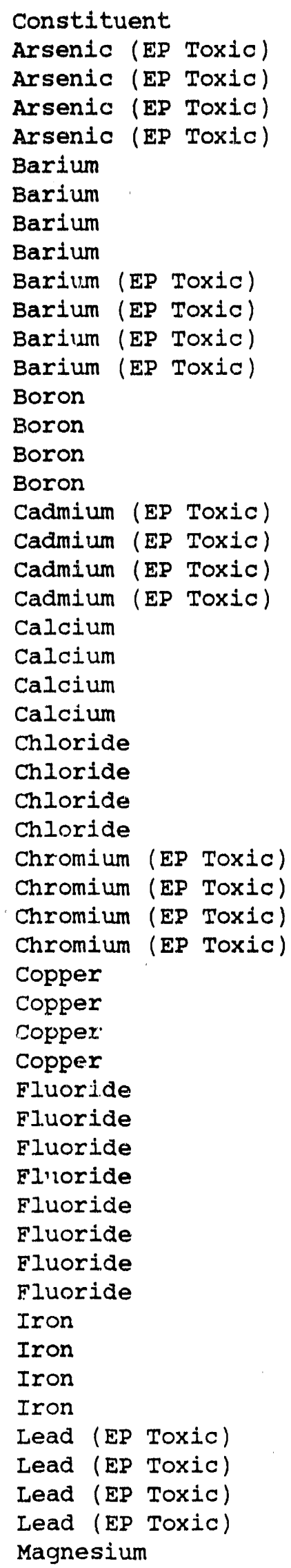

\begin{tabular}{|c|c|c|c|}
\hline Sample \# & Date & Method & Result \\
\hline $50705 \mathrm{E}$ & $10 / 19 / 89$ & ICP & $<5.00 \mathrm{E}+02$ \\
\hline $50752 \mathrm{E}$ & $11 / 02 / 89$ & ICP & $<5.00 \mathrm{E}+02$ \\
\hline $50756 \mathrm{E}$ & $11 / 03 / 89$ & ICP & $<5.00 \mathrm{E}+02$ \\
\hline $50984 \mathrm{E}$ & $2 / 27 / 90$ & ICP & $<5.00 \mathrm{E}+02$ \\
\hline 50705 & $10 / 19 / 89$ & ICP & $2.90 \mathrm{E}+01$ \\
\hline 50752 & $11 / 02 / 89$ & ICP & $3.00 \mathrm{E}+01$ \\
\hline 50756 & $11 / 03 / 89$ & ICP & 3. $10 \mathrm{E}+01$ \\
\hline 50984 & $2 / 27 / 90$ & ICP & $2.70 \mathrm{E}+01$ \\
\hline $50705 \mathrm{E}$ & $10 / 19 / 89$ & ICP & $<1.00 \mathrm{E}+03$ \\
\hline $50752 \mathrm{E}$ & $11 / 02 / 89$ & ICP & $.1 .00 \mathrm{E}+03$ \\
\hline $50756 \mathrm{E}$ & $11 / 03 / 89$ & ICP & $<1.00 E+03$ \\
\hline $50984 \mathrm{E}$ & $2 / 27 / 90$ & ICP & $<1.00 \mathrm{E}+03$ \\
\hline 50705 & $10 / 19 / 89$ & ICP & $<1.00 E+01$ \\
\hline 50752 & $11 / 02 / 89$ & ICP & $1.00 \mathrm{E}+01$ \\
\hline 50756 & $11 / 03 / 89$ & ICP & 1. $20 \mathrm{E}+01$ \\
\hline 50984 & $2 / 27 / 90$ & ICP & 4. $20 \mathrm{E}+01$ \\
\hline $50705 \mathrm{E}$ & $10 / 19 / 89$ & ICP & $<1.00 \mathrm{E}+02$ \\
\hline $50752 E$ & $11 / 02 / 89$ & ICP & $<1.00 \mathrm{E}+02$ \\
\hline $50756 \mathrm{E}$ & $11 / 03 / 89$ & ICP & $<1.00 E+02$ \\
\hline $50984 \mathrm{E}$ & $2 / 27 / 90$ & $I C P$ & $<1.00 \mathrm{E}+02$ \\
\hline 50705 & $10 / 19 / 89$ & ICP & $1.86 \mathrm{E}+04$ \\
\hline 5)752 & $11 / 02 / 89$ & ICP & $1.89 \mathrm{E}+04$ \\
\hline 5) 756 & $11 / 03 / 89$ & ICP & $1.84 \mathrm{E}+04$ \\
\hline 50984 & $2 / 27 / 90$ & ICP & $1.79 \mathrm{E}+0 \mathrm{~A}$ \\
\hline 50705 & $10 / 19 / 89$ & IC & $1.90 \mathrm{E}+03$ \\
\hline 50752 & $11 / 02 / 89$ & IC & $1.50 \mathrm{E}+03$ \\
\hline 50756 & $11 / 03 / 89$ & IC & $1.50 \mathrm{E}+03$ \\
\hline 50984 & $2 / 27 / 90$ & IC & 1.10E+03 \\
\hline $50705 \mathrm{E}$ & $10 / 19 / 89$ & ICP & $<5.00 \mathrm{E}+02$ \\
\hline $50752 \mathrm{E}$ & $11 / 02 / 89$ & ICP & $<5.00 \mathrm{E}+02$ \\
\hline $50756 \mathrm{E}$ & $11 / 03 / 89$ & ICP & $<5.00 \mathrm{E}+02$ \\
\hline $50984 \mathrm{E}$ & $2 / 27 / 90$ & ICP & $<5.00 \mathrm{E}+02$ \\
\hline 50705 & $10 / 19 / 89$ & ICP & $1.30 \mathrm{E}+01$ \\
\hline 50752 & $11 / 02 / 89$ & ICP & $2.10 E+01$ \\
\hline 50756 & $11 / 03 / 89$ & ICP & $3.60 \mathrm{E}+01$ \\
\hline 50984 & $2 / 27 / 90$ & ICP & $1.50 \mathrm{E}+01$ \\
\hline 50705 & $10 / 19 / 89$ & IC & $<5.00 \mathrm{E}+02$ \\
\hline 50705 & $10 / 19 / 89$ & ISE & 1. $40 \mathrm{E}+02$ \\
\hline 50752 & $11 / 02 / 89$ & IC & $<5.00 \mathrm{E}+02$ \\
\hline 50752 & $11 / 02 / 89$ & ISE & 1. $43 \mathrm{E}+02$ \\
\hline 50756 & $11 / 03 / 89$ & IC & $<5.00 E+02$ \\
\hline 50756 & $11 / 03 / 89$ & ISE & 1. $36 \mathrm{E}+02$ \\
\hline 50984 & $2 / 27 / 90$ & IC & $<5.00 E+C 2$ \\
\hline 50984 & 2/27/90 & ISE & $1.37 \mathrm{E}+02$ \\
\hline 50705 & $10 / 19 / 89$ & ICP & $4.60 E+01$ \\
\hline 50752 & $11 / 02 / 89$ & ICP & $<3.00 \mathrm{E}+01$ \\
\hline 50756 & $11 / 03 / 89$ & ICP & $6.70 \mathrm{E}+01$ \\
\hline 50984 & $2 / 27 / 90$ & ICP & $7.00 E+01$ \\
\hline $50705 \mathrm{E}$ & $10 / 19 / 89$ & ICP & $<5.00 \mathrm{D}+02$ \\
\hline $50752 \mathrm{E}$ & $11 / 02 / 89$ & ICP & $<5.00 \mathrm{E}+02$ \\
\hline $50756 \mathrm{E}$ & $11 / 03 / 89$ & ICP & $<5.00 E+02$ \\
\hline $50984 \mathrm{E}$ & $2 / 27 / 90$ & ICP & $<5.00 E+02$ \\
\hline 50705 & $10 / 19 / 89$ & ICP & $4.09 E+03$ \\
\hline
\end{tabular}


WHC-EP-0355, Append1X D

DATA FOR B Plant Chemical sewage-Routine Operation Continued

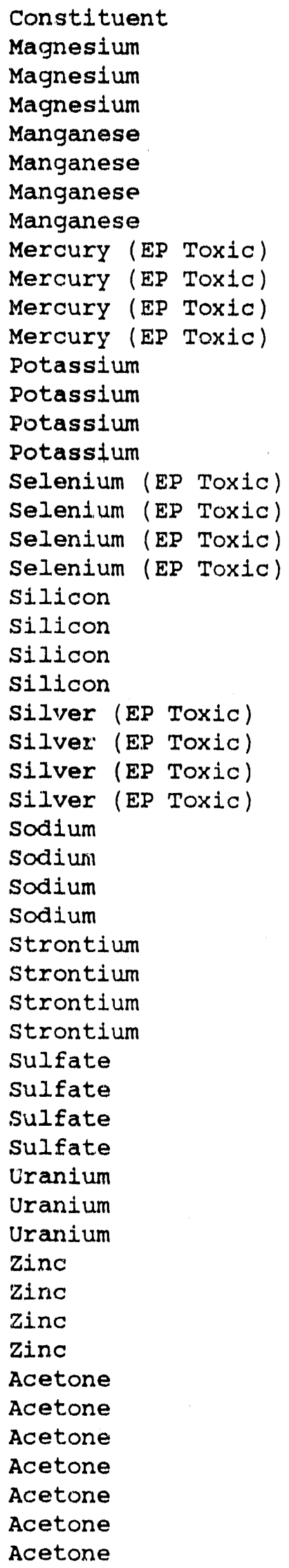

\begin{tabular}{|c|c|c|c|}
\hline ample \# & Date & Method & Result \\
\hline 50752 & $11 / 02 / 89$ & ICP & $4.32 \mathrm{E}+03$ \\
\hline 50756 & $11 / 03 / 89$ & ICP & 4. $23 E+03$ \\
\hline 50984 & $2 / 27 / 90$ & ICP & $3.95 \mathrm{E}+03$ \\
\hline 50705 & $10 / 19 / 89$ & ICP & $7.00 \mathrm{E}+00$ \\
\hline 50752 & $11 / 02 / 89$ & ICP & $<5.00 \mathrm{E}+00$ \\
\hline 50756 & $11 / 03 / 89$ & ICP & $6.00 E+00$ \\
\hline 50984 & $2 / 27 / 90$ & ICP & $<5.00 \mathrm{E}+00$ \\
\hline $50705 \mathrm{E}$ & $10 / 19 / 89$ & CVAA/M & $<2.00 E+01$ \\
\hline $50752 \mathrm{E}$ & $11 / 02 / 89$ & CVAA/M & $<2.00 E+01$ \\
\hline $50756 \mathrm{E}$ & $11 / 03 / 89$ & CVAA/M & $<2.00 \mathrm{E}+01$ \\
\hline $50984 \mathrm{E}$ & $2 / 27 / 90$ & CVAA/M & $<2.00 E+01$ \\
\hline 50705 & $10 / 19 / 89$ & ICP & $6.88 \mathrm{E}+02$ \\
\hline 50752 & $11 / 02 / 89$ & ICP & $7.42 \mathrm{E}+02$ \\
\hline 50756 & $11 / 03 / 89$ & ICP & $8.49 \mathrm{E}+02$ \\
\hline 50984 & $2 / 27 / 90$ & ICP & $9.23 \mathrm{E}+02$ \\
\hline $50705 \mathrm{E}$ & $10 / 19 / 89$ & ICP & $<5.00 \mathrm{E}+02$ \\
\hline $50752 \mathrm{E}$ & $11 / 02 / 89$ & ICP & $<5.00 \mathrm{E}+02$ \\
\hline $50756 \mathrm{E}$ & $11 / 03 / 89$ & ICP & $<5.00 E+02$ \\
\hline $50984 \mathrm{E}$ & $2 / 27 / 90$ & ICP & $<5.00 \mathrm{E}+02$ \\
\hline 50705 & $10 / 19 / 89$ & ICP & $2.20 E+03$ \\
\hline 50752 & $11 / 02 / 89$ & ICP & $2.44 E+03$ \\
\hline 50756 & $11 / 03 / 89$ & ICP & $2.41 E+03$ \\
\hline 50984 & $2 / 27 / 90$ & ICP & 2. $23 \mathrm{E}+03$ \\
\hline $5070 \mathrm{E}$ & $10 / 19 / 89$ & ICP & $<5.00 \mathrm{E}+02$ \\
\hline $50752 \mathrm{E}$ & $11 / 02 / 89$ & ICP & $<5.00 \mathrm{E}+02$ \\
\hline 50756E & $11 / 03 / 89$ & ICP & $<5,00 \mathrm{E}+02$ \\
\hline $50984 \mathrm{E}$ & $2 / 27 / 90$ & ICP & $<5.00 \mathrm{E}+02$ \\
\hline 50705 & $10 / 19 / 89$ & ICP & $2.19 \mathrm{E}+03$ \\
\hline 50752 & $11 / 02 / 89$ & ICP & $2.15 \mathrm{E}+03$ \\
\hline 50756 & $11 / 03 / 89$ & ICP & $2.27 \mathrm{E}+03$ \\
\hline 50984 & $2 / 27 / 90$ & ICP & $1.88 \mathrm{E}+03$ \\
\hline 50705 & $10 / 19 / 89$ & ICF & $9.70 E+01$ \\
\hline 50752 & $11 / 02 / 89$ & ICP & $9.90 \mathrm{E}+01$ \\
\hline 50756 & $11 / 03 / 89$ & $I C P$ & $1.02 \mathrm{E}+02$ \\
\hline 50984 & $2 / 27 / 90$ & ICP & $8.70 E+01$ \\
\hline 50705 & $10 / 19 / 89$ & IC & 1. $22 E+04$ \\
\hline 50752 & $11 / 02 / 89$ & IC & $1.11 E+04$ \\
\hline 50756 & $11 / 03 / 89$ & IC & $1.08 E+04$ \\
\hline 50984 & $2 / 27 / 90$ & IC & 1. $03 E+04$ \\
\hline 50705 & $10 / 19 / 89$ & GEA & $5.36 \mathrm{E}-01$ \\
\hline 50752 & $11 / 02 / 89$ & GEA & $4.54 E-01$ \\
\hline 50756 & $11 / 03 / 89$ & GEA & 4. 23E-01 \\
\hline 50705 & $10 / 19 / 89$ & ICP & 1. $30 \mathrm{E}+01$ \\
\hline 50752 & $11 / 02 / 89$ & ICP & $1.00 \mathrm{E}+0.1$ \\
\hline 50756 & $11 / 03 / 89$ & ICP & $1.60 \mathrm{E}+01$ \\
\hline 50984 & $2 / 27 / 90$ & ICP & 1. $20 \mathrm{E}+01$ \\
\hline 50705 & $10 / 19 / 89$ & VOA & $1.50 \mathrm{E}+01$ \\
\hline 50705 & $10 / 19 / 89$ & $\triangle A B N$ & $<1.00 \mathrm{E}+0.1$ \\
\hline $50705 B$ & $10 / 19 / 89$ & VOA & $2.10 \mathrm{E}+01$ \\
\hline $50705 \mathrm{~T}$ & $10 / 19 / 89$ & VOA & $1.40 E+01$ \\
\hline 50752 & $11 / 02 / 89$ & VOA & $<1.00 \mathrm{E}+01$ \\
\hline 50752 & $11 / 02 / 89$ & $A B N$ & $<1.00 E+01$ \\
\hline $50752 \mathrm{~B}$ & $11 / 02 / 89$ & VOA & $<1.00 E+01$ \\
\hline
\end{tabular}


WHC-EP-0355, Appendix D

DATA FOR B Plant Chemical Sewage-Routine Operation Continued

Constituent
Acetone
Acetone
Acetone
Acetone
Acetone
Acetone
Acetone
Acetone
Acetone
Ammonia
Ammonia
Ammonia
Ammonia

Dichloromethane Dichloromethane Dichloromethane Dichloromethane Dichloronethane Dichloromethane Dichlor methane Dichloromethane Dichloromethane Dichloromethane Dichloromethane Dichloromethane Tetrahydrofuran Tetrahydrofuran Tetrahydrofuran Tetrahydrofuran Tetrahydrofuran Tetrahydrofuran Tetrahyarofurar Tetrahydrofuran Tetrahydrofuran Tetrahydrofuran Tetrahydrofuran Tetrahydrofuran Irichloromethane Trichloromethane Trichlorome thane Trichloromethane Trichloromethane Trichloromethane Trichloromethane Trichloromethane Trichloromethane Trichloromethane Trichloromethane Trichloromethane unknown amide

Alkalinity (Method B)

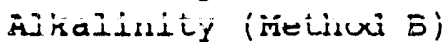
Alkalinity (Method B)

\begin{tabular}{|c|c|c|c|}
\hline Sample \# & & Method & Resiult \\
\hline $50752 \mathrm{~T}$ & $11 / 02 / 89$ & VOA & $<1.00 E+01$ \\
\hline 50756 & $11 / 03 / 89$ & VOA & $<1.00 E+01$ \\
\hline 50756 & $11 / 03 / 89$ & $A B N$ & $<1.0 O E+01$ \\
\hline $50756 B$ & $11 / 03 / 89$ & VOA & $<1.00 E+01$ \\
\hline $50756 \mathrm{~T}$ & $11 / 03 / 89$ & VOA & $<1.0 O E+01$ \\
\hline 50984 & $2 / 27 / 90$ & VOA & $<1.00 E+01$ \\
\hline 50984 & $2 / 27 / 90$ & $A B N$ & $<1.0 O E+01$ \\
\hline $50984 \mathrm{~B}$ & $2 / 27 / 9$ & VOA. & $<1.00 E+01$ \\
\hline $50984 \mathrm{~T}$ & $2 / 27 / 90$ & VOA & $<1.00 E+01$ \\
\hline 50705 & $10 / 19 / 89$ & ISE & $7.10 \mathrm{E}+01$ \\
\hline 50752 & $11 / 02 / 89$ & ISE & $<5.00 \mathrm{E}+01$ \\
\hline 50756 & $11 / 03 / 89$ & ISE & $<5.00 E+01$ \\
\hline 50984 & $2 / 27 / 90$ & ISE & $<5.00 \mathrm{E}+01$ \\
\hline 50705 & $10 / 19 / 89$ & VOA & $<5.00 \mathrm{E}+00$ \\
\hline $5070.5 B$ & $10 / 19 / 89$ & VOA & $<3.00 E+00$ \\
\hline $50705 \mathrm{~T}$ & $10 / 19 / 89$ & VOA & 1. $20 \mathrm{E}+01$ \\
\hline 50752 & $11 / 02 / 89$ & VOA & $<5.00 E+00$ \\
\hline $50752 B$ & $11 / 02 / 89$ & VOA & $<5.00 \mathrm{E}+00$ \\
\hline $50752 \mathrm{~T}$ & $11 / 02 / 89$ & VOA & $7.00 E+00$ \\
\hline 50756 & $11 / 03 / 89$ & VOA & $<5.00 E+00$ \\
\hline $50756 \mathrm{~B}$ & $11 / 03 / 89$ & VOA & $8.00 \mathrm{E}+00$ \\
\hline $50756 \mathrm{~T}$ & $11 / 03 / 89$ & VOA & 8. OOE+CO \\
\hline 50984 & $2 / 27 / 90$ & VOA & $<5,00 E+00$ \\
\hline $50984 B$ & $2 / 27 / 90$ & VOA & $<5,00 \mathrm{E}+00$ \\
\hline $50984 T$ & $2 / 27 / 90$ & VOA & $<5,00 E+00$ \\
\hline 50705 & $10 / 19 / 89$ & VOA & $<1.00 \mathrm{E}+01$ \\
\hline $50705 B$ & $10 / 19 / 89$ & VOA & $1.70 \mathrm{E}+01$ \\
\hline $50705 \mathrm{~T}$ & $10 / 19 / 89$ & VOA & $<6.00 \mathrm{E}+00$ \\
\hline 50752 & $11 / 02 / 89$ & VOA & $<1.00 \mathrm{E}+01$ \\
\hline $50752 \mathrm{~B}$ & $11 / 02 / 89$ & VOA & 1. $40 \mathrm{E}+01$ \\
\hline $50752 \mathrm{~T}$ & $11 / 02 / 89$ & VOA & $<1.00 \mathrm{E}+01$ \\
\hline 50756 & $11 / 03 / 89$ & VOA & $<1.00 E+01$ \\
\hline $50756 \mathrm{~B}$ & $11 / 03 / 89$ & VOA & 1. $20 \mathrm{E}+01$ \\
\hline $50756 \mathrm{~T}$ & $11 / 03 / 89$ & VOA & 1. $20 \mathrm{E}+01$ \\
\hline 50984 & $2 / 27 / 90$ & VOA & $<1.00 E+01$ \\
\hline $50984 B$ & $2 / 27 / 90$ & VOA & $<\epsilon, . O O E+0 O$ \\
\hline $50984 \mathrm{~T}$ & $2 / 27 / 90$ & VOA & $<\epsilon i .0 O E+0 O$ \\
\hline 50705 & $10 / 19 / 89$ & VOA & $<3.00 E+00$ \\
\hline $50705 B$ & $10 / 19 / 89$ & VOA & $<5.00 E+00$ \\
\hline $50705 \mathrm{~T}$ & $10 / 19 / 89$ & VOA & $<\$ .00 E+00$ \\
\hline 50752 & $11 / 02 / 89$ & VOA & $<5.00 E+00$ \\
\hline $50752 \mathrm{~B}$ & $11 / 02 / 89$ & VOA & $<5.00 E+00$ \\
\hline $50752 \mathrm{~T}$ & $11 / 02 / 89$ & VOA & $<3.00 E+00$ \\
\hline 50756 & $11 / 03 / 89$ & VOA & $<3.00 E+00$ \\
\hline $50756 B$ & $11 / 03 / 89$ & VOA & $<3.00 E+00$ \\
\hline $50756 \mathrm{~T}$ & $1.1 / 03 / 89$ & VOA & $<3.00 \mathrm{E}+00$ \\
\hline 50984 & $2 / 27 / 90$ & VOA & $<5.00 E+00$ \\
\hline $50984 \mathrm{~B}$ & $2 / 27 / 90$ & VOA & $1.20 \mathrm{E}+01$ \\
\hline $50984 \mathrm{~T}$ & $2 / 27 / 90$ & VOA & $8.00 E+00$ \\
\hline 50756 & $11 / 03 / 89$ & $A B N$ & $2.30 E+01$ \\
\hline 50705 & $10 / 19 / 89$ & TITRA & $5.40 \mathrm{E}+04$ \\
\hline 50752 & IIj02iล9 & IIIKA & $5.70 \bar{E}+\hat{U} \dot{4}$ \\
\hline 50756 & $11 / 03 / 89$ & TITRA & $5.60 E+04$ \\
\hline
\end{tabular}


DATA FOR B Plant Chemical sewage-Routine operation Continued

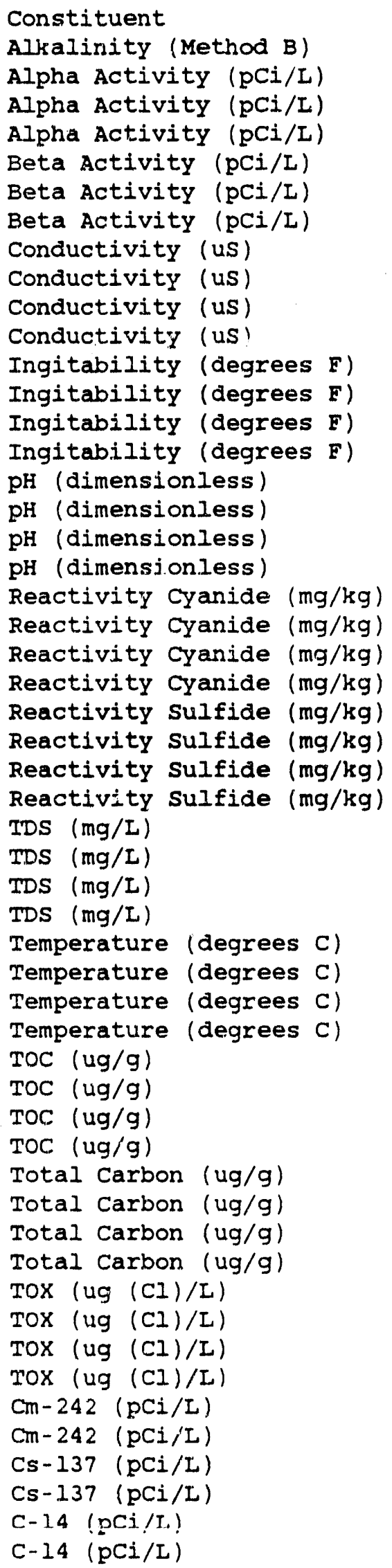

\begin{tabular}{|c|c|c|c|}
\hline iample \# & Date & Method & Result \\
\hline 50984 & $2 / 27 / 90$ & TITRA & $5.70 \mathrm{E}+04$ \\
\hline 50705 & $10 / 19 / 89$ & Alpha & $<2.68 \mathrm{E}-01$ \\
\hline 50752 & $11 / 02 / 89$ & Alpha & $<5.75 \mathrm{E}-01$ \\
\hline 50756 & $11 / 03 / 89$ & Alpha & 8. $22 E-O I$ \\
\hline 50705 & $10 / 19 / 89$ & Beta & $2.97 \mathrm{E}+00$ \\
\hline 50752 & $11 / 02 / 89$ & Beta & $=1.48 \mathrm{E}+00$ \\
\hline 50756 & $11 / 03 / 89$ & Beta & $<2.09 \mathrm{E}+00$ \\
\hline 50705 & $10 / 19 / 89$ & COND-EId & $1.75 \mathrm{E}+02$ \\
\hline 50752 & $11 / 02 / 89$ & COND-FId & 1. $33 E+02$ \\
\hline 50756 & $11 / 03 / 89$ & COND-FId & $1.53 \mathrm{E}+02$ \\
\hline 50984 & $2 / 27 / 90$ & COND-FId & 1. $22 \mathrm{E}+02$ \\
\hline $50705 \mathrm{E}$ & $10 / 19 / 89$ & IGNIT & $2.08 E+02$ \\
\hline $50752 \mathrm{E}$ & $11 / 02 / 89$ & IGNIT & 2. $12 \mathrm{E}+02$ \\
\hline $50756 \mathrm{E}$ & $11 / 03 / 89$ & IGNIT & $2.10 \mathrm{E}+02$ \\
\hline $50984 \mathrm{E}$ & $2 / 27 / 90$ & IGNIT & $2.06 \mathrm{E}+02$ \\
\hline 50705 & $10 / 19 / 89$ & PH-FId & $7.60 \mathrm{E}+00$ \\
\hline 50752 & $11 / 02 / 89$ & $\mathrm{PH}-\mathrm{F} I \mathrm{~d}$ & $7.41 \mathrm{E}+00$ \\
\hline 50756 & $11 / 03 / 89$ & PH-FId & $7.70 \mathrm{E}+00$ \\
\hline 50984 & $2 / 27 / 90$ & PH-FId & $7.10 E+00$ \\
\hline $50705 \mathrm{E}$ & $10 / 19 / 89$ & DSPEC & $<1.00 \mathrm{E}+02$ \\
\hline $50752 \mathrm{E}$ & $11 / 02 / 89$ & DSPEC & $<1.00 E+02$ \\
\hline $50756 \mathrm{E}$ & $11 / 03 / 89$ & DSPEC & $<1.00 \mathrm{E}+02$ \\
\hline $50984 E$ & $2 / 27 / 90$ & DSPEC & $<1.00 \mathrm{E}+02$ \\
\hline $50705 E$ & $10 / 19 / 89$ & DTITRA & $<1.00 \mathrm{E}+02$ \\
\hline $50752 \mathrm{E}$ & $11 / 02 / 89$ & DTITRA & $<1.00 \mathrm{E}+02$ \\
\hline $50756 \mathrm{E}$ & $11 / 03 / 89$ & DTITRA & $<1.00 E+02$ \\
\hline $50984 \mathrm{E}$ & $2 / 27 / 90$ & DTITRA & $<1.00 \mathrm{E}+02$ \\
\hline 50705 & $10 / 19 / 89$ & TDS & $5.00 E+04$ \\
\hline 50752 & $11 / 02 / 89$ & TDS & $4.70 \mathrm{E}+04$ \\
\hline 50756 & $11 / 03 / 89$ & $\operatorname{TDS}$ & $5.20 \mathrm{E}+04$ \\
\hline 50984 & $2 / 27 / 90$ & TDS & $5.40 \mathrm{E}+04$ \\
\hline 50705 & $10 / 19 / 89$ & TEMP-FId & $2.74 \mathrm{E}+01$ \\
\hline 50752 & $11 / 02 / 89$ & TEMP-FId & $1.86 \mathrm{E}+01$ \\
\hline 50756 & $11 / 03 / 89$ & TEMP-EId & $2.03 E+01$ \\
\hline 50984 & $2 / 27 / 90$ & TEMP-FId & $1.71 \mathrm{E}+01$ \\
\hline 50705 & $10 / 19 / 89$ & TOC & $<1.60 \mathrm{E}+03$ \\
\hline 50752 & $11 . / 02 / 89$ & TOC & $<1.20 \mathrm{E}+03$ \\
\hline 50756 & $11 / 03 / 89$ & TOC & $<1.20 \mathrm{E}+03$ \\
\hline 50984 & $2 / 27 / 90$ & TOC & 1. $10 E+03$ \\
\hline 50705 & $10 / 19 / 89$ & $T C$ & $1.60 E+04$ \\
\hline 50752 & $11 / 02 / 89$ & $\mathrm{TC}$ & $1.42 \mathrm{E}+04$ \\
\hline 50756 & $11 / 03 / 89$ & TC & 1. $32 \mathrm{E}+04$ \\
\hline 50984 & $2 / 27 / 90$ & TC & 1. $39 E+04$ \\
\hline 50705 & $10 / 19 / 89$ & LTOX & 4. $30 E+01$ \\
\hline 50752 & $11 / 02 / 89$ & ILTOX & $4.50 E+O 1$ \\
\hline 50756 & $11 / 03 / 89$ & LTOX & $5.90 \mathrm{E}+\mathrm{O}]$ \\
\hline 50984 & $2 / 27 / 90$ & LTOX & $2.60 E+0.1$ \\
\hline 50705 & $10 / 19 / 89$ & $\mathrm{AEA}$ & $<3.81 \mathrm{E}-0.3$ \\
\hline 50756 & $11 / 03 / 89$ & AEA & $5.31 E-03$ \\
\hline 50705 & $10 / 19 / 89$ & GEA & $1.73 E+O C$ \\
\hline 50752 & $11 / 02 / 89$ & GEA & $4.95 E-O I$ \\
\hline 50752 & $11 / 02 / 89$ & LSC & $4.98 E+00$ \\
\hline 50756 & $11 / 03 / 89$ & LSC & $<3.51 E+00$ \\
\hline
\end{tabular}


WHC-EP-0355, Appendix D

DATA FOR B Plant Chemical sewage-Routine Operation Continued

\begin{tabular}{|c|c|}
\hline Const & ituent \\
\hline $11-3$ & $\mathrm{pCi} / \mathrm{I})$ \\
\hline $\mathrm{H}-3$ ( & $\mathrm{pCi} / \mathrm{L})$ \\
\hline $\mathrm{H}-3$ & $\mathrm{pCi} / \mathrm{L})$ \\
\hline Sr -90 & $(\mathrm{pCi} / \mathrm{L})$ \\
\hline Sr-90 & $(\mathrm{pCi} / \mathrm{L})$ \\
\hline Sr -90 & $(\mathrm{pCi} / \mathrm{L})$ \\
\hline$U-234$ & $(\mathrm{pCi} / \mathrm{L})$ \\
\hline$U-234$ & $(\mathrm{pCi} / \mathrm{L})$ \\
\hline$U-234$ & $(\mathrm{pCi} / \mathrm{L})$ \\
\hline$U-238$ & $(\mathrm{pCi} / \mathrm{L})$ \\
\hline$U-238$ & $(\mathrm{pCi} / \mathrm{L})$ \\
\hline & \\
\hline
\end{tabular}

\begin{tabular}{cclr} 
Sample \# & Date & Method & \multicolumn{1}{c}{ Result } \\
50705 & $10 / 19 / 89$ LSC & $<1.99 E+02$ \\
50752 & $11 / 02 / 89$ LSC & $<2.90 E+01$ \\
50756 & $11 / 03 / 89$ LSC & $2.50 E+02$ \\
50705 & $10 / 19 / 89$ Beta & $1.24 E-01$ \\
50752 & $11 / 02 / 89$ Beta & $<9.89 E-02$ \\
50756 & $11 / 03 / 89$ Beta & $2.09 E-01$ \\
50705 & $10 / 19 / 89$ AEA & $1.89 E-01$ \\
50752 & $11 / 02 / 89$ AEA & $1.78 E-01$ \\
50756 & $11 / 03 / 89$ AEA & $1.14 E-01$ \\
50705 & $10 / 19 / 89$ AEA & $1.43 E-01$ \\
50752 & $11 / 02 / 89$ AEA & $1.36 E-01$ \\
50756 & $11 / 03 / 89$ AEA & $1.33 E-01$
\end{tabular}


WHC-EP-0355, Appendix D

DATA FOR 183-D Filter Backflush wastewater

\begin{tabular}{|c|c|c|}
\hline Constitue & lent & \\
\hline Aluminum & & \\
\hline Aluminum & & \\
\hline Aluminum & & \\
\hline Arsenic & & \\
\hline Arsenic & & \\
\hline Arsenic & & \\
\hline Arsenic & & \\
\hline Arsenic & (EP T & Toxic) \\
\hline Arsenic & (EP T & Toxic) \\
\hline Arsenic & (EP T & Toxic) \\
\hline Arsenic & (EP T & Toxic) \\
\hline Barium & & \\
\hline Barium & & \\
\hline Barium & & \\
\hline Barium & & \\
\hline Barium (E & EP TO & oxic) \\
\hline Barium (E & EP TO & oxic) \\
\hline Barium (E & EP TO & oxic) \\
\hline Barium (E & EP TO & oxic) \\
\hline Boron & & \\
\hline Boron & & \\
\hline Boron & & \\
\hline Boron & & \\
\hline Cadmium & (EP T & Toxic) \\
\hline Cadmium & (EF $\mathrm{T}$ & Toxic) \\
\hline Cadmium & (EP T & Toxic) \\
\hline Cadmium & (EP T & Toxic) \\
\hline Calcium & & \\
\hline Calcium & & \\
\hline Calcjum & & \\
\hline Calcium & & \\
\hline Chloride & & \\
\hline Chloride & & \\
\hline Chloride & & \\
\hline Chloride & & \\
\hline Chromium & & \\
\hline Chromium & & \\
\hline Chromium & & \\
\hline Chromium & & \\
\hline Chromium & (EP & Toxic) \\
\hline Chromium & (EP & Toxic) \\
\hline Chromium & (EP & Toxic) \\
\hline Chromium & (EP & Toxic) \\
\hline Copper & & \\
\hline Copper & & \\
\hline Copper & & \\
\hline Copper & & \\
\hline Fluoride & & \\
\hline Fluoride & & \\
\hline Fluoride & & \\
\hline Fluoride & & \\
\hline Elucrida & & \\
\hline
\end{tabular}

\begin{tabular}{|c|c|c|c|}
\hline Sample \# & Date & Method & Result \\
\hline 50861 & $12 / 27 / 89$ & ICP & $2.38 \mathrm{E}+04$ \\
\hline 50976 & $2 / 26 / 90$ & ICP & $2.85 \mathrm{E}+04$ \\
\hline 51044 & $3 / 12 / 90$ & ICP & $3.64 \mathrm{E}+04$ \\
\hline 51099 & $3 / 28 / 90$ & $I C P$ & $1.27 E+03$ \\
\hline 50861 & $12 / 27 / 89$ & GFAA & $7.00 \mathrm{E}+00$ \\
\hline 50976 & $2 / 26 / 90$ & GFAA & $1.00 E+01$ \\
\hline 51044 & $3 / 12 / 90$ & GFAA & 1. $30 \mathrm{E}+01$ \\
\hline 51099 & $3 / 28 / 90$ & GFAA & $<5.00 E+00$ \\
\hline $50861 \mathrm{E}$ & $12 / 27 / 89$ & ICP & $<5.00 E+02$ \\
\hline 50976E & $2 / 26 / 90$ & ICP & $<5.00 \mathrm{E}+02$ \\
\hline $51044 \mathrm{E}$ & $3 / 12 / 90$ & ICP & $<5.00 \mathrm{E}+02$ \\
\hline $51099 \mathrm{E}$ & $3 / 28 / 90$ & ICP & $<5.00 \mathrm{E}+02$ \\
\hline 50861 & $12 / 27 / 89$ & ICP & 4. 20E+OI \\
\hline 50976 & $2 / 26 / 90$ & ICP & 4.70E+01 \\
\hline 51044 & $3 / 12 / 90$ & $I C P$ & $5.70 E+01$ \\
\hline 51099 & $3 / 28 / 90$ & ICP & 3. $20 \mathrm{E}+01$ \\
\hline $50861 \mathrm{E}$ & $12 / 27 / 89$ & ICP & $<1.00 \mathrm{E}+03$ \\
\hline $50976 \mathrm{E}$ & $2 / 26 / 90$ & ICP & $<1.00 \mathrm{E}+03$ \\
\hline $51044 \mathrm{E}$ & $3 / 12 / 90$ & ICP & $<1.00 E+03$ \\
\hline $51099 \mathrm{E}$ & $3 / 28 / 90$ & ICP & $<1.00 E+03$ \\
\hline 50861 & $12 / 27 / 89$ & ICP & $<1.00 E+01$ \\
\hline 50976 & $2 / 26 / 90$ & ICP & $4.00 E+01$ \\
\hline 51044 & $3 / 12 / 90$ & ICP & 3. $40 \mathrm{E}+01$ \\
\hline 51099 & $3 / 28 / 90$ & ICP & $1.60 \mathrm{E}+01$ \\
\hline 50861E & $12 / 27 / 89$ & ICP & $<1.00 \mathrm{E}+02$ \\
\hline $50976 \mathrm{E}$ & $2 / 26 / 90$ & ICP & $<1.00 \mathrm{E}+02$ \\
\hline $5.1044 \mathrm{E}$ & $3 / 12 / 90$ & $I C P$ & $<1.00 \mathrm{E}+02$ \\
\hline $51099 \mathrm{E}$ & $3 / 28 / 90$ & ICP & $<1.00 \mathrm{E}+02$ \\
\hline 50861 & $12 / 27 / 89$ & ICP & $1.98 \mathrm{E}+04$ \\
\hline 50976 & $2 / 26 / 90$ & ICP & $2.14 \mathrm{E}+04$ \\
\hline 51044 & $3 / 12 / 90$ & ICP & $2.17 \mathrm{E}+04$ \\
\hline 51099 & $3 / 28 / 90$ & ICP & $1.92 \mathrm{E}+04$ \\
\hline 50861 & $12 / 27 / 89$ & IC & $3.60 \mathrm{E}+03$ \\
\hline 50976 & $2 / 26 / 90$ & IC & $2.20 E+03$ \\
\hline 51044 & $3 / 12 / 90$ & IC & 2. $90 E+03$ \\
\hline 51099 & $3 / 28 / 90$ & IC & $2.70 \mathrm{E}+03$ \\
\hline 50861 & $12 / 27 / 89$ & ICP & $1.30 \mathrm{E}+01$ \\
\hline 50976 & $2 / 26 / 90$ & ICP & 1. $20 E+01$ \\
\hline 51044 & $3 / 12 / 90$ & ICP & $2.00 E+01$ \\
\hline 51099 & $3 / 28 / 90$ & ICP & $<1.00 \mathrm{E}+01$ \\
\hline $50861 \mathrm{E}$ & $12 / 27 / 89$ & ICP & $<5.00 \mathrm{E}+02$ \\
\hline $50976 \mathrm{E}$ & $2 / 26 / 90$ & ICP & $<5.00 E+02$ \\
\hline $51044 \mathrm{E}$ & $3 / 12 / 90$ & ICP & $<5.00 \mathrm{E}+02$ \\
\hline $51099 \mathrm{E}$ & $3 / 28 / 90$ & ICP & $<5.00 E+02$ \\
\hline 50861 & $12 / 27 / 89$ & ICP & $4.00 E+01$ \\
\hline 50976 & $2 / 26 / 90$ & $I C P$ & $4.70 \mathrm{E}+01$ \\
\hline 51044 & $3 / 12 / 90$ & ICP & $7.30 E+01$ \\
\hline 51099 & $3 / 28 / 90$ & ICP & $<1.00 E+01$ \\
\hline 50861 & $12 / 27 / 89$ & IC & $<5.00 \mathrm{E}+\mathrm{C} 2$ \\
\hline 50861 & $12 / 27 / 89$ & ISE & $1.35 \mathrm{E}+02$ \\
\hline 50976 & $2 / 26 / 90$ & IC & $<5.00 E+02$ \\
\hline 50976 & $2 / 26 / 90$ & ISE & $<5.00 E+01$ \\
\hline 52014 & $3,12,190$ & $I C$ & $<5.00 E+02$ \\
\hline
\end{tabular}


DATA FOR 183-D Filter Backflush Wastewater Continued

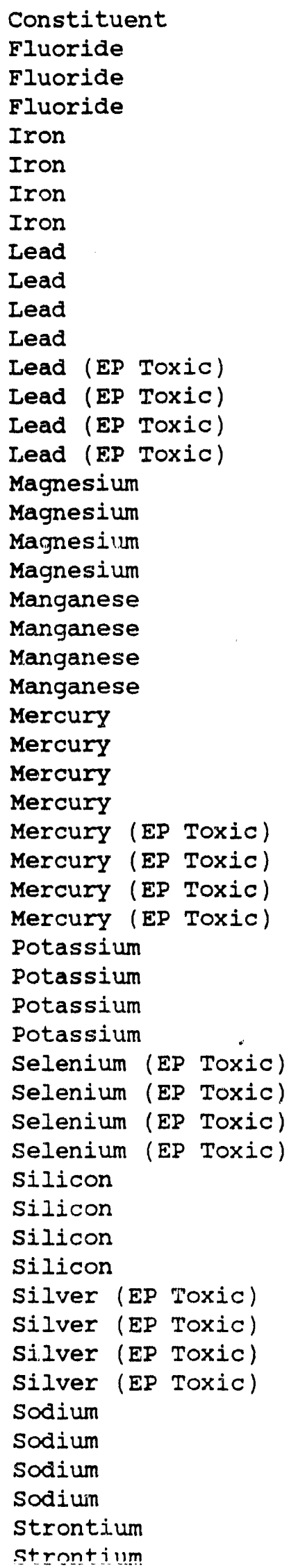

\begin{tabular}{|c|c|c|c|}
\hline & Date & Method & Result \\
\hline 51044 & $3 / 12 / 90$ & ISE & $3.03 E+02$ \\
\hline 51099 & $3 / 28 / 90$ & IC & $<5.00 E+02$ \\
\hline 51099 & $3 / 28 / 90$ & ISE & $1.47 \mathrm{E}+02$ \\
\hline 50861 & $12 / 27 / 89$ & ICP & $3.39 \mathrm{E}+03$ \\
\hline 50976 & $2 / 26 / 90$ & ICP & $3.09 E+03$ \\
\hline 51044 & $3 / 12 / 90$ & ICP & $3.74 \mathrm{E}+03$ \\
\hline 51099 & $3 / 28 / 90$ & ICP & $2.18 E+02$ \\
\hline 50861 & $12 / 27 / 89$ & GFAA & $1.30 \mathrm{E}+01$ \\
\hline 50976 & $2 / 26 / 90$ & GFAA & $9.00 \mathrm{E}+00$ \\
\hline 51044 & $3 / 12 / 90$ & GFAA & $1.30 \mathrm{E}+01$ \\
\hline 51099 & $3 / 28 / 90$ & GFAA & $<5.00 E+00$ \\
\hline $50861 \mathrm{E}$ & $12 / 27 / 89$ & ICP & $<5.00 E+02$ \\
\hline 50976E & $2 / 26 / 90$ & ICP & $<5.00 \mathrm{E}+02$ \\
\hline $51044 \mathrm{E}$ & $3 / 12 / 90$ & ICP & $<5.00 \mathrm{E}+02$ \\
\hline 51099E & $3 / 28 / 90$ & ICP & $<5.00 \mathrm{E}+02$ \\
\hline 50861 & $12 / 27 / 89$ & ICP & 4. $39 \mathbf{E}+03$ \\
\hline 50976 & $2 / 26 / 90$ & $I C P$ & $4.53 E+03$ \\
\hline 51044 & $3 / 12 / 90$ & ICP & $4.71 E+03$ \\
\hline 51099 & $3 / 28 / 90$ & ICP & $4.66 \mathrm{E}+03$ \\
\hline 50861 & $12 / 27 / 89$ & ICP & $9.40 \mathrm{E}+01$ \\
\hline 50976 & $2 / 26 / 90$ & ICP & 1. $03 E+02$ \\
\hline 51044 & $3 / 12 / 90$ & ICP & 1. $23 \mathrm{E}+02$ \\
\hline 51099 & $3 / 28 / 90$ & ICP & $6.00 E+00$ \\
\hline 50861 & $12 / 27 / 89$ & CVAA & $7.50 \mathrm{E}-01$ \\
\hline 50976 & $2 / 26 / 90$ & CVAA & $1.60 \mathrm{E}+00$ \\
\hline 51044 & $3 / 12 / 90$ & CVAA & $2.80 \mathrm{E}-01$ \\
\hline 51099 & $3 / 28 / 90$ & CVAA & $3.20 \mathrm{E}-01$ \\
\hline $50861 E$ & $12 / 27 / 89$ & CVAA/M & $<2.00 \mathrm{E}+01$ \\
\hline $50976 \mathrm{E}$ & $2 / 26 / 90$ & CVAA/M & $<2.00 \mathrm{E}+01$ \\
\hline $51044 \mathrm{E}$ & $3 / 12 / 90$ & CVAA/M & $<2.00 \mathrm{E}+01$ \\
\hline 51099E & $3 / 28 / 90$ & CVAA $/ M$ & $<2.00 \mathrm{E}+01$ \\
\hline 50861 & $12 / 27 / 89$ & ICP & $8.44 \mathrm{E}+02$ \\
\hline 50976 & $2 / 26 / 90$ & ICP & 8. $28 \mathrm{E}+02$ \\
\hline 51044 & $3 / 12 / 90$ & $I C P$ & $8.87 \mathrm{E}+02$ \\
\hline 51099 & $3 / 28 / 90$ & ICP & $8.93 E+02$ \\
\hline $50861 \mathrm{E}$ & $12 / 27 / 89$ & ICP & $<5.00 E+02$ \\
\hline $50976 \mathrm{E}$ & $2 / 26 / 90$ & ICP & $<5.00 \mathrm{E}+02$ \\
\hline $51044 \mathrm{E}$ & $3 / 12 / 90$ & ICP & $<5.00 \mathrm{E}+02$ \\
\hline $51099 \mathrm{E}$ & $3 / 28 / 90$ & ICP & $<5.00 E+02$ \\
\hline 50861 & $12 / 27 / 89$ & ICP & $4.89 E+03$ \\
\hline 50976 & $2 / 26 / 90$ & ICP & $6.08 E+03$ \\
\hline 51044 & $3 / 12 / 90$ & ICP & $7.95 E+03$ \\
\hline 51099 & $3 / 28 / 90$ & ICP & $1.78 \mathrm{E}+03$ \\
\hline $50861 \mathrm{E}$ & $12 / 27 / 89$ & ICP & $<5.00 E+02$ \\
\hline $50976 \mathrm{E}$ & $2 / 26 / 90$ & ICP & $<5.00 \mathrm{E}+02$ \\
\hline $51044 \mathrm{E}$ & $3 / 12 / 90$ & ICP & $<5.00 \mathrm{E}+02$ \\
\hline $51099 E$ & $3 / 28 / 90$ & ICP & $<5.00 \mathrm{E}+02$ \\
\hline 50861 & $12 / 27 / 89$ & ICP & $2.26 \mathrm{E}+03$ \\
\hline 50976 & $2 / 26 / 90$ & $I C P$ & $2.37 E+03$ \\
\hline 51044 & $3 / 12 / 90$ & ICP & $2.33 E+03$ \\
\hline 51099 & $3 / 28 / 90$ & ICP & $2.42 \mathrm{E}+03$ \\
\hline 50861 & $12 / 27 / 89$ & $I C P$ & $1.09 \mathrm{E}+02$ \\
\hline 50976 & $2 / 26 / 90$ & ICD & $1.14 \mathbf{E}+02$ \\
\hline
\end{tabular}


DATA FOR 183-D Filter Backflush wastewater Continued

Constituent
Strontium
Strontium
Sulfate
Sulfate
Sulfate
Sulfate
Uranium
Urarium
Uranium
Vanadium
Vanadium
Vanadium
Vanadium
Zinc
Zinc
zinc
Zinc
Ammonia
Ammonia
Ammonia
Ammonia
2-Butanone
2-Butanone
2-Butanone
2-Butanone
2-Butanone
2-Butanone
2-Butanone
2-Butanone
2-Butanone
2-Butanone
2-Butanone
2-Butanone
Dichloromethane
Dichloromethane
Dichloromethane
Dichloromethane
Dichloromethane
Dichloromethane
Dichloromethane
Dichloromethane
Dichloromethane
Dichloromethane
Dichloromethane
Dichloromethane
Trichloromethane
Trichloromethane
Trichloromethane
Trichloromethane
Trichloromethane

\begin{tabular}{|c|c|c|c|}
\hline Sample \# & Date & Method & Result \\
\hline 51044 & $3 / 12 / 90$ & ICP & 1. $29 \mathrm{E}+02$ \\
\hline 51099 & $3 / 28 / 90$ & ICP & 1.01E+02 \\
\hline 50861 & $12 / 27 / 89$ & IC & $1.83 E+04$ \\
\hline 50976 & $2 / 26 / 90$ & IC & 1. $40 \mathrm{E}+04$ \\
\hline 51044 & $3 / 12 / 90$ & $I C$ & $1.89 \mathrm{E}+04$ \\
\hline 51099 & $3 / 28 / 90$ & IC & $1.76 \mathrm{E}+04$ \\
\hline 50861 & $12 / 27 / 89$ & GEA & $3.94 \mathrm{E}+00$ \\
\hline 51044 & $3 / 12 / 90$ & $G E A$ & $3.68 \mathrm{E}+00$ \\
\hline 51099 & $3 / 28 / 90$ & GEA & $2.98 \mathrm{E}+00$ \\
\hline 50861 & $12 / 27 / 89$ & ICP & $7.00 \mathrm{E}+00$ \\
\hline 50976 & $2 / 26 / 90$ & ICP & $7.00 E+00$ \\
\hline 51044 & $3 / 12 / 90$ & ICP & $1.00 \mathrm{E}+01$ \\
\hline 51099 & $3 / 28 / 90$ & ICP & $<5.00 \mathrm{E}+00$ \\
\hline 50861 & $12 / 27 / 89$ & ICP & 6. 10E+01 \\
\hline 50976 & $2 / 26 / 90$ & ICP & $7.00 \mathrm{E}+01$ \\
\hline 51044 & $3 / 12 / 90$ & ICP & $1.02 \mathrm{E}+02$ \\
\hline 51099 & $3 / 28 / 90$ & ICP & $8.00 \mathrm{E}+00$ \\
\hline 50861 & $12 / 27 / 89$ & ISE & $9.20 \mathrm{E}+01$ \\
\hline 50976 & $2 / 26 / 90$ & ISE & $6.60 \mathrm{E}+01$ \\
\hline 51044 & $3 / 12 / 90$ & ISE & $<5.00 \mathrm{E}+01$ \\
\hline 51039 & $3 / 28 / 90$ & ISE & $<5.00 \mathrm{E}+01$ \\
\hline 50861 & $12 / 27 / 89$ & VOA & $<1.00 \mathrm{E}+01$ \\
\hline $50861 \mathrm{~B}$ & $12 / 27 / 89$ & VOA & $<1.00 \mathrm{E}+01$ \\
\hline $50861 \mathrm{~T}$ & $12 / 27 / 89$ & VOA & $<1.00 E+01$ \\
\hline 50976 & $2 / 26 / 90$ & VOA & $<1.00 \mathrm{E}+01$ \\
\hline $50976 \mathrm{~B}$ & $2 / 26 / 90$ & VOA & $<8.00 \mathrm{E}+00$ \\
\hline $50976 \mathrm{~T}$ & $2 / 26 / 90$ & VOA & $<8.00 \mathrm{E}+00$ \\
\hline 51044 & $3 / 12 / 90$ & VOA & $<1.00 \mathrm{E}+01$ \\
\hline $51044 \mathrm{~B}$ & $3 / 12 / 90$ & VOA & $<1.00 \mathrm{E}+01$ \\
\hline $51044 \mathrm{~T}$ & $3 / 12 / 90$ & VOA & $<1.00 E+01$ \\
\hline 51099 & $3 / 28 / 90$ & VOA & $1.30 \mathrm{E}+01$ \\
\hline $51099 B$ & $3 / 28 / 90$ & VOA & $<1.00 \mathrm{E}+01$ \\
\hline $51099 \mathrm{~T}$ & $3 / 28 / 90$ & VOA & $<1.00 \mathrm{E}+01$ \\
\hline 50861 & $12 / 27 / 89$ & VOA & $<5.00 \mathrm{E}+00$ \\
\hline $50861 B$ & $12 / 27 / 89$ & VOA & $2.00 E+03$ \\
\hline $50861 \mathrm{~T}$ & $12 / 27 / 89$ & VOA & $2.00 \mathrm{E}+03$ \\
\hline 50976 & $2 / 26 / 90$ & VOA & $<5.00 \mathrm{E}+00$ \\
\hline $50976 \mathrm{~B}$ & $2 / 26 / 9 c$ & VOA & $<5.00 E+O O$ \\
\hline $50976 \mathrm{~T}$ & $2 / 26 / 90$ & VOA & $<5.00 E+00$ \\
\hline 51044 & $3 / 12 / 90$ & VOA & $<5.00 E+00$ \\
\hline $51044 \mathrm{~B}$ & $3 / 12 / 90$ & VOA & $<3.00 E+00$ \\
\hline $51044 \mathrm{~T}$ & $3 / 12 / 90$ & VOA & $<5.00 E+00$ \\
\hline 51099 & $3 / 28 / 90$ & VOA & $<5.00 E+00$ \\
\hline $51099 B$ & $3 / 28 / 90$ & VOA & $<3.00 E+O O$ \\
\hline $51099 \mathrm{~T}$ & $3 / 28 / 90$ & VOA & $<5.00 E+00$ \\
\hline 50861 & $12 / 27 / 89$ & VOA & $1.90 \mathrm{E}+01$ \\
\hline 508618 & $12 / 27 / 89$ & VOA & $<5.00 E+00$ \\
\hline $50861 \mathrm{~T}$ & $12 / 27 / 89$ & VOA & $<5.00 E+00$ \\
\hline 50976 & $2 / 26 / 90$ & VOA & $1.90 \mathrm{E}+01$ \\
\hline $50976 \mathrm{~B}$ & $2 / 26 / 90$ & VOA & $<3.00 E+00$ \\
\hline $50976 \mathrm{~T}$ & $2 / 26 / 90$ & VOF & $8.00 \mathrm{E}+00$ \\
\hline 51044 & $3 / 12 / 90$ & VOA & $2.70 E+01$ \\
\hline $51044 B$ & $3 / 12 / 90$ & VOA & $<3.00 E+00$ \\
\hline
\end{tabular}


DATA FOR 183-D Filter Backflush Wastewater Continued

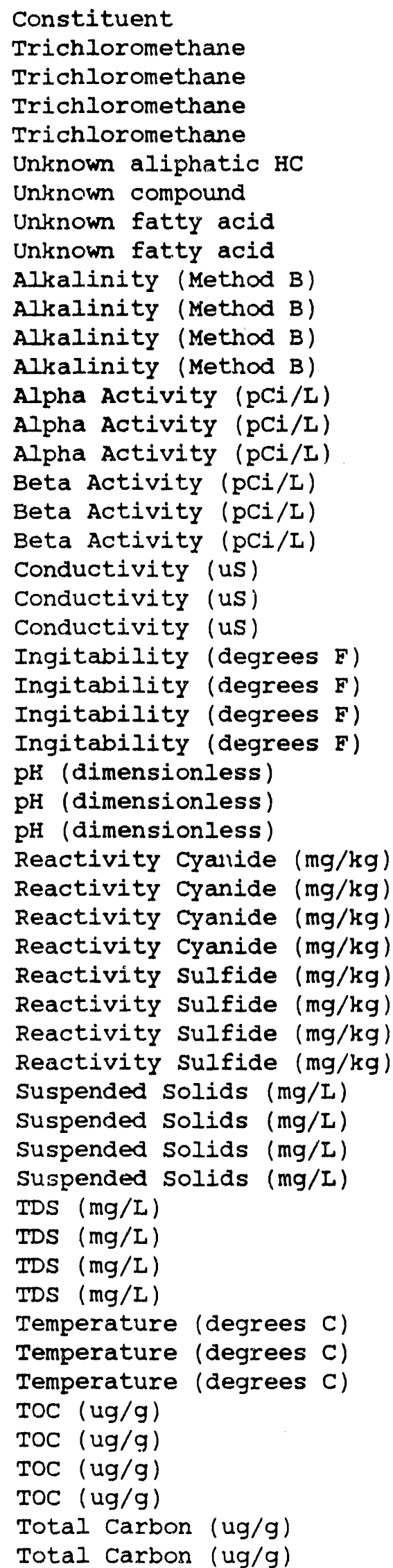

\begin{tabular}{|c|c|c|c|}
\hline ample \# & Date & Method & Result \\
\hline $51044 \mathrm{~T}$ & $3 / 12 / 90$ & VOA & $<5.00 \mathrm{E}+00$ \\
\hline 51099 & $3 / 28 / 90$ & VOA & 1.70E+01 \\
\hline $51099 B$ & $3 / 28 / 90$ & VOA & $5.00 E+00$ \\
\hline $51099 T$ & $3 / 28 / 90$ & VOA & $<4.00 E+00$ \\
\hline 50976 & $2 / 26 / 90$ & $A B N$ & 4. $00 E+02$ \\
\hline 50976 & $2 / 26 / 90$ & $A B N$ & $1.60 \mathrm{E}+03$ \\
\hline 50976 & $2 / 26 / 90$ & $A B N$ & 1. $40 \mathrm{E}+01$ \\
\hline 51044 & $3 / 12 / 90$ & $A B N$ & $1.50 \mathrm{E}+01$ \\
\hline 50861 & $12 / 27 / 89$ & TITRA & $4.90 E+04$ \\
\hline 50976 & $2 / 26 / 90$ & TITRA & 6. $20 \mathrm{E}+04$ \\
\hline 51044 & $3 / 12 / 90$ & TITRA & 5. $40 \mathrm{E}+04$ \\
\hline 51099 & $3 / 28 / 90$ & TITRA & $5.30 \mathrm{E}+04$ \\
\hline 50861 & $12 / 27 / 89$ & Alpha & $4.91 E+00$ \\
\hline 51044 & $3 / 12 / 90$ & Alpha & $2.88 E+00$ \\
\hline 51099 & $3 / 28 / 90$ & Alpha & $3.91 E+00$ \\
\hline 50861 & $12 / 27 / 89$ & Beta & $5.39 E+00$ \\
\hline 51044 & $3 / 12 / 90$ & Beta & $3.38 \mathrm{E}+00$ \\
\hline 51099 & $3 / 28 / 90$ & Beta & $<1.81 \mathrm{E}+00$ \\
\hline 50861 & $12 / 27 / 89$ & COND-FId & 1. $36 \mathrm{E}+02$ \\
\hline 50976 & $2 / 26 / 90$ & COND-FId & $1.59 \mathrm{E}+02$ \\
\hline 51044 & $3 / 12 / 90$ & COND-FId & $1.45 \mathrm{E}+02$ \\
\hline $50861 \mathrm{E}$ & $12 / 27 / 89$ & IGNIT & $2.12 \mathrm{E}+02$ \\
\hline $50976 \mathrm{E}$ & $2 / 26 / 90$ & IGNIT & $2.10 \mathrm{E}+02$ \\
\hline $51044 \mathrm{E}$ & $3 / 12 / 90$ & IGNIT & $2.12 \mathrm{E}+02$ \\
\hline $51099 \mathrm{E}$ & $3 / 28 / 90$ & IGNIT & 2. $10 E+02$ \\
\hline 50861 & $12 / 27 / 89$ & PH-Fld & $6.30 E+00$ \\
\hline 50976 & $2 / 26 / 90$ & $\mathrm{PH}-\mathrm{F} I \mathrm{~d}$ & $7.20 \mathrm{E}+00$ \\
\hline 51044 & $3 / 12 / 90$ & PH-FId & $7.40 \mathrm{E}+00$ \\
\hline $50861 \mathrm{E}$ & $12 / 27 / 89$ & DSPEC & $<1.00 \mathrm{E}+02$ \\
\hline $50976 \mathrm{E}$ & $2 / 26 / 90$ & DSPEC & $<1.0 O E+02$ \\
\hline $51044 \mathrm{E}$ & $3 / 12 / 90$ & DSPEC & $<1.00 \mathrm{E}+02$ \\
\hline $51099 E$ & $3 / 28 / 90$ & DSPEC & $<1.00 E+02$ \\
\hline $50861 \mathrm{E}$ & $12 / 27 / 89$ & DTITRA & $\div 1.00 \mathrm{E}+02$ \\
\hline $50976 \mathrm{E}$ & $2 / 26 / 90$ & DTITRA & $<1.00 E+02$ \\
\hline $51044 \mathrm{E}$ & $3 / 12 / 90$ & DTITRA & $<1.00 \mathrm{E}+02$ \\
\hline $51099 E$ & $3 / 28 / 90$ & DTITRA & $<1.00 E+02$ \\
\hline 50861 & $12 / 27 / 89$ & SSOLID & $1.08 \mathrm{E}+05$ \\
\hline 50976 & $2 / 26 / 90$ & SSOLID & $1.16 \mathrm{E}+05$ \\
\hline 51044 & $3 / 12 / 90$ & SSOLID & 2. $30 E+04$ \\
\hline 51099 & $3 / 28 / 90$ & SSOLID & $<8.00 \mathrm{E}+03$ \\
\hline 50861 & $12 / 27 / 89$ & TDS & $7.50 \mathrm{E}+04$ \\
\hline 50976 & $2 / 26 / 90$ & TDS & $8.00 E+04$ \\
\hline 51044 & $3 / 12 / 90$ & TDS & $7.10 \mathrm{E}+04$ \\
\hline 51099 & $3 / 28 / 90$ & TDS & $8.00 E+04$ \\
\hline 50861 & $12 / 27 / 89$ & TEMP-EId & $6.30 \mathrm{E}+00$ \\
\hline 50976 & $2 / 26 / 90$ & TEMP-FId & 1. $11 E+01$ \\
\hline 51044 & $3 / 12 / 90$ & TEMP-EId & $1.02 \mathrm{E}+01$ \\
\hline 50861 & $12 / 27 / 89$ & TOC & $1.76 \mathrm{E}+04$ \\
\hline 50976 & $2 / 26 / 90$ & TOC & $4.10 E+03$ \\
\hline 51044 & $3 / 12 / 90$ & TOC & $4.50 E+03$ \\
\hline 51099 & $3 / 28 / 90$ & TOC & $2.00 E+03$ \\
\hline 50861 & $12 / 27 / 89$ & $\mathrm{TC}$ & 1. $37 \mathrm{E}+04$ \\
\hline 50976 & $2 / 26 / 90$ & $\mathrm{TC}$ & $1.56 \mathrm{E}+04$ \\
\hline
\end{tabular}


WHC-EP-0355, Appendix D

DATA FOR 183-D Filter Backflush wastewater

Continued

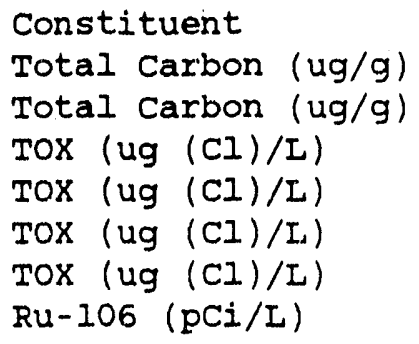

$\begin{array}{crll}\text { Sample \# } & \text { Date } & \text { Method } & \text { Result } \\ 51044 & 3 / 12 / 90 \text { TC } & 1.53 \mathrm{E}+04 \\ 51099 & 3 / 28 / 90 \text { TC } & 1.49 \mathrm{E}+04 \\ 50861 & 12 / 27 / 89 \text { LTOX } & 2.51 \mathrm{E}+02 \\ 50976 & 2 / 26 / 90 \text { LTOX } & 2.02 \mathrm{E}+02 \\ 51044 & 3 / 12 / 90 \text { LTOX } & 2.27 \mathrm{E}+02 \\ 51099 & 3 / 28 / 90 \text { LTOX } & 1.19 \mathrm{E}+02 \\ 51044 & 3 / 12 / 90 \text { GEA } & 1.29 \mathrm{E}+01\end{array}$


DATA FOR 284-E Powerhouse Wastewater-Routine Operation

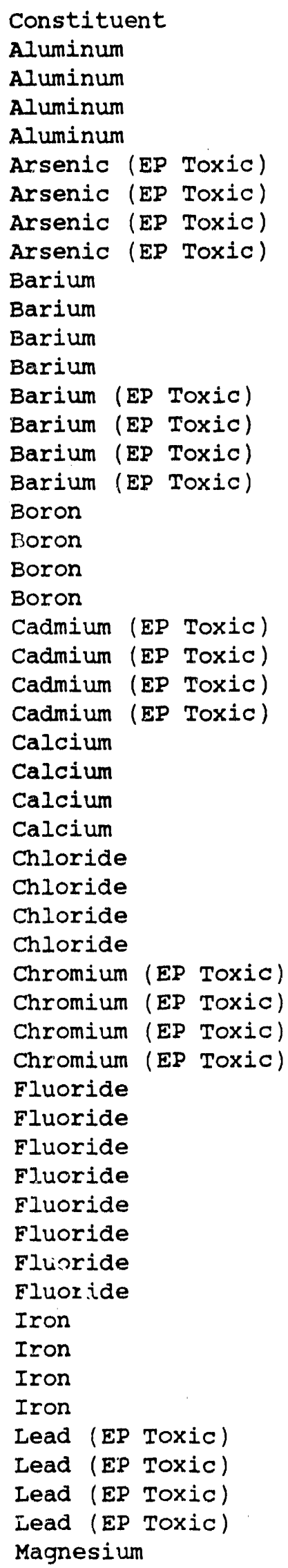

\begin{tabular}{|c|c|c|c|}
\hline Sample \# & Date & Method & Result \\
\hline 50721 & $10 / 24 / 89$ & ICP & $<1.50 \mathrm{E}+02$ \\
\hline 50736 & $10 / 27 / 89$ & ICP & $8.74 \mathrm{E}+02$ \\
\hline 50842 & $12 / 14 / 89$ & ICP & $2.8 I E+02$ \\
\hline 51024 & $3 / 07 / 90$ & ICP & $<1.50 \mathrm{E}+02$ \\
\hline $50721 \mathrm{E}$ & $10 / 24 / 89$ & ICP & $<5.00 \mathrm{E}+02$ \\
\hline $50736 \mathrm{E}$ & $10 / 27 / 89$ & ICP & $<5.00 E+02$ \\
\hline $50842 \mathrm{E}$ & $12 / 14 / 89$ & ICP & $<5.00 E+02$ \\
\hline $51024 \mathrm{E}$ & $3 / 07 / 90$ & ICP & $<5.00 E+02$ \\
\hline 50721 & $10 / 24 / 89$ & ICP & $4.30 E+01$ \\
\hline 50736 & $10 / 27 / 89$ & ICP & $9.60 \mathrm{E}+01$ \\
\hline 50842 & $12 / 14 / 89$ & ICP & $6.00 E+01$ \\
\hline 51024 & $3 / 07 / 90$ & ICP & 4. $20 E+01$ \\
\hline $50721 \mathrm{E}$ & $10 / 24 / 89$ & ICP & $<1.00 \mathrm{E}+03$ \\
\hline $50736 \mathrm{E}$ & $10 / 27 / 89$ & ICP & $<1.00 E+03$ \\
\hline $50842 \mathrm{E}$ & $12 / 14 / 89$ & ICP & $<1.00 \mathrm{E}+03$ \\
\hline $51024 \mathrm{E}$ & $3 / 07 / 90$ & ICP & $<1.00 E+03$ \\
\hline 50721 & $10 / 24 / 89$ & ICP & $4.40 E+01$ \\
\hline 50736 & $10 / 27 / 89$ & ICP & $6.20 E+01$ \\
\hline 50842 & $12 / 14 / 89$ & ICP & $5.50 \mathrm{E}+01$ \\
\hline 51024 & $3 / 07 / 90$ & ICP & $4.90 \mathrm{E}+01$ \\
\hline $50721 E$ & $10 / 24 / 89$ & ICP & $<1.00 \mathrm{E}+02$ \\
\hline $50736 \mathrm{E}$ & $10 / 27 / 89$ & ICP & $<1.00 E+02$ \\
\hline $50842 E$ & $12 / 14 / 89$ & ICP & $<1.00 \mathrm{E}+02$ \\
\hline $51024 \mathrm{E}$ & $3 / 07 / 90$ & ICP & $<1.00 E+02$ \\
\hline 50721 & $10 / 24 / 89$ & ICP & $2.09 E+04$ \\
\hline 50736 & $10 / 27 / 89$ & ICP & $1.88 \mathrm{E}+04$ \\
\hline 50842 & $12 / 14 / 89$ & ICP & $2.00 \mathrm{E}+0 \mathrm{~A}$ \\
\hline 51024 & $3 / 07 / 90$ & ICP & $1.87 \mathrm{E}+04$ \\
\hline 50721 & $10 / 24 / 89$ & IC & $3.30 E+03$ \\
\hline 50736 & $10 / 27 / 89$ & $I C$ & $3.50 \mathrm{E}+03$ \\
\hline 50842 & $12 / 14 / 89$ & IC & $6.00 \mathrm{E}+03$ \\
\hline 51024 & $3 / 07 / 90$ & IC & $2.00 E+03$ \\
\hline $50721 \mathrm{E}$ & $10 / 24 / 89$ & ICP & $<5.00 \mathrm{E}+02$ \\
\hline $50736 \mathrm{E}$ & $10 / 27 / 89$ & ICP & $<5.00 \mathrm{E}+02$ \\
\hline $50842 \mathrm{E}$ & $12 / 14 / 89$ & ICP & $<5.00 \mathrm{E}+02$ \\
\hline $51024 \mathrm{E}$ & $3 / 07 / 90$ & ICP & $<5.00 \mathrm{E}+02$ \\
\hline 50721 & $10 / 24 / 89$ & IC & $<5.00 E+02$ \\
\hline 50721 & $10 / 24 / 89$ & ISE & $1.56 \mathrm{E}+02$ \\
\hline 50736 & $10 / 27 / 89$ & IC & $<5.00 E+02$ \\
\hline 50736 & $10 / 27 / 89$ & ISE & $1.86 \mathrm{E}+02$ \\
\hline 50842 & $12 / 14 / 89$ & IC & $<5.00 E+02$ \\
\hline 50842 & $12 / 14 / 89$ & ISE & $1.49 \mathrm{E}+02$ \\
\hline 51024 & $3 / 07 / 90$ & IC & $<5.00 E+02$ \\
\hline 51024 & $3 / 07 / 90$ & ISE & 1. $36 \mathrm{E}+02$ \\
\hline 50721 & $10 / 24 / 89$ & ICP & $5.20 E+01$ \\
\hline 50736 & $10 / 27 / 89$ & ICP & 3. $30 E+02$ \\
\hline 50842 & $12 / 14 / 89$ & ICP & $1.82 \mathrm{E}+02$ \\
\hline 51024 & $3 / 07 / 90$ & ICP & $5.10 E+O I$ \\
\hline $50721 \mathrm{E}$ & $10 / 24 / 89$ & $I C P$ & $<5.00 E+02$ \\
\hline $50736 \mathrm{E}$ & $10 / 27 / 89$ & ICP & $<5.00 \mathrm{E}+02$ \\
\hline $50842 \mathrm{E}$ & $12 / 14 / 89$ & ICP & $<5.00 E+02$ \\
\hline $51024 \mathrm{E}$ & $3 / 07 / 90$ & ICP & $<5.00 E+02$ \\
\hline 50721 & $10 / 24 / 89$ & ICP & $4.39 E+03$ \\
\hline
\end{tabular}


DATA FOR 284-E Powerhouse wastewater-Routine Operation Continued

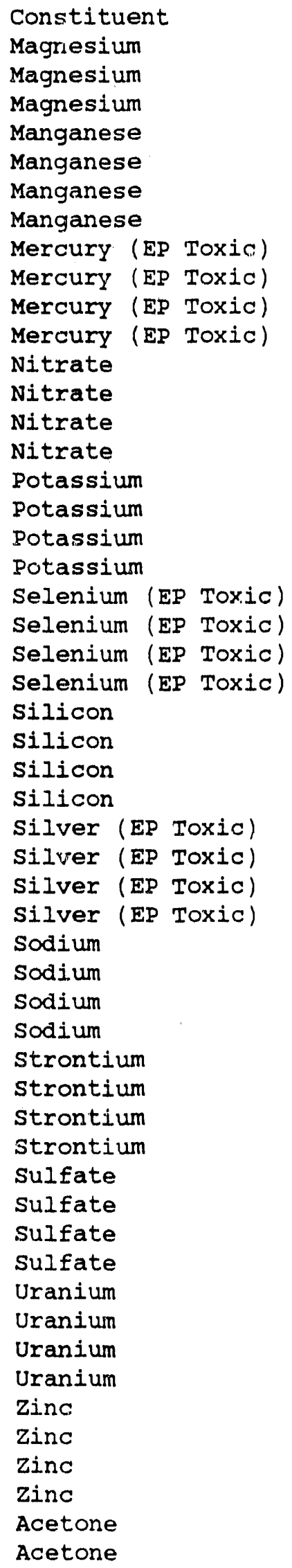

\begin{tabular}{|c|c|c|c|}
\hline ample \# & Date & Method & Result \\
\hline 50736 & $10 / 27 / 89$ & ICP & $4.44 E+03$ \\
\hline 50842 & $12 / 14 / 89$ & ICP & 4.11E+03 \\
\hline 51024 & $3 / 07 / 90$ & ICP & $4.43 E+03$ \\
\hline 50721 & $10 / 24 / 89$ & ICP & $<5.00 E+00$ \\
\hline 50736 & $10 / 27 / 89$ & ICP & $7.00 \mathrm{E}+00$ \\
\hline 50842 & $12 / 14 / 89$ & ICP & $<5.00 \mathrm{E}+00$ \\
\hline 51024 & $3 / 07 / 90$ & ICP & $<5.00 E+00$ \\
\hline $50721 \mathrm{E}$ & $10 / 24 / 89$ & CVAA/M & $<2.00 E+01$ \\
\hline $50736 \mathrm{E}$ & $10 / 27 / 89$ & CVAA $/ M$ & $<2.00 \mathrm{E}+01$ \\
\hline $50842 \mathrm{E}$ & $12 / 14 / 89$ & CVAA/M & $<2.00 E+01$ \\
\hline $51024 \mathrm{E}$ & $3 / 07 / 90$ & $C V A A / M$ & $<2.00 E+01$ \\
\hline 50721 & $10 / 24 / 89$ & IC & $5.00 \mathrm{E}+02$ \\
\hline 50736 & $10 / 27 / 89$ & IC & $5.00 E+02$ \\
\hline 50842 & $12 / 14 / 89$ & IC & $6.00 \mathrm{E}+02$ \\
\hline 51024 & $3 / 07 / 90$ & $I C$ & $5.00 \mathrm{E}+02$ \\
\hline 50721 & $10 / 24 / 89$ & ICP & $7.96 \mathrm{E}+02$ \\
\hline 50736 & $10 / 27 / 89$ & ICP & $8.66 \mathrm{E}+02$ \\
\hline 50842 & $12 / 14 / 89$ & ICP & $1.04 \mathrm{E}+03$ \\
\hline 51024 & $3 / 07 / 90$ & ICP & $7.22 \mathrm{E}+\mathrm{O} 2$ \\
\hline $50721 \mathrm{E}$ & $10 / 24 / 89$ & ICP & $<5.00 \mathrm{E}+02$ \\
\hline $50736 \mathrm{E}$ & $10 / 27 / 89$ & ICP & $<5.00 E+02$ \\
\hline $50842 \mathrm{E}$ & $12 / 14 / 89$ & ICP & $<5.00 E+02$ \\
\hline $51024 \mathrm{E}$ & $3 / 07 / 90$ & $I C P$ & $<5.00 E+02$ \\
\hline 50721 & $10 / 24 / 89$ & ICP & $2.60 E+03$ \\
\hline 50736 & $10 / 27 / 89$ & $I C P$ & $4.06 \mathrm{E}+03$ \\
\hline 50842 & $12 / 14 / 89$ & ICP & $3.22 \mathrm{E}+03$ \\
\hline 51024 & $3 / 07 / 90$ & $I C P$ & $2.53 \mathrm{E}+\mathrm{O}^{2}$ \\
\hline $50721 \mathrm{E}$ & $10 / 24 / 89$ & ICP & $<5.00 E+02$ \\
\hline $50736 \mathrm{E}$ & $10 / 27 / 89$ & ICP & $<5.00 \mathrm{E}+02$ \\
\hline $50842 \mathrm{E}$ & $12 / 14 / 89$ & ICP & $<5.00 \mathrm{E}+02$ \\
\hline $51024 \mathrm{E}$ & $3 / 07 / 90$ & ICP & $<5.00 \mathrm{E}+02$ \\
\hline 50721 & $10 / 24 / 89$ & ICP & $4.18 E+03$ \\
\hline 50736 & $10 / 27 / 89$ & ICP & $1.30 E+04$ \\
\hline 50842 & $12 / 14 / 89$ & ICP & $1.38 E+04$ \\
\hline 51024 & $3 / 07 / 90$ & ICP & $5.17 \mathrm{E}+03$ \\
\hline 50721 & $10 / 24 / 89$ & ICP & $2.58 \mathrm{E}+02$ \\
\hline 50736 & $10 / 27 / 89$ & $I C P$ & $2.00 \mathrm{E}+02$ \\
\hline 50842 & $12 / 14 / 89$ & ICP & $2.39 E+02$ \\
\hline 51024 & $3 / 07 / 90$ & ICP & $2.65 E+02$ \\
\hline 50721 & $10 / 24 / 89$ & $I C$ & $1.43 E+04$ \\
\hline 50736 & $10 / 27 / 89$ & IC & $1.96 \mathrm{E}+04$ \\
\hline 50842 & $12 / 14 / 89$ & $I C$ & 1. $99 E+04$ \\
\hline 51024 & $3 / 07 / 90$ & $I C$ & $1.48 \mathrm{E}+04$ \\
\hline 50721 & $10 / 24 / 89$ & GEA & $3.53 E-01$ \\
\hline 50736 & $10 / 27 / 89$ & GEA & $4.65 \mathrm{E}-01$ \\
\hline 50842 & $12 / 14 / 89$ & GEA & $6.18 E-01$ \\
\hline 51024 & $3 / 07 / 90$ & GEA & $4.53 E-01$ \\
\hline 50721 & $10 / 24 / 89$ & ICP & $<5.00 E+00$ \\
\hline 50736 & $10 / 27 / 89$ & ICP & $1.30 \mathrm{E}+01$ \\
\hline 50842 & $12 / 14 / 89$ & ICP & $6.00 \mathrm{E}+00$ \\
\hline 51024 & $3 / 07 / 90$ & ICP & $<5.00 \mathrm{E}+00$ \\
\hline 50721 & $10 / 24 / 89$ & VOA & $<9.00 \mathrm{E}+00$ \\
\hline 50721 & $10 / 24 / 89$ & $A B N$ & $<1.00 \mathrm{E}+01$ \\
\hline
\end{tabular}


DATA FOR 284-E Powerhouse Wastewater-Routine Operation Continued

Constituent
Acetone
Acetone
Acetone
Acetone
Acetone
Acetone
Acetone
Acetone
Acetone
Acetone
Acetore
Acetone
Acetone
Ammonia
Ammonia
Ammonia
Ammonia
1-Butanol
1-Butanol
1-Butanol
1-Butanol
1-Butanol
1-Butanol
1-Butanol
2-Butanone
2-Butanone
2-Butanone
2-Butanone
2-Butanone
2-Butanone
2-Butanone
2-Butanone
2-Butanone
2-Butanone
2-Butanone
Dichloromethane
Dichloromethane
Dichloromethane
Dichloromethane
Dichloromethane
Dichloromethane
Dichloromethane
Dichloromethane
Dichloromethane
Dichloromethane
Tetrahyoromethane
Tetrahydrofuran

\begin{tabular}{|c|c|c|c|}
\hline Sample \# & Date & Method & Result \\
\hline $50721 \mathrm{~B}$ & $10 / 24 / 89$ & VOA & 1. 10E+01 \\
\hline $50721 T$ & $10 / 24 / 89$ & VOA & $<1.00 E+01$ \\
\hline 50736 & $10 / 27 / 89$ & VOA & $<8.00 \mathrm{E}+00$ \\
\hline 50736 & $10 / 27 / 89$ & $A B N$ & $<1.00 E+01$ \\
\hline $50736 \mathrm{~B}$ & $10 / 27 / 89$ & VOA & $1.30 \mathrm{E}+01$ \\
\hline $50736 \mathrm{~T}$ & $10 / 27 / 89$ & VOA & $<1.00 E+01$ \\
\hline 50842 & $12 / 14 / 89$ & VOA & $<1.00 E+01$ \\
\hline 50842 & $12 / 14 / 89$ & $\mathrm{ABN}$ & $<1.00 \mathrm{E}+01$ \\
\hline $50842 B$ & $12 / 14 / 89$ & VOA & $<1.00 \mathrm{E}+01$ \\
\hline 51024 & $3 / 07 / 90$ & VOA & $<1.00 \mathrm{E}+01$ \\
\hline 51024 & $3 / 07 / 90$ & $A B N$ & $<1.00 E+01$ \\
\hline $51024 \mathrm{~B}$ & $3 / 07 / 90$ & VOA & $<1.00 \mathrm{E}+01$ \\
\hline $51024 \mathrm{~T}$ & $3 / 07 / 90$ & VOA & $<1.00 E+01$ \\
\hline 50721 & $10 / 24 / 89$ & ISE & $5.60 \mathrm{E}+01$ \\
\hline 50736 & $10 / 27 / 89$ & ISE & $5.80 \mathrm{E}+01$ \\
\hline 50842 & $12 / 14 / 89$ & ISE & $<5.00 E+01$ \\
\hline 51024 & $3 / 07 / 90$ & ISE & $<5.00 E+01$ \\
\hline 50721 & $10 / 24 / 89$ & DIGC & $<1.00 \mathrm{E}+04$ \\
\hline 50736 & $10 / 27 / 89$ & DIGC & $<1.00 \mathrm{E}+04$ \\
\hline 50842 & $12 / 14 / 89$ & DIGC & $<1.00 E+04$ \\
\hline 51024 & $3 / 07 / 90$ & VOA & $1.80 \mathrm{E}+01$ \\
\hline 51024 & $3 / 07 / 90$ & DIGC & $<1.00 \mathrm{E}+04$ \\
\hline $51024 B$ & $3 / 07 / 90$ & VOA & $1.80 \mathrm{E}+01$ \\
\hline $51024 \mathrm{~T}$ & $3 / 07 / 90$ & VOA & $1.70 \mathrm{E}+01$ \\
\hline 50721 & $10 / 24 / 89$ & VOA & $<1.00 \mathrm{E}+01$ \\
\hline $50721 \mathrm{~B}$ & $10 / 24 / 89$ & VOA & $1.30 \mathrm{E}+01$ \\
\hline $50721 \mathrm{~T}$ & $10 / 24 / 89$ & VOA & $<1.00 E+01$ \\
\hline 50736 & $10 / 27 / 89$ & VOA & $<1.00 E+01$ \\
\hline $50736 \mathrm{~B}$ & $10 / 27 / 89$ & VOA & $<1.00 E+01$ \\
\hline $50736 \mathrm{~T}$ & $10 / 27 / 89$ & VOA & $<1.00 \mathrm{E}+01$ \\
\hline 50842 & $12 / 14 / 89$ & VOA & $<1.00 \mathrm{E}+01$ \\
\hline $50842 B$ & $12 / 14 / 89$ & VOA & $<1.00 E+01$ \\
\hline 51024 & $3 / 07 / 90$ & VOA & $<1.00 \mathrm{E}+01$ \\
\hline $51024 \mathrm{~B}$ & $3 / 07 / 90$ & VOA & $<1.00 \mathrm{E}+01$ \\
\hline $51024 \mathrm{~T}$ & $3 / 07 / 90$ & VOA & $<1.00 E+01$ \\
\hline 50721 & $10 / 24 / 89$ & VOA & $<5.00 \mathrm{E}+00$ \\
\hline $50721 B$ & $10 / 24 / 89$ & VOA & $5.00 \mathrm{E}+00$ \\
\hline $50721 \mathrm{~T}$ & $10 / 24 / 89$ & VOA & $5.90 \mathrm{E}+01$ \\
\hline 50736 & $10 / 27 / 89$ & VOA & $<5.00 \mathrm{E}+00$ \\
\hline $50736 \mathrm{~B}$ & $10 / 27 / 89$ & VOA & $<4.00 E+00$ \\
\hline $50736 \mathrm{~T}$ & $10 / 27 / 89$ & VOA & $2.40 E+01$ \\
\hline 50842 & $12 / 14 / 89$ & VOA & $<5.00 \mathrm{E}+00$ \\
\hline $5084.2 B$ & $12 / 14 / 89$ & VOA & $5.00 \mathrm{E}+00$ \\
\hline 51024 & $3 / 07 / 90$ & VOA & $<5.00 \mathrm{E}+00$ \\
\hline $51024 \mathrm{~B}$ & $3 / 07 / 90$ & VOA & $<5.00 E+00$ \\
\hline $51024 \mathrm{~T}$ & $3 / 07 / 90$ & VOA & $<5.00 E+00$ \\
\hline 50721 & $10 / 24 / 89$ & VOA & $<1.00 E+01$ \\
\hline $50721 B$ & $10 / 24 / 89$ & VOA & $2.30 \mathrm{E}+01$ \\
\hline $50721 \mathrm{~T}$ & $10 / 24 / 89$ & VOA & $<1.00 \mathrm{E}+01$ \\
\hline 50736 & $10 / 27 / 89$ & VOA & $<1.00 \mathrm{E}+01$ \\
\hline $50736 \mathrm{~B}$ & $10 / 27 / 89$ & VOA & $1.30 E+01$ \\
\hline $50736 \mathrm{~T}$ & $10 / 27 / 89$ & VOA & $<1.00 \mathrm{E}+01$ \\
\hline 50842 & $12 / 14 / 89$ & VOA & $<1.00 E+01$ \\
\hline
\end{tabular}


WHC-EP-0355, Appendix D

DATA FOR 284-E POwerhouse wastewater-Routine Operation Continueä

Constituent

Tetrahydrofuran

Tetrahydrofuran

Tetrahydrofuran

Tetrahydrofuran

Trichloromethane

Trichloromethane

Trichloromethane

Trichloromethane

Trichloromethane

Tilchloromethane

Trichloromethane

Irichloromethane

Trichloromethane

Trichloromethane

Trichloromethane

Alkalinity (Method B)

Alkalinity (Method B)

Alkalinity (Method B)

Alkalinity (Method B)

Alpha Activity ( $\mathrm{pCi} / \mathrm{L}$ )

Alpha Activity (pCi/L)

Alpha Activity (pCi/L)

Alpha Activity (pCi/L)

Beta Activity (pCi/L)

Beta Activity (pCi/L)

Beta Activity (pCi/L)

Beta Activity (pCi/L)

Conductivity (us)

Conductivity (us)

Conductivity (us)

Conductivity (us)

Ingitability (degrees F)

Ingitability (degrees F)

Ingitability (degrees F)

Ingitability (degrees F)

$\mathrm{pH}$ (dimensionless)

$\mathrm{pH}$ (dimensionless)

$\mathrm{pH}$ (dimensionless)

$\mathrm{pH}$ (dimensionless)

Reactivity Cyanide (mg/kg)

Reactivity Cyanide ( $\mathrm{mg} / \mathrm{kg}$ )

Reactivity Cyanide ( $\mathrm{mg} / \mathrm{kg}$ )

Reactivity Cyanide ( $\mathrm{mg} / \mathrm{kg}$ )

Reactivity sulfide ( $\mathrm{mg} / \mathrm{kg}$ )

Reactivity sulfide (mg/kg)

Reactivity sulfide ( $\mathrm{mg} / \mathrm{kg}$ )

Reactivity sulfide ( $\mathrm{mg} / \mathrm{kg}$ )

Suspended solids (mg/L)

Suspended solids $(\mathrm{mg} / \mathrm{L}$ )

suspended solids $(\mathrm{mg} / \mathrm{L})$

suspended solids (mg/L)

TDS $(\mathrm{mg} / \mathrm{L})$

TDS (mg/L)

\begin{tabular}{|c|c|c|c|}
\hline $\begin{array}{r}\text { Imple \# } \\
50842 B\end{array}$ & $\begin{array}{c}\text { Date } \\
12 / 14 / 89\end{array}$ & $\begin{array}{l}\text { Method } \\
\text { VOA }\end{array}$ & $\begin{array}{l}\text { Result } \\
<1.00 \mathrm{E}+01\end{array}$ \\
\hline 51024 & $3 / 07 / 90$ & VOA & $<1.00 \mathrm{E}+01$ \\
\hline $51024 B$ & $3 / 07 / 90$ & VOA & $<1.00 \mathrm{E}+01$ \\
\hline $51024 \mathrm{~T}$ & $3 / 07 / 90$ & VOA & $<1.00 E+01$ \\
\hline 50721 & $10 / 24 / 89$ & VOA & $2.10 \mathrm{E}+01$ \\
\hline $50721 \mathrm{~B}$ & $10 / 24 / 89$ & VOA & $<5.00 \mathrm{E}+00$ \\
\hline $50721 \mathrm{~T}$ & $10 / 24 / 89$ & VOA & $<5.00 E+00$ \\
\hline 50736 & $10 / 27 / 89$ & VOA & $9.00 \mathrm{E}+00$ \\
\hline $36 \mathrm{~B}$ & $10 / 27 / 89$ & VOA & $<5.00 \mathrm{E}+00$ \\
\hline & $10 / 27$ & VOA & $+\infty$ \\
\hline 5084 & $12 / 1$ & VOA & $6.00 E+00$ \\
\hline $50842 B$ & $12 / 14 / 89$ & VOA & $<5.00 \mathrm{E}+00$ \\
\hline 51024 & $3 / 07 / 90$ & VOA & $2.60 \mathrm{E}+01$ \\
\hline $51024 \mathrm{~B}$ & $3 / 07 / 90$ & VOA & $<5.00 E+00$ \\
\hline $51024 \mathrm{~T}$ & $3 / 07 / 90$ & VOA & $<5.00 E+00$ \\
\hline 50721 & $10 / 24 / 89$ & 'TITRA & $5.80 \mathrm{E}+04$ \\
\hline 50736 & $10 / 27 / 89$ & TITRA & $6.60 \mathrm{E}+04$ \\
\hline 50842 & $12 / 1$ & TITRA & +04 \\
\hline 51024 & $3 / 0$ & TIT & $6.00 \mathrm{E}+04$ \\
\hline 50721 & $10 / 2$ & Alpha & $1.18 \mathrm{E}+00$ \\
\hline 50736 & $10 / 27 / 89$ & Alpha & $1.05 \mathrm{E}+00$ \\
\hline 50842 & $12 / 14 / 89$ & Alpha & 1. $22 \mathrm{E}+00$ \\
\hline 51024 & $3 / 07 / 90$ & Alpha & $<1.43 \mathrm{E}-01$ \\
\hline 50721 & $10 / 24 / 89$ & Beta & $<1.61 E+00$ \\
\hline 50736 & $10 / 27 / 89$ & Beta & $<8.76 E-01$ \\
\hline 50842 & $12 / 14 / 89$ & Beta & $8+\infty 0$ \\
\hline 51024 & $1 / 90$ & Beta & $<1$ \\
\hline 50721 & $10 / 2$ & COND - FId & $1.32 \mathrm{E}+02$ \\
\hline 50736 & $10 / 27 / 89$ & COND-FId & $1.91 \mathrm{E}+02$ \\
\hline 50842 & $12 / 14 / 89$ & COND-FId & $2.01 E+02$ \\
\hline 51024 & $3 / 07 / 90$ & COND-FId & $1.48 E+02$ \\
\hline $50721 \mathrm{E}$ & $10 / 24 / 89$ & IGNIT & $2.08 \mathrm{E}+02$ \\
\hline $50736 \mathrm{E}$ & $10 / 27 / 89$ & IGNIT & $2.08 \mathrm{E}+02$ \\
\hline $50842 E$ & $12 / 14 / 89$ & IGNIT & +02 \\
\hline $51024 E$ & $3 / 07$ & IGN & .02 \\
\hline & $10 / 2$ & & \\
\hline 50736 & $10 /$ & PH-Fld & $9.40 E+00$ \\
\hline 50842 & $12 / 14 / 89$ & $\mathrm{PH}-\mathrm{F} I \mathrm{~d}$ & $9.50 \mathrm{E}+00$ \\
\hline 51024 & $3 / 07 / 90$ & $\mathrm{PH}-\mathrm{E}$ Id & $8.80 E+00$ \\
\hline $50721 E$ & $10 / 24 / 89$ & DSPEC & $<1.00 E+02$ \\
\hline $50736 \mathrm{E}$ & $10 / 27 / 89$ & DSPEC & $<1.00 \mathrm{E}+02$ \\
\hline $50842 \mathrm{E}$ & $12 / 14 / 89$ & DSPEC & $<1.00 E+02$ \\
\hline $51024 E$ & $3 / 07 / 90$ & DSPEC & $<1.00 \mathrm{E}+02$ \\
\hline $50721 \mathrm{E}$ & $10 / 24 / 89$ & DTITRA & +02 \\
\hline $736 \mathrm{E}$ & $10 / 2$ & & \\
\hline $5084.2 \mathrm{E}$ & 121 & DTITRA & $<1.00 E+02$ \\
\hline $51024 E$ & $3 / 07 / 90$ & DTITRA & $<1.00 \mathrm{E}+02$ \\
\hline 50721 & $10 / 24 / 89$ & SSOLID & $<5.00 E+03$ \\
\hline 50736 & $10 / 27 / 89$ & SSOLID & $3.90 E+04$ \\
\hline 50842 & $12 / 14 / 89$ & SSOLID & 1. $10 \mathrm{E}+04$ \\
\hline 51024 & $3 / 07 / 90$ & SSOLID & $<5.00 E+03$ \\
\hline & & TDS & $7.60 E+04$ \\
\hline & & & $8.90 \mathrm{E}+\mathrm{C}$ \\
\hline
\end{tabular}

D -36 
DATA FOR 284-E POwerhouse Wastewater-Routine Operation Continued

\begin{tabular}{|c|c|c|c|c|}
\hline $\begin{array}{l}\text { Constituent } \\
\text { TDS (mg/L) }\end{array}$ & $\begin{array}{c}\text { Sample \# } \\
50842\end{array}$ & $\begin{array}{c}\text { Date } \\
12 / 14 / 89\end{array}$ & $\begin{array}{l}\text { Method } \\
\text { TDS }\end{array}$ & $\begin{array}{l}\text { Result } \\
1.03 E+05\end{array}$ \\
\hline $\operatorname{TDS}(m g / L)$ & 51024 & $3 / 07 / 90$ & TDS & $8.00 E+04$ \\
\hline Temperature (degrees C) & 50721 & $10 / 24 / 89$ & TEMP-FId & $1.62 \mathrm{E}+01$ \\
\hline Temperature (degrees C) & 50736 & $10 / 27 / 89$ & TEMP-FId & $1.75 \mathrm{E}+01$ \\
\hline Temperature (degrees C) & 50842 & $12 / 14 / 89$ & TEMP-FId & 1. $11 \mathrm{E}+01$ \\
\hline Temperature (degrees C) & 51024 & $3 / 07 / 90$ & TEMP-FId & $1.01 E+01$ \\
\hline $\operatorname{TOC}(u g / g)$ & 50721 & $10 / 24 / 89$ & TOC & $2.60 \mathrm{E}+03$ \\
\hline TOC (ug/g) & 50736 & $10 / 27 / 89$ & TOC & $<1.20 E+03$ \\
\hline TOC $(u g / g)$ & 50842 & $12 / 14 / 89$ & TOC & $<1.70 \mathrm{E}+03$ \\
\hline $\operatorname{TOC}(u g / g)$ & 51024 & $3 / 07 / 90$ & TOC & $1.30 \mathrm{E}+03$ \\
\hline Total Carbon ( $\mathrm{ug} / \mathrm{g}$ ) & 50721 & $10 / 24 / 89$ & TC & $1.53 \mathrm{E}+04$ \\
\hline Total Carbon (ug/g) & 50736 & $10 / 27 / 89$ & TC & $1.49 \mathrm{E}+04$ \\
\hline Total Carbon (ug/g) & 50842 & $1.2 / 14 / 89$ & TC & $1.55 \mathrm{E}+04$ \\
\hline Total Carbon (ug/g) & 51024 & $3 / 07 / 90$ & TC & $1.52 \mathrm{E}+04$ \\
\hline $\operatorname{TOX}($ ug $(C I) / L)$ & 50721 & $10 / 24 / 89$ & LTOX & $1.15 \mathrm{E}+02$ \\
\hline $\operatorname{Tox}(\mathrm{ug}(\mathrm{C} 1) / \mathrm{L})$ & 50736 & $10 / 27 / 89$ & LTOX & $7.80 E+01$ \\
\hline $\operatorname{Tox}(\operatorname{ug}(\mathrm{Cl}) / \mathrm{L})$ & 50842 & $12 / 14 / 89$ & LTOX & $5.50 \mathrm{E}+01$ \\
\hline$(\mathrm{ug}(\mathrm{Cl}) / \mathrm{L})$ & 51024 & $3 / 07 / 90$ & LTOX & 1. $25 \mathrm{E}+02$ \\
\hline
\end{tabular}


DATA FOR 241-A Tank Farm Cooling Water

Constituent
Arsenic (EP Toxic)
Arsenic (EP Toxic)
Arsenic (EP Toxic)
Arsenic (EP Toxic)
Barium
Barium
Barium
Barium
Barium (EP Toxic)
Barium (EP TOxic)
Barium (EP Toxic)
Barium (EP TOxic)
Boron
Boron
Boron
Boron
Cadmium (EP Toxic)
Cadmium (EP Toxic)
Cadmium (EP Toxic)
Cadmium (EP Toxic)
Calcium
Calcium
Calcium
Calcium
Chloride
Chloride
Chloride
Chloride
Chromium (EP Toxic)
Chromium (EP Toxic)
Chromium (EP Toxic)
Chromium (EP Toxic)
Copper
Copper
Copper
Copper
Eluoride
Fluoride
Fluoride
Fluoride
Fluoride
Fluoride
Fluoride
Fluoride
Iron
Lead
Lead (EP Toxic)

\begin{tabular}{|c|c|c|c|}
\hline Sample \# & Date & Method & Result \\
\hline $50701 \mathrm{E}$ & $10 / 18 / 89$ & ICP & $<5.00 E+02$ \\
\hline $50815 \mathrm{E}$ & $11 / 29 / 89$ & ICP & $<5.00 \mathrm{E}+02$ \\
\hline $50963 \mathrm{E}$ & $2 / 21 / 90$ & ICP & $<5.00 \mathrm{E}+02$ \\
\hline $51028 \mathrm{E}$ & $3 / 08 / 90$ & ICP & $<5.00 \mathrm{E}+02$ \\
\hline 50701 & $10 / 18 / 89$ & ICP & 3. 10E+01 \\
\hline 50815 & $11 / 29 / 89$ & ICP & 3. $20 \mathrm{E}+01$ \\
\hline 50963 & $2 / 21 / 90$ & ICP & $2.70 E+01$ \\
\hline 51028 & $3 / 08 / 90$ & ICP & $3.00 E+01$ \\
\hline $50701 E$ & $10 / 18 / 89$ & ICP & $<1.00 \mathrm{E}+03$ \\
\hline $50815 \mathrm{E}$ & $11 / 29 / 89$ & ICP & $<1.002+03$ \\
\hline $50963 E$ & $2 / 21 / 90$ & ICP & $<1.00 \mathrm{E}+03$ \\
\hline $51028 \mathrm{E}$ & $3 / 08 / 90$ & ICP & $<1.00 \mathrm{E}+03$ \\
\hline 50701 & $10 / 18 / 89$ & ICP & $<1.00 \mathrm{E}+01$ \\
\hline 50815 & $11 / 29 / 89$ & ICP & $<1.00 \mathrm{E}+01$ \\
\hline 50963 & $2 / 21 / 90$ & ICP & 4.10E+01 \\
\hline 51028 & $3 / 08 / 90$ & ICP & $2.30 \mathrm{E}+01$ \\
\hline $50701 E$ & $10 / 18 / 89$ & ICP & $<1.00 \mathrm{E}+02$ \\
\hline $50815 \mathrm{E}$ & $11 / 29 / 89$ & ICP & $<1.00 \mathrm{E}+02$ \\
\hline $50963 \mathrm{E}$ & $2 / 21 / 90$ & ICP & $<1.00 \mathrm{E}+02$ \\
\hline $51028 E$ & $3 / 08 / 90$ & ICP & $<1.00 \mathrm{E}+02$ \\
\hline 50701 & $10 / 18 / 89$ & ICP & $1.86 \mathrm{E}+04$ \\
\hline 50815 & $11 / 29 / 89$ & ICP & $1.89 E+04$ \\
\hline 50963 & $2 / 21 / 90$ & ICP & $1.83 \mathrm{E}+04$ \\
\hline 51028 & $3 / 08 / 90$ & ICP & $1.85 \mathrm{E}+04$ \\
\hline 50701 & $10 / 18 / 89$ & IC & $1.20 \mathrm{E}+03$ \\
\hline 50815 & $11 / 29 / 89$ & IC & $9.00 \mathrm{E}+02$ \\
\hline 50963 & $2 / 21 / 90$ & IC & $8.00 E+02$ \\
\hline 51028 & $3 / 08 / 90$ & IC & 1. 10E+03 \\
\hline $50701 \mathrm{E}$ & $10 / 18 / 89$ & ICP & $<5.00 \mathrm{E}+02$ \\
\hline $508.15 \mathrm{E}$ & $11 / 29 / 89$ & ICP & $<5.00 \mathrm{E}+02$ \\
\hline $50963 \mathrm{E}$ & $2 / 21 / 90$ & ICP & $<5.00 E+02$ \\
\hline $.51028 E$ & $3 / 08 / 90$ & ICP & $<5.00 \mathrm{E}+02$ \\
\hline 50701 & $10 / 18 / 89$ & ICP & 2. $20 \mathrm{E}+01$ \\
\hline 50815 & $11 / 29 / 89$ & ICP & $1.90 \mathrm{E}+01$ \\
\hline 50963 & $2 / 21 / 90$ & ICP & 1. $40 \mathrm{E}+01$ \\
\hline 51028 & $3 / 08 / 90$ & ICP & $1.60 \mathrm{E}+01$ \\
\hline 50701 & $10 / 18 / 89$ & IC & $<5.00 \mathrm{E}+02$ \\
\hline 50701 & $10 / 18 / 89$ & ISE & 1. $76 \mathrm{E}+02$ \\
\hline 50815 & $11 / 29 / 89$ & $I C$ & $<5.00 E+02$ \\
\hline 5081.5 & $11 / 29 / 89$ & ISE & $1.38 \mathrm{E}+02$ \\
\hline 50963 & $2 / 21 / 90$ & IC & $<5.00 E+02$ \\
\hline ro963 & $2 / 21 / 90$ & ISE & 1. $15 \mathrm{E}+02$ \\
\hline 51028 & $3 / 08 / 90$ & IC & $<5.00 \mathrm{E}+02$ \\
\hline 51028 & $3 / 08 / 90$ & ISE & 1. $28 \mathrm{E}+02$ \\
\hline 50701 & $10 / 18 / 89$ & ICP & $7.30 E+01$ \\
\hline 50815 & $11 / 29 / 89$ & ICP & 4. 30E+OI \\
\hline 50963 & $2 / 21 / 90$ & ICP & $3.80 E+01$ \\
\hline 51028 & $3 / 08 / 90$ & ICP & 4. $90 E+01$ \\
\hline 50701 & $10 / 18 / 89$ & GFAA & $<5.00 \mathrm{E}+00$ \\
\hline 50815 & $11 / 29 / 89$ & GFAA & $<5.00 E+00$ \\
\hline 50963 & $2 / 21 / 90$ & GFAA & $<5.00 E+00$ \\
\hline 51028 & $3 / 08 / 90$ & GFAA & $5.00 \mathrm{E}+00$ \\
\hline $50701 \mathrm{E}$ & $10 / 18 / 89$ & ICP & $<5.00 E+02$ \\
\hline
\end{tabular}


WHC-EP-0355, Appendix D

DATA FOR 241-A Tank Farm Cooling water

Continued

Constituent

Lead (EP Toxic)

Lead (EP Toxic)

Lead (EP TOxic)

Magnesium

Magnesium

Magnesium

Magnesium

Manganese

Manganese

Manganese

Manganese

Mercury (EP Toxic)

Mercury (EP Toxic)

Mercury (EP Toxic)

Mercury (EP Toxic)

Nitrate

Nitrate

Nitrate

Nitrate

Potassium

Potassium

Potassium

Potassium

Selenium (EP TOXic)

Selenium (EP TOXic)

Selenium (EP Toxic)

Selenium (EP TOxic)

Silicon

silicon

silicon

silicon

Silver (EP Toxic)

Silver (EF Toxic)

Silver (EP TOxic)

Silver (EP TOXiC)

Sodium

Sodium

sodium

Sodium

strontium

strontium

strontium

strontium

sulfate

sulfate

sulfate

Sulfate

Uranium

Urani um

Uranium

zisic

Zinc

zinc

\begin{tabular}{|c|c|c|c|}
\hline ample \# & Date & Method & Result \\
\hline $50815 E$ & $11 / 29 / 89$ & ICP & $<5.00 E+02$ \\
\hline $50963 E$ & $2 / 21 / 90$ & ICP & $<5.00 \mathrm{E}+02$ \\
\hline $51028 \mathrm{E}$ & $3 / 08 / 90$ & $I C P$ & $<5.00 \mathrm{E}+02$ \\
\hline 50701 & $10 / 18 / 89$ & $I C P$ & $4.11 E+03$ \\
\hline 50815 & $11 / 29 / 89$ & ICP & $4.30 E+03$ \\
\hline 50963 & $2 / 2.1 / 90$ & ICP & $4.33 E+03$ \\
\hline 51028 & $3 / 08 / 90$ & ICP & $4.57 E+03$ \\
\hline 50701 & $10 / 18 / 89$ & ICP & $6.00 E+00$ \\
\hline 50815 & $11 / 29 / 89$ & ICP & $<5.00 E+00$ \\
\hline 50963 & $2 / 21 / 90$ & ICP & $<5.00 \mathrm{E}+00$ \\
\hline 51028 & $3 / 08 / 90$ & ICP & $<5.00 \mathrm{E}+00$ \\
\hline $50701 E$ & $10 / 18 / 89$ & CVAA /M & $<2.00 \mathrm{E}+01$ \\
\hline $50815 E$ & $11 / 29 / 89$ & $C V A A / M$ & $<2.00 \mathrm{E}+0]$. \\
\hline $50963 E$ & $2 / 21 / 90$ & $C V A A / M$ & $O E+01$ \\
\hline $51028 E$ & $3 / 08 / 90$ & CVAA /M & $<2.00 E+01$ \\
\hline 50701 & $10 / 1.8 / 89$ & IC & $<5.00 E+02$ \\
\hline 50815 & $11 / 29 / 89$ & IC & $5.00 E+02$ \\
\hline 50963 & $2 / 21 / 90$ & IC & $6.00 \mathrm{E}+02$ \\
\hline 51028 & $3 / 08 / 90$ & IC & $<5.00 E+02$ \\
\hline 50701 & $10 / 18 / 89$ & $I C P$ & $7.11 E+02$ \\
\hline 50815 & $11 / 29 / 89$ & ICP & $7.78 \mathrm{E}+02$ \\
\hline 50963 & $2 / 21 / 90$ & ICP & $6.97 \mathrm{E}+02$ \\
\hline 51028 & $3 / 08 / 90$ & $I C P$ & $7.31 E+02$ \\
\hline $50701 E$ & $10 / 18 / 89$ & ICP & $<5.00 \mathrm{E}+02$ \\
\hline $50815 E$ & $11 / 29 / 89$ & ICP & $<5.00 E+02$ \\
\hline $50963 E$ & $2 / 21 / 90$ & ICP & $<5.00 \mathrm{E}+02$ \\
\hline $51028 \mathrm{E}$ & $3 / 08 / 90$ & ICP & $<5.00 E+02$ \\
\hline 50701 & $10 / 18 / 89$ & ICP & $2.35 E+03$ \\
\hline 50815 & $11 / 29 / 89$ & ICP & $2.71 \mathrm{E}+03$ \\
\hline 50963 & $2 / 21 / 90$ & ICP & $2.41 \mathrm{E}+03$ \\
\hline 51028 & $3 / 08 / 90$ & ICP & $2.41 \mathrm{E}+03$ \\
\hline $50701 \mathrm{E}$ & $10 / 18 / 89$ & $I C P$ & $<5.00 \mathrm{E}+02$ \\
\hline $50815 E$ & $11 / 29 / 89$ & ICP & $<5.00 E+02$ \\
\hline $50963 E$ & $2 / 21 / 90$ & ICP & $<5.00 \mathrm{E}+02$ \\
\hline $51028 \mathrm{E}$ & $3 / 08 / 90$ & ICP & $<5.00 E+02$ \\
\hline 50701 & $10 / 18 / 89$ & ICP & $2.17 E+03$ \\
\hline 50815 & $11 / 29 / 89$ & ICP & $2.04 E+03$ \\
\hline $51: 03$ & $2 / 21 / 90$ & ICP & $1.89 \mathrm{E}+03$ \\
\hline 51028 & $3 / 08 / 90$ & ICP & $2.08 E+03$ \\
\hline 50701 & $10 / 18 / 89$ & ICP & $9.70 \mathrm{E}+01$ \\
\hline 50815 & $11 / 29 / 89$ & ICP & $9.40 \mathrm{E}+01$ \\
\hline 50963 & $2 / 21 / 90$ & ICP & $9.40 \mathrm{E}+01$ \\
\hline 51028 & $3 / 08 / 90$ & ICP & $9.50 E+O 1$ \\
\hline 50701 & $10,18 / 89$ & IC & $0 E+04$ \\
\hline 50815 & $11 / 29 / 89$ & IC & $9.60 E+03$ \\
\hline 50963 & $2 / 21 / 90$ & IC & $1.01 E+04$ \\
\hline 51028 & $3 / 08 / 90$ & IC & $1.07 \mathrm{E}+04$ \\
\hline 50701 & $10 / 18 / 89$ & GEA & $4.57 \mathrm{E}-01$ \\
\hline 50815 & .11/29/89 & GEA & $3.63 E-C 1$ \\
\hline 51028 & $3 / 08 / 90$ & GEA & $9.21 \mathrm{E}-01$ \\
\hline 50701 & $10 / 10 / 33$ & ICF & $2.30 E \div 01$ \\
\hline 50815 & $11 / 29 / 89$ & ICP & 1. $20 \mathrm{E}+01$ \\
\hline 50963 & $2 / 21 / 90$ & ICP & $1.50 \mathrm{E}+01$ \\
\hline
\end{tabular}


WHC-EP-0355, Appendix D

DATA FOR 241-A Tanl: Farm Cooling water

Continued

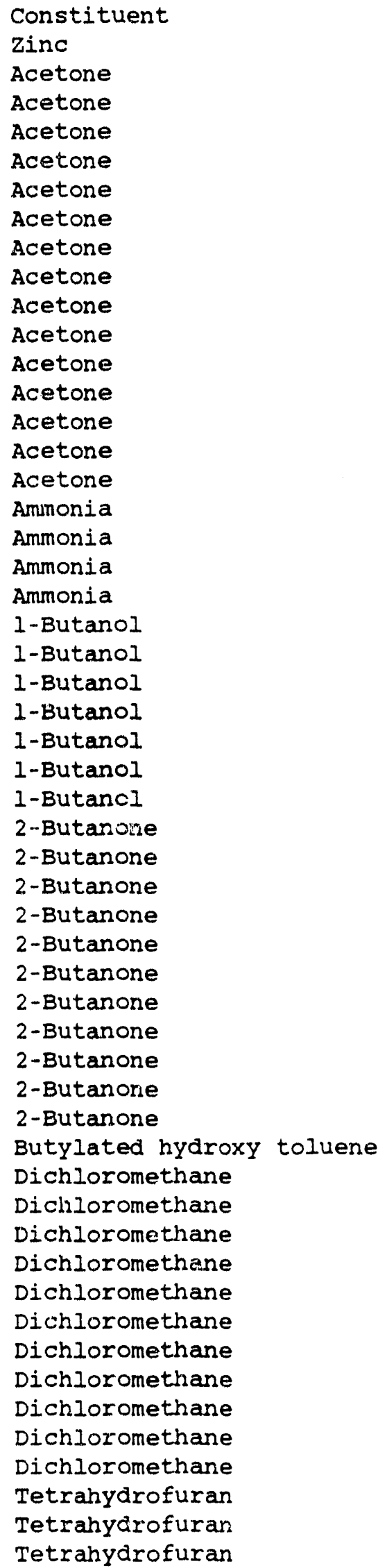

Constituent

zinc

Acetone

Acetone

Acetone

Acetone

Acetone

Acetone

Acetone

Acetone

Acetone

Acetone

Ammonia

1-Butanol

1-Butanol

1 -Butancl

2-Butanone

2-Butanone

2-Butanone

2-Butanone

2-Butanone

Butylated hydroxy toluene

Dichloromethane

Dichloromethane

Dichloromethane

Dichloromethane

Dichloromethane

Dichloromethane

Dichloromethane

Tetrahydrofuran

yarofuran

\begin{tabular}{lrlr} 
Sample \# & Date & Method & \multicolumn{1}{l}{ Result } \\
51028 & $3 / 08 / 90$ ICP & $1.50 E+01$ \\
50701 & $10 / 18 / 89$ VOA & $<9.00 E+00$ \\
50701 & $10 / 18 / 89$ ABN & $<1.00 E+01$ \\
$50701 B$ & $10 / 18 / 89$ VOA & $2.30 E+01$ \\
$50701 T$ & $10 / 18 / 89$ VOA & $<1.00 E+01$ \\
50815 & $11 / 29 / 89$ VOA & $<1.00 E+01$ \\
50815 & $11 / 29 / 89$ ABN & $<1.00 E+01$ \\
$50815 B$ & $11 / 29 / 89$ VOA & $<1.00 E+01$ \\
50963 & $2 / 21 / 90$ VOA & $<1.00 E+01$ \\
50963 & $2 / 21 / 90$ ABN & $<1.05 E+01$ \\
$50963 B$ & $2 / 21 / 90$ VOA & $<1.00 E+01$ \\
$50963 T$ & $2 / 21 / 90$ VOA & $<1.00 E+01$ \\
51028 & $3 / 08 / 90$ VOA & $<1.00 E+01$ \\
51028 & $3 / 08 / 90$ ABN & $<1.00 E+01$ \\
$51028 B$ & $3 / 08 / 90$ VOA & $<1.00 E+01$ \\
$51028 T$ & $3 / 08 / 90$ VOA & $<1.00 E+01$ \\
50701 & $10 / 18 / 89$ ISE & $7.00 E+01$ \\
50815 & $11 / 29 / 89$ ISE & $<5.00 E+01$ \\
50963 & $2 / 21 / 90$ ISE & $<5.00 E+01$ \\
51028 & $3 / 08 / 90$ ISE & $<5.00 E+01$ \\
50701 & $10 / 18 / 89$ DIGC & $<1.00 E+04$ \\
50815 & $11 / 29 / 89$ DIGC & $<1.00 E+04$ \\
50963 & $2 / 21 / 90$ DIGC & $<1.00 E+04$ \\
51028 & $3 / 08 / 90$ VOA & $1.10 E+01$ \\
51028 & $3 / 08 / 90$ DIGC & $<1.00 E+04$ \\
$51028 B$ & $3 / 08 / 90$ VOA & $1.20 E+01$ \\
$51028 T$ & $3 / 08 / 90$ VOA & $1.30 E+01$ \\
50701 & $10 / 18 / 89$ VOA & $<1.00 E+01$ \\
$50701 B$ & $10 / 18 / 89$ VOA & $1.50 E+01$ \\
$50701 T$ & $10 / 18 / 89$ VOA & $<1.00 E+01$ \\
50815 & $11 / 29 / 89$ VOA & $<5.00 E+00$ \\
$50815 B$ & $11 / 29 / 89$ VOA & $<1.00 E+01$ \\
50963 & $2 / 21 / 90$ VOA & $<1.00 E+01$ \\
$50963 B$ & $2 / 21 / 90$ VOA & $<8.00 E+00$ \\
$50963 T$ & $2 / 21 / 90$ VOA & $<1.00 E+01$ \\
51028 & $3 / 08 / 90$ VOA & $<1.00 E+01$ \\
$51028 B$ & $3 / 08 / 90$ VOA & $<1.00 E+01$ \\
$51028 T$ & $3 / 08 / 90$ VOA & $<1.00 E+01$ \\
50963 & $2 / 21 / 90$ ABN & $9.00 E+00$ \\
50701 & $10 / 18 / 89$ VOA & $<5.00 E+00$ \\
$50701 B$ & $10 / 18 / 89$ VOA & $1.10 E+01$ \\
$50701 T$ & $10 / 18 / 89$ VOA & $1.20 E+01$ \\
50815 & $11 / 29 / 89$ VOA & $<5.00 E+00$ \\
$50815 B$ & $11 / 29 / 89$ VOA & $6.00 E+00$ \\
50963 & $2 / 21 / 90$ VOA & $<5.00 E+00$ \\
$50963 B$ & $2 / 21 / 90$ VOA & $<3.00 E+00$ \\
$50963 T$ & $2 / 21 / 90$ VOA & $<5.00 E+00$ \\
51028 & $3 / 08 / 90$ VOA & $<5.00 E+00$ \\
$51028 B$ & $3 / 08 / 90$ VOA & $<5.00 E+00$ \\
$51028 T$ & $3 / 08 / 90$ VOA & $<5.00 E+00$ \\
50701 & $10 / 18 / 89$ VOA & $<1.00 E+01$ \\
$50701 B$ & $10 / 18 / 89$ VOA & $1.10 E+01$ \\
$50701 T$ & $10 / 18 / 89$ VOA & $<1.00 E+01$ \\
& & &
\end{tabular}


DATA FOR 241-A Tank Farm Cooling water Continued

Constituent

Tetrahydrofurin

Tetrahydrofuran

Tetrahydrofuran

Tetrahydrofuran

Tetrahydrofuran

Tetrahydrofuran

Tetrahydrofuran

Tetrahydrofuran

Trichloromethane

Trichloromethane

Trichloromethane

Trichloromethane

rrichloromethane

Trichloromethane

Trichloromethane

Trichloromethane

Trichloromethane

Trichloromethane

Trichloromethane

Alkalinity (Method B)

Alkalinity (Method B)

Alkalinity (Method B)

Alkalinity (Method B)

Alpha Activity ( $\mathrm{pCi} / \mathrm{I}$ )

Alpha Activity (pCi/IN)

Alpha Activity (pCi/L)

Conductivity (us)

Conductivity (us)

Conductivity (us)

Conductivity (us)

Ingitability (degrees F)

Ingitability (degrees F)

Ingitability (degrees F)

Ingitability (degrees $F$ )

$\mathrm{pH}$ (dimensionless)

$\mathrm{pH}$ (dimensionless)

$\mathrm{pH}$ (dimensionless)

pH (aimensionless)

Reactivity Cyanide ( $\mathrm{mg} / \mathrm{kg}$ )

Reactivity cyanide ( $\mathrm{mg} / \mathrm{kg}$ )

Reactivity Cyanide ( $\mathrm{mg} / \mathrm{kg}$ )

Reactivity Cyanide (mg/kg)

Reactivity sulfide (mg/kg)

Reactivity sulfide ( $\mathrm{mg} / \mathrm{kg}$ )

Reactivity sulfide (mg/ikg)

Reactivity Sulfide ( $\mathrm{mg} / \mathrm{kg}$ )

$\operatorname{TDS}(\mathrm{mg} / \mathrm{L}$ )

$\operatorname{TDS}(\mathrm{mg} / \mathrm{L})$

$\operatorname{TDS}(\mathrm{mg} / \mathrm{L})$

TDS (mg/L)

Temperature (degrees C)

Temperature (äegrees $\mathrm{C}$ )

Temperature (degrees C)

\begin{tabular}{|c|c|c|c|}
\hline umple \# & Date & Method & Result \\
\hline 50815 & $11 / 29 / 89$ & VOA & $<1.00 \mathrm{E}+01$ \\
\hline $50815 B$ & $11 / 29 / 89$ & VOA & $<1.00 E+01$ \\
\hline 50963 & $2 / 21 / 90$ & VOA & $<1.00 E+01$ \\
\hline $50963 B$ & $2 / 21 / 90$ & VOA & $<1.00 \mathrm{E}+01$ \\
\hline $50963 \mathrm{~T}$ & $2 / 21 / 90$ & VOA & $<6.00 E+00$ \\
\hline 51028 & $3 / 08 / 90$ & VOA & $<1.00 E+01$ \\
\hline $51028 B$ & $3 / 08 / 90$ & VOA & $<1.00 E+01$ \\
\hline $51028 \mathrm{~T}$ & $3 / 08 / 90$ & VOA & $<1.00 E+01$ \\
\hline 50701 & $10 / 18 / 89$ & VOA & $<5.00 E+00$ \\
\hline 507018 & $10 / 18 / 89$ & VOA & $<5.00 E+00$ \\
\hline $50701 \mathrm{~T}$ & $10 / 18 / 89$ & VOA & $<5.00 \mathrm{E}+00$ \\
\hline 508 & $11 / 29 / 89$ & VOA & $<5.00 \mathrm{E}+00$ \\
\hline $50815 B$ & $11 / 29 / 89$ & VOA & $E+\infty$ \\
\hline 50963 & $2 / 21 / 90$ & VOA & $<5.00 E+00$ \\
\hline $50963 B$ & $2 / 21 / 90$ & VOA & $E+00$ \\
\hline $50963 \mathrm{~T}$ & $2 / 21 / 90$ & VOA & $E+\infty$ \\
\hline 51028 & $3 / 08 / 90$ & VOA & $<5.00 E+00$ \\
\hline $51028 B$ & $3 / 08 / 90$ & VOA & $<5.00 E+00$ \\
\hline $51028 \mathrm{~T}$ & $3 / 08 / 90$ & VOA & $<5.00$ \\
\hline 50701 & $10 / 18 / 89$ & TITRA & $\mathrm{I}+04$ \\
\hline 50815 & $11 / 29 / 89$ & TITRA & +04 \\
\hline 50963 & $2 / 21 / 90$ & TIT & +04 \\
\hline 51028 & $3 / 0$ & TIT & +04 \\
\hline 50701 & $10 / 18 / 89$ & Alpha & $<3.54$ \\
\hline 50815 & $11 / 29 / 89$ & Alpha & $E+\infty$ \\
\hline 51028 & $3 / 08 / 90$ & Alpha & $<3.18 \mathrm{E}-01$ \\
\hline 50701 & $10 / 18 / 89$ & COND-FId & $1.30 \mathrm{E}+02$ \\
\hline 50815 & $11 / 29 / 89$ & COND-Fld & $1.43 \mathrm{E}+02$ \\
\hline 50963 & $2 / 2.1 /$ & COND & $1.47 \mathrm{E}+02$ \\
\hline 51028 & $3 / 08 / 90$ & COND-FId & $E+02$ \\
\hline $50701 \mathrm{E}$ & $10 / 18 / 89$ & IGNIT & $.12 E+02$ \\
\hline $50815 E$ & $11 / 29 / 89$ & IGN & +02 \\
\hline $50963 E$ & $2 / 21 / 90$ & IGN & $i+02$ \\
\hline $51028 \mathrm{E}$ & $3 / 08 / 90$ & IGNIT & $E+02$ \\
\hline 50701 & $10 / 18 / 89$ & PH-Fld & $.95 E+00$ \\
\hline 50815 & $11 / 29 / 89$ & PH - Fld & $7.7: E+00$ \\
\hline 50963 & $2 / 21 / 90$ & PH-Fid & $7.50 \mathrm{E}+00$ \\
\hline 51028 & $3 / 08 / 90$ & $\mathrm{PH}-\mathrm{F} I \mathrm{~d}$ & $3.04 \mathrm{E}+00$ \\
\hline $50701 E$ & $10 / 18 / 39$ & DSPEC & $<1.00 E+02$ \\
\hline $50815 E$ & $11 / 29 / 89$ & DSPEC & $<1.00 \mathrm{E}+02$ \\
\hline $50963 E$ & $2 / 21 / 90$ & DSPEC & $<1.00 \mathrm{E}+02$ \\
\hline $51028 \mathrm{E}$ & $3 / 08 / 90$ & DSPEC & $<1.00 E+02$ \\
\hline $50701 E$ & $10 / 18 / 89$ & DTITRA & $<1.00 \mathrm{E}+02$ \\
\hline $50815 E$ & $11 / 29 / 89$ & DTITRA & $<1.00 \mathrm{E}+02$ \\
\hline $50963 E$ & $2 / 21 / 90$ & DTITRA & $<1.00 \mathrm{E}+02$ \\
\hline $51028 E$ & $3 / 08 / 90$ & DTITRA & $<1.00 \mathrm{E}+02$ \\
\hline 50701 & $10 / 18 / 89$ & TDS & $6.40 E+04$ \\
\hline 50815 & $11 / 29 / 89$ & TDS & $4.40 E+04$ \\
\hline 50963 & $2 / 21 / 90$ & TDS & $5.80 E+04$ \\
\hline 51028 & $3 / 08 / 90$ & TDS & $3.80 E+04$ \\
\hline 50701 & $10 / 18 / 89$ & TEMP $-E]$ & $1.70 E+O 1$ \\
\hline 50815 & $\bar{I} / \angle Y / E \bar{y}$ & 'LEMP-K'Ia & I. $2 U E+\bar{I}$ \\
\hline 0963 & & TEMP-FId & $7.50 \mathrm{E}+00$ \\
\hline
\end{tabular}

$D-4 I$ 
WHC-EP-0355, Appendix D

DATA FOR 241-A Tank Farm Cooling water Continued

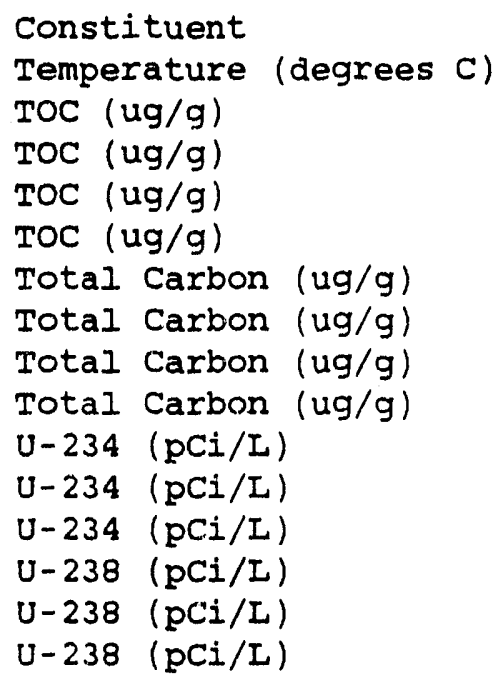

\begin{tabular}{crll} 
Sample \# & \multicolumn{1}{c}{ Date } & Method & \multicolumn{1}{l}{ Result } \\
51028 & $3 / 08 / 90$ TEMP-FId & $6.80 \mathrm{E}+00$ \\
50701 & $10 / 18 / 89$ TOC & $<1.30 \mathrm{E}+03$ \\
50815 & $11 / 29 / 89 \mathrm{TOC}$ & $<1.20 \mathrm{E}+03$ \\
50963 & $2 / 21 / 90 \mathrm{TOC}$ & $1.10 \mathrm{E}+03$ \\
51028 & $3 / 08 / 90 \mathrm{TOC}$ & $1.20 \mathrm{E}+03$ \\
50701 & $10 / 18 / 89 \mathrm{TC}$ & $1.60 \mathrm{E}+04$ \\
50815 & $11 / 29 / 89 \mathrm{TC}$ & $1.58 \mathrm{E}+04$ \\
50963 & $2 / 21 / 90 \mathrm{TC}$ & $1.55 \mathrm{E}+04$ \\
51028 & $3 / 08 / 90 \mathrm{TC}$ & $1.59 \mathrm{E}+04$ \\
50701 & $10 / 18 / 89 \mathrm{AEA}$ & $2.63 \mathrm{E}-01$ \\
50815 & $11 / 29 / 89 \mathrm{AEA}$ & $2.25 \mathrm{E}-01$ \\
51028 & $3 / 08 / 90 \mathrm{AEA}$ & $1.87 \mathrm{E}-01$ \\
50701 & $10 / 18 / 89 \mathrm{AEA}$ & $2.02 \mathrm{E}-01$ \\
50815 & $11 / 29 / 89$ AEA & $1.32 \mathrm{E}-01$ \\
51028 & $3 / 08 / 90 \mathrm{AEA}$ & $1.77 \mathrm{E}-01$
\end{tabular}


WHC-EP-0355, Appendix D

DATA FOR 2724-W Laundry Wastewater

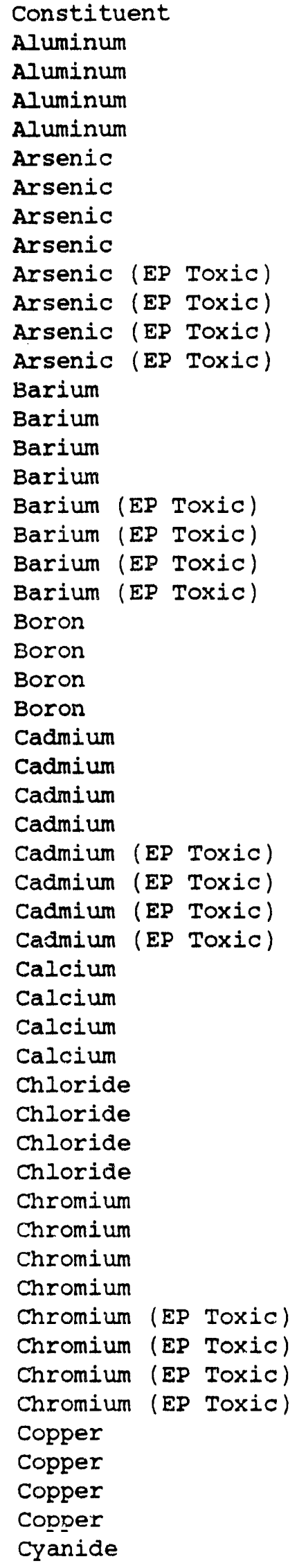

\begin{tabular}{|c|c|c|c|}
\hline Sample \# & Date & Method & Result \\
\hline 50677 & $10 / 12 / 89$ & ICP & $5.51 E+03$ \\
\hline 50772 & $11 / 16 / 89$ & ICP & $3.45 \mathrm{E}+02$ \\
\hline 50780 & $11 / 21 / 89$ & ICP & $6.67 \mathrm{E}+02$ \\
\hline 50857 & $12 / 21 / 89$ & ICP & $5.06 \mathrm{E}+02$ \\
\hline 50677 & $10 / 12 / 89$ & GFAA & $1.00 \mathrm{E}+01$ \\
\hline 50772 & $11 / 16 / 89$ & GFAA & $<5.00 E+00$ \\
\hline 50780 & $11 / 21 / 89$ & GFAA & $<5.00 E+00$ \\
\hline 50857 & $12 / 21 / 89$ & GFAA & $<5.00 \mathrm{E}+00$ \\
\hline $50677 \mathrm{E}$ & $10 / 12 / 89$ & $I C P$ & $<5.00 \mathrm{E}+02$ \\
\hline $50772 \mathrm{E}$ & $11 / 16 / 89$ & $I C P$ & $<5.00 \mathrm{E}+02$ \\
\hline $50780 \mathrm{E}$ & $11 / 21 / 89$ & ICP & $<5.00 E+02$ \\
\hline $50857 \mathrm{E}$ & $12 / 21 / 89$ & ICP & $<5.00 \mathrm{E}+0 ?$ \\
\hline 50677 & $10 / 12 / 89$ & ICP & $1.86 E+03$ \\
\hline 50772 & $11 / 16 / 89$ & ICP & $9.70 E+01$ \\
\hline 50780 & $11 / 21 / 89$ & ICP & $8.50 \mathrm{E}+01$ \\
\hline 50857 & $12 / 21 / 89$ & ICP & I. $36 \mathrm{E}+02$ \\
\hline $50677 \mathrm{E}$ & $10 / 12 / 89$ & ICP & $<1.00 \mathrm{E}+03$ \\
\hline $50772 \mathrm{E}$ & $11 / 16 / 89$ & ICP & $<1.00 E+03$ \\
\hline $50780 \mathrm{E}$ & $11 / 21 / 89$ & ICP & $<1.00 \mathrm{E}+03$ \\
\hline $50857 \mathrm{E}$ & $12 / 21 / 89$ & ICP & $<1.00 E+03$ \\
\hline 50677 & $10 / 12 / 89$ & ICP & $1.08 \mathrm{E}+02$ \\
\hline 50772 & $11 / 16 / 89$ & ICP & $1.64 \mathrm{E}+02$ \\
\hline 50780 & $11 / 21 / 89$ & ICP & $2.13 E+02$ \\
\hline 50857 & $12 / 21 / 89$ & ICP & $1.90 E+01$ \\
\hline 50677 & $10 / 12 / 89$ & ICP & $3.10 \mathrm{E}+01$ \\
\hline 50772 & $11 / 16 / 89$ & ICP & $<2.00 \mathrm{E}+00$ \\
\hline 50780 & $11 / 21 / 89$ & ICP & $7.00 \mathrm{E}+00$ \\
\hline 50857 & $12 / 21 / 89$ & $I C P$ & $<2.00 \mathrm{E}+00$ \\
\hline $50677 \mathrm{E}$ & $10 / 12 / 89$ & ICP & $<1.00 E+02$ \\
\hline $50772 \mathrm{E}$ & $11 / 16 / 89$ & ICP & $<1.00 \mathrm{E}+02$ \\
\hline $50780 \mathrm{E}$ & $11 / 21 / 89$ & ICP & $<1.00 \mathrm{E}+02$ \\
\hline $50857 \mathrm{E}$ & $12 / 21 / 89$ & ICP & $<1.00 E+02$ \\
\hline 50677 & $10 / 12 / 89$ & ICP & $3.18 \mathrm{E}+04$ \\
\hline 50772 & $11 / 16 / 89$ & ICP & $1.74 \mathrm{E}+04$ \\
\hline 50780 & $11 / 21 / 89$ & ICP & $2.09 E+04$ \\
\hline 50857 & $12 / 21 / 89$ & $I C P$ & i. $50 E+04$ \\
\hline 50677 & $10 / 12 / 89$ & IC & $9.00 \mathrm{E}+03$ \\
\hline 50772 & $11 / 16 / 89$ & IC & $3.97 \mathrm{E}+04$ \\
\hline 50780 & $11 / 21 / 89$ & $I C$ & $9.00 E+03$ \\
\hline 50857 & $12 / 21 / 89$ & IC & $2.60 \mathrm{E}+03$ \\
\hline 50677 & $10 / 12 / 89$ & ICP & 9. 10E+O1 \\
\hline 50772 & $11 / 16 / 89$ & ICP & $<1.00 E+01$ \\
\hline 50780 & $11 / 21 / 89$ & ICP & $1.70 \mathrm{E}+01$ \\
\hline 50857 & $12 / 21 / 89$ & ICP & $<1.00 \mathrm{E}+01$ \\
\hline $50677 \mathrm{E}$ & $10 / 12 / 89$ & ICP & $<5.00 \mathbf{E}+02$ \\
\hline $50772 \mathrm{E}$ & $11 / 16 / 89$ & $I C P$ & $<5.00 \mathrm{E}+02$ \\
\hline 50780E & $11 / 21 / 89$ & ICP & $<5.00 \mathrm{E}+02$ \\
\hline $50857 \mathrm{E}$ & $12 / 21 / 89$ & $I C P$ & $<5.00 E+02$ \\
\hline 50677 & $10 / 12 / 89$ & ICP & $6.78 E+02$ \\
\hline 50772 & $11 / 16 / 89$ & ICP & $6.70 E+01$ \\
\hline 50780 & $11 / 21 / 89$ & ICP & $7.30 E+01$ \\
\hline 50857 & $12 / 21 / 89$ & ICP & $7.40 E+01$ \\
\hline 50677 & $10 / 12 / 89$ & SPEC & $<1.00 \mathrm{E}+01$ \\
\hline
\end{tabular}


WHC-EP-0355, Appendix D

DATA FOR 2724-W Laundry Wastewater

Continued

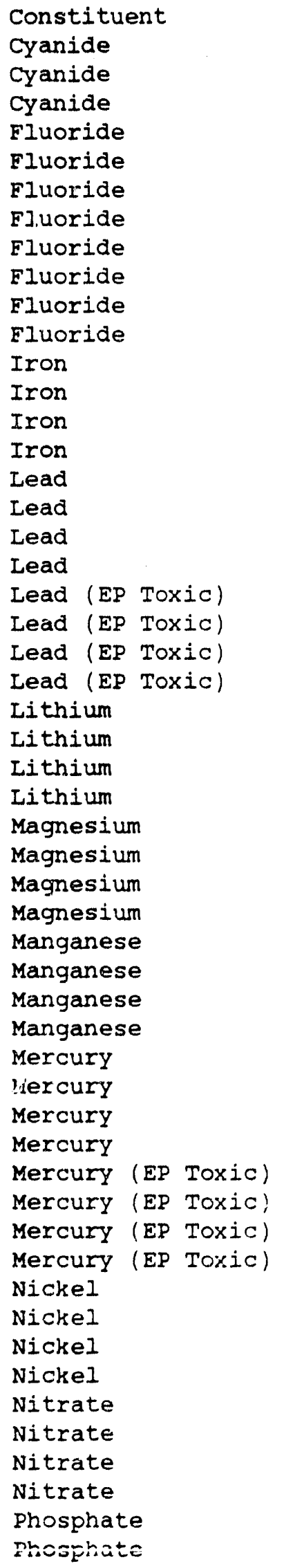

\begin{tabular}{|c|c|c|c|}
\hline Sample \# & Date & Method & Result \\
\hline 50772 & $11 / 16 / 89$ & SPEC & 4. $45 E+01$ \\
\hline 50780 & $11 / 21 / 89$ & SPEC & 2. $93 E+01$ \\
\hline 50857 & $12 / 21 / 89$ & SPEC & $2.22 \mathrm{E}+01$ \\
\hline 50677 & $10 / 12 / 89$ & $I C$ & $2.10 \mathrm{E}+03$ \\
\hline 50677 & $10 / 12 / 89$ & ISE & 2. $25 \mathrm{E}+02$ \\
\hline 50772 & $11 / 16 / 89$ & IC & $1.60 \mathrm{E}+03$ \\
\hline 50772 & $11 / 16 / 89$ & ISE & $1.75 E+02$ \\
\hline 50780 & $11 / 21 / 89$ & IC & $1.10 \mathrm{E}+03$ \\
\hline 50780 & $11 / 21 / 89$ & ISE & 2. $26 \mathrm{E}+02$ \\
\hline 50857 & $12 / 21 / 89$ & IC & $<5.00 \mathrm{E}+02$ \\
\hline 50857 & $12 / 21 / 89$ & ISE & $1.47 \mathrm{E}+02$ \\
\hline 50677 & $10 / 12 / 89$ & ICP & $1.72 E+04$ \\
\hline 50772 & $11 / 16 / 89$ & ICP & $6.66 E+02$ \\
\hline 50780 & $11 / 21 / 89$ & ICP & $1.36 \mathrm{E}+03$ \\
\hline 50857 & $12 / 21 / 89$ & ICP & $1.01 E+03$ \\
\hline 50677 & $10 / 12 / 89$ & GFAA & $3.19 \mathrm{E}+02$ \\
\hline 50772 & $11 / 16 / 89$ & GFAA & $1.80 E+01$ \\
\hline 50780 & $11 / 21 / 89$ & GFAA & $7.70 E+01$ \\
\hline 50857 & $12 / 21 / 89$ & GEAA & $1.90 \mathrm{E}+01$ \\
\hline $50677 \mathrm{E}$ & $10 / 12 / 89$ & $I C P$ & $<5.00 E+02$ \\
\hline $50772 \mathrm{E}$ & $11 / 16 / 89$ & $I C P$ & $<5.00 \mathrm{E}+02$ \\
\hline $50780 \mathrm{E}$ & $11 / 21 / 89$ & ICP & $<5.00 \mathrm{E}+02$ \\
\hline $50857 \mathrm{E}$ & $12 / 21 / 89$ & ICP & $<5.00 E+02$ \\
\hline 50677 & $10 / 12 / 89$ & ICP & $1.56 \mathrm{E}+02$ \\
\hline 50772 & $11 / 16 / 89$ & ICP & $1.59 \mathrm{E}+03$ \\
\hline 50780 & $11 / 21 / 89$ & ICP & $2.30 \mathrm{E}+01$ \\
\hline 50857 & $12 / 21 / 89$ & ICP & $<1.00 \mathrm{E}+01$ \\
\hline 50677 & $10 / 12 / 89$ & ICP & $7.57 \mathrm{E}+03$ \\
\hline 50772 & $11 / 16 / 89$ & ICP & $5.74 E+03$ \\
\hline 50780 & $11 / 21 / 89$ & ICP & $7.44 \mathrm{E}+03$ \\
\hline 50857 & $12 / 21 / 89$ & ICP & $3.16 E+03$ \\
\hline 50677 & $10 / 12 / 89$ & ICP & $1.90 \mathrm{E}+02$ \\
\hline 50772 & $11 / 16 / 89$ & $I C P$ & $2.20 E+01$ \\
\hline 50780 & $11 / 21 / 89$ & $I C P$ & $3.60 \mathrm{E}+01$ \\
\hline 50857 & $12 / 21 / 89$ & ICP & $1.80 \mathrm{E}+01$ \\
\hline 50677 & $10 / 12 / 89$ & CVAA & $6.50 \mathrm{E}-01$ \\
\hline 50772 & $11 / 16 / 89$ & CVAA & $<1.00 \mathrm{E}-01$ \\
\hline 50780 & $11 / 21 / 89$ & CVAA & 3. $30 \mathrm{E}-01$ \\
\hline 50857 & $12 / 21 / 89$ & CVAA & $1.60 \mathrm{E}-01$ \\
\hline $50677 \mathrm{E}$ & $10 / 12 / 89$ & CVAA/M & $<1.00 \mathrm{E}+02$ \\
\hline $50772 \mathrm{E}$ & $11 / 16 / 89$ & CVAA/M & $<2.00 \mathrm{E}+01$ \\
\hline $50780 E$ & $11 / 21 / 89$ & CVAA/M & $<2.00 E+01$ \\
\hline $50857 \mathrm{E}$ & $12 / 21 / 89$ & $C V A A / M$ & $<2.00 E+0 I$ \\
\hline 50677 & $10 / 12 / 89$ & $I C P$ & $1.63 E+02$ \\
\hline 50772 & $11 / 16 / 89$ & ICP & $=1.00 \mathrm{E}+01$ \\
\hline 50780 & $11 / 21 / 89$ & ICP & $1.10 E+01$ \\
\hline 50857 & $12 / 21 / 89$ & $I C P$ & $<1.005+01$ \\
\hline 50677 & $10 / 12 / 89$ & IC & $1.00 E+03$ \\
\hline 50772 & $11 / 16 / 89$ & IC & $2.60 E+03$ \\
\hline 50780 & $11 / 21 / 89$ & $I C$ & $1.40 E+03$ \\
\hline 50857 & $12 / 21 / 89$ & IC & $5.00 E+02$ \\
\hline 50677 & $10 / 12 / 89$ & IC & $1.83 E+05$ \\
\hline $50 ? 72$ & 11,16/89 & $I C$ & $7.285 \div 01$ \\
\hline
\end{tabular}


DATA FOR 2724-W Laundry Wastewater

Continued

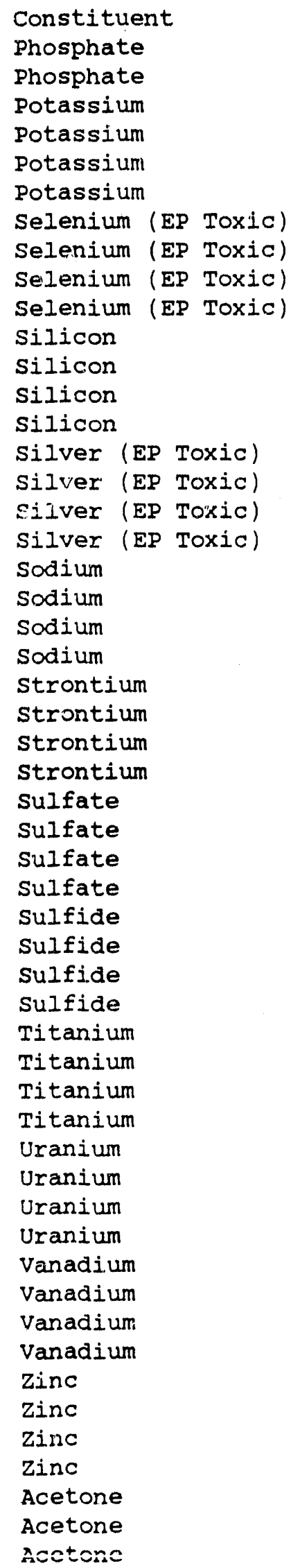

\begin{tabular}{|c|c|c|c|}
\hline Sample \# & & Method & Resillt \\
\hline 50780 & $11 / 21 / 89$ & IC & $7.33 E+04$ \\
\hline 50857 & $12 / 21 / 89$ & IC & 4. $33 E+04$ \\
\hline 50677 & $10 / 12 / 89$ & ICP & $4.53 E+03$ \\
\hline 50772 & $11 / 16 / 89$ & ICP & $1.82 \mathrm{E}+04$ \\
\hline 50780 & $11 / 21 / 89$ & $I C P$ & 1. $35 \mathrm{E}+04$ \\
\hline 50857 & $12 / 21 / 89$ & ICP & $1.14 \mathrm{E}+03$ \\
\hline $50677 \mathrm{E}$ & $10 / 12 / 89$ & ICP & $<5.00 \mathrm{E}+02$ \\
\hline $50772 \mathrm{E}$ & $11 / 16 / 89$ & ICP & $<5.00 E+02$ \\
\hline $50780 \mathrm{E}$ & $11 / 21 / 89$ & ICP & $<5.0 O E+02$ \\
\hline $50857 \mathrm{E}$ & $12 / 21 / 89$ & ICP & $<5.00 \mathrm{E}+02$ \\
\hline 50677 & $10 / 12 / 89$ & ICP & $3.97 \mathrm{E}+04$ \\
\hline 50772 & $11 / 16 / 89$ & ICP & $2.48 \mathrm{E}+04$ \\
\hline 50780 & $11 / 21 / 89$ & ICP & 3. $23 E+04$ \\
\hline 50857 & $12 / 21 / 89$ & ICP & 1. $20 \mathrm{E}+04$ \\
\hline $50677 \mathrm{E}$ & $10 / 12 / 89$ & ICP & $<5.00 \mathrm{E}+02$ \\
\hline $50772 \mathrm{E}$ & $11 / 16 / 89$ & ICP & $<5.00 \mathrm{E}+02$ \\
\hline $50780 \mathrm{E}$ & $11 / 21 / 89$ & ICP & $<5.00 E+02$ \\
\hline $50857 \mathrm{E}$ & $12 / 21 / 89$ & ICP & $<5.00 \mathrm{E}+02$ \\
\hline 50677 & $10 / 12 / 89$ & ICP & $1.27 \mathrm{E}+05$ \\
\hline 50772 & $11 / 16 / 89$ & ICP & $1.23 E+05$ \\
\hline 50780 & $11 / 21 / 89$ & ICP & $1.42 \mathrm{E}+05$ \\
\hline 50857 & $12 / 21 / 89$ & ICP & $4.51 E+04$ \\
\hline 50677 & $10 / 12 / 89$ & ICP & $1.83 E+02$ \\
\hline 50772 & $11 / 16 / 89$ & ICP & $8.90 \mathrm{E}+01$ \\
\hline 50780 & $11 / 21 / 89$ & ICP & $9.70 E+O I$ \\
\hline 50857 & $12 / 21 / 89$ & ICP & $7.40 \mathrm{E}+01$ \\
\hline 50677 & $10 / 12 / 89$ & IC & $1.87 \mathrm{E}+04$ \\
\hline 50772 & $11 / 16 / 89$ & IC & $2.30 \mathrm{E}+04$ \\
\hline 50780 & $11 / 21 / 89$ & IC & $1.85 E+04$ \\
\hline 50857 & $12 / 21 / 89$ & IC & $1.08 \mathrm{E}+04$ \\
\hline 50677 & $10 / 12 / 89$ & TITRA & $1.97 \mathrm{E}+03$ \\
\hline 50772 & $11 / 16 / 83$ & TITRA & $<1.00 E+03$ \\
\hline 50780 & $11 / 21 / 89$ & TITRA & $1.65 \mathrm{E}+03$ \\
\hline 50857 & $12 / 21 / 89$ & '.ITRA & $<1.00 E+03$ \\
\hline 50677 & $10 / 12 / 89$ & ICP & $5.42 \mathrm{E}+02$ \\
\hline 50772 & $11 / 16 / 89$ & ICP & $<6.00 E+01$ \\
\hline 50780 & $11 / 21 / 89$ & ICP & $<6.00 E+01$ \\
\hline 50857 & $12 / 21 / 89$ & ICP & $<6.00 \mathrm{E}+01$ \\
\hline 50677 & $10 / 12 / 89$ & GEA & $5.44 E+01$ \\
\hline 50772 & $11 / 16 / 89$ & GEA & $3.83 E+02$ \\
\hline 50780 & $11 / 21 / 89$ & GEA & 3. $28 \mathrm{E}+00$ \\
\hline 50857 & $12 / 21 / 89$ & GEA & $1.06 \mathrm{E}+01$ \\
\hline 50677 & $10 / 12 / 89$ & ICP & $1.70 E+01$ \\
\hline 50772 & $11 / 16 / 89$ & $I C P$ & $<5.00 E+00$ \\
\hline 50780 & $11 / 21 / 89$ & ICP & $<5.00 E+00$ \\
\hline 50857 & $12 / 21 / 89$ & ICP & $<5.00 E+00$ \\
\hline 50677 & $10 / 12 / 89$ & ICP & $1.73 E+03$ \\
\hline 50772 & $11 / 16 / 89$ & ICP & 1. $95 \mathrm{E}+02$ \\
\hline 50780 & $11 / 21 / 89$ & $I C P$ & $3.68 \mathrm{E}+02$ \\
\hline 50857 & $12 / 21 / 89$ & $I C P$ & 1. $40 \mathrm{E}+02$ \\
\hline 50677 & $10 / 12 / 89$ & VOA & $2.30 \mathrm{E}+01$ \\
\hline 50677 & $10 / 12 / 89$ & $A B N$ & $<1.00 E+01$ \\
\hline $5057 ? \mathrm{P}$ & $10,12,89$ & YOX & $-5.00 E \div 00$ \\
\hline
\end{tabular}


DATA FOR 2724-W Laundry Wastewater

Continued

\begin{tabular}{|c|c|c|c|c|}
\hline Constituent & Sample \# & Date & Method & Result \\
\hline Acetone & $50677 \mathrm{~T}$ & $10 / 12 / 89$ & VOA & 1. $20 \mathrm{E}+O 1$ \\
\hline Acetone & 50772 & $11 / 16 / 89$ & VOA & $1.60 E+01$ \\
\hline Acetone & 50772 & $11 / 16 / 89$ & $A B N$ & $<1.00 E+J I$ \\
\hline Acetone & $50772 \mathrm{~B}$ & $11 / 16 / 89$ & VOA & $<1.00 E+01$ \\
\hline Acetone & $50772 \mathrm{~T}$ & $11 / 16 / 89$ & VOA & $<1.00 E+01$ \\
\hline Acetone & 50780 & $11 / 21 / 89$ & VOA & $47.00 E+00$ \\
\hline Acetone & 50780 & $11 / 21 / 89$ & $A B N$ & $<1.00 \mathrm{E}+01$ \\
\hline Acetone & $50780 B$ & $11 / 21 / 89$ & VOA & $<1.00 E+01$ \\
\hline Acetone & $50780 \mathrm{~T}$ & $11 / 21 / 89$ & VOA & $<1.00 E+01$ \\
\hline Acetone & 50857 & $12 / 21 / 89$ & VOA & $<1.00 E+01$ \\
\hline Acetone & 50857 & $12 / 21 / 89$ & $A B N$ & $<1.00 E+01$ \\
\hline Acetone & $50857 \mathrm{~B}$ & $12 / 21 / 89$ & VOA & $<1.00 E+01$ \\
\hline Acetone & $50857 \mathrm{~T}$ & $12 / 21 / 89$ & VOA & $<1.00 E+01$ \\
\hline Ammonia & 50677 & $10 / 12 / 89$ & ISE & $1.71 \mathrm{E}+04$ \\
\hline Ammonia & 50772 & $11 / 16 / 89$ & ISE & $5.99 E+03$ \\
\hline Ammonia & 50780 & $11 / 21 / 89$ & ISE & $1.71 E+04$ \\
\hline Ammonia & 50857 & $12 / 21 / 89$ & ISE & $3.27 \mathrm{E}+04$ \\
\hline Benzoic acid & 50772 & $11 / 16 / 89$ & $A B N$ & 1. $22 E+02$ \\
\hline Benzoic acid & 50780 & $11 / 21 / 89$ & $A B N$ & $2.80 \mathrm{E}+02$ \\
\hline Benzoic acid & 50857 & $12 / 21 / 89$ & $A B N$ & $6.00 \mathrm{E}+01$ \\
\hline Benzyl a.lcohol & 50677 & $10 / 12 / 89$ & $A B N$ & 3. $22 \mathrm{E}+02$ \\
\hline Benzyl alcohol & 50772 & $11 / 16 / 89$ & $A B N$ & $5.80 E+01$ \\
\hline Benzyl alcohol & 50780 & $11 / 21 / 89$ & $A B N$ & $1.04 \mathrm{E}+02$ \\
\hline Benzyl alcohol & 50857 & $12 / 21 / 89$ & $A B N$ & $9.00 \mathrm{E}+01$ \\
\hline Bis(2-ethylhexyl) phthalate & 50677 & $10 / 12 / 89$ & $A B N$ & $8.80 E+01$ \\
\hline Bis(2-ethylhexyl) phthalate & 50772 & $11 / 16 / 89$ & $A B N$ & 3. $10 \mathrm{E}+01$ \\
\hline Bis(2-ethylhexyl) phthalate & 50780 & $11 / 21 / 89$ & $A B N$ & $1.00 \mathrm{E}+02$ \\
\hline Bis(2-ethylhexyl) phthalate & 50857 & $12 / 21 / 89$ & $A B N$ & $6.70 E+01$ \\
\hline 1-Butoxy-2-propanol & 50857 & $12 / 21 / 89$ & $A B N$ & 1. $20 \mathrm{E}+02$ \\
\hline Butylbenzyl phthalate & 50677 & $10 / 12 / 89$ & $A B N$ & $7.80 E+01$ \\
\hline Butylbenzyl phthalate & 50772 & $11 / 16 / 89$ & $A B N$ & $1.80 E+01$ \\
\hline Butylbenzyl phthalate & 50780 & $11 / 21 / 89$ & $A B N$ & $3.20 \mathrm{E}+01$ \\
\hline Butylbenzyl phthalate & 50857 & $12 / 21 / 89$ & $A B N$ & $3.00 \mathrm{E}+01$ \\
\hline Dichloromethane & 50677 & $10 / 12 / 89$ & VOA & $<5.00 \mathrm{E}+00$ \\
\hline Dichloromethane & $50677 \mathrm{~B}$ & $10 / 12 / 89$ & VOA & $3.90 \mathrm{E}+02$ \\
\hline Dichloromethane & $50677 \mathrm{~T}$ & $10 / 12 / 89$ & VOA & 4. $20 \mathrm{E}+02$ \\
\hline Dichloromethane & 50772 & $11 / 16 / 89$ & VOA & $<5.00 E+00$ \\
\hline Dichloromethane & $50772 B$ & $11 / 16 / 89$ & VOA & $<5.00 E+00$ \\
\hline Dichloromethane & $50772 \mathrm{~T}$ & $1.1 / 16 / 89$ & VOA & $<5.0 O E+00$ \\
\hline Dichloromethane & 50780 & $11 / 21 / 89$ & VOA & $<5.00 \mathrm{E}+00$ \\
\hline Dichloromethane & $50780 \mathrm{~B}$ & $11 / 21 / 89$ & VOA & $<3.00 \mathrm{E}+00$ \\
\hline Dichloromethane & $50780 \mathrm{~T}$ & $11 / 21 / 89$ & VOA & $<3.00 E+00$ \\
\hline Dichloromethane & 50857 & $12 / 21 / 89$ & VOA & $<5.00 \mathrm{E}+00$ \\
\hline Dichloromethane & $50857 \mathrm{~B}$ & $12 / 21 / 89$ & VOA & 1. $30 \mathrm{E}+03$ \\
\hline Dichloromethane & $50857 \mathrm{~T}$ & $12 / 21 / 89$ & VOA & $<3.00 \mathrm{E}+00$ \\
\hline Di-n-octy 1 phthalate & 50677 & $10 / 12 / 89$ & $A B N$ & $1.86 \mathrm{E}+02$ \\
\hline Di-n-octyl phthalate & 50772 & $11 / 16 / 89$ & $A B N$ & $3.20 E+01$ \\
\hline Di-n-octyl phthalate & 50780 & $11 / 21 / 89$ & $A B N$ & $1.18 \mathrm{E}+02$ \\
\hline Di-n-octyl phthalate & 50857 & $12 / 21 / 89$ & $A B N$ & 1. $10 \mathrm{E}+02$ \\
\hline Hydrazine & 50677 & $10 / 12 / 89$ & SPEC & $<3.00 E+01$ \\
\hline Hydrazine & 50772 & $11 / 16 / 89$ & SPEC & $7.20 E+01$ \\
\hline Hydrazine & 50780 & $11 / 21 / 89$ & SPEC & $7.50 E+01$ \\
\hline Hydrazine & 50857 & $12 / 21 / 89$ & SPEC & $3.60 E+01$ \\
\hline
\end{tabular}


DATA FOR 2724-W Laundry Wastewater

Continued

Constituent

Phthalic anhydride

Trichloromethane

Trichloromethane

Trichloromethane

Trichloromethane

Trichloromethane

Trichloromethane

Trichloromethane

Trichloromethane

Trichloromethane

Trichloromethane

Trichloromethane

Trichloromethane

Unknown

Untenown

Unknown

Unknown

Unknown acid

Unknown aliphatic alcohol

Unknown aliphatic HC

Unknown aliphatic HC

Unknown aliphatic HC

Unknown aliphatic HC

Unknown amine

Unknown fatty acid

Unknown fatty acid

Unknown fatty acid

Unknown fatty acid

Unknown glycol

Unknown hydrocarbon

Unknown phthalate

Unknown phthalate

Unknown phthalate

Unknown phthalate

Unknown polycycll= HC

Unknown polycyclic HC

Unknown sulfur compound

Alkalinity (Method B)

Alkalinity (Method B)

Alkalinity (Method B)

Alkalinity (Method B)

Alpha Activity (pCi/L)

Alpha Activity (pCi/L)

Alpha Activity (pCi/L)

Alpha Activity (pCi/I)

Beta Activity (pCi/L)

Beta Activity (pCi/L)

Beta Activity ( $\mathrm{pCi} / \mathrm{L}$ )

Beta Activity (pCi/L)

Conductivity (us)

Conductivity (us)

Conductivity (us)

Conductivity (us)

\begin{tabular}{|c|c|c|c|}
\hline Sample \# & Date & Method & Result \\
\hline 50857 & $12 / 21 / 89$ & $A B N$ & $1.20 \mathrm{E}+01$ \\
\hline 50677 & $10 / 12 / 89$ & VOA & $2.20 E+01$ \\
\hline $50677 \mathrm{~B}$ & $10 / 12 / 89$ & VOA & $<5.00 \mathrm{E}+00$ \\
\hline $50677 \mathrm{~T}$ & $10 / 12 / 89$ & VOA & $<5.00 \mathrm{E}+00$ \\
\hline 50772 & $11 / 16 / 89$ & VOA & $9.50 \mathrm{E}+01$ \\
\hline $50772 \mathrm{E}$ & $11 / 16 / 89$ & VOA & $5.00 E+00$ \\
\hline $50772 \mathrm{~T}$ & $11 / 16 / 89$ & VOA & $5.00 E+00$ \\
\hline 50780 & $11 / 21 / 89$ & VOA & $6.00 \mathrm{E}+00$ \\
\hline $50780 \mathrm{~B}$ & $11 / 21 / 89$ & VOA & $<4.00 E+00$ \\
\hline $.50780 \mathrm{~T}$ & $11 / 21 / 89$ & VOA & $9.00 \mathrm{E}+00$ \\
\hline 50857 & $12 / 21 / 89$ & VOA & $1.00 E+01$ \\
\hline $50857 \mathrm{~B}$ & $12 / 21 / 89$ & VOA & $<5.00 \mathrm{E}+00$ \\
\hline $50857 \mathrm{~T}$ & $12 / 21 / 89$ & VOA & $5.00 \mathrm{E}+00$ \\
\hline 50677 & $10 / 12 / 89$ & $A B N$ & $5.60 \mathrm{E}+01$ \\
\hline 50772 & $11 / 16 / 89$ & $A B N$ & $5.20 E+01$ \\
\hline 50780 & $11 / 21 / 89$ & $A B N$ & $3.60 \mathrm{E}+01$ \\
\hline 50857 & $12 / 21 / 89$ & $A B N$ & $7.70 \mathrm{E}+01$ \\
\hline 50677 & $10 / 12 / 89$ & $\mathrm{ABN}$ & 2. $20 \mathrm{E}+01$ \\
\hline 50857 & $12 / 21 / 89$ & $A B N$ & $1.60 \mathrm{E}+01$ \\
\hline 50677 & $10 / 12 / 89$ & $A B N$ & $3.60 \mathrm{E}+01$ \\
\hline 50772 & $11 / 16 / 89$ & $A B N$ & $2.80 \mathrm{E}+01$ \\
\hline 50780 & $11 / 21 / 89$ & $A B N$ & $2.70 E+01$ \\
\hline 50857 & $12 / 21 / 89$ & $A B N$ & $6.00 \mathrm{E}+00$ \\
\hline 50772 & $11 / 16 / 89$ & $A B N$ & 2. $10 \mathrm{E}+01$ \\
\hline 50677 & $10 / 12 / 89$ & $A B N$ & 4. $10 \mathrm{E}+02$ \\
\hline 50772 & $11 / 16 / 89$ & $A B N$ & $7.30 \mathrm{E}+01$ \\
\hline 50780 & $11 / 21 / 89$ & $\mathrm{ABN}$ & $6.20 E+01$ \\
\hline 50857 & $12 / 21 / 89$ & $A B N$ & $9.10 \mathrm{E}+01$ \\
\hline 50857 & $12 / 21 / 89$ & $A B N$ & $9.20 E+01$ \\
\hline 50857 & $12 / 21 / 89$ & $\mathrm{ABN}$ & $9.00 E+01$ \\
\hline 50677 & $10 / 12 / 89$ & $A B N$ & $9.10 \mathrm{E}+01$ \\
\hline $5 c 772$ & $11 / 16 / 89$ & $\mathrm{ABN}$ & $2.20 \mathrm{E}+01$ \\
\hline 50780 & $11 / 21 / 89$ & $A B N$ & $4.80 E+01$ \\
\hline 50857 & $12 / 21 / 89$ & $A B N$ & $2.70 E+01$ \\
\hline 50772 & $11 / 16 / 89$ & $\mathrm{ABN}$ & 3. $20 E+01$ \\
\hline 50780 & $11 / 21 / 89$ & $A B N$ & $7.60 \mathrm{E}+01$ \\
\hline 50857 & $12 / 21 / 89$ & $A B N$ & $1.30 \mathrm{E}+01$ \\
\hline 50677 & $10 / 12 / 89$ & TITRA & 4. $36 E+05$ \\
\hline 50772 & $11 / 16 / 89$ & TITRA & $2.18 E+05$ \\
\hline 50780 & $11 / 21 / 89$ & TITRA & $2.50 \mathrm{E}+05$ \\
\hline 50857 & $12 / 21 / 89$ & TITRA & 1. $26 \mathrm{E}+05$ \\
\hline 50677 & $10 / 12 / 89$ & Alpha & $6.34 E+02$ \\
\hline 50772 & $11 / 16 / 89$ & Alpha & $1.84 \mathrm{E}+02$ \\
\hline 50780 & $11 . / 21 / 89$ & Alpha & $1.90 \mathrm{E}+00$ \\
\hline 50857 & $12 / 21 / 89$ & Alpha & $1.68 \mathrm{E}+01$ \\
\hline 50677 & $10 / 12 / 89$ & Beta & $3.70 \mathrm{E}+03$ \\
\hline 50772 & $11 / 16 / 89$ & Beta & $2.91 \mathrm{E}+03$ \\
\hline 50780 & $11 / 21 / 89$ & Beta & 1. $23 \mathrm{E}+01$ \\
\hline 50857 & $12 / 21 / 89$ & Beta & $3.17 \mathrm{E}+01$ \\
\hline 50677 & $10 / 12 / 89$ & COND-FId & $8.00 E+02$ \\
\hline 50772 & $11 / 16 / 89$ & COND-FId & $9.71 E+02$ \\
\hline 50780 & $11 / 21 / 89$ & COND-EId & $1.01 \mathrm{E}+03$ \\
\hline 50857 & $12 / 21 / 89$ & COND-FId & $2.97 \mathrm{E}+02$ \\
\hline
\end{tabular}

$D-47$ 
DATA FOR 2724-W Laundry Wastewater

Continued

Constituent

Ingitability (degrees F)

Ingitability (degrees F)

Ingitability (degrees F)

Ingitability (degrees F)

$\mathrm{pH}$ (dimensionless)

$\mathrm{pH}$ (dimensionless)

$\mathrm{pH}$ (dimensionless)

pH (dimensionless)

Reactivity Cyanide (mg/kg)

Reactivity Cyanide ( $\mathrm{mg} / \mathrm{kg}$ )

Reactivity Cyanide ( $\mathrm{mg} / \mathrm{kg}$ )

Reactivity Cyanide ( $\mathrm{mg} / \mathrm{kg}$ )

Reactivity Sulfide ( $\mathrm{mg} / \mathrm{kg}$ )

Reactivity Sulfide (mg/kg)

Reactivity sulfide (mg/kg)

Reactivity Sulfide ( $\mathrm{mg} / \mathrm{kg}$ )

Suspended Solids ( $\mathrm{mg} / \mathrm{L})$

suspended solids $(\mathrm{mg} / \mathrm{L}$ )

Suspended solids $(\mathrm{mg} / \mathrm{L}$ )

suspended solids (mg/L)

$\operatorname{TDS}(\mathrm{mg} / \mathrm{L})$

$\operatorname{TDS}(\mathrm{mg} / \mathrm{L})$

TDS $(\mathrm{mg} / \mathrm{L})$

TDS (mg/L)

Temperature (degrees C)

Temperature (degrees C)

Temperature (degrees $C$ )

Temperature (degrees $\mathrm{C}$ )

TOC (ug/g)

TOC $(u g / g)$

TOC (ug/g)

TOC (ug/g)

Total Carbon (ug/g)

Total Carbon ( $u g / g$ )

Total Carbon (ug/g)

Total Carbon (ug/g)

TOX (ug $(C 1) / L)$

$\operatorname{Tox}(u g(\mathrm{Cl}) / \mathrm{L})$

TOX (ug $(C I) / L)$

TOX (ug $(C 1) / L)$

Am-241 (pCi/L)

Am- 241 (pCi/L)

Am-241 (pCi/L)

Cm-244 (pCi/L)

$\mathrm{Cm}-244$ (pCi/L)

Co-60 ( $\mathrm{pCi} / \mathrm{L})$

Co-60 ( $\mathrm{pCi} / \mathrm{L})$

Co-60 (pCi/L)

Co-60 (pCi/L)

Cs-137 (pCi/L)

$\mathrm{Cs}-1.37$ (pCi/L)

Cs-137 (pCi/L)

Cs-137 (pCi/L)

\begin{tabular}{|c|c|c|c|}
\hline & & & \\
\hline $50677 \mathrm{E}$ & $10 / 12 / 89$ & IGNIT & $2.12 \mathrm{E}+02$ \\
\hline $50772 \mathrm{E}$ & $11 / 16 / 89$ & IGNIT & $2.06 \mathrm{E}+02$ \\
\hline $50780 \mathrm{E}$ & $11 / 21 / 89$ & IGNIT & 2. $12 \mathrm{E}+02$ \\
\hline $50857 \mathrm{E}$ & $12 / 21 / 89$ & IGNIT & 2. $10 \mathrm{E}+\mathrm{O} 2$ \\
\hline 50677 & $10 / 12 / 89$ & $\mathrm{PH}-\mathrm{F} I \mathrm{~d}$ & $9.49 \mathrm{E}+00$ \\
\hline 50772 & $11 / 16 / 89$ & PH-FIC & $9.55 \mathrm{E}+00$ \\
\hline 50780 & $11 / 21 / 89$ & $P H-F I d$ & $8.02 \mathrm{E}+00$ \\
\hline 50857 & $12 / 21 / 89$ & $\mathrm{PH}-\mathrm{F} I \mathrm{~d}$ & $6.75 \mathrm{E}+00$ \\
\hline $50677 \mathrm{E}$ & $10 / 12 / 89$ & DSPEC & $<1.00 \mathrm{E}+02$ \\
\hline $50772 \mathrm{E}$ & $11 / 16 / 89$ & DSPEC & $<1.00 \mathrm{E}+02$ \\
\hline $50780 \mathrm{E}$ & $11 / 21 / 89$ & DSPEC & $<1.00 E+02$ \\
\hline $50857 \mathrm{E}$ & $12 / 21 / 89$ & DSPEC & $<1.00 E+02$ \\
\hline $50677 \mathrm{E}$ & $10 / 12 / 89$ & DTITRA & $<1.00 E+02$ \\
\hline $50772 \mathrm{E}$ & $11 / 1$ & DTITRA & $\mathrm{OE}+\mathrm{O} 2$ \\
\hline $50780 \mathrm{E}$ & $11 / 21 / 89$ & DTITRA & $<1.00 \mathrm{E}+02$ \\
\hline $50857 \mathrm{E}$ & $12 / 21 / 89$ & DTITRA & $<1.00 \mathrm{E}+02$ \\
\hline 50677 & $10 / 12 / 89$ & SSOLID & $3.85 E+05$ \\
\hline 50772 & $11 / 16 / 89$ & SSOLID & $9.90 \mathrm{E}+04$ \\
\hline 50780 & $11 / 21 / 89$ & SSOLID & $8.40 \mathrm{E}+04$ \\
\hline 50857 & $12 / 21 / 89$ & SSOLID & 4. $26 E+04$ \\
\hline 50677 & $10 / 12 / 89$ & TDS & $6.33 \mathrm{E}+05$ \\
\hline 50772 & $11 / 1$ & TDS & $9 \mathrm{E}+05$ \\
\hline 50780 & $11 / 21 / 89$ & TDS & $7.51 \mathrm{E}+05$ \\
\hline 50857 & $12 / 21 / 89$ & TDS & $1.77 \mathrm{E}+05$ \\
\hline 50677 & $10 / 12 / 89$ & TEMP-FId & $4.65 \mathrm{E}+01$ \\
\hline 50772 & $11 / 16 / 89$ & TEMP-FId & $4.10 \mathrm{E}+01$ \\
\hline 50780 & $11 / 21 / 89$ & TEMP-FId & $5.06 \mathrm{E}+01$ \\
\hline 50857 & $12 / 21 / 89$ & TEMP-FId & $4.48 E+01$ \\
\hline 50677 & $10 / 12 / 89$ & TOC & $3.94 E+04$ \\
\hline 50772 & $11 / 1$ & TOC & $8.71 \mathrm{E}+04$ \\
\hline 50780 & $11 / 2$ & TOC & $.77 E+04$ \\
\hline 50857 & $12 / 21 / 89$ & TOC & $4.02 \mathrm{E}+04$ \\
\hline 50677 & $10 / 12 / 89$ & $T C$ & 1. $36 \mathrm{E}+05$ \\
\hline 50772 & $11 / 16 / 89$ & $\mathrm{TC}$ & 1. $21 E+05$ \\
\hline 50780 & $11 / 21 / 89$ & $\mathrm{TC}$ & $9.20 \mathrm{E}+04$ \\
\hline 50857 & $12 / 21 / 89$ & $\mathrm{TC}$ & $8.97 \mathrm{E}+04$ \\
\hline 50677 & $10 / 12 / 89$ & LTOX & $1.84 \mathrm{E}+02$ \\
\hline 50772 & $11 / 16 / 89$ & LTOX & 1. $23 \mathrm{E}+03$ \\
\hline 50780 & $11 /$ & LTOX & $9 \mathrm{E}+02$ \\
\hline 50857 & $12 / 2$ & LTOX & . $23 . \mathrm{E}+02$ \\
\hline 50677 & $10 / 12 / 89$ & $\mathrm{AEA}$ & 1. $23 \mathrm{E}+01$ \\
\hline 50772 & $11 / 16 / 89$ & $\mathrm{AEA}$ & $1.20 \mathrm{E}+02$ \\
\hline 50857 & $12 / 21 / 89$ & AEA & $<9.21 E-04$ \\
\hline 50677 & $10 / 12 / 89$ & $\mathrm{AEA}$ & $1.19 \mathrm{E}-01$ \\
\hline 30772 & $11 / 16 / 89$ & $A E A$ & $1.09 \mathrm{E}+00$ \\
\hline 50677 & $10 / 12 / 89$ & GEA & $5.76 \mathrm{E}+02$ \\
\hline 50772 & $11 / 16 / 89$ & GEA & $12 \mathrm{E}+02$ \\
\hline 50780 & $11 / 21 / 89$ & GEA & $<4.82 \mathrm{E}-02$ \\
\hline 50857 & $12 / 21 / 89$ & GEA & $5.35 \mathrm{E}+01$ \\
\hline 50677 & $10 / 12 / 89$ & GEA & 1. $35 \mathrm{E}+02$ \\
\hline 50772 & $11 / 16 / 89$ & GEA & $5.02 \mathrm{E}+02$ \\
\hline 50780 & $11 / 21 / 89$ & GEA & $<2.00 E-01$ \\
\hline 50857 & $12, / 21 / 89$ & GEA & $4.33 E+01$ \\
\hline
\end{tabular}

$D-48$ 
DATA FOR 2724-W Laundry Wastewater Continued

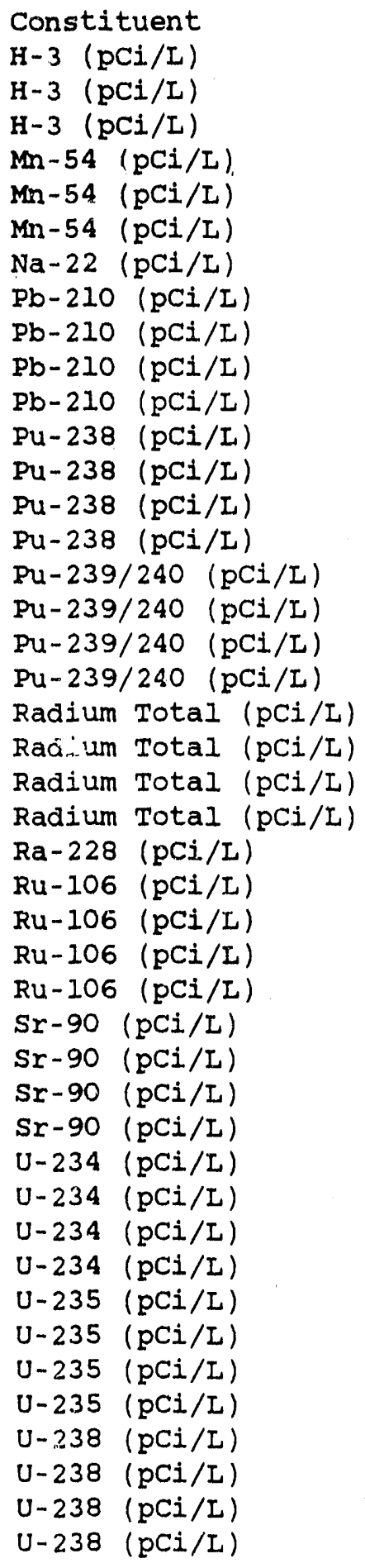

\begin{tabular}{|c|c|c|c|}
\hline & & & Resu \\
\hline 50677 & $10 / 12 / 89$ & LSC & $3.78 \mathrm{E}+03$ \\
\hline 50780 & $11 / 21 / 89$ & LSC & $<2.68 \mathrm{E}+01$ \\
\hline 50857 & $12 / 21 / 89$ & LSC & $<1.78 \mathrm{E}+01$ \\
\hline 50677 & $10 / 12 / 89$ & GEA & $6.18 \mathrm{E}+01$ \\
\hline 50772 & $11 / 16 / 89$ & GEA & $5.49 \mathrm{E}+01$ \\
\hline 50857 & $12 / 21 / 89$ & GEA & $4.47 \mathrm{E}+00$ \\
\hline 50772 & $11 / 16 / 89$ & GEA & $8.88 \mathrm{E}+01$ \\
\hline 50677 & $10 / 12 / 89$ & GEA & $1.09 \mathrm{E}+00$ \\
\hline 50772 & $11 / 16 / 89$ & GEA & $2.63 E+00$ \\
\hline 50780 & $11 / 21 / 89$ & GEA & $<8.18 \mathrm{E}-01$ \\
\hline 50857 & $12 / 21 / 89$ & GEA & $<3.47 \mathrm{E}-01$ \\
\hline 50677 & $10 / 12 / 89$ & AEA & $3.36 \mathrm{E}+01$ \\
\hline 50772 & $11 / 16 / 89$ & AEA & $3.61 \mathrm{E}+01$ \\
\hline 50780 & $11 / 21 / 89$ & AEA & $1.22 \mathrm{E}-01$ \\
\hline 50857 & $12 / 21 / 89$ & AEA & $5.15 \mathrm{E}-01$ \\
\hline 50677 & $10 / 12 / 89$ & AEA & 4. $16 \mathrm{E}+02$ \\
\hline 50772 & $11 / 16 / 89$ & $A E A$ & $3.95 E+02$ \\
\hline 50780 & $11 / 21 / 89$ & AEA & 1. $23 \mathrm{E}+00$ \\
\hline 50857 & $12 / 21 / 89$ & AEA & $5.58 \mathrm{E}+00$ \\
\hline 50677 & $10 / 12 / 89$ & Alpha-Ra & $4.87 \mathrm{E}-0.1$ \\
\hline 50772 & $11 / 16 / 89$ & Alpha - Ra & $1.41 \mathrm{E}+00$ \\
\hline 50780 & $11 / 21 / 89$ & Alpha-Ra & $<9.24 E-01$ \\
\hline 50857 & $12 / 21 / 89$ & Alpha-Ra & $1.82 \mathrm{E}-01$ \\
\hline 50772 & $11 / 16 / 89$ & GEA & $2.50 \mathrm{E}+02$ \\
\hline 50677 & $10 / 12 / 89$ & GEA & $<3.53 E+01$ \\
\hline 50772 & $11 / 16 / 89$ & GEA & $7.79 \mathrm{E}+01$ \\
\hline 50780 & $11 / 21 / 89$ & GEA & $<1.96 \mathrm{E}+00$ \\
\hline 50857 & $12 / 21 / 89$ & GEA & $<1.57 \mathrm{E}+01$ \\
\hline 50677 & $10 / 12 / 89$ & Beta & $1.25 \mathrm{E}+03$ \\
\hline 50772 & $11 / 16 / 89$ & Beta & $4.56 \mathrm{E}+0.3$ \\
\hline 50780 & $11 / 21 / 89$ & Beta & $8.07 \mathrm{E}+00$ \\
\hline 50857 & $12 / 21 / 89$ & Beta & $1.61 \mathrm{E}+01$ \\
\hline 50677 & $10 / 12 / 89$ & AEA & $1.58 \mathrm{E}+01$ \\
\hline 50772 & $11 / 16 / 89$ & $A E A$ & $2.12 \mathrm{E}+02$ \\
\hline 50780 & $11 / 21 / 89$ & $A E A$ & $1.77 \mathrm{E}+00$ \\
\hline 50857 & $12 / 21 / 89$ & $A E A$ & $3.87 \mathrm{E}+00$ \\
\hline 50677 & $10 / 12 / 89$ & $A E A$ & $5.31 \mathrm{E}-01$ \\
\hline 50772 & $11 / 16 / 89$ & $A E A$ & $2.31 \mathrm{E}+01$ \\
\hline 50780 & $11 / 21 / 89$ & $A E A$ & $<2.35 \mathrm{E}-02$ \\
\hline 50857 & $12 / 21 / 89$ & AEA & $1.90 \mathrm{E}-01$ \\
\hline 50677 & $10 / 12 / 89$ & $A E A$ & $1.60 \mathrm{E}+01$ \\
\hline 50772 & $11 / 16 / 89$ & AEA & $2.43 \mathrm{E}+02$ \\
\hline 50780 & $11 / 21 / 89$ & AEA & $1.45 \mathrm{E}+00$ \\
\hline 50857 & $12 / 21 / 89$ & AEA & $3.44 \mathrm{E}+00$ \\
\hline
\end{tabular}


DATA FOR N Reactor Effluent

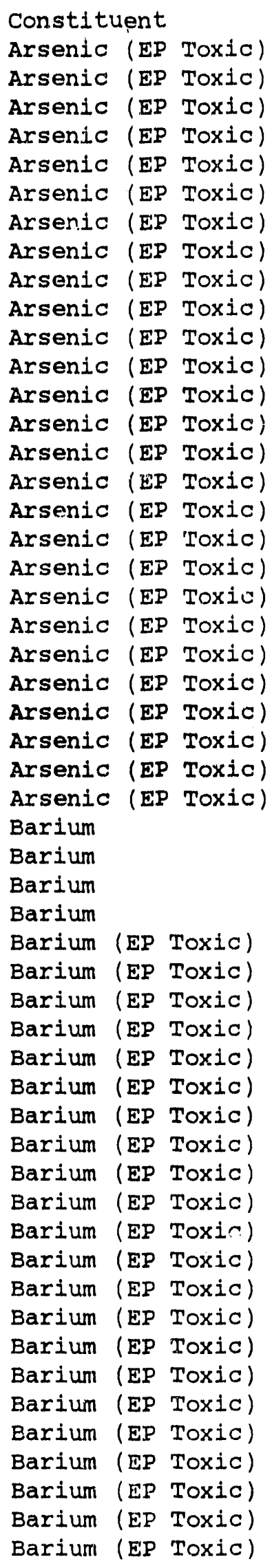

\begin{tabular}{|c|c|c|c|}
\hline ample \# & & & Result \\
\hline $25158 \mathrm{E}$ & $10 / 03 / 89$ & $I C P$ & $<5.00 \mathrm{E}+02$ \\
\hline $25160 E$ & $10 / 10 / 89$ & ICP & $<5.00 \mathrm{E}+02$ \\
\hline $25162 \mathrm{E}$ & $10 / 17 / 89$ & ICP & $<5.00 \mathrm{E}+02$ \\
\hline $25167 \mathrm{E}$ & $10 / 24 / 89$ & $I C P / M$ & $<5.00 E+02$ \\
\hline $25169 \mathrm{E}$ & $10 / 31 / 89$ & $I C P / M$ & $<5.00 \mathrm{E}+02$ \\
\hline $25171 \mathrm{E}$ & $11 / 07 / 89$ & $\mathrm{ICP} / \mathrm{M}$ & $<5.00 \mathrm{E}+02$ \\
\hline $25173 E$ & $11 / 14 / 89$ & $I C P / M$ & $<5.00 \mathrm{E}+02$ \\
\hline $25175 \mathrm{E}$ & $11 / 21 / 89$ & $\mathrm{ICP} / \mathrm{M}$ & $<5.00 \mathrm{E}+02$ \\
\hline $25177 \mathrm{E}$ & $11 / 28 / 89$ & $I C P / M$ & $<5.00 E+02$ \\
\hline $25179 \mathrm{E}$ & $12 / 05 / 89$ & $I C P / M$ & $<5.00 \mathrm{E}+02$ \\
\hline 25181E & $12 / 12 / 89$ & $I C P / M$ & $<5.00 \mathrm{E}+02$ \\
\hline $25183 E$ & $12 / 19 / 89$ & $\operatorname{ICP} / \mathrm{M}$ & $<5.00 \mathrm{E}+02$ \\
\hline $25185 \mathrm{E}$ & $1 / 02 / 90$ & $\operatorname{ICP} / \mathrm{M}$ & $<5.00 \mathrm{E}+02$ \\
\hline $25187 \mathrm{E}$ & $1 / 09 / 90$ & $\operatorname{ICP} / \mathrm{M}$ & $<5.00 E+02$ \\
\hline $25189 \mathrm{E}$ & $1 / 16 / 90$ & $I C P / M$ & $<5.00 \mathrm{E}+02$ \\
\hline 25191E & $1 / 23 / 90$ & $I C P / M$ & $<5.00 \mathrm{E}+02$ \\
\hline 25193E & $1 / 30 / 90$ & $I C P / M$ & $<5.00 \mathrm{E}+02$ \\
\hline $25195 E$ & $2 / 06 / 90$ & $I C P / M$ & $<5.00 \mathrm{E}+02$ \\
\hline $25197 \mathrm{E}$ & $2 / 13 / 90$ & $I C P / M$ & $<5.00 \mathrm{E}+02$ \\
\hline 25199E & $2 / 20 / 90$ & $I C P / M$ & $<5.00 E+02$ \\
\hline $25201 \mathrm{E}$ & $2 / 27 / 90$ & $I C P / M$ & $<5.00 E+02$ \\
\hline $25203 E$ & $3 / 06 / 90$ & $\operatorname{ICP} / \mathrm{M}$ & $<5.00 \mathrm{E}+02$ \\
\hline $25205 E$ & $3 / 13 / 90$ & $I C P / M$ & $<5.00 \mathrm{E}+02$ \\
\hline $50873 E$ & $1 / 03 / 90$ & ICP & $<5.00 \mathrm{E}+02$ \\
\hline 50959E & $2 / 21 / 90$ & ICP & $<5.00 \mathrm{E}+02$ \\
\hline $51012 \mathrm{E}$ & $3 / 05 / 90$ & ICP & $<5.00 \mathrm{E}+02$ \\
\hline $51091 \mathrm{E}$ & $3 / 26 / 90$ & ICP & $<5.00 \mathrm{E}+02$ \\
\hline 50873 & $1 / 03 / 90$ & ICP & 3. 10E+O1 \\
\hline 50959 & $2 / 21 / 90$ & ICP & $3.80 \mathrm{E}+01$ \\
\hline 51012 & $3 / 05 / 90$ & ICP & 4. $40 \mathrm{E}+01$ \\
\hline 51091 & $3 / 26 / 90$ & ICP & 3. $40 \mathrm{E}+01$ \\
\hline 25158E & $10 / 03 / 89$ & $I C P$ & $<1.00 \mathrm{E}+03$ \\
\hline $25160 E$ & $10 / 10 / 89$ & ICP & $<1.00 \mathrm{E}+03$ \\
\hline $25162 \mathrm{E}$ & $10 / 17 / 89$ & ICP & $<1.00 \mathrm{E}+03$ \\
\hline $25167 \mathrm{E}$ & $10 / 24 / 89$ & $I C P / M$ & $<1.00 \mathrm{E}+03$ \\
\hline $25169 E$ & $10 / 31 / 89$ & $I C P / M$ & $<1.00 \mathrm{E}+03$ \\
\hline $25171 \mathrm{E}$ & $11 / 07 / 89$ & $I C P / M$ & $<1.00 E+03$ \\
\hline $25173 E$ & $11 / 14 / 89$ & $I C P / M$ & $<1.00 \mathrm{E}+03$ \\
\hline $25175 \mathrm{E}$ & $11 / 21 / 89$ & $I C P / M$ & $<1.00 \mathrm{E}+03$ \\
\hline $25177 \mathrm{E}$ & $11 / 28 / 89$ & $I C P / M$ & $<1.00 \mathrm{E}+03$ \\
\hline $25179 E$ & $12 / 05 / 89$ & $I C P / M$ & $<1.00 \mathrm{E}+03$ \\
\hline $25181 \mathrm{E}$ & $12 / 12 / 89$ & $I C P / M$ & $<1.00 \mathrm{E}+03$ \\
\hline $25183 E$ & $12 / 19 / 89$ & $I C P / M$ & $<1.00 \mathrm{E}+03$ \\
\hline $25185 E$ & $1 / 02 / 90$ & $I C P / M$ & $<1.00 \mathrm{E}+03$ \\
\hline 25187E & $1 / 09 / 90$ & $\operatorname{ICP} / \mathrm{M}$ & $<1.00 E+03$ \\
\hline $25189 \mathrm{E}$ & $1 / 16 / 90$ & $I C P / M$ & $<1.00 \mathrm{E}+03$ \\
\hline 25191E & $1 / 23 / 90$ & $I C P / M$ & $<1.00 E+03$ \\
\hline $25193 E$ & $1 / 30 / 90$ & $I C P / M$ & $<1.00 \mathrm{E}+03$ \\
\hline $25195 E$ & $2 / 06 / 90$ & $I C P / M$ & $<1.00 \mathrm{E}+03$ \\
\hline $25197 \mathrm{E}$ & $2 / 13 / 90$ & $I C P / M$ & $<1.00 \mathrm{E}+03$ \\
\hline $25199 E$ & $2 / 20 / 90$ & $\mathrm{ICP} / \mathrm{M}$ & $<1.00 \mathrm{E}+03$ \\
\hline $25201 \mathrm{E}$ & $2 / 27 / 90$ & $I C P / M$ & $<1.00 E+03$ \\
\hline $25203 E$ & $3 / 06 / 90$ & $\mathrm{ICP} / \mathrm{M}$ & $<1.00 E+03$ \\
\hline
\end{tabular}


DATA FOR N Reactor Effluent Continued

Constituent

Barium (EP TOxic)

Barium (EP Toxic)

Barium (EP Toxic)

Barium (EP Toxic)

Barium (EP TOxic)

Boron

Boron

Boron

Boron

Cadmium

Cadmium

Cadmium

Cadmium

Cadmium (EP Toxic)

Cadmium (EP TOxic)

Cadmium (EP TOxic)

Cadmium (EP Toxic)

Cadmium (EP TOxic)

Cadmium (EP TOXic)

Cadmium (EP TOXic)

Cadmium (EP TOxiC)

Cadmium (EP Toxic)

Cadmium (EP TOXic)

Cadmium (EP Toxic)

Cadmium (EP TOxic)

Cadmium (EP TOxic)

Cadmium (EP Toxic)

Cadmium (EP Toxic)

Cadmium (EP Toxic)

Cadmium (EP TOxic)

Cadmium (EP TOXic)

Cadmium (EP TOxic)

Cadmium (EP TOXic)

Cadmium (EP Toxic)

Cadmium (EP TOXic)

Cadmium (EP Toxic)

Cadmium (EP Toxic)

Cadmium (EP TOxic)

Cadmium (EP TOXic)

Cadmium (EP Toxic)

Calcium

Calcium

Calcium

Calcium

Chloride

Chloride

Chloride

Chloride

Chloride

Chloride

Chloride

Chloride

Chloride

\begin{tabular}{|c|c|c|c|}
\hline & & Method & Result \\
\hline & $3 / 13 / 90$ & $I C P / M$ & $<1.00 \mathrm{E}+03$ \\
\hline $50873 E$ & $1 / 03 / 90$ & ICP & $<1.00 \mathrm{E}+03$ \\
\hline $50959 \mathrm{E}$ & $2 / 21 / 90$ & ICP & $<1.00 \mathrm{E}+03$ \\
\hline $51012 \mathrm{E}$ & $3 / 05 / 90$ & ICP & $<1.00 \mathrm{E}+03$ \\
\hline $51091 \mathrm{E}$ & $3 / 26 / 90$ & ICP & $<1.00 \mathrm{E}+03$ \\
\hline 50873 & $1 / 03 / 90$ & ICP & $5.00 \mathrm{E}+01$ \\
\hline 50959 & $2 / 21 / 90$ & ICP & $6.00 \mathrm{E}+01$ \\
\hline 51012 & $3 / 05 / 90$ & ICP & $9.90 \mathrm{E}+01$ \\
\hline 51091 & $3 / 26 / 90$ & ICP & $3.60 \mathrm{E}+01$ \\
\hline 50873 & $1 / 03 / 90$ & ICP & $<2.00 E+00$ \\
\hline 50959 & $2 / 21 / 90$ & ICP & $<2.00 E+00$ \\
\hline 51012 & $3 / 05 / 90$ & ICP & $<2.00 E+00$ \\
\hline 51091 & $3 / 26 / 90$ & ICP & $2,00 \mathrm{E}+00$ \\
\hline $25158 \mathrm{E}$ & $10 / 03 / 89$ & ICP & $<1.00 \mathrm{E}+02$ \\
\hline $25160 \mathrm{E}$ & $10 / 10 / 89$ & ICP & $<1.00 \mathrm{E}+02$ \\
\hline $25162 E$ & $10 / 17 / 89$ & ICP & $<1.00 \mathrm{E}+02$ \\
\hline $25167 \mathrm{E}$ & $10 / 24 / 89$ & $I C P / M$ & $<1.00 \mathrm{E}+02$ \\
\hline $25169 \mathrm{E}$ & $10 / 31 / 89$ & $I C P / M$ & $<1.00 E+02$ \\
\hline $25171 \mathrm{E}$ & $11 / 07 / 89$ & $I C P / M$ & $<1.00 \mathrm{E}+02$ \\
\hline $25173 E$ & $11 / 14 / 89$ & $I C P / M$ & $<1.00 \mathrm{E}+02$ \\
\hline $25175 \mathrm{E}$ & $11 / 21 / 89$ & $I C P / M$ & $<1.00 \mathrm{E}+02$ \\
\hline $25177 \mathrm{E}$ & $11 / 28 / 89$ & $I C P / M$ & $<1.00 \mathrm{E}+02$ \\
\hline $25179 \mathrm{E}$ & $12 / 05 / 89$ & ICP $/ \mathrm{M}$ & $<1.00 \mathrm{E}+02$ \\
\hline $25181 \mathrm{E}$ & $12 / 12 / 89$ & $I C P / M$ & $<1.00 \mathrm{E}+02$ \\
\hline $25183 \mathrm{E}$ & $12 / 19 / 89$ & $\mathrm{ICP} / \mathrm{M}$ & $<1.00 \mathrm{E}+02$ \\
\hline $25185 \mathrm{E}$ & $1 / 02 / 90$ & $I C P / M$ & $<1.00 \mathrm{E}+02$ \\
\hline $25187 E$ & $1 / 09 / 90$ & $I C P / M$ & $<1.00 \mathrm{E}+02$ \\
\hline $25189 \mathrm{E}$ & $1 / 16 / 90$ & $I C P / M$ & $\mathrm{E}+02$ \\
\hline $25191 \mathrm{E}$ & $1 / 23 / 90$ & $I C P / M$ & $<1.00 \mathrm{E}+02$ \\
\hline 25193E & $1 / 30 / 90$ & $I C P / M$ & $<1.00 \mathrm{E}+02$ \\
\hline $25195 \mathrm{E}$ & $2 / 06 / 90$ & $I C P / M$ & $<1.00 \mathrm{E}+02$ \\
\hline 25197E & $2 / 13 / 90$ & ICP $/ \mathrm{M}$ & $<1.00 E+02$ \\
\hline $25199 \mathrm{E}$ & $2 / 20 / 90$ & $I C P / M$ & $<1.00 \mathrm{E}+02$ \\
\hline $25201 E$ & $2 / 27 / 90$ & $I C P / M$ & $<1.00 \mathrm{E}+02$ \\
\hline $25203 \mathrm{E}$ & $3 / 06 / 90$ & $I C P / M$ & $<1.00 \mathrm{E}+02$ \\
\hline $25205 E$ & $3 / 13 / 90$ & ICP $/ \mathrm{M}$ & $<1.00 \mathrm{E}+02$ \\
\hline $50873 E$ & $1 / 03 / 90$ & ICP & $<1.00 \mathrm{E}+02$ \\
\hline $50959 \mathrm{E}$ & $2 / 21 / 90$ & ICP & $<1.00 \mathrm{E}+02$ \\
\hline $51012 \mathrm{E}$ & $3 / 05 / 90$ & ICP & $<1.00 \mathrm{E}+02$ \\
\hline $51091 \mathrm{E}$ & $3 / 26$ & ICP & $<1.00 \mathrm{E}+02$ \\
\hline 50873 & $1 / 03 / 90$ & ICP & $5.36 \mathrm{E}+03$ \\
\hline 50959 & $2 / 21 / 90$ & ICP & $7.44 \mathrm{E}+03$ \\
\hline 51012 & $3 / 05 / 90$ & ICP & $1.58 \mathrm{E}+04$ \\
\hline 51091 & $3 / 26 / 90$ & ICP & $1.80 \mathrm{E}+04$ \\
\hline 25160 & $10 / 10 / 89$ & IC & $1.10 \mathrm{E}+03$ \\
\hline 25162 & $10 / 17 / 89$ & IC & $6.00 \mathrm{E}+02$ \\
\hline 25167 & $10 / 24 / 89$ & IC & $1.50 E+03$ \\
\hline 25169 & $10 / 31 / 89$ & IC & $8.00 \mathrm{E}+02$ \\
\hline 25171 & $11 / 07 / 89$ & IC & $1.30 \mathrm{E}+03$ \\
\hline 25173 & $11 / 14 / 89$ & IC & $1.40 \mathrm{E}+03$ \\
\hline 25175 & $11 / 21 / 89$ & IC & $7.00 \mathrm{E}+02$ \\
\hline 25177 & $11 / 28 / 89$ & IC & $1.00 \mathrm{E}+03$ \\
\hline 25179 & $12 / 05 / 89$ & IC & $1.00 E+03$ \\
\hline
\end{tabular}

D -51 
WHC-EP-0355, Appendix D

DATA FOR N Reactor Effluent

Continued

\begin{tabular}{|c|c|c|}
\hline $\begin{array}{l}\text { Constitu } \\
\text { Chloride }\end{array}$ & & \\
\hline & & \\
\hline Chloride & & \\
\hline Chloride & & \\
\hline Chloride & & \\
\hline Chloride & & \\
\hline Chloride & & \\
\hline Chloride & & \\
\hline Chloride & & \\
\hline Chloride & & \\
\hline Chloride & & \\
\hline Chloride & & \\
\hline Chloride & & \\
\hline Chloride & & \\
\hline Chloride & & \\
\hline Chloride & & \\
\hline Chloride & & \\
\hline Chromium & (EP & Toxic) \\
\hline Chromium & (EP & Toxic) \\
\hline Chromium & (EP & Toxic) \\
\hline Chromium & (EP & Toxic) \\
\hline Chromium & (EP & Toxic) \\
\hline Chromium & (EP & 'Toxic) \\
\hline Chromium & (EP & Toxic) \\
\hline Chroinium & (EP & Toxic) \\
\hline Chromium & (EP & Toxic) \\
\hline Chromium & (EP & Toxic) \\
\hline Chromium & (EP & Toxic) \\
\hline Chromium & (EP & Toxic) \\
\hline Chromium & (EP & Toxic) \\
\hline Chromium & (EP & Toxic) \\
\hline Chromium & (EP & Toxic) \\
\hline Chromium & (EP & Toxic) \\
\hline Chromium & (EP & Toxic) \\
\hline Chromium & (EP & Toxic) \\
\hline Chromium & (EP & Toxic) \\
\hline Chromium & (EP & Toxic) \\
\hline Chromium & (EP & Toxic) \\
\hline Chromium & (EP & Toxic) \\
\hline Chromium & (EP & Toxic) \\
\hline Chromium & (EP & Toxic) \\
\hline Chromium & (EP & Toxic) \\
\hline Chromium & (EP & Toxic) \\
\hline Chromium & (EP & Toxic) \\
\hline Fluoride & & \\
\hline Fluoride & & \\
\hline Fluoride & & \\
\hline Fluoride & & \\
\hline Fluoride & & \\
\hline Fluoride & & \\
\hline Fluoride & & \\
\hline Fluoride & & \\
\hline Fluoride & & \\
\hline
\end{tabular}

\begin{tabular}{|c|c|c|c|}
\hline imple \# & Date & Method & Result \\
\hline 25181 & $12 / 12 / 89$ & IC & $1.10 \mathrm{E}+03$ \\
\hline 25183 & $12 / 19 / 89$ & IC & $1.10 \mathrm{E}+03$ \\
\hline 25185 & $1 / 02 / 90$ & IC & $<5.00 \mathrm{E}+02$ \\
\hline 25187 & $1 / 09 / 90$ & IC & $6.00 \mathrm{E}+02$ \\
\hline 25189 & $1 / 16 / 90$ & IC & $1.70 \mathrm{E}+03$ \\
\hline 25191 & $1 / 23 / 90$ & IC & $1.50 E+03$ \\
\hline 25193 & $1 / 30 / 90$ & IC & $2.30 \mathrm{E}+03$ \\
\hline 25195 & $2 / 06 / 90$ & IC & $9.00 \mathrm{E}+02$ \\
\hline 25197 & $2 / 13 / 90$ & IC & $<5.00 \mathrm{E}+02$ \\
\hline 25199 & $2 / 20 / 90$ & IC & $7.00 \mathrm{E}+02$ \\
\hline 25201 & $2 / 27 / 90$ & IC & $1.70 E+03$ \\
\hline 25203 & $3 / 06 / 90$ & IC & $1.50 \mathrm{E}+03$ \\
\hline 25205 & $3 / 13 / 90$ & IC & $1.70 \mathrm{E}+03$ \\
\hline 50873 & $1 / 03 / 90$ & IC & $5.00 \mathrm{E}+02$ \\
\hline 50959 & $2 / 21 / 90$ & IC & $<5.00 \mathrm{E}+02$ \\
\hline 51012 & $3 / 05 / 90$ & IC & $D E+03$ \\
\hline 51091 & $3 / 26 / 90$ & IC & $1.70 E+03$ \\
\hline $25158 \mathrm{E}$ & $10 / 03 / 89$ & ICP & $<5.00 \mathrm{E}+02$ \\
\hline $25160 E$ & $10 / 10 / 89$ & ICP & $<5.00 \mathrm{E}+02$ \\
\hline $25162 \mathrm{E}$ & $10 / 27 / 89$ & $I C P$ & $<5.00 E+02$ \\
\hline $25167 E$ & $10 / 24 / 39$ & $I C P / M$ & $<5.00 \mathrm{E}+02$ \\
\hline $25169 \mathrm{E}$ & $10 / 31 / 89$ & $I C P / M$ & $<5.00 \mathrm{E}+02$ \\
\hline 25171E & $11 / 07 / 89$ & ICP $/ \mathrm{M}$ & $<5.00 \mathrm{E}+02$ \\
\hline $25173 E$ & $11 / 14 / 89$ & ICP $/ \mathrm{M}$ & $<5.00 \mathrm{E}+02$ \\
\hline $25175 E$ & $11 / 21 / 89$ & $I C P / M$ & $<5.00 E+02$ \\
\hline $25177 \mathrm{E}$ & $11 / 28 / 89$ & $I C P / M$ & $<5.00 \mathrm{E}+02$ \\
\hline $25179 \mathrm{E}$ & $12 / 05 / 89$ & $I C P / M$ & $<5.00 \mathrm{E}+02$ \\
\hline $25181 \mathrm{E}$ & $12 / 12 / 89$ & $I C P / M$ & $<5.00 \mathrm{E}+02$ \\
\hline 25183E & $12 / 19 / 89$ & ICP $/ \mathrm{M}$ & $<5.00 \mathrm{E}+02$ \\
\hline $25185 E$ & $1 / 02 / 90$ & $I C P / M$ & $<5.00 E+02$ \\
\hline $25187 \mathrm{E}$ & $1 / 09 / 90$ & $I C P / M$ & $<5.00 \mathrm{E}+02$ \\
\hline $25189 E$ & $1 / 16 / 90$ & $I C P / M$ & $<5.00 \mathrm{E}+02$ \\
\hline 25191E & $1 / 23 / 90$ & $I C P / M$ & $<5.00 \mathrm{E}+02$ \\
\hline 25193E & $1 / 30 / 90$ & $I C P / M$ & $<5.00 \mathrm{E}+02$ \\
\hline 25195E & $2 / 06 / 90$ & ICP $/ M$ & $<5.00 \mathrm{E}+02$ \\
\hline $25197 \mathrm{E}$ & $2 / 13 / 90$ & ICP $/ \mathrm{M}$ & $<5.00 \mathrm{E}+02$ \\
\hline $25199 \mathrm{E}$ & $2 / 20 / 90$ & $I C P / M$ & $<5.00 \mathrm{E}+02$ \\
\hline $25201 \mathrm{E}$ & $2 / 27 / 90$ & $I C P / M$ & $<5.00 \mathrm{E}+02$ \\
\hline $25203 E$ & $3 / 06 / 90$ & $I C P / M$ & $<5.00 \mathrm{E}+02$ \\
\hline $25205 E$ & $3 / 13 / 90$ & $I C P / M$ & $<5.00 \mathrm{E}+02$ \\
\hline 50873E & $1 / 03 / 90$ & ICP & $<5.00 \mathrm{E}+02$ \\
\hline 50959E & $2 / 21 / 90$ & ICP & $<5.00 E+02$ \\
\hline $51012 \mathrm{E}$ & $3 / 05 / 90$ & ICP & $<5.00 \mathrm{E}+02$ \\
\hline $51091 \mathrm{E}$ & $3 / 26 / 90$ & ICP & $<5.00 \mathrm{E}+02$ \\
\hline 25160 & $10 / 10 / 89$ & IC & $<5.00 \mathrm{E}+02$ \\
\hline 25162 & $10 / 17 / 89$ & IC & $<5.00 \mathrm{E}+02$ \\
\hline 25167 & $10 / 24 / 89$ & IC & $<5,00 E+02$ \\
\hline 25169 & $10 / 31 / 89$ & IC & $<5.00 \mathrm{E}+02$ \\
\hline 25171 & $11 / 07 / 89$ & IC & $<5.00 E+02$ \\
\hline 25173 & $11 / 14 / 89$ & IC & $<5.00 E+02$ \\
\hline 25175 & $11 / 21 / 89$ & I.C & $<5.00 \mathrm{E}+02$ \\
\hline 25177 & $11 / 28 / 89$ & IC & $<5.00 \mathrm{E}+02$ \\
\hline 25179 & $12 / 05 / 89$ & IC & $<5.00 \mathrm{E}+02$ \\
\hline
\end{tabular}


DATA FOR N Reactor Effluent

Continued

\begin{tabular}{|c|c|c|}
\hline Const & itue & \\
\hline Fluor & ide & \\
\hline Eluor & ide & \\
\hline $\begin{array}{l}\text { Fluor } \\
\text { Fluor }\end{array}$ & $\begin{array}{l}\text { idae } \\
\text { idee }\end{array}$ & \\
\hline Fluor & ide & \\
\hline Fluos & side & \\
\hline Fiuos & eide & \\
\hline Fluor & :ide & \\
\hline$F=2 x$ & :ide & \\
\hline Fluor & eide & \\
\hline Eluor & cide & \\
\hline Fluor & cide & \\
\hline Fluor & Eide & \\
\hline Fluor & ide & \\
\hline Fluor & ide & \\
\hline Fluod & ide & \\
\hline Eluor & ide & \\
\hline Fluor & :ide & \\
\hline Fluor & ride & \\
\hline Fluor & ride & \\
\hline Fluol & ride & \\
\hline Iron & & \\
\hline Iron & & \\
\hline Iron & & \\
\hline Iron & & \\
\hline Lead & (EP & 10xic) \\
\hline Lead & (EP & Toxic) \\
\hline Lead & (EP & Toxic) \\
\hline Lead & (EP & Toxic) \\
\hline Lead & (EP & Toxic) \\
\hline Lead & (EP & Toxic) \\
\hline Lead & (EP & Toxic) \\
\hline I.ead & (EP & Toxic) \\
\hline Lead & (EP & Toxic) \\
\hline Lead & (EP & Toxic) \\
\hline Lead & (EP & Toxic) \\
\hline Lead & (EP & Toxic) \\
\hline Iead & (EP & Toxic) \\
\hline Lead & (EP & Toxic) \\
\hline Lead & (EP & Toxic) \\
\hline Lead & (EP & Toxic) \\
\hline Lead & (EP & Toxic) \\
\hline Lead & $(E P$ & Toxic) \\
\hline Lead & (EP & Toxic) \\
\hline Lead & (EP & Toxic) \\
\hline Lead & (EP & Toxic) \\
\hline Lead & (EP & Toxic) \\
\hline I.ead & (EP & Toxic) \\
\hline Lead & (EP & Toxic) \\
\hline LEad & (E) & Tcxic) \\
\hline Lead & (EP & Toxic) \\
\hline Lead & (EP & Toxic) \\
\hline Magn & esiur & \\
\hline
\end{tabular}

\begin{tabular}{|c|c|c|c|}
\hline Sample \# & Date & Method & Result \\
\hline 25181 & $12 / 12 / 89$ & $I C$ & $<5.00 E+02$ \\
\hline 25183 & $12 / 19 / 89$ & IC & $<5.00 E+02$ \\
\hline 25185 & $1 / 02 / 90$ & IC & $<5.00 E+02$ \\
\hline 25187 & $1 / 09 / 90$ & IC & $<5.00 \mathrm{E}+02$ \\
\hline 25189 & $1 / 16 / 90$ & IC & $15.00 \mathrm{E}+02$ \\
\hline 25191 & $1 / 23 / 90$ & IC & $: 5.00 \mathrm{E}+02$ \\
\hline 25193 & $1 / 30 / 90$ & IC & $45.00 E+02$ \\
\hline 25195 & $2 / 06 / 90$ & IC & $<5.00 \mathrm{E}+02$ \\
\hline 25197 & $2 / 13 / 90$ & IC & $<5.00 \mathrm{E}+02$ \\
\hline 25199 & $2 / 20 / 90$ & IC & $<5.00 \mathrm{x}+02$ \\
\hline 25201 & $2 / 27 / 90$ & IC & $<5.00 \mathrm{E}+02$ \\
\hline 25203 & $3 / 06 / 90$ & IC & $<5.0 O E+02$ \\
\hline 25205 & $3 / 13 / 90$ & $\therefore \mathrm{C}$ & $<5.00 E+02$ \\
\hline 50873 & $1 / 03 / 90$ & IC & $<5.00 \mathrm{E}+02$ \\
\hline 50873 & $1 / 03 / 90$ & ISE & $8.60 \mathrm{E}+01$ \\
\hline 50959 & $2 / 21 / 90$ & IC & $<5.00 E+02$ \\
\hline 50959 & $2 / 21 / 90$ & ISE & $9.90 \mathrm{E}+01$ \\
\hline 51012 & $3 / 05 / 90$ & IC & $<5.00 E+02$ \\
\hline 51012 & $3 / 05 / 90$ & ISE & $1.75 \mathrm{E}+02$ \\
\hline 51091 & $3 / 26 / 90$ & IC & $<5.00 \mathrm{E}+02$ \\
\hline 51091 & $3 / 26 / 90$ & ISE & $2.32 \mathrm{E}+02$ \\
\hline 50873 & $1 / 03 / 90$ & ICP & $2.04 E+02$ \\
\hline 50959 & $2 / 21 / 90$ & ICP & 3. $27 \mathrm{E}+02$ \\
\hline 51012 & $3 / 05 / 90$ & ICP & 2. $29 E+02$ \\
\hline 51091 & $3 / 26 / 90$ & ICP & $2.52 \mathrm{E}+02$ \\
\hline $25158 \mathrm{E}$ & $10 / 03 / 89$ & ICP & $<5.00 \mathrm{E}+02$ \\
\hline 25L6OE & $10 / 10 / 89$ & ICP & $<5 . C \cup E+02$ \\
\hline $25162 \mathrm{E}$ & $10 / 17 / 89$ & ICP & $\angle 5.00 \mathrm{E}+02$ \\
\hline $25167 \mathrm{E}$ & $10 / 24 / 89$ & $I C P / M$ & $<5.00 \mathrm{E}+02$ \\
\hline $25169 \mathrm{E}$ & $10 / 31 / 89$ & $I C P / M$ & $<5.00 E+C 2$ \\
\hline $25171 E$ & $11 / 07 / 89$ & $I C P / M$ & $<5.0 O E+C 2$ \\
\hline $25173 \mathrm{E}$ & $11 / 14 / 89$ & $I C P / M$ & $<5.00 E+02$ \\
\hline $25175 E$ & $11 / 21 / 89$ & $I C P / M$ & $<5.00 \mathrm{E}+02$ \\
\hline $25177 \mathrm{E}$ & $1] / / 28 / 89$ & $I C P / M$ & $<5.00 E+02$ \\
\hline $25179 \mathrm{E}$ & $12 / 05 / 89$ & $I C P / M$ & $<5.00 E+02$ \\
\hline $25181 \mathrm{E}$ & $12 / 12 / 89$ & $I C P / M$ & $<5.00 \mathrm{E}+02$ \\
\hline $25183 E$ & $12 / 19 / 89$ & $\mathrm{CCP} / \mathrm{M}$ & $\because 5.00 E+02$ \\
\hline $25185 \mathrm{E}$ & $1 / 02 / 90$ & $I C P / M$ & $<5.00 \mathrm{E}+02$ \\
\hline $25187 \mathrm{E}$ & $1 / 09 / 90$ & $\operatorname{ICP} / \mathrm{M}$ & $<5.00 E+02$ \\
\hline $25189 E$ & $1 / 16 / 90$ & $I C P / M$ & $<5.00 E+02$ \\
\hline $25191 E$ & $1 / 23 / 90$ & $I C P / M$ & $.5 .00 E+02$ \\
\hline $25193 E$ & $1 / 30 / 90$ & $I C P / M$ & $<5,-7 \mathrm{E}+02$ \\
\hline 25195E & $2 / 06 / 90$ & $I C P / M$ & $<5 . \cup O E+02$ \\
\hline $25197 \mathrm{E}$ & $2 / 13 / 90$ & $I C P / M$ & $<5.00 E+02$ \\
\hline $25199 E$ & $2 / 20 / 90$ & $I C P / M$ & $<5 . C O E+02$ \\
\hline 25201E & $2 / 27 / 90$ & $I C P / M$ & $<5.00 \mathrm{E}+02$ \\
\hline $25203 E$ & $3 / 06 / 90$ & $I C P / M$ & $<5.00 \mathrm{E}+02$ \\
\hline $25205 E$ & $3 / 13 / 90$ & $I C P / M$ & $<5.00 \mathrm{E}+02$ \\
\hline $50873 E$ & $1 / 03 / 90$ & ICP & $<5.0 O E+02$ \\
\hline 509595 & $2,21,900$ & 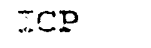 & $-5.005 \div 02$ \\
\hline $51012 \mathrm{E}$ & $3 / 05 / 90$ & ICP & $<5.00 E+02$ \\
\hline $51091 \mathrm{E}$ & $3 / 26 / 90$ & ICP & $<5.00 E+02$ \\
\hline 50873 & $1 / 03 / 90$ & ICP & $1.19 E+23$ \\
\hline
\end{tabular}


DATA FOR N Reactor Effluent Continued

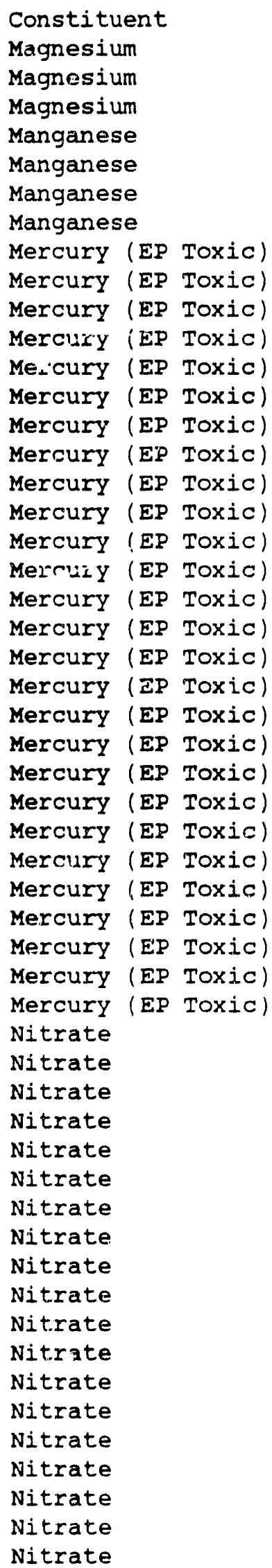

\begin{tabular}{|c|c|c|c|}
\hline Sample \# & Date & Method & Result \\
\hline 50959 & $2 / 21 / 90$ & ICP & 2, $75 \mathrm{E}+03$ \\
\hline 51012 & $3 / 05 / 90$ & ICP & $3.77 E+03$ \\
\hline$=2091$ & $3 / 26 / 90$ & ICP & 1.91E+03 \\
\hline 50373 & $1 / 03 / 90$ & ICP & $<5.00 E+00$ \\
\hline 50959 & $2 / 21 / 90$ & ICP & $7.00 \mathrm{E}+00$ \\
\hline 51012 & $3 / 05 / 90$ & ICP & $<5.00 E+00$ \\
\hline 51091 & $3 / 26 / 90$ & ICP & $1.00 \mathrm{E}+01$ \\
\hline $25158 \mathrm{E}$ & $10 / 03 / 89$ & $C V A A / M$ & $<2.00 \mathrm{E}+01$ \\
\hline $25160 \mathrm{E}$ & $10 / 10 / 89$ & $C V A A / M$ & $<2.00 \mathrm{E}+01$ \\
\hline $25162 \mathrm{E}$ & $10 / 17 / 89$ & CVAA $/ M$ & $<2.00 E+01$ \\
\hline $25167 \mathrm{E}$ & $10 / 24 / 89$ & CVAA $/ M$ & $<2.00 E+01$ \\
\hline $25169 \mathrm{E}$ & $10 / 31 / 89$ & CVAA/M & $<2.00 E+01$ \\
\hline $25171 E$ & $11 / 07 / 89$ & $C V A A / M$ & $<2.00 E+01$ \\
\hline $25173 E$ & $11 / 14 / 89$ & CVAA/M & $<2.00 E+01$ \\
\hline $25 \geq 75 E$ & $11 / 21 / 89$ & CVAA $/ M$ & $<2.00 E+01$ \\
\hline $25177 \mathrm{E}$ & $1.1 / 28 / 89$ & $C V A A / M$ & $<2.00 E+01$ \\
\hline $25179 E$ & $12 / 05 / 89$ & $C V A A / M$ & $<2.00 E+01$ \\
\hline $25181 E$ & $12 / 12 / 89$ & CVAA/M & $<2.00 E+01$ \\
\hline $25183 E$ & $12 / 19 / 89$ & CVAA/M & $<2.00 E+01$ \\
\hline $25185 E$ & $1 . / 02 / 90$ & CVAA $/ M$ & $<2.00 \mathrm{E}+01$ \\
\hline $25187 \mathrm{E}$ & $1 / 09 / 90$ & $C V A A / M$ & $<2.00 E+01$ \\
\hline $25189 E$ & $1 / 16 / 90$ & CVAA /M & $<2.00 E+01$ \\
\hline $25191 \mathrm{E}$ & $1 / 23 / 90$ & CVAA/M & $<2.00 \mathrm{E}+01$ \\
\hline $25193 \mathrm{E}$ & $1 / 30 / 90$ & CVAA/M & $<2.00 E+01$ \\
\hline 25195E & $2 / 06 / 90$ & CVAA $/ M$ & $<2.00 E+01$ \\
\hline 25197E & $2 / 13 / 90$ & CVAA $/ M$ & $<2.00 \mathrm{E}+01$ \\
\hline 25199E & $? / 20 / 90$ & CVAA/M & $<2.00 E+01$ \\
\hline $25201 \mathrm{E}$ & $2 / 27 / 90$ & CVAA $/ M$ & $<2.00 E+01$ \\
\hline $25203 E$ & $3 / 06 / 90$ & CVAA/M & $<2.00 \mathrm{E}+01$ \\
\hline $25205 E$ & $3 / 13 / 90$ & $\mathrm{CVAA} / \mathrm{M}$ & $<2.00 E+01$ \\
\hline $50873 E$ & $1 / 03 / 90$ & $C V A A / M$ & $<2.00 E+01$ \\
\hline 50959E & $2 / 21 / 90$ & CVAA/M & $<2.00 E+01$ \\
\hline $51012 \mathrm{E}$ & $3 / 05 / 90$ & CVAA/M & $<2.00 \mathrm{E}+01$ \\
\hline $51091 \mathrm{E}$ & $3 / 26 / 90$ & CVAA/M & $<2.00 E+01$ \\
\hline 25160 & $10 / 10 / 89$ & IC & $<5.00 E+02$ \\
\hline 25162 & $10 / 17 / 89$ & IC & $<5.00 \mathrm{E}+02$ \\
\hline 25167 & $10 / 24 / 89$ & IC & $7.00 \mathrm{E}+02$ \\
\hline 25169 & $10 / 31 / 89$ & IC & $<5.00 E+02$ \\
\hline 25171 & $11 / 07 / 89$ & IC & $6.00 E+02$ \\
\hline 25173 & $11 / 14 / 89$ & IC & $6.00 E+02$ \\
\hline 25175 & $11 / 21 / 89$ & IC & $<5.00 E+02$ \\
\hline 25177 & $11 / 28 / 89$ & IC & $<5.00 E+02$ \\
\hline 25179 & $12 / 05 / 89$ & IC & $<5.00 E+02$ \\
\hline 25181 & $12 / 12 / 89$ & IC & $<5.00 E+02$ \\
\hline 25183 & $12 / 19 / 89$ & IC & $<5.00 E+02$ \\
\hline 25185 & $1 / 02 / 90$ & IC & $<5.00 E+02$ \\
\hline 25187 & $1 / 09 / 90$ & IC & $<5.00 E+02$ \\
\hline 25189 & 1/16/90 & IC & $<5.0 O E+02$ \\
\hline 25191 & $1 / 23 / 90$ & IC & $<5.00 \mathrm{E}+02$ \\
\hline 25193 & $1 / 30 / 90$ & IC & $6.00 \mathrm{E}+02$ \\
\hline 25195 & $2 / 06 / 30$ & IS & $<5.00 E+02$ \\
\hline 25197 & $2 / 13 / 90$ & IC & $<5.00 E+02$ \\
\hline 25199 & $2 / 20 / 90$ & IC & $<5.00 E+02$ \\
\hline
\end{tabular}


DATA FOR $N$ Reactor Effluent Continued

\begin{tabular}{|c|c|c|}
\hline \multicolumn{3}{|c|}{ Constituent } \\
\hline \multicolumn{3}{|c|}{ Nitrate } \\
\hline \multicolumn{3}{|l|}{ Nitrate } \\
\hline \multicolumn{3}{|l|}{ Nitrate } \\
\hline \multicolumn{3}{|l|}{ Nitrate } \\
\hline \multicolumn{3}{|l|}{ Nitrate } \\
\hline \multicolumn{3}{|l|}{ Nitrate } \\
\hline \multicolumn{3}{|l|}{ Nitrate } \\
\hline \multicolumn{3}{|l|}{ Potassium } \\
\hline \multicolumn{3}{|l|}{ Potassium } \\
\hline \multicolumn{3}{|l|}{ Potassium } \\
\hline \multicolumn{3}{|l|}{ Potassium } \\
\hline Selenium & (EP & Toxic) \\
\hline Selenium ( & (EP & Toxic) \\
\hline Selenium ( & ( EP & Toxic) \\
\hline Selenium & (EP & Toxic) \\
\hline Selenium ( & (EP & Toxic) \\
\hline Selenium & (EP & Toxic) \\
\hline Selenium & ( EP & Toxic) \\
\hline Selentum ( & (EP & Toxic) \\
\hline Selenium ( & (EP & Toxic) \\
\hline Selenium & (EP & Toxic) \\
\hline SeIerdum & (ER & Toxic) \\
\hline Seianium ( & $(\mathrm{EP}$ & Toxic) \\
\hline Selenium & (EP & Toxic) \\
\hline Selenium ( & (EP & Toxic) \\
\hline Selenium & (EP & Toxic) \\
\hline Selenium & (EP & Toxic) \\
\hline Selenium & (EP & Toxic) \\
\hline Selenium & (EP & Toxic) \\
\hline Selenium & (EP & Toxic) \\
\hline Selenium & (EP & Toxic) \\
\hline Selenium & (EP & Toxic) \\
\hline Selenium & (EP & Toxic) \\
\hline Selenium & (EP & Toxic) \\
\hline Selenium & (EP & Toxic) \\
\hline Selenium & (EP & Toxic) \\
\hline Selenium & (EP & Toxic \\
\hline Selenium & (EF & Toxic \\
\hline Silic & & \\
\hline Sili & & \\
\hline SiIic & & \\
\hline Silic & & \\
\hline Silver (EF & $P T C$ & oxic) \\
\hline Silver & $\mathrm{PTC}$ & oxic) \\
\hline Silver & $\mathrm{P} T \mathrm{C}$ & oxic) \\
\hline Silver & TC & oxic) \\
\hline Silver & & oxic) \\
\hline Silver & $\mathrm{P} T \mathrm{C}$ & oxic) \\
\hline Silver & $\mathrm{P} T \mathrm{C}$ & oxic) \\
\hline Silver & $\mathrm{PTC}$ & oxic) \\
\hline SiIver & & oxicj \\
\hline Silver (EF & & oxic) \\
\hline Silver (EF & & oxic) \\
\hline
\end{tabular}

\begin{tabular}{|c|c|c|c|}
\hline Sample \# & Date & Method & Result \\
\hline 25201 & $2 / 27 / 90$ & IC & $5.00 E+02$ \\
\hline 25203 & $3 / 06 / 90$ & IC & $5.00 E+02$ \\
\hline 25205 & $3 / 13 / 90$ & IC & $6.00 \mathrm{E}+02$ \\
\hline 50873 & $1 / 03 / 90$ & IC & $<5.00 E+02$ \\
\hline 50959 & $2 / 21 / 90$ & $I C$ & $<5.00 \mathrm{E}+02$ \\
\hline 51.012 & $3 / 05 / 90$ & IC & $5.00 \mathrm{E}+02$ \\
\hline 51091 & $3 / 26 / 90$ & IC & $<5.00 \mathrm{E}+02$ \\
\hline 50873 & $1 / 03 / 90$ & ICP & $2=24 \mathrm{E}+02$ \\
\hline 50959 & $2 / 21 / 90$ & ICP & 3. $10 \mathrm{E}+02$ \\
\hline 51012 & $3 / 05 / 90$ & ICP & $6.12 \mathrm{E}+02$ \\
\hline 51091 & $3 / 26 / 90$ & I.CP & $7.53 \mathrm{E}+02$ \\
\hline $25158 \mathrm{E}$ & $10 / 03 / 89$ & ICP & $<5.00 \mathrm{E}+02$ \\
\hline 25160E & $10 / 10 / 89$ & ICP & $<5.00 \mathrm{E}+02$ \\
\hline $25162 \mathrm{E}$ & $10 / 17 / 89$ & ICP & $<5.00 \mathrm{E}+02$ \\
\hline $25167 \mathrm{E}$ & $10 / 24 / 89$ & $I C P / M$ & $<5.00 \mathrm{E}+02$ \\
\hline $25169 \mathrm{E}$ & $10 / 31 / 89$ & $I C P / M$ & $<5.00 E+02$ \\
\hline 25171E & $11 / 07 / 89$ & $I C P / M$ & $<5.00 \mathrm{E}+02$ \\
\hline $25173 E$ & $11 / 14 / 89$ & $I C P / M$ & $<5.00 \mathrm{E}+02$ \\
\hline $25175 \mathrm{E}$ & $11 / 21 / 89$ & $I C P / M$ & $<5.00 E+02$ \\
\hline $25177 \mathrm{E}$ & $11 / 28 / 89$ & $I C P / M$ & $<5.00 E+02$ \\
\hline $25179 \mathrm{E}$ & $12 / 05 / 89$ & $I C P / M$ & $<5.00 E+02$ \\
\hline $25181 \mathrm{E}$ & $12 / 12 / 89$ & $I C P / M$ & $<5.00 E+02$ \\
\hline $25183 E$ & $12 / 19 / 89$ & $I C P / M$ & $<5.00 E+02$ \\
\hline $25185 E$ & $1 / 02 / 90$ & $I C P / M$ & $<5.00 \mathrm{E}+02$ \\
\hline $25187 \mathrm{E}$ & $1 / 09 / 90$ & $I C P / M$ & $<5.00 E+02$ \\
\hline $25189 E$ & $1 / 16 / 90$ & $I C P / M$ & $<5.00 \mathrm{E}+02$ \\
\hline 25191E & $=/ 23 / 90$ & $I C P / M$ & $<5.00 \mathrm{E}+02$ \\
\hline $25193 \mathrm{E}$ & $1 / 30 / 90$ & $I C P / M$ & $<5: 00 E+02$ \\
\hline $25195 E$ & $2 / 06 / 90$ & $I C P / M$ & $<5.00 E+02$ \\
\hline $25197 \mathrm{E}$ & $2 / 13 / 90$ & $I C P / M$ & $<5.00 \mathrm{E}+02$ \\
\hline $25199 E$ & $2 / 20 / 90$ & $\operatorname{ICP} / \mathrm{M}$ & $<5.00 E+02$ \\
\hline 25201E & $2 / 27 / 90$ & $I C P / M$ & $<5.00 E+02$ \\
\hline $25203 E$ & $3 / 06 / 90$ & $I C P / M$ & $<5.00 E+02$ \\
\hline $25205 \mathrm{E}$ & $3 / 13 / 90$ & $I C P / M$ & $<5.00 \mathrm{E}+02$ \\
\hline $50873 E$ & $1 / 03 / 90$ & ICP & $<5.00 E+02$ \\
\hline $50959 E$ & $2 / 21 / 90$ & ICP & $<5.00 \mathrm{E}+02$ \\
\hline $51012 \mathrm{E}$ & $3 / 05 / 90$ & ICP & $<5.00 E+02$ \\
\hline 51091E & $3 / 26 / 90$ & ICP & $<5.00 E+02$ \\
\hline 50873 & $1 / 03 / 90$ & $I C P$ & $7.70 E+02$ \\
\hline 50959 & $2 / 21 / 90$ & ICP & $1.04 \mathrm{E}+03$ \\
\hline 51012 & $3 / 05 / 90$ & ICP & $1.94 \mathrm{E}+03$ \\
\hline 51091 & $3 / 26 / 90$ & ICP & $2.17 \mathrm{E}+03$ \\
\hline $25158 \mathrm{E}$ & $10 / 03 / 89$ & $I C P$ & $<5.00 \mathrm{E}+02$ \\
\hline $25160 \mathrm{E}$ & $10 / 10 / 89$ & ICP & $<5.00 \mathrm{E}+02$ \\
\hline $25162 \mathrm{E}$ & $10 / 17 / 89$ & ICP & $<5.00 \mathrm{E}+02$ \\
\hline $25167 \mathrm{E}$ & $10 / 24 / 89$ & $I C P / M$ & $<5.00 \mathrm{E}+02$ \\
\hline $25169 E$ & $10 / 31 / 89$ & $I C P / M$ & $<5.00 E+02$ \\
\hline 25171E & $11 / 07 / 89$ & $I C P / M$ & $<5.00 \mathrm{E}+02$ \\
\hline $25173 E$ & $11 / 14 / 89$ & $I C P / M$ & $<5.00 \mathrm{E}+02$ \\
\hline $25175 E$ & $11,21 / 89$ & $I C P / M$ & $<5.00 \mathrm{E}+02$ \\
\hline 25I77E & エエ/டも/89 & Iしマ/M & $<5 . \overline{U U E}+\bar{U} 2$ \\
\hline $25179 E$ & $12 / 05 / 89$ & $I C P / M$ & $<5.00 E+02$ \\
\hline $25181 \mathrm{E}$ & $12 / 12 / 89$ & $I C P / M$ & $<5.00 E+02$ \\
\hline
\end{tabular}

D -55 
DATA FOR N Reactor Effluent

Continued

\begin{tabular}{|c|c|c|}
\hline \multicolumn{3}{|c|}{ Constituent } \\
\hline Silver & (EP & Toxic) \\
\hline silver & (EP & Toxic) \\
\hline Silver & (EP & Toxic) \\
\hline Silver & (EP & Toxic) \\
\hline Silver & ( $E P$ & Toxic) \\
\hline Silver & (EP & Toxic \\
\hline Silver & (EP & Toxic) \\
\hline Silver & (EP & Toxic) \\
\hline Silver & (EP & Toxic) \\
\hline Silver & ( $\mathrm{EP}$ & Toxic) \\
\hline Silver & (EP & Toxic) \\
\hline Silver & (EP & Toxic) \\
\hline Silver & (EP & Toxic) \\
\hline Silver & (EP & Toxic) \\
\hline silver & (EP & Toxic) \\
\hline Silver & (EP & Toxic \\
\hline Sodium & & \\
\hline sodium & & \\
\hline sodium & & \\
\hline sodium & & \\
\hline stronti & & \\
\hline stronti & & \\
\hline stronti & & \\
\hline stronti & & \\
\hline Sulfate & & \\
\hline sulfate & & \\
\hline Sulfate & & \\
\hline Sulfate & & \\
\hline Sulfate & & \\
\hline Sulfate & & \\
\hline sulfate & & \\
\hline Sulfate & & \\
\hline Sulfate & & \\
\hline Sulfate & & \\
\hline Sulfate & & \\
\hline Sulfate & & \\
\hline Sulfate & & \\
\hline Sulfate & & \\
\hline Sulfate & & \\
\hline Sulfate & & \\
\hline sulfate & & \\
\hline Sulfate & & \\
\hline sulfate & & \\
\hline sulfate & & \\
\hline sulfate & & \\
\hline Sulfate & & \\
\hline Sulfate & & \\
\hline Sulfate & & \\
\hline Sulfate & & \\
\hline Sulfate & & \\
\hline Iranium & & \\
\hline Uranium & & \\
\hline Uranium & & \\
\hline
\end{tabular}

\begin{tabular}{|c|c|c|c|}
\hline Sample \# & Date & Method & Result \\
\hline $25183 E$ & $12 / 19 / 89$ & $I C P / M$ & $<5.00 \mathrm{E}+02$ \\
\hline $25185 \mathrm{E}$ & $1 / 02 / 90$ & $I C P / M$ & $<5.00 \mathrm{E}+02$ \\
\hline $25187 E$ & $1 / 09 / 90$ & $I C P / M$ & $<5.00 \mathrm{E}+02$ \\
\hline $25189 \mathrm{E}$ & $1 / 16 / 90$ & $I C P / M$ & $<5.00 \mathrm{E}+02$ \\
\hline 25191E & $1 / 23 / 90$ & $I C P / M$ & $<5.00 \mathrm{E}+02$ \\
\hline $25193 E$ & $1 / 30 / 90$ & $I C P / M$ & $<5.00 \mathrm{E}+02$ \\
\hline $25195 E$ & $2 / 06 / 90$ & $I C P / M$ & $<5.00 E+02$ \\
\hline $25197 \mathrm{E}$ & $2 / 13 / 90$ & $I C P / M$ & $<5.00 \mathrm{E}+02$ \\
\hline $25199 E$ & $2 / 20 / 90$ & $I C P / M$ & $<5.00 \mathrm{E}+02$ \\
\hline $25201 E$ & $2 / 27 / 90$ & $I C P / M$ & $<5.00 \mathrm{E}+02$ \\
\hline $25203 E$ & $3 / 06 / 90$ & $I C P / M$ & $<5.00 E+02$ \\
\hline $25205 \mathrm{E}$ & $3 / 13 / 90$ & $I C P / M$ & $<5.00 E+02$ \\
\hline $50873 E$ & $1 / 03 / 90$ & ICP & $<5.00 \mathrm{E}+02$ \\
\hline $50959 E$ & $2 / 21 / 90$ & $I C P$ & $<5.00 \mathrm{E}+02$ \\
\hline $51012 \mathrm{E}$ & $3 / 05 / 90$ & ICP & $<5.00 \mathrm{E}+02$ \\
\hline $51091 \mathrm{E}$ & $3 / 26 / 90$ & ICP & $<5.00 \mathrm{E}+02$ \\
\hline 50873 & $1 / 03 / 90$ & ICP & $1.08 E+03$ \\
\hline 50959 & $2 / 21 / 90$ & ICP & $9.59 \mathrm{E}+02$ \\
\hline 5101.2 & $3 / 05 / 90$ & ICP & $1.87 \mathrm{E}+03$ \\
\hline 51091 & $3 / 26 / 90$ & ICP & 2. $21 \mathrm{E}+03$ \\
\hline 50873 & $1 / 03 / 90$ & ICP & $2.50 \mathrm{E}+01$ \\
\hline 50959 & $2 / 21 / 90$ & ICP & $3.70 E+01$ \\
\hline 51012 & $3 / 05 / 90$ & ICP & $8.00 E+01$ \\
\hline 5109.1 & $3 / 26 / 90$ & ICP & $9.60 \mathrm{E}+01$ \\
\hline 25160 & $10 / 10 / 89$ & IC & $1.00 \mathrm{E}+04$ \\
\hline 25162 & $10 / 17 / 89$ & IC & $5.20 \mathrm{E}+03$ \\
\hline 25167 & $10 / 24 / 89$ & IC & $9.40 \mathrm{E}+03$ \\
\hline 25169 & $10 / 31 / 89$ & IC & $5.70 E+03$ \\
\hline 25171 & $11 / 07 / 89$ & IC & $8.50 E+03$ \\
\hline 25173 & $11 / 14 / 89$ & IC & $1.02 \mathrm{E}+04$ \\
\hline 25175 & $11 / 21 / 89$ & IC & $4.30 \mathrm{E}+03$ \\
\hline 25177 & $11 / 28 / 89$ & IC & $5.20 E+03$ \\
\hline 25179 & $12 / 05 / 89$ & IC & $1.01 E+04$ \\
\hline 25181 & $12 / 12 / 89$ & IC & $4.70 E+03$ \\
\hline 25183 & $12 / 19 / 89$ & IC & $1.12 \mathrm{E}+04$ \\
\hline 25185 & $1 / 02 / 90$ & IC & $4.00 E+03$ \\
\hline 25187 & $1 / 09 / 90$ & IC & $6.80 \mathrm{E}+03$ \\
\hline 25189 & $1 / 16 / 90$ & IC & $1.49 \mathrm{E}+04$ \\
\hline 25191 & $1 / 23 / 90$ & IC & $1.67 \mathrm{E}+04$ \\
\hline 25193 & $1 / 30 / 90$ & IC & $1.72 \mathrm{E}+04$ \\
\hline 25195 & $2 / 06 / 90$ & IC & $1.03 E+04$ \\
\hline 25197 & $2 / 13 / 90$ & IC & $4.90 E+03$ \\
\hline 25199 & $2 / 20 / 90$ & IC & $6.30 \mathrm{E}+03$ \\
\hline 25201. & $2 / 27 / 90$ & IC & $1.66 \mathrm{E}+04$ \\
\hline 25203 & $3 / 06 / 90$ & IC & $1.91 \mathrm{E}+04$ \\
\hline 25205 & $3 / 13 / 90$ & IC & $2.12 \mathrm{E}+04$ \\
\hline 50873 & $1 / 03 / 90$ & $I C$ & $5.40 E+03$ \\
\hline 50959 & $2 / 21 / 90$ & $I C$ & $7.40 \mathrm{E}+03$ \\
\hline 51012 & $3 / 05 / 90$ & $I C$ & $1.80 \mathrm{E}+04$ \\
\hline 51091 & $3 / 26 / 90$ & IC & $2.32 E+04$ \\
\hline 50873 & $1,03,90$ & SEE & $<1.06 E+O O$ \\
\hline 51012 & $3 / 05 / 90$ & GEA & $2.62 \mathrm{E}+00$ \\
\hline 51091 & $3 / 26 / 90$ & GEA & $2.21 E+00$ \\
\hline
\end{tabular}


DATA FOR N Reactor Effluent

Continued

\begin{tabular}{|c|c|c|c|c|}
\hline Constituent & Sample \# & Date & Method & Result \\
\hline zinc & 50873 & $1 / 03 / 90$ & ICP & $9.00 \mathrm{E}+00$ \\
\hline Zinc & 50959 & $2 / 21 / 90$ & ICP & $1.00 E+01$ \\
\hline Zinc & 51012 & $3 / 05 / 90$ & ICP & 1. $10 \mathrm{E}+01$ \\
\hline Zinc & 51091 & $3 / 26 / 90$ & ICP & 1.30E+01 \\
\hline Acetone & 25158 & $10 / 03 / 89$ & $\mathrm{ABN}$ & $<1.00 \mathrm{E}+01$ \\
\hline Acetone & 25160 & $10 / 10 / 89$ & VOA & $<1.00 \mathrm{E}+01$ \\
\hline Acetone & 25160 & $10 / 10 / 89$ & $A B N$ & $<1.00 \mathrm{E}+01$ \\
\hline Acetone & 25162 & $10 / 17 / 89$ & VOA & $1.40 \mathrm{E}+01$ \\
\hline Acetone & 25162 & $10 / 17 / 89$ & $\mathrm{ABN}$ & $<1.00 E+01$ \\
\hline Acetone & 25167 & $10 / 24 / 89$ & VOA & $<1.00 \mathrm{E}+01$ \\
\hline Acetone & 25167 & $10 / 24 / 89$ & $A B N$ & $<1.00 E+01$ \\
\hline Acetone & 25169 & $10 / 31 / 89$ & VOA & $<1.00 \mathrm{E}+0 \mathrm{I}$. \\
\hline Acetone & 25169 & $10 / 31 / 89$ & $\mathrm{ABN}$ & $<1.00 \mathrm{E}+01$ \\
\hline Acetone & 25171 & $11 / 07 / 89$ & VOA & $<1.00 E+01$ \\
\hline Acetone & 25171 & $11 / 07 / 89$ & $A B N$ & $<1.00 \mathrm{E}+01$ \\
\hline Acetone & 25173 & $11 / 14 / 89$ & VOA & $<1.00 \mathrm{E}+01$ \\
\hline 7.cetone & 25173 & $11 / 14 / 89$ & $A B N$ & $<1.20 \mathrm{E}+01$ \\
\hline Acetone & 25175 & $11 / 21 / 89$ & VOA & $<1.00 \mathrm{E}+01$ \\
\hline Acetone & 25175 & $11 / 21 / 89$ & $\mathrm{ABN}$ & $<1.16 \mathrm{E}+01$ \\
\hline Acetone & 25177 & $11 / 28 / 89$ & VOA & $1.20 \mathrm{E}+01$ \\
\hline Acetone & 25177 & $11 / 28 / 89$ & $A B N$ & $=1.00 E+01$ \\
\hline Acetone & 25173 & $12 / 05 / 89$ & VOA & $1.00 E+01$ \\
\hline Acetone & 25179 & $12 / 05 / 89$ & $A B N$ & $<1.00 E+01$ \\
\hline Acetone & 25181 & $12 / 12 / 89$ & VOA & $<1.00 E+01$ \\
\hline Acetone & 25181 & $12 / 12 / 89$ & $A B N$ & $<1.00 E+01$ \\
\hline Acetone & 25183 & $12 / 19 / 89$ & VOA & $3.70 \mathrm{E}+01$ \\
\hline Acetone & 25183 & $12 / 19 / 89$ & $A B N$ & $<1.00 E+01$ \\
\hline Acetone & 25185 & $1 / 02 / 90$ & VOA & $<1.00 E+01$ \\
\hline Acetone & 25185 & $1 / 02 / 90$ & $A B N$ & $<1.00 E+01$ \\
\hline Acetone & 25187 & $1 / 09 / 90$ & VOA & 1. 1OE+01 \\
\hline Acetone & 25187 & $1 / 09 / 90$ & $A B N$ & $<1.00 E+01$ \\
\hline Acetone & 25189 & $1 / 16 / 90$ & VOA & $<5.00 E+00$ \\
\hline Acetone & 25189 & $1 / 16 / 90$ & $A B N$ & $<1.00 E+01$ \\
\hline Acetone & 25191 & $1 / 23 / 90$ & VOA & $<1.00 E+01$ \\
\hline Acetone & 25191 & $1 / 23 / 90$ & $A B N$ & $<1.00 E+01$ \\
\hline Acetone & 25133 & $1 / 30 / 90$ & VOA & $<1.00 \mathrm{E}+01$ \\
\hline Acctone & 25193 & $1 / 30 / 90$ & $\mathrm{ABN}$ & $<1.20 \mathrm{E}+01$ \\
\hline Acetone & 25195 & $2 / 06 / 90$ & VOA & $<1.00 \mathrm{E}+01$ \\
\hline Acetone & 25195 & $2 / 06 / 90$ & $A B N$ & $<1.12 \mathrm{E}+01$ \\
\hline Acetone & 25197 & $2 / 13 / 90$ & VOA & $2.90 \mathrm{E}+01$ \\
\hline Acetone & 25197 & $2 / 13 / 90$ & $A B N$ & $<1.00 E+01$ \\
\hline Acetone & 25199 & $2 / 20 / 90$ & VOA & $<1.00 E+01$ \\
\hline Acetone & 25199 & $2 / 20 / 90$ & $A B N$ & $<1.14 \mathrm{E}+01$ \\
\hline Acetone & 25201 & $2 / 27 / 90$ & VOA & $1.51 E+02$ \\
\hline Acetone & 25201 & $2 / 27 / 90$ & $\mathrm{ABN}$ & $<1.10 E+01$ \\
\hline Acetone & 25203 & $3 / 06 / 90$ & VOA & $<6.00 \mathrm{E}+00$ \\
\hline Acetone & 25203 & $3 / 06 / 90$ & $A B N$ & $<1.10 E+01$ \\
\hline Acetone & 25205 & $3 / 13 / 90$ & VOA & $<1.00 E+01$ \\
\hline Acetore & 25205 & $3 / 13 / 90$ & $\mathrm{ABN}$ & $<1.30 E+01$ \\
\hline Acetone & 50873 & $1 / 03 / 90$ & VOA & $<1.00 E+01$ \\
\hline Acetone & 50873 & $1 / 03 / 90$ & $A B N$ & $<1.00 \mathrm{E}+01$ \\
\hline Acetone & $50873 B$ & $1 / 03 / 90$ & VOA & $<1.00 E+01$ \\
\hline Acetone & 50959 & $2 / 21 / 90$ & VOA & $<1.00 E+01$ \\
\hline
\end{tabular}


WHC-EP-0355, Appendix D

DATA FOR N Reactor Effluent

Continued

\begin{tabular}{|c|c|c|c|c|}
\hline Constituent & Sample \# & Date & Method & Result \\
\hline Acetone & 50959 & $2 / 21 . / 90$ & $\mathrm{ABN}$ & $<1.11 E+01$ \\
\hline Acetone & $50959 B$ & $2 / 21 / 90$ & VOA & $<1.00 E+01$ \\
\hline Acetone & $50959 \mathrm{~T}$ & $2 / 21 / 90$ & VOA & $<1.00 E+01$ \\
\hline Acetone & 51012 & $3 / 05 / 90$ & VOA & $<1.00 E+01$ \\
\hline Acetone & 51012 & $3 / 05 / 90$ & $A B N$ & $\because 1.00 E+01$ \\
\hline Acetone & $51012 B$ & $3 / 05 / 90$ & VOA & $<1.00 E+01$ \\
\hline Acetone & $51012 \mathrm{~T}$ & $3 / 05 / 90$ & VOA & $<1.00 E+01$ \\
\hline Acetone & 51091 & $3 / 26 / 90$ & VOA & $<1.00 \mathrm{E}+01$ \\
\hline Acetone & 51091 & $3 / 26 / 90$ & $\mathrm{ABN}$ & $<1.00 E+01$ \\
\hline Acetone & $51091 B$ & $3 / 26 / 90$ & VOA & $6.00 \mathrm{E}+00$ \\
\hline Acetone & $51091 \mathrm{~T}$ & $3 / 26 / 90$ & VOA & $<1.00 \mathrm{E}+01$ \\
\hline Ammonia & 25158 & $10 / 03 / 89$ & ISE & $1.58 \mathrm{E}+02$ \\
\hline Ammonia & 25160 & $10 / 10 / 89$ & ISE & 1. $40 \mathrm{E}+02$ \\
\hline Ammonia & 25162 & $10 / 17 / 89$ & ISE & $2.47 \mathrm{E}+02$ \\
\hline Ammonia & 25167 & $10 / 24 / 89$ & ISE & $3.72 E+02$ \\
\hline Ammonia & 25169 & $10 / 31 / 89$ & ISE & $2.89 E+02$ \\
\hline Ammonia & 25171 & $11 / 07 / 89$ & ISE & 3. $45 \mathrm{E}+02$ \\
\hline Ammonia & 25173 & $11 / 14 / 89$ & ISE & $<5.00 \mathrm{E}+01$ \\
\hline Ammonia & 25175 & $11 / 21 / 89$ & ISE & $1.98 \mathrm{E}+02$ \\
\hline Ammonia & 25177 & $11 / 28 / 89$ & ISE & $6.03 E+02$ \\
\hline Ammonia & 25179 & $12 / 05 / 89$ & ISE & $1.91 E+02$ \\
\hline Ammonia & 25181 & $12 / 12 / 89$ & ISE & $2.42 \mathrm{E}+02$ \\
\hline Ammonia & 25183 & $12 / 19 / 89$ & ISE & $2.65 \mathrm{E}+02$ \\
\hline Anmonia & 25185 & $1 / 02 / 90$ & ISE & $7.50 \mathrm{E}+01$ \\
\hline Ammonia & 25187 & $1 / 09 / 90$ & ISE & $7.70 \mathrm{E}+01$ \\
\hline Ammonia & 25189 & $1 / 16 / 90$ & ISE & $5.67 E+02$ \\
\hline Ammonia & 25191 & $1 / 23 / 90$ & ISE & $5.50 \mathrm{E}+01$ \\
\hline Ammonia & 25193 & $1 / 30 / 90$ & ISE & $4.02 \mathrm{E}+02$ \\
\hline Ammonia & 25195 & $2 / 06 / 90$ & ISE & $9.20 \mathrm{E}+01$ \\
\hline Ammonia & 25197 & $2 / 13 / 90$ & ISE & 1. 24E+02 \\
\hline Ammonia & 25199 & $2 / 20 / 90$ & ISE & $1.02 \mathrm{E}+02$ \\
\hline Ammonia & 25201 & $2 / 27 / 90$ & ISE & $9.60 \mathrm{E}+01$ \\
\hline Ammonia & 25203 & $3 / 06 / 90$ & ISE & $<5.00 E+01$ \\
\hline Ammonia & 25205 & $3 / 13 / 90$ & ISE & $<5.00 E+01$ \\
\hline Ammonia & 50873 & $1 / 03 / 90$ & ISE & $<5.00 \mathrm{E}+01$ \\
\hline Ammonia & 50959 & $2 / 21 / 90$ & ISE & $<5.00 E+01$ \\
\hline Ammonia & 51012 & $3 / 05 / 90$ & ISE & $<5.00 E+01$ \\
\hline Ammonia & 51091 & $3 / 26 / 90$ & ISE: & $<5.00 E+01$ \\
\hline Benzoic acid & 25203 & $3 / 06 / 90$ & P.EN & $1.10 E+02$ \\
\hline 1-Butanol & 25203 & $3 / 06 / 90$ & $\operatorname{VOA}$ & $3.00 E+01$ \\
\hline 1-Butanol & 50873 & $1 / 03 / 90$ & DIGS & $<1.00 E+04$ \\
\hline 1-Butanol & 50959 & $2 / 21 / 90$ & DIGC & $<1.00 E+04$ \\
\hline 1-Butanol & 51012 & $3 / 05 / 90$ & VOA & $2.40 \mathrm{E}+01$ \\
\hline 1-Butanol & 51012 & $3 / 05 / 90$ & DIGC & $<1.00 E+04$ \\
\hline 1-Butanol & 51091 & $3 / 26 / 90$ & DIGC & $<1.00 E+04$ \\
\hline 2-Butanone & 25160 & $10 / 10 / 89$ & VOA & $1.65 \mathrm{E}+02$ \\
\hline 2-Butanone & 25162 & $10 / 17 / 89$ & VOA & $<1.00 \mathrm{E}+01$ \\
\hline 2-Butanone & 25167 & $10 / 24 / 89$ & VOA & $<1.00 E+01$ \\
\hline 2-Butanone & 25169 & $10 / 31 / 89$ & VOA & $<1.00 E+01$ \\
\hline 2-Butanone & 25171 & $11 / 07 / 89$ & VOA & I. $78 E+02$ \\
\hline 2-Butanone & 25173 & $11 / 14 / 89$ & VOA & $2.30 E+01$ \\
\hline 2-Butanone & 25175 & $11 / 21,89$ & YִDA & < ? . OOE +OO \\
\hline 2-Butanone & 25177 & $11 / 28 / 89$ & VOA & $<1.00 E+01$ \\
\hline
\end{tabular}


DATA FOR N Reactor Effluert

Continued

\begin{tabular}{|c|c|c|c|c|}
\hline Constituent & Sample \# & Date & Method & Result \\
\hline 2-Butanone & 25179 & $12 / 05 / 89$ & VOA & $<5.00 \mathrm{E}+00$ \\
\hline 2-Butanone & 25181 & $12 / 12 / 89$ & VOA & $<1.00 E+01$ \\
\hline 2-Butanone & 25183 & $12 / 19 / 89$ & VOA & $<8.00 E+00$ \\
\hline 2-Butanone & 25185 & $1 / 02 / 90$ & VOA & $<1.00 \mathrm{E}+01$ \\
\hline 2-Butanone & 25187 & $1 / 09 / 90$ & VOA & $<1.00 \mathrm{E}+01$ \\
\hline 2-Butanone & 25189 & $1 / 16 / 90$ & VOA & $<1: 00 E+01$ \\
\hline 2-Butanone & 25191 & $1 / 2.3 / 90$ & VOA & $<1.00 E+01$ \\
\hline 2-Butanone & 25193 & $1 / 30 / 90$ & VOA & $<9.00 \mathrm{E}+00$ \\
\hline 2-Butanone & 25195 & $2 / 06 / 90$ & VOA & $1.00 \mathrm{E}+01$ \\
\hline 2-Butanone & 25197 & $2 / 13 / 90$ & VOA & $1.90 \mathrm{E}+01$ \\
\hline 2-Butanone & 25199 & $2 / 20 / 90$ & VOA & $<1.00 E+01$ \\
\hline 2-Butanone & 25201 & $2 / 27 / 90$ & VOA & $1.90 \mathrm{E}+01$ \\
\hline 2-Butanone & 25203 & $3 / 06 / 90$ & VOA & $1.10 \mathrm{E}+01$ \\
\hline 2-Butanone & 25205 & $3 / 13 / 90$ & VOA & $2.30 E+01$ \\
\hline 2-Butanone & 50873 & $1 / 03 / 90$ & VOA & $<1.00 E+01$ \\
\hline 2-Butanone & $50873 B$ & $1 / 03 / 90$ & VOA & $<1.00 E+01$ \\
\hline 2-Butanone & 50959 & $2 / 21 / 90$ & VOA & $<1.00 \mathrm{E}+01$ \\
\hline 2-Butanone & $50959 B$ & $2 / 21 / 90$ & VOA & $<1.00 \mathrm{E}+01$ \\
\hline 2-Butanone & $50959 \mathrm{~T}$ & $2 / 21 / 90$ & VOA & $<1.00 \mathrm{E}+01$ \\
\hline 2-Butanone & 51012 & $3 / 05 / 90$ & VOA & $<1.00 \mathrm{E}+01$ \\
\hline 2-Butanone & $51012 \mathrm{~B}$ & $3 / 05 / 90$ & VOA & $<1.00 E+01$ \\
\hline 2-Butanone & $51012 \mathrm{~T}$ & $3 / 05 / 90$ & VOA & $<1.00 \mathrm{E}+01$ \\
\hline 2-Butanone & 51091 & $3 / 26 / 90$ & VOA & $<1.00 E+01$ \\
\hline 2-Butanone & $51091 \mathrm{~B}$ & $3 / 26 / 90$ & VOA & $<1.00 \mathrm{E}+01$ \\
\hline 2-Butanone & $51091 T$ & $3 / 26 / 90$ & VOA & $<1.00 E+01$ \\
\hline Butylated hydroxy toluene & 25193 & $1 / 30 / 90$ & $A B N$ & $1.10 E+01$ \\
\hline Butylated hydroxy toluene & 25195 & $2 / 06 / 90$ & $A B N$ & $2.00 E+01$ \\
\hline Butylated hydroxy toluene & 25197 & $2 / 13 / 90$ & $A B N$ & $8.00 \mathrm{E}+00$ \\
\hline Butylated hydroxy toluene & 25199 & $2 / 20 / 90$ & $\mathrm{ABN}$ & $8.00 \mathrm{E}+00$ \\
\hline Butylated hydroxy toluene & 25201 & $2 / 27 / 90$ & $A B N$ & $8.00 E+00$ \\
\hline Butylated hydroxy toluene & 50959 & $2 / 21 / 90$ & $\mathrm{ABN}$ & $9.00 E+00$ \\
\hline Dichloromethane & 25160 & $10 / 10 / 89$ & VOA & $<5.00 \mathrm{E}+00$ \\
\hline Dichloromethane & 25162 & $10 / 17 / 89$ & VOA & $<5.00 \mathrm{E}+00$ \\
\hline Dichloromethane & 25167 & $10 / 24 / 89$ & VOA & $<5.0 O E+O C$ \\
\hline Dichloromethane & 25169 & $10 / 31 / 89$ & VOA & $<5.00 E+00$ \\
\hline Dichloromethane & 25171 & $11 / 07 / 89$ & VOA & $<5.00 E+00$ \\
\hline Dichloromethane & 25173 & $11 / 14 / 89$ & VOA & $<5.00 E+00$ \\
\hline Dichloromethane & 25175 & $11 / 21 / 89$ & VOA & $<5.00 \mathrm{E}+00$ \\
\hline Dichloromethane & 25177 & $11 / 28 / 89$ & VOA & $<5.00 E+00$ \\
\hline Dichloromethane & 25179 & $12 / 05 / 89$ & VOA & $<5.00 \mathrm{E}+00$ \\
\hline Dichloromethane & 25181 & $12 / 12 / 89$ & VOA & $<5.00 E+00$ \\
\hline Dichloromethane & 25183 & $12 / 19 / 89$ & VOA & $<5.00 E+00$ \\
\hline Dichloromethane & 25185 & $1 / 02 / 90$ & VOA & $<5.00 \mathrm{E}+00$ \\
\hline Dichloromethane & 25187 & $1 / 09 / 90$ & VOA & $<5.00 E+00$ \\
\hline Dichloromethane & 25189 & $1 / 16 / 90$ & VOA & $<5.00 \mathrm{E}+00$ \\
\hline Dichloromethane & 25191 & $1 / 23 / 90$ & VOA & $<5.00 \mathrm{E}+00$ \\
\hline Dichloromethane & 25193 & $1 / 30 / 90$ & VOA & $<5.00 E+00$ \\
\hline Dichloromethane & 25195 & $2 / 06 / 90$ & VOA & $<5.00 E+00$ \\
\hline Dichloromethane & 25197 & $2 / 13 / 90$ & VOA & $<5.00 \mathrm{E}+00$ \\
\hline Dichloromethane & 25199 & $2 / 20 / 90$ & VOA & $<5.00 \mathrm{E}+00$ \\
\hline Dichloromethane & 25201 & $2 / 27 / 90$ & VOA & $<5.00 E+00$ \\
\hline Di chloromethane & 2520.3 & $3 / 06 / 90$ & ỴDA & $<5.0 O E+00$ \\
\hline Dichloromethane & 25205 & $3 / 13 / 90$ & VOA & $<5.00 E+00$ \\
\hline
\end{tabular}


DATA FOR N Reactor Effluent

Continued

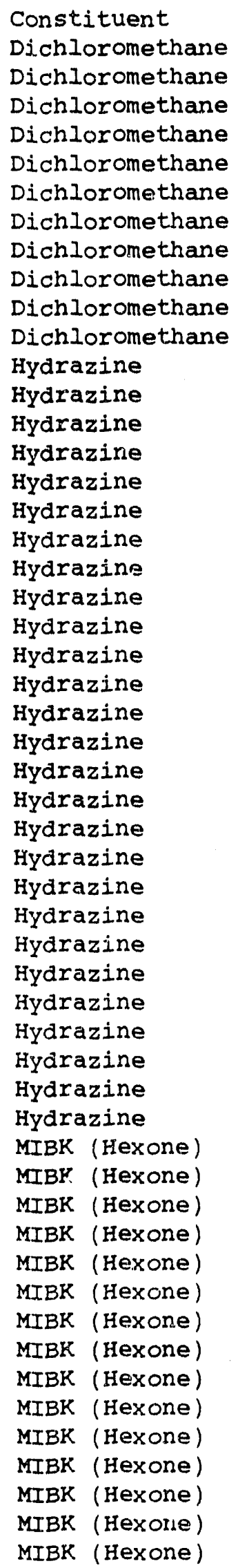

\begin{tabular}{|c|c|c|c|}
\hline Sample \# & Date & Method & Result \\
\hline 50873 & $1 / 03 / 90$ & VOA & $<5.00 \mathrm{E}+00$ \\
\hline $50873 B$ & $1 / 03 / 90$ & VOA & $<5.00 \mathrm{E}+00$ \\
\hline 50959 & $2 / 21 / 90$ & VOA & $<5.00 E+00$ \\
\hline $50959 B$ & $2 / 21 / 90$ & VOA & $<3.00 \mathrm{E}+00$ \\
\hline $50959 \mathrm{~T}$ & $2 / 21 / 90$ & VOA & $<5.00 E+00$ \\
\hline 51012 & $3 / 05 / 90$ & VOA & $<5.00 E+00$ \\
\hline $51012 \mathrm{~B}$ & $3 / 05 / 90$ & VOA & $<5.00 E+00$ \\
\hline $51012 \mathrm{~T}$ & $3 / 05 / 90$ & VOA & $<5.00 \mathrm{E}+00$ \\
\hline 51091 & $3 / 26 / 90$ & VOA & $<5.00 \mathrm{E}+00$ \\
\hline $51091 B$ & $3 / 26 / 90$ & VOA & $5.0 O E \perp 00$ \\
\hline $51091 \mathrm{~T}$ & $3 / 26 / 90$ & VOA & $<5.00 E+00$ \\
\hline 25158 & $10 / 03 / 89$ & SPEC & $<3.00 E+01$ \\
\hline 25160 & $10 / 10 / 89$ & SPEC & $<3.00 \mathrm{E}+01$ \\
\hline 25162 & $10 / 17 / 89$ & SPEC & $4.40 E+01$ \\
\hline 25167 & $10 / 24 / 89$ & SPEC & $<3.00 E+01$ \\
\hline 25169 & $10 / 31 / 89$ & SPEC & $<3.00 E+01$ \\
\hline 25171 & $11 / 07 / 89$ & SPEC & $<3.00 \mathrm{E}+01$ \\
\hline 25173 & $11 / 14 / 89$ & SPEC & $<3.00 E+01$ \\
\hline 25175 & $11 / 21 / 89$ & SPEC & $<3.00 \mathrm{E}+01$ \\
\hline 25177 & $11 / 28 / 89$ & SPEC & $<3.00 \mathrm{E}+01$ \\
\hline 25179 & $12 / 05 / 89$ & SPEC & $<3.00 \mathrm{E}+01$ \\
\hline 25181 & $12 / 12 / 89$ & SPEC & $<3.00 \mathrm{E}+01$ \\
\hline 25183 & $12 / 19 / 89$ & SPEC & $<3.00 \mathrm{E}+01$ \\
\hline 25185 & $1 / 02 / 90$ & SPEC & $<3.00 E+01$ \\
\hline 25187 & $1 / 09 / 90$ & SPEC & $<3.00 \mathrm{E}+01$ \\
\hline 25189 & $1 / 16 / 90$ & SPEC & $<3.00 E+01$ \\
\hline 25191 & $1 / 23 / 90$ & SPEC & $<3.00 E+01$ \\
\hline 25193 & $1 / 30 / 90$ & SPEC & $<3.00 E+01$ \\
\hline 25195 & $2 / 06 / 90$ & SPEC & $<3.00 E+01$ \\
\hline 25197 & $2 / 13 / 90$ & SPEC & $<3.00 E+01$ \\
\hline 25199 & $2 / 20 / 90$ & SPEC & $<3.00 E+01$ \\
\hline 25201 & $2 / 27 / 90$ & SPEC & $<3.00 \mathrm{E}+01$ \\
\hline 25203 & $3 / 06 / 90$ & SPEC & $<3.00 E+01$ \\
\hline 25205 & $3 / 13 / 90$ & SPEC & $<3.00 E+01$ \\
\hline 50873 & $1 / 03 / 90$ & SPEC & $<3.00 \mathrm{E}+01$ \\
\hline 50959 & $2 / 21 / 90$ & SPEC & $<3.00 \mathrm{E}+01$ \\
\hline 51012 & $3 / 05 / 90$ & SPEC & $<3.00 E+01$ \\
\hline 51091 & $3 / 26 / 90$ & SPEC & $<3.00 E+01$ \\
\hline 25160 & $10 / 10 / 89$ & VOA & $<1.00 E+01$ \\
\hline 25162 & $10 / 17 / 89$ & VOA & $<1.00 \mathrm{E}+01$ \\
\hline 25167 & $10 / 24 / 89$ & VOA & $<1.00 E+01$ \\
\hline 25169 & $10 / 31 / 89$ & YOA & $<1.00 E+01$ \\
\hline 25171 & $11 / 07 / 89$ & VOA & $<1.00 E+01$ \\
\hline 25173 & $11 / 14 / 89$ & VOA & $<1.00 \mathrm{E}+01$ \\
\hline 25175 & $11 / 21 / 89$ & VOA & $<1.00 E+01$ \\
\hline 25177 & $11 / 28 / 89$ & VOA & $<1.00 E+01$ \\
\hline 25179 & $12 / 05 / 89$ & VOA & $-1.00 \mathrm{E}+01$ \\
\hline 25181 & $12 / 12 / 89$ & VOA & $<1.00 \mathrm{E}+01$ \\
\hline 25183 & $12 / 19 / 89$ & VOA & $<1.00 E+01$ \\
\hline 25185 & $1 / 02 / 90$ & VOA & $<1.00 \mathrm{E}+01$ \\
\hline 25187 & $1 / 09 / 90$ & VOA & $<1.00 E+01$ \\
\hline 25189 & $1 / 16 / 90$ & VOA & $<1.00 E+01$ \\
\hline 25191 & $1 / 23 / 90$ & VOA & $<1.00 E_{r} 01$ \\
\hline
\end{tabular}


DATA FOR N Reactor Effluent Continued

Constituent
MIBK (Hexone)
MIBK (Hexone)
MIBK (Hexone)
MIBK (Hexone)
MIBK (Hexone)
MIBK (Hexone)
MIBK (Hexone)
MIBK (Hexone)
MIBK (Hexone)
MIBK (Hexone)
MIBK (Hexone)
MIBK (Hexone)
MIBK (Hexone)
MIBK (Hexone)
MrBK (Hexone)
MIBK (Hexone)
MIBK (Hexone)
MIBK (Hexone)
Toluene
Toluene
Toluene
Toluene
Toluene
Toluene
Toluene
Toluene
Toluene
Toluene
Toluene
Toluene
Toluene
Toluene
Toluene
Toluene
Toluene
Toluene
Toluene
Toluene
Toluene
Toluene
Toluene
Toluene
Toluene
Toluene
Toluene
Toluene
Toluene
Trichluene
Iricilurometilaine

\begin{tabular}{|c|c|c|c|}
\hline Sample \# & Date & Method & Result \\
\hline 25193 & $1 / 30 / 90$ & VOA & 1. $30 \mathrm{E}+01$ \\
\hline 25195 & $2 / 06 / 90$ & VOA & $<1.00 E+01$ \\
\hline 25197 & $2 / 13 / 90$ & VOA & $<1.00 E+01$ \\
\hline 25199 & $2 / 20 / 90$ & VOA & $<1.00 \mathrm{E}+01$ \\
\hline 25201 & $2 / 27 / 90$ & VOA & $<1.00 E+01$ \\
\hline 25203 & $3 / 06 / 90$ & VOA & $<1.00 E+01$ \\
\hline 25205 & $3 / 13 / 90$ & VOA & $<1.00 E+01$ \\
\hline 50873 & $1 / 03 / 90$ & VOA & $<1.00 E+01$ \\
\hline $50873 B$ & $1 / 03 / 90$ & VOA & $<1.00 E+01$ \\
\hline 50959 & $2 / 21 / 90$ & VOA & $<1.00 \mathrm{E}+01$ \\
\hline $50959 B$ & $2 / 21 / 90$ & VOA & $<1.00 \mathrm{E}+01$ \\
\hline $50959 \mathrm{~T}$ & $2 / 21 / 90$ & VOA & $<1.00 E+01$ \\
\hline 51012 & $3 / 05 / 90$ & VOA & $<1.00 \mathrm{E}+01$ \\
\hline $51012 \mathrm{~B}$ & $3 / 05 / 90$ & VOA & $<1.00 \mathrm{E}+01$ \\
\hline $51012 \mathrm{~T}$ & $3 / 05 / 90$ & VOA & $<1.00 \mathrm{E}+01$ \\
\hline 51091 & $3 / 26 / 90$ & VOA & $<1.00 E+01$ \\
\hline 51091B & $3 / 26 / 90$ & VOA & $<1.00 \mathrm{E}+01$ \\
\hline $51091 \mathrm{~T}$ & $3 / 26 / 90$ & VOA & $<1.00 E+01$ \\
\hline 25160 & $10 / 10 / 89$ & VOA & $<5.00 \mathrm{E}+00$ \\
\hline 25162 & $10 / 17 / 89$ & VOA & $<5.00 E+00$ \\
\hline 25167 & $10 / 24 / 89$ & VOA & $<5.00 \mathrm{E}+00$ \\
\hline 25169 & $10 / 31 / 89$ & VOA & $<5.00 \mathrm{E}+00$ \\
\hline 25171 & $11 / 07 / 89$ & VOA & $<5.00 E+00$ \\
\hline 25173 & $11 / 14 / 89$ & VOA & $<5.00 E+00$ \\
\hline 25175 & $11 / 21 / 89$ & VOA & $<3.00 E+00$ \\
\hline 25177 & $11 / 28 / 89$ & VOA & $<5.00 \mathrm{E}+00$ \\
\hline 25179 & $12 / 05 / 89$ & VOA & $5.00 \mathrm{E}+00$ \\
\hline 25181 & $12 / 12 / 89$ & VOA & $1.00 \mathrm{E}+01$ \\
\hline 25183 & $12 / 19 / 89$ & VOA & $<4.00 E+00$ \\
\hline 25185 & $1 / 02 / 90$ & VOA & $<5.00 E+00$ \\
\hline 25187 & $1 / 09 / 90$ & VOA & $<5.00 \mathrm{E}+00$ \\
\hline 25189 & $1 / 16 / 90$ & VOA & $<5.00 \mathrm{E}+00$ \\
\hline 25191 & $1 / 23 / 90$ & VOA & $<5.00 \mathrm{E}+00$ \\
\hline 25193 & $1 / 30 / 90$ & VOA & $<5.00 \mathrm{E}+00$ \\
\hline 25195 & $2 / 06 / 90$ & VOA & $<5.00 \mathrm{E}+00$ \\
\hline 25197 & $2 / 13 / 90$ & VOA & $<5.00 E+00$ \\
\hline 25199 & $2 / 20 / 90$ & VOA & $<5.00 \mathrm{E}+00$ \\
\hline 25201 & $2 / 27 / 90$ & VOA & $<5.00 \mathrm{E}+00$ \\
\hline 25203 & $3 / 06 / 90$ & VOA & $<5.00 \mathrm{E}+00$ \\
\hline 25205 & $3 / 13 / 90$ & VOA & $<5.00 \mathrm{E}+00$ \\
\hline 50873 & $1 / 03 / 90$ & VOA & $<5.00 \mathrm{E}+00$ \\
\hline $50873 \mathrm{~B}$ & $1 / 03 / 90$ & VOA & $<5.00 \mathrm{E}+00$ \\
\hline 50959 & $2 / 21 / 90$ & VOA & $<5.00 E+00$ \\
\hline $50959 \mathrm{~B}$ & $2 / 21 / 90$ & VOA & $<5.00 \mathrm{E}+00$ \\
\hline $50959 \mathrm{~T}$ & $2 / 21 / 90$ & VOA & $<5.00 \mathrm{E}+00$ \\
\hline 51012 & $3 / 05 / 90$ & VOA & $<5.00 \mathrm{E}+00$ \\
\hline $51012 \mathrm{~B}$ & $3 / 05 / 90$ & VOA & $<5.00 E+00$ \\
\hline $51012 \mathrm{~T}$ & $3 / 05 / 90$ & VOA & $<5.00 E+00$ \\
\hline 51091 & $3 / 26 / 90$ & VOA & $<5.00 E+00$ \\
\hline $51091 \mathrm{~B}$ & $3 / 26 / 90$ & VOA & $<5.00 \mathrm{E}+00$ \\
\hline 51091T & $3 / 26 / 90$ & VOA & $<5.00 \mathrm{E}+00$ \\
\hline 25160 & $10 / 10 / 89$ & VOA & $<5.00 E+00$ \\
\hline 25162 & I0/I7/85 & $\bar{V} \overline{O A}$ & צ. UิUিE+UิU \\
\hline
\end{tabular}


DATA FOR N Reactor Effluent Continued

Constituent

Trichloromethane

Trichloromethane

Trichloromethane

Trichloromethane

Trichloromethane

Trichloromethane

Trichloromethane

Trichloromethane

Trichloromethane

Trichloromethane

Trichloromethane

Trichloromethane

Trichloromethane

Trichloromethane

Trichloromethane

Trichloromethane

Trichloromethane

Trichloromethane

Trichloromethane

Trichloromethane

Trichloromethane

Trichloromethane

Trichloromethane

Trichloromethane

Trichloromethane

Trichloromethane

Trichloromethane

Trichloromethane

Trichloromethane

Trichloromethane

Trichloromethane

Unknown

Unknown

Unknown

Unknown gly if

Unknown hy ocarbon

Unknown oxygenated $\mathrm{PAH}$

Unknown phthalate

Alkalinity (Method B)

Alkalinity (Method B)

Alkalinity (Method B)

Alkalinity (Method B)

Alkalinity (Method B)

Alkalinity (Method B)

Alkalinity (Method B)

Alkalinity (Method B)

Alralinity (Method B)

Alkalinity (Method B)

Alkalinity (Method B)

Alkalinity (Method B)

Alkalinity (Method B)

Alkalinity (Method B)

Ausuinity (sothod $D$ )

\begin{tabular}{|c|c|c|c|}
\hline Sample \# & Date & Method & Result \\
\hline 25167 & $10 / 24 / 89$ & VOA & $8.00 \mathrm{E}+00$ \\
\hline 25169 & $10 / 31 / 89$ & VOA & $6.00 \mathrm{E}+00$ \\
\hline 25171 & $11 / 07 / 89$ & VOA & $<4.00 \mathrm{E}+00$ \\
\hline 25173 & $11 / 14 / 89$ & VOA & $5.00 E+00$ \\
\hline 25175 & $11 / 21 / 89$ & VOA & $<5.00 \mathrm{E}+00$ \\
\hline 25177 & $11 / 28 / 89$ & VOA & $<4.00 E+00$ \\
\hline 25179 & $12 / 05 / 89$ & VOA & $<4.00 \mathrm{E}+00$ \\
\hline 25181 & $12 / 12 / 89$ & VOA & $<4.00 E+00$ \\
\hline 25183 & $12 / 19 / 89$ & VOA & $<3.00 \mathrm{E}+00$ \\
\hline 25185 & $1 / 02 / 90$ & VOA & $<5.00 \mathrm{E}+00$ \\
\hline 25187 & $1 / 09 / 90$ & VOA & $<5.00 \mathrm{E}+00$ \\
\hline 25189 & $1 / 16 / 90$ & VOA & $<5.00 \mathrm{E}+00$ \\
\hline 25191 & $1 / 23 / 90$ & VOA & $<5.00 E+00$ \\
\hline 25193 & $1 / 30 / 90$ & VOA & $<5.00 E+00$ \\
\hline 25195 & $2 / 06 / 90$ & VOA & $<3.00 \mathrm{E}+00$ \\
\hline 25197 & $2 / 13 / 90$ & VOA & $<5.00 E+00$ \\
\hline 25199 & $2 / 20 / 90$ & VOA & $<5.00 \mathrm{E}+00$ \\
\hline 25201 & $2 / 27 / 90$ & VOA & $<3.00 E+00$ \\
\hline 25203 & $3 / 06 / 90$ & VOA & $<3.00 E+00$ \\
\hline 25205 & $3 / 13 / 90$ & VOA & $<5.00 \mathrm{E}+00$ \\
\hline 50873 & $1 / 03 / 90$ & VOA & $<5.00 E+00$ \\
\hline $50873 \mathrm{~B}$ & $1 / 03 / 90$ & VOA & $<5.00 E+00$ \\
\hline 50959 & $2 / 21 / 90$ & VOA & $<5.00 \mathrm{E}+00$ \\
\hline $50959 B$ & $2 / 21 / 90$ & VOA & $6.00 \mathrm{E}+00$ \\
\hline $50959 \mathrm{~T}$ & $2 / 21 / 90$ & VOA & $6.00 E+00$ \\
\hline 51012 & $3 / 05 / 90$ & VOA & $<5.00 \mathrm{E}+00$ \\
\hline $51012 \mathrm{~B}$ & $3 / 05 / 90$ & VOA & $<5.00 E+00$ \\
\hline $51012 \mathrm{~T}$ & $3 / 05 / 90$ & VOA & $<5.00 E+00$ \\
\hline 51091 & $3 / 26 / 90$ & VOA & $<5.00 \mathrm{E}+00$ \\
\hline $51091 \mathrm{~B}$ & $3 / 26 / 90$ & VOA & $<4.00 E+00$ \\
\hline $51091 \mathrm{~T}$ & $3 / 26 / 90$ & VOA & $6.00 \mathrm{E}+00$ \\
\hline 25160 & $10 / 10 / 89$ & $\mathrm{ABN}$ & $9.50 \mathrm{E}+01$ \\
\hline 25173 & $11 / 14 / 89$ & $A B N$ & $2.40 \mathrm{E}+01$ \\
\hline 25191 & $1 / 23 / 90$ & $A B N$ & 2. $20 \mathrm{E}+01$ \\
\hline 25203 & $3 / 06 / 90$ & $\mathrm{ABN}$ & $2.80 E+01$ \\
\hline 25191 & $1 / 23 / 90$ & $A B N$ & $1.90 \mathrm{E}+\mathrm{O} 2$ \\
\hline 25203 & $3 / 06 / 90$ & $A B N$ & $9.00 E+00$ \\
\hline 25197 & $2 / 1.3 / 90$ & $A B N$ & $9.00 \mathrm{E}+00$ \\
\hline 25167 & $10 / 24 / 89$ & TITRA & 3. $40 \mathrm{E}+04$ \\
\hline 25169 & $10 / 31 / 89$ & TITRA & $2.10 E+04$ \\
\hline 25171 & $11 / 07 / 89$ & TITRA & $2.60 E+04$ \\
\hline 25173 & $11 / 14 / 89$ & TITRA & 3. $20 E+04$ \\
\hline 25175 & $11 / 21 / 89$ & TITRA & $<2.00 E+04$ \\
\hline 25177 & $11 / 28 / 89$ & TITRA & $<2.00 E+04$ \\
\hline 25179 & $12 / 05 / 89$ & TITRA & $3.00 E+04$ \\
\hline 25.181 & $12 / 12 / 89$ & TITRA & $<2.00 E+04$ \\
\hline 25183 & $12 / 19 / 89$ & TITRA & 2. $40 \mathrm{E}+04$ \\
\hline 25185 & $1 / 02 / 90$ & TITRA & $<2.00 \mathrm{E}+04$ \\
\hline 25187 & $1 / 09 / 90$ & TITRA & $<2.00 \mathrm{E}+04$ \\
\hline 25189 & $1 / 16 / 90$ & IIIILRA & 4. $10 \mathrm{E}+04$ \\
\hline 25191 & $1 / 23 / 90$ & TITRA & 4. $90 \mathrm{E}+04$ \\
\hline 25193 & $1 / 30 / 90$ & TITRA & $5.60 E+04$ \\
\hline 25235 & $2,06 / 30$ & TITPA & $2.705 \div 01$ \\
\hline
\end{tabular}


DATA FOR N Reactor Effluent

Continued

Constituent

Alkalinity (Method B)

Alkalinity (Method B)

Alkalinity (Method B)

Alkalinity (Method B)

Alkalinity (Method B)

Aralinity (Method B)

Alralinity (Method B)

Alkalinity (Method B)

Alkalinity (Method B)

Alpha Activity ( $\mathrm{pCi} / \mathrm{L}$ )

Alpha Activity (pCi/L)

Alpha Activity (pCi/L)

Beta Activity (pCi/L)

Beta Activity (pCi/L)

Beta Activity ( $\mathrm{pCi} / \mathrm{L}$ )

Conductivity (US)

Conductivity (us)

Conductivity (us)

Conductivity (us)

Ingitability (degrees F)

Ingitability (degrees F)

Ingitability (degrees E)

Ingitability (degrees F)

pH (dimensionless)

$\mathrm{pH}$ (dimensionless)

pH (dimensionless)

E. (dimensionless)

pH (dimensionless)

pH (dimensionless)

$\mathrm{pH}$ (dimensionless)

pH (dimensionless)

pH (dimensionless)

$\mathrm{pH}$ (dimensionless)

pH (dimensionless)

pH (dimensionless)

pH (dimensionless)

pH (dimensionless)

pH (dimensionless)

pH (dimensionless)

pH (dimensionless)

pH (dimensionless)

$\mathrm{pH}$ (dimensionless)

$\mathrm{pH}$ (dimensionless)

$\mathrm{pH}$ (dimensionless)

$\mathrm{pH}$ (dimensionless)

pH (dimensionless)

$\mathrm{pH}$ (dimensionless)

pH idiriensionless)

pH (dimensionless)

Reactivity Cyanide ( $\mathrm{mg} / \mathrm{kg}$ )

Reactivity Cyanide ( $\mathrm{mg} / \mathrm{kg}$ )

Reactivity Cyanide ( $\mathrm{mg} / \mathrm{kg}$ )

Reactivity Cyanide (mg/lrg)

\begin{tabular}{|c|c|c|c|}
\hline Sample \# & Date & Method & Result \\
\hline 25197 & $2 / 13 / 90$ & TITRA & $<2.00 \mathrm{E}+04$ \\
\hline 25199 & $2 / 20 / 90$ & TITRA & $<2.00 \mathrm{E}+04$ \\
\hline 25201 & $2 / 27 / 90$ & TITRA & $6.50 \mathrm{E}+04$ \\
\hline 25203 & $.3 / 06 / 90$ & TITRA & $4.10 \mathrm{E}+04$ \\
\hline 25205 & $3 / 13 / 90$ & TITRA & $4.90 E+04$ \\
\hline 50873 & $1 / 03 / 90$ & TITRA & $<2.00 E+04$ \\
\hline 50959 & $2 / 21 / 90$ & TITRA & 2. $40 E+04$ \\
\hline 51012 & $3 / 05 / 90$ & TITRA & 3. $90 E+04$ \\
\hline 51091. & $3 / 26 / 90$ & TITRA & $4.80 \mathrm{E}+04$ \\
\hline 50873 & $1 / 03 / 90$ & Alpha & $7.76 \mathrm{E}+00$ \\
\hline 51012 & $3 / 05 / 90$ & Alpha & 3. $93 \mathrm{E}+01$ \\
\hline 51091 & $3 / 26 / 90$ & Alpha & 4. $12 \mathrm{E}+01$ \\
\hline 50873 & $1 / 03 / 90$ & Beta & 1. $25 E+04$ \\
\hline 51012 & $3 / 05 / 90$ & Beta & $1.70 E+05$ \\
\hline 51091 & $3 / 26 / 90$ & Beta & $2.83 \mathrm{E}+04$ \\
\hline 50873 & $1 / 03 / 90$ & COND-FId & $5.60 \mathrm{E}+01$ \\
\hline 50959 & $2 / 21 / 90$ & COND-FId & $6.00 \mathrm{E}+0.1$ \\
\hline 51012 & $3 / 05 / 90$ & COND-FId & $1.57 \mathrm{E}+02$ \\
\hline 51091 & $3 / 26 / 90$ & COND-FId & $1.50 \mathrm{E}+02$ \\
\hline $50873 E$ & $1 / 03 / 90$ & IGNIT & 2. $12 \mathrm{E}+02$ \\
\hline 50959E & $2 / 21 / 90$ & IGNIT & $2.14 \mathrm{E}+02$ \\
\hline $51012 \mathrm{E}$ & $3 / 05 / 90$ & IGNIT & 2. $10 \mathrm{E}+02$ \\
\hline $51091 \mathrm{E}$ & $3 / 26 / 90$ & IGNIT & $1.94 \mathrm{E}+02$ \\
\hline 25160 & $10 / 10 / 89$ & $P H-L a b$ & $7.90 \mathrm{E}+00$ \\
\hline 25162 & $10 / 17 / 89$ & PH-Lab & $7.30 \mathrm{E}+00$ \\
\hline 25167 & $10 / 24 / 89$ & PH - Lab & $7.30 \mathrm{E}+00$ \\
\hline 25169 & $10 / 31 / 89$ & PH-Lab & $7.40 \mathrm{E}+00$ \\
\hline 25171 & $11 / 07 / 89$ & PH-Lab & $7.30 \mathrm{E}+00$ \\
\hline 25173 & $11 / 14 / 89$ & PH-Iab & $7.30 E+00$ \\
\hline 25175 & $11 / 21 / 89$ & PH-Lab & $6.90 \mathrm{E}+00$ \\
\hline 25177 & $11 / 28 / 89$ & PH-Lab & $7.10 \mathrm{E}+00$ \\
\hline 25179 & $12 / 05 / 89$ & PH-Lab & $7.00 E+00$ \\
\hline 25181 & $12 / 12 / 89$ & PH-Lab & $6.80 \mathrm{E}+00$ \\
\hline 25.1 .33 & $12 / 19 / 89$ & PH - Lab & $7.00 \mathrm{E}+00$ \\
\hline 25185 & $1 / 02 / 90$ & $\mathrm{PH}-\mathrm{Lab}$ & $6.90 E+00$ \\
\hline 25187 & $1 / 09 / 90$ & PH-Lab & $7.30 \mathrm{E}+00$ \\
\hline 25189 & $1 / 16 / 90$ & $\mathrm{PH}-\mathrm{Lab}$ & $7.30 E+00$ \\
\hline 25191 & $1 / 23 / 90$ & PH-Lab & $7.70 \mathrm{E}+00$ \\
\hline 25193 & $1 / 30 / 90$ & PH-Lab & $7.50 E+00$ \\
\hline 25195 & $2 / 06 / 90$ & PH-Lab & $7.20 \mathrm{E}+00$ \\
\hline 25197 & $2 / 13 / 90$ & PH.Lab & $7.10 \mathrm{E}+00$ \\
\hline 25199 & $2 / 20 / 90$ & $\mathrm{PH}-\mathrm{Lab}$ & $6.90 \mathrm{E}+00$ \\
\hline 25201 & $2 / 27 / 90$ & $\mathrm{PH}-\mathrm{Lab}$ & $7.40 \mathrm{E}+00$ \\
\hline 25203 & $3 / 06 / 90$ & PH-Lab & $7.50 \mathrm{E}+00$ \\
\hline 25205 & $3 / 13 / 90$ & PH-Lab & $7.60 \mathrm{E}+00$ \\
\hline 50873 & $1 / 03 / 90$ & $\mathrm{PH}-\mathrm{FIC}$ & $7.30 \mathrm{E}+00$ \\
\hline 50959 & $2 / 21 / 90$ & $I H-F I d$ & $7.10 \mathrm{E}+00$ \\
\hline 51012 & $3 / 05 / 90$ & $\mathrm{PH}-\mathrm{F} I \mathrm{~d}$ & $7.15 \mathrm{E}+00$ \\
\hline 51091 & $3 / 26 / 90$ & PH-FId & $8.00 \mathrm{E}+00$ \\
\hline $50873 E$ & $1 / 03 / 90$ & DSPEC & $<1.00 \mathrm{E}+02$ \\
\hline $50959 E$ & $2 / 21 / 90$ & DSPEC & $<1.00 \mathrm{E}+02$ \\
\hline $51012 \mathrm{E}$ & $3 / 05 / 90$ & DSPEC & $<1.00 \mathrm{E}+02$ \\
\hline $51091 \mathrm{E}$ & $3 / 26 / 90$ & DSPEC & $<1.00 \mathrm{E}+02$ \\
\hline
\end{tabular}

D -63 
DATA FOR $N$ Reactor Effluent

Continued

Constituent

Reactivity Sulfide ( $\mathrm{mg} / \mathrm{kg})$

Reactivity sulfide (mg/kg)

Reactivity sulfide ( $\mathrm{mg} / \mathrm{kg}$ )

Reactivity sulfide ( $\mathrm{mg} / \mathrm{kg}$ )

$\operatorname{TDS}(\mathrm{mg} / \mathrm{L})$

$\operatorname{TDS}(\mathrm{mg} / \mathrm{L})$

$\operatorname{TDS}(m g / L)$

$\operatorname{TDS}(\mathrm{mg} / \mathrm{L})$

$\operatorname{TDS}(\mathrm{mg} / \mathrm{L})$

$\operatorname{TDS}(\mathrm{mg} / \mathrm{L})$

$\operatorname{TDS}(\mathrm{mg} / \mathrm{L})$

$\operatorname{TDS}(\mathrm{mg} / \mathrm{L})$

$\operatorname{TDS}(\mathrm{mg} / \mathrm{L})$

$\operatorname{TDS}(\mathrm{mg} / \mathrm{L})$

$\operatorname{TDS}(m g / L)$

$\operatorname{TDS}(\mathrm{mg} / \mathrm{L})$

TDS $(\mathrm{mg} / \mathrm{L})$

$\operatorname{TDS}(\mathrm{mg} / \mathrm{L})$

$\operatorname{TDS}(\mathrm{mg} / \mathrm{L})$

$\operatorname{TDS}(\mathrm{mg} / \mathrm{L})$

$\operatorname{TDS}(\mathrm{mg} / \mathrm{I})$

$\operatorname{TDS}(\mathrm{mg} / \mathrm{L})$

$\operatorname{TDS}(\mathrm{mg} / \mathrm{L})$

TDS $(\mathrm{mg} / \mathrm{L})$

Temperature (degrees C)

Temperature (degrees C)

Temperature (degrees C)

Temperature (degrees $C$ )

TOC (ug/g)

TOC ( $\mathrm{ig} / \mathrm{g}$ )

TOC (ug/g)

TCC (ug/g)

Total Carbon (ug/g)

Total Carbon (ug/g)

Total Carbon (ug/g)

Total Carbon (ug/g)

$\operatorname{TOX}(\mathrm{ug}(\mathrm{Cl}) / \mathrm{L})$

$\operatorname{Tox}(\mathrm{ug}(\mathrm{Cl}) / \mathrm{L})$

$\operatorname{Tax}(u g(C l) / L$ )

$\operatorname{TOX}(\mathrm{ug}(\mathrm{Cl}) / \mathrm{L})$

Am-24I (pCi.L $)$

Am-2.11 (pCi/L)

Am-24i (pCi/L)

$\mathrm{Cm}-242$ (pCi/L)

$\mathrm{Cm}-242(\mathrm{pCi} / \mathrm{I})$

$\mathrm{Cm}-244 \quad(\mathrm{pCi} / \mathrm{L})$

$\mathrm{Cm}-244$ (pCi/L)

$\mathrm{Cm}-244$ (pCi/L)

$\mathrm{Co}-60(\mathrm{pCi} / \mathrm{L})$

Co-60 (pCi/s)

Co.60 (pCi/L)

$\mathrm{Cs}-134 \cdot(\mathrm{pCi} / \mathrm{L})$

$\mathrm{Cs}-134(\mathrm{pCi} / \mathrm{L})$
Sample \# Date Method Result

50873E 1/03/90 DTITRA <1.00E+02

50959E 2/21/90 DTITRA <1.00E+02

51012E 3/05/90 DTITRA <1.00E+02

51.091E 3/26/90 DTITRA <1.00E+02

$2517511 / 21 / 89$ TDS $6.00 \mathrm{E}+03$

$2517711 / 28 / 89$ TDS 3.30E+04

$2517912 / 05 / 89$ TDS 4.10E+04

$25181 \quad 12 / 12 / 89$ TDS $\quad 6.00 \mathrm{E}+03$

$2518312 / 19 / 89$ TDS $4.80 \mathrm{E}+04$

$251851 / 02 / 90$ TDS $1.00 \mathrm{E}+04$

$25187 \quad 1 / 09 / 90$ TDS $3.00 \mathrm{E}+04$

$25189 \quad 1 / 16 / 90$ TDS $5.70 \mathrm{E}+04$

$25191 \quad 1 / 23 / 90 \mathrm{TDS} \quad 4.70 \mathrm{E}+04$

$25193 \quad 1 / 30 / 90$ TDS $\quad 7.60 \mathrm{E}+04$

$251952 / 06 / 90$ TDS 3.80E+04

$251972 / 13 / 90$ TDS $\quad 1.70 \mathrm{E}+04$

$251992 / 20 / 90$ TDS $1.60 \mathrm{E}+04$

$252012 / 27 / 90$ TDS $6.70 \mathrm{E}+04$

$25203 \quad 3 / 06 / 90$ TDS $7.30 \mathrm{E}+04$

$252053 / 13 / 90$ TDS 7.50E+04

$508731 / 03 / 90$ TDS $1.40 \mathrm{E}+04$

$509592 / 21 / 90$ TDS $\quad 1.30 \mathrm{E}+04$

$510123 / 05 / 90$ IDS $6.20 \mathrm{E}+04$

$510913 / 26 / 90$ TDS $6.60 \mathrm{E}+04$

$508731 / 03 / 90$ TEMP-FId 1.92E+O1

$509592 / 21 / 90$ TEMP-FId 1.80E+OI

$510123 / 05 / 90$ TEMP-FId 1.33E+01

$510913 / 26 / 90$ TEMP-FId 1.25E+01

$508731 / 03 / 90$ TOC $<6.00 \mathrm{E}+02$

$509592 / 21 / 90$ TOC $<6.00 E+02$

$510123 / 05 / 90$ TOC $\quad 1.00 \mathrm{E}+03$

$510913 / 26 / 90$ TOC $<7.00 \mathrm{E}+02$

$508731 / 03 / 90 \mathrm{TC} \quad 4.30 \mathrm{E}+03$

$509592 / 21 / 90 \mathrm{TC} \quad 5.90 \mathrm{E}+03$

$51012 \quad 3 / 05 / 90 \mathrm{TC} \quad 1.09 \mathrm{E}+04$

$510913 / 26 / 90 \mathrm{TC} \quad 1.30 \mathrm{E}+04$

$508731 / 03 / 90$ ITOX 1.60E+01

$509592 / 21 / 90$ LTOX $<9.00 E+00$

$510123 / 05 / 90$ LTOX $2.00 \mathrm{E}+01$

$510913 / 26 / 90$ LTOX 2.70E+01

$50873 \quad 1 / 03 / 90$ AEA $7.24 \mathrm{E}+01$

$510123 / 05 / 90$ AEA $1.55 \mathrm{E}+01$

$510913 / 26 / 90$ AEA $1.64 \mathrm{E}+01$

$508731 / 03 / 90 \mathrm{AEA} \quad 2.73 \mathrm{E}-01$

$510913 / 26 / 90 \mathrm{AEA} \quad<6.17 \mathrm{E}-02$

$508731 / 03 / 90$ AEA $9.24 \mathrm{E}-01$

$510123 / 05 / 90$ AEA < 26 . 26E-02

$510913 / 26 / 90$ AEA 8.75E-02

$50873 \quad 1 / 03 / 90 \mathrm{GEA} \quad 1.61 \mathrm{E}+02$

$510123 / 05 / 90 \mathrm{GEA} \quad 5.23 \mathrm{E}+02$

$510913 / 26 / 90 \mathrm{GEA} \quad 6.23 \mathrm{E}+02$

$50873 \quad 1 / 03 / 90 \mathrm{GEA} \quad 7.07 \mathrm{E}+01$

$510913 / 26 / 90 \mathrm{GEA} \quad 1.18 \mathrm{E}+02$ 
DATA FOR N Reactor Effluent Continued

\begin{tabular}{|c|c|c|c|c|}
\hline Constituent & Sample \# & Date & Method & Result \\
\hline $\mathrm{Cs}-137(\mathrm{pCi} / \mathrm{L})$ & 50873 & $1 / 03 / 90$ & GEA & $7.44 \mathrm{E}+03$ \\
\hline $\mathrm{Cs}-137(\mathrm{pCi} / \mathrm{L})$ & 51012 & $3 / 05 / 90$ & GEA & $4.83 E+04$ \\
\hline $\mathrm{Cs}-137(\mathrm{pCi} / \mathrm{L})$ & 51091 & $3 / 26 / 90$ & GEA & $1.31 \mathrm{E}+04$ \\
\hline $\mathrm{C}-14(\mathrm{pCi} / \mathrm{I})$ & 50873 & $1 / 03 / 90$ & LSC & $<5.41 \mathrm{E}-01$ \\
\hline $\mathrm{C}-14(\mathrm{pC} 1 / \mathrm{L})$ & 51012 & $3 / 05 / 90$ & LSC & $<1.91 \mathrm{E}+00$ \\
\hline$C \cdots 14(p C i / L)$ & 51091 & $3 / 26 / 90$ & LSC & 4. $21 \mathrm{E}+00$ \\
\hline $\mathrm{H}-3 \quad(\mathrm{pCI} / \mathrm{L})$ & 50873 & $1 / 03 / 90$ & LSC & $1.56 \mathrm{E}+05$ \\
\hline $\mathrm{H}-3(\mathrm{pCi} / \mathrm{L})$ & 51012 & $3 / 05 / 90$ & LSC & $1.50 \mathrm{E}+05$ \\
\hline $\mathrm{H}-3(\mathrm{pCi} / \mathrm{L})$ & 51091 & $3 / 26 / 90$ & LSC & 4. $76 \mathrm{E}+02$ \\
\hline $\mathrm{Mn}-54(\mathrm{pCi} / \mathrm{L})$ & 50873 & $1 / 03 / 90$ & GEA & $6.31 \mathrm{E}+01$ \\
\hline $\mathrm{Mn}-54(\mathrm{pCi} / \mathrm{I})$ & 51012 & $3 / 05 / 90$ & GEA & $3.08 E+02$ \\
\hline$M n-54$ (pCi/L) & 51091 & $3 / 26 / 90$ & GEA & $5.86 \mathrm{E}+02$ \\
\hline $\mathrm{Pb}-210(\mathrm{pCi} / \mathrm{L})$ & 50873 & $1 / 03 / 90$ & GEA & $3.07 E+00$ \\
\hline $\mathrm{Pb}-210(\mathrm{pCi} / \mathrm{L})$ & 51012 & $3 / 05 / 90$ & GEA & $<3.58 \mathrm{E}-01$ \\
\hline $\mathrm{Pb}-210(\mathrm{pCI} / \mathrm{L})$ & 51091 & $3 / 26 / 90$ & GEA & $<2.23 E-01$ \\
\hline $\mathrm{Pu}-238(\mathrm{pCi} / \mathrm{L})$ & 50873 & $1 / 03 / 90$ & AEA & $1.11 \mathrm{E}+01$ \\
\hline $\mathrm{Pu}-238(\mathrm{pCI} / \mathrm{I})$ & 51012 & $3 / 05 / 90$ & AEA & $2.79 \mathrm{E}+00$ \\
\hline $\mathrm{Pu}-238 \quad(\mathrm{pCi} / \mathrm{I})$ & 51091 & $3 / 26 / 90$ & AEA & $5.77 \mathrm{~g}-01$ \\
\hline $\mathrm{Pu}-239 / 240(\mathrm{pCi} / \mathrm{I})$ & 50873 & $1 / 03 / 90$ & AEA & $6.83 E+01$ \\
\hline $\mathrm{Pu}-239 / 240(\mathrm{pCi} / \mathrm{L})$ & 5.1012 & $3 / 05 / 90$ & AEA & $2.25 \mathrm{E}+01$ \\
\hline $\mathrm{Pu}-239 / 240 \quad(\mathrm{pCi} / \mathrm{\tau})$ & 51091 & $3 / 26 / 90$ & AEA & $1.78 \mathrm{E}+01$ \\
\hline Radium Total (pci/L) & 50873 & $1 / 03 / 90$ & Alpha-Ra & $2.27 \mathrm{E}-01$ \\
\hline Radium Total (pci/L) & 51091 & $3 / 26 / 90$ & Alpha-Ra & $<1.28 \mathrm{E}-01$ \\
\hline$R u-106(p C i / L)$ & 50873 & $1 / 03 / 90$ & GEA & $<5.63 \mathrm{E}+01$ \\
\hline$R u-106(\mathrm{pCi} / \mathrm{L})$ & 51012 & $3 / 05 / 90$ & GEA & 8. 70E+02 \\
\hline $5 x-90(p C i / L)$ & 50873 & $1 / 03 / 90$ & Beta & $1.14 \mathrm{E}+05$ \\
\hline $\operatorname{sr}-90(\mathrm{pCi} / \mathrm{L})$ & 51012 & $3 / 05 / 90$ & Beta & $5.41 E+04$ \\
\hline $\mathrm{U}-234(\mathrm{pCi} / \mathrm{L})$ & 50873 & $1 / 03 / 90$ & AEA & $1.53 E+00$ \\
\hline $\mathrm{U}-234(\mathrm{pCi} / \mathrm{L})$ & 51012 & $3 / 05 / 90$ & AEA & $1.42 \mathrm{E}+00$ \\
\hline $\mathrm{U}-234 \quad(\mathrm{pCi} / \mathrm{I})$ & 51091 & $3 / 26 / 90$ & AEA & $1.06 \mathrm{E}+00$ \\
\hline$U-235(\mathrm{pCi} / \mathrm{L})$ & 50873 & $1 / 03 / 90$ & AEA & $2.10 \mathrm{E}-01$ \\
\hline$U-235(\mathrm{pCi} / \mathrm{L})$ & 51012 & $3 / 05 / 90$ & AEA & $8.86 E-02$ \\
\hline$U-235 \quad\left(\mathrm{pCi} / \mathrm{I}_{1}\right)$ & 51091 & $3 / 26 / 90$ & AEA & $1.53 \mathrm{E}-01$ \\
\hline$U-238 \quad(p C i / L)$ & 50873 & $1 / 03 / 90$ & AEA & $8.93 E-01$ \\
\hline $\mathrm{U}-238 \quad(\mathrm{pCi} / \mathrm{L})$ & 51012 & $3 / 05 / 90$ & AEA & $1.12 \mathrm{E}+00$ \\
\hline $\mathrm{U}-238 \quad(\mathrm{pCi} / \mathrm{L})$ & 51091 & $3 / 26 / 90$ & AEA & $7.23 \mathrm{E}-01$ \\
\hline
\end{tabular}


WHC-EP-0355, Appendix D

DATA FOR 163-N Demineralizer Plant Wastewater-Demineralizer Bypas

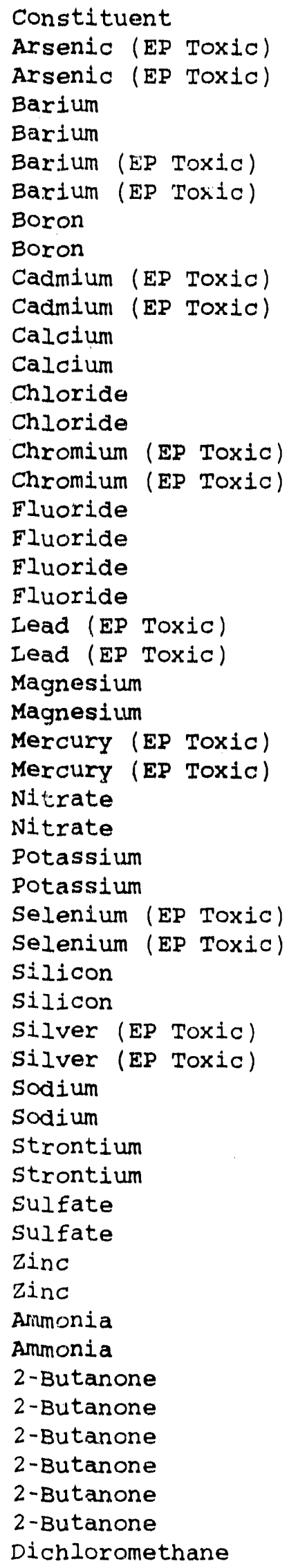

\begin{tabular}{|c|c|c|c|}
\hline Sample \# & Date & Method & Result \\
\hline $50869 \mathrm{E}$ & $1 / 03 / 90$ & ICP & $<5.00 \mathrm{E}+02$ \\
\hline $50923 E$ & $2 / 09 / 90$ & ICP & $<5.00 \mathrm{E}+02$ \\
\hline 50869 & $1 / 03 / 90$ & ICP & $2.50 \mathrm{E}+01$ \\
\hline 50923 & $2 / 09 / 90$ & ICP & $2.00 E+01$ \\
\hline 50869E & $1 / 03 / 90$ & ICP & $<1.00 \mathrm{E}+03$ \\
\hline $50923 E$ & $2 / 09 / 90$ & ICP & $<1.00 \mathrm{E}+03$ \\
\hline 50869 & $1 / 03 / 90$ & ICP & $1.30 \mathrm{EI}+01$ \\
\hline 50923 & $2 / 05 / 90$ & ICP & $4.40 \mathrm{E}+01$. \\
\hline $50869 \mathrm{E}$ & $1 / 03 / 90$ & ICP & $<1.00 \mathrm{E}+02$ \\
\hline $50923 \mathrm{E}$ & $2 / 09 / 90$ & ICP & $<1.00 \mathrm{E}+02$ \\
\hline 50869 & $1 / 03 / 90$ & ICP & $1.53 \mathrm{E}+04$ \\
\hline 50923 & $2 / 09 / 90$ & ICP & $2.31 \mathrm{~J}+04$ \\
\hline 50869 & $1 / 03 / 90$ & IC & $1.40 \mathrm{E}+03$ \\
\hline 50923 & $2 / 09 / 90$ & IC & 1. $102+03$ \\
\hline 50869E & $1 / 03 / 90$ & ICP & $<5.00 \mathrm{E}+02$ \\
\hline $50923 \mathrm{E}$ & $2 / 09 / 90$ & ICP & $<5.00 \mathrm{E}+02$ \\
\hline 50869 & $1 / 03 / 90$ & IC & $<5.00 E+02$ \\
\hline 50869 & $1 / 03 / 90$ & ISE & $1.74 \mathrm{E}+02$ \\
\hline 50923 & $2 / 09 / 90$ & IC & $<5.00 E+02$ \\
\hline 50923 & $2 / 09 / 90$ & ISE & $1.66 \mathrm{E}+02$ \\
\hline $50869 \mathrm{E}$ & $1 / 03 / 90$ & ICP & $<5.00 \mathrm{E}+02$ \\
\hline $50923 E$ & $2 / 09 / 90$ & $I C P$ & $<5.00 \mathrm{E}+02$ \\
\hline 50869 & $1 / 03 / 90$ & ICP & $3.70 \mathrm{E}+03$ \\
\hline 50923 & $2 / 09 / 90$ & $I C P$ & $3.19 \mathrm{E}+03$ \\
\hline $50869 \mathrm{E}$ & $1 / 03 / 90$ & CVAA/M & $<2.00 E+01$ \\
\hline 50923E & $2 / 09 / 90$ & CVAA/M & $<2.00 \mathrm{E}+01$ \\
\hline 50869 & $1 / 03 / 90$ & IC & $5.00 \mathrm{E}+02$ \\
\hline 50923 & $2 / 09 / 90$ & IC & $<5.00 \mathrm{E}+02$ \\
\hline 50869 & $1 / 03 / 90$ & ICP & $5.38 \mathrm{E}+02$ \\
\hline 50923 & $2 / 09 / 90$ & ICP & $4.84 E+02$ \\
\hline 50869E: & $1 / 03 / 90$ & ICP & $<5.00 \mathrm{E}+02$ \\
\hline $50923 E$ & $2 / 09 / 90$ & ICP & $<5.00 E+02$ \\
\hline 50869 & $1 / 03 / 90$ & ICP & $1.96 \mathrm{E}+03$ \\
\hline 50923 & $2 / 09 / 90$ & ICP & $1.76 \mathrm{E}+03$ \\
\hline 50869E & $1 / 03 / 90$ & ICP & $<5.00 E+02$ \\
\hline $50923 \mathrm{E}$ & $2 / 09 / 90$ & ICP & $<5.00 E+02$ \\
\hline 50869 & $1 / 03 / 90$ & ICP & $\therefore 78 \mathrm{E}+03$ \\
\hline 50923 & $2 / 09 / 90$ & ICP & 1. $60 \mathrm{E}+03$ \\
\hline 50869 & $1 / 03 / 90$ & ICP & $7.00 E+01$ \\
\hline 50923 & $2 / 09 / 90$ & ICP & $6.20 \mathrm{E}+01$ \\
\hline 50869 & $1 / 03 / 90$ & IC & $1.74 E+04$ \\
\hline $50 \subseteq 23$ & $2 / 09 / 90$ & IC & 1. $43 \mathrm{E}+04$ \\
\hline 50869 & $1 / 03 / 90$ & ICP & 1. $30 \mathrm{E}+01$ \\
\hline 50923 & $2 / 09 / 90$ & ICP & 1. $10 \mathrm{E}+01$ \\
\hline 50869 & $1 / 03 / 90$ & ISE & $1.60 \mathrm{E} \cdot 02$ \\
\hline 50923 & $2 / 09 / 90$ & ISE & $3.12 \mathrm{E}+02$ \\
\hline 50869 & $1 / 03 / 90$ & VOA & $<1.00 E+01$ \\
\hline $50869 B$ & $1 / 03 / 90$ & VOA & $1.40 E+01$ \\
\hline $50869 \mathrm{~T}$ & $1 / 03 / 90$ & VOA & 1. $50 \mathrm{E}+01$ \\
\hline 50923 & $2 / 09 / 90$ & VOA & $<1.00 E+01$ \\
\hline $50923 B$ & $2 / 09 / 90$ & VOA & $<6.00 E+D O$ \\
\hline $50923 \mathrm{~T}$ & $2 / 09 / 90$ & VOA & $<6.00 \mathrm{E}+00$ \\
\hline 50869 & $1 / 03 / 90$ & $\angle O A$ & $<5.00 E+00$ \\
\hline
\end{tabular}


WHC-EP-0355, Appendix D

DATA FOR 163-N Demineralizer Plant Wastewater-Demineralizer Bypas Continued

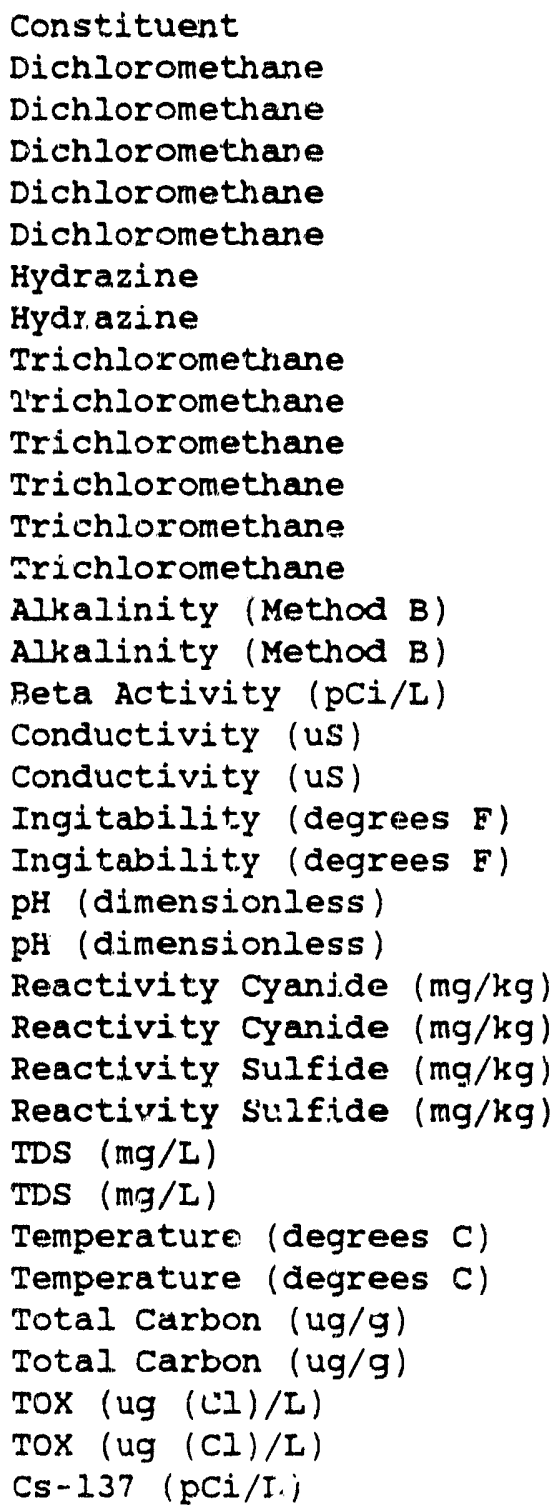

\begin{tabular}{|c|c|c|c|}
\hline Sample \# & Date & Method & ResuIt \\
\hline $50869 B$ & $1 / 03 / 90$ & VOA & $<5.00 E+00$ \\
\hline $50869 \mathrm{~T}$ & $1 / 03 / 90$ & VOA & $1.30 \mathrm{E}+01$ \\
\hline 50923 & $2 / 09 / 90$ & VOA & $<5.00 \mathrm{E}+00$ \\
\hline $50923 B$ & $2 / 09 / 90$ & VOA & $<5.005+00$ \\
\hline $50923 T$ & $2 / 09 / 90$ & VOA & $<5.00 E+00$ \\
\hline 50869 & $1 / 03 / 90$ & SPEC & $<3.00 E+01$ \\
\hline 50923 & $2 / 09 / 90$ & SPEC & $8.80 \mathrm{E}+01$ \\
\hline 50869 & $1 / 03 / 30$ & VOA & $1.10 E+01$ \\
\hline $50869 B$ & $1 / 03 / 90$ & VOA & $<3.00 E+00$ \\
\hline $50869 \mathrm{I}$ & $1 / 03 / 90$ & VOA & $<5.00 E+00$ \\
\hline 50923 & $2 / 09 / 90$ & VOA & $1.10 \mathrm{E}+01$ \\
\hline $50923 B$ & $2 / 09 / 90$ & VOA & $<5.00 E+00$ \\
\hline $50923 \mathrm{~T}$ & $2 / 09 / 90$ & VOA & $<5.00 \mathrm{E}+00$ \\
\hline 50869 & $1 / 03 / 90$ & IITRA & 4. $10 E+04$ \\
\hline 50923 & $2 / 09 / 90$ & TITRA & $3.70 \mathrm{E}+04$ \\
\hline 50869 & $1 / 03 / 50$ & Eeta & $2.85 \mathrm{E}+01$ \\
\hline 50865 & $1 / 03 / 90$ & COND-FId & $2.43 E+02$ \\
\hline 923 & $2 / 09 / 90$ & COND-FIA & $1.22 \mathrm{E}+02$ \\
\hline 50869E & $1 / 03 / 90$ & IGNIT & 2. $12 \mathrm{E}+02$ \\
\hline $50923 E$ & $2 / 09 / 90$ & IGNIT & $2.06 \mathrm{E}+02$ \\
\hline 50869 & $1 / 03 / 90$ & PH-FId & $6.45 \mathrm{E}+00$ \\
\hline 50923 & $2 / 09 / 90$ & PH-FId & $6.25 E+00$ \\
\hline 50869E & $1 / 03 / 90$ & DSPEC & $<1.00 E+02$ \\
\hline $50923 \mathrm{E}$ & $2 / 09 / 90$ & DSPEC & $<1.00 \mathrm{E}+02$ \\
\hline $50869 E$ & $1 / 03 / 90$ & DTITRA & $<1.00 \mathrm{E}+02$ \\
\hline $50923 E$ & $2 / 09 / 90$ & DTITRA & $<1.00 E+02$ \\
\hline 50869 & $1 / 03 / 90$ & TDS & $3.60 \mathrm{E}+04$ \\
\hline 50923 & $2 / 09 / 90$ & TDS & $5.80 E+04$ \\
\hline 50869 & $1 / 03 / 90$ & TEMP-FId & $2.23 E+01$ \\
\hline 50923 & $2 / 09 / 90$ & TEMP - E Id & $2.31 \mathrm{E}+01$ \\
\hline 50869 & $1 / 03 / 90$ & TC & $1.68 \mathrm{E}+04$ \\
\hline 50923 & $2 / 09 / 90$ & TC & $1.40 E+04$ \\
\hline 50869 & $1 / 03 / 90$ & ITOX & $4.80 E+01$ \\
\hline 50523 & $2 / 09 / 90$ & ITOX & $3.80 E+01$ \\
\hline 50869 & $1 / 03 / 90$ & GEA & 1. $29 \mathrm{E}+0.1$ \\
\hline
\end{tabular}




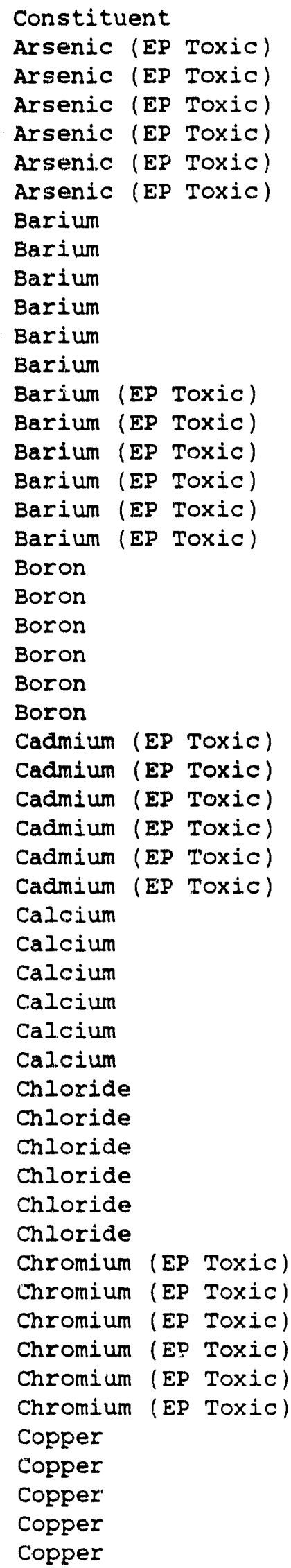

\begin{tabular}{|c|c|c|c|}
\hline ample \# & Date & Method & Result \\
\hline $50725 E$ & $10 / 25 / 89$ & ICP & $<5.00 \mathrm{E}+02$ \\
\hline $50748 E$ & $11 / 02 / 89$ & ICP & $<5.00 \mathrm{E}+02$ \\
\hline $50764 \mathrm{E}$ & $11 / 14 / 89$ & ICP & $<5.00 E+02$ \\
\hline $50903 E$ & $1 / 26 / 90$ & ICP & $<5.00 E+02$ \\
\hline $50943 \mathrm{E}$ & $2 / 16 / 90$ & ICP & $<5.00 E+02$ \\
\hline $50967 \mathrm{E}$ & $2 / 22 / 90$ & $I C P$ & $<5.00 E+02$ \\
\hline 50725 & $10 / 25 / 89$ & ICP & $3.10 E+01$ \\
\hline 50748 & $11 / 02 / 89$ & ICP & $3.205+01$ \\
\hline 50764 & $11 / 14 / 89$ & ICP & $3.20 \mathrm{E}+01$ \\
\hline 50903 & $1 / 26 / 90$ & ICP & $3.20 \mathrm{E}+0.1$ \\
\hline 50943 & $2 / 16 / 90$ & ICP & $2.80 E+01$ \\
\hline 50967 & $2 / 22 / 90$ & ICP & $2.80 E+01$ \\
\hline $50725 E$ & $10 / 25 / 89$ & ICP & $<1.00 E+03$ \\
\hline $50748 \mathrm{E}$ & $11 / 02 / 89$ & ICP & $<1.00 E+03$ \\
\hline $50764 \mathrm{E}$ & $11 / 14 / 89$ & ICP & $<1.00 E+03$ \\
\hline $50903 \mathrm{E}$ & $1 / 26 / 90$ & ICP & $<1.00 E+03$ \\
\hline $50943 E$ & $2 / 16 / 90$ & ICP & $<1.00 E+03$ \\
\hline $50967 \mathrm{E}$ & $2 / 22 / 90$ & ICP & $<1.00 \mathrm{E}+03$ \\
\hline 50725 & $10 / 25 / 89$ & $I C P$ & $1.50 \mathrm{E}+01$ \\
\hline 50748 & $11 / 02 / 89$ & ICP & $1.30 \mathrm{E}+01$ \\
\hline 50764 & $11 / 14 / 89$ & ICP & $<1.00 E+01$ \\
\hline 50903 & $1 / 26 / 90$ & ICP & 1. $20 \mathrm{E}+01$ \\
\hline 50943 & $2 / 16 / 90$ & ICP & $3.90 \mathrm{E}+01$ \\
\hline 50967 & $2 / 22 / 90$ & ICP & $2.20 \mathrm{E}+0.1$ \\
\hline 50725E & $10 / 25 / 89$ & ICP & $<1.00 E+02$ \\
\hline $50748 \mathrm{E}$ & $1.1 / 02 / 89$ & ICP & $<1.00 E+02$ \\
\hline $50764 \mathrm{E}$ & $11 / 14 / 89$ & ICP & $<1.00 \mathrm{E}+02$ \\
\hline $50903 E$ & $1 / 26 / 90$ & ICP & $<1.00 E+02$ \\
\hline $50943 E$ & $2 / 16 / 90$ & ICP & $<1.00 E+02$ \\
\hline $50967 \mathrm{E}$ & $2 / 22 / 90$ & ICP & $<1.00 \mathrm{E}+02$ \\
\hline 50725 & $10 / 25 / 89$ & ICP & $1.96 \mathrm{E}+04$ \\
\hline 50748 & $11 / 02 / 89$ & ICP & $1.93 E+04$ \\
\hline 50764 & $11 / 14 / 89$ & ICP & $1.88 \mathrm{E}+04$ \\
\hline 50903 & $1 / 26 / 90$ & ICP & 1. $99 E+04$ \\
\hline 50943 & $2 / 16 / 90$ & ICP & $1.85 \mathrm{E}+04$ \\
\hline 50967 & $2 / 22 / 90$ & ICP & $1.87 \mathrm{E}+04$ \\
\hline 50725 & $10 / 25 / 89$ & IC & 1. $20 E+03$ \\
\hline 50748 & $11 / 02 / 89$ & IC & $1.00 E+03$ \\
\hline 50764 & $11 / 14 / 89$ & IC & $1.10 E+03$ \\
\hline 50903 & $1 / 26 / 90$ & IC & 1. $30 E+03$ \\
\hline 50943 & $2 / 26 / 90$ & IC & $9.00 E+02$ \\
\hline 50967 & $2 / 22 / 90$ & IC & $8.00 E+02$ \\
\hline $50725 E$ & $10 / 25 / 89$ & ICP & $<5.00 E+02$ \\
\hline $50748 \mathrm{E}$ & $11 / 02 / 89$ & ICP & $<5.00 E+02$ \\
\hline $50764 E$ & $11 / 14 / 89$ & ICP & $<5.00 E+02$ \\
\hline $50903 \mathrm{E}$ & $1 / 26 / 90$ & ICP & $<5.00 E+02$ \\
\hline $50943 E$ & $2 / 16 / 90$ & ICP & $<5.00 E+02$ \\
\hline $50967 \mathrm{E}$ & $2 / 22 / 90$ & ICP & $<5.00 E+02$ \\
\hline 50725 & $10 / 25 / 89$ & ICP & $<1.00 E+01$ \\
\hline 50748 & $11 / 02 / 89$ & ICP & $1.30 \mathrm{E}+01$ \\
\hline 50764 & $11 / 14 / 89$ & ICP & $<1.00 E+01$ \\
\hline 50903 & $1 / 26 / 90$ & ICP & $<1.00 E+01$ \\
\hline 50943 & $2 / 16 / 90$ & ICP & $<1.00 E+01$ \\
\hline
\end{tabular}


DATA FOR PUREX Cooling water

Continued

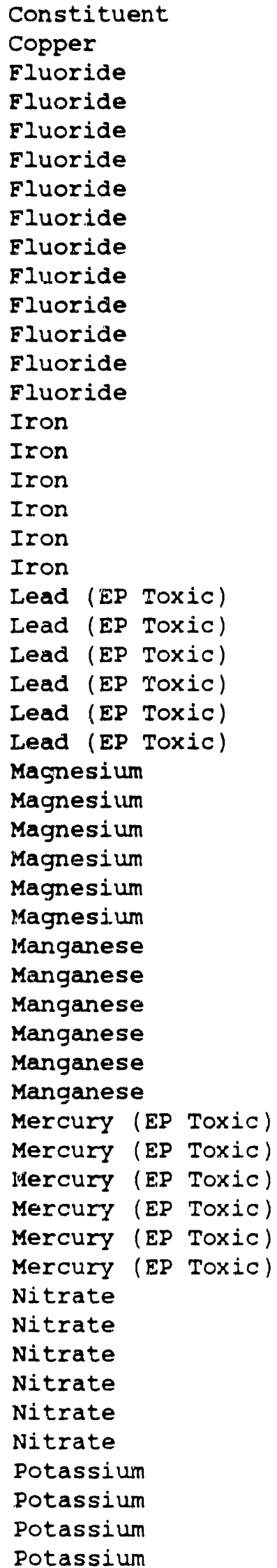

\begin{tabular}{|c|c|c|c|}
\hline Sample \# & Date & Method & Result \\
\hline 50967 & $2 / 22 / 90$ & ICP & $<1.00 \mathrm{E}+01$ \\
\hline 50725 & $10 / 25 / 89$ & IC & $<5.00 E+02$ \\
\hline 50725 & $10 / 25 / 89$ & ISE & $1.55 \mathrm{E}+02$ \\
\hline 50748 & $11 / 02 / 89$ & IC & $<5.00 E+02$ \\
\hline 50748 & $11 / 02 / 89$ & ISE & $1.58 \mathrm{E}+02$ \\
\hline 50764 & $11 / 14 / 89$ & IC & $<5.00 E+02$ \\
\hline 50764 & $11 / 14 / 89$ & ISE & $1.25 E+012$ \\
\hline 50903 & $1 / 26 / 90$ & IC & $<5.00 E+02$ \\
\hline 50903 & $1 / 26 / 90$ & ISE & 1.30E+02 \\
\hline 50943 & $2 / 16 / 90$ & IC & $<5.00 \mathrm{E}+02$ \\
\hline 50943 & $2 / 16 / 90$ & ISE & $1.27 \mathrm{E}+02$ \\
\hline 50967 & $2 / 22 / 90$ & $I C$ & $<5.00 E+02$ \\
\hline 50967 & $2 / 22 / 90$ & ISE & $1.18 \mathrm{E}+02$ \\
\hline 50725 & $10 / 25 / 89$ & ICP & $3.30 E+01$ \\
\hline 50748 & $11 / 02 / 89$ & ICP & $5.10 \mathrm{E}+01$ \\
\hline 50764 & $11 / 14 / 89$ & ICP & 4. $30 \mathrm{E}+01$ \\
\hline 50903 & $1 / 26 / 90$ & ICP & $4.70 E+01$ \\
\hline 50943 & $2 / 16 / 90$ & $I C P$ & $5.10 \mathrm{E}+01$ \\
\hline 50967 & $2 / 22 / 90$ & ICP & $6.00 \mathrm{E}+01$ \\
\hline $50725 \mathrm{E}$ & $10 / 25 / 89$ & $I C P$ & $<5.00 \mathrm{E}+02$ \\
\hline $50748 E$ & $11 / 02 / 89$ & ICP & $<5.00 \mathrm{E}+02$ \\
\hline $50764 \mathrm{E}$ & $11 / 14 / 89$ & ICP & $<5.00 E+02$ \\
\hline $50903 E$ & $1 / 26 / 90$ & $I C P$ & $<5.00 E+02$ \\
\hline $50943 E$ & $2 / 16 / 90$ & ICP & $<5.00 \mathrm{E}+02$ \\
\hline $50967 \mathrm{E}$ & $2 / 22 / 90$ & ICP & $<5.00 \mathrm{E}+02$ \\
\hline 50725 & $10 / 25 / 89$ & ICP & $4.26 \mathrm{E}+03$ \\
\hline 50748 & $11 / 02 / 89$ & ICP & $4.44 E+03$ \\
\hline 50764 & $11 / 14 / 89$ & ICP & $4.21 E+03$ \\
\hline 50903 & $1 / 26 / 90$ & ICP & $4.60 E+03$ \\
\hline 50943 & $2 / 16 / 90$ & ICP & $4.41 E+03$ \\
\hline 50967 & $2 / 22 / 90$ & ICP & $4.42 \mathrm{E}+013$ \\
\hline 50725 & $10 / 25 / 89$ & ICP & $<5.00 \mathrm{E}+00$ \\
\hline 50748 & $11 / 02 / 89$ & ICP & $8.00 \mathbf{E}+00$ \\
\hline 50764 & $11 / 14 / 89$ & ICP & $6.00 E+00$ \\
\hline 50903 & $1 / 26 / 90$ & ICP & $<5.00 \mathrm{E}+00$ \\
\hline 50943 & $2 / 16 / 90$ & ICP & $<5.00 \mathrm{E}+00$ \\
\hline 50967 & $2 / 22 / 90$ & ICP & $8.00 E+00$ \\
\hline $50725 \mathrm{E}$ & $10 / 25 / 89$ & CVAA/M & $<2.00 \mathrm{E}+01$ \\
\hline $50748 E$ & $11 / 02 / 89$ & CVAA/M & $<2.00 \mathrm{E}+01$ \\
\hline $50764 \mathrm{E}$ & $11 / 14 / 89$ & CVAA $/ M$ & $<2.00 E+01$ \\
\hline $50903 E$ & $1 / 26 / 90$ & CVAA/M & $<2.00 \mathrm{E}+01$ \\
\hline $50943 E$ & $2 / 16 / 90$ & CVAA/M & $<2.00 \mathrm{E}+01$ \\
\hline $50967 \mathrm{E}$ & $2 / 22 / 90$ & CVAA $/ M$ & $<2.00 E+01$ \\
\hline 50725 & $10 / 25 / 89$ & IC & $6.00 E+02$ \\
\hline 50748 & $11 / 02 / 89$ & IC & $? .00 \mathrm{E}+02$ \\
\hline 50764 & $11 / 14 / 89$ & $I C$ & $<5.00 \mathrm{E}+02$ \\
\hline 50903 & $1 / 26 / 90$ & IC & $6.00 E+02$ \\
\hline 50943 & $2 / 16 / 90$ & IC & $5.00 \mathrm{E}+02$ \\
\hline 50967 & $2 / 22 / 90$ & IC & $6.00 E+02$ \\
\hline 50725 & $10 / 25 / 89$ & $I C P$ & $7.78 E+02$ \\
\hline 50748 & $11 / 02 / 89$ & TCP & $8.11 E+02$ \\
\hline 50764 & $11 / 14 / 89$ & ICP & $7.24 E+02$ \\
\hline 50903 & $1 / 26 / 90$ & $I C P$ & $7.09 \mathrm{E}+02$ \\
\hline
\end{tabular}


DATA FOR PUREX Cooling water

Continued

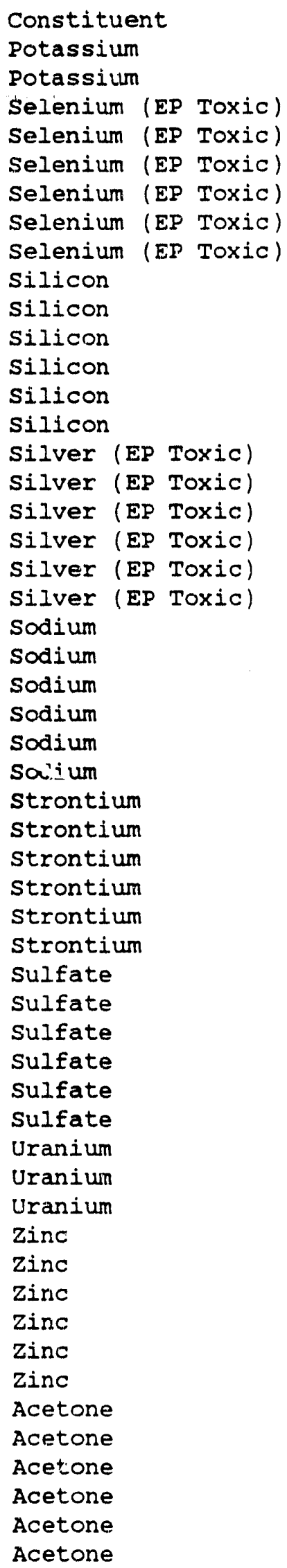

\begin{tabular}{|c|c|c|c|}
\hline Sample \# & Date & Method & Result \\
\hline 50943 & $2 / 16 / 90$ & $I C P$ & $7.29 E+02$ \\
\hline 50967 & $2 / 22 / 90$ & $I C P$ & $7.40 \mathrm{E}+02$ \\
\hline $50725 \mathrm{E}$ & $10 / 25 / 89$ & $I C P$ & $<5.00 \mathrm{E}+02$ \\
\hline $50748 \mathrm{E}$ & $11 / 02 / 89$ & ICP & $<5.00 E+02$ \\
\hline $50764 \mathrm{E}$ & $11 / 14 / 89$ & $I C P$ & $<5.00 \mathrm{E}+02$ \\
\hline $50903 E$ & $1 / 26 / 90$ & ICP & $<5.00 E+02$ \\
\hline $50943 E$ & $2 / 16 / 90$ & ICP & $<5.00 E+02$ \\
\hline $50967 \mathrm{E}$ & $2 / 22 / 90$ & ICP & $<5.00 E+02$ \\
\hline 50725 & $10 / 25 / 89$ & ICP & $2.56 \mathrm{E}+03$ \\
\hline 50748 & $11 / 02 / 89$ & ICP & $2.54 \mathrm{E}+03$ \\
\hline 50764 & $11 / 14 / 89$ & ICP & 2. $26 E+03$ \\
\hline 50903 & $1 / 26 / 90$ & ICP & $2.58 \mathrm{E}+03$ \\
\hline 50943 & $2 / 16 / 90$ & ICP & $2.55 \mathrm{E}+03$ \\
\hline 50967 & $2 / 22 / 90$ & ICP & $2.43 \mathrm{E}+03$ \\
\hline $50725 E$ & $10 / 25 / 89$ & ICP & $<5.00 E+02$ \\
\hline $50748 E$ & $11 / 02 / 89$ & ICP & $<5.00 \mathrm{E}+02$ \\
\hline $50764 \mathrm{E}$ & $11 / 14 / 89$ & ICP & $<5.00 E+02$ \\
\hline $50903 \mathrm{E}$ & $1 / 26 / 90$ & ICP & $<5.00 \mathrm{E}+02$ \\
\hline $50943 \mathrm{E}$ & $2 / 16 / 90$ & ICP & $<5.00 \mathrm{E}+02$ \\
\hline $50967 \mathrm{E}$ & $2 / 22 / 90$ & ICP & $<5.00 \mathrm{E}+02$ \\
\hline 50725 & $10 / 25 / 89$ & ICP & $2.29 \mathrm{E}+03$ \\
\hline 50748 & $11 / 02 / 89$ & ICP & $2.23 E+03$ \\
\hline 50764 & $11 / 14 / 89$ & ICP & $2.08 \mathrm{E}+03$ \\
\hline 50903 & $1 / 26 / 90$ & ICP & $2.17 \mathrm{E}+03$ \\
\hline 50943 & $2 / 16 / 90$ & ICP & $2.03 \mathrm{E}+03$ \\
\hline 50967 & $2 / 22 / 90$ & ICP & $1.97 \mathrm{E}+03$ \\
\hline 50725 & $10 / 25 / 89$ & ICP & $1.00 \mathrm{E}+02$ \\
\hline 50748 & $11 / 02 / 89$ & ICP & $1.03 E+02$ \\
\hline 50764 & $11 / 14 / 89$ & ICP & $1.01 E+02$ \\
\hline 50903 & $1 / 26 / 90$ & ICP & $9.00 E+01$ \\
\hline 50943 & $2 / 1.6 / 90$ & ICP & $9.10 \mathrm{E}+01$ \\
\hline 50967 & $2 / 22 / 90$ & ICP & $9.60 \mathrm{E}+01$ \\
\hline 50725 & $10 / 25 / 89$ & IC & $1.10 \mathrm{E}+04$ \\
\hline 50748 & $11 / 02 / 89$ & IC & $1.03 E+04$ \\
\hline 50764 & $11 / 14 / 89$ & IC & $1.00 E+04$ \\
\hline 50903 & $1 / 26 / 90$ & IC & $1.13 E+04$ \\
\hline 50943 & $2 / 16 / 90$ & IC & $1.02 \mathrm{E}+04$ \\
\hline 50967 & $2 / 22 / 90$ & IC & $1.02 \mathrm{E}+04$ \\
\hline 50725 & $10 / 25 / 89$ & GEA & $5.21 E-01$ \\
\hline 50748 & $11 / 02 / 89$ & GEA & $5.26 \mathrm{E}-01$ \\
\hline 50764 & $11 / 14 / 89$ & GEA & $4.89 E-01$ \\
\hline 50725 & $10 / 25 / 89$ & ICP & $5.00 \mathrm{E}+00$ \\
\hline 50748 & $11 / 02 / 89$ & ICP & $<5.00 E+00$ \\
\hline 50764 & $11 / 14 / 89$ & Irsp & $9.00 E+00$ \\
\hline 50903 & $1 / 26 / 90$ & $I C P$ & $6.00 F+00$ \\
\hline 50943 & $2 / 16 / 90$ & ICP & $8.00 \mathrm{E}+00$ \\
\hline 50967 & $2 / 22 / 90$ & $I C P$ & $1.00 E+01$ \\
\hline 5072.5 & $10 / 25 / 39$ & VOA & $1.30 E+01$ \\
\hline 50725 & $10 / 25 / 89$ & $A B N$ & $<1.00 \mathrm{E}+01$ \\
\hline $50725 B$ & $10 / 25 / 89$ & VOA & $<1.00 \mathrm{E}+01$ \\
\hline $50725 \mathrm{~T}$ & $10 / 25 / 89$ & VOA & $<1.00 \mathrm{E}+01$ \\
\hline 50748 & $11 / 02 / 89$ & VOA & $<1.00 E+01$ \\
\hline 50748 & $11 / 02 / 89$ & $A B N$ & $<1.00 E+01$ \\
\hline
\end{tabular}


WHC-EP-0355, Appendix D

DATA FOR PUREX COOling water

Continued

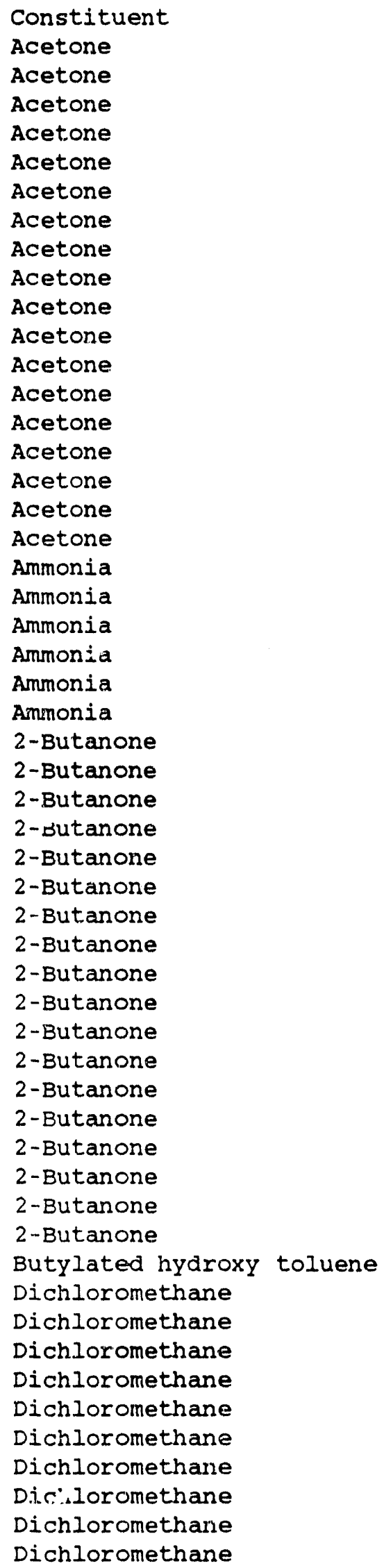

\begin{tabular}{|c|c|c|c|}
\hline Sample \# & Date & Method & Result \\
\hline $50748 B$ & $11 / 02 / 89$ & VOA & $<1.00 E+01$ \\
\hline $50748 \mathrm{~T}$ & $11 / 02 / 89$ & VOA & $<1.00 \mathrm{E}+01$ \\
\hline 50764 & $11 / 14 / 89$ & VOA & $<1.00 E+01$ \\
\hline 50764 & $11 / 14 / 89$ & $A B N$ & $<1.00 \mathrm{E}+01$ \\
\hline $50764 \mathrm{~B}$ & $11 / 14 / 89$ & VOA & $<1.008+01$ \\
\hline $50764 \mathrm{~T}$ & $11 / 14 / 89$ & VOA & $1.001 \mathrm{i}+01$ \\
\hline 50903 & $1 / 26 / 90$ & VOA & $<1.00 \mathrm{E}+01$ \\
\hline 50903 & $1 / 26 / 90$ & $A B N$ & $<1.00 \mathrm{E}+01$ \\
\hline $50903 \mathrm{~B}$ & $1 / 26 / 90$ & VOA & $<1.00 \mathrm{E}+01$ \\
\hline $50903 T$ & $1 / 26 / 90$ & VOA & $<1.00 \mathrm{E}+01$ \\
\hline 50943 & $2 / 16 / 90$ & VOA & $<1.00 E+01$ \\
\hline 50943 & $2 / 16 / 90$ & $A B N$ & $<1.00 \mathrm{E}+01$ \\
\hline $50943 B$ & $2 / 16 / 90$ & VOA & $<1.00 E+01$ \\
\hline $50943 \mathrm{~T}$ & $2 / 16 / 90$ & VOA & $<1.00 E+01$ \\
\hline 50967 & $2 / 22 / 90$ & VOA & $<1.00 \mathrm{E}+01$ \\
\hline 50967 & $2 / 22 / 90$ & AEN & $<1.00 E+01$ \\
\hline $50967 \mathrm{~B}$ & $2 / 22 / 90$ & VOA & $<1.00 E+01$ \\
\hline $50957 T$ & $2 / 22 / 90$ & VOA & $1.90 \mathrm{E}+01$ \\
\hline 50725 & $10 / 25 / 89$ & ISE & $5.40 \mathrm{E}+0.2$ \\
\hline 50748 & $11 / 02 / 89$ & ISE & $<5.00 \mathrm{E}+01$ \\
\hline 50764 & $11 / 14 / 89$ & ISE & $<5.00 E+01$ \\
\hline 50903 & $1 / 26 / 90$ & ISE & $<5.00 \mathrm{E}+01$ \\
\hline 50943 & $2 / 16 / 90$ & ISE & $<5.00 \mathrm{E}+01$ \\
\hline 50967 & $2 / 22 / 90$ & ISE & $<5.00 E+01$ \\
\hline 50725 & $10 / 25 / 89$ & VOA & $1.00 E+01$ \\
\hline $50725 B$ & $10 / 25 / 89$ & VOA & $1.40 \mathrm{E}+01$ \\
\hline $50725 \mathrm{~T}$ & $10 / 25 / 89$ & VOA & $<1.00 \mathrm{E}+01$ \\
\hline 50748 & $11 / 02 / 89$ & VOA & $<1.00 \mathrm{E}+01$ \\
\hline $50748 B$ & $11 / 02 / 89$ & VOA & $<1.00 \mathrm{E}+01$ \\
\hline $50748 \mathrm{~T}$ & $11 / 02 / 89$ & VOA & $<1.00 \mathrm{E}+01$ \\
\hline 50764 & $1 i / 14 / 89$ & VOA & $<1.00 E+01$ \\
\hline $50764 \mathrm{~B}$ & $11 / 14 / 89$ & VOA & $<1.00 \mathrm{E}+01$ \\
\hline $50764 \mathrm{~T}$ & $11 / 14 / 89$ & VDA & $<1.00 \mathrm{E}+01$ \\
\hline 50903 & $1 / 26 / 90$ & VOA & $<1.00 \mathrm{E}+01$ \\
\hline $50903 \mathrm{~B}$ & $1,26 / 90$ & VOA & $1.30 \mathrm{E}+01$ \\
\hline $50903 \mathrm{~T}$ & $1 / 26 / 90$ & VOA & $2.10 \mathrm{E}+01$ \\
\hline 50943 & $2 / 16 / 90$ & VOA & $<1.00 E+01$ \\
\hline $50943 B$ & $2 / 16 / 90$ & VOA & $<1.00 \mathrm{E}+01$ \\
\hline $50943 \mathrm{~T}$ & $2 / 16 / 90$ & VOA & $<6.00 E+00$ \\
\hline 50967 & $2 / 22 / 90$ & VOA & $<1.00 \mathrm{E}+01$ \\
\hline $50967 \mathrm{~B}$ & $2 / 22 / 90$ & VOA & $<9.00 E+00$ \\
\hline $50967 \mathrm{~T}$ & $2 / 22 / 90$ & VOA & $1.20 \mathrm{E}+01$ \\
\hline 50943 & $2 / 16 / 90$ & $A B N$ & $1.00 \mathrm{E}+01$ \\
\hline 50725 & $10 / 25 / 89$ & VOA & $<5.00 \mathrm{E}+00$ \\
\hline $50725 B$ & $10 / 25 / 89$ & VOA & $9.00 \mathrm{E}+00$ \\
\hline $50725 \mathrm{~T}$ & $10 / 25 / 89$ & VOA & $9.00 \mathrm{E}+00$ \\
\hline 50748 & $11 / 02 / 89$ & VOA & $<5.00 E+00$ \\
\hline $50748 B$ & $11 / 02 / 89$ & VOA & $<5.00 E+00$ \\
\hline $50748 \mathrm{~T}$ & $11 / 02 / 89$ & VOA & $8.00 E+00$ \\
\hline 50764 & $11 / 14 / 89$ & VOA & $<5.00 \mathrm{E}+00$ \\
\hline 507643 & $11 / 14 / 89$ & VOA & $<5.00 E+00$ \\
\hline $50764 \mathrm{~T}$ & $11 / 14 / 89$ & VOA & $<5.00 E+00$ \\
\hline 50903 & $1 / 26 / 90$ & VOA & $7.00 \mathrm{E}+00$ \\
\hline
\end{tabular}


DATA FOR PUREX Cooling water

Continued

Constituent

Dichloromethane

Dichloromethane

Dichloromethane

Dichloromethane

Dichloromethare

Dichloromethane

Dichloromethane

Dichloromethane

Tetrahydrofuran

Tetrahydrofuran

Tetrahydrofuran

Tetrahydrofuran

Tetrahydrofuran

Tetrahydrofuran

Tetrahydrofuran

Tetrahydrofuran

Tetrahydrofuran

Tetrahyarofuran

Tetrahydrofuran

Tetrahydrofuran

Tetrahydrofuran

Tetrahydrofuran

Tetrahydrofuran

Tetrahydrofuran

Tetrahydrofuran

Tetrahydrofuran

Trichloromethane

Trichloromethane

Trichloromethane

Trichloromethane

Trichloromethane

Trichloromethane

Trichloromethane

Trichloromethane

Trichloromethane

Trichloromethane

Trichloromethane

Trichloromethane

Trichloromethane

Trichloromethane

Trichloromethane

Trichloromethane

Trichloromethane

Trichloromethane

Alkalinity (Method B)

Alkalinity (Method B)

Alkalinity (Method B)

Alkalinity (Method B)

Alkalinity (Method B)

Alkalinity (Method B)

Conductivity (us)

Conductivity (us)

conductivity (us)

\begin{tabular}{|c|c|c|c|}
\hline Sample \# & Date & Method & Result \\
\hline $50903 B$ & $1 / 26 / 90$ & VOA & $<5.00 \mathrm{E}+00$ \\
\hline $50903 T$ & $1 / 26 / 90$ & VOA & $<4.00 E+00$ \\
\hline 50943 & $2 / 16 / 90$ & VOA & $<5.00 \mathrm{E}+00$ \\
\hline $50943 B$ & $2 / 16 / 90$ & VOA & $<5.00 E+00$ \\
\hline $50943 T$ & $2 / 16 / 90$ & VOA & $<5.00 E+00$ \\
\hline 50967 & $2 / 22 / 90$ & VOA & $<5.00 \mathrm{E}+00$ \\
\hline $50967 \mathrm{~B}$ & $2 / 22 / 90$ & VOA & $5.00 \mathrm{E}+00$ \\
\hline $50967 \mathrm{~T}$ & $2 / 22 / 90$ & VOA & $<5.00 \mathrm{E}+00$ \\
\hline 50725 & $10 / 25 / 89$ & VOA & $<1.00 E+01$ \\
\hline $50725 B$ & $10 / 25 / 89$ & VOA & $<1.00 E+01$ \\
\hline $50725 \mathrm{~T}$ & $10 / 25 / 89$ & VOA & $<1.00 \mathrm{E}+01$ \\
\hline 50748 & $11 / 02 / 89$ & VOA & $<1.00 \mathrm{E}+01$ \\
\hline $50748 B$ & $11 / 02 / 89$ & VOA & $1.00 \mathrm{E}+01$ \\
\hline $50748 T$ & $11 / 02 / 89$ & VOA & $<1.00 E+01$ \\
\hline 50764 & $11 / 14 / 89$ & VOA & $<1.00 \mathbf{E}+01$ \\
\hline $50764 \mathrm{~B}$ & $11 / 14 / 89$ & VOA & $<1.00 E+01$ \\
\hline $50764 \mathrm{~T}$ & $11 / 14 / 89$ & VOA & $<1.00 \mathrm{E}+01$ \\
\hline 50903 & $1 / 26 / 90$ & VOA & $<1.00 \mathrm{E}+01$ \\
\hline $50903 B$ & $1 / 26 / 90$ & VOA & $<1.00 E+01$ \\
\hline $50903 \mathrm{~T}$ & $1 / 26 / 90$ & VOA & $<8.00 \mathrm{E}+00$ \\
\hline 50943 & $2 / 16 / 90$ & VOA & $<1.00 E+01$ \\
\hline $50943 B$ & $2 / 16 / 90$ & VOA & $<1.00 E+01$ \\
\hline $50943 \mathrm{~T}$ & $2 / 16 / 90$ & VOA & $<1.00 E+01$ \\
\hline 50967 & $2 / 22 / 90$ & VOA & $<1.00 E+01$ \\
\hline $50967 \mathrm{~B}$ & $2 / 22 / 90$ & VOA & $<1.00 \mathrm{E}+01$ \\
\hline $50967 \mathrm{~T}$ & $2 / 22 / 90$ & VOA & $<6.00 E+00$ \\
\hline 50725 & $10 / 25 / 89$ & VOA & $<5.00 \mathrm{E}+00$ \\
\hline $50725 B$ & $10 / 25 / 89$ & VOA & $<5.00 \mathrm{E}+00$ \\
\hline $50725 \mathrm{~T}$ & $10 / 25 / 89$ & VOA & $<5.00 \mathrm{E}+00$ \\
\hline 50748 & $11 / 02 / 89$ & VOA & $<5.00 E+00$ \\
\hline $50748 B$ & $11 / 02 / 89$ & VOA & $<5.00 E+00$ \\
\hline $50748 \mathrm{~T}$ & $11 / 02 / 89$ & VOA & $<3.00 \mathrm{E}+00$ \\
\hline 50764 & $11 / 14 / 89$ & VOA & $<5.00 \mathrm{E}+00$ \\
\hline $50764 \mathrm{~B}$ & $11 / 14 / 89$ & VOA & $<4.00 E+00$ \\
\hline $50764 \mathrm{~T}$ & $11 / 14 / 89$ & VOA & $<4.00 \mathrm{E}+00$ \\
\hline 50903 & $1 / 26 / 90$ & VOA & $<5.00 E+00$ \\
\hline $50903 B$ & $1 / 26 / 90$ & VOA & $<5.00 E+00$ \\
\hline $50903 \mathrm{~T}$ & $1 / 26 / 90$ & VOA & $<5.00 E+00$ \\
\hline 50943 & $2 / 16 / 90$ & VOA & $<5.00 E+00$ \\
\hline $50943 B$ & $2 / 16 / 90$ & VOA & $5.00 \mathrm{E}+00$ \\
\hline $50943 T$ & $2 / 16 / 90$ & VOA & $<3.00 \mathrm{E}+00$ \\
\hline 50967 & $2 / 22 / 90$ & VOA & $<5.00 E+00$ \\
\hline $50967 \mathrm{~B}$ & $2 / 22 / 90$ & VOA & $7.00 E+00$ \\
\hline $50967 \mathrm{~T}$ & $2 / 22 / 90$ & VOA & $<4.00 E+00$ \\
\hline 50725 & $10 / 25 / 89$ & TITRA & $5.90 E+04$ \\
\hline 50748 & $11 / 02 / 89$ & TITRA & $5.80 E+04$ \\
\hline 50764 & $11 / 14 / 89$ & TITRA & $5.90 E+04$ \\
\hline 50903 & $1 / 26 / 90$ & TITRA & $6.50 \mathrm{E}+04$ \\
\hline 50943 & $2 / 16 / 90$ & TITRA & $6.00 E+04$ \\
\hline 50967 & $2 / 22 / 90$ & TITRA & $6.10 E+04$ \\
\hline 50725 & $10 / 25 / 89$ & COND-FId & $1.35 \mathrm{E}+02$ \\
\hline 50748 & $11 / 02 / 89$ & COND-FId & $1.40 \mathrm{E}+02$ \\
\hline 50764 & $11 / 14 / 89$ & COND-FId & $1.43 E+02$ \\
\hline
\end{tabular}


DATA FOR PUREX Cooling water

Continued

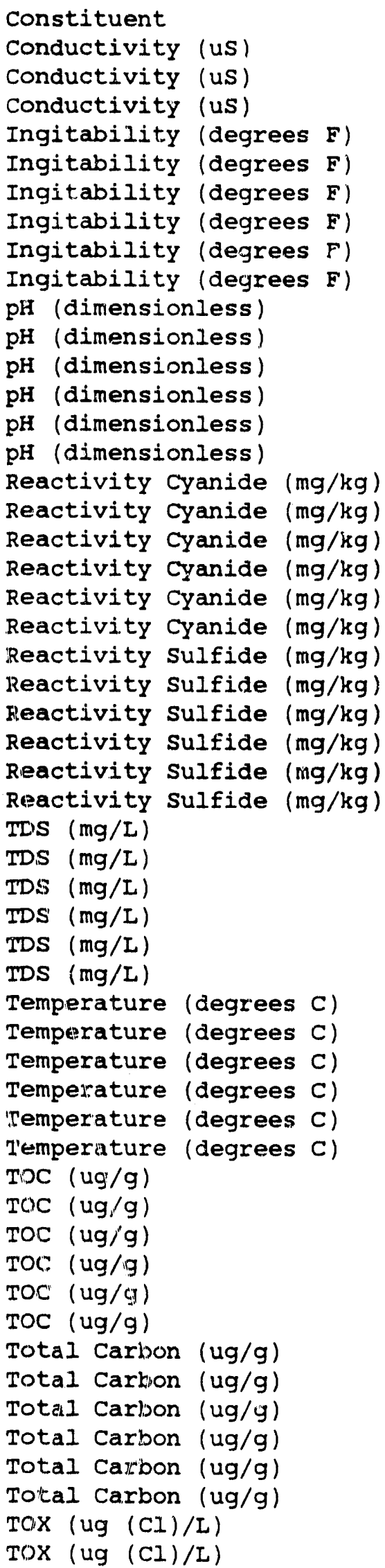

\begin{tabular}{|c|c|c|c|}
\hline ample \# & Date & Method & Result \\
\hline 50903 & $1 / 26 / 90$ & COND-FId & $1.54 \mathrm{E}+02$ \\
\hline 943 & $2 / 16 / 90$ & COND-Fld & $1.67 \mathrm{E}+02$ \\
\hline 50967 & $2 / 22 / 90$ & COND-FId & $1.43 \mathrm{E}+02$ \\
\hline $0725 \mathrm{E}$ & $10 / 25 / 89$ & IGNIT & $2.12 \mathrm{E}+02$ \\
\hline $50748 E$ & $11 / 02 / 89$ & IGNIT & $2.12 \mathrm{E}+02$ \\
\hline $50764 \mathrm{E}$ & $11 / 14 / 89$ & IGNIT & $2.08 \mathrm{E}+02$ \\
\hline $50903 \mathrm{E}$ & $1 / 26 / 90$ & IGNIT & $2.12 \mathrm{E}+02$ \\
\hline $50943 E$ & $2 / 16 / 90$ & IGNIT & $2.06 \mathrm{E}+02$ \\
\hline $50967 \mathrm{E}$ & $2 / 22 / 90$ & IGNIT & $2.12 \mathrm{E}+02$ \\
\hline 50725 & $10 / 25 / 89$ & PH-FId & $8.06 \mathbf{E}+00$ \\
\hline 50748 & $11 / 02 / 89$ & $\mathrm{PH}-\mathrm{F} I \mathrm{~d}$ & $7.69 \mathrm{E}+00$ \\
\hline 50764 & $11 / 14 / 89$ & PH-FId & $7.64 \mathrm{E}+00$ \\
\hline 50903 & $1 / 26 / 90$ & PH-FId & $7.76 \mathrm{E}+00$ \\
\hline 50943 & $2 / 16 / 90$ & PH-FId & $7.70 \mathrm{E}+00$ \\
\hline 50967 & $2 / 22 / 90$ & PH-EId & $7.90 E+00$ \\
\hline $725 E$ & $10 / 25 / 89$ & DSPEC & $<1.00 E+02$ \\
\hline $50748 \mathrm{E}$ & $11 / 0: / 89$ & DSPEC & $<1.00 \mathrm{E}+02$ \\
\hline $764 \mathrm{E}$ & $11 / 14 / 89$ & DSPEC & $<1.00 \mathrm{E}+02$ \\
\hline $50903 E$ & $1 / 26 / 90$ & DSPEC & $<1.00 \mathrm{E}+02$ \\
\hline $50943 \mathrm{E}$ & $2 / 16 / 90$ & DSPEC & $<1.00 \mathrm{E}+02$ \\
\hline $50967 \mathrm{E}$ & $2 / 22 / 90$ & DSPEC & $<1.00 \mathrm{E}+02$ \\
\hline $5072.5 \mathrm{E}$ & $10 / 25 / 89$ & DTITRA & $<1.00 \mathrm{E}+02$ \\
\hline $50748 \mathrm{E}$ & $11 / 02 / 89$ & DTITRA & $<1.00 E+02$ \\
\hline $764 \mathrm{E}$ & $11 / 14 / 89$ & DTITRA & $<1.00 \mathrm{E}+02$ \\
\hline $50903 \mathrm{E}$ & $1 / 26 / 90$ & DTITRA & $<1.00 \mathrm{E}+02$ \\
\hline $50943 E$ & $2 / 16 / 90$ & DTITRA & $<1.00 \mathrm{E}+02$ \\
\hline $50967 \mathrm{E}$ & & DTITRA & $<1.00 \mathrm{E}+02$ \\
\hline 50725 & $10 / 25 / 89$ & TDS & $7.90 \mathrm{E}+04$ \\
\hline 50748 & $11 / 02 / 89$ & TDS & $5.50 \mathrm{E}+04$ \\
\hline 50764 & $11 / 14 / 89$ & TIDS & $5.90 \mathrm{E}+04$ \\
\hline 50903 & $1 / 2 \epsilon / 90$ & TDS & $7.20 \mathrm{E}+04$ \\
\hline & $2 / 16 / 90$ & TDS & $6.40 \mathrm{E}+04$ \\
\hline 50967 & $2 / 22 / 90$ & TDS & $6.80 E+04$ \\
\hline 50725 & $10 / 25 / 89$ & TEMP - F1d & $1.72 \mathrm{E}+01$ \\
\hline 50748 & $11 / 02 / 89$ & TEMP-FId & 1. $45 \mathrm{E}+01$ \\
\hline 50764 & $11 / 14 / 89$ & T'EMP - Eld & $1.36 \mathrm{E}+01$ \\
\hline & $1 / 26 / 90$ & T'EMP - Fld & $2.37 \mathrm{E}+01$ \\
\hline 943 & $2 / 16 / 90$ & T'EMP - FId & $1.91 \mathrm{E}+01$ \\
\hline 50967 & $2 / 22 / 90$ & I'EMP - EId & $1.94 \mathrm{E}+01$ \\
\hline 50725 & $10 / 25 / 89$ & IיOC & $<1.70 \mathrm{E}+03$ \\
\hline 50748 & $11 / 02 / 89$ & צים & $<1.30 E+03$ \\
\hline 50764 & $11 / 14 / 89$ & IOOC & $<1.10 \mathrm{E}+03$ \\
\hline 50903 & $1 / 26 / 90$ & TOOC & $<1.20 \mathrm{E}+03$ \\
\hline 50943 & $2 / 16 / 90$ & roc & $<1.40 E+03$ \\
\hline 50967 & $2 / 22 / 90$ & rTOC & $1.10 \mathrm{E}+03$ \\
\hline 50725 & $10 / 25 / 89$ & TC & $1.63 E+04$ \\
\hline 50748 & $11 / 02 / 89$ & TC & $1.45 \mathrm{E}+04$ \\
\hline 50764 & $11 / 14 / 89$ & TC & $1.47 \mathrm{E}+04$ \\
\hline 50903 & $1 / 26 / 90$ & TC & 1. $36 E+04$ \\
\hline 50943 & $2 / 16 / 90$ & $\mathrm{TC}$ & $1.56 \mathrm{E}+04$ \\
\hline 50967 & $2 / 22 / 90$ & TC & $1.54 \mathrm{E}+04$ \\
\hline 50725 & $10 / 25 / 89$ & LTOX & $1.00 \mathrm{E}+01$ \\
\hline 50748 & $11 / 02 / 89$ & LTOX & $1.00 \mathrm{E}+01$ \\
\hline
\end{tabular}


WHC-EP-0355, Appendix D

DATA FOR PUREX Cooling water

Continued

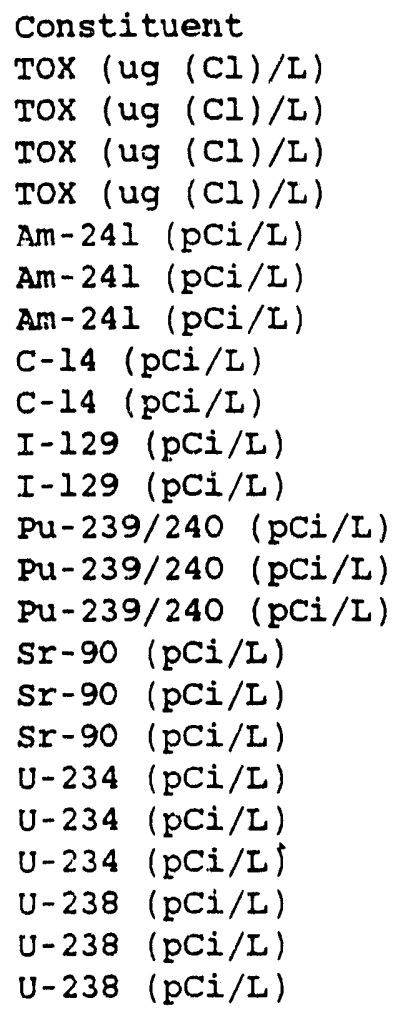

\begin{tabular}{cclr} 
Sample \# & \multicolumn{1}{c}{ Date } & Method & \multicolumn{1}{c}{$\begin{array}{c}\text { Result } \\
<0764 \\
11 / 14 / 89 \text { LTOX }\end{array}$} \\
50903 & $1 / 26 / 90$ LTOX & $1.10 \mathrm{E}+01$ \\
50943 & $2 / 16 / 90$ LTOX & $1.00 \mathrm{E}+01$ \\
50967 & $2 / 22 / 90$ LTOX & $1.10 \mathrm{E}+01$ \\
50725 & $10 / 25 / 89$ AEA & $<2.07 \mathrm{E}-03$ \\
50748 & $11 / 02 / 89$ AEA & $<1.37 \mathrm{E}-03$ \\
50764 & $11 / 14 / 89$ AEA & $4.93 \mathrm{E}-03$ \\
50748 & $11 / 02 / 89$ LSC & $<3.09 \mathrm{E}+00$ \\
50764 & $11 / 14 / 89$ LSC & $4.64 \mathrm{E}+00$ \\
50748 & $11 / 02 / 89$ LEPD & $<2.40 \mathrm{E}-02$ \\
50764 & $11 / 14 / 89$ LEPD & $8.52 \mathrm{E}-02$ \\
50725 & $10 / 25 / 89$ AEA & $3.14 \mathrm{E}-03$ \\
50748 & $11 / 02 / 89$ AEA & $<7.40 \mathrm{E}-04$ \\
50764 & $11 / 14 / 89$ AEA & $2.52 \mathrm{E}-03$ \\
50725 & $10 / 25 / 89$ Beta & $3.63 \mathrm{E}-01$ \\
50748 & $11 / 02 / 89$ Beta & $<9.49 \mathrm{E}-02$ \\
50764 & $11 / 14 / 89$ Beta & $2.57 \mathrm{E}-01$ \\
50725 & $10 / 25 / 89$ AEA & $2.59 \mathrm{E}-01$ \\
50748 & $11 / 02 / 89$ AEA & $1.69 \mathrm{E}-01$ \\
50764 & $11 / 14 / 89$ AEA & $2.17 \mathrm{E}-01$ \\
50725 & $10 / 25 / 89$ AEA & $1.87 \mathrm{E}-01$ \\
50748 & $11 / 02 / 89$ AEA & $1.46 \mathrm{E}-01$ \\
50764 & $11 / 14 / 89$ AEA & $1.62 \mathrm{E}-01$
\end{tabular}


DATA FOR PUREX Chemical sewage-Routine Operation

\begin{tabular}{|c|c|c|}
\hline Constitue & dent & \\
\hline Aluminum & & \\
\hline Aluminum & & \\
\hline Aluminum & & \\
\hline Aluminum & & \\
\hline Aluminum & & \\
\hline Aluminum & & \\
\hline Arsenic ( & (EP & Toxic) \\
\hline Arsenic ( & (EP & Toxic) \\
\hline Arsenic ( & (EP & Toxic) \\
\hline Arsenic ( & (EP & Toxic) \\
\hline Arsenic ( & (EP & Toxic) \\
\hline Arsenic ( & (EP & Toxic) \\
\hline Barium & & \\
\hline Barium & & \\
\hline Barium & & \\
\hline Bariun & & \\
\hline Barium & & \\
\hline Barium & & \\
\hline Barium (E & EP T & (oxic) \\
\hline Barium (E & EP T & (oxic) \\
\hline Barium (E & $\operatorname{EP} T$ & Toxic) \\
\hline Barium (E & EP $T$ & (oxic) \\
\hline Barium (E & EP $T$ & roxic) \\
\hline Barium (E & EP T & roxic) \\
\hline Boron & & \\
\hline Boron & & \\
\hline Boron & & \\
\hline Boron & & \\
\hline Boron & & \\
\hline Boron & & \\
\hline Cadmium ( & (EP & Toxic) \\
\hline Cadmium ( & ( EP & Toxic) \\
\hline Cadmium ( & (EP & Toxic) \\
\hline Cadmium ( & (EP & Toxic) \\
\hline Cadmium & (EP & Toxic) \\
\hline Cadmium ( & (EP & Toxic) \\
\hline CaIcium & & \\
\hline Calcium & & \\
\hline Calcium & & \\
\hline Calcium & & \\
\hline Calcium & & \\
\hline Calcium & & \\
\hline Chloride & & \\
\hline Chloride & & \\
\hline Chloride & & \\
\hline Chloride & & \\
\hline Chloride & & \\
\hline Chloride & & \\
\hline Chrom:um & (EP & Toxic) \\
\hline Chromium & (EP & Toxic) \\
\hline Chromium & (EP & Toxic) \\
\hline Chromium & (EP & P Toxic) \\
\hline Chromium & $n$ (EP & P Toxic) \\
\hline
\end{tabular}

\begin{tabular}{|c|c|c|c|}
\hline sample \# & Date & Method & Result \\
\hline 50689 & $10 / 16 / 89$ & IC.P & $6.14 \mathrm{E}+02$ \\
\hline 50709 & $10 / 19 / 89$ & ICP & $<1.50 E+02$ \\
\hline 50796 & $11 / 27 / 89$ & ICP & $<1.50 E+1) 2$ \\
\hline 50891 & $1 / 17 / 90$ & ICP & $<1.50 \mathrm{E}+02$ \\
\hline 50939 & $2 / 13 / 90$ & ICP & $<1.50 \mathrm{E}+02$ \\
\hline 50955 & $2 / 20 / 90$ & ICP & $<1.50 \mathrm{E}+02$ \\
\hline $50689 \mathrm{E}$ & $10 / 16 / 89$ & ICP & $<5.00 \mathrm{E}+02$ \\
\hline $50709 \mathrm{E}$ & $10 / 19 / 89$ & ICP & $<5.00 \mathrm{E}+02$ \\
\hline $50796 \mathrm{E}$ & $11 / 27 / 89$ & ICP & $<5.00 E+02$ \\
\hline 50891E & $1 / 17 / 90$ & ICP & $<5.00 \mathrm{E}+02$ \\
\hline 50939E & $2 / 13 / 90$ & ICP & $<5.00 E+02$ \\
\hline $50955 \mathrm{E}$ & $2 / 20 / 90$ & ICP & $<5.00 \mathrm{E}+02$ \\
\hline 50689 & $10 / 16 / 89$ & ICP & $3.90 \mathrm{E}+01$ \\
\hline 50709 & $10 / 19 / 89$ & ICP & 3. $20 \mathrm{E}+01$ \\
\hline 50796 & $11 / 27 / 89$ & ICP & $2.90 \mathrm{E}+01$ \\
\hline 50891 & $1 / 17 / 90$ & ICP & 3. $20 \mathrm{E}+01$ \\
\hline 509.39 & $2 / 13 / 90$ & ICP & 2. $90 \mathrm{E}+01$ \\
\hline 50955 & $2 / 20 / 90$ & ICP & $2.70 \mathrm{E}+01$ \\
\hline $50689 \mathrm{E}$ & $10 / 16 / 89$ & ICP & $<1.00 \mathrm{E}+?$ \\
\hline $50709 E$ & $10 / 19 / 89$ & ICP & $<1.00 \mathrm{E}+\mathrm{U} 3$ \\
\hline $50796 \mathrm{E}$ & $11 / 27 / 89$ & ICP & $<1.00 \mathrm{E}+03$ \\
\hline $50891 \mathrm{E}$ & $1 / 17 / 90$ & ICP & $<1.00 \mathrm{E}+03$ \\
\hline 50939E & $2 / 13 / 90$ & ICP & $<1.00 E+03$ \\
\hline $50955 E$ & $2 / 20 / 90$ & ICP & $<1.00 \mathrm{E}+03$ \\
\hline 50689 & $10 / 16 / 89$ & ICP & $<1.00 \mathrm{E}+01$ \\
\hline 50709 & $10 / 19 / 89$ & ICP & $<1.00 \mathrm{E}+01$ \\
\hline 50796 & $11 / 27 / 89$ & ICP & $<1.00 \mathrm{E}+01$ \\
\hline 50891 & $1 / 17 / 90$ & ICP & $<1.00 \mathrm{E}+01$ \\
\hline 50939 & $2 / 13 / 90$ & ICP & $3.50 \mathbf{E}+01$ \\
\hline 50955 & $2 / 20 / 90$ & ICP & $2.10 \mathrm{E}+01$ \\
\hline $50689 E$ & $10 / 16 / 89$ & ICP & $<1.00 \mathrm{E}+02$ \\
\hline $50709 E$ & $10 / 19 / 89$ & ICP & $<1.00 \mathrm{E}+02$ \\
\hline $50: 96 \mathrm{E}$ & $11 / 27 / 89$ & ICP & $<1.00 \mathrm{E}+02$ \\
\hline 50891E & $1 / 17 / 90$ & ICP & $<1.00 \mathrm{E}+02$ \\
\hline $50939 E$ & $2 / 13 / 90$ & ICP & $<1.00 \mathrm{E}+02$ \\
\hline 50955E & $2 / 20 / 90$ & ICP & $<1.00 E+02$ \\
\hline 50689 & $10 / 16 / 89$ & ICP & $1.86 \mathrm{E}+04$ \\
\hline 50709 & $10 / 19 / 89$ & ICP & $1.91 E+04$ \\
\hline 50796 & $11 / 27 / 89$ & ICP & $1.68 \mathrm{E}+04$ \\
\hline 50891 & $1 / 17 / 90$ & ICP & $1.94 \mathrm{E}+04$ \\
\hline 50939 & $2 / 13 / 90$ & ICP & $1.85 \mathrm{E}+04$ \\
\hline 50955 & $2 / 20 / 90$ & ICP & $1.8 I E+04$ \\
\hline 50689 & $10 / 16 / 89$ & IC & 1. $40 \mathrm{E}+03$ \\
\hline 50709 & $10 / 19 / 89$ & IC & $1.50 \mathrm{E}+03$ \\
\hline 50796 & $11 / 27 / 89$ & IC & $1.90 \mathrm{E}+03$ \\
\hline 50891 & $1 / 17 / 90$ & IC & $2.00 E+03$ \\
\hline 50939 & $2 / 13 / 90$ & IC & $1.20 \mathrm{E}+03$ \\
\hline 50955 & $2 / 20 / 90$ & IC & $1.30 \mathrm{E}+03$ \\
\hline $50689 \mathrm{E}$ & $10 / 16 / 89$ & ICF & $<5.00 E+02$ \\
\hline $50709 \mathrm{E}$ & $10 / 19 / 89$ & ICP & $<5.00 \mathrm{E}+02$ \\
\hline $50796 \mathrm{E}$ & $11 / 27 / 89$ & ICP & $<5.00 \mathrm{E}+02$ \\
\hline $50891 E$ & $1 / 17 / 90$ & ICP & $<5.00 E+02$ \\
\hline $50939 E$ & $2 / 13 / 90$ & ICP & $<5.00 E+02$ \\
\hline
\end{tabular}


DATA FOR PUREX Chemical Sewage-Routine operation Continued

Constituent
Chromium (EP Toxic)
Copper
Copper
Copper
Copper
Copper
Copper
Fluoride
Fluoride
Fluoride
Fluoride
Fluoride
Fluoride
Fluoride
Fluoride
Fluoride
Fluoride
Fluoride
Fluoride
Iron
Iron
Iron
Iron
Iron
Iron
Lead
Lead
Lead
Lead
Lead
Lead
Lead (EP Toxic)
Lead (EP Toxic)
Lead (EP Toxic)
Lead (EP Toxic)
Lead (EP Toxic)
Lead (EP Toxic)
Magnesium
Magnesium
Magnesium
Magnesium
Magnesium
Magnesium
Manganese
Manganganese
Mercury
Mercury
Mercury
Mercury

\begin{tabular}{|c|c|c|c|}
\hline Sample \# & Date & Method & Result \\
\hline $50955 \mathrm{E}$ & $2 / 20 / 90$ & ICP & $<5.00 E+02$ \\
\hline 50689 & $10 / 16 / 89$ & ICP & $5.30 \mathrm{E}+01$ \\
\hline 50709 & $10 / 19 / 89$ & ICP & $3.50 \mathrm{E}+0 \mathrm{~J}$ \\
\hline 50796 & $11 / 27 / 89$ & ICP & 3. $90 \mathrm{E}+01$ \\
\hline 50891 & $1 / 17 / 90$ & ICP & $2.90 \mathrm{E}+01$ \\
\hline 50939 & $2 / 13 / 90$ & ICP & $<1.00 E+01$ \\
\hline 50955 & $2 / 20 / 90$ & ICP & $<1.00 E+01$ \\
\hline 50689 & $10 / 16 / 89$ & IC & $<5.00 \mathrm{E}+02$ \\
\hline 50689 & $10 / 16 / 89$ & ISE & $1.80 \mathrm{E}+02$ \\
\hline 50709 & $10 / 19 / 89$ & IC & $<5.00 \mathrm{E}+02$ \\
\hline 50709 & $10 / 19 / 89$ & ISE & 1. $44 \mathrm{E}+02$ \\
\hline 50796 & $11 / 27 / 89$ & IC & $<5.00 \mathrm{E}+02$ \\
\hline 50796 & $11 / 27 / 89$ & ISE & $1.44 \mathrm{E}+02$ \\
\hline 50891 & 1/17/90 & IC & $<5.00 \mathrm{E}+02$ \\
\hline 50891 & $1 / 17 / 90$ & ISE & $1.37 \mathrm{E}+02$ \\
\hline 50939 & $2 / 13 / 90$ & IC & $<5.00 \mathrm{E}+02$ \\
\hline 50939 & $2 / 13 / 90$ & ISE & 1. $17 \mathrm{E}+02$ \\
\hline 50955 & $2 / 20 / 90$ & IC & $<5.00 E+02$ \\
\hline 50955 & $2 / 20 / 90$ & ISE & $1.18 \mathrm{E}+02$ \\
\hline 50689 & $10 / 16 / 89$ & ICP & $9.80 \mathrm{E}+02$ \\
\hline 50709 & $10 / 19 / 89$ & ICP & 1. $26 \mathrm{E}+02$ \\
\hline 50796 & $11 / 27 / 89$ & ICP & 4. $60 \mathrm{E}+01$ \\
\hline 50891 & $1 / 17 / 90$ & ICP & 3. $20 E+01$ \\
\hline 50939 & $2 / 13 / 90$ & ICP & $5.20 E+01$ \\
\hline 50955 & $2 / 20 / 90$ & $I C P$ & $6.20 E+01$ \\
\hline 50689 & $10 / 16 / 89$ & GEAA & $8.00 E+00$ \\
\hline 50709 & $10 / 19 / 89$ & GFAA & $<5.00 \mathrm{E}+00$ \\
\hline 50796 & $11 / 27 / 89$ & GFAA & $<5.00 E+00$ \\
\hline 50891 & $1 / 17 / 90$ & GFAA & $<5.00 \mathrm{E}+00$ \\
\hline 50939 & $2 / 13 / 90$ & GFAA & $<5.00 \mathrm{E}+00$ \\
\hline 50955 & $2 / 20 / 90$ & GFAA & $<5.00 \mathrm{E}+00$ \\
\hline 50689E & $10 / 16 / 89$ & ICP & $<5.00 \mathrm{E}+02$ \\
\hline 50709E & $10 / 19 / 89$ & ICP & $<5.00 \mathrm{E}+02$ \\
\hline $50796 \mathrm{E}$ & $11 / 27 / 89$ & ICP & $<5.00 \mathrm{E}+02$ \\
\hline 50891E & $1 / 17 / 90$ & $I C P$ & $<5.00 \mathrm{E}+02$ \\
\hline 50939E & $2 / 13 / 90$ & ICP & $<5.00 \mathrm{E}+02$ \\
\hline $50955 \mathrm{E}$ & $2 / 20 / 90$ & ICP & $<5.00 \mathrm{E}+02$ \\
\hline 50689 & $10 / 16 / 89$ & ICY & $4.21 E+03$ \\
\hline 50709 & $10 / 19 / 89$ & ICP & 4. 19E+03 \\
\hline 50796 & $11 / 27 / 89$ & ICP & $3.80 E+03$ \\
\hline 50891 & $1 / 17 / 90$ & ICP & $4.52 \mathrm{E}+03$ \\
\hline 50939 & $2 / 13 / 90$ & ICP & 4. $28 E+03$ \\
\hline 50955 & $2 / 20 / 90$ & ICP & $4.28 E+03$ \\
\hline 50689 & $10 / 16 / 89$ & ICP & $6.30 \mathrm{E}+01$ \\
\hline 50709 & $10 / 19 / 89$ & ICF & 1. 10E+01 \\
\hline 50796 & $11 / 27 / 89$ & ICP & $<5.00 E+C O$ \\
\hline 50891. & $1 / 17 / 90$ & ICP & $<5.00 \mathrm{E}+00$ \\
\hline 50939 & $2 / 13 / 90$ & ICP & $<5.00 E+00$ \\
\hline 50955 & $2 / 20 / 90$ & $I C P$ & $<5.00 \mathrm{E}+00$ \\
\hline 50689 & $10 / 16 / 89$ & CVAA & $1.60 \mathrm{E}-01$ \\
\hline 50709 & $10 / 19 / 89$ & CVAA & $<1.00 \mathrm{E}-01$ \\
\hline 50796 & $11 / 27 / 89$ & CVAA & $<1.00 E-01$ \\
\hline 50891 & $1 / 17 / 90$ & CVAA & $<1.00 E-01$ \\
\hline
\end{tabular}


DATA FOR PUREX Chemical Sewage-Routine Operation Continued

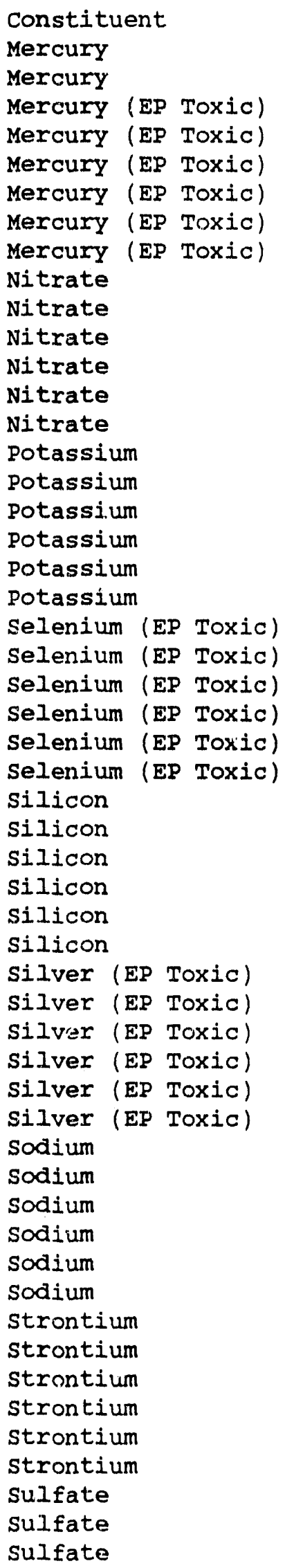

\begin{tabular}{|c|c|c|c|}
\hline Sample \# & Date & Method & Result \\
\hline 50939 & $2 / 13 / 50$ & CVAA & $<1.00 \mathrm{E}-01$ \\
\hline 50955 & $2 / 20 / 90$ & CVAA & $<1.00 \mathrm{E}-01$ \\
\hline $50689 E$ & $10 / 16 / 89$ & CVAA/M & $<2.00 E+01$ \\
\hline $50709 \mathrm{E}$ & $10 / 19 / 89$ & $C V A A / M$ & $<2.00 E+0 !$ \\
\hline $50796 \mathrm{E}$ & $11 / 27 / 89$ & $C V A A / M$ & $<2.00 \mathrm{E}+0 . \mathrm{L}$ \\
\hline 50891E & $1 / 17 / 90$ & $C V A A / M$ & $<2.00 E+01$ \\
\hline $50939 E$ & $2 / 13 / 90$ & CVAA/M & $<2.00 E+01$ \\
\hline $50955 \mathrm{E}$ & $2 / 20 / 90$ & $C V A A / M$ & $<2.00 E+01$ \\
\hline 50689 & $10 / 16 / 89$ & IC & $5.00 \mathrm{E}+02$ \\
\hline 50709 & $10 / 19 / 89$ & IC & $<5.00 E+02$ \\
\hline 50796 & $11 / 27 / 89$ & IC & $5.00 E+02$ \\
\hline 50891 & $1 / 17 / 90$ & IC & $\tau .00 E+02$ \\
\hline 50939 & $2 / 13 / 90$ & IC & $<5.00 \mathrm{E}+02$ \\
\hline 50955 & $2 / 20 / 90$ & IC & $6.00 \mathrm{E}+02$ \\
\hline 50689 & $10 / 16 / 89$ & ICP & $7.63 E+02$ \\
\hline 50709 & $10 / 19 / 89$ & ICP & $7.18 \mathrm{E}+02$ \\
\hline 50796 & $11 / 27 / 89$ & ICP & $6.98 \mathrm{E}+02$ \\
\hline 50891 & $1 / 17 / 90$ & ICP & $7.60 \mathrm{E}+02$ \\
\hline 50939 & $2 / 13 / 90$ & ICP & $6.78 E+02$ \\
\hline 50955 & $2 / 20 / 90$ & ICP & $6.98 \mathrm{E}+02$ \\
\hline $50689 E$ & $10 / 16 / 89$ & ICP & $<5.00 \mathrm{E}+02$ \\
\hline 50709E & $10 / 19 / 89$ & ICP & $<5.00 \mathrm{E}+02$ \\
\hline 50796E & $11 / 27 / 89$ & ICP & $<5.00 \mathrm{E}+02$ \\
\hline 50891E & $1 / 17 / 90$ & ICP & $<5.00 E+02$ \\
\hline $50939 E$ & $2 / 13 / 90$ & ICP & $<5.00 \mathrm{E}+02$ \\
\hline 50955E & $2 / 20 / 90$ & ICP & $<5,00 E+02$ \\
\hline 50689 & $10 / 16 / 89$ & ICP & $3.71 \mathrm{E}+03$ \\
\hline 50709 & $10 / 19 / 89$ & ICP & $2.4 \cup E+03$ \\
\hline 50796 & $11 / 27 / 89$ & ICP & $2.18 E+03$ \\
\hline 50891 & $1 / 17 / 90$ & ICP & $2.41 E+03$ \\
\hline 50939 & $2 / 13 / 90$ & ICP & $2.36 E+03$ \\
\hline 50955 & $2 / 20 / 90$ & ICP & $2.38 E+03$ \\
\hline 50689E & $10 / 16 / 89$ & ICP & $<5.00 \mathrm{E} \times 02$ \\
\hline 50709E & $10 / 19 / 89$ & ICP & $<5.00 \mathrm{E}+02$ \\
\hline $50796 \mathrm{E}$ & $11 / 27 / 89$ & $I C P$ & $<5.00 E+02$ \\
\hline 50891E & $1 / 17 / 90$ & ICP & $<5.00 \mathrm{E}+02$ \\
\hline 50939E & $2 / 13 / 90$ & ICP & $<5.00 \mathrm{E}+02$ \\
\hline $50955 \mathrm{E}$ & $2 / 20 / 90$ & ICP & $<5.00 \mathrm{E}+02$ \\
\hline 50689 & $10 / 16 / 89$ & ICP & $2.14 \mathrm{E}+03$ \\
\hline 50709 & $10 / 19 / 89$ & ICP & $2.19 \mathrm{E}+03$ \\
\hline 50796 & $11 / 27 / 89$ & ICF & $1.96 \mathrm{E}+03$ \\
\hline 50891 & $1 / 17 / 90$ & ICP & $2.26 \mathrm{E}+03$ \\
\hline 50939 & $2 / 13 / 90$ & ICP & $2.00 \mathrm{E}+03$ \\
\hline 50955 & $2 / 20 / 90$ & ICP & $1.87 \mathrm{E}+03$ \\
\hline 50689 & $10 / 16 / 89$ & $I C P$ & $1.00 \mathrm{E}+02$ \\
\hline 50709 & $10 / 19 / 89$ & ICP & $9.90 \mathrm{E}+01$ \\
\hline 50796 & $11 / 27 / 89$ & ICP & $8.40 E+01$ \\
\hline 50891 & $1 / 17 / 90$ & ICP & 8. $60 \mathrm{E}+01$ \\
\hline 50939 & $2 / 13 / 9 C$ & ICP & $8.50 E+01$ \\
\hline 50955 & $2 / 20 / 90$ & ICP & $9.10 \mathrm{E}+01$ \\
\hline 50689 & $1.0 / 16 / 89$ & $I C$ & 1.11E+04 \\
\hline 50709 & $1.0 / 19 / 89$ & IC & 1. $14 \mathrm{E}+04$ \\
\hline 50796 & $11 / 27 / 89$ & IC & $1.06 \mathrm{E}+04$ \\
\hline
\end{tabular}


DATA FOR PUREX Chemical Sewage-Routine operation Continued

Constituent
Sulfite
Sulfate
Sulfate
Uranium
Uranium
Uranium
Uranium
zinc
Zinc
zinc
zinc
Zinc
Zinc
Acetone
Acetone
Acetone
Acetone
Acetone
Acetone
Acetone
Acetone
Acetone
Acetone
Acetone
Acetone
Acetone
Acetone
Acetone
Acetone
Acetone
Acetone
Acetone
Acetone
Acetone
Acetone
Acetone
Acetone
Ammonia
Ammonia
Ammonia
Ammonia
Ammonia
Ammonia
l-Butananolanol
l-Butanol
l-Butanol
l-Butanol
2-Butanone
2-Butanone

\begin{tabular}{|c|c|c|c|}
\hline & Date & Method & Result \\
\hline 50891 & $1 / 17 / 90$ & IC & 1. $28 \mathrm{E}+04$ \\
\hline 50939 & $2 / 13 / 90$ & IC & $1.08 \mathrm{E}+04$ \\
\hline 50955 & $2 / 20 / 90$ & IC & $1.04 \mathrm{E}+04$ \\
\hline 50689 & $10 / 16 / 89$ & GEA & $3.93 E-01$ \\
\hline 50709 & $10 / 19 / 89$ & GEA & $4.31 \mathrm{E}-01$ \\
\hline 50796 & $11 / 27 / 89$ & GEA & $3.95 \mathrm{E}-01$ \\
\hline 50891 & $1 / 17 / 90$ & GEA & $6.40 E-01$ \\
\hline 50689 & $10 / 16 / 89$ & $\mathrm{ICP}$ & $4.90 \mathrm{E}+01$ \\
\hline 50709 & $10 / 19 / 89$ & ICP & $1.00 \mathrm{E}+01$ \\
\hline 50796 & $11 / 27 / 89$ & ICP & $<5.00 \mathrm{E}+00$ \\
\hline 50891 & $1 / 17 / 90$ & ICP & $6.00 \mathrm{E}+00$ \\
\hline 50939 & $2 / 13 / 90$ & ICP & $1.20 \mathrm{E}+01$ \\
\hline 50955 & $2 / 20 / 90$ & ICP & $8.00 E+00$ \\
\hline 50689 & $10 / 16 / 89$ & VOA & $<9.00 E+00$ \\
\hline 50689 & $10 / 16 / 89$ & $\mathrm{ABN}$ & $<1.00 E+01$ \\
\hline $50689 B$ & $10 / 16 / \varepsilon 9$ & VOA & $1.40 \mathrm{E}+01$ \\
\hline $50689 \mathrm{~T}$ & $10 / 16 / 89$ & VOA & $<1.00 \mathrm{E}+01$ \\
\hline 50709 & $10 / 19 / 89$ & VOA & $<9.00 \mathrm{E}+00$ \\
\hline 50709 & $10 / 19 / 89$ & $\mathrm{ARN}$ & $<1.00 \mathrm{E}+01$ \\
\hline $50709 \mathrm{~B}$ & $10 / 19 / 89$ & VOA & $<1.00 E+01$ \\
\hline $50709 \mathrm{~T}$ & $10 / 19 / 89$ & VOA & $<1.00 \mathrm{E}+01$ \\
\hline 50796 & $11 / 27 / 89$ & VOA & $<1.00 \mathrm{E}+01$ \\
\hline 50796 & $11 / 27 / 89$ & $\mathrm{ABNN}$ & $<1.00 \mathrm{E}+01$ \\
\hline $50796 \mathrm{E}$ & $11 / 27 / 89$ & VOA & $<1.00 E+01$ \\
\hline 50796I: & $11 / 27 / 89$ & VOA & $<7.00 E+00$ \\
\hline 50891 & $1 / 17 / 90$ & VOA & $<7.00 \mathrm{E}+00$ \\
\hline 50891 & $1 / 17 / 90$ & $A B N$ & $<1.00 E+01$ \\
\hline $50891 B$ & $1 / 17 / 90$ & VOA & $<1.00 \mathrm{E}+01$ \\
\hline $50891 \mathrm{~T}$ & $1 / 17 / 90$ & VOA & $<1.00 E+01$ \\
\hline 50939 & $2 / 13 / 90$ & VOA & $<1.00 \mathrm{E}+0 \mathrm{~J}$. \\
\hline 50939 & $2 / 13 / 90$ & $A B N$ & $<1.00 \mathrm{E}+01$ \\
\hline $50939 B$ & $2 / 13 / 90$ & VOA & $<1.00 E+01$ \\
\hline $50939 \mathrm{~T}$ & $2 / 13 / 90$ & VOA & $<1.00 \mathrm{E}+01$ \\
\hline 50955 & $2 / 20 / 90$ & VOA & $<1.00 \mathrm{E}+01$ \\
\hline 5095.5 & $2 / 20 / 90$ & $A B N$ & $<1.00 E+01$ \\
\hline $50955 B$ & $2 / 20 / 90$ & VOA & $1.30 E+01$ \\
\hline $50955 \mathrm{~T}$ & $2 / 20 / 90$ & VOA & $<1.00 E+01$ \\
\hline 50689 & $10 / 16 / 89$ & ISE & $6.60 \mathrm{E}+01$ \\
\hline 50709 & $10 / 19 / 89$ & ISE & $6.10 \mathrm{E}+01$ \\
\hline 50796 & $11 / 27 / 89$ & ISE & $<5.00 \mathrm{E}+01$ \\
\hline 50891 & $1 / 17 / 90$ & ISE & $6.90 E+01$ \\
\hline 50939 & $2 / 13 / 90$ & ISE & $<5.00 \mathrm{E}+01$ \\
\hline 50955 & $2 / 20 / 90$ & ISE & $<5.00 \mathrm{E}+01$ \\
\hline 50689 & $10 / 16 / 89$ & DIGC & $<1.00 E+04$ \\
\hline 50709 & $10 / 19 / 89$ & DIGC & $<1.00 \mathrm{E}+04$ \\
\hline 50796 & $1.1 / 27 / 89$ & DIGC & $<1.00 \mathrm{E}+04$ \\
\hline 50891 & $1 / 17 / 90$ & DIGC & $<1.00 E+04$ \\
\hline 50939 & $2 / 13 / 90$ & DIGC & $<1.00 E+04$ \\
\hline 50955 & $2 / 20 / 90$ & DIGC & $<1.00 E+04$ \\
\hline $50955 B$ & $2 / 20 / 90$ & VOA & $7.00 \mathrm{E}+00$ \\
\hline $50955 \mathrm{~T}$ & $2 / 20 / 90$ & VOA & $8.00 E+00$ \\
\hline 50689 & $10 / 16 / 89$ & VOA & $<1.00 E+01$ \\
\hline $50689 B$ & $10 / 16 / 89$ & VOA & $<1.00 \mathrm{E}+01$ \\
\hline
\end{tabular}


DATA FOR PUREX Chemical Sewage-Routine operation Continued

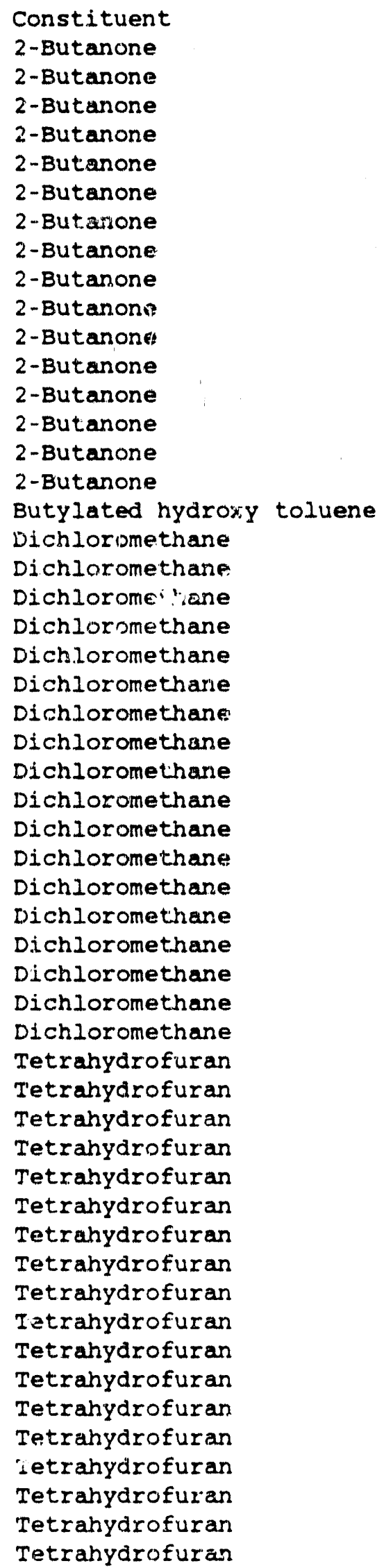

\begin{tabular}{|c|c|c|c|}
\hline Sample \# & Date & Method & Result \\
\hline $50689 T$ & $10 / 16 / 89$ & VOA & $<1.00 E+01$ \\
\hline 50709 & $10 / 19 / 89$ & VOA & $<7.00 E+00$ \\
\hline $50709 \mathrm{~B}$ & $10 / 19 / 89$ & VOA & $<1.00 E+01$ \\
\hline 507091 & $10 / 19 / 89$ & VOA & $<1.00 E+01$ \\
\hline 50796 & $11 / 27 / 89$ & VOA & $<1.00 \mathrm{E}+01$ \\
\hline $50736 \mathrm{~B}$ & $11 / 27 / 89$ & VOA & $<5.00 E+00$ \\
\hline $50796 \mathrm{~T}$ & $11 / 27 / 89$ & VOA & $<5.00 E+00$ \\
\hline 50891 & $1 / 17 / 90$ & VOA & $<1.00 \mathrm{E}+01$ \\
\hline $50891 B$ & $1 / 17 / 90$ & VOA & $<1.00 \mathrm{E}+01$ \\
\hline $50891 \mathrm{~T}$ & $1 / 17 / 90$ & VOA & $<1.00 E+01$ \\
\hline 50939 & $2 / 13 / 90$ & VOA & $<1.00 E+01$ \\
\hline $50939 \mathrm{~B}$ & $2 / 13 / 90$ & VOA & $<8.00 E+00$ \\
\hline 509.392 & $2 / 13 / 90$ & VOA & $<8.00 E+00$ \\
\hline 50955 & $2 / 20 / 90$ & VOA & $<1.00 E+01$ \\
\hline 5095.5 .3 & $2 / 20 / 90$ & VOA & $1.40 \mathrm{E}+01$ \\
\hline 5095 & $2 / 20 / 90$ & VOA & $1.30 \mathrm{E}+01$ \\
\hline 50955 & $2 / 20 / 90$ & $A B N$ & $1.00 E+01$ \\
\hline 50689 & $10 / 16 / 89$ & VOA & $<5.00 E+00$ \\
\hline $50689 B$ & $10 / 16 / 89$ & VOA & 4. $40 \mathrm{E}+02$ \\
\hline $50689 \mathrm{~T}$ & $10 / 16 / 89$ & VOA & 5. $41 E+02$ \\
\hline 50709 & $10 / 19 / 89$ & VOA & $<5.00 E+00$ \\
\hline $50709 B$ & $10 / 19 / 89$ & VOA & $<4.00 E+00$ \\
\hline $50709 \mathrm{~T}$ & $10 / 19 / 89$ & VOA & 1. $30 \mathrm{E}+01$ \\
\hline 50796 & $11 / 27 / 89$ & VOA & $<5.00 E+00$ \\
\hline $50796 B$ & $11 / 27 / 89$ & VOA & $<3,00 E+00$ \\
\hline $50796 \mathrm{~T}$ & $11 / 27 / 89$ & VOA & $<4.00 \mathrm{E}+00$ \\
\hline 50891 & $1 / 17 / 90$ & VOA & $<5.00 E+00$ \\
\hline $50891 B$ & $1 / 17 / 90$ & VOA & $<4.00 \mathrm{E}+00$ \\
\hline $50891 \mathrm{~T}$ & $1 / 17 / 90$ & VOA & $1.50 \mathrm{E}+01$ \\
\hline 50939 & $2 / 13 / 90$ & VOA & $<5.00 E+00$ \\
\hline $50939 B$ & $2 / 13 / 90$ & VOA & $<5.00 \mathrm{E}+00$ \\
\hline $50939 T$ & $2 / 13 / 90$ & VOA & $<5.00 \mathrm{E}+00$ \\
\hline 50955 & $2 / 20 / 90$ & VOA & $<5.00 \mathrm{E}+00$ \\
\hline $50955 B$ & $2 / 20 / 90$ & VOA & $6.00 \mathrm{E}+00$ \\
\hline $50955 \mathrm{~T}$ & $2 / 20 / 90$ & VOA & $<5.00 E+00$ \\
\hline 50689 & $10 / 16 / 89$ & VOA & $<1.00 E+01$ \\
\hline 506898 & $10 / 16 / 89$ & VOA & $<1.00 \mathrm{E}+01$ \\
\hline $50689 \mathrm{~T}$ & $10 / 16 / 89$ & VOA & $<1.00 E+01$ \\
\hline 50709 & $10 / 19 / 89$ & VOA & $<1.00 E+0.1$ \\
\hline $50709 B$ & $10 / 19 / 89$ & VOA & 1. . $90 \mathrm{E}+01$ \\
\hline $50709 \mathrm{~T}$ & $10 / 19 / 89$ & VOA & $<1.00 \mathrm{E}+01$ \\
\hline 50796 & $11 / 27 / 89$ & VOA & $<1.00 E+01$ \\
\hline $50796 \mathrm{~B}$ & $11 / 27 / 89$ & VOA & $<1.00 \mathrm{E}+01$ \\
\hline $50796 \mathrm{~T}$ & $11 / 27 / 89$ & VOA & $<1.00 E+01$ \\
\hline 50891 & $1 / 17 / 90$ & VOA & $<1.00 E+01$ \\
\hline $50891 \mathrm{~B}$ & $1 / 17 / 90$ & VOA & $<5.00 \mathrm{E}+00$ \\
\hline $50891 \mathrm{~T}$ & $1 / 17 / 90$ & VOD. & $<1.00 \mathrm{E}+01$ \\
\hline 50939 & $2 / 23 / 90$ & VOA & $<1.00 E+01$ \\
\hline $50939 B$ & $2 / 13 / 90$ & VOA & $<7.00 E+00$ \\
\hline $50939 \mathrm{~T}$ & $2 / 13 / 90$ & VOA & $<9.00 E+00$ \\
\hline .50955 & $2 / 20 / 90$ & VOA & $<1.00 E+01$ \\
\hline $50955 \mathrm{~B}$ & $2 / 20 / 90$ & VOA & $.1 .00 E+01$ \\
\hline $50955 \mathrm{~T}$ & $2 / 20 / 90$ & VOA & $\angle 8.00 E+00$ \\
\hline
\end{tabular}


DATA FOR PUREI: Chemical Sewage-Routine Operation Continued

Constituent

Trichloromethane

Trichloromethane

Trichioromethane

Trichloromethane

Trichloromethane

Trichluromethane

Trichloromethane

Trichloromethane

Trichloromethane

Trichloromethane

Trichloromethane

Trichloromethane

Trichloromethane

Trichloromethane

Trichloromethane

Trichloromethane

Trichloromethane

Irichloromethane

Alkalinity (Method B)

Alkalinity (Method B)

Alkalinity (Method B)

Alkalinity (Method B)

Alkalinity (Method B)

Alralinity (Method B)

Alpha Activity (pCi/L)

Alpha Activity (pCi/L)

Alphe Activity (pCi/L)

Alpha Activity (pCi/L)

Beta Activity (pCi/L)

Beta Activity (pCi/L)

Eeta Activity (pCi/L)

Beta Activity (pCi/L)

Conductivity (uS)

Conductivity (us)

Conductivity (uS)

Conductivity (us)

Conductivity (us)

Ingitability (degrees $F$ )

Ingitability (degrees F)

Ingitability (degrees $F$ )

Ingitability (degrees $F$ )

Ingitability (degrees F)

Ingitability (degrees F)

$\mathrm{pH}$ (dimensionless)

$\mathrm{pH}$ (dimensionless)

$\mathrm{pH}$ (dimensionless)

$\mathrm{pH}$ (dimensionless)

$\mathrm{pH}$ (dimensionless)

Reactivity Cyanide (mg/kg)

Reactivity Cyanide $(\mathrm{mg} / \mathrm{kg})$

Reactivity Cyanide ( $\mathrm{mg} / \mathrm{kg}$ )

Reactivity Cyanide ( $\mathrm{mg} / \mathrm{kg}$ )

Reactivity Cyanide (mg/kg)

\begin{tabular}{|c|c|c|c|}
\hline & Date & Method & Result \\
\hline 50689 & $10 / 16 / 89$ & VOA & $<3.00 \mathrm{E}+00$ \\
\hline $50689 B$ & $10 / 16 / 89$ & VOA & $<5.00 \Xi+00$ \\
\hline $50689 \mathrm{~T}$ & $10 / 16 / 89$ & VOA & $<5.00 \mathrm{E}+00$ \\
\hline 50709 & $10 / 19 / 89$ & VOA & $<3.00 E+00$ \\
\hline $50709 B$ & $10 / 19 / 89$ & VOA & $<5.00 E+00$ \\
\hline $50709 \mathrm{~T}$ & $10 / 19 / 89$ & VOA & $<5.00 E+00$ \\
\hline 50796 & $11 / 27 / 89$ & VOA & $2.20 \mathrm{E}+01$ \\
\hline $50796 \mathrm{~B}$ & $11 / 27 / 89$ & VOA & $<5.00 E+00$ \\
\hline $50796 \mathrm{~T}$ & $11 / 27 / 89$ & VDA & $1.20 \mathrm{E}+01$ \\
\hline 50891 & $1 / 17 / 90$ & VOA & $1.70 \mathrm{E}+01$ \\
\hline 508918 & $1 / 17 / 90$ & VOA & $<5.00 \mathrm{E}+00$ \\
\hline $50891 \mathrm{~T}$ & $1 / 17 / 90$ & VOA & $5.00 E+C 0$ \\
\hline 50939 & $2 / 13 / 90$ & VOA & $<3.00 E+00$ \\
\hline $50939 B$ & $2 / 13 / 90$ & VOA & $<3.00 E+00$ \\
\hline $50939 \mathrm{~T}$ & $2 / 13 / 90$ & $\mathrm{~V}, \mathrm{~A}$ & $<5.00 \mathrm{E}+\infty 0$ \\
\hline 50955 & $2 / 20 / 90$ & Vox & $5.00 E+0$ \\
\hline $50955 B$ & $2 / 20 / 90$ & VOA & $.00 E+J O$ \\
\hline $50955 \mathrm{~T}$ & $2 / 20 / 90$ & VOA & $5.00 E+L O$ \\
\hline 50689 & $10 / 16 / 89$ & TITRA & $5.60 E+04$ \\
\hline 50709 & $10 / 19 / 89$ & TITRA & $5.60 \mathrm{E}+04$ \\
\hline 50796 & $11 / 27 / 89$ & TITRA & $5.00 E+04$ \\
\hline 50891 & $1 / 17 / 90$ & TITRA & $5.80 \mathrm{E}+04$ \\
\hline 50939 & $2 / 13 / 90$ & TITRA & $5.80 E+04$ \\
\hline 50955 & $2 / 20 / 90$ & TIIRA & $7.90 \mathrm{E}+04$ \\
\hline 50689 & $10 / 16 / 89$ & Alpha & $1.36 \mathrm{E}+00$ \\
\hline 50709 & $10 / 19 / 89$ & Alpha & $1.33 \mathrm{E}+00$ \\
\hline 50796 & $11 / 27 / 89$ & Alpha & $5 E+00$ \\
\hline 50891 & $1 / 17 / 90$ & Alpha & $<9.62 \mathrm{E}-02$ \\
\hline 50689 & $10 / 16 / 89$ & Beta & $2.19 \mathrm{E}+00$ \\
\hline 50709 & $10 / 1$ & Beta & $<1.85 \mathrm{E}+00$ \\
\hline 50796 & $11 / 27 / 89$ & 3eta & $2.49 E+00$ \\
\hline 50891 & $1 / 17 / 90$ & Reta & $2.35 \mathrm{E}+00$ \\
\hline 50689 & $10 / 16 / 89$ & COND $-F$ & $1.47 \mathrm{E}+02$ \\
\hline 50709 & $10 / 19 / 89$ & COND $-F$ & $1.40 \mathrm{E}+02$ \\
\hline 50796 & $11 / 27 / 89$ & COND $-F$ & $1.61 E+02$ \\
\hline 50891 & $1 / 17 / 90$ & COND-FId & $1.60 \mathrm{E}+02$ \\
\hline 50955 & $2 / 20 / 90$ & $\operatorname{COND}-\mathrm{F} \perp \mathrm{d}$ & $1.49 E+02$ \\
\hline $50689 E$ & $10 / 16 / 89$ & IGNIT & $2.10 E+02$ \\
\hline $50709 E$ & $10 / 19 / 89$ & IGNIT & $2.10 E+02$ \\
\hline $50796 \mathrm{E}$ & $11 / 27 / 89$ & IGNIT & $2.10 E+02$ \\
\hline $50891 \mathrm{E}$ & $1 / 17 / 90$ & IGNIT & $2.14 E+02$ \\
\hline & $2 / 13 / 90$ & IGNIT & $2.04 E+02$ \\
\hline $50955 \mathrm{E}$ & $2 / 20 / 90$ & IGNIT & $2.10 \mathrm{E}+02$ \\
\hline 50689 & $10 / 16 / 89$ & PH-Fld & $7.70 E+00$ \\
\hline 50709 & $10 / 19 / 89$ & $P H-F I d$ & $8.10 E+00$ \\
\hline 50796 & $11 / 27 / 89$ & PH-Fld & $7.60 \mathrm{E}+00$ \\
\hline 50891 & $1 / 17 / 90$ & PH-Eld & $7.50 E+00$ \\
\hline 50955 & $2 / 20 / 90$ & $P H-F I d$ & $7.34 \mathrm{E}+00$ \\
\hline $50689 E$ & $10 / 16 / 89$ & DSPEC & $<1.00 E+02$ \\
\hline $50709 \mathrm{E}$ & $10 / 19 / 89$ & DSPEC & $<1.00 E+02$ \\
\hline $50796 E$ & $11 / 27 / 89$ & DSPEC & $<1.00 \mathrm{E}+02$ \\
\hline J0891E & $1 / 17 / 90$ & DSPEC & $<1.00 E+02$ \\
\hline $50939 E$ & $2 / 13 / 90$ & DSPEC & $<1.00 E+02$ \\
\hline
\end{tabular}


DATA FOR PUREX Chemical Sewage-Routine Operation Continued

Constituent

Reactivity Cyanide ( $\mathrm{mg} / \mathrm{kg}$ )

Reactivity sulfide ( $\mathrm{mg} / \mathrm{kg})$

Reactivity sulfide ( $\mathrm{mg} / \mathrm{kg}$ )

Reactivity Sulfide (mg/kg)

Reactivity sulfide ( $\mathrm{mg} / \mathrm{kg}$ )

Reaclivity sulfide ( $\mathrm{mg} / \mathrm{kg}$ )

Reactivity Sulfide (mg/kg)

IDS (mg/L)

$\operatorname{TDS}(\mathrm{mg} / \mathrm{L})$

TDS (mg/L)

TDS $(\mathrm{mg} / \mathrm{L})$

IDS (mg/L)

TDS (mg/L)

Temperature (degrees $c$ )

Temperature (degrees $c$ )

Temperature (degrees C)

Temperature (degrees $C$ )

Temper ture (degrees $c$ )

Total C.rbon (ug/g)

Total Carbon (ug/g)

Totai Carbon ( $u g / g$ )

Total Carbon (ug/g)

lotal Carbon (ug/g)

Total Carbon (ug/g)

$\operatorname{Tox}(g(C I) / I)$

$\operatorname{Tox}(\operatorname{ug}(C I) / L)$

$\operatorname{TOX}(\mathrm{ug}(\mathrm{Cl}) / \mathrm{L})$

$\operatorname{Tox}(u g(C 1) / L)$

$\operatorname{Tox}($ ug $(C L) / L)$

$\operatorname{TOX}(\mathrm{ug}(\mathrm{Cl}) / \mathrm{L})$

Am-241 (pCi/L)

An- 241 (pCi/L)

Am-241 (pCi/L)

Am-241 (pCi/L)

Cs-137 (pCi/L)

$\mathrm{Cs}-137$ (pCi/L)

Cs-1.37 (pCi/I)

$\mathrm{Cs}-137$ (pCi/L)

C- $14(\mathrm{pCi} / \mathrm{L})$

$\mathrm{C}-14(\mathrm{pCi} / \mathrm{I})$

$\mathrm{C}-14$ (pCi/L)

$\mathrm{H}-3(\mathrm{pCl} / \mathrm{L})$

$\mathrm{H}-3$ ( $\mathrm{pCi} / \mathrm{L})$

Pu-238 (pCi/L)

pu-238 (pCi/L)

pu-2.38 (pCi/L)

$\mathrm{Pu}-239 / 240$ (pCi/L)

$\mathrm{Pu}-239 / 240(\mathrm{pCi} / \mathrm{L})$

Pu-239/240 (pCi/L)

Pu $239 / 240$ (pCi/L)

Radium Total (pCi/L)

Radium Total (pci/L)

Radium Total (pCi/L)
Sariple \# Date Method Result 50955E 2/20/90 DSPEC <1.00E+02 50689 10/16/89 DTITRA < 1.00E+02 50709E 10/19/89 DTITRA <1.00E+02 50796E 11/27/89 DTITRA <1.00E+02 5089.1 $1 / 17 / 90$ DTITRA $<1.00 E+02$ 50939E 2/13/90 DTITRA <1. OOE+02 50955E V/20/90 DTITRA <I. OCE+02 $5068910 / 16 / 89$ TDS 3.90E+04 $50700 \quad 10 / 15 / 89$ IDS 3.80E+04 $5079611 / 27 / 89$ TDS $5.90 \mathrm{E}+04$

$50891 \quad 1 / 17 / 90$ TDS $6.90 E+04$

$509392 / 13 / 90$ TDS $7.00 E+04$

$509552.20 / 90$ TDS 6.30E+04

$5068910 / 16 / 89$ TEMP-FId 3.00E+01

$5070910 / 19 / 89$ TEMP-FId 2.50E+01

$5079611 / 27 / 89$ '

$508911 / 17 / 90$ TEMP-FId 2.24E+OI

$509552 / 20 / 90$ TEMP-FId $1.90 E+01$

$5068910 / 16 / 89 \mathrm{TC} \quad 1.50 \mathrm{E}+04$

$5070910 / 19 / 89 \mathrm{TC} \quad 1.60 \mathrm{E}+04$

$5079611 / 27 / 89$ TC $\quad 1.35 \mathrm{E}+04$

$50891 \quad 1 / 17 / 90$ TC $1.27 \mathrm{E}+04$

$509392 / 13 / 90 \mathrm{TC} \quad 1.44 \mathrm{E}+04$

$509552 / 20 / 90 \mathrm{TC} \quad 1.50 \mathrm{E}+04$

$5068910 / 16 / 89$ LTOX 2.90E+OI

$5070910 / 19 / 89$ LTOX $3.50 \mathrm{E}+01$

$5079611 / 27 / 89$ ITOX $1.50 E+02$

$508911 / 17 / 90$ LTOX $1.06 \mathrm{E}+02$

$509392 / 13 / 90$ LTOX 4. 1OE+OI

$509552 / 20 / 90$ LTOX 6.20E+0I

$5068910 / 16 / 89$ AEA $1.67 \mathrm{E}-02$

$5070910 / 19 / 89$ AEA I.12E-01

$5079611 / 27 / 89$ AEA 2.73E-01

$508911 / 17 / 90$ AEA $9.98 \mathrm{E}-03$

$5068910 / 16 / 89 \mathrm{GEA}<2.85 \mathrm{E}-01$

$5070910 / 19 / 89 \mathrm{GEA}<1.18 \mathrm{E}-01$

$5079611 / 27 / 89$ GEA $<1.59 E-01$

$50891 \quad 1 / 17 / 90$ GEA $5.78 E-01$

$5068910 / 16 / 89$ ISC $<2.41 E+00$

$5079611 / 27 / 89$ ILSC $<3.45 \mathrm{E}+00$

$508911 / 17 / 90$ LSC $4.14 \mathrm{E}+00$

$5070910 / 19 / 89$ ISC $<1.46 E+02$

$50891 \quad 1 / 17 / 90$ LSC $3.13 \mathrm{E}+02$

$5068910 / 16 / 89$ AEA $<9.34 \mathrm{E}-05$

$5070910 / 19 / 89$ AEA $6.97 E-03$

$5079611 / 27 / 89$ AEA $1.58 E-02$

$5068910 / 16 / 89$ AEA $1.86 E-01$

$5070910 / 19 / 89$ AEA $3.71 E-01$

$5079611 / 27 / 89 \mathrm{AEA} \quad 6.59 \mathrm{E}-01$

$50891 \quad 1 / 17 / 90$ AEA 2.17E-02

$5068910 / 16 / 89$ Alpha-Ra $<3.58 E-02$

50709 10/19/89 A.lpha-Ra <8.00E-02

$5079611 / 27 / 89$ Alpha-Ra 2.66E-01 
WHC-EP-0355, Appendix D

DATA FOR PUREX Chemical Sewage-Routine operation Continued

Constituent
Radium Total (pCi/L)
$U-234(\mathrm{pCi} / \mathrm{L})$
$U-234(\mathrm{pCi} / \mathrm{L})$
$U-234(\mathrm{pCi} / \mathrm{L})$
$U-234(\mathrm{pCi} / \mathrm{L})$
$U-238(\mathrm{pCi} / \mathrm{L})$
$U-238(\mathrm{pCi} / \mathrm{L})$
$U-238(\mathrm{pri} / \mathrm{L})$
$U-238(\mathrm{i} / \mathrm{L})$

Constituent

Radium Total ( $\mathrm{pCi} / \mathrm{L}$ )

$\mathrm{U}-234(\mathrm{pCi} / \mathrm{L})$

$\mathrm{U}-234(\mathrm{pCi} / \mathrm{L})$

U-234 (pCi/L

U-234 (pCi/I

$\mathrm{U}-238(\mathrm{pCi} / \mathrm{L})$

$\mathrm{U}-238(\mathrm{pCi} / \mathrm{L})$

$\mathrm{U}-238($, : $\mathrm{i} / \mathrm{L})$

\begin{tabular}{|c|c|c|c|}
\hline $\begin{array}{c}\text { Sample \# } \\
50891\end{array}$ & $\begin{array}{l}\text { Date } \\
1 / 17 / 90\end{array}$ & $\begin{array}{l}\text { Method } \\
\text { Alpha-Ra }\end{array}$ & $\begin{array}{l}\text { Result } \\
<7.68 \mathrm{E}-03\end{array}$ \\
\hline 50689 & $10 / 16 / 89$ & AEA & $1.53 \mathrm{E}-01$ \\
\hline 50709 & $10 / 19 / 89$ & $A E A$ & $1.80 \mathrm{E}-01$ \\
\hline 50796 & $11 / 27 / 89$ & AEA & $1.45 \mathrm{E}-01$ \\
\hline 50891 & $1 / 17 / 90$ & AEA & $2.06 \mathrm{E}-01$ \\
\hline 50689 & $10 / 16 / 89$ & AEA & $1.40 \mathrm{E}-01$ \\
\hline 50709 & $10 / 19 / 89$ & AEA & $1.55 \mathrm{E}-01$ \\
\hline 50796 & $11 / 27 / 89$ & AEA & $1.36 \mathrm{E}-01$ \\
\hline 50891 & $1 / 17 / 90$ & AEA & $1.70 \mathrm{E}-01$ \\
\hline
\end{tabular}


WHC-EP-0355, Appendj.x D

DATA FOR PUREX process Condensate

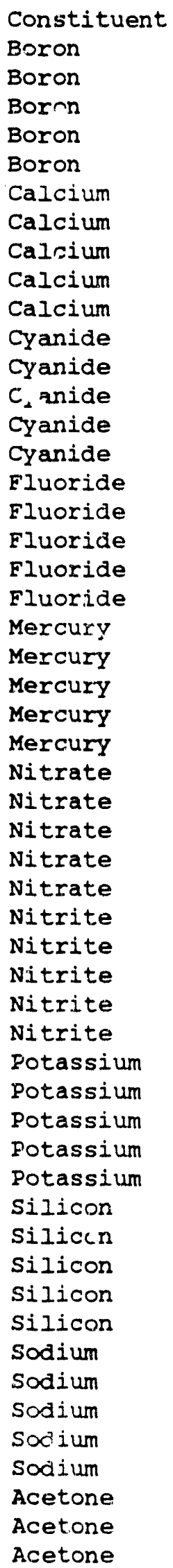

\begin{tabular}{|c|c|c|c|}
\hline Sample \# & Date & Method & Result \\
\hline 50895 & $1 / 23 / 90$ & ICP & $<1.00 \mathrm{E}+01$ \\
\hline 50935 & $2 / 13 / 90$ & ICP & $<1.00 \mathrm{E}+01$ \\
\hline 50951 & $2 / 20 / 90$ & ICP & $<1.00 \mathrm{E}+01$ \\
\hline 50988 & $2 / 27 / 90$ & ICP & $2.70 E+01$ \\
\hline 51020 & $3 / 06 / 90$ & ICP & $2.50 \mathrm{E}+01$ \\
\hline 50895 & $1 / 23 / 90$ & ICP & $<5.00 E+01$ \\
\hline 50935 & $2 / 13 / 90$ & ICP & E. $10 \mathrm{E}+01$ \\
\hline 50951 & $2 / 20 / 90$ & ICP & $<5.00 E+01$ \\
\hline 50988 & $2 / 27 / 90$ & ICP & $<5.00 E+01$ \\
\hline 5.1020 & $3 / 06 / 90$ & ICP & $<5.00 \mathrm{E}+01$ \\
\hline 50895 & $1 / 23 / 90$ & SPEC & $3.74 \mathrm{E}+01$ \\
\hline 50935 & $2 / 13 / 90$ & SPEC & $4.65 E+01$ \\
\hline 50951 & $2 / 20 / 90$ & SPEC & $3.30 \mathrm{E}+01$ \\
\hline 50988 & $2 / 27 / 90$ & SPEC & $1.58 \mathrm{E}+01$ \\
\hline 51020 & $3 / 06 / 90$ & SPEC & $4.58 E+01$ \\
\hline 50895 & $1 / 23 / 90$ & IC & $<5.00 E+02$ \\
\hline 50935 & $2 / 13 / 90$ & IC & $1.20 E+03$ \\
\hline 50951 & $2 / 20 / 90$ & IC & $<5.00 E+02$ \\
\hline 50988 & $2 / 27 / 90$ & IC & $1.60 \mathrm{E}+03$ \\
\hline 51020 & $3 / 06 / 90$ & IC & $<5.00 E+02$ \\
\hline 50895 & $1 / 23 / 90$ & CVAA & $<3.40 E-01$ \\
\hline 50935 & $2 / 13 / 90$ & CVAA & $8.40 \mathrm{E}-01$ \\
\hline 50951 & $2 / 20 / 90$ & CVAA & 1. $20 \mathrm{E}+00$ \\
\hline 50988 & $2 / 27 / 90$ & CVAA & $5.50 \mathrm{E}-01$ \\
\hline 5.1020 & $3 / 06 / 90$ & CVAA & 1. $90 \mathrm{E}+00$ \\
\hline 50895 & $1 / 23 / 90$ & $I C$ & $4.66 \mathrm{E}+04$ \\
\hline 50935 & $2 / 13 / 90$ & IC & $2.17 \mathrm{E}+05$ \\
\hline 50951 & $2 / 20 / 90$ & IC & $3.90 \mathrm{E}+03$ \\
\hline 50988 & $2 / 27 / 90$ & IC & $4.80 \mathrm{E}+03$ \\
\hline 51020 & $3 / 06 / 90$ & IC & $5.50 \mathrm{E}+03$ \\
\hline 50895 & $1.23 / 90$ & IC & $5.79 \mathrm{E}+04$ \\
\hline 50935 & $2 / 13 / 90$ & IC & $1.65 E+05$ \\
\hline 50951 & $2 / 20 / 90$ & IC & $1.01 \mathrm{E}+04$ \\
\hline $5 C 988$ & $2 / 27 / 90$ & IC & $<9.30 E+03$ \\
\hline 51020 & $3 / 06 / 90$ & IC & 4. $30 \mathrm{E}+03$ \\
\hline 50895 & $1 / 23 / 90$ & ICP & $<1.00 \mathrm{E}+02$ \\
\hline 50935 & $2 / 13 / 90$ & ICP & $1.04 \mathrm{E}+02$ \\
\hline 509.51 & $2 / 20 / 90$ & ICP & $<1.00 \mathrm{E}+02$ \\
\hline 50988 & $2 / 27 / 90$ & ICP & $4.95 E+02$ \\
\hline 51020 & $3 / 06 / 90$ & ICP & $1.74 \mathrm{E}+03$ \\
\hline 50895 & $1 / 23 / 90$ & ICP & $<2.00 E+02$ \\
\hline 50935 & $2 / 13 / 90$ & ICP & $2.80 \mathrm{E}+02$ \\
\hline 5095.1 & $2 / 20 / 90$ & ICP & $<2.00 \mathrm{E}+02$ \\
\hline 50988 & $2 / 27 / 90$ & ICP & $2.17 \mathrm{E}+02$ \\
\hline 51020 & $3 / 06 / 90$ & ICP & $<2.00 E+02$ \\
\hline 50895 & $1 / 23 / 90$ & ICP & $9.83 E+03$ \\
\hline 50935 & $2 / 13 / 90$ & ICP & $2.94 \mathrm{E}+04$ \\
\hline 50951 & $2 / 20 / 90$ & ICP & $1.60 E+04$ \\
\hline 50988 & $2,27 / 90$ & ICP & $7.81 E+03$ \\
\hline 51020 & $3 / 06 / 90$ & ICP & $1.37 \mathrm{E}+03$ \\
\hline 50895 & $1 / 23 / 90$ & VOA & $6.70 E+01$ \\
\hline 50395 & $1 / 23 / 90$ & $A B N$ & $<1.00 \mathrm{E}+02$ \\
\hline $50895 B$ & $1 / 23 / 90$ & VOA & $<1.00 E+01$ \\
\hline
\end{tabular}


WHC-EP-0355, Appendix D

DATA FOR PUREX Process Condensate Continued

Constituent
Acetone
Acetone
Acetone
Acetone
Acetone
Acetone
Acetone
Acetone
Acetone
Ace one
Acetone
Acetone
Acetorie
Acetone
Acetone
Acetone
Acetone
Acetone
Acetone
Acetone
Acetone
Acetone
Acetone
Acetone
Acetone
Acetone
Acetone
Acetone
Acetone
Acetone
Ammonia
Ammonia
Ammonia
Ammonia
Ammonia
1-Butanol
1-Butanol
l-Butanol
l-Butanol
l-Butanol
l-Butanol
1-Butanol
l-Butanol
l-Butanol
1-Butanol
2-Butanone
2-Butanone
2-Butanone
2-Butanore
2-Butanone
2-Butanone
2-Butanone
2-Butanone

\begin{tabular}{|c|c|c|c|}
\hline Sample \# & Date & Method & Result \\
\hline $50895 \mathrm{~T}$ & $1 / 23 / 90$ & VOA & $<1.00 E+01$ \\
\hline 50935 & $2 / 13 / 90$ & $\mathrm{ABN}$ & $<1.00 E+03$ \\
\hline $50935 B$ & $2 / 13 / 90$ & VOA & $<1.00 E+01$ \\
\hline $50935 \mathrm{~T}$ & $2 / 13 / 90$ & VOA & $<1.00 E+01$ \\
\hline 59171 & $1 / 17 / 90$ & VOA & $<5.00 E+02$ \\
\hline 59171 & $1 / 17 / 90$ & $\mathrm{ABN}$ & $<1.00 \mathrm{E}+02$ \\
\hline $59171 B$ & $1 / 17 / 90$ & VOA & $<3.00 E+02$ \\
\hline $59171 \mathrm{~B}$ & $1 / 17 / 90$ & $A B N$ & $<1.00 \mathrm{E}+03$ \\
\hline $59171 \mathrm{~T}$ & $1 / 17 / 90$ & VOA & $<5.00 E+02$ \\
\hline 59176 & $1 / 22 / 90$ & VOA & $<5.00 E+02$ \\
\hline 59176 & $1 / 22 / 90$ & $A B N$ & $<1.00 \mathrm{E}+02$ \\
\hline $59176 \mathrm{~B}$ & $1 / 22 / 90$ & VOA & $<5.00 E+02$ \\
\hline $59176 \mathrm{~B}$ & $1 / 22 / 90$ & $A B N$ & $0 E+03$ \\
\hline $59176 \mathrm{~T}$ & $1 / 22 / 90$ & VOA & $<5.00 \mathrm{E}+02$ \\
\hline 59181 & $1 / 25 / 90$ & VOA & $<5.00 E+02$ \\
\hline 59181 & $1 / 25 / 90$ & $\mathrm{ABN}$ & $<1.00 \mathrm{E}+02$ \\
\hline $59181 B$ & $1 / 25 / 90$ & VOA & $<5.00 E+02$ \\
\hline $59181 \mathrm{~T}$ & $1 / 25 / 90$ & VOA & $<5.00 E+02$ \\
\hline 50951 & $2 / 20 / 90$ & VOA & $7.90 E+01$ \\
\hline 50951 & $2 / 20 / 90$ & $A B N$ & $<1.11 E+02$ \\
\hline $50951 B$ & $2 / 20 / 90$ & VOA & $<1.00 E+01$ \\
\hline $50951 \mathrm{~T}$ & $2 / 20 / 90$ & VOA & $<1.00 E+01$ \\
\hline 50988 & $2 / 27 / 90$ & VOA & $<1.00 E+01$ \\
\hline 50988 & $2 / 27 / 90$ & $A B N$ & $<1.00 E+03$ \\
\hline $50988 B$ & $2 / 27 / 90$ & VOA & $<1.00 \mathrm{E}+01$ \\
\hline $50988 \mathrm{~T}$ & $2 / 27 / 90$ & VOA & $<1.00 E+01$ \\
\hline 51020 & $3 / 06 / 90$ & VOA & $7.40 \mathrm{E}+01$ \\
\hline 51020 & $3 / 06 / 90$ & $A B N$ & $<1.00 E+04$ \\
\hline $51020 B$ & $3 / 06 / 90$ & VOA & $<1.00 \mathrm{E}+01$ \\
\hline $51020 \mathrm{~T}$ & $3 / 06 / 90$ & VOA & $<1.00 \mathrm{E}+01$ \\
\hline 50895 & $1 / 23 / 90$ & ISE & $<5.00 E+01$ \\
\hline 50935 & $2 / 13 / 90$ & ISE & $<5.00 E+01$ \\
\hline 50951 & $2 / 20 / 90$ & ISE & $<5.00 E+01$ \\
\hline 50988 & $2 / 27 / 90$ & ISE & $6.60 \mathrm{E}+01$ \\
\hline 51020 & $3 / 06 / 90$ & ISE & $<5.00 \mathrm{E}+01$ \\
\hline 50935 & $2 / 13 / 90$ & DIGC & $<1.00 E+04$ \\
\hline 50951 & $2 / 20 / 90$ & $\mathrm{VOA}$ & $1.00 \mathrm{E}+01$ \\
\hline 50951 & $2 / 20 / 90$ & DIGC & $<1.00 \mathrm{E}+04$ \\
\hline 50988 & $2 / 27 / 90$ & VOA & $1.30 \mathrm{E}+01$ \\
\hline 50988 & $2 / 27 / 90$ & DIGC & $<1.00 E+04$ \\
\hline $50988 B$ & $2 / 27 / 90$ & VOA & $5.00 E+00$ \\
\hline $50988 \mathrm{~T}$ & $2 / 27 / 90$ & VOA & $7.00 \mathrm{E}+00$ \\
\hline 51020 & $3 / 06 / 90$ & VOA & $3.40 E+01$ \\
\hline 51020 & $3 / 06 / 90$ & DIGC & $<1.00 E+04$ \\
\hline $51020 \mathrm{~T}$ & $3 / 06 / 90$ & VOA & $0 E+01$ \\
\hline 50895 & $1 / 2.3 / 90$ & VOA & $1.40 \mathrm{E}+01$ \\
\hline $50895 B$ & $1 / 23 / 90$ & VOA & $<9.00 E+00$ \\
\hline $50895 \mathrm{~T}$ & $1 / 23 / 90$ & VOA & $<1.00 \mathrm{E}+0]$ \\
\hline $50935 B$ & $2 / 13 / 90$ & VOA & $<7.00 \mathrm{E}+00$ \\
\hline $50935 \mathrm{~T}$ & $2 / 13 / 90$ & VOA & $<8.00 E+00$ \\
\hline 59171 & $1 / 17 / 90$ & VOA & $<5.00 E+02$ \\
\hline 591718 & $1 / 17 / 90$ & VOA & $<5.00 E+02$ \\
\hline $59171 \mathrm{~T}$ & $1 / 1 i / 90$ & VOA & $<5.00 \mathrm{E}+02$ \\
\hline
\end{tabular}


DATA FOR PUREX Process Condensate Continued

Constituent
2-Butanone
2-Butanone
2-Butanone
2-Butanone
2-Butanone
2-Butanone
2-Butanone
2-Butanone
2-Butanone
2-Butanone
2-Butanone
2-Butanone
2-Butanone
2-Butanone
2-Butanone

Butylated hydroxy toluene Dibutylphosphate Dibutylphosphate Dibutylphosphate Dibutylphosphate Dodecane Dodecane Dodecane Dodecane Dodecane Dodecane

Dodecane

Dodecane

Tetradecane Tetradecane Tetradecane Tetradecane Tetradecane Tetradecane Tetradecane Tetradecane Tetradecane Tetradecane Tetrahydrofuran Tetrahydrofuran Tetrahydrofuran Tetrahydrofuran Tetrahydrofuran Tetrahydrofuran Tetrahydrofuran Tetrahydrofuran Tetrahydrofuran Tetrahydrofuran Tetrahydrofuran Tetrahydrofuran Tetrahydrofuran Tetrahydrofuran Tetrahydrofuran

\begin{tabular}{|c|c|c|c|}
\hline Sample \# & Date & Method & Result \\
\hline 59176 & $1 / 22 / 90$ & VOA & $<5.00 \mathrm{E}+02$ \\
\hline $59176 B$ & $1 / 22 / 90$ & VOA & $<5.00 E+02$ \\
\hline $59176 \mathrm{~T}$ & $1 / 22 / 90$ & VOA & $<5.00 E+02$ \\
\hline 59181 & $1 / 25 / 90$ & VOA & $<5.00 \mathrm{E}+02$ \\
\hline $59181 B$ & $1 / 25 / 90$ & VOA & $<5.00 E+02$ \\
\hline $59181 \mathrm{~T}$ & $1 / 25 / 90$ & VOA & $<5.00 \mathrm{E}+02$ \\
\hline 50951 & $2 / 20 / 90$ & VOA & $1.40 \mathrm{E}+01$ \\
\hline $50951 B$ & $2 / 20 / 90$ & VOA & $1.00 \mathrm{E}+01$ \\
\hline $50951 \mathrm{~T}$ & $2 / 20 / 90$ & VOA & $<6.00 \mathrm{E}+00$ \\
\hline 50988 & $2 / 27 / 90$ & VOA & $7.20 E+01$ \\
\hline $50988 B$ & $2 / 27 / 90$ & VOA & $<1.00 E+01$ \\
\hline $50988 T$ & $2 / 27 / 90$ & VOA & $<1.00 \mathrm{E}+01$ \\
\hline 51020 & $3 / 06,90$ & VOA & $1.40 \mathrm{E}+01$ \\
\hline $51020 E$ & $3 / 06 / 90$ & VOA & $<1.00 E+01$ \\
\hline $51020 \mathrm{~T}$ & $3 / 06 / 90$ & VOA & $<1.00 E+01$ \\
\hline 59181 & $1 / 25 / 90$ & $A B N$ & $1.00 \mathrm{E}+02$ \\
\hline 50935 & $2 / 13 / 90$ & DIGC & $<1.00 E+04$ \\
\hline 50951 & $2 / 20 / 90$ & DIGC & $2.81 \mathrm{E}+04$ \\
\hline 50988 & $2 / 27 / 90$ & DIGC & $1.89 E+04$ \\
\hline 51020 & $3 / 06 / 90$ & DIGC & $1.26 \mathrm{E}+04$ \\
\hline 50895 & $1 / 23 / 90$ & $A B N$ & $6.50 \mathrm{E}+01$ \\
\hline 50935 & $2 / 13 / 90$ & $\mathrm{ABN}$ & $1.60 \mathrm{E}+03$ \\
\hline 59171 & $1 / 17 / 90$ & $A B N$ & 1. $20 \mathrm{E}+04$ \\
\hline $59171 B$ & $1 / 17 / 90$ & $A B N$ & $1.48 \mathrm{E}+04$ \\
\hline 59181 & $1 / 25 / 90$ & $A B N$ & $2.40 \mathrm{E}+02$ \\
\hline 50951 & $2 / 20 / 90$ & $A B N$ & $1.50 \mathrm{E}+04$ \\
\hline 50988 & $2 / 27 / 90$ & $A B N$ & $5.00 E+01$ \\
\hline 51020 & $3 / 06 / 90$ & $A B N$ & $3.50 \mathrm{E}+04$ \\
\hline 50895 & $1 / 23 / 90$ & $A B N$ & $3.50 E+02$ \\
\hline 50935 & $2 / 13 / 90$ & $A B N$ & $6.50 \mathrm{E}+03$ \\
\hline 59171 & $1 / 17 / 90$ & $A B N$ & $4.80 E+04$ \\
\hline $59171 B$ & $1 / 17 / 90$ & $A B N$ & $6.10 \mathrm{E}+04$ \\
\hline 59176 & $1 / 22 / 90$ & $A B N$ & $1.70 \mathrm{E}+03$ \\
\hline $59176 \mathrm{~B}$ & $1 / 22 / 90$ & $\mathrm{ABN}$ & $2.00 \mathrm{E}+03$ \\
\hline 59181 & $1 / 25 / 90$ & $A B N$ & $1.50 \mathrm{E}+03$ \\
\hline 50951 & $2 / 20 / 90$ & ABN & $3.30 \mathrm{E}+04$ \\
\hline 50988 & $2 / 27 / 90$ & $A B N$ & $6.00 E+01$ \\
\hline 51020 & $3 / 06 / 90$ & $A B N$ & $7.70 E+04$ \\
\hline 50895 & $1 / 23 / 90$ & VOA & $5.70 E+01$ \\
\hline $50895 B$ & $1 / 23 / 90$ & $V \cap \cap 1$ & $<6.00 E+00$ \\
\hline $50895 \mathrm{~T}$ & $1 / 23 / 90$ & VUA & $<1.00 \mathrm{E}+01$ \\
\hline $50935 B$ & $2 / 13 / 90$ & VOA & $<9.00 \mathrm{E}+00$ \\
\hline $50935 T$ & $2 / 13 / 90$ & VOA & $<9.00 \mathrm{E}+00$ \\
\hline 59171 & $1 / 17 / 90$ & VOA & $<5.00 E+02$ \\
\hline $59171 B$ & $1 / 17 / 90$ & VOA & $<5.00 E+02$ \\
\hline $59171 \mathrm{~T}$ & $1 / 17 / 90$ & VOA & $<5.00 \mathrm{E}+02$ \\
\hline 59176 & $1 / 22 / 90$ & VOA & $<5.00 E+02$ \\
\hline $59176 \mathrm{~B}$ & $1 / 22 / 90$ & VOA & $<5.00 \mathrm{E}+02$ \\
\hline $59176 \mathrm{~T}$ & $1 / 22 / 90$ & VOA & $<5.00 \mathrm{E}+02$ \\
\hline 59181 & $1 / 25 / 90$ & VOA & $<5.00 \mathrm{E}+02$ \\
\hline $59181 \mathrm{~B}$ & $1 / 25 / 90$ & VOA & $<5.00 E+02$ \\
\hline $59181 T$ & $1 / 25 / 90$ & VOA & $<5.00 \mathrm{E}+02$ \\
\hline 50951 & $2 / 20 / 90$ & VOA & $1.03 E+02$ \\
\hline
\end{tabular}


DATA FOR PUREX Process Condensate

Continued

Sonstituent

Tetrahydrofurar

Tetrahydrofuran

Tetrahydrofuran

Tetrahydrofuran

Tetrahydrofuran

Tetrahydrofuran

Tetrahydrofuran

Tetrahydrofuran

Tributylphosphate

Tributylphosphate

Tributylphosphate

Tributy Iphosphate

Tributylphosphate

Tributylphosphate

Tributylphosphate

Tributy Iphosphate

Tributylphosphate

Tributylphospizate

Trichloromethane

Trichloromethane

Trichloromethane

Trichloromethane

Trichloromethane

Trichloromethane

Trichloromethane

Trichloromethane

Trichloromethane

Trichloromethane

Trichloromethane

rrichloromethane

Trichloromethane

Trichloromethane

Trichloromethane

Trichloromethane

Trichloromethane

Irichloromethane

Trichloromethane

Trichloromethane

Trichloromethane

Trichloromethane

Trichloromethane

Tridecane

Tridecane

Tridecane

Tridecane

Tridecane

Tridecane

Tridecane

Tridecane

Tridecane

Tridecane

Undecane

Unknown

\begin{tabular}{|c|c|c|c|}
\hline $\begin{array}{r}\text { Sample \# } \\
50951 B\end{array}$ & $\begin{array}{l}\text { Date } \\
2 / 20 / 90\end{array}$ & $\begin{array}{l}\text { Method } \\
\text { VOA }\end{array}$ & $\begin{array}{l}\text { Result } \\
<1.00 \mathrm{E}+01\end{array}$ \\
\hline $50951 \mathrm{~T}$ & $2 / 20 / 90$ & VOA & $<1.00 \mathrm{E}+01$ \\
\hline 50988 & $2 / 27 / 90$ & VOA & $9.50 \mathrm{E}+01$ \\
\hline $50988 B$ & $2 / 27 / 90$ & VOA & $<5.00 \mathrm{E}+00$ \\
\hline $50988 \mathrm{~T}$ & $2 / 27 / 90$ & VOA & $<7.00 E+00$ \\
\hline 51020 & $3 / 06 / 90$ & VOA & 4.30E+OI \\
\hline $51020 B$ & $3 / 06 / 90$ & VOA & $1.00 \mathrm{E}+01$ \\
\hline $51020 \mathrm{~T}$ & $3 / 06 / 90$ & VOA & $<8.00 \mathrm{E}+00$ \\
\hline 50895 & $1 / 23 / 90$ & $A B N$ & $3.76 \mathrm{E}+04$ \\
\hline 50935 & $2 / 13 / 90$ & $A B N$ & $8.00 \mathrm{E}+04$ \\
\hline 59171 & $1 / 17 / 90$ & $\mathrm{ABN}$ & $3.92 E+04$ \\
\hline $59171 B$ & $1 / 17 / 90$ & $A B N$ & $3.84 \mathrm{E}+04$ \\
\hline 59176 & $1 / 22 / 90$ & $A B N$ & 1. $56 \mathrm{E}+04$ \\
\hline $59176 \mathrm{~B}$ & $1 / 22 / 90$ & $A B N$ & $1.56 \mathrm{E}+04$ \\
\hline 59181 & $1 / 2, / 90$ & $A B N$ & $1.16 \mathrm{E}+05$ \\
\hline 50951 & $2 / 20 / 90$ & $\mathrm{ABN}$ & $6.80 E+04$ \\
\hline 50988 & $2 / 27 / 90$ & $A B N$ & $6.80 E+04$ \\
\hline 51020 & $3 / 06 / 9=$ & $A B N$ & $1.98 \mathrm{E}+05$ \\
\hline 50895 & $1 / 23 / 90$ & VOA & $<5.00 E+00$ \\
\hline $50895 B$ & $1 / 23 / 90$ & VOA & $<5.00 E+00$ \\
\hline $50895 \mathrm{~T}$ & $1 / 23 / 90$ & VOA. & $<5.00 E+00$ \\
\hline $50935 B$ & $2 / 13 / 90$ & VOA & $<3.00 \mathrm{E}+00$ \\
\hline $50935 \mathrm{~T}$ & $2 / 13 / 90$ & VOA & $<5.00 \mathrm{E}+00$ \\
\hline 59171 & $1 / 17 / 90$ & VOA & $<2.50 \mathrm{E}+02$ \\
\hline $59171 B$ & $1 / 17 / 90$ & VOA & $<2.50 \mathrm{E}+02$ \\
\hline $59171 \mathrm{~T}$ & $1 / 17 / 90$ & VOA & $<2.50 \mathrm{E}+02$ \\
\hline 59176 & $1 / 22 / 90$ & VOA & $<2.50 \mathrm{E}+02$ \\
\hline $59176 B$ & $1 / 22 / 90$ & VOA & $<2.50 \mathrm{E}+02$ \\
\hline $59176 \mathrm{~T}$ & $1 / 22 / 90$ & VOA & $<2.50 \mathrm{E}+02$ \\
\hline 59181 & $1 / 25 / 90$ & VOA & $<2.50 \mathrm{E}+02$ \\
\hline $59181 B$ & $1 / 25 / 90$ & VOA & $<2.50 \mathrm{E}+02$ \\
\hline $59181 \mathrm{~T}$ & $1 / 25 / 90$ & VOA & $<2.50 E+02$ \\
\hline 50951 & $2 / 20 / 90$ & VOA & $<5.0 O E+O O$ \\
\hline $50951 \mathrm{~B}$ & $2 / 20 / 90$ & VOA & $6.00 \mathrm{E}+00$ \\
\hline $50951 \mathrm{~T}$ & $2 / 20 / 90$ & VOA & $<3.00 E+00$ \\
\hline 50988 & $2 / 27 / 90$ & VOA & $<5.00 \mathrm{E}+00$ \\
\hline $50988 B$ & $2 / 27 / 90$ & VOA & $1.00 \mathrm{E}+01$ \\
\hline $50988 \mathrm{~T}$ & $2 / 27 / 90$ & VOA & $1.00 E+01$ \\
\hline 51020 & $3 / 06 / 90$ & VOA & $<5.00 E+00$ \\
\hline $51020 B$ & $3 / 06 / 90$ & VOA & $<5.00 \mathrm{E}+00$ \\
\hline $51020 \mathrm{~T}$ & $3 / 06 / 90$ & VOA & $<5.00 \mathrm{E}+00$ \\
\hline 50895 & $1 / 23 / 90$ & $A B N$ & 4. $10 \mathrm{E}+02$ \\
\hline 50935 & $2 / 13 / 90$ & $A B N$ & $9.70 \mathrm{E}+03$ \\
\hline 59171 & $1 / 17 / 90$ & $A B N$ & $7.10 E+O 4$ \\
\hline $59171 \mathrm{~B}$ & $1 / 17 / 90$ & $A B N$ & $8.80 E+04$ \\
\hline 59176 & $1 / 22 / 90$ & $A B N$ & $2.40 E+03$ \\
\hline $59176 \mathrm{~B}$ & $1 / 22 / 90$ & $\mathrm{ABN}$ & 2. $70 E+03$ \\
\hline 59181 & $1 / 25 / 90$ & $A B N$ & $1.50 \mathrm{E}+03$ \\
\hline 50951 & $2 / 20 / 90$ & $\mathrm{ABN}$ & $5.70 E+04$ \\
\hline 50988 & $2 / 27 / 90$ & $A B N$ & 1. $70 \mathrm{E}+02$ \\
\hline 51020 & $3 / 06 / 90$ & $A B N$ & $1.20 \mathrm{E}+05$ \\
\hline 50951 & $2 / 20 / 90$ & $A B N$ & 1. $20 \mathrm{E}+\mathrm{O} 2$ \\
\hline 50895 & $1 / 23 / 90$ & VOA & $7.00 \mathrm{E}+00$ \\
\hline
\end{tabular}


DATA FOR PUREX Process Condensate Continued

\begin{tabular}{|c|c|c|c|c|}
\hline Constituent & sample \# & Date & Method & Result \\
\hline Unknown & 50895 & $1 / 23 / 90$ & ABN & $1.20 \mathrm{E}+02$ \\
\hline Unknown & 50935 & $2 / 13 / 90$ & $A B N$ & $1.50 E+04$ \\
\hline Unknown & $59176 B$ & $1 / 22 / 90$ & $A B N$ & $1.30 \mathrm{E}+03$ \\
\hline Unknown & 50951 & $2 / 20 / 90$ & VOA & $3.60 \mathrm{E}+01$ \\
\hline Untrnown & 51020 & $3 / 06 / 90$ & VOA & $5.00 \mathrm{E}+00$ \\
\hline Unknown aliphatic HC & 59171 & $1 / 17 / 90$ & $A B N$ & $1.70 E+03$ \\
\hline Unknown aliphatic HC & $59171 \mathrm{~B}$ & $1 / 17 / 90$ & $A B N$ & $2.40 \mathrm{E}+03$ \\
\hline Unknown aliphatic HC & 50951 & $2 / 20 / 90$ & $\mathrm{ABN}$ & $6.90 \mathrm{E}+02$ \\
\hline unknown ester & 50895 & $1 / 23 / 90$ & $A B N$ & $9.60 \mathrm{E}+01$ \\
\hline Unknown ester & 59171 & $1 / 17 / 90$ & $A B N$ & $1.70 E+03$ \\
\hline Unknown ester & $59171 B$ & $1 / 17 / 90$ & $A B N$ & $1.40 \mathrm{E}+03$ \\
\hline Unknown ester & 59181 & $1 / 25 / 90$ & $A B N$ & $1.00 \mathrm{E}+02$ \\
\hline Unknown ester & 50951 & $2 / 20 / 90$ & $A B N$ & $2.00 \mathrm{E}+02$ \\
\hline Unknown ester & 50951 & $2 / 20 / 90$ & VOA & $3.00 E+01$ \\
\hline Unknown ester & 50988 & $2 / 27 / 90$ & VOA & 4. $20 \mathrm{E}+01$ \\
\hline Unknown ester & 51020 & $3 / 06 / 90$ & VOA & $2.00 \mathrm{E}+01$ \\
\hline Unknown hydrocarbon & 50895 & $1 / 23 / 90$ & $A B N$ & $4.50 E+01$ \\
\hline Unknown hydrocarbon & 50935 & $2 / 13 / 90$ & $A B N$ & 3. $10 \mathrm{E}+04$ \\
\hline Alpha Activity (pCi/L) & 50895 & $1 / 23 / 90$ & Alpha & $1.74 \mathrm{E}+00$ \\
\hline Alpha Activity (pCi/L) & 51020 & $3 / 06 / 90$ & Alpha & $1.34 \mathrm{E}+01$ \\
\hline Beta Activity (pCi/L) & 50895 & $1 / 23 / 90$ & Beta & $6.07 \mathrm{E}+00$ \\
\hline Beta Activity (pCi/L) & 51020 & $3 / 06 / 90$ & Beta & $5.83 \mathrm{E}+00$ \\
\hline Conductivity (us) & 50895 & $1 / 23 / 90$ & COND-FId & $3.95 \mathrm{E}+02$ \\
\hline Conductivity (us) & 50935 & $2 / 13 / 90$ & COND-FId & $1.50 \mathrm{E}+03$ \\
\hline Conductivity (us) & 50988 & $2 / 27 / 90$ & COND-EId & $5.70 E+01$ \\
\hline Conductivity (us) & 51020 & $3 / 06 / 90$ & COND-FId & $7.90 \mathrm{E}+01$ \\
\hline Ingitability (degrees E) & $50895 \mathrm{E}$ & $1 / 23 / 90$ & IGNIT & $2.10 \mathrm{E}+02$ \\
\hline Ingitability (degrees $\mathbf{F}$ ) & $50935 \mathrm{E}$ & $2 / 13 / 90$ & IGNIT & $2.12 \mathrm{E}+02$ \\
\hline Ingitability (degrees $\mathrm{F}$ ) & $50951 \mathrm{E}$ & $2 / 20 / 90$ & IGNIT & $2.10 \mathrm{E}+02$ \\
\hline Ingitability (degrees E) & 50988E & $2 / 27 / 90$ & IGNIT & $2.04 E+02$ \\
\hline Ingitability (degrees F) & $51020 \mathrm{E}$ & $3 / 06 / 90$ & IGNIT & $2.04 \mathrm{E}+02$ \\
\hline $\mathrm{pH}$ (dimensionless) & 50895 & $1 / 23 / 90$ & PH-FId & $2.88 \mathrm{E}+00$ \\
\hline $\mathrm{pH}$ (dimensionless) & 50935 & $2 / 13 / 90$ & PH-EId & $2.60 \mathrm{E}+00$ \\
\hline pH (dimensionless) & 50988 & $2 / 27 / 90$ & PH-FId & 4. $40 \mathrm{E}+00$ \\
\hline pH (dimensionless) & 51020 & $3 / 06 / 90$ & PH-EId & $2.30 E+00$ \\
\hline Temperature (degrees C) & 50895 & $1 / 23 / 90$ & TEMP-EId & $2.30 \mathrm{E}+01$ \\
\hline Temperature (degrees C) & 50988 & $2 / 27 / 90$ & TEMP-FId & $3.78 \mathrm{E}+01$ \\
\hline Temperature (degrees C) & 51020 & $3 / 06 / 911$ & TEMP-EId & $7.90 \mathrm{E}+01$ \\
\hline $\operatorname{TOC}(u g / g)$ & 50895 & $1 / 23 / 50$ & I'OC & $6.60 \mathrm{E}+04$ \\
\hline $\operatorname{TOC}(u g / g)$ & 50935 & $2 / 13 / 90$ & TOC & $8.02 \mathrm{E}+04$ \\
\hline TOC $(u g / g)$ & 50951 & $2 / 20 / 90$ & TOC & $1.02 \mathrm{E}+05$ \\
\hline TOC $(u g / g)$ & 50988 & $2 / 27 / 90$ & TOC & $1.41 \mathrm{E}+05$ \\
\hline $\operatorname{TOC}(\mathrm{ug} / \mathrm{g})$ & 51020 & $3 / 06 / 90$ & TOC & $1.42 \mathrm{E}+05$ \\
\hline $\operatorname{TOX}(\mathrm{ug}(\mathrm{Cl}) / \mathrm{L})$ & 50895 & $1 / 23 / 90$ & ITOX & $6.40 \mathrm{E}+01$ \\
\hline $\operatorname{TOX}(\mathrm{ug}(\mathrm{Cl}) / \mathrm{L})$ & 50935 & $2 / 13 / 90$ & LTOX & $5.60 \mathrm{E}+01$ \\
\hline $\operatorname{TOX}(\operatorname{ug}(\mathrm{CI}) / I)$ & 50951 & $2 / 20 / 90$ & LTOX & $2.60 \mathrm{E}+01$ \\
\hline $\operatorname{TOX}(\operatorname{ug}(C I) / L)$ & 50988 & $2 / 27 / 90$ & LTOX & $4.70 E+01$ \\
\hline $\operatorname{TOX}(\operatorname{ug}(\mathrm{Cl}) / \mathrm{I})$ & 51020 & $3 / 06 / 90$ & ITOX & $4.70 E+0 I$ \\
\hline
\end{tabular}


WHC-EP-0355, Appendix D

DATA FOR PUREX Steam Condensate

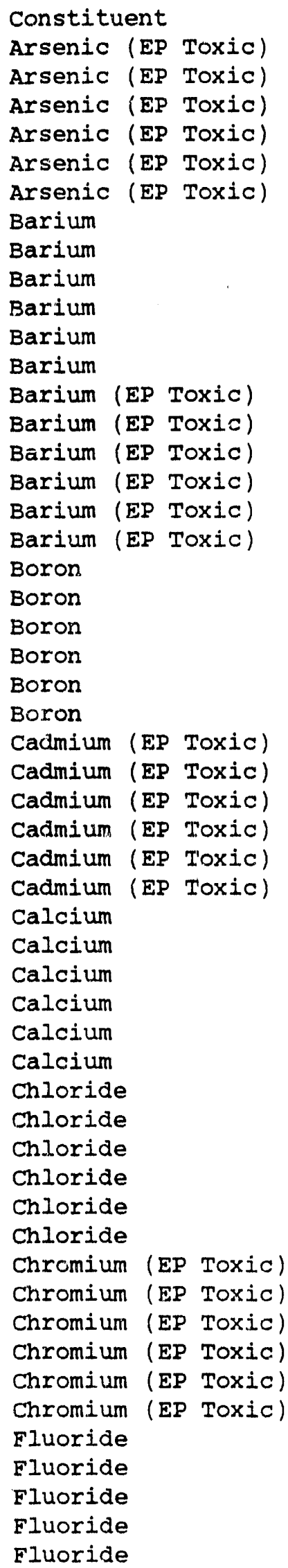

\begin{tabular}{|c|c|c|c|}
\hline Sample \# & Date & Method & Result \\
\hline $50792 \mathrm{E}$ & $11 / 27 / 89$ & $I C P$ & $<5.00 E+02$ \\
\hline $50883 E$ & $1 / 16 / 90$ & ICP & $<5.00 \mathrm{E}+02$ \\
\hline 50887E & $1 / 17 / 90$ & $I C P$ & $<5.00 E+02$ \\
\hline 30899E & $1 / 24 / 90$ & ICP & $<5.00 \mathrm{E}+02$ \\
\hline $51016 \mathrm{E}$ & $3 / 06 / 90$ & ICP & $<5.00 E+02$ \\
\hline $51075 \mathrm{E}$ & $3 / 20 / 90$ & ICP & $<5.00 E+02$ \\
\hline 50792 & $1.1 / 27 / 89$ & ICP & $3.00 \mathrm{E}+01$ \\
\hline 50883 & $1 / 16 / 90$ & ICP & $2.80 E+01$ \\
\hline 50887 & $1 / 17 / 90$ & ICP & $3.00 E+01$ \\
\hline 50899 & $1 / 24 / 90$ & ICP & $2.80 E+01$ \\
\hline 51016 & $3 / 06 / 90$ & ICP & $2.40 E+01$ \\
\hline 51075 & $3 / 20 / 90$ & ICP & $2.90 E+01$ \\
\hline $50792 E$ & $11 / 27 / 89$ & ICP & $<1.00 \mathrm{E}+03$ \\
\hline 50883E & $1 / 16 / 90$ & ICP & $<1.00 \mathrm{E}+03$ \\
\hline $50887 \mathrm{E}$ & $1 / 17 / 90$ & ICP & $<1.00 \mathrm{E}+03$ \\
\hline 50899E & $1 / 24 / 90$ & ICP & $<1.00 E+03$ \\
\hline $51016 \mathrm{E}$ & $3 / 06 / 90$ & ICP & $\cdot 1.00 E+03$ \\
\hline $51075 \mathrm{E}$ & $3 / 20 / 90$ & ICP & $<1.00 E+03$ \\
\hline 50792 & $11 / 27 / 89$ & ICP & $<1.00 E+01$ \\
\hline 50883 & $1 / 16 / 90$ & ICP & $<1.00 \mathrm{E}+01$ \\
\hline 50887 & $1 / 17 / 90$ & ICP & 1. $40 \mathrm{E}+01$ \\
\hline 50899 & $1 / 24 / 90$ & ICP & $<1.00 E+01$ \\
\hline 51016 & $3 / 06 / 90$ & ICP & $3.20 E+01$ \\
\hline 51075 & $3 / 20 / 90$ & ICP & $2.90 \mathrm{E}+01$ \\
\hline $50792 \mathrm{E}$ & $11 / 27 / 89$ & $I C P$ & $<1.00 E+02$ \\
\hline 50883E & $1 / 16 / 90$ & ICP & $<1.00 \mathrm{E}+02$ \\
\hline 50887E & $1 / 17 / 90$ & ICP & $<1.00 \mathrm{E}+02$ \\
\hline $50899 E$ & $1 / 24 / 90$ & ICP & $<1.00 \mathrm{E}+02$ \\
\hline $51016 \mathrm{E}$ & $3 / 06 / 90$ & ICP & $<1.00 \mathrm{E}+02$ \\
\hline 51075E & $3 / 20 / 90$ & ICP & $<1.00 \mathrm{E}+02$ \\
\hline 50792 & $11 / 27 / 89$ & ICP & 1.71E+04 \\
\hline 50883 & $1 / 16 / 90$ & ICP & $1.73 \mathrm{E}+04$ \\
\hline 50887 & $1 / 17 / 90$ & ICP & $1.81 E+04$ \\
\hline 50899 & $1 / 24 / 90$ & ICP & $1.79 E+04$ \\
\hline 5.1016 & $3 / 06 / 90$ & ICP & 1. $49 \mathrm{E}+04$ \\
\hline 51075 & $3 / 20 / 90$ & ICP & $1.80 \mathrm{E}+04$ \\
\hline 50792 & $11 / 27 / 89$ & IC & $9.00 \mathrm{E}+02$ \\
\hline 50883 & $1 / 16 / 90$ & IC & $1.10 \mathrm{E}+03$ \\
\hline 50887 & $1 / 17 / 90$ & $I C$ & $1.10 \mathrm{E}+03$ \\
\hline 50899 & $1 / 24 / 90$ & IC & $1.00 \mathrm{E}+03$ \\
\hline 51016 & $3 / 06 / 90$ & IC & $8.00 \mathrm{E}+02$ \\
\hline 51075 & $3 / 20 / 90$ & IC & $8.00 E+02$ \\
\hline $50792 \mathrm{E}$ & $11 / 27 / 89$ & ICP & $<5.00 \mathrm{E}+02$ \\
\hline $50883 E$ & $1 / 16 / 90$ & ICP & $<5.00 E+02$ \\
\hline $50887 \mathrm{E}$ & $1 / 17 / 90$ & ICP & $<5.00 E+02$ \\
\hline 50899E & $1 / 24 / 90$ & ICP & $<5.00 \mathrm{E}+02$ \\
\hline $51016 \mathrm{E}$ & $3 / 06 / 90$ & ICP & $<5.00 E+02$ \\
\hline 51075E & $3 / 20 / 90$ & ICP & $<5.00 E+02$ \\
\hline 50792 & $11 / 27 / 89$ & IC & $<5.00 \mathrm{E}+02$ \\
\hline 50792 & $1] / 27 / 89$ & ISE & 1. $36 \mathrm{E}+02$ \\
\hline 50883 & $1 / 16 / 90$ & $I C$ & $<5.00 E+02$ \\
\hline 50883 & $1 / 16 / 90$ & ISE & $1.17 \mathrm{E}+02$ \\
\hline 50887 & $1 / 17 / 90$ & IC & $<5.00 E+02$ \\
\hline
\end{tabular}


DATA FOR PUREX steam Condensate Continued

\begin{tabular}{|c|c|c|}
\hline Constitu & & \\
\hline Fluoride & & \\
\hline Eluoride & & \\
\hline Eluoride & & \\
\hline Fluoride & & \\
\hline Fluoride & & \\
\hline Fluoride & & \\
\hline Fluoride & & \\
\hline Iron & & \\
\hline Iron & & \\
\hline Iron & & \\
\hline Iron & & \\
\hline Iron & & \\
\hline Iron & & \\
\hline Lead (EP & Toxi & ic) \\
\hline Lead (EP & Toxi & ic) \\
\hline Lead (EF & Toxi & ic) \\
\hline Lead (EP & Toxi & ic) \\
\hline Lead (EP & Toxi & ic) \\
\hline Lead (EP & Toxi & ic) \\
\hline Magnesiu & & \\
\hline Magnesiu & & \\
\hline Magnesiu & & \\
\hline Magnesiu & & \\
\hline Magnesiu & & \\
\hline Magnesiu & & \\
\hline Mercury & (EP T & Toxic) \\
\hline Mercury & (EP I & Toxic) \\
\hline Mercury & (EP I & Toxic) \\
\hline Mercury & (EP I & Toxic) \\
\hline Mercury & (EP I & Toxic) \\
\hline Mercury & (EP I & Toxic) \\
\hline Nitrate & & \\
\hline Nitrate & & \\
\hline Nitrate & & \\
\hline Nitrate & & \\
\hline Nitrate & & \\
\hline Nitrate & & \\
\hline Potassiu & & \\
\hline Potassiu & & \\
\hline Potassiu & & \\
\hline Potassiu & & \\
\hline Potassiu & & \\
\hline Potassiu & & \\
\hline Selenium & (EP & Toxic) \\
\hline Selenium & (EP & Toxic) \\
\hline Selenium & (EP & Toxic) \\
\hline Selenium & (EP & Toxic) \\
\hline Selenium & (EP & Toxic) \\
\hline Selenium & $(E P$ & Toxic) \\
\hline Silicon & & \\
\hline silicon & & \\
\hline silicon & & \\
\hline Silicon & & \\
\hline
\end{tabular}

\begin{tabular}{|c|c|c|c|}
\hline Sample \# & Dáte & Method & Result \\
\hline 50887 & $1 / 17 / 90$ & ISE & $1.13 \mathrm{E}+02$ \\
\hline 50899 & $1 / 24 / 90$ & IC & $<5.00 \mathrm{E}+02$ \\
\hline 50899 & $1 / 24 / 90$ & ISE & $1.14 \mathrm{E}+02$ \\
\hline 51016 & $3 / 06 / 90$ & IC & $<5.00 \mathrm{E}+02$ \\
\hline 51016 & $3 / 06 / 90$ & ISE & $1.15 \mathrm{E}+02$ \\
\hline 51075 & $3 / 20 / 90$ & IC & $<5.00 E+02$ \\
\hline 51075 & $3 / 20 / 90$ & ISE & $1.14 \mathrm{E}+02$ \\
\hline 50792 & $11 / 27 / 89$ & ICP & $3.30 \mathrm{E}+01$ \\
\hline 50883 & $1 / 16 / 90$ & ICP & $<3.00 E+01$ \\
\hline 50887 & $1 / 17 / 90$ & ICP & $<3.00 E+01$ \\
\hline 50899 & $1 / 24 / 90$ & ICP & 3. $20 \mathrm{E}+01$ \\
\hline 51016 & $3 / 06 / 90$ & ICP & $3.30 \mathrm{E}+01$ \\
\hline 51075 & $3 / 20 / 90$ & ICP & $<3.00 \mathrm{E}+01$ \\
\hline $50792 \mathrm{E}$ & $11 / 27 / 89$ & ICP & $<5.00 \mathrm{E}+02$ \\
\hline $50883 E$ & $1 / 16 / 90$ & ICP & $<5.00 \mathrm{E}+02$ \\
\hline $50887 \mathrm{E}$ & $1 / 17 / 90$ & ICP & $<5.00 \mathrm{E}+02$ \\
\hline 50899E & $1 / 24 / 90$ & ICP & $<5.00 \mathrm{E}+02$ \\
\hline $51016 \mathrm{E}$ & $3 / 06 / 90$ & ICP & $<5.00 E+02$ \\
\hline $51075 \mathrm{E}$ & $3 / 20 / 90$ & ICP & $<5.00 \mathrm{E}+02$ \\
\hline 50792 & $11 / 27 / 89$ & ICP & $3.94 \mathrm{E}+03$ \\
\hline 50883 & $1 / 16 / 90$ & ICP & $4.06 \mathrm{E}+03$ \\
\hline 50887 & $1 / 17 / 90$ & ICP & 4. $25 \mathrm{E}+03$ \\
\hline 50899 & $1 / 24 / 90$ & ICP & $4.08 E+03$ \\
\hline 51016 & $3 / 06 / 90$ & ICP & $3.59 \mathrm{E}+03$ \\
\hline 51075 & $3 / 20 / 90$ & ICP & $4.492+03$ \\
\hline $50792 \mathrm{E}$ & $11 / 27 / 89$ & CVAA/M & $<2.00 \mathrm{E}+01$ \\
\hline $50883 \mathrm{E}$ & $1 / 16 / 90$ & CVAA/M & $<2.00 \mathrm{E}+01$ \\
\hline $50887 \mathrm{E}$ & $1 / 17 / 90$ & CVAA/M & $<2.00 \mathrm{E}+01$ \\
\hline 50899E & $1 / 24 / 90$ & $C V A A / M$ & $<2.00 E+01$ \\
\hline $51016 \mathrm{E}$ & $3 / 06 / 90$ & CVAA/M & $<2.00 \mathrm{E}+01$ \\
\hline $51075 \mathrm{E}$ & $3 / 20 / 90$ & CVAA/M & $<2.00 \mathrm{E}+01$ \\
\hline 50792 & $11 / 27 / 89$ & IC & $6.00 E+02$ \\
\hline 50883 & $1 / 16 / 90$ & IC & $6.00 E+02$ \\
\hline 50887 & $1 / 17 / 90$ & IC & $6.00 E+02$ \\
\hline 50899 & $1 / 24 / 90$ & IC & $5.00 \mathrm{E}+02$ \\
\hline 51016 & $3 / 06 / 90$ & IC & $<5 ., 0 \mathrm{E}+02$ \\
\hline 51075 & $3 / 20 / 90$ & IC & $<5.00 \mathrm{E}+02$ \\
\hline 50792 & $11 / 27 / 89$ & ICP & $7.24 \mathrm{E}+02$ \\
\hline 50883 & $1 / 16 / 90$ & ICP & $6.67 \mathrm{E}+02$ \\
\hline 50887 & $1 / 17 / 90$ & ICP & $6.94 E+02$ \\
\hline 50899 & $1 / 24 / 90$ & ICP & $6.60 \mathrm{E}+02$ \\
\hline 51016 & $3 / 06 / 90$ & ICP & $6.02 \mathrm{E}+02$ \\
\hline 51075 & $3 / 20 / 90$ & ICP & $7.45 \mathrm{E}+02$ \\
\hline 50792.E & $11 / 27 / 89$ & ICP & $<5.00 \mathrm{E}+02$ \\
\hline $50883 \mathbf{E}$ & $1 / 16 / 90$ & ICP & $<5.00 E+02$ \\
\hline $50887 \mathrm{E}$ & $1 / 17 / 90$ & ICP & $<5.00 \mathrm{E}+02$ \\
\hline $50899 \mathrm{E}$ & $1 / 24 / 90$ & ICP & $<5.00 \mathbf{E}+02$ \\
\hline $51016 \mathrm{E}$ & $3 / 06 / 90$ & ICP & $<5.00 \mathrm{E}+02$ \\
\hline $51075 \mathrm{E}$ & $3 / 20 / 90$ & ICP & $<5.00 \mathrm{E}+02$ \\
\hline 50792 & $11 / 27 / 89$ & ICP & $2.26 \mathrm{E}+03$ \\
\hline 50883 & $1 / 16 / 90$ & ICP & $2.27 E+03$ \\
\hline 50887 & $1 / 17 / 90$ & ICP & $2.35 \mathrm{E}+03$ \\
\hline 50899 & $1 / 24 / 90$ & ICP & $2.30 \mathrm{E}+03$ \\
\hline
\end{tabular}


DATA FOR PUREX Steum Condensate

Continued

Constituent
Silicon
Silicon
Silver (EP Toxic)
Silver (EP TOxiC)
Silver (EP Toxic)
Silver (EP TOxic)
Silver (EP Toxic)
Silver (EP Toxic)
Sodium
Sodium
Sodium
Sodium
Sodium
Sodium
Strontium
Strontium
Strontium
Strontium
Strontium
Strontium
Sulfate
Sulfate
Sulfate
Sulfate
Sulfate
Sulfate
Uranium
Uranium
Uranium
Uranium
Uranium
Uranium
zinc
Zinc
Zinc
Zinc
Zinc
Zinc
Acetone
Acetone
Acetone
Acetone
Acetone
Acetone
Acetone
Acetonetone
Acetone
Acetone
Acetone

\begin{tabular}{|c|c|c|c|}
\hline mole \# & & Method & Result \\
\hline 51016 & $3 / 06 / 90$ & ICP & $1.87 E+03$ \\
\hline 51075 & $3 / 20 / 90$ & ICP & $2.23 E+03$ \\
\hline $50792 \mathrm{E}$ & $11 / 27 / 89$ & ICP & $<5.00 \mathrm{E}+02$ \\
\hline $50883 E$ & $1 / 16 / 90$ & ICP & $<5.00 \mathrm{E}+02$ \\
\hline $50887 \mathrm{E}$ & $1 / 17 / 90$ & ICP & $<5.00 \mathrm{E}+02$ \\
\hline 50899E & $1 / 24 / 90$ & ICP & $<5.00 E+02$ \\
\hline $51016 \mathrm{E}$ & $3 / 06 / 90$ & ICP & $<5.00 E+02$ \\
\hline $51075 \mathrm{E}$ & $3 / 20 / 90$ & ICP & $<5.00 E+02$ \\
\hline 50792 & $11 / 27 / 89$ & I P & $2.01 E+03$ \\
\hline 50883 & $1 / 16 / 90$ & ICP & $2.05 E+03$ \\
\hline 50887 & $1 / 17 / 90$ & ICP & $2.07 E+03$ \\
\hline 50899 & $1 / 24 / 90$ & ICP & $1.99 \mathrm{E}+03$ \\
\hline 51016 & $3 / 06 / 90$ & ICP & $1.76 \mathrm{E}+03$ \\
\hline 51075 & $3 / 20 / 90$ & ICP & $2.03 \mathrm{E}+03$ \\
\hline 50792 & $11 / 27 / 89$ & ICP & $8.60 \mathrm{E}+01$ \\
\hline 50883 & $1 / 16 / 90$ & ICP & $7.80 \mathrm{E}+01$ \\
\hline 50887 & $1 / 17 / 90$ & ICP & $8.10 \mathrm{E}+01$ \\
\hline 50899 & $1 / 24 / 90$ & $I C P$ & $8.20 E+01$ \\
\hline 51016 & $3 / 06 / 90$ & ICP & $7.70 \mathrm{E}+01$ \\
\hline 51075 & $3 / 20 / 90$ & $I C_{x}$ & $9.60 \mathrm{E}+01$ \\
\hline 50792 & $11 / 27 / 89$ & IC & $9.00 \mathrm{E}+03$ \\
\hline 50883 & $1 / 16 / 90$ & IC & $9.70 E+03$ \\
\hline 50887 & $1 / 17 / 90$ & IC & $9.70 \mathrm{E}+03$ \\
\hline 50899 & $1 / 24 / 90$ & IC & $9.50 \mathrm{E}+03$ \\
\hline 51016 & $3 / 06 / 90$ & $I C$ & $8.70 E+03$ \\
\hline 51.075 & $3 / 20 / 90$ & IC & $1.02 \mathrm{E}+04$ \\
\hline 50792 & $11 / 27 / 89$ & GEA & $5.17 \mathrm{E}-01$ \\
\hline 50883 & $1 / 16 / 90$ & GEA & $6.07 \mathrm{E}-01$ \\
\hline 50887 & $1 / 17 / 90$ & GEA & $6.11 E-01$ \\
\hline 50899 & $1 / 24 / 90$ & GEA & $5.16 \mathrm{E}-01$ \\
\hline 51016 & $3 / 06 / 90$ & GEA & $4.61 E-0.1$ \\
\hline 51075 & $3 / 20 / 90$ & GEA & $4.40 \mathrm{E}-01$ \\
\hline 50792 & $11 / 27 / 89$ & ICP & $<5.00 \mathrm{E}+00$ \\
\hline 50883 & $1 / 16 / 90$ & ICP & $<5.00 E+00$ \\
\hline 50887 & $1 / 17 / 90$ & $I C P$ & $<5.00 E+00$ \\
\hline 50899 & $1 / 24 / 90$ & ICP & $6.00 \mathrm{E}+00$ \\
\hline 51016 & $3 / 06 / 90$ & ICP & $<5.00 \mathrm{E}+00$ \\
\hline 51075 & $3 / 20 / 90$ & ICP & $6.00 E+00$ \\
\hline 50792 & $11 / 27 / 89$ & VOA & $<1.00 \mathrm{E}+01$ \\
\hline 50792 & $11 / 27 / 89$ & $A B N$ & $<1.00 \mathrm{E}+01$ \\
\hline $50792 B$ & $11 / 27 / 89$ & VOA & $<1.00 \mathrm{E}+01$ \\
\hline 50883 & $1 / 16 / 90$ & VOA & $<1.00 \mathrm{E}+01$ \\
\hline 50883 & $1 / 16 / 90$ & $A B N$ & $<1.00 \mathrm{E}+01$ \\
\hline $50883 B$ & $1 / 16 / 90$ & VOA & $<6.00 \mathrm{E}+00$ \\
\hline $50883 T$ & $1 / 16 / 90$ & VOA & $<7.00 E+00$ \\
\hline 50887 & $1 / 17 / 90$ & VOA & $<1.00 \mathrm{E}+01$ \\
\hline 50887 & $1 / 17 / 90$ & $\mathrm{ABN}$ & $<1.00 \mathrm{E}+01$ \\
\hline $50887 \mathrm{~B}$ & $1 / 17 / 90$ & VOA & $<1.00 E+01$ \\
\hline $50887 \mathrm{~T}$ & $1 / 17 / 90$ & VOA & $<1.00 \mathrm{E}+01$ \\
\hline 50899 & $1 / 24 / 90$ & VOA & $<1.00 E+01$ \\
\hline 50899 & $1 / 24 / 90$ & $A B N$ & $<1.00 \mathrm{E}+01$ \\
\hline $50899 B$ & $1 / 24 / 90$ & VOA & $<6.00 \mathrm{E}+00$ \\
\hline $50899 \mathrm{~T}$ & $1 / 24 / 90$ & VOA & $<6.00 \mathrm{E}+00$ \\
\hline
\end{tabular}


DATA FOR PUREX steam Condensate

Continued

Constituent
Acetone
Acetone
Acetone
Acetone
Acetone
Acetone
Acetone
Acetone
1-Butanol
1-Butanol
1-Butanol
1-Butanol
1-Butanol
1-Butanol
1-Butanol
1-Butanol
1-Butanol
2-Butanone
2-Butanone
2-Butanone
2-Butanone
2-Butanone
2-Butanone
2-Butanone
2-Butanone
2-Butanone
2-Butanone
2-Butanone
2-Butanone
2-Butanone
2-Butanone
2-Butanone
2-Butanone
2-Butanone
Dichloromethane
Dichloromethane
Dichloromethane
Dichloromethane
Dichloromethane
Dichloromethane
Dichloromethane
Dichloromethane
Dichloromethane
Dichloromethane
Dichloromethane
Dichloromethane
Dichloromethane
Dichloromethane
Dichloromethane
Dichloromethane
Dichloromethane
Tetrahydrofuran
Tetrahydrofuran

\begin{tabular}{|c|c|c|c|}
\hline sample \# & Date & Method & Result \\
\hline 51016 & $3 / 06 / 90$ & VOA & $1.00 E+01$ \\
\hline 51016 & $3 / 06 / 90$ & $A B N$ & $<1.00 \mathrm{E}+01$ \\
\hline $51016 \mathrm{~B}$ & $3 / 06 / 90$ & VOA & $<5.00 E+00$ \\
\hline $51016 \mathrm{~T}$ & $3 / 06 / 90$ & VOA & $<1.00 E+01$ \\
\hline 51075 & $3 / 20 / 90$ & VOA & $<1.00 E+01$ \\
\hline 51075 & $3 / 20 / 90$ & $A B N$ & $<1.00 E+01$ \\
\hline $51075 B$ & $3 / 20 / 90$ & VOA & $<1.00 E+01$ \\
\hline $51075 \mathrm{~T}$ & $3 / 20 / 90$ & VOA & $<1.00 E+01$ \\
\hline 50792 & $11 / 27 / 89$ & DIGC & $<1.00 \mathrm{E}+04$ \\
\hline 50883 & $1 / 16 / 90$ & DIGC & $<1.00 E+04$ \\
\hline 50887 & $1 / 17 / 90$ & DIGC & $<1.00 \mathrm{E}+04$ \\
\hline 50899 & $1 / 24 / 90$ & DIGC & $<1.00 \mathrm{E}+04$ \\
\hline 51016 & $3 / 06 / 90$ & VOA & $2.40 E+01$ \\
\hline 51016 & $3 / 06 / 90$ & DIGC & $<1.00 E+04$ \\
\hline $51016 \mathrm{~EB}$ & $3 / 06 / 90$ & VOA & $2.30 E+01$ \\
\hline 51016x & $3 / 06 / 90$ & VOA & 2. $30 \mathrm{E}+01$ \\
\hline 51075 & $3 / 20 / 90$ & DIGC & $<1.00 \mathrm{E}+04$ \\
\hline 50792 & $11 / 27 / 89$ & VOA & $<1.00 \mathrm{E}+01$ \\
\hline $50792 \mathrm{E}$ & $11 / 27 / 89$ & VOA & $<1.00 E+01$ \\
\hline 50883 & $1 / 16 / 90$ & VOA & $<1.00 E+01$ \\
\hline $50833 \mathrm{E}$ & $1 / 16 / 90$ & VOA & $<1.00 E+01$ \\
\hline $50883 \mathrm{~T}$ & $1 / 16 / 90$ & VOA & $<1.00 \mathrm{E}+01$ \\
\hline 50887 & $1 / 17 / 90$ & VOA & $<1.00 E+01$ \\
\hline $50887 \mathrm{E}$ & $1 / 17 / 90$ & VOA & $<6.00 \mathrm{E}+00$ \\
\hline 50887J? & $1 / 17 / 90$ & VOA & $<1.00 \mathrm{E}+01$ \\
\hline 50899 & $1 / 24 / 90$ & VOA & $<1.00 E+01$ \\
\hline 50899E3 & $1 / 24 / 90$ & VOA & $1.50 \mathrm{E}+01$ \\
\hline 50899I! & $1 / 24 / 90$ & VOA & $1.60 \mathrm{E}+01$ \\
\hline 51016 & $3 / 06 / 90$ & VOA & $<1.00 \mathrm{E}+01$ \\
\hline $51016 \mathrm{E} 3$ & $3 / 06 / 90$ & VOA & $<1.00 E+01$ \\
\hline 51016'! & $3 / 06 / 90$ & VOA & $<1.00 \mathrm{E}+01$ \\
\hline 51075 & $3 / 20 / 90$ & VOA & $<1.00 \mathrm{E}+01$ \\
\hline $51075 E 3$ & $3 / 20 / 90$ & VOA & $<1.00 E+01$ \\
\hline 51075J" & $3 / 20 / 90$ & VOA & $<1.00 E+01$ \\
\hline 50792 & $11 / 27 / 89$ & VOA & $<5.00 \mathrm{E}+00$ \\
\hline $50792 \mathrm{~B}$ & $11 / 27 / 89$ & VOA & $<5.00 E+00$ \\
\hline 50883 & $1 / 16 / 90$ & VOA & $<5.00 E+O O$ \\
\hline 5088313 & $1 / 16 / 90$ & VOA & $<5.00 E+00$ \\
\hline $50883 \mathrm{~T}$ & $1 / 16 / 90$ & VOA & $<5.00 E+00$ \\
\hline 50887 & $1 / 17 / 90$ & VOA & $<5.00 E+00$ \\
\hline $50887 B$ & $1 / 17 / 90$ & VOA & $<3.00 \mathrm{E}+00$ \\
\hline $50887 ' \mathrm{I}$ & $1 / 17 / 90$ & VOA & $1.50 \mathrm{E}+01$ \\
\hline 50899 & $1 / 24 / 90$ & VOA & $<5.00 \mathrm{E}+00$ \\
\hline $50899 B$ & $1 / 24 / 90$ & VOA & $<4.00 E+00$ \\
\hline $50899 \mathrm{~T}$ & $1 / 24 / 90$ & VOA & $<3.00 \mathrm{E}+00$ \\
\hline 51016 & $3 / 06 / 90$ & VOA & $<5.00 \mathrm{E}+00$ \\
\hline $51016 \mathrm{~B}$ & $3 / 06 / 90$ & VOA & $5.0 O E+00$ \\
\hline $51016 \mathrm{~T}$ & $3 / 06 / 90$ & VOA & $<5.00 E+00$ \\
\hline 51075 & $3 / 20 / 90$ & VOA & $<5.00 \mathrm{E}+00$ \\
\hline $51075 B$ & $3 / 20 / 90$ & VOA & $<5.00 E+00$ \\
\hline $51075 \mathrm{~T}$ & $3 / 20 / 90$ & VOA & $<5.00 E+00$ \\
\hline 50792 & $11 / 27 / 89$ & VOA & $<1.00 E+01$ \\
\hline $50792 . \mathrm{B}$ & $11 / 27 / 89$ & VOA & $<1.00 E+01$ \\
\hline
\end{tabular}


DATA FOR PUREX steam Condersate

Continued

Constituent

Tetrahyarofuran

Tetrahydrofuran

Tetrahydrofuran

Tetrahydrofurar

Tetrahydrofuran

Tetrahydrofuran

Tetrahydrofuran

Tetrahydrofuran

Tetrahyarofuran

Tetrahydrofuran

Tetrahycirofuran

Tetrahydrofuran

Tetrahydrofuran

Tetrahydrofuran

Tetrahydrofuran

Tributylphosphate

Tributylphosphate

Tributylphosphate

Tributylphosphate

Tributylphosphate

Tributylphosphate

Trichloromethane

Trichloromethane

Trichloromethane

Trichloromethane

Trichloromethane

Trichloromethane

Trichloromethane

Trichloromethane

Trichloromethane

Trichloromethane

Trichloromethane

Trichloromethane

Trichloromethane

Trichloromethane

Trichloromethane

Trichloromethane

Trichloromethane

Unknown aliphatic HC

Alkalinity (Method B)

Alkalinity (Method B)

Alkalinity (Method B)

Alkalinity (Method B)

alkalinity (Method B)

Alkalinity (Method B)

Alpha Activity (pCi/L)

Alpha Activity (pCi/L)

Alpha Activity (pCi/L)

Alpha Activity (pCi/L)

Alpha Activity (pCi/L)

Alpha Activity (pCi/L)

Beta Activity (pCi/L)

Beta Activity (pCi/L)

\begin{tabular}{|c|c|c|c|}
\hline Sample \# & Date & Method & Result \\
\hline 50883 & $1 / 16 / 90$ & VOA & $<1.00 \mathrm{E}+01$ \\
\hline $50883 B$ & $1 / 16 / 90$ & TOA & $<7.00 \mathrm{E}+00$ \\
\hline $50883 \mathrm{~T}$ & $1 / 16 / 90$ & VOA & $<9.00 \mathrm{E}+00$ \\
\hline 50887 & $1 / 17 / 90$ & VOA & $<1.00 \mathrm{E}+01$ \\
\hline $50887 \mathrm{~B}$ & $1 / 17 / 90$ & VOA & $<1.00 \mathrm{E}+01$ \\
\hline $50887 \mathrm{~T}$ & $1 / 17 / 90$ & VOA & $<1.00 \mathrm{E}+01$ \\
\hline 50899 & $1 / 24 / 90$ & VOA & $<1.00 E+01$ \\
\hline 508998 & $1 / 24 / 90$ & VOA & $<5.00 E+00$ \\
\hline $5 C 899 \mathrm{~T}$ & $1 / 24 / 90$ & VOA & $<6.00 \mathrm{E}+00$ \\
\hline 51016 & $3 / 06 / 90$ & VOA & $<1.00 \mathrm{E}+01$ \\
\hline $51016 \mathrm{~B}$ & $3 / 06 / 90$ & VOA & $1.00 \mathrm{E}+01$ \\
\hline $51016 \mathrm{~T}$ & $3 / 06 / 90$ & VOA & $<1.00 \mathrm{E}+01$ \\
\hline 51075 & $3 / 20 / 90$ & VOA & $<1.00 E+01$ \\
\hline $51075 B$ & $3 / 20 / 90$ & VOA & $<1.00 \mathrm{E}+01$ \\
\hline $51075 \mathrm{~T}$ & $3 / 20 / 90$ & VOA & $<1.00 E+01$ \\
\hline 50792 & $11 / 27 / 89$ & $A B N$ & $<1.00 E+01$ \\
\hline 50883 & $1 / 16 / 90$ & $\mathrm{ABN}$ & $\angle 1.00 E+01$ \\
\hline 50887 & $1 / 17 / 90$ & $\mathrm{ABN}$ & $<1.00 \mathrm{E}+01$ \\
\hline 50899 & $1 / 24 / 90$ & $\mathrm{ABN}$ & $1.40 \mathrm{E}+01$ \\
\hline 51016 & $3 / 06 / 90$ & $A B N$ & $<1.00 E+01$ \\
\hline 51075 & $3 / 20 / 90$ & $\mathrm{ABN}$ & $<1.00 \mathrm{E}+01$ \\
\hline 50792 & $11 / 27 / 89$ & $\mathrm{VCA}$ & $<5.00 E+00$ \\
\hline $50792 B$ & $11 / 27 / 89$ & VOA & $<5.00 \mathrm{E} /+00$ \\
\hline 50883 & $1 / 16 / 90$ & VOA & $<5.00 \mathrm{E}+00$ \\
\hline $50883 B$ & $1 / 16 / 90$ & VOA & $8.00 E+00$ \\
\hline $50883 T$ & $1 / 16 / 90$ & VOA & $7.00 \mathrm{E}+00$ \\
\hline 50887 & $1 / 17 / 90$ & VOA & $<5,00 E+00$ \\
\hline $50887 \mathrm{~B}$ & $1 / 17 / 90$ & VOA & $<5.00 \mathrm{E}+00$ \\
\hline $50887 \mathrm{~T}$ & $1 / 17 / 90$ & VOA & $5.00 \mathrm{E}+00$ \\
\hline 50899 & $1 / 24 / 90$ & VOA & $<5.00 E+00$ \\
\hline $50899 B$ & $1 / 24 / 90$ & VOA. & $<5.00 E+00$ \\
\hline $50899 \mathrm{~T}$ & $1 / 24 / 90$ & VOA & $<5.00 E+00$ \\
\hline 51016 & $3 / 06 / 90$ & VOA & $<5.00 \mathrm{E}+00$ \\
\hline $51016 \mathrm{~B}$ & $3 / 06 / 90$ & VOA & $<5.00 \mathrm{E}+00$ \\
\hline $51016 \mathrm{~T}$ & $3 / 06 / 90$ & VOA & $<5.00 E+00$ \\
\hline 51075 & $3 / 20 / 90$ & VOA & $<5.00 \mathrm{E}+00$ \\
\hline $51075 \mathrm{~B}$ & $3 / 20 / 90$ & VOA & $<3.00 E+00$ \\
\hline $51075 \mathrm{~T}$ & $3 / 20 / 90$ & $\mathrm{VOA}$ & $<5.00 \mathrm{E}+00$ \\
\hline $51016 \mathrm{~T}$ & $3 / 06 / 90$ & VOA & $.00 \mathrm{E}+00$ \\
\hline 50792 & $11 / 27 / 89$ & "IITRA & $5.60 \mathrm{E}+04$ \\
\hline 50883 & $1 / 16 / 90$ & TITRA & $5.70 \mathrm{E}+04$ \\
\hline 50887 & $1 / 17 / 90$ & TITRA & $5.60 E+04$ \\
\hline 50899 & $1 / 24 / 90$ & TITRA & $5.70 E+04$ \\
\hline 51016 & $3 / 06 / 90$ & TITRA & $4.70 \mathrm{E}+04$ \\
\hline 51075 & $3 / 20 / 90$ & TITRA & $5.80 E+04$ \\
\hline 50792 & $11 / 27 / 89$ & Alpha & $2.91 E+00$ \\
\hline 50883 & $1 / 16 / 90$ & Alpha & $9.92 \mathrm{E}+00$ \\
\hline 50887 & $1 / 17 / 90$ & Alpha & $7.72 \mathrm{E}+00$ \\
\hline 50899 & $1 / 24 / 90$ & Alpha & 4. $18 E+00$ \\
\hline 51016 & $3 / 06 / 90$ & Alpha & $2.18 \mathrm{E}+00$ \\
\hline 51075 & $3 / 20 / 90$ & Alpha & $5.40 \mathrm{E}+00$ \\
\hline 50792 & $11 / 27 / 89$ & Beta & $6.85 \mathrm{E}+01$ \\
\hline 50883 & $1 / .16 / 90$ & Beta & 2. $28 \mathrm{E}+02$ \\
\hline
\end{tabular}


DATA FOR PUREX Steam Condensate

Continued

Constituen:

Beta Activity (pCi/L)

Beta Activity (pci/L)

Beta Activity (pCi/L)

Beta Activity ( $\mathrm{pCi} / \mathrm{L}$ )

Conductivity (us)

Conductivity (us)

Conductivity (us)

Conductivity (us)

Conductivity (us)

Conductivity (us)

Ingitability (degrees F)

Ingitability (degrees F)

Ingitaility (degrees $\mathrm{F}$ )

Ingitabijity (degrees F)

Ingitability (degrees F)

Ingitability (degrees $\mathrm{F}$ )

$\mathrm{pH}$ (dimensionless)

pH (dimensionless)

pH (dimensionless)

$\mathrm{pH}$ (dimensionless)

$\mathrm{pH}$ (dimensionless)

$\mathrm{pH}$ (dimensionless)

Reactivity Cyanide (mg/kg)

Reactivity Cyanide (mg/ $\mathrm{kg}$ )

Reactivity Cyanide (mg/kg)

Reactivity Cyanide (mg/kg)

Reactivity Cyanide ( $\mathrm{mg} / \mathrm{kg}$ )

Reactivity Cyanide (mg/kg)

Reactivity sulfide (mg/kg)

Reactivity sulficle (mg/kg)

Reactivity Sulfide ( $\mathrm{mg} / \mathrm{kg}$ )

Reactivity sulfide ( $\mathrm{mg} / \mathrm{kg}$ )

Reactivity sulfide ( $\mathrm{mg} / \mathrm{kg}$ )

Reactivity sulfide ( $\mathrm{mg} / \mathrm{kg}$ )

$\operatorname{TDS}(\mathrm{mg} / \mathrm{L})$

$\operatorname{TDS}(\mathrm{mg} / \mathrm{L})$

$\operatorname{TDS}(\mathrm{mg} / \mathrm{L}$ )

TDS $(\mathrm{mg} / \mathrm{L})$

TDS (mg/L)

$\operatorname{TDS}(\mathrm{mg} / \mathrm{L}$ )

Temperature (degrees $C$ )

Temperature (degrees C)

Temperature (degrees $C$ )

Temperature (degrees C)

Temperature (degrees $C$ )

Temperature (degrees C)

TOC (ug/g)

TOC (ug/g)

TOC (ug/g)

$\operatorname{TOC}(u g / g)$

TOC (ug/g)

$\operatorname{TaC}(u g / g)$

Total Carbon (ug/g)

\begin{tabular}{|c|c|c|}
\hline Sample \# & Datee & Method \\
\hline 50887 & $1 / 17 / 90$ & Beta \\
\hline 50899 & $1 / 24 / 90$ & Beta \\
\hline 51016 & $3 / 06 / 90$ & Beta \\
\hline 51075 & $3 / 20 / 90$ & Beta \\
\hline 50792 & $11 / 27 / 89$ & COND-FId \\
\hline 50883 & $1 / 16 / 90$ & COND-FId \\
\hline 50887 & $1 / 17 / 90$ & COND-FId \\
\hline 50899 & $1 / 24 / 90$ & COND-FId \\
\hline 51016 & $3 / 06 / 90$ & COND-FId \\
\hline 51075 & $3 / 20 / 90$ & COND-FId \\
\hline $50792 \mathrm{E}$ & $11 / 27 / 89$ & IGNIT \\
\hline $50883 \mathrm{E}$ & $1 / 15 / 90$ & IGNIT \\
\hline $50887 \mathrm{E}$ & $1 / 17 / 90$ & IGNII \\
\hline $50899 E$ & $1 / 24 / 90$ & IGNIT \\
\hline $51016 \mathrm{E}$ & $3 / 06 / 90$ & IGNIT \\
\hline $51075 \mathrm{E}$ & $3 / 20 / 90$ & IGNIT \\
\hline 50792 & $11 / 27 / 89$ & PH - FId \\
\hline 50883 & $1 / 15 / 90$ & $P H-F I d$ \\
\hline 50887 & $1 / 17 / 90$ & $P H-E^{\prime} I d$ \\
\hline 50899 & $1 / 24 / 90$ & $P H-F I d$ \\
\hline 51016 & $3 / 06 / 90$ & PH-Eld \\
\hline 51075 & $3 / 20 / 90$ & PH-FId \\
\hline $50792 \mathrm{E}$ & $11 / 27 / 89$ & DSPEC \\
\hline $50883 \mathrm{E}$ & $1 / 16 / 90$ & DSPEC \\
\hline $50887 \mathrm{E}$ & $1 / 17 / 90$ & DSPEC \\
\hline $50899 \mathrm{E}$ & $1 / 24 / 90$ & DSPEC \\
\hline $51016 \mathrm{E}$ & $3 / 06 / 90$ & DSPEC \\
\hline $51075 \mathrm{E}$ & $3 / 20 / 90$ & DSPEC \\
\hline $50792 \mathrm{E}$ & $11 / 27 / 89$ & DTITRA \\
\hline $50883 \mathrm{E}$ & $1 / 16 ; 90$ & DTITRA \\
\hline $50887 \mathrm{E}$ & $1 / 17 / 90$ & DTITRA \\
\hline $50899 \mathrm{E}$ & $1 / 24 / 90$ & DTITRA \\
\hline $51016 \mathrm{E}$ & $3 / 06 / 90$ & DTITRA \\
\hline $51075 \mathrm{E}$ & $3 / 20 / 90$ & DTITRA \\
\hline 50792 & $11 / 27 / 89$ & TDS \\
\hline 50883 & $1 / 16 / 90$ & TDS \\
\hline 50887 & $1 / 17 / 90$ & TDS \\
\hline 50899 & $1 / 24 / 90$ & TDS \\
\hline 51016 & $3 / 06 / 90$ & TDS \\
\hline 51075 & $3 / 20 / 90$ & TDS \\
\hline 50792 & $11 / 27 / 89$ & TEMD-FId \\
\hline 50883 & $1 / 16 / 90$ & TEMP-FId \\
\hline 50887 & $1 / 17 / 90$ & TEMP $-F I d$ \\
\hline 50899 & $1 / 24 / 90$ & TEMP - FId \\
\hline 51016 & $3 / 06 / 90$ & TEMPP-EId \\
\hline 51075 & $3 / 20 / 90$ & TEMP-FId \\
\hline 50792 & $11 / 27 / 89$ & mえu \\
\hline 50883 & $1 / 16 / 90$ & TOC \\
\hline 50887 & $1 / 17 / 90$ & TOC \\
\hline 50899 & $1 / 24 / 90$ & TOC \\
\hline 51016 & $3 / 06 / 90$ & TOC \\
\hline 51075 & $3 / 20 / 90$ & TOC \\
\hline 50792 & $11 / 27 / 89$ & $\mathrm{TC}$ \\
\hline
\end{tabular}

Result

2. $26 E+02$

9. $34 E+O I$

3. $54 \mathrm{E}+01$

3. $04 \mathrm{E}+02$

1. $49 \mathrm{E}+02$

1. $41 \mathrm{E}+02$

1. $51 \mathrm{E}+02$

1. $41 \mathrm{E}+02$

1. $21 \mathrm{E}+02$

1. $29 \mathrm{E}+02$

2. $12 \mathrm{E}+02$

2. $10 \mathrm{E}+02$

2. $12 \mathrm{E}+02$

2. $10 \mathrm{E}+\mathrm{O} 2$

2. $06 \mathrm{E}+02$

2. $12 \mathrm{E}+02$

7. 20E+00

7. $40 \mathrm{E}+00$

7. $50 \mathrm{E}+00$

7. $37 \mathrm{E}+00$

$7.60 \mathrm{E}+00$

$8.09 E+00$

$<1.00 E+02$

$<1.00 E+02$

$<1.00 \mathrm{E}+02$

$<1.00 E+02$

$<1.00 \mathrm{E}+02$

$<1.00 E+02$

$<1.00 \mathrm{E}+02$

$<1.00 E+02$

$<1.00 E+02$

$<1.00 E+02$

$<1.00 E+02$

$<1.00 \mathrm{E}+02$

6. $20 E+04$

5. $90 \mathrm{E}+04$

$6.00 \mathrm{E}+04$

6. $40 \mathrm{E}+04$

5. $60 \mathrm{E}+04$

7. $40 \mathrm{E}+04$

2. $00 \mathrm{E}+01$

2. $03 \mathrm{E}+01$

2. $10 \mathrm{E}+01$

2. $37 \mathrm{E}+01$

$2.07 \mathrm{E}+01$

1. $04 \mathrm{E}+01$

<1. $30 E+03$

$<1.00 E+03$

$<1.00 E+03$

$<1.00 E+03$

$<9.00 E+02$

1. $20 \mathrm{E}+03$

1. $52 \mathrm{E}+04$ 
WHC-EP-0355, Appendix D

DATA FOR PUREX steam Condensate

Continued

Constituent

Total Carbon (ug/g)

Total Carbon (ug/g)

Total Carbon (ug/g)

Total Carbon (ug/g)

Total Carbon (ug/g)

Tox ( ug ( Cl)/L)

$\operatorname{TOX}(\operatorname{ug}(\mathrm{Cl}) / \mathrm{L})$

TOX (ug $(\mathrm{CI}) / \mathrm{L}$ )

$\operatorname{Tox}(u g(C l) / L$ )

$\operatorname{TOX}(\mathrm{ug}(\mathrm{Cl}) / \mathrm{L})$

Tox (ug (Cl)/L)

Unidentified constituent

Unidentified constituent

Unidentified constituent

Unidentified constituent

Unidentified constituent

Am-241 (pCi/L)

Am-24I (pCi/I)

Am-24I (pCi/L)

$\mathrm{Am}-241$ (pCi/L)

$\mathrm{Am}-241$ (pCi/L)

Am-241 (pCi/L)

Cs-137 (pCi/L)

Cs-137 (pCi/L)

Cs-137 (pCi/L)

Cs-137 (pCi/I)

Cs-137 (pCi/L)

Cs-137 (pCi/L)

Pu-238 (pCi/I)

Pu-238 (pCi/L)

$\mathrm{Pu}-238(\mathrm{pCi} / \mathrm{L})$

Pu-238 (pCi/L)

Pu-238 (pCi/L)

$\mathrm{Pu}-238$ (pCi/I)

Pu-239/240 (PCi/L)

$\mathrm{Pu}-239 / 240(\mathrm{pCi} / \mathrm{L})$

Pu-239/240 (pCi/L)

Pu-239/240 (pCi/L)

Pu-239/240 (pCi/L)

Pu-239/240 (pCi/L)

Radium Total (pCi/L)

Radium Total (pCi/L)

Radium Total ( $\mathrm{pCi} / \mathrm{L}$ )

Radium Total (pCi/I)

Radium Total ( $\mathrm{pCi} / \mathrm{L}$ )

Ralium Total (pCi/L)

Sr-90 (pCi/L)

$\operatorname{sr}-90(\mathrm{p} C \mathrm{I} / \mathrm{L})$

sr-90 (pCi/L)

$s r-90\left(\mathrm{pCi} / \mathrm{L}_{1}\right)$

sr-90 (pCi/L)

Sr-90 (pCi/L)

U-234 (pCi/I)

\begin{tabular}{|c|c|c|c|}
\hline Sample \# & Date & Method & Result \\
\hline 50883 & $1 / 16 / 90$ & $\mathrm{TC}$ & 1. $25 \mathrm{E}+04$ \\
\hline 50887 & $1 / 17 / 90$ & $\mathrm{TC}$ & $1.24 \mathrm{E}+04$ \\
\hline 50899 & $1 / 24 / 90$ & TC & 1. $24 \mathrm{E}+04$ \\
\hline 51016 & $3 / 06 / 90$ & TC & $1.35 \mathrm{E}+04$ \\
\hline 51075 & $3 / 20 / 90$ & $\mathrm{TC}$ & $1.59 \mathrm{E}+04$ \\
\hline 50792 & $11 / 27 / 89$ & ITOX & $<7.00 E+00$ \\
\hline 50883 & $1 / 16 / 90$ & ITOX & $<4.00 \mathrm{E}+00$ \\
\hline 50887 & $1 / 17 / 90$ & ITOX & $<5.00 E+00$ \\
\hline 50899 & $1 / 24 / 90$ & LTOX & $1.20 E+O I$ \\
\hline 51016 & $3 / 06 / 90$ & ITOX & $<4.00 E+00$ \\
\hline 51075 & $3 / 20 / 90$ & LTOX & $<5.00 E+00$ \\
\hline 50883 & $1 / 16 / 90$ & GEA & $2.43 \mathrm{E}+02$ \\
\hline 50887 & $1 / 17 / 90$ & GEA & $2.64 \mathrm{E}+02$ \\
\hline 50899 & $1 / 24 / 90$ & GEA & $9.15 \mathrm{E}+01$. \\
\hline 51016 & $3 / 06 / 90$ & GEA & $4.35 \mathbf{E}+01$ \\
\hline 51075 & $3 / 20 / 90$ & GEA & $4.69 \mathrm{E}+02$ \\
\hline 50792 & $11 / 27 / 89$ & AEA & $3.17 \mathrm{E}-01$ \\
\hline 50883 & $1 / 16 / 90$ & $A E A$ & $4.87 E-01$ \\
\hline 50887 & $1 / 17 / 90$ & $A E A$ & $5.60 \mathrm{E}-01$ \\
\hline 50899 & $1 / 24 / 90$ & AEA & $1.76 \mathrm{E}-01$ \\
\hline 51016 & $3 / 06 / 90$ & AEA & $9.62 \mathrm{E}-02$ \\
\hline 51075 & $3 / 20 / 90$ & $A E A$ & 1. $60 \mathrm{E}+00$ \\
\hline 50792 & $11 / 27 / 89$ & GEA & $2.34 \mathrm{E}+00$ \\
\hline 50883 & $1 / 16 / 90$ & GEA & $2.20 \mathrm{E}+01$ \\
\hline $5088^{\prime} 7$ & $1 / 17 / 90$ & GEA & $1.65 \mathrm{E}+01$ \\
\hline 50899 & $1 / 24 / 90$ & GEA & 1. $74 \mathrm{E}+01$ \\
\hline 51016 & $3 / 06 / 90$ & GEA & $7.85 \mathrm{E}-01$ \\
\hline 51075 & $3 / 20 / 90$ & GEA & $<2.43 \mathrm{E}+00$ \\
\hline 50797. & $11 / 27 / 89$ & AEA & $2.02 E-01$ \\
\hline 50883 & $1 / 16 / 90$ & AEA & $4.60 \mathbf{E}-01$ \\
\hline 50887 & $1 / 17 / 90$ & AEA & $5.48 E-01$ \\
\hline 50899 & $1 / 24 / 90$ & AEA & $3.45 \mathrm{E}-01$ \\
\hline 51016 & $3 / 06 / 90$ & AEA. & $2.41 E-01$ \\
\hline 51075 & $3 / 20 / 90$ & AEA & $7.60 \mathrm{E}-01$ \\
\hline 50792 & $11 / 27 / 89$ & AEA & $2.33 E+00$ \\
\hline 50883 & $1 / 16 / 90$ & AEA & $6.32 \mathrm{E}+00$ \\
\hline 50887 & $1 / 17 / 90$ & AEA & $6.94 E+00$ \\
\hline 50899 & $1 / 24 / 90$ & AEA & $4.40 E+00$ \\
\hline 51016 & $3 / 06 / 90$ & $A E A$ & $3.07 E+00$ \\
\hline 51075 & $3 / 20 / 90$ & AEA & $9.88 \mathrm{E}+00$ \\
\hline 50792 & $11 / 27 / 89$ & Alpha-Ra & $6.05 \mathrm{E}-01$ \\
\hline 50883 & $1 / 16 / 90$ & Alpha-Ra & $1.82 \mathrm{E}-01$ \\
\hline 50887 & $1 / 17 / 90$ & Alpha-Ra & $1.59 \mathrm{E}-01$ \\
\hline 50899 & $1 / 24 / 90$ & Alpha-Ra & $2.01 E-01$ \\
\hline 51016 & $3 / 06 / 90$ & Alpha-Ra & $9.10 \mathrm{E}-01$ \\
\hline 51075 & $3 / 20 / 90$ & Alpha-Ra & $1.00 E+01$ \\
\hline 50792 & $11 / 27 / 89$ & Beta & $7.76 \mathrm{E}-01$ \\
\hline 50883 & $1 / 16 / 90$ & Beta & $6.99 \mathrm{E}+00$ \\
\hline 50887 & $1 / 17 / 90$ & Beta & $1.50 \mathrm{E}+00$ \\
\hline 50899 & $1 / 24 / 90$ & Beta & $4.93 \mathrm{E}-01$ \\
\hline 51016 & $3 / 06 / 90$ & Beta & $1.79 E-01$ \\
\hline 51075 & $3 / 20 / 90$ & Beta & $<6.01 E-01$ \\
\hline 50792 & $11 / 27 / 89$ & $A E A$ & $2.02 E-01$ \\
\hline
\end{tabular}


WHC-EP-0355, Appendix D

DATA FOR PUREX Steam Condensate Continued

$$
\begin{array}{ll}
\multicolumn{3}{c}{\text { Constituent }} \\
\mathrm{U}-234 & \text { (pCi/L) } \\
\mathrm{U}-234 & (\mathrm{pCi} / \mathrm{L}) \\
\mathrm{U}-234 & (\mathrm{pCi} / \mathrm{L}) \\
\mathrm{U}-234 & (\mathrm{pCi} / \mathrm{L}) \\
\mathrm{U}-234 & (\mathrm{pCi} / \mathrm{L}) \\
\mathrm{U}-238 & (\mathrm{pCi} / \mathrm{L}) \\
\mathrm{U}-238 & (\mathrm{pCi} / \mathrm{I}) \\
\mathrm{U}-238 & (\mathrm{pCi} / \mathrm{L}) \\
\mathrm{U}-238 & (\mathrm{pCi} / \mathrm{L}) \\
\mathrm{U}-238 & (\mathrm{pCi} / \mathrm{L}) \\
\mathrm{U}-238 & (\mathrm{pCi} / \mathrm{L})
\end{array}
$$

\begin{tabular}{crll} 
Sample \# & \multicolumn{1}{c}{ Date } & Method & Result \\
50883 & $1 / 16 / 90$ AEA & $1.84 E-01$ \\
50887 & $1 / 17 / 90$ AEA & $2.01 E-01$ \\
50899 & $1 / 24 / 90$ AEA & $2.94 E-01$ \\
51016 & $3 / 06 / 90$ AEA & $2.22 E-01$ \\
51075 & $3 / 20 / 90$ AEA & $2.99 E-01$ \\
50792 & $11 / 27 / 89$ AEA & $1.71 E-01$ \\
50883 & $1 / 16 / 90$ AEA & $1.92 E-01$ \\
50887 & $1 / 17 / 90$ AEA & $1.01 E-01$ \\
50899 & $1 / 24 / 90$ AEA & $1.69 E-01$ \\
51016 & $3 / 06 / 90$ AEA & $1.27 E-01$ \\
51075 & $3 / 20 / 90$ AEA & $1.82 E-01$
\end{tabular}


WHC-EP-0355, Appendix D

DATA FOR 222-S Laboratory Wastewater

Constituent

Arsenic (EP Toxic)

Arsenic (EP Toxic)

Arsenic (EP TOxic)

Arsenic (EP Toxic)

Barium

Barium

Barium

Barium

Barium (EP Toxic)

Barium (EP TOxic)

iarium (EP Toxic)

Barium (EP TOxic)

Boron

Boron

Boron

Boron

Cadmium (EP Toxic)

Cadmium (EP TOXic)

Cadmium (EP TOXic)

Cadmium (EP TOXic)

Calcium

Calcium

Calcium

Calcium

Chloride

Chloride

Chloride

Chloride

Chromium (EP Toxic)

Chromium (EP Toxic)

Chromium (EP Toxic)

Chromium (EP Toxic)

Copper

Copper

Copper

Copper

Fluoride

Fluoride

Fluoride

Fluoride

Fluoride

Fluoride

Fluoride

Fluoride

Iron

Iron

Iron

Iron

Lead (EP Toxic)

Lead (EP Toxic)

Lead (EP Toxic)

Lead (EP Toxic)

Magnesium

\begin{tabular}{|c|c|c|c|}
\hline Sample \# & Date & Method & Result \\
\hline $50804 \mathrm{E}$ & $11 / 28 / 89$ & ICP & $<5.00 \mathrm{E}+02$ \\
\hline $50661 \mathrm{E}$ & $10 / 09 / 89$ & ICP & $<5.00 \mathrm{E}+02$ \\
\hline $50673 E$ & $10 / 11 / 89$ & ICP & $<5.00 \mathrm{E}+02$ \\
\hline $51079 E$ & $3 / 21 / 90$ & ICP & $<5.00 E+02$ \\
\hline 50804 & $11 / 28 / 89$ & ICP & $2.50 E+01$ \\
\hline 50661 & $10 / 09 / 89$ & ICP & $3.30 E+01$ \\
\hline 50673 & $10 / 11 / 89$ & ICP & $3.00 \mathrm{E}+01$ \\
\hline 51079 & $3 / 21 / 90$ & $I C P$ & $2.50 E+O 1$ \\
\hline $50804 \mathrm{E}$ & $11 / 28 / 89$ & $I C P$ & $<1.00 \mathrm{E}+03$ \\
\hline $50661 \mathrm{E}$ & $10 / 09 / 89$ & $I C P$ & $<1.00 \mathrm{E}+03$ \\
\hline $50673 \mathrm{E}$ & $10 / 11 / 89$ & ICP & $<1.00 E+03$ \\
\hline $51079 E$ & $3 / 21 / 90$ & ICP & $<1.00 E+03$ \\
\hline 50804 & $11 / 28 / 89$ & ICP & $<1.00 E+01$ \\
\hline 50661 & $10 / 09 / 89$ & ICP & $1.70 \mathrm{E}+01$ \\
\hline 50673 & $10 / 11 / 89$ & ICP & $1.60 \mathrm{E}+01$ \\
\hline 51079 & $3 / 21 / 90$ & $I C P$ & $2.30 \mathrm{E}+01$ \\
\hline $50804 \mathrm{E}$ & $11 / 28 / 89$ & ICP & $<1.00 E+02$ \\
\hline $50661 E$ & $10 / 09 / 89$ & ICP & $<1.00 E+02$ \\
\hline $50673 E$ & $10 / 11 / 89$ & ICP & $<1.00 E+02$ \\
\hline $51079 E$ & $3 / 21 / 90$ & ICP & $<1.00 \mathrm{E}+02$ \\
\hline 50804 & $11 / 28 / 89$ & ICP & $1.55 \mathrm{E}+04$ \\
\hline 50661 & $10 / 09 / 89$ & ICP & $1.90 \mathrm{E}+04$ \\
\hline 50673 & $10 / 11 / 89$ & ICP & $1.76 \mathrm{E}+04$ \\
\hline 51079 & $3 / 21 / 90$ & ICP & $1.57 \mathrm{E}+04$ \\
\hline 50804 & $11 / 28 / 89$ & IC & $2.30 \mathrm{E}+03$ \\
\hline 50661 & $10 / 09 / 89$ & IC & $5.20 \mathrm{E}+03$ \\
\hline 50673 & $10 / 11 / 89$ & $I C$ & $5.10 \mathrm{E}+03$ \\
\hline 51079 & $3 / 21 / 90$ & IC & $2.20 \mathrm{E}+03$ \\
\hline $50804 \mathrm{E}$ & $11 / 28 / 89$ & ICP & $<5.00 E+02$ \\
\hline $50661 \mathrm{E}$ & $10 / 09 / 89$ & ICP & $<5.00 \mathrm{E}+02$ \\
\hline $50673 \mathrm{E}$ & $10 / 11 / 89$ & ICP & $<5.00 \mathrm{E}+02$ \\
\hline $51079 \mathrm{E}$ & $3 / 21 / 90$ & $I C P$ & $<5.00 E+02$ \\
\hline 50804 & $11 / 28 / 89$ & ICP & $2.47 \mathrm{E}+02$ \\
\hline 50661 & $10 / 09 / 89$ & ICP & $9.20 \mathrm{E}+01$ \\
\hline 50673 & $10 / 11 / 89$ & ICP & $1.05 \mathrm{E}+02$ \\
\hline 51079 & $3 / 21 / 90$ & ICP & $1.65 \mathrm{E}+02$ \\
\hline 50804 & $11 / 28 / 89$ & IC & $<5.00 \mathrm{E}+02$ \\
\hline 50804 & $11 / 28 / 89$ & ISE & $1.19 \mathrm{E}+02$ \\
\hline 50661 & $10 / 09 / 89$ & IC & $<5.00 E+02$ \\
\hline 50561 & $10 / 09 / 89$ & ISE & $1.42 \mathrm{E}+02$ \\
\hline 50673 & $10 / 11 / 89$ & IC & $<5.00 E+02$ \\
\hline 50673 & $10 / 11 / 89$ & ISE & 1. $35 \mathrm{E}+02$ \\
\hline 51079 & $3 / 21 / 90$ & $I C$ & $<5.00 E+02$ \\
\hline 51079 & $3 / 21 / 90$ & ISE & $1.04 \mathrm{E}+02$ \\
\hline 50804 & $11 / 28 / 89$ & ICP & $4.50 E+01$ \\
\hline 50661 & $10 / 09 / 89$ & ICP & $1.10 \mathrm{E}+02$ \\
\hline 50673 & $10 / 11 / 89$ & ICP & $2.25 E+02$ \\
\hline 51079 & $3 / 21 / 90$ & $I C P$ & $1.25 \mathrm{E}+02$ \\
\hline $50804 E$ & $11 / 28 / 89$ & ICP & $<5.00 E+02$ \\
\hline $50661 \mathrm{E}$ & $10,09 / 89$ & ICP & $<5.00 E+02$ \\
\hline $50673 E$ & $10 / 11 / 89$ & ICP & $<5.00 \mathrm{E}+02$ \\
\hline $51079 \mathrm{E}$ & $3 / 21 / 90$ & ICP & $<5.00 E+02$ \\
\hline 50804 & $11 / 28 / 89$ & ICP & $3.53 E+03$ \\
\hline
\end{tabular}


DATA FOR 222-S Laboratory Wastewater Continued

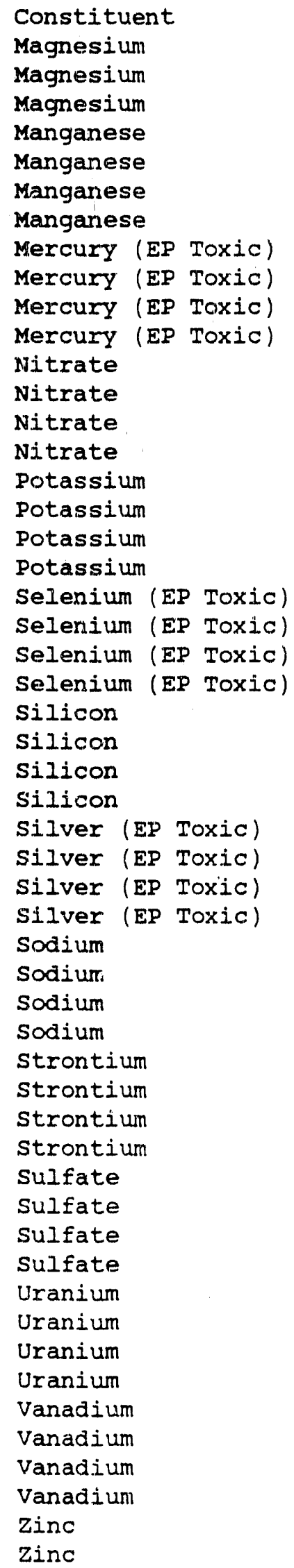

\begin{tabular}{|c|c|c|c|}
\hline ample \# & Date & Method & Result \\
\hline 50661 & $10 / 09 / 89$ & ICP & $4.35 E+03$ \\
\hline 50673 & $10 / 11 / 89$ & ICP & $4.07 \mathrm{E}+03$ \\
\hline 51079 & $3 / 21 / 90$ & ICP & $3.83 E+03$ \\
\hline 50804 & $11 / 28 / 89$ & ICP & $<5.0 C E+00$ \\
\hline 50661 & $10 / 09 / 89$ & ICP & $1.00 E+01$ \\
\hline 50673 & $10 / 11 / 89$ & ICP & $9.00 \mathrm{E}+00$ \\
\hline 51079 & $3 / 21 / 90$ & ICP & $<5.00 \mathrm{E}+00$ \\
\hline $50804 \mathrm{E}$ & $11 / 28 / 89$ & CVAA $/ M$ & $<2.00 E+01$ \\
\hline $50661 \mathrm{E}$ & $10 / 09 / 89$ & CVAA/M & $<2.00 E+01$ \\
\hline $50673 \mathrm{E}$ & $10 / 11 / 89$ & CVAA $/ M$ & $<2.00 E+01$ \\
\hline $51079 E$ & $3 / 21 / 90$ & $C V A A / M$ & $<2.00 E+0.1$ \\
\hline 50804 & $11 / 28 / 89$ & IC & $<5.00 E+02$ \\
\hline 50661 & $10 / 09 / 89$ & IC & $<5.00 \mathrm{E}+02$ \\
\hline 50673 & $10 / 11 / 89$ & IC & $<5.00 E+02$ \\
\hline 51079 & $3 / 21 . / 90$ & IC & $8.00 \mathrm{E}+02$ \\
\hline 50804 & $11 / 28 / 89$ & $I C P$ & $6.93 \mathrm{E}+02$ \\
\hline 50661 & $10 / 09 / 89$ & ICP & $8.56 \mathrm{E}+02$ \\
\hline 50673 & $10 / 11 / 89$ & ICP & $8.43 E+02$ \\
\hline 51079 & $3 / 21 / 90$ & ICP & $6.64 \mathrm{E}+02$ \\
\hline $50804 \mathrm{E}$ & $11 / 28 / 89$ & ICP & $<5.00 \mathrm{E}+02$ \\
\hline $50661 \mathrm{E}$ & $10 / 09 / 89$ & $I C P$ & $<5.00 E+02$ \\
\hline $50673 \mathrm{E}$ & $10 / 11 / 89$ & ICP & $<5.00 \mathrm{E}+02$ \\
\hline $5.1079 E$ & $3 / 21 / 90$ & ICP & $<5.00 \mathrm{E}+02$ \\
\hline 50804 & $11 / 28 / 89$ & ICP & $1.94 \mathrm{E}+03$ \\
\hline 50661 & $10 / 09 / 89$ & ICP & $2.05 E+03$ \\
\hline 50673 & $10 / 11 / 89$ & ICP & $2.06 E+03$ \\
\hline 51079 & $3 / 21 / 90$ & ICP & $1.74 \mathrm{E}+03$ \\
\hline $50804 \mathrm{E}$ & $11 / 28 / 89$ & ICP & $<5.00 \mathrm{E}+02$ \\
\hline 50661E & $10 / 09 / 89$ & ICP & $<5.00 E+02$ \\
\hline 50673E & $10 / 11 / 89$ & ICP & $<5.00 E+02$ \\
\hline $51079 E$ & $3 / 21 / 90$ & ICP & $<5.00 \mathrm{E}+02$ \\
\hline 50804 & $11 / 28 / 89$ & ICP & $1.82 \mathrm{E}+03$ \\
\hline 50661 & $10 / 09 / 89$ & ICP & $2.55 E+03$ \\
\hline 50673 & $10 / 11 / 89$ & ICP & $2.32 \mathrm{E}+03$ \\
\hline 51079 & $3 / 21 / 90$ & ICP & $1.83 E+03$ \\
\hline 50804 & $11 / 28 / 89$ & ICP & $7.80 \mathrm{E}+01$ \\
\hline 50661 & $10 / 09 / 89$ & ICP & $1.07 \mathrm{E}+02$ \\
\hline 50673 & $10 / 11 / 89$ & ICP & $9.80 \mathrm{E}+01$ \\
\hline 51079 & $3 / 21 / 90$ & ICP & $8.30 \mathrm{E}+01$ \\
\hline 50804 & $11 / 28 / 89$ & IC & $1.06 \mathrm{E}+04$ \\
\hline 50661 & $10 / 09 / 89$ & IC & $1.48 \mathrm{E}+04$ \\
\hline 50673 & $10 / 11 / 89$ & IC & $1.49 \mathrm{E}+04$ \\
\hline 51079 & $3 / 21 / 90$ & IC & $1.20 \mathrm{E}+04$ \\
\hline 50804 & $11 / 28 / 89$ & GEA & $3.46 \mathrm{E}-01$ \\
\hline 50661 & $10 / 09 / 89$ & GEA & $9.58 \mathrm{E}-01$ \\
\hline 50673 & $10 / 11 / 89$ & GEA & $4.81 \mathrm{E}-01$ \\
\hline 51079 & $3 / 21 / 90$ & GEA & $3.11 \mathrm{E}-01$ \\
\hline 50804 & $11 / 28 / 89$ & ICP & $<5.00 \mathrm{E}+00$ \\
\hline 50661 & $10 / 09 / 89$ & ICP & $7.00 \mathrm{E}+00$ \\
\hline 50673 & $10 / 11 / 89$ & ICP & $<5.00 \mathrm{E}+00$ \\
\hline 51079 & $3 / 21 / 90$ & ICP & $<5.00 \mathrm{E}+00$ \\
\hline 50804 & $11 / 28 / 89$ & ICP & $<5.0 O E+0 O$ \\
\hline 50661 & $10 / 09 / 89$ & ICP & $1.80 \mathrm{E}+01$ \\
\hline
\end{tabular}


DATA FOR 222-S Laboratory Wastewater

Continued

Constituent
zinc
zinc
Acetone
Acetone
Acetone
Acetone
Acetone
Acetone
Acetone
Acetone
Acetone
Acetone
Acetone
Acetone
Acetone
Acetone
Acetone
Acetone
Ammonia
Ammonia
Ammonia
Ammonia

Dichloromethane Dichloromethane Dichloromethane Dichloromethane Dichloromethane Dichloromethane Dichloromethane Dichloromethane Dichloromethane Dichloromethane Dichloromethane Dichluromethane Tetrahyarofuran Tetrahyarofuran Tetrahydrofuran Tetrahydrofuran Tetrahydrofuran Tetrahydrofuran Tetrahydrofuran Tetrahydrofuran Tetrahydrofuran Tetrahydrofuran Tetrahydrofuran Tetrahydrofuran Trichloromethane Trichloromethane Trichloromethane Trichloromethane Trichloromethane Trichloromethane Trichloromethane

\begin{tabular}{|c|c|c|c|}
\hline Sample \# & Date & Method & Result \\
\hline 50673 & $10 / 11 / 89$ & ICP & $2.30 \mathrm{E}+01$ \\
\hline 51079 & $3 / 21 / 90$ & ICP & $1.50 \mathrm{E}+01$ \\
\hline 50804 & $11 / 28 / 89$ & VOA & $1.40 \mathrm{E}+01$ \\
\hline 50804 & $11 / 28 / 89$ & $A B N$ & $<1.00 \mathrm{E}+01$ \\
\hline $50804 B$ & $11 / 28 / 89$ & VOA & $<1.00 \mathrm{E}+01$ \\
\hline $50804 \mathrm{~T}$ & $11 / 28 / 89$ & VOA & $<1.00 \mathrm{E}+01$ \\
\hline 50561 & $10 / 09 / 89$ & VOA & $<1.00 \mathrm{E}+01$ \\
\hline 50661 & $10 / 09 / 89$ & $A B N$ & $<1.00 \mathrm{E}+01$ \\
\hline $50661 \mathrm{~B}$ & $10 / 09 / 89$ & VOA & $<1.00 \mathrm{E}+01$ \\
\hline $50661 \mathrm{~T}$ & $10 / 09 / 89$ & VOA & $<1.00 \mathrm{E}+01$ \\
\hline 50673 & $10 / 11 / 89$ & VOA & $<1.00 \mathrm{E}+01$ \\
\hline 50673 & $10 / 11 / 89$ & AEN & $<1.00 E+01$ \\
\hline $50673 \mathrm{~B}$ & $10 / 11 / 89$ & VOA & $<8.00 E+00$ \\
\hline $50673 \mathrm{~T}$ & $10 / 11 / 89$ & VOA & $<1.00 \mathrm{E}+01$ \\
\hline 51079 & $3 / 21 / 90$ & VOA & $<7.00 \mathrm{E}+00$ \\
\hline 51079 & $3 / 21 / 90$ & $A B N$ & $<1.00 E+01$ \\
\hline $51079 B$ & $3 / 21 / 90$ & VOA & $<1.00 \mathrm{E}+01$ \\
\hline $51079 T$ & $3 / 21 / 90$ & VOA & $<1.00 \mathrm{E}+01$ \\
\hline 50804 & $11 / 28 / 89$ & ISE & $<5.00 E+01$ \\
\hline 50661 & $10 / 09 / 89$ & ISE & $9.10 \mathrm{E}+01$ \\
\hline 50673 & $10 / 11 / 89$ & ISE & $7.80 \mathrm{E}+01$ \\
\hline 51079 & $3 / 21 / 90$ & ISE & $<5.00 \mathrm{E}+01$ \\
\hline 50804 & $11 / 28 / 89$ & VOA & $<5.00 E+00$ \\
\hline $50804 \mathrm{~B}$ & $11 / 28 / 89$ & VOA & $7.00 \mathrm{E}+00$ \\
\hline $50804 T$ & $11 / 28 / 89$ & VOA & $6.00 E+00$ \\
\hline 50661 & $10 / 09 / 89$ & VOA & $<5.00 E+00$ \\
\hline $50661 B$ & $10 / 09 / 89$ & VOA & $6.00 \mathrm{E}+02$ \\
\hline $50661 \mathrm{i}$ & $10 / 09 / 89$ & VOA & $6.30 \mathrm{E}+02$ \\
\hline 50673 & $10 / 11 / 89$ & VOA & $<5.00 \mathrm{E}+00$ \\
\hline $50673 B$ & $10 / 11 / 89$ & VOA & 4:70E+02 \\
\hline $50673 \mathrm{~T}$ & $10 / 11 / 89$ & VOA & $4.90 \mathrm{E}+02$ \\
\hline 51079 & $3 / 21 / 90$ & VOA & $<5.00 \mathrm{E}+00$ \\
\hline $51079 \mathrm{~B}$ & $3 / 21 / 90$ & VOA & $<5.00 E+00$ \\
\hline $51079 \mathrm{~T}$ & $3 / 21 / 90$ & VOA & $<5.00 E+00$ \\
\hline 50804 & $11 / 28 / 89$ & VCA & $<1.00 E+01$ \\
\hline $50804 B$ & $11 / 28 / 89$ & VOA & $<6.00 \mathrm{E}+00$ \\
\hline $50804 \mathrm{~T}$ & $11 / 28 / 89$ & VOA & $<5.00 E+00$ \\
\hline 50661 & $10 / 09 / 89$ & VOA & $<1.00 \mathrm{E}+01$ \\
\hline 5066 IB & $10 / 09 / 89$ & VOA & $<1.00 \mathrm{E}+01$ \\
\hline $50661 \mathrm{~T}$ & $10 / 09 / 89$ & VOA & $<1.00 \mathrm{E}+01$ \\
\hline 50673 & $10 / 11 / 89$ & VOA & $<1.00 E+01$ \\
\hline $50673 B$ & $10 / 11 / 89$ & VOA & $<1.00 \mathrm{E}+01$ \\
\hline $50673 \mathrm{~T}$ & $10 / 11 / 89$ & VOA & $<1.00 \mathrm{E}+01$ \\
\hline 51079 & $3 / 21 / 90$ & VOA & $<1.00 E+01$ \\
\hline $51079 \mathrm{~B}$ & $3 / 21 / 90$ & VOA & $1.00 \mathrm{E}+01$ \\
\hline $51079 \mathrm{~T}$ & $3 / 21 / 90$ & VOA & $1.00 \mathrm{E}+01$ \\
\hline 50804 & $11 / 28 / 89$ & VOA & $2.10 E+01$ \\
\hline $50804 \mathrm{~B}$ & $11 / 28 / 89$ & VOA & $10 O E+01$ \\
\hline $50804 \mathrm{~T}$ & $11 / 28 / 89$ & VOA & $9.00 \mathrm{E}+00$ \\
\hline 50661 & $10 / 09 / 89$ & VOA & $2.50 \mathrm{E}+01$ \\
\hline $50661 B$ & $10 / 09 / 89$ & VOA & $<5.00 \mathrm{E}+00$ \\
\hline $50661 \mathrm{~T}$ & $10 / 09 / 89$ & VOA & $<5.00 E+00$ \\
\hline 50673 & $10 / 11 / 89$ & VOA & $2.00 E+01$ \\
\hline
\end{tabular}

D -98 
DATA FOR 222-S Laboratory Wastewater Continued

Constituent

Trichloromethane Trichloromethane Trichloromethane Trichloromethane Trichloromethane Unknown amide

Alkalinity (Method Alkalinity (Method B) Alkalinity (Method B) Alkalinity (Method B) Alpha Activity ( $\mathrm{pCi} / \mathrm{I}$ ) Alpha Activity (pCi/L) Alpha Activity (pCi/L) Alpha Activity (pCi/L) Beta Activity (pCi/L) Beta Activity (pCi/L) Beta Activity (pCi/L) Beta Activity (pCi/L) Conductivity (us) Conductivity (us) Conductivity (us) Conductivity (us) Ingitability (degrees F) Ingitability (degrees F) Ingitabiljty (degrees F) Ingitability (degrees F) pH (dimensionless) $\mathrm{pH}$ (dimensionless) $\mathrm{pH}$ (dimensionless) pH (dimensionless)

Reactivity Cyanide ( $\mathrm{mg} / \mathrm{kg}$ ) Reactivity Cyanide ( $\mathrm{mg} / \mathrm{kg}$ ) Reactivity Cyanide ( $\mathrm{mg} / \mathrm{kg}$ ) Reactivity Cyanide ( $\mathrm{mg} / \mathrm{kg}$ ) Reactivity sulfide ( $\mathrm{mg} / \mathrm{kg}$ ) Reactivi.ty sulfide (mg/kg) Reactivity sulfide (mg/kg) Reactitiij sulfide (mg/kg) Susponded solids (mg/L) Suspended solids (mg/L) Suspended Solids (mg/z) Suspended Solids ( $\mathrm{mg} / \mathrm{L}$ ) $\operatorname{TDS}(\mathrm{mg} / \mathrm{L})$

$\operatorname{TDS}(\mathrm{mg} / \mathrm{L})$

$\operatorname{TDS}(\mathrm{mg} / \mathrm{L})$

IDS (mg/L)

Tempenature (degrees C)

Temp-_ture (degrees C)

Temperature (degrees C)

Temperature (degrees $C$ )

$\operatorname{TOC}(u g / g)$

$\operatorname{TOC}(u g / g)$

$\operatorname{roc}(u g / g$ )

\begin{tabular}{|c|c|c|c|}
\hline ample \# & & Method & Result \\
\hline $50673 B$ & $10 / 11 / 89$ & VOA & $<5.00 E+00$ \\
\hline $50673 \mathrm{~T}$ & $10 / 11 / 89$ & VOA & $<5.00 E+00$ \\
\hline 51079 & $3 / 21 / 90$ & VOA & $1.30 \mathrm{E}+01$ \\
\hline $51079 B$ & $3 / 21 / 90$ & VOA & $<4.00 E+00$ \\
\hline $51079 \mathrm{~T}$ & $3 / 21 / 90$ & VOA & $<4.00 \mathrm{E}+00$ \\
\hline 50804 & $11 / 28 / 89$ & $A B N$ & $2.60 E+01$ \\
\hline 50804 & $11 / 28 / 89$ & TITRA & $4.40 E+04$ \\
\hline 50661 & $10 / 09 / 89$ & TITRA & $4.70 E+04$ \\
\hline 50673 & $10 / 11 / 89$ & TITRA & $5.30 E+04$ \\
\hline 51079 & $3 / 21 / 90$ & TITRA & $4.60 \mathrm{E}+04$ \\
\hline 50804 & $11 / 28 / 89$ & Alpha & $7.78 \mathrm{E}-01$ \\
\hline 50661 & $.10 / 09 / 89$ & Alpha & $5.88 \mathrm{E}+00$ \\
\hline 50673 & $10 / 11 / 89$ & Alpha & $1.06 \mathrm{E}+00$ \\
\hline 51079 & $3 / 21 / 90$ & Alpha & $<2.89 E-01$ \\
\hline 50804 & $11 / 28 / 89$ & Beta & $<1.04 \mathrm{E}+00$ \\
\hline 50661 & $10 / 09 / 89$ & Beta & $5.00 \mathrm{E}+00$ \\
\hline 50673 & $10 / 11 / 89$ & Beta & $<4.72 \mathrm{E}-01$ \\
\hline 51079 & $3 / 21 / 90$ & Beta & $3.88 \mathrm{E}+00$ \\
\hline 50804 & $11 / 28 / 89$ & COND-FId & 1. $33 E+02$ \\
\hline 50661 & $10 / 09 / 89$ & COND-EId & $5.46 \mathrm{E}+02$ \\
\hline 50673 & $10 / 11 / 89$ & COND-FId & $1.37 E+02$ \\
\hline 51079 & $3 / 21 / 90$ & COND-FId & $1.37 \mathrm{E}+02$ \\
\hline $50804 \mathrm{E}$ & $11 / 28 / 89$ & IGNIT & $2.12 \mathrm{E}+02$ \\
\hline $50661 \mathrm{E}$ & $10 / 09 / 89$ & IGNIT & $2.10 E+02$ \\
\hline $50673 E$ & $10 / 11 / 89$ & NIT & 2. $10 \mathrm{E}+02$ \\
\hline 51079E & $3 / 21 / 90$ & IGNIT & $2.06 \mathrm{E}+02$ \\
\hline 50804 & $11 / 28 / 89$ & PH-FId & $7.19 E+00$ \\
\hline 50661 & $10 / 09 / 89$ & PH-FId & $6.73 \mathrm{E}+00$ \\
\hline 50673 & $10 / 11 / 89$ & PH-Eld & $6.60 E+00$ \\
\hline & $3 / 21 / 90$ & PH-FId & $7.34 E+00$ \\
\hline $50804 \mathrm{E}$ & $11 / 28 / 89$ & DSPEC & $<1.00 E+02$ \\
\hline $50661 \mathrm{E}$ & $10 / 09 / 89$ & DSPEC & $<1.00 E+02$ \\
\hline $50673 E$ & $10 / 11 / 89$ & DSPEC & $<1.00 \mathrm{E}+02$ \\
\hline $51079 \mathrm{E}$ & $3 / 21 / 90$ & DSPEC & $<1.00 E+02$ \\
\hline & $11 / 28 / 89$ & DTITRA & $<1.00 E+02$ \\
\hline $50661 \mathrm{E}$ & $10 / 09 / 89$ & DTITRA & $<1.00 \mathrm{E}+02$ \\
\hline $50673 E$ & $10 / 11 / 89$ & DTITRA & $<1.00 E+02$ \\
\hline $51079 E$ & $3 / 21 / 90$ & DTITRA & $<1.00 E+02$ \\
\hline 50804 & $12 / 28 / 89$ & SSOLID & $<5.00 E+03$ \\
\hline 50661 & $10 / 09 / 89$ & SSOLID & $<5.00 E+03$ \\
\hline 50673 & $10 / 11 / 89$ & SSOLID & $2.20 \mathrm{E}+04$ \\
\hline 51079 & $3 / 21 / 90$ & SSOLID & $<5.00 E+03$ \\
\hline 50804 & $11 / 28 / 89$ & TDS & $5.90 \mathrm{E}+04$ \\
\hline 50661 & $10 / 09 / 89$ & TDS & $7.80 \mathrm{E}+04$ \\
\hline 5067.3 & $10 / 11 / 89$ & TDS & $6.20 E+04$ \\
\hline 51079 & $3 / 21 / 90$ & TDS & $3.40 \mathrm{E}+04$ \\
\hline 50804 & $11 / 28 / 89$ & TEMP - FId & $1.50 E+01$ \\
\hline 50661 & $10 / 09 / 89$ & TEMPP-FId & $2.13 E+01$ \\
\hline 50673 & $10 / 11 / 89$ & TEMP-FId & $2.26 E+01$ \\
\hline 51079 & $3 / 21 / 90$ & TEMP - FId & $1.73 \mathrm{E}+01$ \\
\hline 50804 & $11 / 28 / 89$ & TOC & $<1.00 E+03$ \\
\hline 50661 & $10 / 09 / 89$ & TOC & $<1.30 E+03$ \\
\hline 50673 & $10 / 11 / 89$ & TOC & $<1.90 \mathrm{E}+03$ \\
\hline
\end{tabular}


WHC-EP-0355, Appendix D

DATA FOR 222-S Laboratory Wastewater

Continued

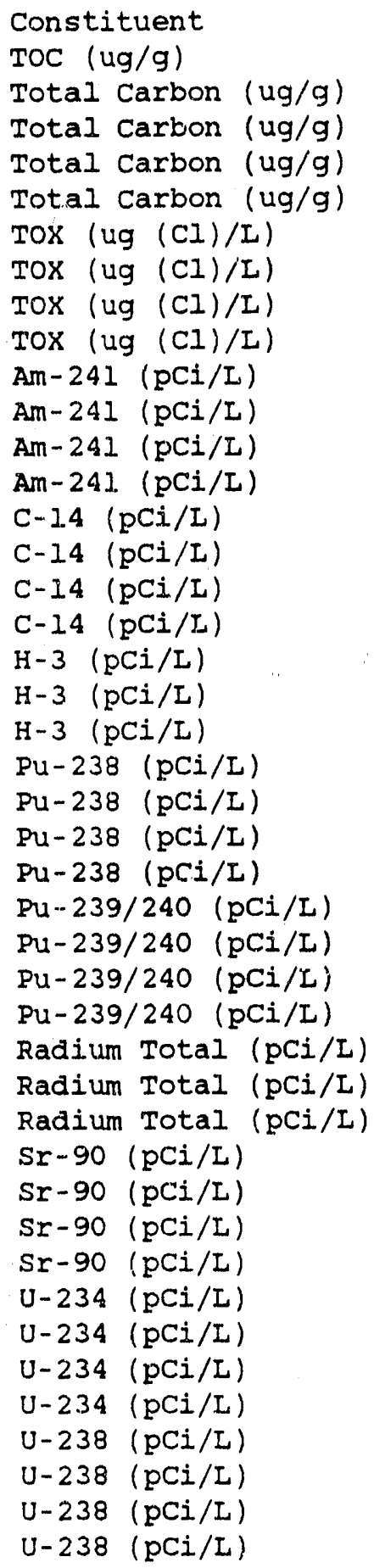

\begin{tabular}{|c|c|c|c|}
\hline Sample \# & Date & Method & Result \\
\hline 51079 & $3 / 21 / 90$ & TOC & 1. $10 \mathrm{E}+03$ \\
\hline 50804 & $11 / 28 / 89$ & $\mathrm{TC}$ & 1. 19E+04 \\
\hline 50661 & $10 / 09 / 89$ & TC & 1. $30 \mathrm{E}+04$ \\
\hline 50673 & $10 / 11 / 89$ & $\mathrm{TC}$ & 1. $20 \mathrm{E}+04$ \\
\hline 51079 & $3 / 21 / 90$ & $\mathrm{TC}$ & $1.30 \mathrm{E}+04$ \\
\hline 50804 & $11 / 28 / 89$ & ITOX & 1. 11.E+O2 \\
\hline 50661 & $10 / 09 / 89$ & LTOX & 1. $27 \mathrm{E}+02$ \\
\hline 50673 & $10 / 11 / 89$ & ITOX & $1.33 E+02$ \\
\hline 51079 & $3 / 21 / 90$ & LTOX & $8.40 E+O 1$ \\
\hline 50804 & $11 / 28 / 89$ & $A E A$ & $3.96 \mathrm{E}-02$ \\
\hline 50661 & $10 / 09 / 89$ & AEA & $2.95 \mathrm{E}+00$ \\
\hline 50673 & $10 / 11 / 89$ & AEA & $7.96 \mathrm{E}-02$ \\
\hline 51079 & $3 / 21 / 90$ & AEA & $7.44 \mathrm{E}-02$ \\
\hline 50804 & $11 / 28 / 89$ & LSC & $5.59 \mathrm{E}+00$ \\
\hline 50661 & $10 / 09 / 89$ & LSC & $<1.49 \mathrm{E}+00$ \\
\hline 50673 & $10 / 11 / 89$ & LSC & $<1.80 E+00$ \\
\hline 51079 & $3 / 21 / 90$ & LSC & $<1.55 \mathrm{E}+00$ \\
\hline 50661 & $10 / 09 / 89$ & LSC & $2.90 \mathrm{E}+02$ \\
\hline 50673 & $10 / 11 / 89$ & ISC & $<9.74 \mathrm{E}+01$ \\
\hline 51079 & $3 / 21 / 90$ & LSC & $<1.37 \mathrm{E}+02$ \\
\hline 50804 & $11 / 28 / 89$ & $A E A$ & $4.17 \mathrm{E}-03$ \\
\hline 50661 & $10 / 09 / 89$ & AEA & 4. 31E-02 \\
\hline 50673 & $10 / 11 / 89$ & AEA & $1.53 \mathrm{E}-02$ \\
\hline 51079 & $3 / 21 / 90$ & $A E A$ & $<1.75 \mathrm{E}-03$ \\
\hline 50804 & $11 / 28 / 89$ & AEA & $2.79 \mathrm{E}-02$ \\
\hline 50661 & $10 / 09 / 89$ & AEA & $7.42 \mathrm{E}-01$ \\
\hline 50673 & $10 / 11 / 89$ & AEA & $7.70 \mathrm{E}-02$ \\
\hline 51079 & $3 / 21 / 90$ & AEA & $3.78 \mathrm{E}-02$ \\
\hline 50804 & $11 / 28 / 89$ & Alpha-Ra & $1.74 \mathrm{E}-01$ \\
\hline 50661 & $10 / 09 / 89$ & Alpha-Ra & $<1.05 \mathrm{E}-01$ \\
\hline 51079 & $3 / 21 / 90$ & Alpha-Ra & $<4.54 \mathrm{E}-03$ \\
\hline 50804 & $11 / 28 / 89$ & Beta & $<9.50 \mathrm{E}-02$ \\
\hline 50661 & $10 / 09 / 89$ & Beta & $2.93 E-O I$ \\
\hline 50673 & $10 / 11 / 89$ & Beta & $<7.30 \mathrm{E}-03$ \\
\hline 51079 & $3 / 21 / 90$ & Beta & $<9.93 \mathrm{E}-02$ \\
\hline 50804 & $11 / 28 / 89$ & $A E A$ & $1.14 \mathrm{E}-01$ \\
\hline 50661 & $10 / 09 / 89$ & AEA & $9.08 \mathrm{E}-02$ \\
\hline 50673 & $10 / 11 / 89$ & AEA & $6.20 \mathrm{E}-02$ \\
\hline 51079 & $3 / 21 / 90$ & $A E A$ & $1.55 \mathrm{E}-\mathrm{OI}$ \\
\hline 50804 & $11 / 28 / 89$ & $A E A$ & $8.90 \mathrm{E}-02$ \\
\hline 50661 & $10 / 09 / 89$ & AEA & $1.28 \mathrm{E}-01$ \\
\hline 50673 & $10 / 11 / 89$ & AEA & $5.97 \mathrm{E}-02$ \\
\hline 51079 & $3 / 21 / 90$ & AEA & $1.30 \mathrm{E}-0$ \\
\hline
\end{tabular}


DATA FOR S Plant Wastewater

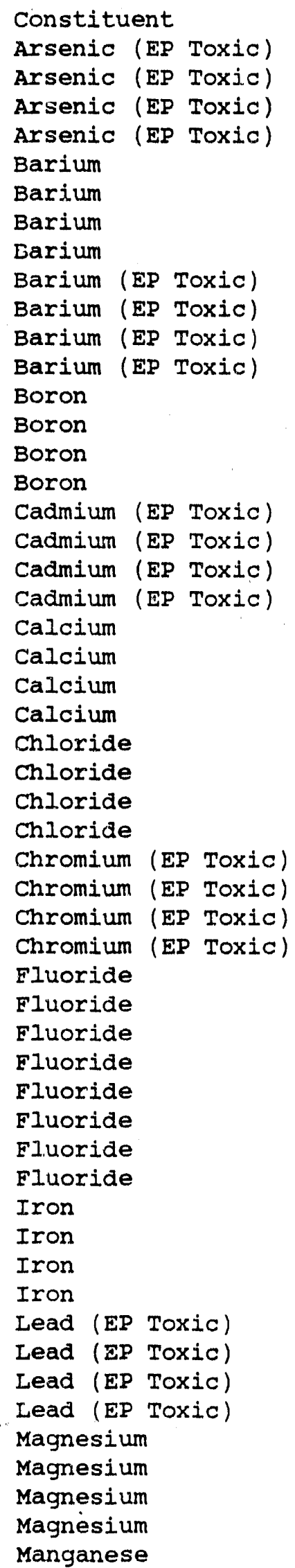

\begin{tabular}{|c|c|c|c|}
\hline Sample \# & Date & Method & Result \\
\hline $50744 \mathrm{E}$ & $10 / 31 / 89$ & $I C P$ & $<5.00 \mathrm{E}+02$ \\
\hline $50845 \mathrm{E}$ & $12 / \angle \dot{L} / 89$ & ICP & $<5.00 \mathrm{E}+02$ \\
\hline $50911 \mathrm{E}$ & $1 / 31 / 90$ & ICP & $<5.00 \mathrm{E}+02$ \\
\hline $51072 \mathrm{~T}$ & $3 / 20 / 90$ & ICP & $<5.00 \mathrm{E}+02$ \\
\hline 50744 & $10 / 31 / 89$ & ICP & $2.80 E+01$ \\
\hline 50845 & $12 / 20 / 89$ & ICP & $2.80 \mathrm{E}+01$ \\
\hline 50911 & $1 / 31 / 90$ & ICP & $3.20 E+01$ \\
\hline 51072 & $3 / 20 / 90$ & ICP & $2.90 \mathrm{E}+01$ \\
\hline $50744 \mathrm{E}$ & $10 / 31 / 89$ & ICP & $<1.00 \mathrm{E}+03$ \\
\hline $50845 \mathrm{E}$ & $12 / 20 / 89$ & ICP & $<1.00 \mathrm{E}+03$ \\
\hline $50911 \mathrm{E}$ & $1 / 31 / 90$ & ICP & $<1.00 \mathrm{E}+03$ \\
\hline $51072 \mathrm{~T}$ & $3 / 20 / 90$ & ICP & $<1.00 \mathrm{E}+03$ \\
\hline 50744 & $10 / 31 / 89$ & ICP & $<1.00 E+01$ \\
\hline 50845 & $12 / 20 / 89$ & ICP & $<1.00 E+01$ \\
\hline 50911 & $1 / 31 / 90$ & ICP & $<1.00 E+01$ \\
\hline 51072 & $3 / 20 / 90$ & ICP & $2.00 E+01$ \\
\hline $50744 E$ & $10 / 31 / 89$ & ICP & $<1.00 E+02$ \\
\hline $50845 \mathrm{E}$ & $12 / 20 / 89$ & ICP & $<1.00 \mathrm{E}+02$ \\
\hline $50911 E$ & $1 / 31 / 90$ & ICP & $<1.00 \mathrm{E}+02$ \\
\hline $51072 \mathrm{~T}$ & $3 / 20 / 90$ & ICP & $<1.00 \mathrm{E}+02$ \\
\hline 50744 & $10 / 31 / 89$ & ICP & $1.80 \mathrm{E}+04$ \\
\hline 50845 & $12 / 20 / 89$ & ICP & $1.74 \mathrm{E}+04$ \\
\hline 50911 & $1 / 31 / 90$ & ICP & $2.02 \mathrm{E}+04$ \\
\hline 51072 & $3 / 20 / 90$ & ICP & $1.80 \mathrm{E}+04$ \\
\hline 50744 & $10 / 31 / 89$ & IC & $1.80 \mathrm{E}+03$ \\
\hline 50845 & $12 / 20 / 89$ & IC & $1.30 \mathrm{E}+03$ \\
\hline 50911 & $1 / 31 / 90$ & IC & $1.30 \mathrm{E}+03$ \\
\hline 51072 & $3 / 20 / 90$ & IC & 1. 10E+03 \\
\hline $50744 \mathrm{E}$ & $10 / 31 / 89$ & ICP & $<5.00 \mathrm{E}+02$ \\
\hline $50845 \mathrm{E}$ & $12 / 20 / 89$ & ICP & $<5.00 \mathrm{E}+02$ \\
\hline 5091 LE & $1 / 31 / 90$ & ICP & $<5.00 \mathrm{E}+02$ \\
\hline $51072 \mathrm{~T}$ & $3 / 20 / 90$ & ICP & $<5.00 \mathrm{E}+02$ \\
\hline 50744 & $10 / 31 / 89$ & IC & $<5.00 E+02$ \\
\hline $5 C 744$ & $10 / 31 / 89$ & ISE & $1.42 \mathrm{E}+02$ \\
\hline 50845 & $12 / 20 / 89$ & IC & $<5.00 \mathrm{E}+02$ \\
\hline 50845 & $12 / 20 / 89$ & ISE & $1.16 \mathrm{E}+02$ \\
\hline 50911 & $1 / 31 / 90$ & IC & $<5.00 E+02$ \\
\hline 50911 & $1 / 31 / 90$ & ISE & $1.24 \mathrm{E}+02$ \\
\hline 51072 & $3 / 20 / 90$ & IC & $<5.00 \mathrm{E}+02$ \\
\hline 51072 & $3 / 20 / 90$ & ISE & 1. $24 \mathrm{E}+02$ \\
\hline 50744 & $10 / 31 / 89$ & ICP & 4.2OE+OI \\
\hline 50845 & $12 / 20 / 89$ & ICP & $<3.00 \mathrm{E}+01$ \\
\hline 50911 & $1 / 31 / 90$ & ICP & $<3.00 E+01$ \\
\hline 51072 & $3 / 20 / 90$ & ICP & $<3.00 E+01$ \\
\hline $50744 \mathrm{E}$ & $10 / 31 / 89$ & ICP & $<5.00 \mathrm{E}+02$ \\
\hline $50845 \mathrm{E}$ & $12 / 20 / 89$ & ICP & $<5.00 \mathrm{E}+02$ \\
\hline $50911 \mathrm{E}$ & $1 / 31 / 90$ & ICP & $<5.00 \mathrm{E}+02$ \\
\hline $51072 \mathrm{~T}$ & $3 / 20 / 90$ & ICP & $<5.00 \mathrm{O}+02$ \\
\hline 50744 & $10 / 31 / 89$ & ICP & $4.16 \mathrm{E}+03$ \\
\hline 50845 & $12 / 20 / 89$ & ICP & $4.03 E+03$ \\
\hline 50911 & $1 / 31 / 90$ & ICP & $4.52 \mathrm{E}+03$ \\
\hline 51072 & $3 / 20 / 90$ & ICP & $4.48 E+03$ \\
\hline 50744 & $10 / 31 / 89$ & ICP & $6.00 E+00$ \\
\hline
\end{tabular}

$D-101$ 
DATA FOR S Plant Wastewater Continued

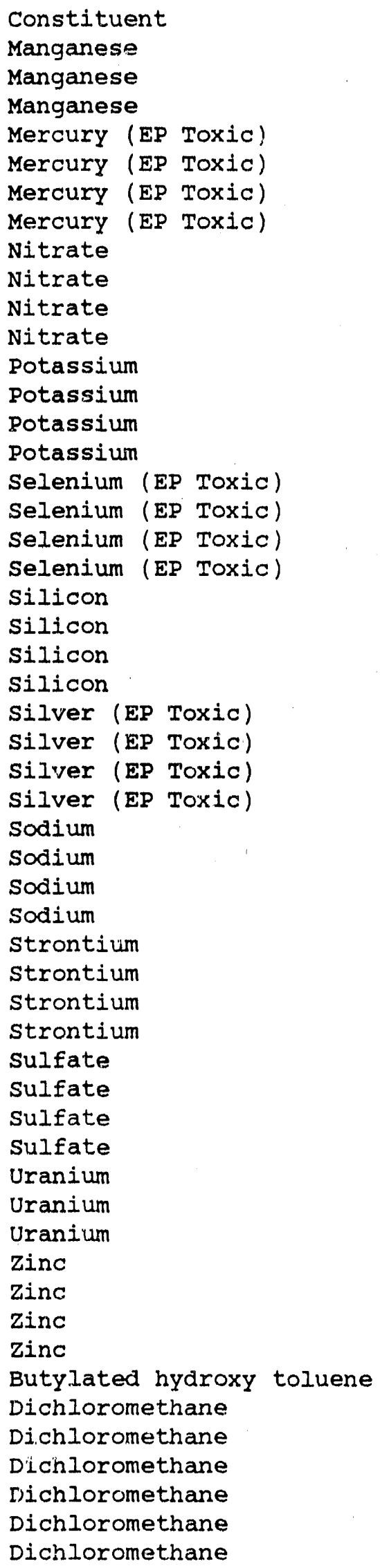

\begin{tabular}{|c|c|c|c|}
\hline ample \# & & Method & Result \\
\hline 50845 & $12 / 20 / 89$ & ICP & $<5.00 E+00$ \\
\hline 50311 & $1 / 31 / 90$ & ICP & $<5.00 \mathrm{E}+00$ \\
\hline 51072 & $3 / 20 / 90$ & ICP & $<5.00 \mathrm{E}+00$ \\
\hline $50744 E$ & $10 / 31 / 89$ & $\mathrm{CVAA} / \mathrm{M}$ & $<2.00 \mathrm{E}+01$ \\
\hline $50845 \mathrm{E}$ & $12 / 20 / 89$ & CVAA/M & $<2.00 E+01$ \\
\hline $50911 \mathrm{E}$ & $1 / 31 / 90$ & CVAA/M & $<2.00 \mathrm{E}+01$ \\
\hline $51072 \mathrm{~T}$ & $3 / 20 / 90$ & CVAA/M & $<2.00 E+01$ \\
\hline 50744 & $10 / 31 / 89$ & IC & $<5.00 \mathrm{E}+02$ \\
\hline 50845 & $12 / 20 / 89$ & $I C$ & $5.00 E+02$ \\
\hline 50911 & $1 / 31 / 90$ & IC & $<5.00 E+02$ \\
\hline 51072 & $3 / 20 / 90$ & IC & $<5.00 E+02$ \\
\hline 50744 & $10 / 31 / 89$ & ICP & $7.56 \mathrm{E}+02$ \\
\hline 50845 & $12 / 20 / 89$ & ICP & $7.09 \mathrm{E}+02$ \\
\hline 50911 & $1 / 31 / 90$ & ICP & $7.03 E+02$ \\
\hline 51072 & $3 / 20 / 90$ & ICP & $7.60 \mathrm{E}+02$ \\
\hline $50744 \mathrm{E}$ & $10 / 31 / 89$ & ICP & $<5.00 \mathrm{E}+02$ \\
\hline $50845 E$ & $12 / 20 / 89$ & ICP & $<5.00 \mathrm{E}+02$ \\
\hline $50911 E$ & $1 / 31 / 90$ & ICP & $<5.00 E+02$ \\
\hline $51072 \mathrm{~T}$ & $3 / 20 / 90$ & ICP & $<5.00 \mathrm{E}+02$ \\
\hline 50744 & $10 / 31 / 89$ & ICP & $2.17 \mathrm{E}+03$ \\
\hline 50845 & $12 / 20 / 89$ & ICP & $2.26 E+03$ \\
\hline 50911 & $1 / 31 / 90$ & ICP & $2.36 E+03$ \\
\hline 51072 & $3 / 20 / 90$ & ICP & $2.10 \mathrm{E}+03$ \\
\hline $50744 \mathrm{E}$ & $10 / 31 / 89$ & ICP & $<5.00 \mathrm{E}+02$ \\
\hline $50845 \mathrm{E}$ & $12 / 20 / 89$ & ICP & $<5.00 E+02$ \\
\hline 50911E & $1 / 31 / 90$ & ICP & $<5.00 \mathrm{D}+02$ \\
\hline $51072 \mathrm{~T}$ & $3 / 20 / 90$ & ICP & $<5.00 E+02$ \\
\hline 50744 & $10 / 31 / 89$ & ICP & $2.17 \mathrm{E}+03$ \\
\hline 50845 & $12 / 20 / 89$ & ICP & $1.90 \mathrm{E}+03$ \\
\hline 50911 & $1 / 31 / 90$ & ICP & $2.13 E+03$ \\
\hline 51072 & $3 / 20 / 90$ & ICP & $2.01 E+03$ \\
\hline 50744 & $10 / 31 / 89$ & ICP & $9.70 \mathrm{E}+01$ \\
\hline 50845 & $12 / 20 / 89$ & ICP & $8.40 \mathrm{E}+01$ \\
\hline 50911 & $1 / 31 / 90$ & IrP & $8.90 \mathrm{E}+01$ \\
\hline 51072 & $3 / 20 / 90$ & ICP & $9.60 \mathrm{E}+01$ \\
\hline 50744 & $10 / 31 / 89$ & IC & 1. $10 \mathrm{E}+04$ \\
\hline 50845 & $12 / 20 / 89$ & IC & $1.04 \mathrm{E}+04$ \\
\hline 50911 & $1 / 31 / 90$ & IC & 1. 10E+04 \\
\hline 51072 & $3 / 20 / 90$ & IC & $1.08 E+04$ \\
\hline 50744 & $10 / 31 / 89$ & GEA & $4.70 \mathrm{E}-01$ \\
\hline 50845 & $12 / 20 / 89$ & GEA & $6.82 \mathrm{E}-01$ \\
\hline 51072 & $3 / 20 / 90$ & GEA & $4.17 \mathrm{E}-01$ \\
\hline 50744 & $10 / 31 / 89$ & $I C P$ & $<5.00 E+00$ \\
\hline 50845 & $12 / 20 / 89$ & ICP & $6.00 \mathrm{E}+00$ \\
\hline 50911 & $1 / 31 / 90$ & ICP & 8. OOE +00 \\
\hline 51072 & $3 / 20 / 90$ & ICP & $<5.00 \mathrm{E}+00$ \\
\hline 50911 & $1 / 31 / 90$ & $A B N$ & $8.00 E+00$ \\
\hline 50744 & $10 / 31 / 89$ & VOA & $<5.0 O E+00$ \\
\hline $50744 \mathrm{~B}$ & $10 / 31 / 89$ & VOA & $<5.00 \mathrm{E}+00$ \\
\hline $50744 \mathrm{~T}$ & $10 / 31 / 89$ & VOA & $2.40 \mathrm{E}+01$ \\
\hline 50845 & $12 / 20 / 89$ & VOA & $<5.00 E+00$ \\
\hline $50845 B$ & $12 / 20 / 89$ & VOA & $1.10 \mathrm{E}+03$ \\
\hline $50845 \mathrm{~T}$ & $12 / 20 / 89$ & VOA & $9.50 \mathrm{E}+02$ \\
\hline
\end{tabular}


DATA FOR S Plant Wastewater Continued

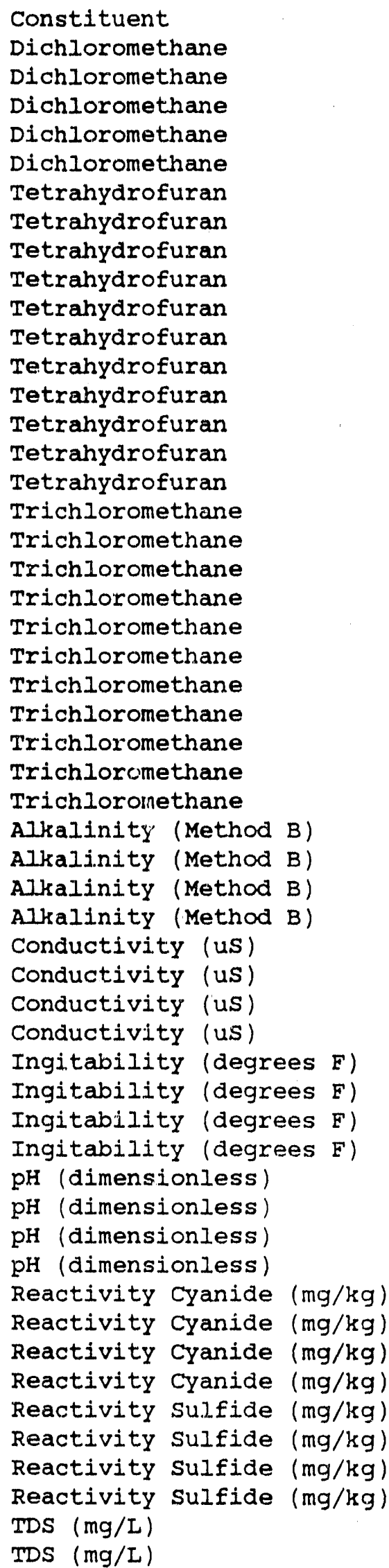

\begin{tabular}{|c|c|c|c|}
\hline Sample \# & Date & Method & Result \\
\hline 50911 & $1 / 31 / 90$ & VOA & $<5.00 \mathrm{E}+00$ \\
\hline $50911 B$ & $1 / 31 / 90$ & VOA & $<5.00 E+00$ \\
\hline $50911 \mathrm{~T}$ & $1 / 31 / 90$ & VCI & $<5.00 \mathrm{E}+00$ \\
\hline 51072 & $3 / 20 / 90$ & VOA & $<5.00 \mathrm{E}+00$ \\
\hline $51072 B$ & $3 / 20 / 90$ & VOA & $<5.00 \mathrm{E}+00$ \\
\hline 50744 & $10 / 31 / 89$ & VOA & $<1.00 E+01$ \\
\hline $.50744 \mathrm{~B}$ & $10 / 31 / 89$ & VOA & 1.30E+01 \\
\hline $50744 \mathrm{~T}$ & $10 / 31 / 89$ & VOA & 1. $20 \mathrm{E}+01$ \\
\hline 50845 & $12 / 20 / 89$ & VOA & $<1.00 \mathrm{E}+01$ \\
\hline $50845 B$ & $12 / 20 / 89$ & VOA & $<1.00 \mathrm{E}+01$ \\
\hline $50845 \mathrm{~T}$ & $12 / 20 / 89$ & VOA & $<1.00 E+01$ \\
\hline 50911 & $1 / 31 . / 90$ & VOA & $<1.00 \mathrm{E}+\cup 1$ \\
\hline $50911 \mathrm{~B}$ & $1 / 31 / 90$ & VOA & $<7.00 \mathrm{E}+00$ \\
\hline $50911 \mathrm{~T}$ & $1 / 31 / 90$ & VOA. & $<7.00 \mathrm{E}+00$ \\
\hline 51072 & $3 / 20 / 90$ & VOA & $<1.00 \mathrm{E}+01$ \\
\hline $51072 B$ & $3 / 20 / 90$ & VOA & $<1.00 \mathrm{E}+01$ \\
\hline 50744 & $10 / 31 / 89$ & VOA & $9.00 \mathrm{E}+00$ \\
\hline $50744 \mathrm{~B}$ & $10 / 31 / 89$ & VOA & $<5.00 \mathrm{E}+00$ \\
\hline $50744 \mathrm{~T}$ & $10 / 31 / 89$ & VOA & $<5.00 \mathrm{E}+00$ \\
\hline 50845 & $12 / 20 / 89$ & VOA & $8.00 E+00$ \\
\hline $50845 B$ & $12 / 20 / 89$ & VOA & $<5.00 E+00$ \\
\hline $50845 \mathrm{~T}$ & $12 / 20 / 89$ & VOA & $<5.00 \mathrm{E}+00$ \\
\hline 50911 & $1 / 31 / 90$ & VOA & $<3.00 \mathrm{E}+00$ \\
\hline $50911 B$ & $1 / 31 / 90$ & VOA & $<5.00 \mathrm{E}+00$ \\
\hline $50911 \mathrm{~T}$ & $1 / 3.1 / 90$ & VOA & $<5.00 \mathrm{E}+00$ \\
\hline 51072 & $3 / 20 / 90$ & VOA & $<5.00 \mathrm{E}+00$ \\
\hline $51072 B$ & $3 / 20 / 90$ & VOA & $<3.00 \mathrm{E}+00$ \\
\hline 50744 & $10 / 31 / 89$ & TITRA & $5.70 \mathrm{E}+04$ \\
\hline 50845 & $12 / 20 / 89$ & TITRA & $5.60 \mathrm{E}+04$ \\
\hline 50911 & $1 / 31 / 90$ & TITRA & $6.20 E+04$ \\
\hline 51072 & $3 / 20 / 90$ & TITRA & $5.70 E+04$ \\
\hline 50744 & $10 / 31 / 89$ & COND-FId & 1. $14 \mathrm{E}+02$ \\
\hline 50845 & $12 / 20 / 89$ & COND-EId & 1. $40 \mathrm{E}+02$ \\
\hline 50911 & $1 / 31 / 90$ & COND-FId & $1.32 \mathrm{E}+02$ \\
\hline 51072 & $3 / 20 / 90$ & COND-FId & 1. $50 \mathrm{E}+02$ \\
\hline $50744 \mathrm{E}$ & $10 / 31 / 89$ & IGNIT & $2.08 \mathrm{E}+02$ \\
\hline $50845 \mathrm{E}$ & $12 / 20 / 89$ & IGNIT & $2.08 E+02$ \\
\hline $50911 \mathrm{E}$ & $1 / 31 / 90$ & IGNIT & $2.12 \mathrm{E}+02$ \\
\hline $51072 \mathrm{~T}$ & $3 / 20 / 90$ & IGNIT & $2.08 E+02$ \\
\hline 50744 & $10 / 31 / 89$ & PH-FId & $8.00 E+00$ \\
\hline 50845 & $12 / 20 / 89$ & $P H-F I d$ & $7.80 \mathrm{E}+00$ \\
\hline 50911 & $1 / 31 / 90$ & PH-FId & $7.46 \mathrm{E}+00$ \\
\hline 51072 & $3 / 20 / 90$ & PH-FId & $7.43 \mathrm{E}+00$ \\
\hline $50744 E$ & $10 / 31 / 89$ & DSPEC & $<1.00 \mathrm{E}+02$ \\
\hline $50845 \mathrm{E}$ & $12 / 20 / 89$ & DSPEC & $<1.00 \mathrm{E}+02$ \\
\hline $50911 \mathrm{E}$ & $1 / 31 / 90$ & DSPEC & $<1.00 \mathrm{E}+02$ \\
\hline $51072 \mathrm{~T}$ & $3 / 20 / 90$ & DSPEC & $<1.00 \mathrm{E}+02$ \\
\hline $50744 \mathrm{E}$ & $10 / 31 / 89$ & DTITRA & $<1.00 \mathrm{E}+02$ \\
\hline $50845 \mathrm{E}$ & $12 / 20 / 89$ & DTITRA & $<1.00 E+02$ \\
\hline $50911 \mathrm{E}$ & $1 / 31 / 90$ & DTITRA & $<1.00 \mathrm{E}+02$ \\
\hline $51072 \mathrm{~T}$ & $3 / 20 / 90$ & DTITRA & $<1.00 \mathrm{z}+02$ \\
\hline $50 ? 44$ & $10 / 31 / 89$ & TDS & $8.30 E+04$ \\
\hline 50845 & $12 / 20 / 89$ & TDS & $7.00 \mathrm{E}+04$ \\
\hline
\end{tabular}


WHC-EP-0355, Appendix D

DATA EOR S Plant Wastewater Continued

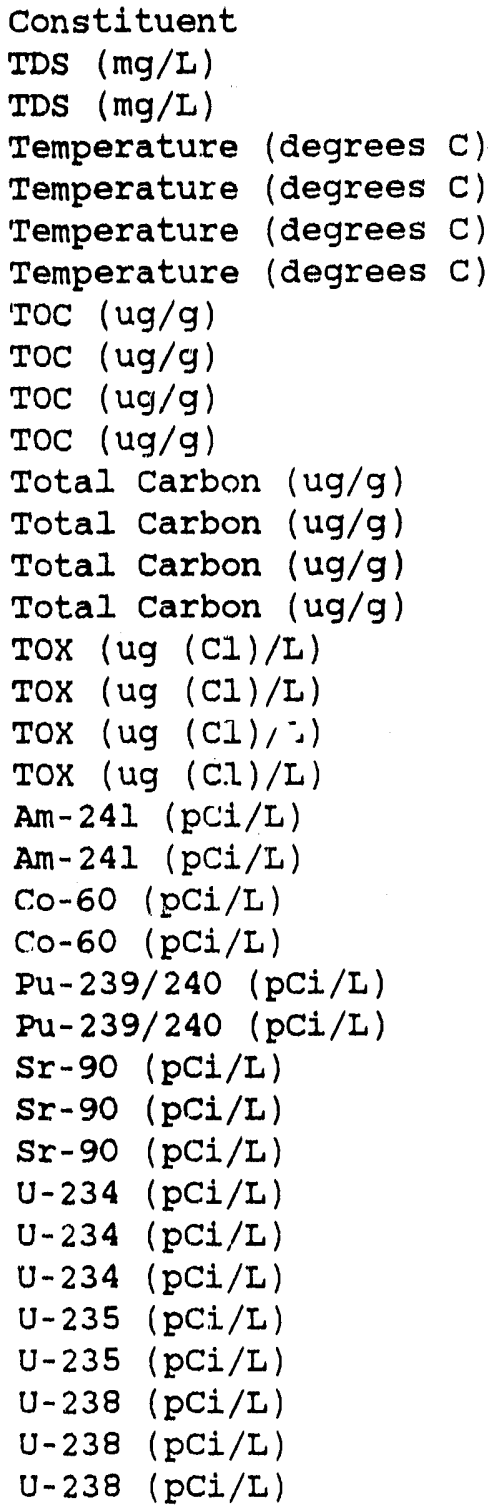

\begin{tabular}{|c|c|c|c|}
\hline Sample \# & Date & Method & Result \\
\hline 50911 & $1 / 31 / 90$ & TDS & $7.60 \mathrm{E}+04$ \\
\hline 51072 & $3 / 20 / 90$ & TDS & $7.40 \mathrm{E}+04$ \\
\hline 50744 & $10 / 31 / 89$ & TEMPP-FId & $1.52 \mathrm{E}+01$ \\
\hline 50845 & $12 / 20 / 89$ & TEMP-EId & $1.10 \mathrm{E}+01$ \\
\hline 50911 & $1 / 31 / 90$ & TEMP - EId & $2.37 \mathrm{E}+01$ \\
\hline 51072 & $3 / 20 / 90$ & TEMP - FId & 1. $22 \mathrm{E}+01$ \\
\hline 50744 & $10 / 31 / 89$ & TOC & $<1.40 \mathrm{E}+03$ \\
\hline 50845 & $12 / 20 / 89$ & TOC & $<1.20 \mathrm{E}+03$ \\
\hline 50911 & $1 / 31 / 90$ & $\mathrm{~T} D \mathrm{C}$ & $<1.20 \mathrm{E}+03$ \\
\hline 51072 & $3 / 20 / 90$ & TOC & $1.00 E+03$ \\
\hline 50744 & $10 / 31 / 89$ & TC & $1.39 E+04$ \\
\hline 50845 & $12 / 20 / 89$ & $\mathrm{TC}$ & 1. 50E+04 \\
\hline 50911 & $1 / 31 / 90$ & TC & 1. $33 \mathrm{E}+04$ \\
\hline 51072 & $3 / 20 / 90$ & $T C$ & $1.56 \mathrm{E}+04$ \\
\hline 50744 & $10 / 31 / 89$ & LTOX & $7.10 \mathrm{E}+01$ \\
\hline 50845 & $12 / 20 / 89$ & LTOX & $5.10 \mathrm{E}+01$ \\
\hline 50911 & $1 / 31 / 90$ & LTOX & 4. $50 \mathrm{E}+01$ \\
\hline 51072 & $3 / 20 / 90$ & ITOX & 4. $30 E+01$ \\
\hline 50744 & $10 / 31 / 89$ & $A E A$ & $<3.23 E-03$ \\
\hline 50845 & $12 / 20 / 89$ & AEA & $2.00 \mathrm{E}-02$ \\
\hline 50845 & $12 / 20 / 89$ & GEA & $<2.35 E-01$ \\
\hline 51072 & $3 / 20 / 90$ & GEA & $1.64 \mathrm{E}+00$ \\
\hline 50845 & $12 / 20 / 89$ & AEA & $<1.17 \mathrm{E}-03$ \\
\hline 51072 & $3 / 20 / 90$ & AEA & $4.68 \mathrm{E}-03$ \\
\hline 50744 & $10 / 31 / 89$ & Beta & 3. 31E-01 \\
\hline $50 \varepsilon 45$ & $12 / 20 / 89$ & Beta & $<1.68 \mathrm{E}-01$ \\
\hline 51072 & $3 / 20 / 90$ & Beta & $<1.80 \mathrm{E}-01$ \\
\hline 50744 & $10 / 31 / 89$ & AEA & $1.76 \mathrm{E}-01$ \\
\hline 50845 & $12 / 20 / 89$ & AEA & $1.71 \mathrm{E}-01$ \\
\hline 51072 & $3 / 20 / 90$ & AEA & $2.18 \mathrm{E}-01$ \\
\hline 50845 & $12 / 20 / 89$ & AEA & $1.36 \mathrm{E}-02$ \\
\hline 51072 & $3 / 20 / 90$ & $A E A$ & $<2.39 \mathrm{E}-03$ \\
\hline 50744 & $10 / 31 / 89$ & AEA & 1. 22E-01 \\
\hline 50845 & $12 / 20 / 89$ & AEA & $1.67 \mathrm{E}-01$ \\
\hline 51072 & $3 / 20 / 90$ & AEA & $1.39 \mathrm{E}-01$ \\
\hline
\end{tabular}


WHC-EP-0355, Appendix D

DATA FOR 242-S Evaporator Steam Condensate

\begin{tabular}{|c|c|c|}
\hline Constitue & dent & \\
\hline Arsenic & (EP & Toxia) \\
\hline Arsenic & (EP & Toxic) \\
\hline Arsenic & $(\operatorname{EP}$ & Toxic) \\
\hline Arsenic & (EP & Toxic) \\
\hline Barium & & \\
\hline Barium & & \\
\hline Barium & & \\
\hline Barium & & \\
\hline Barium ( & (EP T & Toxic) \\
\hline Barium & (EP T & Toxic) \\
\hline Barium ( & (EP T & Toxic) \\
\hline Barium ( & (EP T & Toxic) \\
\hline Boron & & \\
\hline Boron & & \\
\hline Boron & & \\
\hline Boron & & \\
\hline Cadmium & (E'P & Toxic) \\
\hline Cadmium & $(\operatorname{EP}$ & Toxic) \\
\hline Cadmium & (EP & Toxic) \\
\hline Cadmium & $(E P$ & Toxic) \\
\hline Calcium & & \\
\hline Calcium & & \\
\hline Calcium & & \\
\hline Calcium & & \\
\hline Chloride & & \\
\hline ChIoride & & \\
\hline Chloride & & \\
\hline Chloride & & \\
\hline Chromium & $n$ (EP & P Toxic) \\
\hline Chromium & $n$ (EF & P Toxic) \\
\hline Chromium & $n$ (EP & P Toxic) \\
\hline Chromium & $n(E P$ & P Toxic) \\
\hline Copper & & \\
\hline Copper & & \\
\hline Copper & & \\
\hline Copper & & \\
\hline Eluoride & & \\
\hline Fluoride & & \\
\hline FIuoride & & \\
\hline Fluoride & & \\
\hline Fluoxide & & \\
\hline Fluoride & & \\
\hline Fluoride & & \\
\hline Fluoride & & \\
\hline Lead (EP & p Tox & xic) \\
\hline Lead (EP & P TOX & $x i c)$ \\
\hline Lead (EP & $P$ Tox & xic) \\
\hline Lead (EP & P Tox & $x i c)$ \\
\hline Magnesiun & & \\
\hline Magnesiun & & \\
\hline Magnesiun & & \\
\hline Magnesiun & & \\
\hline Mercury & (EP & Toxic) \\
\hline
\end{tabular}

\begin{tabular}{|c|c|c|c|}
\hline Sample \# & Date & Method & Result \\
\hline $50729 \mathrm{E}$ & $10 / 26 / 89$ & $\operatorname{ICP}$ & $<5.00 \mathrm{E}+02$ \\
\hline $50820 \mathrm{E}$ & $11 / 30 / 89$ & ICP & $<5.00 E+02$ \\
\hline $50915 \mathrm{E}$ & $1 / 31 / 90$ & ICP & $<5.00 \mathrm{E}+02$ \\
\hline 51060E & $3 / 16 / 90$ & ICP & $<5.00 \mathrm{E}+02$ \\
\hline 50729 & $10 / 26 / 89$ & ICP & $3.00 E+01$ \\
\hline 50820 & $11 / 30 / 89$ & ICP & $3.00 E+01$ \\
\hline 50915 & $1 / 31 / 90$ & ICP & 3. $20 E+01$ \\
\hline 51060 & $3 / 16 / 90$ & ICP & $2.80 E+01$ \\
\hline $50729 \mathrm{E}$ & $10 / 26 / 89$ & ICP & $\because 1.00 E+03$ \\
\hline $50820 \mathrm{E}$ & $11 / 30 / 89$ & ICP & $<1.00 \mathrm{E}+03$ \\
\hline 50915E & $1 / 31 / 90$ & ICP & $<1.00 E+03$ \\
\hline $51060 x$ & $3 / 16 / 90$ & ICP & $<1.00 \mathrm{E}+03$ \\
\hline 50729 & $10 / 26 / 89$ & ICP & $<1.00 \mathrm{E}+01$ \\
\hline 50820 & $11 / 30 / 89$ & ICP & 1. $20 \mathrm{E}+01$ \\
\hline 50915 & $1 / 31 / 90$ & ICP & $1.90 \mathrm{E}+01$ \\
\hline 51060 & $3 / 16 / 90$ & ICP & $2.30 E+01$ \\
\hline $50729 \mathrm{E}$ & $10 / 26 / 89$ & ICP & $<1.00 \mathrm{E}+02$ \\
\hline $50820 \mathrm{E}$ & $11 / 30 / 89$ & ICP & $<1.00 \mathrm{E}+02$ \\
\hline 50915E: & $1 / 31 / 90$ & ICP & $<1.00 \mathrm{E}+02$ \\
\hline 51060E: & $3 / 16 / 90$ & ICP & $<1.00 \mathrm{E}+02$ \\
\hline 50729 & $10 / 26 / 89$ & ICP & $2.01 E+04$ \\
\hline 50820 & $11 / 30 / 89$ & ICP & 1. $95 \mathrm{E}+04$ \\
\hline 50915 & $1 / 31 / 90$ & ICP & $1.98 \mathrm{E}+04$ \\
\hline 51060 & $3 / 16 / 90$ & ICP & $1.76 \mathrm{E}+04$ \\
\hline 50729 & $10 / 26 / 89$ & $I C$ & $4.30 \mathrm{E}+03$ \\
\hline 50820 & $11 / 30 / 89$ & IC & $2.90 \mathrm{E}+03$ \\
\hline 50915 & $1 / 31 / 90$ & IC & $5.50 \mathrm{E}+03$ \\
\hline 51060 & $3 / 16 / 90$ & $I C$ & $2.30 \mathrm{E}+03$ \\
\hline $50729 \mathrm{E}$ & $10 / 26 / 89$ & ICP & $<5.00 E+02$ \\
\hline $50820 E$ & $11 / 30 / 89$ & ICP & $<5.00 \mathrm{~N}+02$ \\
\hline 50915E & $1 / 31 / 90$ & ICP & $<5.00 \mathrm{E}+02$ \\
\hline 51060E & $3 / 16 / 90$ & ICP & $<5.00 E+02$ \\
\hline 50729 & $10 / 26 / 89$ & ICP & $<1.00 E+01$ \\
\hline 50820 & $11 / 30 / 89$ & ICP & $<1.00 \mathrm{E}+01$ \\
\hline 50915 & $1 / 31 / 90$ & ICP & 1. 30E+01 \\
\hline 51060 & $3 / 16 / 90$ & ICP & $<1.00 \mathrm{E}+01$ \\
\hline 50729 & $10 / 26 / 89$ & I.C & $<5.00 E+02$ \\
\hline 50729 & $10 / 26 / 89$ & ISE & $1.66 \mathrm{E}+02$ \\
\hline 50820 & $11 / 30 / 89$ & $I C$ & $<5.00 \mathrm{E}+02$ \\
\hline 50820 & $11 / 30 / 89$ & ISE & $1.44 \mathrm{E}+02$ \\
\hline 50915 & $1 / 31 / 90$ & IC & $1.00 \mathrm{E}+03$ \\
\hline 50915 & $1 / 31 / 90$ & ISE & 1.13E+02 \\
\hline 51060 & $3 / 16 / 90$ & IC & $<5.00 \mathrm{E}+02$ \\
\hline 51060 & $3 / 16 / 90$ & ISE & $1.32 \mathrm{E}+02$ \\
\hline $50729 \mathrm{E}$ & $10 / 26 / 89$ & ICP & $<5.00 E+02$ \\
\hline $50820 \mathrm{E}$ & $11 / 30 / 89$ & ICP & $<5.00 \mathrm{E}+02$ \\
\hline $50915 \mathrm{E}$ & $1 / 31 / 90$ & ICP & $<5.00 E+02$ \\
\hline $51060 \mathrm{E}$ & $3 / 16 / 90$ & ICP & $<5.0 O E+02$ \\
\hline 50729 & $10 / 26 / 89$ & ICP & $4.05 E+03$ \\
\hline 50820 & $11 / 30 / 89$ & ICP & $4.21 E+03$ \\
\hline 50915 & $1 / 31 / 90$ & ICP & $4.69 \mathrm{E}+03$ \\
\hline 51060 & $3 / 16 / 90$ & ICP & $4.32 E+03$ \\
\hline $50729 \mathrm{E}$ & $10 / 26 / 89$ & CVAA $/ M$ & $<2.00 E+01$ \\
\hline
\end{tabular}


DATA FOR 242-S Evaporator Steam Condensate

Continued

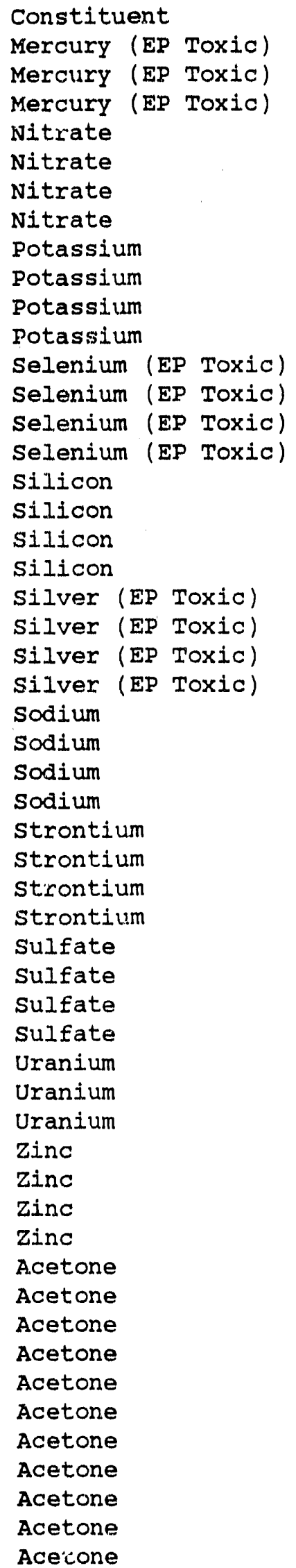

\begin{tabular}{|c|c|c|c|}
\hline Sample \# & Date & Method & Result \\
\hline $508,20 \mathrm{E}$ & $11 / 30 / 89$ & CVAA/M & $<2.00 \mathrm{E}+01$ \\
\hline $50915 \mathrm{E}$ & $1 / 31 / 90$ & $\mathrm{CVAA} / \mathrm{M}$ & $<2.00 E+01$ \\
\hline $51.060 \mathrm{E}$ & $3 / 16 / 90$ & $C V A A / M$ & $<2.00 E+O I$ \\
\hline 50729 & $10 / 26 / 89$ & IC & $<5.00 \mathrm{E}+02$ \\
\hline 50820 & $11 / 30 / 89$ & IC & $5.00 \mathrm{E}+02$ \\
\hline 50915 & $1 / 31 / 90$ & IC & $5.00 \mathrm{E}+02$ \\
\hline 51060 & $3 / 16 / 90$ & IC & $<5.00 \mathrm{E}+02$ \\
\hline 50729 & $10 / 26 / 89$ & ICP & $7.49 \mathrm{E}+02$ \\
\hline 50820 & $11 / 30 / 89$ & ICP & $7.54 \mathrm{E}+02$ \\
\hline 50915 & $1 / 31 / 90$ & ICP & $7.27 \mathrm{E}+02$ \\
\hline 51060 & $3 / 16 / 90$ & ICP & $7.22 \mathrm{E}+02$ \\
\hline $50729 \mathrm{E}$ & $10 / 26 / 89$ & ICP & $<5.00 E+02$ \\
\hline 50820E & $11 / 30 / 89$ & ICP & $<5.00 \mathrm{E}+02$ \\
\hline $50915 \mathrm{E}$ & $1 / 31 / 90$ & ICP & $<5.00 \mathrm{E}+02$ \\
\hline 51060E & $3 / 16 / 90$ & ICP & $<5.00 \mathrm{E}+02$ \\
\hline 50729 & $10 / 26 / 89$ & ICP & $2.10 \mathrm{E}+03$ \\
\hline 50820 & $11 / 30 / 89$ & ICP & 2. $22 E+03$ \\
\hline 50915 & $1 / 31 / 90$ & ICP & $2.32 E+03$ \\
\hline 51060 & $3 / 16 / 90$ & ICP & $1.99 \mathrm{E}+03$ \\
\hline $50729 \mathrm{E}$ & $10 / 26 / 89$ & ICP & $<5.00 \mathrm{E}+02$ \\
\hline $50820 \mathrm{E}$ & $11 / 30 ! 89$ & ICP & $<5.00 \mathrm{E}+02$ \\
\hline 50915E & $1 / 31 / 90$ & ICP & $<5.00 \mathrm{E}+02$ \\
\hline 51060E & $3 / 16 / 90$ & ICP & $<5.00 \mathrm{E}+02$ \\
\hline 50729 & $10 / 26 / 89$ & $I C P$ & $2.29 E+03$ \\
\hline 50820 & $11 / 30 / 89$ & ICP & $2.09 E+03$ \\
\hline 50915 & $1 / 31 / 90$ & ICP & $2.25 E+03$ \\
\hline 51060 & $3 / 16 / 90$ & ICP & $2.02 \mathrm{E}+03$ \\
\hline 50729 & $10 / 26 / 89$ & ICP & $9.90 \mathrm{E}+01$ \\
\hline 50820 & $11 / 30 / 89$ & ICP & $9.60 \mathrm{E}+01$ \\
\hline 50915 & $1 / 31 / 90$ & ICP & $9.50 \mathrm{E}+0.1$ \\
\hline 51060 & $3 / 16 / 90$ & ICP & $9.40 \mathrm{E}+01$ \\
\hline 50729 & $10 / 26 / 89$ & IC & $1.39 \mathrm{E}+04$ \\
\hline 50820 & $11 / 30 / 89$ & IC & 1. 30E+04 \\
\hline 50915 & $1 / 31 / 90$ & IC & $1.47 \mathrm{E}+04$ \\
\hline 51060 & $3 / 16 / 90$ & $I C$ & $1.48 \mathrm{E}+04$ \\
\hline 50729 & $10 / 26 / 89$ & GEA & $4.04 \mathrm{E}-01$ \\
\hline 50820 & $11 / 30 / 89$ & GEA & $1.94 \mathrm{E}-01$ \\
\hline 51060 & $3 / 16 / 90$ & GEA & $2.98 \mathrm{E}-01$ \\
\hline 50729 & $10 / 26 / 89$ & ICP & $4.50 E+01$ \\
\hline 50820 & $11 / 30 / 89$ & ICP & $3.10 \mathrm{E}+01$ \\
\hline 50915 & $1 / 31 / 90$ & ICP & $3.00 E+01$ \\
\hline 51060 & $3 / 16 / 90$ & ICP & $1.80 \mathrm{E}+01$ \\
\hline 50729 & $10 / 26 / 89$ & VOA & $<1.00 E+01$ \\
\hline 50729 & $10 / 26 / 89$ & $A B N$ & $<1.00 E+01$ \\
\hline $50729 B$ & $10 / 26 / 89$ & VOA & 1. $40 \mathrm{E}+01$ \\
\hline $50729 \mathrm{~T}$ & $10 / 26 / 89$ & VOA & $1.60 \mathrm{E}+01$ \\
\hline 50820 & $11 / 30 / 89$ & VOA & $<8.00 \mathrm{E}+00$ \\
\hline 50820 & $11 / 30 / 89$ & $A B N$ & $<1.00 \mathrm{E}+01$ \\
\hline 50915 & $1 / 31 / 50$ & VOA & $<1.00 E+01$ \\
\hline 50915 & $1 / 31 / 90$ & $\mathrm{ABN}$ & $<1.00 E+01$ \\
\hline $50915 B$ & $1 / 31 / 90$ & VOA & $<1.00 E+01$ \\
\hline $50915 \mathrm{~T}$ & $1 / 31 / 90$ & VOA & $<1.00 E+01$ \\
\hline 51060 & $3 / 16 / 90$ & VOA & $<1.00 \mathrm{E}+01$ \\
\hline
\end{tabular}


DATA FOR 242-S Evaporator steam Condensate Continued

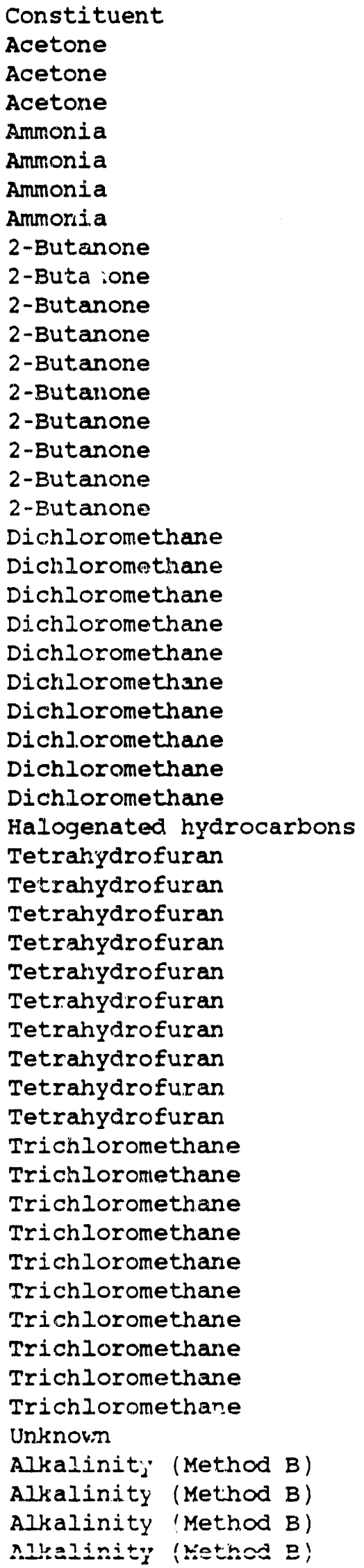

\begin{tabular}{|c|c|c|c|}
\hline ample \# & Date & Method & Result \\
\hline 51060 & $3 / 16 / 90$ & $A B N$ & $<1.10 E+01$ \\
\hline $51060 B$ & $3 / 16 / 90$ & VOA & $<1.00 E+01$ \\
\hline 51060T & $3 / 16 / 90$ & VOA & $<1.00 \mathrm{E}+01$ \\
\hline 50729 & $10 / 26 / 89$ & ISE & $5.00 \mathrm{E}+01$ \\
\hline 50820 & $11 / 30 / 89$ & ISE & $<5.00 \mathrm{E}+01$ \\
\hline 50915 & $1 / 31 / 90$ & ISE & $<5.00 E+01$ \\
\hline 51060 & $3 / 16 / 90$ & ISE & $<5.00 \mathrm{E}+01$ \\
\hline 50729 & $10 / 26 / 89$ & VOA & $<1.00 \mathrm{E}+01$ \\
\hline $50729 B$ & $10 / 26 / 89$ & VOA & $<1.00 \mathrm{E}+01$ \\
\hline $50729 \mathrm{I}$ & $10 / 26 / 89$ & VOA & $<1.00 E+01$ \\
\hline 50820 & $1.1 / 30 / 89$ & VOA & $<6.00 E+00$ \\
\hline 50915 & $1 / 31 / 90$ & VOA & $<1.00 \mathrm{E}+01$ \\
\hline $50915 B$ & $1 / 31 / 90$ & VOA & $1.00 E+01$ \\
\hline $50915 \mathrm{~T}$ & $1 / 31 / 90$ & VOA & $<9.00 E+00$ \\
\hline 51060 & $3 / 16 / 90$ & VOA & $<1.00 \mathrm{E}+01$ \\
\hline 510608 & $3 / 26 / 90$ & VOA & $<1.00 E+01$ \\
\hline $51060 \mathrm{~T}$ & $3 / 16 / 90$ & VOA & $<1.00 E+01$ \\
\hline 50729 & $10 / 26 / 89$ & VOA & $=5.00 E+00$ \\
\hline $50729 B$ & $10 / 26 / 89$ & VOA & $<4.00 E+00$ \\
\hline $50729 \mathrm{~T}$ & $10 / 26 / 89$ & VOA & $2.60 \mathrm{E}+01$ \\
\hline 50820 & $11 / 30 / 89$ & VOA & $<5.00 E+00$ \\
\hline 50915 & $1 / 31 / 90$ & VOA & $<5.00 \mathrm{E}+00$ \\
\hline $50915 B$ & $1 / 31 / 90$ & VOA & $<3.00 \mathrm{E}+00$ \\
\hline $50915 \mathrm{~T}$ & $1 / 31 / 90$ & VOA & $<3.00 E+00$ \\
\hline 51060 & $3 / 16 / 90$ & VOA & $<5.00 \mathrm{E}+00$ \\
\hline $51060 \mathrm{~B}$ & $3 / 16 / 90$ & VOA & $<5.00 \mathrm{E}+00$ \\
\hline $51060 \mathrm{~T}$ & $3 / 16 / 90$ & VOA & $<3.00 E+00$ \\
\hline 50820 & $11 / 30 / 89$ & $A B N$ & $3.40 E+01$ \\
\hline 50729 & $10 / 26 / 89$ & VOA & $<1.00 E+01$ \\
\hline $50729 \mathrm{~B}$ & $10 / 26 / 89$ & VOA & $1.70 \mathrm{E}+01$ \\
\hline $50729 \mathrm{~T}$ & $10 / 26 / 89$ & VOA & $<1.00 \mathrm{E}+01$ \\
\hline 50820 & $11 / 30 / 89$ & VOA & $<1.00 E+01$ \\
\hline 50915 & $1 / 31 / 90$ & VOA & $<1.00 E+01$ \\
\hline $5091.5 B$ & $1 / 31 / 90$ & VOA & 1. 10E+01 \\
\hline $50915 \mathrm{~T}$ & $1 / 31 / 90$ & VDA & $<9.00 \mathrm{E}+00$ \\
\hline 51060 & $3 / 16 / 90$ & VOA & $<1.00 \mathrm{E}+01$ \\
\hline $51060 B$ & $3 / 16 / 90$ & VOA & $<1.00 E+01$ \\
\hline $51060 \mathrm{~T}$ & $3 / 16 / 90$ & VOA & $<7.00 E+00$ \\
\hline 50729 & $10 / 26 / 89$ & VOA & $1.50 \mathrm{E}+01$ \\
\hline $50729 B$ & $10 / 26 / 89$ & VOA & $<5.00 \mathrm{E}+00$ \\
\hline $50729 \mathrm{~T}$ & $10 / 26 / 89$ & VOA & $<5.00 \mathrm{E}+00$ \\
\hline 50820 & $11 / 30 / 89$ & VOA & $2.70 \mathrm{E}+01$ \\
\hline 50915 & $1 / 31 / 90$ & VOA & $1.50 \mathrm{E}+0 \mathrm{I}$ \\
\hline $50915 B$ & $1 / 31 / 90$ & VOA & $<5.00 E+00$ \\
\hline $50915 \mathrm{~T}$ & $1 / 31 / 90$ & VOA & $<5.00 \mathrm{E}+00$ \\
\hline 51060 & $3 / 16 / 90$ & VOA & $1.30 \mathrm{E}+01$ \\
\hline $51060 \mathrm{~B}$ & $3 / 16 / 90$ & VOA & $<5.00 \mathrm{E}+00$ \\
\hline $51060 \mathrm{~T}$ & $3 / 16 / 90$ & VOA & $<4.00 E+00$ \\
\hline 50915 & $1 / 31 / 90$ & $A B R^{7}$ & $5.20 E+01$ \\
\hline 50729 & $10 / 26 / 89$ & TITRA & $5.30 E+04$ \\
\hline 50820 & $11 / 30 / 89$ & TITRA & $5.60 E+04$ \\
\hline 50915 & $1 / 31 / 90$ & TITRA & $6.10 \mathrm{E}+04$ \\
\hline 510s0 & $3,15,190$ & TITPX & $5.50 E \div 04$ \\
\hline
\end{tabular}


WHC-EP-0355, Appendix D

DATA FOR 242-S Evaporator steam Condensate

Continued

Constituent

Alpha Activity (pCi/L)

Alpha Activity (pCi/L)

Alpha Activity (pCi/L)

Beta Activity (pCi/L)

Beta Activity ( $\mathrm{pCi} / \mathrm{I}$ )

Beta Activity ( $\mathrm{pCi} / \mathrm{L}$ )

Conductivity (us)

conductivity (us)

conductivity (us)

conductivity (us)

Ingitability (degrees F)

Ingitability (degrees F)

Ingitability (degrees F)

Ingitability (degrees F)

pH (dimensionless)

pH (dimensionless)

pH (dimensionless)

pH (dimensionless)

Reactivity Cyanide (mg/kg)

Reactivity Cyanide ( $\mathrm{mg} / \mathrm{kg}$ )

Reactivity Cyanide ( $\mathrm{mg} / \mathrm{kg}$ )

Reactivity Cyanide ( $\mathrm{mg} / \mathrm{kg}$ )

Reactivity sulfide (mg/kg)

Reactivity Sulfide (mg/kg)

Reactivit: sulfide (mg/kg)

Reactivity sulfide ( $\mathrm{mg} / \mathrm{kg}$ )

TDS (mg/L)

$\operatorname{TDS}(\mathrm{mg} / \mathrm{L})$

$\operatorname{TDS}(\mathrm{mg} / \mathrm{L})$

TDS (mg/L)

Temperature (degrees C)

Temperature (degrees $C$ )

Temperature (degrees C)

Temperature (degrees C)

Total Carbon (ug/g)

Total Carbon (ug/g)

Total Carbon (ug/g)

Total Carbon (ug/g)

$\operatorname{TOX}(\mathrm{ug}(\mathrm{Cl}) / \mathrm{L})$

$\operatorname{Tox}(\mathrm{ug}(\mathrm{Cl}) / \mathrm{L})$

TOX (ug (CI)/L)

$\operatorname{TOX}(\mathrm{ug}(\mathrm{Cl}) / \mathrm{L})$

Co-60 (pCi/L

Co-60 (pCi.L)

$\mathrm{sr}-90(\mathrm{pCj} / \mathrm{L})$

$\mathrm{Sr}-90(\mathrm{pCi} / \mathrm{L})$

$\mathrm{sr}-90(\mathrm{pCi} / \mathrm{L})$

$\mathrm{U}-234$ (pCi/L)

$\mathrm{U}-234(\mathrm{pCi} / \mathrm{L})$

U-234 (pCi/L)

$\mathrm{U}-233$ ( $\mathrm{DCi} / \mathrm{L})$

U-238 (pCi/L)

$\mathrm{U}-238(\mathrm{pCi} / \mathrm{L})$

\begin{tabular}{|c|c|c|c|}
\hline $\begin{array}{c}\text { ample \# } \\
50729\end{array}$ & $\begin{array}{c}\text { Date } \\
10 / 26 / 89\end{array}$ & $\begin{array}{l}\text { Method } \\
\text { Alpha }\end{array}$ & $\begin{array}{l}\text { Result } \\
<3.39 \mathrm{E}-01\end{array}$ \\
\hline 50820 & $11 / 30 / 89$ & Alpha & $1.05 \mathrm{E}+00$ \\
\hline 51060 & $3 / 16 / 90$ & Alpha & $<2.10 E-01$ \\
\hline 50729 & $10 / 26 / 99$ & Beta & $<3.19 \mathrm{E}-01$ \\
\hline 50820 & $11, / 30 / 89$ & Beta & $2.36 E+00$ \\
\hline 51060 & $3 / 16 / 90$ & Beta & $<5.00 \mathrm{E}-01$ \\
\hline 50729 & $10 / 26 / 89$ & COND-FId & $1.60 \mathrm{E}+02$ \\
\hline 50820 & $11 / 30 / 89$ & COND-FId & $1.68 \mathrm{E}+02$ \\
\hline 50915 & $1 / 31 / 90$ & COND-FId & $1.58 \mathrm{E}+02$ \\
\hline 51060 & $3 / 16 / 90$ & COND-FId & $1.58 \mathrm{E}+02$ \\
\hline $50729 \mathrm{E}$ & $10 / 26 / 89$ & IGNIT & $2.12 E+02$ \\
\hline $50820 \mathrm{E}$ & $11 / 30 / 89$ & IGNIT & $2.14 \mathrm{E}+02$ \\
\hline $50915 \mathrm{E}$ & $1 / 31 / 90$ & IGNIT & $2.10 E+02$ \\
\hline $51060 \mathrm{E}$ & $3 / 16 / 90$ & IGNIT & $2.08 \mathrm{E}+02$ \\
\hline 50729 & $10 / 26 / 89$ & $\mathrm{PH}-\mathrm{F} I \mathrm{~d}$ & $7.90 E+00$ \\
\hline 50820 & $11 / 30 / 89$ & PH-FId & $7.51 E+00$ \\
\hline 50915 & $1 / 31 / 90$ & PH-FId & $8.00 E+00$ \\
\hline 51060 & $3 / 16 / 90$ & $P K-F^{\prime} I d$ & $8.00 E+00$ \\
\hline $50729 E$ & $10 / 26 / 89$ & DSPEC & $<1.00 E+02$ \\
\hline $50820 \mathrm{E}$ & $11 / 30 / 89$ & DSPEC & $<1.00 \mathrm{E}+02$ \\
\hline $50915 E$ & $1 / 31 / 90$ & DSPEC & $<1.00 E+02$ \\
\hline $51060 E$ & $3 / 16 / 90$ & DSPEC & $<1.00 E+02$ \\
\hline $50729 E$ & $10 / 26 / 89$ & DTITRA & $<1.00 E+02$ \\
\hline $50820 \mathrm{E}$ & $1.1 / 30 / 89$ & DTITRA & $<1.00 \mathrm{E}+02$ \\
\hline $50915 \mathrm{E}$ & $1 / 31 / 90$ & DTITRA & $<1.00 E+02$ \\
\hline $51060 E$ & $3 / 16 / 90$ & DTITRA & $<1.00 \mathrm{E}+02$ \\
\hline 50729 & $10 / 26 / 89$ & IDS & $7.60 \mathrm{E}+04$ \\
\hline 50820 & $11 / 30 / 89$ & TDS & $7.70 E+04$ \\
\hline 50915 & $1 / 31 / 90$ & TDS & $8.40 E+04$ \\
\hline 51060 & $3 / 16 / 90$ & TDS & $5.70 E+04$ \\
\hline 50729 & $10 / 2$ & TEMP-FId & $2.45 E+O I$ \\
\hline 50820 & $11 / 30 / 89$ & TEMP-FId & $1.72 \mathrm{E}+01$ \\
\hline 50915 & $1 / 31 / 90$ & TEMP-FId & $2.37 E+01$ \\
\hline 51060 & $3 / 16 / 90$ & TEMP-FId & 1. $92 \mathrm{E}+01$ \\
\hline 50729 & $10 / 26 / 89$ & $\mathrm{TC}$ & $1.49 \mathrm{E}+04$ \\
\hline 50820 & $11 / 30 / 89$ & $\mathrm{TC}$ & $1.41 E+04$ \\
\hline 50915 & $1 / 31 / 90$ & TC & 1. $26 \mathrm{E}+04$ \\
\hline 510r: & $3 / 16 / 90$ & TC & $1.44 E+04$ \\
\hline 50729 & $10 / 26 /$ & LTOX & $1.94 \mathrm{E}+02$ \\
\hline 50820 & $11 / 30 / 89$ & ITOX & $1.65 \mathrm{E}+02$ \\
\hline 50915 & $1 / 31 / 90$ & LTOX & $1.46 \mathrm{E}+02$ \\
\hline 51060 & $3 / 16 / 90$ & LTOX & $1.18 \mathrm{E}+02$ \\
\hline 50729 & $10 / 26 / 89$ & GEA & $6.14 \mathrm{E}-01$ \\
\hline 51060 & $3 / 16 / 90$ & GEA & $1.03 E+00$ \\
\hline 50729 & $10 / 26 / 89$ & Beta & $<1.59 \mathrm{E}-01$ \\
\hline 50820 & $11 / 30 / 89$ & Beta & $1.99 E-01$ \\
\hline 51060 & $3 / 16 / 90$ & Beta & $<2.93 \mathrm{E}-01$ \\
\hline 50729 & $10 / 26 / 89$ & $A E A$ & $7.58 E-02$ \\
\hline 50820 & $11 / 30 / 89$ & $A E A$ & $1.41 \mathrm{E}-01$ \\
\hline 51060 & $3 / 16 / 90$ & $A E A$ & $1.89 E-01$ \\
\hline 50729 & $10 / 26 / 89$ & $A E A$ & $9.78 E-02$ \\
\hline 50820 & $11 / 30 / 89$ & $A E A$ & $1.09 \mathrm{E}-01$ \\
\hline 51060 & $3 / 16 / 90$ & $A E A$ & $1.17 \mathrm{E}-01$ \\
\hline
\end{tabular}


WHC-EP-0355, Appendix D

DATA FOR T Plant Laboratory Wastewater-Plasmu Torch Operation

Constituent
Arsenic (EP Toxic)
Barium
Barium (EP Toxic)
Boron
Cadmium (EP Toxic)
Calcium
Chloride
Chromium (EP Toxic)
Fluoride
Fluoride
Iron
Lead (EP Toxic)
Magnesium
Manganese
Mercury (EP Toxic)
Potassium
Selenjum (EP Toxic)
Silicon
Silver (EF Toxic)
Sodium
Strontium
Sulfate
Zinc
Ammonia
2-Butanone
2-Butanone
2-Butanone
Dichloromethane
Dichloromethane
Dichloromethane
Trichloromethane
Trichloromethane
Trichloromethane
Unknown
Alkalinity (Method B)
Conductivity (us)
Ingitability (degrees F)
pH (dimensionless)
Reactivity Cyanide (mg/kg)
Reactivity Sulfide (mg/kg)
TDS (mg/I)
Temperature (degrees C)
Total Carbon (ug/g)
Tox (ug (Cl)/L)

\begin{tabular}{|c|c|c|c|}
\hline ample \# & Date & Method & Result \\
\hline $50971 \mathrm{E}$ & $2 / 22 / 90$ & ICP & $<5.00 \mathrm{E}+02$ \\
\hline 50971 & $2 / 22 / 90$ & ICP & $2.50 E+O 1$ \\
\hline $50971 \mathrm{E}$ & $2 / 22 / 90$ & ICP & $<1.00 E+03$ \\
\hline 50971 & $2 / 22 / 90$ & ICP & $2.40 E+01$ \\
\hline 50971E & $2 / 22 / 90$ & ICP & $<3.00 \mathrm{E}+02$ \\
\hline 50971 & $2 / 22 / 90$ & ICP & $1.78 \mathrm{E}+04$ \\
\hline 50971 & $2 / 22 / 90$ & IC & $2.70 \mathrm{E}+03$ \\
\hline 50971E & $2 / 22 / 90$ & ICP & $<5.00 E+02$ \\
\hline 50971 & $2 / 22 / 90$ & IC & $<5.00 E+02$ \\
\hline 50971 & $2 / 22 / 90$ & ISE & $1.13 \mathrm{E}+02$ \\
\hline 50971 & $2 / 22 / 90$ & ICP & 1. $25 \mathrm{E}+02$ \\
\hline $50971 \mathrm{E}$ & $2 / 22 / 90$ & ICP & $<5.00 E+02$ \\
\hline 50971 & $2 / 22 / 90$ & ICP & $4.10 \mathrm{E}+03$ \\
\hline 50971 & $2 / 22 / 90$ & ICP & 4. $50 E+01$ \\
\hline 50971E & $2 / 22 / 90$ & CVAA/M & $<2.00 E+01$ \\
\hline 50971 & $2 / 22 / 90$ & ICP & $7.01 \mathrm{E}+02$ \\
\hline $50971 E$ & $2 / 22 / 90$ & ICP & $<5.00 E+02$ \\
\hline 50971 & $2 / 22 / 90$ & ICP & $2.11 \mathrm{E}+03$ \\
\hline $50971 \mathrm{E}$ & $2 / 22 / 90$ & ICP & $<5.00 E+02$ \\
\hline 50971 & $2 / 22 / 90$ & ICP & $1.93 E+03$ \\
\hline 50971 & $2 / 22 / 90$ & ICP & $8.70 E+01$ \\
\hline 50971 & $2 / 22 / 90$ & $I C$ & $1.36 \mathrm{E}+04$ \\
\hline 50971 & $2 / 22 / 90$ & ICP & $2.90 E+01$ \\
\hline 50971 & $2 / 22 / 90$ & ISE & 6.90E+01 \\
\hline 50971 & $2 / 22 / 90$ & VOA & $<1.00 E+01$ \\
\hline $50971 B$ & $2 / 22 / 90$ & VOA & $1.30 \mathrm{E}+01$ \\
\hline $50971 \mathrm{~T}$ & $2 / 22 / 90$ & VOA & 1.30E+01 \\
\hline 50971 & $2 / 22 / 90$ & VOA & $<5.00 \mathrm{E}+00$ \\
\hline $50971 \mathrm{~B}$ & $2 / 22 / 90$ & VOA & $5.00 \mathrm{E}+00$ \\
\hline $50971 \mathrm{~T}$ & $2 / 22 / 90$ & VOA & $<5.00 \mathrm{E}+00$ \\
\hline 50971 & $2 / 22 / 90$ & VOA & $1.50 \mathrm{E}+01$ \\
\hline $50971 B$ & $2 / 22 / 90$ & VOA & $7.00 \mathrm{E}+00$ \\
\hline $50971 \mathrm{~T}$ & $2 / 22 / 90$ & VOA & $<4.00 \mathrm{E}+00$ \\
\hline 50971 & $2 / 22 / 90$ & $A B N$ & $2.20 E+01$ \\
\hline 50971 & $2 / 22 / 90$ & TITRA & $5.40 \mathrm{E}+04$ \\
\hline 50971 & $2 / 22 / 90$ & COND-FId & $1.49 \mathrm{E}+02$ \\
\hline 5097IE & $2 / 22 / 90$ & IGNIT & $2.10 \mathrm{E}+02$ \\
\hline 50971 & $2 / 22 / 90$ & PH-FId & $7.47 \mathrm{E}+00$ \\
\hline $50971 \mathrm{E}$ & $2 / 22 / 90$ & DSPEC & $<1.00 E+02$ \\
\hline $50971 \mathrm{E}$ & $2 / 22 / 90$ & DIITRA & $<1.00 \mathrm{E}+02$ \\
\hline 50971 & $2 / 22 / 90$ & TDS & $7.80 \mathrm{E}+04$ \\
\hline 50971 & $2 / 22 / 90$ & TEMP-FId & $2.44 E+01$ \\
\hline 50971 & $2 / 22 / 90$ & $\mathrm{TC}$ & $1.39 \mathrm{E}+04$ \\
\hline 50971 & $2 / 22 / 90$ & LTOX & $8.00 \mathrm{E}+01$ \\
\hline
\end{tabular}


WHC-EP-0355, Appendix D

DATA FOR T Plant Laboratory Wastewater-Plasma Torch standby

\begin{tabular}{|c|c|c|}
\hline Constitu & uent & \\
\hline Aluminun & & \\
\hline Aluminum & & \\
\hline Aluminu & & \\
\hline Aluminun & & \\
\hline Arsenic & (EP 7 & Toxic) \\
\hline Arsenic & (EP T & Toxic) \\
\hline Arsenic & (EP 3 & Toxic) \\
\hline Arsenic & (EP 7 & Toxic) \\
\hline Barium & & \\
\hline Barium & & \\
\hline Barium & & \\
\hline Barium & & \\
\hline Barium ( & (EP TC & roxic) \\
\hline Barium ( & (EP TC & (oxic) \\
\hline Barium ( & (EP TC & [oxic) \\
\hline Barium ( & (EP TC & (oxic) \\
\hline Boron & & \\
\hline Boron & & \\
\hline Boron & & \\
\hline Boron & & \\
\hline Cadmium & (EP I & Toxic) \\
\hline Cadmium & (EP I & Toxic) \\
\hline C'admium & (EP : & Toxic) \\
\hline Cadmium & (EP & Toxic) \\
\hline Calcium & & \\
\hline Calcium & & \\
\hline Calcium & & \\
\hline Calcium & & \\
\hline Chloride & & \\
\hline Chloride & & \\
\hline Chloride & & \\
\hline Chloride & & \\
\hline Chromium & m (EP & Toxic) \\
\hline Chromium & $m$ (EP & Toxic) \\
\hline Chromium & $m$ (EP & Toxic) \\
\hline Chromium & $m$ (EP & Toxic) \\
\hline Copper & & \\
\hline Copper & & \\
\hline Copper & & \\
\hline Copper & & \\
\hline Fluoride & & \\
\hline Fluoride & & \\
\hline Fluoride & & \\
\hline Fluoride & & \\
\hline Fluoride & & \\
\hline Fluoride & & \\
\hline Fluoride & & \\
\hline Fluoride & & \\
\hline Iron & & \\
\hline Iron & & \\
\hline Iron & & \\
\hline Iron & & \\
\hline Lead & & \\
\hline
\end{tabular}

\begin{tabular}{|c|c|c|c|}
\hline Sample \# & Date & Method & Result \\
\hline 50733 & $10 / 26 / 89$ & ICP & $1.98 E+02$ \\
\hline 50776 & $11 / 17 / 89$ & $I C P$ & $<1.50 \mathrm{E}+02$ \\
\hline 50800 & $11 / 28 / 89$ & ICP & $<1.50 \mathrm{E}+02$ \\
\hline 50838 & $12 / 14 / 89$ & ICP & $<1.50 \mathrm{E}+02$ \\
\hline $50733 \mathrm{E}$ & $10 / 26 / 89$ & ICP & $<5.00 \mathrm{E}+02$ \\
\hline $50776 \mathrm{E}$ & $11 / 17 / 89$ & ICP & $<5.00 E+02$ \\
\hline $50800 E$ & $11 / 28 / 89$ & ICP & $<5.00 \mathrm{E}+02$ \\
\hline $50838 \mathrm{E}$ & $12 / 14 / 89$ & ICP & $<5.00 E+02$ \\
\hline 50733 & $10 / 26 / 89$ & ICP & $2.60 \mathrm{E}+01$ \\
\hline 50776 & $11 / 17 / 89$ & ICP & $2.90 \mathrm{E}+01$ \\
\hline 50800 & $11 / 28 / 89$ & ICP & 2. $90 E+01$ \\
\hline 50838 & $12 / 14 / 89$ & ICP & $2.40 \mathrm{E}+01$ \\
\hline $50733 \mathrm{E}$ & $10 / 26 / 89$ & ICP & $<1.00 E+03$ \\
\hline $50776 \mathrm{E}$ & $11 / 17 / 89$ & ICP & $<1.00 \mathrm{E}+03$ \\
\hline $50800 \mathrm{E}$ & $11 / 28 / 89$ & ICP & $<1.00 \mathrm{E}+03$ \\
\hline $50838 \mathrm{E}$ & $12 / 14 / 89$ & ICP & $<1,00 \mathrm{E}+03$ \\
\hline 50733 & $10 / 26 / 89$ & ICP & $1.50 \mathrm{E}+01$ \\
\hline 50776 & $11 / 17 / 89$ & ICP & $<1.00 E+01$ \\
\hline 50800 & $11 / 28 / 89$ & ICP & $1.40 \mathrm{E}+01$ \\
\hline 50838 & $12 / 14 / 89$ & ICP & 1. $40 \mathrm{E}+01$ \\
\hline $50733 E$ & $10 / 26 / 89$ & ICP & $<1.00 E+02$ \\
\hline $50776 \mathrm{E}$ & $11 / 17 / 89$ & ICP & $<1.00 \mathrm{E}+02$ \\
\hline $50800 \mathrm{E}$ & $11 / 28 / 89$ & ICP & $<1.00 \mathrm{E}+02$ \\
\hline $50838 \mathrm{E}$ & $12 / 14 / 89$ & ICP & $<1.00 \mathrm{E}+02$ \\
\hline 50733 & $10 / 26 / 89$ & ICP & $1.77 \mathrm{E}+04$ \\
\hline 50776 & $11 / 17 / 89$ & ICP & $1.85 \mathrm{E}+04$ \\
\hline 50800 & $11 / 28 / 89$ & ICP & $1.81 \mathrm{E}+04$ \\
\hline 50838 & $12 / 14 / 89$ & ICP & $1.52 \mathrm{E}+04$ \\
\hline 50733 & $10 / 26 / 89$ & $I C$ & $4.10 E+03$ \\
\hline 50776 & $11 / 17 / 89$ & IC & $3.20 E+03$ \\
\hline 50800 & $11 / 28 / 89$ & IC & $2.90 E+03$ \\
\hline 50838 & $12 / 14 / 89$ & IC & $2.80 \mathrm{E}+03$ \\
\hline $50733 E$ & $10 / 26 / 89$ & ICP & $<5.00 \mathrm{E}+02$ \\
\hline $50776 \mathrm{E}$ & $11 / 17 / 89$ & ICP & $<5.00 \mathrm{E}+02$ \\
\hline $50800 \mathrm{E}$ & $11 / 28 / 89$ & ICP & $<5.00 E+02$ \\
\hline $50838 E$ & $12 / 14 / 89$ & ICP & $<5.00 E+02$ \\
\hline 50733 & $10 / 26 / 89$ & ICP & $2.30 \mathrm{E}+01$ \\
\hline 50776 & $11 / 17 / 89$ & ICP & 1. $10 \mathrm{E}+01$ \\
\hline 50800 & $11 / 28 / 89$ & ICP & 1. $20 \mathrm{E}+01$ \\
\hline 50838 & $12 / 14 / 89$ & ICP & 1. $20 \mathrm{E}+01$ \\
\hline 50733 & $10 / 26 / 89$ & IC & $<5.00 E+02$ \\
\hline 50733 & $10 / 26 / 89$ & ISE & $1.54 \mathrm{E}+02$ \\
\hline 50776 & $11 / 17 / 89$ & $I C$ & $<5.00 E+02$ \\
\hline 50776 & $11 / 17 / 89$ & ISE & 1. $33 \mathrm{E}+02$ \\
\hline 50800 & $11 / 28 / 89$ & IC & $<5.00 E+02$ \\
\hline 50800 & $11 / 28 / 89$ & ISE & $1.23 E+02$ \\
\hline 50838 & $12 / 14 / 89$ & IC & $<5.00 \mathrm{E}+02$ \\
\hline 50838 & $12 / 14 / 89$ & ISE & $1.11 E+02$ \\
\hline 50733 & $10 / 26 / 89$ & ICP & $5.01 \mathrm{E}+02$ \\
\hline 50776 & $11 / 17 / 89$ & $I C P$ & $1.85 \mathrm{E}+02$ \\
\hline 50800 & $11 / 28 / 89$ & JCP & 2. $31 E+02$ \\
\hline 50838 & $12 / 14 / 89$ & ICP & $1.36 \mathrm{E}+02$ \\
\hline 50733 & $10 / 26 / 89$ & GFAA & $1.30 \mathrm{E}+01$ \\
\hline
\end{tabular}


DATA FOR T Plant Laboratory Wastewater-Plasma Torch Standby Continued

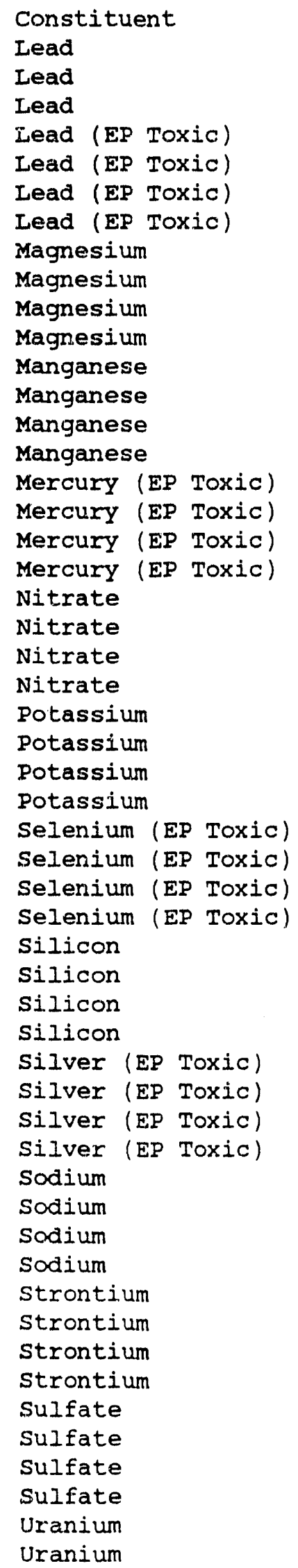

\begin{tabular}{|c|c|c|c|}
\hline ample \# & Date & Method & Result \\
\hline 50776 & $11 / 17 / 89$ & GFAA & $<5.00 \mathrm{E}+00$ \\
\hline 50800 & $11 / 28 / 89$ & GFAA & $<5.00 E+00$ \\
\hline 50838 & $12 / 14 / 89$ & GFAA & $<5.00 E+00$ \\
\hline $50733 \mathrm{E}$ & $10 / 26 / 89$ & IC̣P & $<5.00 E+02$ \\
\hline $50776 \mathrm{E}$ & $11 / 17 / 89$ & ICP & $<5.00 \mathrm{E}+02$ \\
\hline $50800 \mathrm{E}$ & $11 / 28 / 89$ & ICP & $<5.00 \mathrm{E}+02$ \\
\hline 50838E & $12 / 14 / 89$ & ICP & $<5.00 \mathrm{E}+02$ \\
\hline 50733 & $10 / 26 / 89$ & ICP & $3.87 \mathrm{E}+03$ \\
\hline 50776 & $11 / 17 / 89$ & ICP & $4.20 E+03$ \\
\hline 50800 & $11 / 28 / 89$ & ICP & $3.97 \mathrm{E}+03$ \\
\hline 50838 & $12 / 14 / 89$ & ICP & 3. $24 \mathrm{E}+03$ \\
\hline 50733 & $10 / 26 / 89$ & ICP & $2.28 E+02$ \\
\hline 50776 & $11 / 17 / 89$ & ICP & $1.03 \mathrm{E}+02$ \\
\hline 501800 & $11 / 28 / 89$ & $I C P$ & $9.00 \mathrm{E}+01$ \\
\hline 50838 & $12 / 14 / 89$ & ICP & $7.20 E+01$ \\
\hline $50733 E$ & $10 / 26 / 89$ & CVAA/M & $<2.00 E+01$ \\
\hline $50776 \mathrm{E}$ & $11 / 17 / 89$ & CVAA/M & $<2.00 E+01$ \\
\hline $50800 \mathrm{E}$ & $11 / 28 / 89$ & CVAA $/ M$ & $<2.00 E+01$ \\
\hline $50838 E$ & $12 / 14 / 89$ & CVAA/M & $<2.00 E+01$ \\
\hline 50733 & $10 / 26 / 89$ & IC & 6.0OE+02 \\
\hline 50776 & $11 / 17 / 89$ & IC & $<5.00 E+02$ \\
\hline 50800 & $11 / 28 / 89$ & IC & $<5.00 E+02$ \\
\hline 50838 & $12 / 14 / 89$ & IC & $<5.00 E+02$ \\
\hline 50733 & $10 / 26 / 89$ & ICP & $7.62 \mathrm{E}+02$ \\
\hline 50776 & $11 / 17 / 89$ & ICP & $7.19 \mathrm{E}+02$ \\
\hline 50800 & $11 / 28 / 89$ & ICP & $6.93 E+02$ \\
\hline 50838 & $12 / 14 / 89$ & ICP & $5.65 E+02$ \\
\hline $50733 E$ & $10 / 26 / 89$ & ICP & $<5.00 E+02$ \\
\hline $50776 \mathrm{E}$ & $11 / 17 / 89$ & ICP & $<5.0 O E+02$ \\
\hline $50800 \mathrm{E}$ & $11 / 28 / 89$ & ICP & $<5.0 O E+02$ \\
\hline $50838 \mathrm{E}$ & $12 / 14 / 89$ & ICP & $<5.00 \mathrm{E}+02$ \\
\hline 50733 & $10 / 26 / 89$ & ICP & $2.08 \mathrm{E}+03$ \\
\hline 50776 & $11 / 17 / 89$ & ICP & $2.04 \mathrm{E}+03$ \\
\hline 50800 & $11 / 28 / 89$ & ICP & $2.10 \mathrm{E}+0.3$ \\
\hline 50838 & $12 / 14 / 89$ & ICP & $1.77 \mathrm{E}+03$ \\
\hline $50733 E$ & $10 / 26 / 89$ & ICP & $<5.00 E+02$ \\
\hline $50776 \mathrm{E}$ & $11 / 17 / 89$ & ICP & $<5.0 O E+02$ \\
\hline $50800 \mathrm{E}$ & $11 / 28 / 89$ & ICP & $<5.00 E+02$ \\
\hline $50838 \mathrm{E}$ & $12 / 14 / 89$ & ICP & $<5.00 E+02$ \\
\hline 50733 & $10 / 26 / 89$ & ICF & $2.19 \mathrm{E}+03$ \\
\hline 50776 & $11 / 17 / 89$ & ICP & $2.14 \mathrm{E}+03$ \\
\hline 50800 & $11 / 28 / 89$ & ICP & $1.93 E+03$ \\
\hline 50838 & $12 / 14 / 89$ & ICP & $1.55 \mathrm{E}+03$ \\
\hline 50733 & $10 / 26 / 89$ & ICP & $9.10 \mathrm{E}+01$ \\
\hline 50776 & $11 / 17 / 89$ & ICP & $9.10 \mathrm{E}+01$ \\
\hline 50800 & $11 / 28 / 89$ & ICP & $9.00 \mathrm{E}+01$ \\
\hline 50838 & $12 / 14 / 89$ & ICP & $7.20 \mathrm{E}+01$ \\
\hline 50733 & $10 / 26 / 89$ & IC & $1.30 \mathrm{E}+04$ \\
\hline 50776 & $11 / 17 / 89$ & IC & $1.27 \mathrm{E}+04$ \\
\hline 50800 & $11 / 28 / 89$ & IC & $1.30 \mathrm{E}+04$ \\
\hline 50838 & $12 / 14 / 89$ & IC & $1.01 \mathrm{E}+04$ \\
\hline 50733 & $10 / 26 / 89$ & GEA & $3.44 \mathrm{E}-01$ \\
\hline 50776 & $11 / 17 / 89$ & GEA & $2.37 \mathrm{E}-01$ \\
\hline
\end{tabular}


WHC-EP-0355, Appendix D

DATA FOR T Plant Laboratory Wastewater-Plasma Torch Standby Continued

Constituent
Uranium
Uranium
zinc
zinc
Zinc
zinc
Acetone
Acetone
Acetone
Acetone
Acetore
Acetone
Acetone
Acetone
Acetone
Acetone
Acetone
Acetone
Acetone
Acetone
Ammonia
Ammonia
Ammonia
Ammonia
2-Butanone
2-Butanone
2-Butanone
2-Butanone
2-Butanone
2-Butanone
2-Butanone
2-Butanone
2-Butanone
2-Butanone
Dichloromethane
Dichloromethane
Dichloromethane
Dichloromethane
Dichloromethane
Dichloromethane
Dichloromethane
Dichloromethane
Dichloromethane
Dichloromethane
Trichloromethane
Trichloromethane
Trichloromethane
Trichloromethane
Trichloromethane
Trichloromethane
Trichloromethane
Trichloromethane

\begin{tabular}{|c|c|c|c|}
\hline Sample \# & Date & Method & Result \\
\hline 50800 & $11 / 28 / 89$ & GEA & $2.56 \mathrm{E}-01$ \\
\hline 50838 & $12 / 14 / 89$ & GEA & $7.08 E-01$ \\
\hline 50733 & $10 / 26 / 89$ & ICP & $1.82 \mathrm{E}+02$ \\
\hline 50776 & $11 / 17 / 89$ & ICP & $1.10 E+01$ \\
\hline 50800 & $11 / 28 / 89$ & ICP & $2.10 E+01$ \\
\hline 50838 & $12 / 14 / 89$ & ICP & $2.70 \mathrm{E}+01$ \\
\hline 50733 & $10 / 26 / 89$ & VOA & $1.70 \mathrm{E}+01$ \\
\hline 50733 & $10 / 26 / 89$ & $A B N$ & $<1.00 \mathrm{E}+01$ \\
\hline $50733 B$ & $10 / 26 / 89$ & VOA & $<1.00 \mathrm{E}+01$ \\
\hline 50776 & $11 / 17 / 89$ & VOA & $<1.00 \mathrm{E}+01$ \\
\hline 50776 & $11 / 17 / 89$ & $A B N$ & $<1.00 E+01$ \\
\hline $50776 \mathrm{~B}$ & $11 / 17 / 89$ & VOA & $<1.00 \mathrm{E}+01$ \\
\hline 50800 & $11 / 28 / 89$ & VOA & $<1.00 \mathrm{E}+01$ \\
\hline 50800 & $11 / 28 / 89$ & $A B N$ & $<1.00 E+01$ \\
\hline $50800 B$ & $11 / 28 / 89$ & VOA & $<1.00 \mathrm{E}+01$ \\
\hline $50800 \mathrm{~T}$ & $11 / 28 / 89$ & VOA & $<1.00 \mathrm{E}+01$ \\
\hline 50838 & $12 / 14 / 89$ & VOA & $<1.00 \mathrm{E}+01$ \\
\hline 50838 & $12 / 14 / 89$ & $A B N$ & $<1.00 \mathrm{E}+01$ \\
\hline $50838 B$ & $12 / 14 / 89$ & VOA & $<1.00 \mathrm{E}+01$ \\
\hline $50838 \mathrm{~T}$ & $12 / 14 / 89$ & VOA & $<1.00 E+01$ \\
\hline 5073.3 & $10 / 26 / 89$ & ISE & $5.60 \mathrm{E}+01$ \\
\hline 50776 & $11 / 17 / 89$ & ISE & $<5.00 E+01$ \\
\hline 50800 & $11 / 28 / 89$ & ISE & $<5.00 E+01$ \\
\hline 50838 & $12 / 14 / 89$ & ISE & $<5.00 E+01$ \\
\hline 50733 & $10 / 26 / 89$ & VOA & $<1.00 \mathrm{E}+01$ \\
\hline $50733 B$ & $10 / 26 / 89$ & VOA & $<1.00 \mathrm{E}+01$ \\
\hline 50776 & $11 / 17 / 89$ & VOA & $<1.00 E+01$ \\
\hline $50776 \mathrm{~B}$ & $11 / 17 / 89$ & VOA & $<1.00 \mathrm{E}+01$ \\
\hline 50800 & $11 / 28 / 89$ & VOA & $<1.00 \mathrm{~F}+01$ \\
\hline $50800 B$ & $11 / 28 / 89$ & VCA & $<1.00 \mathrm{E}+01$ \\
\hline $50800 T$ & $11 / 28 / 89$ & VOA & $<1.00 \mathrm{E}+01$ \\
\hline 50838 & $12 / 14 / 89$ & VOA & $<1.00 \mathrm{E}+01$ \\
\hline $50838 B$ & $12 / 14 / 89$ & VOA & $1.40 \mathrm{E}+01$ \\
\hline $50838 \mathrm{~T}$ & $12 / 14 / 89$ & VOA & $<1.00 \mathrm{E}+01$ \\
\hline 50733 & $10 / 26 / 89$ & VOA & $<5.00 \mathrm{E}+00$ \\
\hline $50733 B$ & $10 / 26 / 89$ & VOA & $5.00 E+00$ \\
\hline 50776 & $11 / 17 / 89$ & VOA & $<5.00 \mathrm{E}+00$ \\
\hline $50776 B$ & $11 / 17 / 89$ & VOA & $<5.00 \mathrm{E}+00$ \\
\hline 50800 & $11 / 28 / 89$ & VOA & $<5.00 \mathrm{E}+00$ \\
\hline $50800 B$ & $11 / 28 / 89$ & VOA & $6.00 E+00$ \\
\hline $50800 T$ & $11 / 28 / 89$ & VOA & $5.00 E+00$ \\
\hline 50838 & $12 / 14 / 89$ & VOA & $<5.00 E+00$ \\
\hline $50838 B$ & $12 / 14 / 89$ & VOA & $6.00 E+00$ \\
\hline $50838 \mathrm{~T}$ & $12 / 14 / 89$ & VOA & $1.40 \mathrm{E}+03$ \\
\hline 50733 & $10 / 26 / 89$ & VOA & $3.50 E+01$ \\
\hline $50733 B$ & $10 / 26 / 89$ & VOA & $<5.00 E+00$ \\
\hline 50776 & $11 / 17 / 89$ & VOA & $2.90 \mathrm{E}+01$ \\
\hline $50776 \mathrm{~B}$ & $11 / 17 / 89$ & VOA & $<4.00 E+00$ \\
\hline 50800 & $11 / 28 / 89$ & VOA & $2.60 \mathrm{E}+01$ \\
\hline $50800 \mathrm{~B}$ & $11 / 28 / 89$ & VOA & 1. $10 \mathrm{E}+01$ \\
\hline $50800 \mathrm{~T}$ & $.11 / 28 / 89$ & VOA & $8.00 \mathrm{E}+00$ \\
\hline 50838 & $12 / 14 / 89$ & VOA & $1.60 \mathrm{E}+01$ \\
\hline $50838 B$ & $12 / 14 / 89$ & VOA & $<5.00 \mathrm{E}+00$ \\
\hline
\end{tabular}


DATA FOR I Plant Laboratory Wastewater-plasma Torch standby Continued

Constituent

Trichloromethane

Unknown

Auralinity (Method B)

Alkalinity (Method B)

Alralinity (Method B)

Alkalinity (Method B)

Alpha Activity ( $\mathrm{pCi} / \mathrm{L}$ )

Alpha Activity (pCi/L)

Alpha Activity (pCi/L)

Alpha Activity ( $\mathrm{pCi} / \mathrm{L}$ )

Beta Activity (pCi/L)

Beta Activity (pCi/L)

Beta Activity (pCi/L)

Conductivity (us)

Conductivity (uS)

Conductivity (us)

Conductivity (us)

Irgitability (degrees $\mathbf{F}$ )

Ingitability (degrees F)

Ingitability (degrees F)

Ingitability (degrees F)

$\mathrm{pH}$ (dimensionless)

$\mathrm{pH}$ (dimensionless)

$\mathrm{pH}$ (dimensionless)

$\mathrm{pH}$ (dimensionless)

Reactivity Cyanide (mg/ $/ \mathrm{kg}$ )

Reactivity Cyanide ( $\mathrm{mg} / \mathrm{kg}$ )

Reactivity Cyanide ( $\mathrm{mg} / \mathrm{kg}$ )

Reactivity Cyanide ( $\mathrm{mg} / \mathrm{kg}$ )

Reactivity Sulfide (mg/kg)

Reactivity Sulfide ( $\mathrm{mg} / \mathrm{kg}$ )

Reactivity sulfide ( $\mathrm{mg} / \mathrm{kg}$ )

Reactivity sulfide ( $\mathrm{mg} / \mathrm{kg}$ )

$\operatorname{TDS}(\mathrm{mg} / \mathrm{L})$

$\operatorname{TDS}$ (mg/L)

TDS $(\mathrm{mg} / \mathrm{L})$

TDS (mg/I)

Temperature (degrees C)

Temperature (degrees C)

Temperature (degrees C)

Temperature (degrees $C$ )

Total Carbon (ug/g)

Total Carbon (ug/g)

Total Carbon (ug/g)

Total Carbon (ug/g)

$\operatorname{Tox}(\operatorname{ug}(\mathrm{Cl}) / \mathrm{L})$

$\operatorname{TOX}(\operatorname{ug}(C . I) / I)$

TOX (ug $(C I) / I)$

$\operatorname{TOX}(u g(C l) / L)$

Co-60 ( pCi/L)

Co-60 (pCi/L)

Co-60 ( pCi/L)

Co-60 (pCi/L)

\begin{tabular}{|c|c|c|c|}
\hline & D & Method & Result \\
\hline $50838 \mathrm{~T}$ & $12 / 14 / 89$ & VOA & $<5.00 E+00$ \\
\hline 50776 & $11 / 17 / 89$ & $\mathrm{ABN}$ & $4.50 E+01$ \\
\hline 50733 & $10 / 26 / 89$ & TITRA & $5.00 \mathrm{E}+04$ \\
\hline 50776 & $11 / 17 / 89$ & TITRA & $.20 E+04$ \\
\hline 50800 & $11 / 28 / 89$ & TITRA & $5.20 E+04$ \\
\hline 50838 & $12 / 14 / 89$ & TITRA & $4.10 E+04$ \\
\hline 50733 & $10 / 26 / 89$ & Alpha & $<1.20 \mathrm{E}-01$ \\
\hline 50776 & $11 / 17 / 89$ & Alpha & $<3.91 E-01$ \\
\hline 50800 & $11 / 28 / 89$ & A]pha & $1.64 \mathrm{E}+00$ \\
\hline 50838 & $12 / 14 / 89$ & Alpha & $8.98 \mathrm{E}-01$ \\
\hline 50776 & $11 / 17 / 89$ & Beta & $.40 \mathrm{E}+00$ \\
\hline 50800 & $11 / 28 / 89$ & Beta & $2.41 \mathrm{E}+00$ \\
\hline 50838 & $12 / 14 / 89$ & Beta & $5.53 \mathrm{E}+00$ \\
\hline 50733 & $10 / 26 / 89$ & COND-FId & $1.53 \mathrm{E}+02$ \\
\hline 50776 & $11 / 1$ & COND $-F$ & $E+02$ \\
\hline 50800 & $11 / 28 / 89$ & COND-FId & $1.69 \mathrm{E}+02$ \\
\hline 50838 & $12 / 14 / 89$ & COND-Fld & $1.65 \mathrm{E}+02$ \\
\hline $50733 \mathrm{E}$ & $10 / 26 / 89$ & IGNIT & $2.12 \mathrm{E}+02$ \\
\hline $50776 \mathrm{E}$ & $11 / 17 / 89$ & IGNIT & $2.10 E+02$ \\
\hline $50800 \mathrm{E}$ & $11 / 28 / 89$ & IGNIT & $2.12 \mathrm{E}+02$ \\
\hline $50838 \mathrm{E}$ & $12 / 14 / 89$ & IGNIT & $2.12 \mathrm{E}+02$ \\
\hline 50733 & $10 / 26 / 89$ & PH-Fld & $7.60 \mathrm{E}+00$ \\
\hline 50776 & $11 / 17 / 89$ & PH-Fld & $6.47 E+00$ \\
\hline 50800 & $11 / 2$ & PH $-F l d$ & $7.60 \mathrm{E}+00$ \\
\hline 50838 & $12 / "$ & PH $-F l d$ & $7.80 \mathrm{E}+00$ \\
\hline $50733 E$ & 10 & DSPEC & $E+02$ \\
\hline $50776 \mathrm{E}$ & $11 / 1$ & DSPEC & $<1.00 \mathrm{E}+02$ \\
\hline $50800 \mathrm{E}$ & $11 / 28 / 89$ & DSPEC & $<1.00 E+02$ \\
\hline $5.0838 \mathrm{E}$ & $12 / 14 / 89$ & DSPEC & $<1.00 \mathrm{E}+02$ \\
\hline $50733 E$ & $10 / 26 / 89$ & DTITRA & $<1.00 E+02$ \\
\hline $50776 \mathrm{E}$ & $11 / 17 / 89$ & DTITRA & $<1.00 E+02$ \\
\hline $50800 \mathrm{E}$ & $11 / 28 / 89$ & DTITRA & $<1.00 E+02$ \\
\hline $50838 \mathrm{E}$ & $12 / 14 / 89$ & DTITRA & $<1.00 \mathrm{E}+02$ \\
\hline 50733 & $10 / 26 /$ & TDS & $E+03$ \\
\hline & $11 / 17$ & TDS & +04 \\
\hline & $11 / 2$ & TDS & +04 \\
\hline 8 & $1.2 / 1$ & TDS & $0 E+04$ \\
\hline 50733 & $10 / 26 / 89$ & TEMP-F & $2.89 E+01$ \\
\hline 50776 & $11 / 17 / 89$ & TEMP-FId & $1.97 \mathrm{E}+01$ \\
\hline 50800 & $11 / 28 / 89$ & TEMP-FId & $2.76 \mathrm{E}+01$ \\
\hline 50838 & $12 / 14 / 89$ & TEMP-FId & $4.76 \mathrm{E}+01$ \\
\hline 50733 & $10 / 26 / 89$ & TC & $1.35 \mathrm{E}+04$ \\
\hline 50776 & $11 / 17 / 89$ & TC & $1.35 \mathrm{E}+04$ \\
\hline 50800 & $11 / 28 / 89$ & TC & ]. $38 \mathrm{E}+04$ \\
\hline 2838 & $12 / 14 / 89$ & TC & $1.09 E+04$ \\
\hline & $10 / 26 / 89$ & LTOX & $2.04 E+02$ \\
\hline & $11 / 17 / 89$ & LTOX & 1. $82 \mathrm{E}+02$ \\
\hline & $11 / 28 / 89$ & LTOX & $1.54 \mathrm{E}+02$ \\
\hline 50838 & $.22 / 14 / 89$ & LTOX & $2.55 \mathrm{E}+02$ \\
\hline & $10 / 26 / 89$ & GEA & $<2.77 \mathrm{E}-01$ \\
\hline 50776 & $11 / 17 / 89$ & GEA & $2.83 E+00$ \\
\hline & $11 / 28 / 89$ & GEA & $<9.74 \mathrm{E}-01$ \\
\hline 50838 & $12 / 14 / 89$ & GEA & $<4.60 \mathrm{E}-01$ \\
\hline
\end{tabular}

D -113 
WHC-EP-0355, Appendix D

DATA FOR T Plant Laboratory Wastewater-Plasma Forch Standby Continued

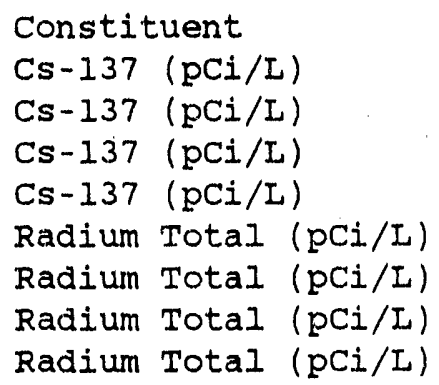

\begin{tabular}{cclr} 
Sample \# & $\begin{array}{c}\text { Date } \\
50733\end{array}$ & $10 / 26 / 89 \mathrm{GEA}$ & \multicolumn{1}{c}{ Result } \\
$53.93 \mathrm{E}-01$ \\
50776 & $11 / 17 / 89 \mathrm{GEA}$ & $<8.32 \mathrm{E}-01$ \\
50800 & $11 / 28 / 89 \mathrm{GEA}$ & $<6.76 \mathrm{E}-01$ \\
50838 & $12 / 14 / 89 \mathrm{GEA}$ & $3.46 \mathrm{E}+00$ \\
50733 & $10 / 26 / 89 \mathrm{Alpha}-\mathrm{Ra}$ & $<6.09 \mathrm{E}-02$ \\
50776 & $11 / 17 / 89$ Alpha-Ra & $3.10 \mathrm{E}-01$ \\
50800 & $11 / 28 / 89$ Alpha-Ra & $<6.59 \mathrm{E}-02$ \\
50838 & $12 / 14 / 89$ Alpha-Ra & $<9.77 \mathrm{E}-02$
\end{tabular}


DATA FOR T Plant Wastewater

\begin{tabular}{|c|c|c|}
\hline Constitue & dent & \\
\hline Arsenic & (EP & Toxic) \\
\hline Arsenic & (EP & Toxic) \\
\hline Arsenic & (EP & Toxic) \\
\hline Arsenic & (EP & Toxic) \\
\hline Barium & & \\
\hline arium & & \\
\hline Barium & & \\
\hline Barium & & \\
\hline Barium & (EP T & Toxic) \\
\hline Barium & (EP T & Toxic) \\
\hline Barium (E & (EP T & Toxic) \\
\hline Barium & (EP T & Toxic) \\
\hline Boron & & \\
\hline Boron & & \\
\hline Boron & & \\
\hline Boron & & \\
\hline Cadmium & & \\
\hline Cadmium & & \\
\hline Cadmium & & \\
\hline Cadmium & & \\
\hline Cadmium & (EP & Toxic) \\
\hline Cadmium & (EP & Toxic) \\
\hline Cadmium & (EP & Toxic) \\
\hline Cadmium & (EP & Toxic) \\
\hline Calcium & & \\
\hline Calcium & & \\
\hline Calcium & & \\
\hline Calcium & & \\
\hline Chloride & & \\
\hline Chloride & & \\
\hline Chloride & & \\
\hline Chloride & & \\
\hline Chromium & m (EP & Poxic) \\
\hline Chromium & m (EP & (Poxic) \\
\hline Chromium & $m(E P$ & P Toxic) \\
\hline Chromium & $m$ (EP & Poxic) \\
\hline Copper & & \\
\hline Copper & & \\
\hline Copper & & \\
\hline Copper & & \\
\hline Fluoride & & \\
\hline Fluoride & & \\
\hline Fluoride & & \\
\hline Fluoride & & \\
\hline Fluoride & & \\
\hline Fluoride & & \\
\hline Fluoride & & \\
\hline Fluoride & & \\
\hline Iron & & \\
\hline Iron & & \\
\hline Iron & & \\
\hline con & & \\
\hline Lead (EP & $P$ Tox & xic) \\
\hline
\end{tabular}

\begin{tabular}{|c|c|c|c|}
\hline Sample \# & Date & Method & Result \\
\hline $50681 \mathrm{E}$ & $10 / 13 / 89$ & ICP & $<5.00 E+02$ \\
\hline $50697 \mathrm{E}$ & $10 / 17 / 89$ & ICP & $<5.00 E+02$ \\
\hline 50808E & $11 / 28 / 89$ & ICP & $<5.00 \mathrm{E}+02$ \\
\hline $51036 \mathrm{E}$ & $3 / 09 / 90$ & ICP & $<5.00 \mathrm{E}+02$ \\
\hline 50681 & $10 / 13 / 89$ & ICP & $3.30 E+01$ \\
\hline 50697 & $10 / 17 / 89$ & ICP & $3.00 \mathrm{E}+01$ \\
\hline 50808 & $11 / 28 / 89$ & ICP & $2.70 E+01$ \\
\hline 51036 & $3 / 09 / 90$ & ICP & $3.00 E+01$ \\
\hline $50681 E$ & $10 / 13 / 89$ & ICP & $<1.00 \mathrm{E}+03$ \\
\hline $50697 \mathrm{E}$ & $10 / 17 / 89$ & ICP & $<1.00 \mathrm{E}+03$ \\
\hline 50808E & $11 / 28 / 89$ & ICP & $<1.00 \mathrm{E}+03$ \\
\hline $51036 \mathrm{E}$ & $3 / 09 / 90$ & ICP & $<1.00 \mathrm{E}+03$ \\
\hline 50681 & $10 / 13 / 89$ & ICP & 2. 20E+O1 \\
\hline 50697 & $10 / 17 / 89$ & ICP & $<1.00 \mathrm{E}+01$ \\
\hline 50808 & $11 / 28 / 89$ & ICP & 1. $30 E+01$ \\
\hline 51.036 & $3 / 09 / 90$ & ICP & $3.50 \mathrm{E}+01$ \\
\hline 50681 & $10 / 13 / 89$ & ICP & $2.00 \mathrm{E}+00$ \\
\hline 50697 & $10 / 17 / 89$ & ICP & $<2.00 \mathrm{E}+00$ \\
\hline 50808 & $11 / 28 / 89$ & ICP & $<2.00 E+00$ \\
\hline 51036 & $3 / 09 / 90$ & ICP & $<2.00 E+00$ \\
\hline $50681 \mathrm{E}$ & $10 / 13 / 89$ & ICP & $<1.00 \mathrm{E}+02$ \\
\hline $50697 \mathrm{E}$ & $10 / 17 / 89$ & ICP & $<1.00 \mathrm{E}+02$ \\
\hline $50808 E$ & $11 / 28 / 89$ & ICP & $<1.00 \mathrm{E}+02$ \\
\hline $51036 \mathrm{E}$ & $3 / 09 / 90$ & ICP & $<1.00 E+02$ \\
\hline 50681 & $10 / 13 / 89$ & ICP & $2.18 E+04$ \\
\hline 50697 & $10 / 17 / 89$ & ICP & $1.84 \mathrm{E}+04$ \\
\hline 50808 & $11 / 28 / 89$ & ICP & $1.50 \mathrm{E}+04$ \\
\hline 51036 & $3 / 09 / 90$ & ICP & 2. $10 E+04$ \\
\hline 50681 & $10 / 13 / 89$ & IC & 1. $30 \mathrm{E}+03$ \\
\hline 50697 & $10 / 17 / 89$ & IC & $1.10 \mathrm{E}+03$ \\
\hline 50808 & $11 / 28 / 89$ & IC & $1.10 \mathrm{E}+03$ \\
\hline 51036 & $3 / 09 / 90$ & IC & $1.20 E+03$ \\
\hline $50681 \mathrm{E}$ & $10 / 13 / 89$ & ICP & $<5.00 \mathrm{E}+02$ \\
\hline $50697 \mathrm{E}$ & $10 / 17 / 89$ & ICP & $<5.00 E+02$ \\
\hline $50808 \mathrm{E}$ & $11 / 28 / 89$ & ICP & $<5.00 E+02$ \\
\hline $51036 \mathrm{E}$ & $3 / 09 / 90$ & ICP & $<5.00 \mathrm{E}+02$ \\
\hline 50681 & $10 / 13 / 89$ & ICP & $<1.00 E+0]$ \\
\hline 50697 & $10 / 17 / 89$ & ICP & $1.80 \mathrm{E}+0$ \\
\hline 50808 & $11 / 28 / 89$ & ICP & $3.20 E+O I$ \\
\hline 510.36 & $3 / 09 / 90$ & ICP & $<1.00 \mathrm{E}+01$ \\
\hline 50681 & $10 / 13 / 89$ & $I C$ & $<5.00 \mathrm{E}+02$ \\
\hline 50681 & $10 / 13 / 89$ & ISE & 1.71E+0 \\
\hline 50697 & $10 / 17 / 89$ & IC & $<5.00 \mathrm{E}+0$ \\
\hline 50697 & $10 / 17 / 89$ & ISE & $1.46 \mathrm{E}+02$ \\
\hline 50808 & $11 / 28 / 89$ & $I C$ & $<5.00 E+0$ \\
\hline 50808 & $11 / 28 / 89$ & ISE & 1. $29 \mathrm{E}+0$ \\
\hline 51036 & $3 / 09 / 90$ & IC & $<5.00 \mathrm{O}+0$ \\
\hline 51036 & $3 / 09 / 90$ & ISE & $1.33 \mathrm{E}+02$ \\
\hline 50681 & $10 / 13 / 89$ & ICP & $9.20 \mathrm{E}+0 \mathrm{I}$ \\
\hline 50697 & $10 / 17 / 89$ & ICP & $3.40 E+O 1$ \\
\hline 50808 & $11 / 28 / 89$ & ICP & 3. $10 \mathrm{E}+0$. \\
\hline 51036 & $3 / 09 / 90$ & ICP & $5.90 \mathrm{E}+0$. \\
\hline 50681E & $10 / 13 / 89$ & $\mathrm{ICP}$ & $<5.00 E+0$ \\
\hline
\end{tabular}

D -115 
DATA FOR T Plant Wastewater

Continued

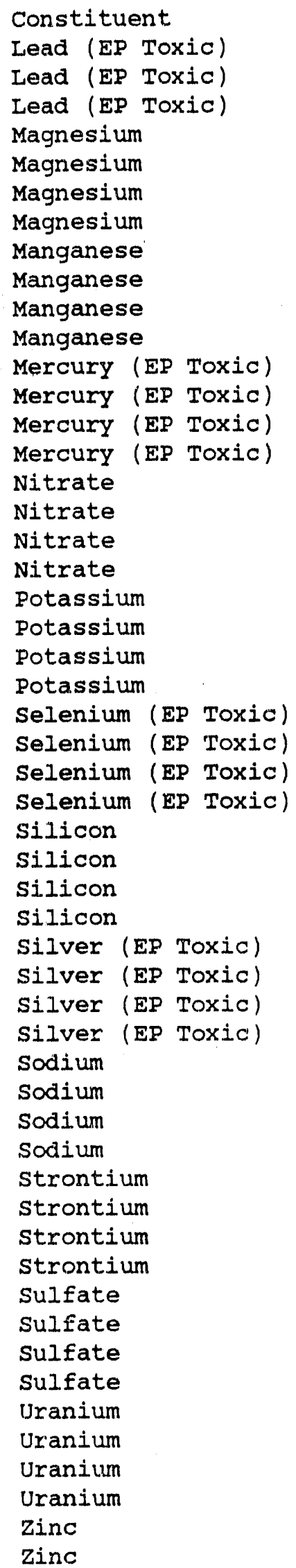

\begin{tabular}{|c|c|c|c|}
\hline Sample \# & Date & Method & Result \\
\hline 50697E & $10 / 17 / 89$ & ICP & $<5.00 E+02$ \\
\hline $50808 \mathrm{E}$ & $11 / 28 / 89$ & ICP & $<5.00 E+02$ \\
\hline $51036 \mathrm{E}$ & $3 / 09 / 90$ & ICP & $<5.00 \mathrm{E}+02$ \\
\hline 50681 & $10 / 13 / 89$ & ICP & $4.09 E+03$ \\
\hline 50697 & $10 / 17 / 89$ & ICP & $3.98 E+03$ \\
\hline 50808 & $11 / 28 / 89$ & ICP & $3.35 \mathrm{E}+03$ \\
\hline 51036 & $3 / 09 / 90$ & ICP & $4.48 E+03$ \\
\hline 50681 & $10 / 13 / 89$ & ICP & 2. $10 \mathrm{E}+01$ \\
\hline 50697 & $10 / 17 / 89$ & ICP & $<5.00 \mathrm{E}+00$ \\
\hline 50808 & $11 / 28 / 89$ & ICP & $<5.00 \mathrm{E}+00$ \\
\hline 51036 & $3 / 09 / 90$ & ICP & $<5.00 \mathrm{E}+00$ \\
\hline $50681 \mathrm{E}$ & $10 / 13 / 89$ & CVAA/M & $<2.00 E+01$ \\
\hline 50697E & $10 / 17 / 89$ & CVAA/M & $<2.00 \mathrm{E}+01$ \\
\hline $50808 \mathrm{E}$ & $11 / 28 / 89$ & CVAA/M & $<2.00 \mathrm{E}+01$ \\
\hline $51036 \mathrm{E}$ & $3 / 09 / 90$ & CVAA/M & $<2.00 \mathrm{E}+01$ \\
\hline 50681 & $10 / 13 / 89$ & IC & $<5.00 \mathrm{E}+02$ \\
\hline 50697 & $10 / 17 / 89$ & IC & $<5.00 \mathrm{E}+02$ \\
\hline 50808 & $11 / 28 / 89$ & IC & $5.00 \mathrm{E}+02$ \\
\hline 51036 & $3 / 09 / 90$ & IC & $<5.00 \mathrm{E}+02$ \\
\hline 50681 & $10 / 13 / 89$ & ICP & $8.89 E+02$ \\
\hline 50697 & $10 / 17 / 89$ & ICP & $6.70 E+02$ \\
\hline 50808 & $11 / 28 / 89$ & ICP & $7.07 \mathrm{E}+02$ \\
\hline 51036 & $3 / 09 / 90$ & ICP & $7.62 \mathrm{E}+02$ \\
\hline 50681E & $10 / 13 / 89$ & ICP & $<5.00 \mathrm{E}+02$ \\
\hline $50697 \mathrm{E}$ & $10 / 17 / 89$ & ICP & $<5.00 E+02$ \\
\hline $50808 E$ & $11 / 28 / 89$ & ICP & $<5.00 \mathrm{E}+02$ \\
\hline $51036 \mathrm{E}$ & $3 / 09 / 90$ & ICP & $<5.00 \mathrm{E}+02$ \\
\hline 50681 & $10 / 13 / 89$ & ICP & $1.98 E+03$ \\
\hline 50697 & $10 / 17 / 89$ & ICP & $1.96 \mathrm{E}+03$ \\
\hline 50808 & $11 / 28 / 89$ & ICP & $2.06 \mathrm{E}+03$ \\
\hline 51036 & $3 / 09 / 90$ & ICP & $2.21 \mathrm{E}+03$ \\
\hline $50681 \mathrm{E}$ & $10 / 13 / 89$ & ICP & $<5.00 E+02$ \\
\hline $50697 \mathrm{E}$ & $10 / 17 / 89$ & ICP & $<5.00 \mathrm{E}+02$ \\
\hline $50808 E$ & $11 / 28 / 89$ & ICP & $<5.00 E+02$ \\
\hline $51036 \mathrm{E}$ & $3 / 09 / 90$ & ICP & $<5.00 E+02$ \\
\hline 50681 & $10 / 13 / 89$ & ICP & $2.27 \mathrm{E}+03$ \\
\hline 50697 & $10 / 17 / 89$ & ICP & $2.04 \mathrm{E}+03$ \\
\hline 50808 & $11 / 28 / 89$ & ICP & $1.73 \mathrm{E}+03$ \\
\hline 51036 & $3 / 09 / 90$ & ICP & $2.09 E+03$ \\
\hline 50681 & $10 / 13 / 89$ & ICP & 1. $12 \mathrm{E}+02$ \\
\hline 50697 & $10 / 17 / 89$ & ICP & $9.50 \mathrm{E}+01$ \\
\hline 50808 & $11 / 28 / 89$ & $I C P$ & $7.70 \mathrm{E}+01$ \\
\hline 51036 & $3 / 09 / 90$ & $\operatorname{ICP}$ & $9.80 \mathrm{E}+01$ \\
\hline 50681 & $10 / 13 / 89$ & $I C$ & $1.05 \mathrm{E}+04$ \\
\hline 50697 & $10 / 17 / 89$ & IC & $1.02 \mathrm{E}+04$ \\
\hline 50808 & $11 / 28 / 89$ & IC & $9.10 \mathrm{E}+03$ \\
\hline 51036 & $3 / 09 / 90$ & IC & $1.08 \mathrm{E}+04$ \\
\hline 50681 & $10 / 13 / 89$ & GEA & $6.88 E-01$ \\
\hline 50697 & $10 / 17 / 89$ & GEA & $2.33 E-01$ \\
\hline 50808 & $11 / 28 / 89$ & GEA & $4.88 \mathrm{E}-01$ \\
\hline 51036 & $3 / 09 / 90$ & GEA & 4.70E-01 \\
\hline 50681 & $10 / 13 / 89$ & ICP & $1.72 \mathrm{E}+02$ \\
\hline 50697 & $10 / 17 / 89$ & ICP & $7.00 E+00$ \\
\hline
\end{tabular}


WHC-EP-0355, Appendix D

DATA FOR T Plant Wastewater

Continued

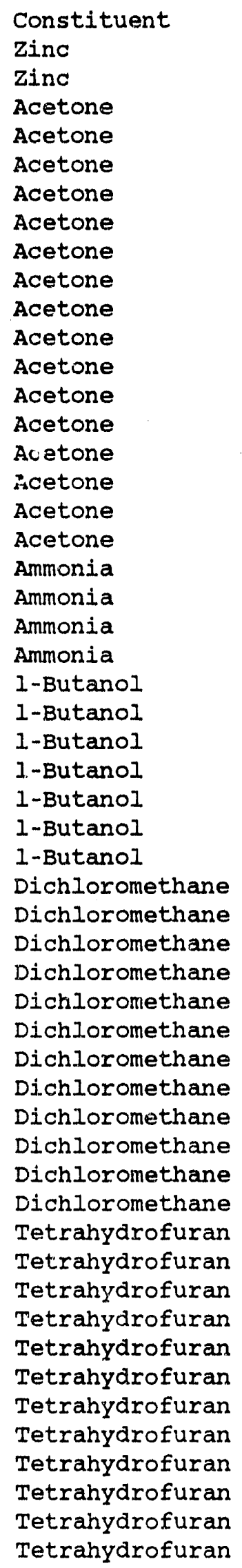

\begin{tabular}{|c|c|c|c|}
\hline iample \# & Date & Method & Result \\
\hline 50808 & $11 / 28 / 89$ & ICP & $<5.00 \mathrm{E}+00$ \\
\hline 51036 & $3 / 09 / 90$ & ICP & $3.30 \mathrm{E}+01$ \\
\hline 50681 & $10 / 13 / 89$ & VOA & $<1.00 \mathrm{E}+01$ \\
\hline 50681 & $10 / 13 / 89$ & $A B N$ & $<1.00 E+01$ \\
\hline $5068 \mathrm{AB}$ & $10 / 13 / 89$ & VOA & $<6.00 \mathrm{E}+00$ \\
\hline $50681 \mathrm{~T}$ & $10 / 13 / 89$ & VOA & $1.70 \mathrm{E}+01$ \\
\hline 50697 & $10 / 17 / 89$ & VOA & $<6.00 \mathrm{E}+00$ \\
\hline 50697 & $10 / 17 / 89$ & $A B N$ & $<1.00 E+01$ \\
\hline $50697 \mathrm{~B}$ & $10 / 17 / 89$ & VOA & $<1.00 \mathrm{E}+01$ \\
\hline $50697 \mathrm{~T}$ & $10 / 17 / 89$ & VOA & $<1.00 E+01$ \\
\hline 50808 & $11 / 28 / 89$ & VOA & $<1.00 \mathrm{E}+01$ \\
\hline 50808 & $11 / 28 / 89$ & $A B N$ & $<1.00 E+01$ \\
\hline $50808 B$ & $11 / 28 / 89$ & VOA & $<1.00 \mathrm{E}+01$ \\
\hline $50808 T$ & $11 / 28 / 89$ & VOA & $<1.00 \mathrm{E}+01$ \\
\hline 51036 & $3 / 09 / 90$ & VOA & $<1.00 E+01$ \\
\hline 51036 & $3 / 09 / 90$ & $A B N$ & $<1.00 E+01$ \\
\hline $51036 \mathrm{~B}$ & $.3 / 09 / 90$ & VOA & $<1.00 \mathrm{E}+01$ \\
\hline $51036 \mathrm{~T}$ & $3 / 09 / 90$ & VOA & $<5.00 E+00$ \\
\hline 50681 & $10 / 13 / 89$ & ISE & $6.20 \mathrm{E}+01$ \\
\hline 50697 & $10 / 17 / 89$ & ISE & $5.40 \mathrm{E}+01$ \\
\hline 50808 & $11 / 28 / 89$ & ISE & $<5.00 E+01$ \\
\hline 51036 & $3 / 09 / 90$ & ISE & $<5.00 E+01$ \\
\hline 50681 & $10 / 13 / 89$ & DIGC & $<1.00 \mathrm{E}+04$ \\
\hline 50697 & $10 / 17 / 89$ & DIGC & $<1.00 \mathrm{E}+04$ \\
\hline 50808 & $11 / 28 / 89$ & DIGC & $<1.00 E+04$ \\
\hline 51036 & $3 / 09 / 90$ & VOA & 1.20E+01 \\
\hline 51036 & $3 / 09 / 90$ & DIGC & $<1.00 \mathrm{E}+04$ \\
\hline $51036 \mathrm{~B}$ & $3 / 09 / 90$ & VOA & $1.00 \mathrm{E}+01$ \\
\hline $51036 \mathrm{~T}$ & $3 / 09 / 90$ & VOA & $1.10 \mathrm{E}+01$ \\
\hline 50681 & $10 / 13 / 89$ & VOA & $<5.00 \mathrm{E}+00$ \\
\hline $50681 \mathrm{~B}$ & $10 / 13 / 89$ & VOA & $4.10 \mathrm{E}+02$ \\
\hline $50681 \mathrm{~T}$ & $10 / 13 / 89$ & VOA & $4.20 \mathrm{E}+02$ \\
\hline 50697 & $10 / 17 / 89$ & VOA & $<5.00 \mathrm{E}+00$ \\
\hline $50697 \mathrm{~B}$ & $10 / 17 / 89$ & VOA & $<3.00 E+00$ \\
\hline $50697 \mathrm{~T}$ & $10 / 17 / 89$ & VOA & $1.20 \mathrm{E}+01$ \\
\hline 50808 & $11 / 28 / 89$ & VOA & $<5.00 E+00$ \\
\hline $50808 B$ & $11 / 28 / 89$ & VOA & $6.00 \mathrm{E}+00$ \\
\hline $50808 \mathrm{~T}$ & $1.1 / 28 / 89$ & VOA & $6.00 E+00$ \\
\hline 51036 & $3 / 09 / 90$ & VOA & $<5.00 \mathrm{E}+00$ \\
\hline $51036 \mathrm{~B}$ & $3 / 09 / 90$ & VOA & $<5.00 \mathrm{E}+00$ \\
\hline $51036 \mathrm{~T}$ & $3 / 09 / 90$ & VOA & $<5.00 E+00$ \\
\hline 50681 & $10 / 13 / 89$ & VOA & $<1.00 \mathrm{E}+01$ \\
\hline $50681 \mathrm{~B}$ & $10 / 13 / 89$ & VOA & $<1.00 \mathrm{E}+01$ \\
\hline $50681 \mathrm{~T}$ & $10 / 13 / 89$ & VOA & $<1.00 \mathrm{E}+01$ \\
\hline 50697 & $10 / 17 / 89$ & VOA & $<1.00 \mathrm{E}+01$ \\
\hline $50697 \mathrm{~B}$ & $10 / 17 / 89$ & VOA & $1.40 \mathrm{E}+01$ \\
\hline $50697 \mathrm{~T}$ & $10 / 17 / 89$ & VOA & $<1.00 \mathrm{E}+01$ \\
\hline 50808 & $11 / 28 / 89$ & VOA & $<1.00 \mathrm{E}+01$ \\
\hline $50808 B$ & $1.1 / 28 / 89$ & VOA & $<8.00 \mathrm{E}+00$ \\
\hline $50808 \mathrm{~T}$ & $11 / 28 / 89$ & VOA & $<6.00 \mathrm{E}+00$ \\
\hline 51036 & $3 / 09 / 90$ & VOA & $<1.00 E+01$ \\
\hline $51036 \mathrm{~B}$ & $3 / 09 / 90$ & VOA & $<1.00 E+01$ \\
\hline $51036 \mathrm{~T}$ & $3 / 09 / 90$ & VOA & $<6.00 \mathrm{E}+00$ \\
\hline
\end{tabular}


DATA FOR T Plant Wastewater

Continued

Constituent

Trichloromethane

Trichloromethane

Trichloromethane

Trichloromethane

Trichloromethane

Trichloromethane

Trichloromethane

Trichloromethane

Trichloromethane

Trichloromethane

Trichloromethane

Trichloromethane

unknown amide

Alkalinity (Method B)

Alkalinity (Method B)

Alkalinity (Method B)

Alkalinity (Method B)

Beta Activity (pCi/L)

Beta Activity (pCi/L)

Beta Activity ( $\mathrm{pCi} / \mathrm{L})$

Beta Activity ( $\mathrm{pCi} / \mathrm{L})$

Conductivity (us)

Conductivity (us)

Conductivity (us)

Conductivity (us)

Ingitability (degrees F)

Ingitability (degrees F)

Ingitability (degrees F)

Ingitability (degrees F)

$\mathrm{pH}$ (dimensionless)

pH (dimensionless)

pH (dimensionless)

pH (dimensionless)

Reactivity Cyanide ( $\mathrm{mg} / \mathrm{kg}$ )

Reactivity Cyanide ( $\mathrm{mg} / \mathrm{kg}$ )

Reactivity Cyanide (mg/ $\mathrm{kg}$ )

Reactivity Cyanide ( $\mathrm{mg} / \mathrm{kg}$ )

Reactivity Sulfide (mg/kg)

Reactivity Sulfide (mg/ $\mathrm{kg}$ )

Reactivity Sulfide (mg/ $\mathrm{hg}$ )

Reactivity sulfide (ms/kg)

TDS $(\mathrm{mg} / \mathrm{L})$

TDS $(\mathrm{mg} / \mathrm{L})$

$\operatorname{TDS}(\mathrm{mg} / \mathrm{L})$

$\operatorname{TDS}(\mathrm{mg} / \mathrm{L})$

Temperature (degrees C)

Temperature (degrees C)

Temperature (degrees C)

Temperature (degrees C)

TOC (ug/g)

TOC (ug/g)

TOC (ug/g)

TOC (ug/g)

\begin{tabular}{|c|c|c|c|}
\hline & Date & Method & Result \\
\hline 5068.1 & $10 / 13 / 89$ & VOA & $<5.00 \mathrm{E}+00$ \\
\hline $50681 \mathrm{~B}$ & $10 / 13 / 89$ & VOA & $<5.00 \mathrm{E}+00$ \\
\hline $50681 \mathrm{~T}$ & $10 / 13 / 89$ & VOA & $<5.00 \mathrm{E}+00$ \\
\hline 50597 & $10 / 17 / 89$ & VOA & $<5.00 E+00$ \\
\hline $50697 \mathrm{~B}$ & $10 / 17 / 89$ & VOA & $<5.00 \mathrm{E}+00$ \\
\hline $50697 \mathrm{~T}$ & $10 / 17 / 89$ & VOA & $<5.00 E+00$ \\
\hline 50808 & $11 / 28 / 89$ & VOA & $<5.00 E+00$ \\
\hline $50808 \mathrm{~B}$ & $11 / 28 / 89$ & VOA & $7.00 \mathrm{E}+00$ \\
\hline $50808 \mathrm{~T}$ & $11 / 28 / 89$ & VOA & $6.00 \mathrm{E}+00$ \\
\hline 51036 & $3 / 09 / 90$ & VOA & $<5.00 E+00$ \\
\hline $51036 \mathrm{~B}$ & $3 / 09 / 90$ & VOA & $<5.00 E+00$ \\
\hline $51036 \mathrm{~T}$ & $3 / 09 / 90$ & VOA & $<5.00 E+00$ \\
\hline 50697 & $10 / 17 / 89$ & $\mathrm{ABN}$ & $2.60 \mathrm{E}+01$ \\
\hline 50681 & $10 / 13 / 89$ & TITRA & $5.80 E+04$ \\
\hline 50697 & $10 / 17 / 89$ & TIT & $0 E+04$ \\
\hline 50808 & $11 / 28 / 89$ & TIT & $E+04$ \\
\hline 51036 & $3 / 09 / 90$ & TITRA & $E+04$ \\
\hline 50681 & $10 / 13 / 89$ & Beta & $<1.93 E+00$ \\
\hline 50697 & $10 / 17 / 89$ & Beta & $<7.96 \mathrm{E}-01$ \\
\hline 50808 & $11 / 28 / 89$ & Beta & $4.01 E+00$ \\
\hline 51036 & $3 / 09 / 90$ & Beta & $3.63 \mathrm{E}+00$ \\
\hline 50681 & $10 / 13 / 89$ & COND-FId & $1.21 E+02$ \\
\hline 50697 & $10 / 17 / 89$ & COND -1 & 1. $22 \mathrm{E}+02$ \\
\hline 50808 & $11 / 28 / 89$ & COND-E & $2.00 \mathrm{E}+02$ \\
\hline 1036 & $3 / 09 / 90$ & COND-FId & $1.47 \mathrm{E}+02$ \\
\hline $50681 \mathrm{E}$ & $10 / 13 / 89$ & IGNIT & $.12 \mathrm{E}+02$ \\
\hline $0697 E$ & $10 / 17 / 89$ & IGN & $E+02$ \\
\hline $50808 \mathrm{E}$ & $11 / 2$ & IGN & $\mathrm{E}+02$ \\
\hline $51036 \mathrm{E}$ & $3 / 0$ & IGN & $14 E+02$ \\
\hline 50681 & $10 / 13 / 89$ & PH-FId & $6.80 \mathrm{E}+00$ \\
\hline 50697 & $10 / 17 / 89$ & PH-FId & $7.20 \mathrm{E}+00$ \\
\hline 50808 & $11 / 28 / 89$ & PH-FId & $7.55 \mathrm{E}+00$ \\
\hline 51036 & $3 / 09 / 90$ & PH-Fld & $7.70 \mathrm{E}+00$ \\
\hline $50681 \mathrm{E}$ & $10 / 13 / 89$ & DSPEC & $<1.00 \mathrm{E}+02$ \\
\hline $50697 \mathrm{E}$ & $10 / 17 / 89$ & DSPEC & $\mathrm{E}+02$ \\
\hline $50808 \mathrm{E}$ & $11 / 28 / 89$ & DSPEC & $<1.00 \mathrm{E}+02$ \\
\hline $51036 \mathrm{E}$ & $3 / 09 / 90$ & DSPEC & $E+02$ \\
\hline 50681E & $10 / 13 / 89$ & DTITRA & $E+02$ \\
\hline $597 \mathrm{E}$ & $10 / 1$ & DTITRA & $\mathrm{E}+02$ \\
\hline OBE & $11 /$ & DTITRA & $\mathrm{OE}+\mathrm{O} 2$ \\
\hline $036 \mathrm{E}$ & $3 / C$ & DTITRA & $<1.00 \mathrm{E}+02$ \\
\hline 681 & $10 / 1$ & IDS & $5.80 E+04$ \\
\hline 50697 & $10 / 17 / 89$ & TDS & $6.10 \mathrm{E}+04$ \\
\hline 50808 & $11 / 28 / 89$ & TDS & $6.30 \mathrm{E}+04$ \\
\hline 51036 & $3 / 09 / 90$ & TDS & $6.00 \mathrm{E}+04$ \\
\hline 50681 & $10 / 13 / 89$ & TEMP-Fld & $1.75 \mathrm{E}+01$ \\
\hline 50697 & $10 / 17 / 89$ & TEMP-FId & $1.81 \mathrm{E}+01$ \\
\hline 50808 & $11 / 28 / 39$ & TEMP-Fld & $1.56 \mathrm{E}+01$ \\
\hline 51036 & $3 / 09 / 90$ & TEMP-FId & $1.40 \mathrm{E}+01$ \\
\hline 50681 & $10 / 13 / 89$ & TOC & $<1.80 \mathrm{E}+03$ \\
\hline 50697 & $10 / 17 / 89$ & TOC & $<1.50 \mathrm{E}+03$ \\
\hline 0808 & $11 / 28 / 89$ & TOC & $<1,20 E+03$ \\
\hline 1036 & $3 / 09 / 90$ & TOC & $1.00 E+0$ \\
\hline
\end{tabular}

D- 118 
WHC-EP-0355, Appendix D

DATA FOR T Plant Wastewater Continued

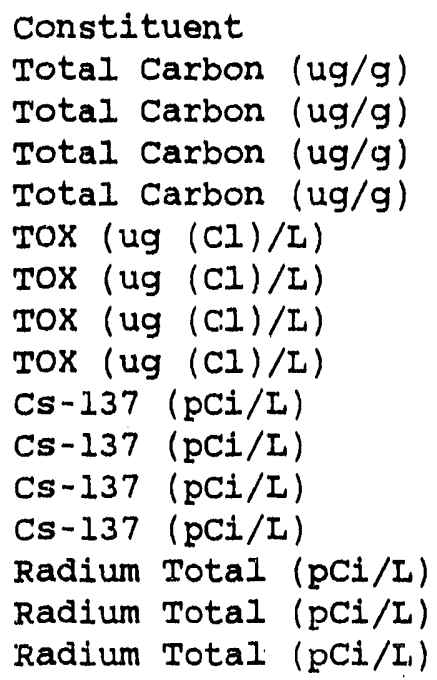

\begin{tabular}{crlr} 
Sample \# & \multicolumn{1}{c}{ Date } & Method & \multicolumn{1}{l}{ Result } \\
50681 & $10 / 13 / 89$ TC & $1.50 \mathrm{E}+04$ \\
50697 & $10 / 17 / 89 \mathrm{TC}$ & $1.60 \mathrm{E}+04$ \\
50808 & $11 / 28 / 89 \mathrm{TC}$ & $1.54 \mathrm{E}+04$ \\
51036 & $3 / 09 / 90 \mathrm{TC}$ & $1.54 \mathrm{E}+04$ \\
50681 & $10 / 13 / 89$ LTOX & $1.40 \mathrm{E}+01$ \\
50697 & $10 / 17 / 89$ LTOX & $<8.00 \mathrm{E}+00$ \\
50808 & $11 / 28 / 89$ LTOX & $2.00 \mathrm{E}+01$ \\
51036 & $3 / 09 / 90$ LTOX & $<9.00 \mathrm{E}+00$ \\
50681 & $10 / 13 / 89 \mathrm{GEA}$ & $<4.34 \mathrm{E}-02$ \\
50697 & $10 / 17 / 89 \mathrm{GEA}$ & $<1.89 \mathrm{E}-01$ \\
50808 & $11 / 28 / 89$ GEA & $<9.76 \mathrm{E}-01$ \\
51036 & $3 / 09 / 90$ GEA & $1.86 \mathrm{E}+00$ \\
50681 & $10 / 13 / 89$ Alpha-Ra & $<1.39 \mathrm{E}-01$ \\
50697 & $10 / 17 / 89$ Alpha-Ra & $1.48 \mathrm{E}-01$ \\
51036 & $3 / 09 / 90$ Alpha-Ra $<3.79 \mathrm{E}-02$
\end{tabular}


WHC-EP-0355, Appendix D

DATA FOR UO3/U Plants Wastewater

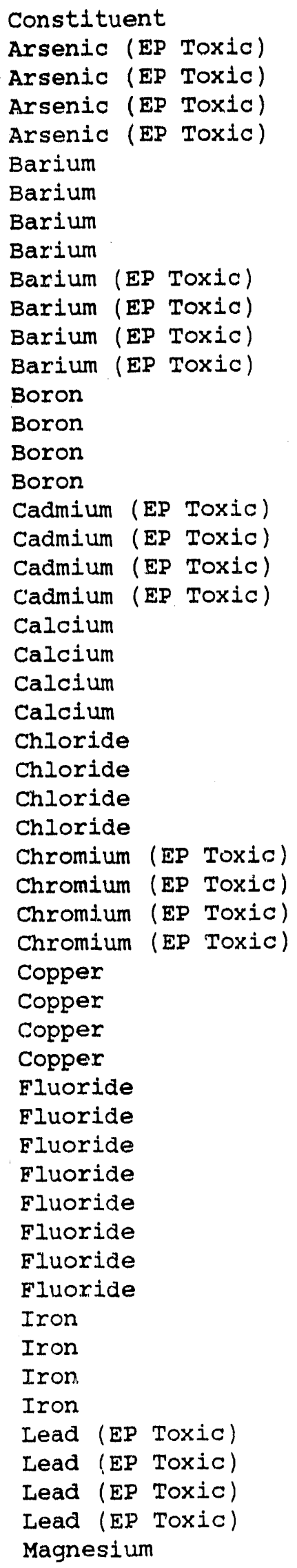

\begin{tabular}{|c|c|c|c|}
\hline Sample \# & Date & Method & Result \\
\hline $50822 \mathrm{E}$ & $12 / 01 / 89$ & ICP & $<5.00 \mathrm{E}+02$ \\
\hline $51000 \mathrm{E}$ & $3 / 02 / 90$ & ICP & $<5.00 \mathrm{E}+02$ \\
\hline $51048 \mathrm{E}$ & $3 / 14 / 90$ & ICP & $<5.00 \mathrm{E}+02$ \\
\hline $51083 E$ & $3 / 22 / 90$ & ICP & $<5.00 \mathrm{E}+02$ \\
\hline 50822 & $12 / 01 / 89$ & ICP & $3.00 E+01$ \\
\hline 51000 & $3 / 02 / 90$ & ICP & $2.80 E+01$ \\
\hline 51048 & $3 / 14 / 90$ & ICP & $3.00 \mathrm{E}+01$ \\
\hline 51083 & $3 / 22 / 90$ & ICP & $2.90 E+01$ \\
\hline $50822 \mathrm{E}$ & $12 / 01 / 89$ & ICP & $<1.00 \mathrm{E}+03$ \\
\hline 51000E & $3 / 02 / 90$ & ICP & $<1.00 \mathrm{E}+03$ \\
\hline $51048 \mathrm{E}$ & $3 / 14 / 90$ & ICP & $<1.00 \mathrm{E}+03$ \\
\hline $51083 \mathrm{E}$ & $3 / 22 / 90$ & ICP & $<1.00 \mathrm{E}+03$ \\
\hline 50822 & $12 / 01 / 89$ & ICP & $1.10 \mathrm{E}+01$ \\
\hline 51000 & $3 / 02 / 90$ & ICP & $3.30 \mathrm{E}+01$ \\
\hline 51048 & $3 / 14 / 90$ & ICP & 2. $40 E+01$ \\
\hline 51083 & $3 / 22 / 90$ & ICP & $1.00 \mathrm{E}+01$ \\
\hline $50822 \mathrm{E}$ & $12 / 01 / 89$ & ICP & $<1.00 E+02$ \\
\hline $51000 \mathrm{E}$ & $3 / 02 / 90$ & ICP & $<1.00 \mathrm{E}+02$ \\
\hline $510 \Delta 8 E$ & $3 / 14 / 90$ & ICP & $<1.00 \mathrm{E}+02$ \\
\hline $51083 \mathrm{E}$ & $3 / 22 / 90$ & ICP & $<1.00 \mathrm{E}+02$ \\
\hline 50822 & $12 / 01 / 89$ & ICP & $1.77 \mathrm{E}+04$ \\
\hline 51000 & $3 / 02 / 90$ & ICP & 1. $78 \mathrm{E}+04$ \\
\hline 51048 & $3 / 14 / 90$ & ICP & $1.82 \mathrm{E}+04$ \\
\hline 51083 & $3 / 22 / 90$ & ICP & $1.79 E+04$ \\
\hline 50822 & $12 / 01 / 89$ & IC & $9.00 \mathrm{E}+02$ \\
\hline 51000 & $3 / 02 / 90$ & IC & $9.20 \mathrm{E}+02$ \\
\hline 51048 & $3 / 1.4 / 90$ & IC & $1.00 \mathrm{E}+03$ \\
\hline 51083 & $3 / 22 / 90$ & IC & $9.00 \mathrm{E}+02$ \\
\hline $50822 E$ & $12 / 01 / 89$ & $I C P$ & $<5,00 \mathrm{E}+02$ \\
\hline 51000E & $3 / 02 / 90$ & ICP & $<5.00 E+02$ \\
\hline $51048 \mathrm{E}$ & $3 / 14 / 90$ & ICP & $<5.00 E+02$ \\
\hline 51083E & $3 / 22 / 90$ & ICP & $<5.00 \mathrm{E}+02$ \\
\hline 50822 & $12 / 01 / 89$ & ICP & $3.10 E+01$ \\
\hline 51000 & $3 / 02 / 90$ & ICP & $2.20 \mathrm{E}+01$ \\
\hline 51048 & $3 / 14 / 90$ & ICP & $<1.00 E+01$ \\
\hline 51083 & $3 / 22 / 90$ & ICP & 1. $20 \mathrm{E}+01$ \\
\hline 50822 & $12 / 01 / 89$ & $I C$ & $<5.00 \mathrm{E}+02$ \\
\hline 50822 & $12 / 01 / 89$ & ISE & 1. $33 \mathrm{E}+02$ \\
\hline 51000 & $3 / 02 / 90$ & $I C$ & $<5.00 \mathrm{E}+02$ \\
\hline 51000 & $3 / 02 / 90$ & ISE & 1. $29 \mathrm{E}+02$ \\
\hline 51048 & $3 / 14 / 90$ & $I C$ & $<5.00 E+02$ \\
\hline 51048 & $3 / 14 / 90$ & ISE & 1. $16 \mathrm{E}+02$ \\
\hline 51083 & $3 / 22 / 90$ & IC & $<5.00 \mathrm{E}+02$ \\
\hline 51083 & $3 / 22 / 90$ & ISE & 1. $38 E+02$ \\
\hline 50822 & $12 / 01 / 89$ & ICP & $<3.00 E+01$ \\
\hline 51000 & $3 / 02 / 90$ & ICP & $3.50 \mathrm{E}+01$ \\
\hline 51048 & $3 / 14 / 90$ & $I C P$ & $<3.00 \mathrm{E}+01$ \\
\hline 51083 & $3 / 22 / 90$ & ICP & $<3.00 \mathrm{E}+01$ \\
\hline $50822 \mathrm{E}$ & $12 / 01 / 89$ & ICP & $<5.00 E+02$ \\
\hline 51000E & $3 / 02 / 90$ & ICP & $<5.00 \mathrm{E}+02$ \\
\hline $51048 \mathrm{E}$ & $3 / 14 / 90$ & ICP & $<5.00 E+02$ \\
\hline $51083 \mathrm{E}$ & $3 / 22 / 90$ & $I C P$ & $<5.00 \mathrm{E}+02$ \\
\hline 50822 & $12 / 01 / 89$ & ICP & $3.98 \mathrm{E}+03$ \\
\hline
\end{tabular}


DATA FOR U03/U Plants Wastewater Continued

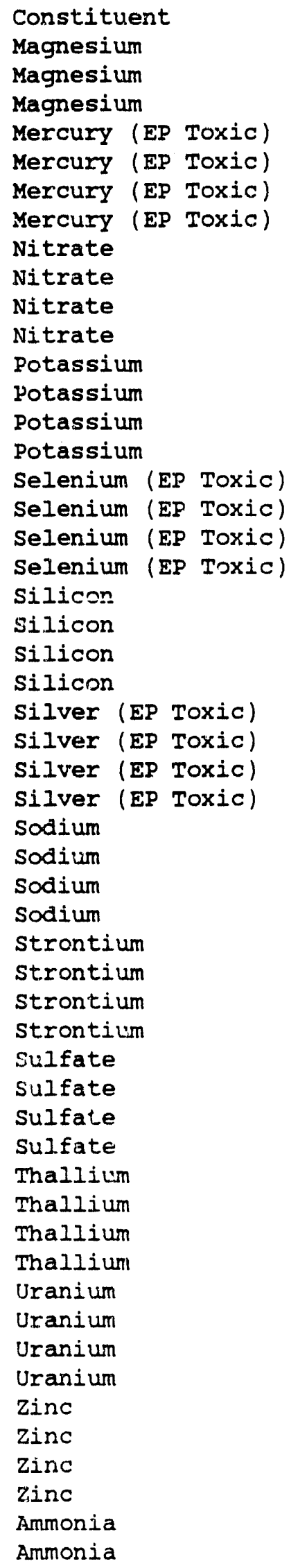

\begin{tabular}{|c|c|c|c|}
\hline Sample \# & Date & Method & Result \\
\hline $5: 000$ & $3 / 02 / 90$ & ICP & $4.17 \mathrm{E}+03$ \\
\hline 51048 & $3 / 14 / 90$ & ICP & $4.54 \mathrm{E}+03$ \\
\hline 51083 & $3 / 22 / 90$ & ICP & $4.55 E+03$ \\
\hline $50822 \mathrm{E}$ & $12 / 01 / 89$ & $C V A A / M$ & $<2.00 \mathrm{E}+01$ \\
\hline 51000E & $3 / 02 / 90$ & CVAA/M & $<2.00 \mathrm{E}+01$ \\
\hline $51048 E$ & $3 / 14 / 90$ & CVAA/M & $<2.00 E+0 I$ \\
\hline $51083 \mathrm{E}$ & $3 / 22 / 90$ & $C V A A / M$ & $<2.00 E+01$ \\
\hline 50822 & $12 / 01 / 89$ & IC & $5.00 \mathrm{E}+02$ \\
\hline 51000 & $3 / 02 / 90$ & IC & $5.97 \mathrm{E}+02$ \\
\hline 51048 & $3 / 14 / 90$ & IC & $<5.00 E+02$ \\
\hline 51083 & $3 / 22 / 90$ & $I C$ & $<5.00 E+02$ \\
\hline 50822 & $12 / 01 / 89$ & ICP & $6.86 \mathrm{E}+02$ \\
\hline 51000 & $3 / 02 / 90$ & ICP & $6.85 E+02$ \\
\hline 51048 & $3 / 14 / 90$ & ICP & $7.52 \mathrm{E}+02$ \\
\hline 51083 & $3 / 22 / 90$ & ICP & $7.40 E+02$ \\
\hline $50822 \mathrm{E}$ & $12 / 01 / 89$ & ICP & $<5.00 \mathrm{E}+02$ \\
\hline 51000E & $3 / 02 / 90$ & ICP & $<5.00 E+02$ \\
\hline $51048 E$ & $3 / 14 / 90$ & ICP & $<5.00 E+02$ \\
\hline $51083 \mathrm{E}$ & $3 / 22 / 90$ & $I C P$ & $<5.00 \mathrm{E}+02$ \\
\hline 50822 & $12 / 01 / 89$ & ICP & $2.22 \mathrm{E}+03$ \\
\hline 51000 & $3 / 02 / 90$ & ICP & $2.17 E+03$ \\
\hline 51048 & $3 / 14 / 90$ & ICP & $2.19 \mathrm{E}+03$ \\
\hline 51083 & $3 / 22 / 90$ & ICP & $2.03 E+03$ \\
\hline $50822 \mathrm{E}$ & $12 / 01 / 89$ & ICP & $<5.00 \mathrm{E}+02$ \\
\hline 51000E & $3 / 02 / 90$ & ICP & $<5.00 E+02$ \\
\hline $51048 \mathrm{E}$ & $3 / 14 / 90$ & $I C P$ & $<5.00 \mathrm{E}+02$ \\
\hline $51083 E$ & $3 / 22 / 90$ & ICP & $<5.00 E+02$ \\
\hline 50822 & $12 / 01 / 89$ & ICP & $1.89 \mathrm{E}+03$ \\
\hline 51000 & $3 / 02 / 90$ & $I C P$ & $1.88 E+03$ \\
\hline 51048 & $3 / 14 / 90$ & ICP & $2.11 E+03$ \\
\hline 51083 & $3 / 22 / 90$ & ICP & $2.03 E+03$ \\
\hline 50822 & $12 / 01 / 89$ & ICP & $8.80 E+01$ \\
\hline 51000 & $3 / 02 / 90$ & ICP & $9.50 E+01$ \\
\hline 51048 & $3 / 14 / 90$ & ICP & $9.80 E+O I$ \\
\hline 51083 & $3 / 22 / 90$ & ICP & $9.60 E+01$ \\
\hline 50822 & $12 / 01 / 89$ & IC & $8.90 E+03$ \\
\hline 51000 & $3 / 02 / 90$ & IC & $1.03 E+04$ \\
\hline 51048 & $3 / 14 / 90$ & IC & $1.06 \mathrm{E}+04$ \\
\hline 51083 & $3 / 22 / 90$ & IC & $1.02 \mathrm{E}+04$ \\
\hline 50822 & $12 / 01 / 89$ & GFAA & $7.00 \mathrm{E}+00$ \\
\hline 51000 & $3 / 02 / 90$ & GFAA & $<5.00 \mathrm{E}+00$ \\
\hline 51048 & $3 / 14 / 90$ & GFAA & $<5.00 E+00$ \\
\hline 51083 & $3 / 22 / 90$ & GFAA & $<5.00 E+00$ \\
\hline 50822 & $12 / 01 / 89$ & GEA & $3.27 \mathrm{E}+00$ \\
\hline 51000 & $3 / 02 / 90$ & GEA & $5.75 E-01$ \\
\hline 51048 & $3 / 14 / 90$ & GEA & $7.36 E-01$ \\
\hline 51083 & $3 / 22 / 90$ & GEA & $6.88 \mathrm{E}-01$ \\
\hline 50822 & $12 / 01 / 89$ & ICP & $<5.00 \mathrm{E}+00$ \\
\hline 51000 & $3 / 02 / 90$ & ICP & $6.00 E+00$ \\
\hline 51048 & $3 / 14 / 90$ & ICP & $5.00 \mathrm{E}+00$ \\
\hline 51083 & $3 / 22 / 90$ & ICP & $<5.00 E+00$ \\
\hline 50822 & $12 / 01 / 89$ & ISE & $6.30 \mathrm{E}+01$ \\
\hline 51000 & $3 / 02 / 90$ & ISE & $<5.00 E+01$ \\
\hline
\end{tabular}


DATA FOR UD3/U Plants Wastewater

Continued

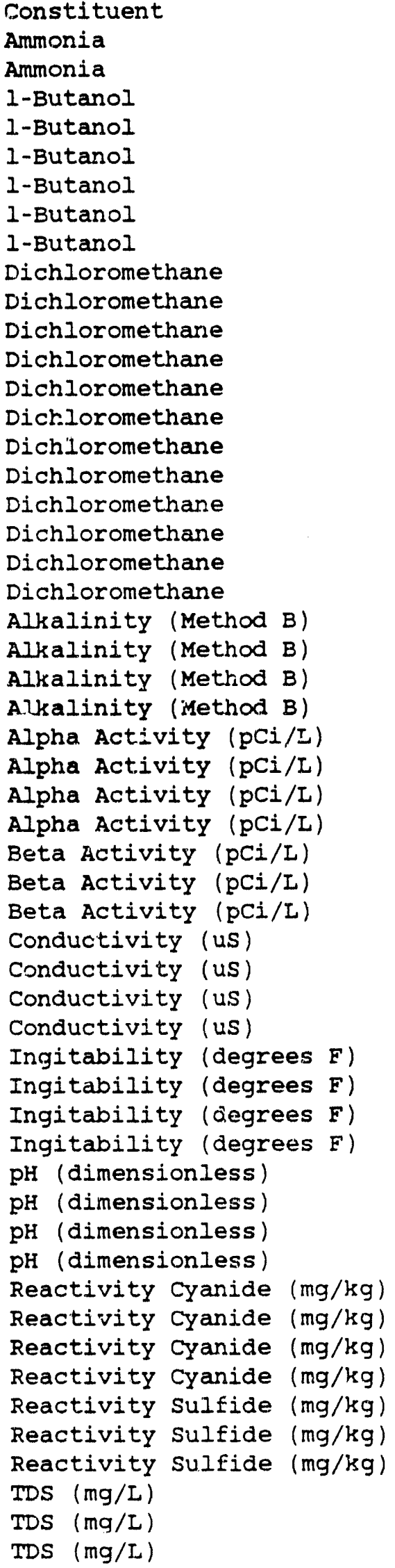

\begin{tabular}{|c|c|c|c|}
\hline Sample \# & Date & Method & Result \\
\hline 51048 & $3 / 14 / 90$ & ISE & $<5.00 \mathrm{E}+01$ \\
\hline 51083 & $3 / 22 / 90$ & ISE & $<5.00 E+01$ \\
\hline 50822 & $12 / 01 / 89$ & DIGC & $<1.00 \mathrm{E}+04$ \\
\hline 51000 & $3 / 02 / 90$ & DIGC & $<1.00 E+04$ \\
\hline $51000 B$ & $3 / 02 / 90$ & VOA & $4.00 E+01$ \\
\hline $51000 \mathrm{~T}$ & $3 / 02 / 90$ & VOA & $3.90 \mathrm{E}+01$ \\
\hline 51048 & $3 / 14 / 90$ & DIGC & $<1.00 E+04$ \\
\hline 51083 & $3 / 22 / 90$ & DIGC & $<1.00 E+04$ \\
\hline 50822 & $12 / 01 / 89$ & VOA & $<5.00 E+00$ \\
\hline $50822 B$ & $12 / 01 / 89$ & VOA & $1.55 \mathrm{E}+03$ \\
\hline $50822 \mathrm{~T}$ & $12 / 01 / 89$ & VOA & 1. $70 \mathrm{E}+03$ \\
\hline 51000 & $3 / 02 / 90$ & VOA & $<5.00 \mathrm{E}+00$ \\
\hline $51000 \mathrm{~B}$ & $3 / 02 / 90$ & VOA & $<5.00 \mathrm{E}+00$ \\
\hline 51000T & $3 / 02 / 90$ & VOA & $<5.00 \mathrm{E}+00$ \\
\hline 51048 & $3 / 14 / 90$ & VOA & $<5.00 E+00$ \\
\hline $51048 B$ & $3 / 14 / 90$ & VOA & $<3.00 E+00$ \\
\hline $51048 \mathrm{~T}$ & $3 / 14 / 90$ & VOA & $<5.00 E+00$ \\
\hline 51083 & $3 / 22 / 90$ & VOA & $<5.00 \mathrm{E}+00$ \\
\hline $51083 B$ & $3 / 22 / 90$ & VOA & $<5.00 E+00$ \\
\hline $51083 \mathrm{~T}$ & $3 / 22 / 90$ & VOA & $<5.00 \mathrm{E}+00$ \\
\hline 50822 & $12 / 01 / 89$ & TITRA & $5.40 E+04$ \\
\hline 51000 & $3 / 02 / 90$ & TIIRA & $5.80 E+04$ \\
\hline 51048 & $3 / 14 / 90$ & TITRA & $5.90 \mathrm{E}+04$ \\
\hline 51083 & $3 / 22 / 90$ & TITRA & $5.80 \mathrm{E}+04$ \\
\hline 50822 & $12 / 01 / 89$ & Alpha & $5.09 E+00$ \\
\hline 51000 & $3 / 02 / 90$ & Alpha & $9.48 \mathrm{E}-01$ \\
\hline 51048 & $3 / 14 / 90$ & Alpha & $<8.65 \mathrm{E}-01$ \\
\hline 51083 & $3 / 22 / 90$ & Alpha & $<3.76 \mathrm{E}-01$ \\
\hline 50822 & $12 / 01 / 89$ & Beta & 2. $37 \mathrm{E}+00$ \\
\hline 51048 & $3 / 14 / 90$ & Beta & $2.31 \mathrm{E}+00$ \\
\hline 51083 & $3 / 22 / 90$ & Beta & $<1.92 \mathrm{E}+00$ \\
\hline 50822 & $12 / 01 / 89$ & COND-FId & 1. $38 E+02$ \\
\hline 51000 & $3 / 02 / 90$ & COND-FId & 1. $23 \mathrm{E}+02$ \\
\hline 51048 & $3 / 14 / 90$ & COND-Fld & 1. $35 \mathrm{E}+02$ \\
\hline 51083 & $3 / 22 / 90$ & COND-FId & 1. $27 \mathrm{E}+02$ \\
\hline $50822 \mathrm{E}$ & $12 / 01 / 89$ & IGNIT & 2. $10 \mathrm{E}+02$ \\
\hline 51000E & $3 / 02 / 90$ & IGNIT & 2. $10 \mathrm{E}+02$ \\
\hline $51048 \mathrm{E}$ & $3 / 14 / 90$ & IGNIT & $2.02 E+02$ \\
\hline $51083 E$ & $3 / 22 / 90$ & IGNIT & $1.96 \mathrm{E}+02$ \\
\hline 5082.2 & $12 / 01 / 89$ & PH-FId & $6.25 E+00$ \\
\hline 51000 & $3 / 02 / 90$ & $\mathrm{PH}-\mathrm{F} I \mathrm{~d}$ & $7.40 \mathrm{E}+00$ \\
\hline 510.48 & $3 / 14 / 90$ & PH-FId & 7. $22 \mathrm{E}+00$ \\
\hline 51083 & $3 / 22 / 90$ & $\mathrm{PH}-\mathrm{F} I \mathrm{~d}$ & $7.23 E+00$ \\
\hline $50822 E$ & $12 / 01 / 89$ & DSPEC & $<1.00 \mathrm{E}+02$ \\
\hline $51000 \mathrm{E}$ & $3 / 02 / 90$ & DSPEC & $<1.00 \mathrm{E}+02$ \\
\hline $51048 \mathrm{E}$ & $3 / 14 / 90$ & DSPEC & $<1.00 E+02$ \\
\hline $51083 \mathrm{E}$ & $3 / 22 / 90$ & DSPEC & $<1.00 \mathrm{E}+02$ \\
\hline $50822 \mathrm{E}$ & $12 / 01 / 89$ & DTITRA & $<1.00 \mathrm{E}+02$ \\
\hline 51000E & $3 / 02 / 90$ & DTITRA & $<1.00 \mathrm{E}+02$ \\
\hline $51048 E$ & $3 / 14 / 90$ & DTITRA & $<1.00 E+02$ \\
\hline 50822 & $12 / 01 / 89$ & TDS & $6.60 \mathrm{E}+04$ \\
\hline 51000 & $3 / 02 / 90$ & TDS & $6.40 E+04$ \\
\hline 51048 & $3 / 14 / 90$ & TDS & $6.90 E+04$ \\
\hline
\end{tabular}


DATA FOR U03/U Plants Wastewater Continued

\begin{tabular}{|c|c|c|c|c|}
\hline & $\begin{array}{c}\text { Sample \# } \\
51083\end{array}$ & $\begin{array}{l}\text { Date } \\
3 / 22 / 90\end{array}$ & $\begin{array}{l}\text { Method } \\
\text { TDS }\end{array}$ & $\begin{array}{l}\text { Result } \\
7.90 \mathrm{E}+04\end{array}$ \\
\hline $\begin{array}{l}\text { TDS (mg/L) } \\
\text { Temperature, legrees } C\end{array}$ & 50822 & $12 / 01 / 89$ & TEMP-FId & $1.61 \mathrm{E}+01$ \\
\hline Temperature (degrees & 51000 & $3 / 02 / 90$ & $T E M P-F I d$ & $9.70 \mathrm{E}+00$ \\
\hline Temperature (degrees $\mathrm{C}$ & 51048 & $3 / 14 / 90$ & TEMP-FId & $1.02 \mathrm{E}+01$ \\
\hline Temperature (degrees & 51083 & $3 / 22 / 90$ & TEMP-FId & $1.27 \mathrm{E}+01$ \\
\hline $\operatorname{TOC}(\mathrm{ug} / \mathrm{g})$ & 50822 & $12 / 01 / 89$ & TOC & $<1.20 E+03$ \\
\hline TOC $(u g / g)$ & 51000 & $3 / 02 / 90$ & TOC & $<9.00 E+02$ \\
\hline $\operatorname{TOC}(u g / g)$ & 51048 & $3 / 14 / 90$ & TOC & $1.10 \mathrm{E}+03$ \\
\hline $\operatorname{TOC}(u g / g)$ & 51083 & $3 / 22 / 90$ & TOC & $1.00 E+03$ \\
\hline Total Carbon (ug/g) & 50822 & $12 / 01 / 89$ & TC & $1.45 \mathrm{E}+04$ \\
\hline Total Carbon (ug/g) & 51000 & $3 / 02 / 90$ & TC & $1.51 E+04$ \\
\hline Total Carbon (ug/g) & 51048 & $3 / 14 / 90$ & TC & $1.55 \mathrm{E}+04$ \\
\hline Total Carbon (ug/g) & 51083 & $3 / 22 / 90$ & TC & $1.55 \mathrm{E}+04$ \\
\hline $\operatorname{rox}($ ug $(C 1) / L)$ & 50822 & $12 / 01 / 89$ & ITOX & 1. $10 E+01$ \\
\hline $\operatorname{Tox}(\operatorname{ug}(C I) / L)$ & 51000 & $3 / 02 / 90$ & ITOX & 1. $20 \mathrm{E}+01$ \\
\hline $\operatorname{Tox}($ ug $(C I) / L)$ & 51048 & $3 / 14 / 90$ & LTOX & 1. $10 E+01$ \\
\hline $\operatorname{Tox}(u g(C l) / L)$ & 51083 & $3 / 22 / 90$ & ITOX & $1.50 E+01$ \\
\hline $\mathrm{Co}-60(\mathrm{pCi} / \mathrm{L})$ & 50822 & $12 / 01 / 89$ & GEA & $<1.18 \mathrm{E}-01$ \\
\hline Co-60 (pCi/L) & 51000 & $3 / 02 / 90$ & GEA & $<5.33 E-02$ \\
\hline $\mathrm{Co}-60(\mathrm{pCi} / \mathrm{L})$ & 51048 & $3 / 14 / 90$ & GEA & $1.23 \mathrm{E}+00$ \\
\hline $\mathrm{Co}-60(\mathrm{pCi} / L)$ & 51083 & $3 / 22 / 90$ & GEA & $1.10 \mathrm{E}+00$ \\
\hline $\mathrm{PL}-239 / 240 \quad(\mathrm{pCi} / \mathrm{L})$ & 51000 & $3 / 02 / 90$ & AEA & $<1.75 E-03$ \\
\hline $\mathrm{Pu}-239 / 240(\mathrm{pCi} / \mathrm{L})$ & 51048 & $3 / 14 / 90$ & AEA & $<2.65 E-03$ \\
\hline $\mathrm{Pu}-239 / 240(\mathrm{pCi} / \mathrm{L})$ & 51083 & $3 / 22 / 90$ & AEA & $6.24 \mathrm{E}-03$ \\
\hline $\mathrm{U}-234(\mathrm{pCi} / \mathrm{L})$ & 50822 & $12 / 01 / 89$ & AEA & $1.59 \mathrm{E}+00$ \\
\hline$U-234(\mathrm{pCi} / L)$ & 51000 & $3 / 02 / 90$ & AEA & $3.43 E-01$ \\
\hline$U-231(\mathrm{pCi} / \mathrm{L})$ & 51048 & $3 / 14 / 90$ & $A E A$ & $3.41 E-01$ \\
\hline U-234 (pCi/L) & 51083 & $3 / 22 / 90$ & AEA & $3.36 \mathrm{E}-01$ \\
\hline$U-235(\mathrm{PC} i / L)$ & 50822 & $12 / 01 / 89$ & AEA & $1.90 \mathrm{E}-01$ \\
\hline $\mathrm{U}-235(\mathrm{pCi} / \mathrm{L})$ & 51000 & $3 / 02 / 90$ & AEA & $2.41 E-02$ \\
\hline $\mathrm{U}-235(\mathrm{pCi} / \mathrm{L})$ & 51048 & $3 / 14 / 90$ & $A E A$ & $<5.56 \mathrm{E}-03$ \\
\hline$U-235(\mathrm{pCi} / \mathrm{L})$ & 51083 & $3 / 22 / 90$ & AEA & $<5.75 E-03$ \\
\hline$U-238(p C i / L)$ & 50822 & $12 / 01 / 89$ & $A E A$ & $1.17 \varepsilon+00$ \\
\hline $\mathrm{U}-238 \quad(\mathrm{PCi} / \mathrm{L})$ & 51000 & $3 / 02 / 90$ & AEA & $3.16 \mathrm{E}-01$ \\
\hline$U-238 \quad(\mathrm{pCi} / \mathrm{L})$ & 51048 & $3 / 14 / 90$ & AEA & $2.82 \mathrm{E}-\mathrm{Cl}$ \\
\hline $\mathrm{U}-238(\mathrm{pCi} / \mathrm{L})$ & 52083 & $3 / 22 / 90$ & AEA & $2.66 \mathrm{E}-01$ \\
\hline
\end{tabular}


Constituent

Arsenic (EP Toxic)

Arsenic (EP TOxic)

Arsenic (EP Toxic)

Arsenic (EP 'Toxic)

Barium

Barium

Barium

Barium

Barium (EP Toxis)

Barium (EP Toxic)

Barium (EP Toxic)

Barium (EP Toxic)

Boron

Boron

Boron

Boron

Cadmium (EP TOxic)

Cadmium (EP TOxic)

Cadmium (EP TOXic)

Cadmium (EP TOxic)

Calcium

Calcium

Calcium

Calcium

Chloride

ChIoride

Chloride

Chloride

Chromium (EP Toxic)

Chromium (EP Toxic)

Chromium (EP Toxic)

Chromium (EP Toxic)

Copper

Copper

Copper

Copper

Fluoride

Eluoride

Fluoride

Fluoride

Fluoride

Fluoride

Fluoride

Fluoride

Iron

Iron

Iron

Iron

Lead (EP Toxic)

Lead (EP Toxic)

Lead (EP Toxic)

Lead (EP TOXic)

Maqnesium

\begin{tabular}{|c|c|c|c|}
\hline Sample \# & Date & Method & Result \\
\hline $50685 \mathrm{E}$ & $10 / 13 / 89$ & ICP & $<5.00 \mathrm{E}+02$ \\
\hline $50693 E$ & $10 / 17 / 89$ & ICP & $<5.00 E+02$ \\
\hline $50717 \mathbf{E}$ & $10 / 24 / 89$ & ICP & $<5.00 E+02$ \\
\hline $50947 \mathrm{E}$ & $2 / 16 / 90$ & ICP & $<5.00 \mathrm{E}+02$ \\
\hline 50685 & $10 / 13 / 89$ & ICP & $3.60 \mathrm{E}+01$ \\
\hline 50693 & $10 / 17 / 89$ & ICP & 3. $20 \mathrm{E}+01$ \\
\hline 50717 & $10 / 24 / 89$ & ICP & $3.00 E+01$ \\
\hline 50947 & $2 / 16 / 90$ & ICP & $2.90 E+01$ \\
\hline $50685 E$ & $10 / 13 / 89$ & ICP & $<1.00 \mathrm{E}+03$ \\
\hline $50693 E$ & $10 / 17 / 89$ & ICP & $<1.00 E+03$ \\
\hline $50717 \mathrm{E}$ & $10 / 24 / 89$ & ICP & $<1.00 E+03$ \\
\hline $50947 \mathrm{E}$ & $2 / 16 / 90$ & ICP & $<1.00 E+03$ \\
\hline 50685 & $10 / 13 / 89$ & ICP & $1.50 \mathrm{E}+01$ \\
\hline 50693 & $10 / 17 / 89$ & ICP & $<1.00 E+01$ \\
\hline 50717 & $10 / 24 / 89$ & ICP & $1.40 \mathrm{E}+01$ \\
\hline 50947 & $2 / 16 / 90$ & ICP & $2.20 E+01$ \\
\hline $50685 \mathrm{E}$ & $10 / 13 / 89$ & ICP & $<1.00 \mathrm{E}+02$ \\
\hline $50693 \mathrm{E}$ & $10 / 17 / 89$ & ICP & $<1.00 E+02$ \\
\hline $50717 \mathrm{E}$ & $10 / 24 / 89$ & ICP & $<1.00 \mathrm{E}+02$ \\
\hline $50947 \mathrm{E}$ & $2 / 16 / 90$ & ICP & $<1.00 \mathrm{E}+02$ \\
\hline 50685 & $10 / 13 / 89$ & ICP & $1.84 \mathrm{E}+04$ \\
\hline 50693 & $10 / 17 / 89$ & ICP & $1.92 \mathrm{E}+04$ \\
\hline 50717 & $10 / 24 / 89$ & ICP & $1.88 E+04$ \\
\hline 50947 & $2 / 16 / 90$ & ICP & $1.79 \mathrm{E}+04$ \\
\hline 50685 & $10 / 13 / 89$ & IC & $1.00 \mathrm{E}+03$ \\
\hline 50693 & $10 / 17 / 89$ & IC & $1.00 E+03$ \\
\hline 50717 & $10 / 24 / 89$ & IC & 1. $20 E+03$ \\
\hline 50947 & $2 / 16 / 90$ & IC & $9.00 \mathrm{E}+02$ \\
\hline 50685E & $10 / 13 / 89$ & ICP & $<5.00 \mathrm{E}+02$ \\
\hline $50693 \mathrm{E}$ & $10 / 17 / 89$ & ICP & $<5.00 E+02$ \\
\hline $50717 E$ & $10 / 24 / 89$ & ICP & $<5.00 E+02$ \\
\hline $50947 E$ & $2 / 16 / 90$ & ICP & $<5.00 E+02$ \\
\hline 50685 & $10 / 13 / 89$ & ICP & $<1.00 E+01$ \\
\hline 50693 & $10 / 17 / 89$ & ICP & $<1.00 E+01$ \\
\hline 50717 & $10 / 24 / 89$ & ICP & $<1.00 E+01$ \\
\hline 50947 & $2 / 16 / 90$ & ICP & $3.01 \mathrm{E}+02$ \\
\hline 50685 & $10 / 13 / 89$ & IC & $<5.00 \mathrm{E}+02$ \\
\hline 50685 & $10 / 13 / 89$ & ISE & $1.75 \mathrm{E}+02$ \\
\hline 50693 & $10 / 17 / 89$ & $I C$ & $<5.00 E+02$ \\
\hline 50693 & $10 / 17 / 89$ & ISE & $1.51 E+02$ \\
\hline 50717 & $10 / 24 / 89$ & IC & $<5.00 E+02$ \\
\hline 50717 & $10 / 24 / 89$ & ISE & $1.63 \mathrm{E}+02$ \\
\hline 50947 & $2 / 16 / 90$ & $I C$ & $<5.00 E+02$ \\
\hline 50947 & $2 / 16 / 90$ & ISE & 1. $28 \mathrm{E}+02$ \\
\hline 50685 & $10 / 13 / 89$ & ICP & 1. $28 \mathrm{E}+02$ \\
\hline 50693 & $10 / 17 / 89$ & ICP & 4. $20 E+01$ \\
\hline 50717 & $10 / 24 / 89$ & ICP & 3. $20 E+01$ \\
\hline 50947 & $2 / 16 / 90$ & ICP & $6.58 E+02$ \\
\hline $50685 \mathrm{E}$ & $10 / 13 / 89$ & ICP & $<5.00 \mathrm{E}+02$ \\
\hline 50693E & $10 / 17 / 89$ & ICP & $<5.00 \mathrm{E}+02$ \\
\hline $50717 \mathrm{E}$ & $10 / 24 / 89$ & ICP & $<5,00 \mathrm{E}+02$ \\
\hline $50947 \mathrm{E}$ & $2 / 16 / 90$ & ICP & $<5,00 E+02$ \\
\hline 50685 & $10 / 13 / 89$ & ICP & $4.17 \mathrm{E}+03$ \\
\hline
\end{tabular}

$<5.00 \mathrm{E}+02$

$<5.00 \mathrm{E}+02$

< $5.00 \mathrm{E}+02$

3. $60 \mathrm{E}+01$

3. $20 \mathrm{E}+01$

3. $00 \mathrm{E}+01$

$<1.00 E+03$

$<1.00 \mathrm{E}+03$

$<1.00 \mathrm{E}+03$

$<1.00 E+03$

1. 00101

1. $40 \mathrm{E}+01$

2. $20 \mathrm{E}+01$

$<1.00 \mathrm{E}+02$

$<1.00 E+02$

$1.00 \mathrm{E}+02$

1. $84 \mathrm{E}+04$

1. $92 \mathrm{E}+04$

1. $88 \mathrm{E}+04$

1. $79 \mathrm{E}+04$

$1.00 \mathrm{E}+03$

1. $20 \mathrm{E}+03$

9. $00 \mathrm{E}+02$

$<5.00 \mathrm{E}+02$

$<5.0 O E+02$

$<5.00 E+02$

$5.00 E+02$

$1.00 \mathrm{E}+01$

$<1.00 E+01$

3. $01 E+02$

$<5.00 \mathrm{E}+02$

$5.00 \mathrm{E}+02$

1. $51 \mathrm{E}+02$

$<5.00 \mathrm{E}+02$

1. $63 \mathrm{E}+02$

$<5.00 \mathrm{E}+02$

1. $28 E+02$

4. $20 E+01$

3. $20 E+01$

$<5.00 E+02$

$5.00 \mathrm{E}+02$

4. $17 \mathrm{E}+03$
6. $58 \mathrm{E}+02$ 
DATA FOR 244-AR Vault Cooling water

Continued

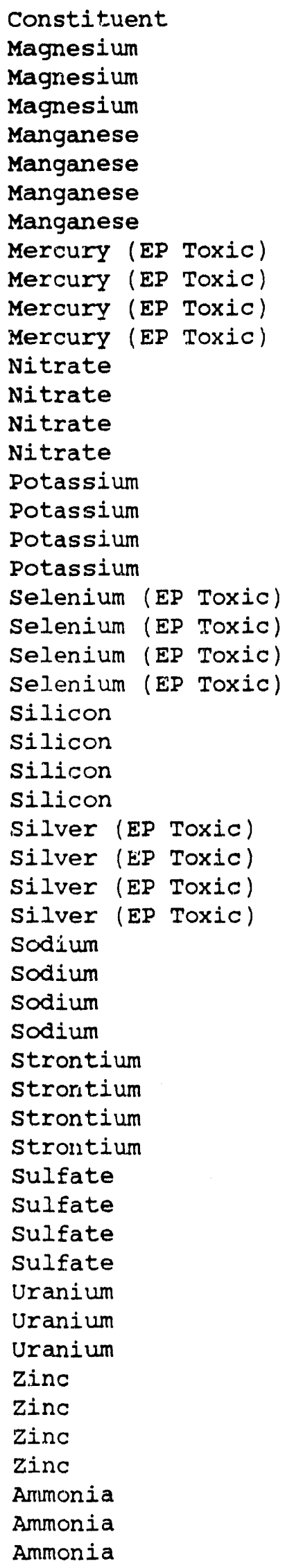

\begin{tabular}{|c|c|c|c|}
\hline Sample \# & Date & Method & Result \\
\hline 50693 & $10 / 17 / 89$ & ICP & $4.16 \mathrm{E}+03$ \\
\hline 50717 & $10 / 24 / 89$ & ICP & $4.03 E+03$ \\
\hline 50947 & $2 / 16 / 90$ & ICP & 4. $23 E+03$ \\
\hline 50685 & $10 / 13 / 89$ & ICP & $<5.00 \mathrm{E}+00$ \\
\hline 50693 & $10 / 17 / 89$ & $I(P$ & $<5.00 E+00$ \\
\hline 50717 & $10 / 24 / 89$ & ICP & $<5.00 E+00$ \\
\hline 50947 & $2 / 16 / 90$ & ICP & $2.30 \mathrm{E}+01$ \\
\hline $50685 \mathrm{E}$ & $10 / 13 / 89$ & CVAA/M & $<2.00 E+01$ \\
\hline $50693 \mathrm{E}$ & $10 / 17 / 89$ & CVAA/M & $<2.00 E+01$ \\
\hline $50717 \mathrm{E}$ & $10 / 24 / 89$ & CVAA/M & $<2.00 \mathrm{E}+01$ \\
\hline $50947 \mathrm{E}$ & $2 / 16 / 90$ & CVAA $/ M$ & $<2.00 \mathrm{E}+01$ \\
\hline 50685 & $10 / 13 / 89$ & $I C$ & $<5.00 \mathrm{E}+02$ \\
\hline 50693 & $10 / 17 / 89$ & IC & $<5.00 \mathrm{E}+02$ \\
\hline 50717 & $10 / 24 / 89$ & IC & $6.00 \mathrm{E}+02$ \\
\hline 50947 & $2 / 16 / 90$ & IC & $6.00 \mathrm{E}+02$ \\
\hline 50685 & $10 / 13 / 89$ & ICP & $8.00 E+02$ \\
\hline 50693 & $10 / 17 / 89$ & ICP & $7.27 E+02$ \\
\hline 50717 & $10 / 24 / 89$ & ICP & $7.54 \mathrm{E}+02$ \\
\hline 50947 & $2 / 16 / 90$ & ICP & $7.50 \mathrm{E}+02$ \\
\hline $50685 \mathrm{E}$ & $10 / 13 / 89$ & ICP & $<5.00 \mathrm{E}+02$ \\
\hline $50693 E$ & $10 / 17 / 89$ & ICP & $<5.00 \mathrm{E}+02$ \\
\hline $50717 \mathrm{E}$ & $10 / 24 / 89$ & ICP & $<5.00 \mathbf{E}+02$ \\
\hline $50947 \mathrm{E}$ & $2 / 16 / 90$ & ICP & $<5.00 \mathrm{E}+02$ \\
\hline 50685 & $10 / 13 / 89$ & ICP & 2. $38 \mathrm{E}+03$ \\
\hline 50693 & $10 / 17 / 89$ & ICP & $2.31 E+03$ \\
\hline 50717 & $10 / 24 / 89$ & ICP & $2.40 \mathrm{E}+03$ \\
\hline 50947 & $2 / 16 / 90$ & ICP & $2.55 E+03$ \\
\hline $50685 E$ & $10 / 13 / 89$ & ICP & $<5.00 E+02$ \\
\hline $50693 \mathrm{E}$ & $10 / 17 / 89$ & ICP & $<5.00 \mathrm{E}+02$ \\
\hline $50717 \mathrm{E}$ & $10 / 24 / 89$ & ICP & $<5.00 \mathrm{E}+02$ \\
\hline $50947 \mathrm{E}$ & $2 . / 16 / 90$ & ICP & $<5.00 \mathrm{E}+02$ \\
\hline 50685 & $10 / 13 / 89$ & ICP & 2. $24 \mathrm{E}+03$ \\
\hline 50693 & $10 / 17 / 89$ & ICP & $2.21 E+03$ \\
\hline 50717 & $10 / 24 / 89$ & ICP & $2.24 \mathrm{E}+03$ \\
\hline 50947 & $2 / 16 / 90$ & ICP & $2.03 E+03$ \\
\hline 50685 & $10 / 13 / 89$ & ICP & $1.05 \mathrm{E}+02$ \\
\hline 50693 & $10 / 17 / 89$ & ICP & $1.01 \mathrm{E}+02$ \\
\hline 50717 & $10 / 24 / 89$ & ICP & $9.60 \mathrm{E}+01$ \\
\hline 50947 & $2 / 16 / 90$ & ICP & $8.70 E+01$ \\
\hline 5068.5 & $10 / 13 / 89$ & $I C$ & $1.02 \mathrm{E}+04$ \\
\hline 50693 & $10 / 17 / 89$ & IC & $1.05 E+04$ \\
\hline 50717 & $10 / 24 / 89$ & IC & $1.08 \mathrm{E}+04$ \\
\hline $5094 ?$ & $2 / 16 / 90$ & IC & $1.00 E+04$ \\
\hline 50685 & $10 / 13 / 89$ & GEA & $5.04 \mathrm{E}-01$ \\
\hline 50693 & $10 / 17 / 89$ & GEA & $5.59 E-01$ \\
\hline 50717 & $10 / 24 / 89$ & GEA & $5.93 E-01$ \\
\hline 50685 & $10,13 / 89$ & ICP & $7.00 E+00$ \\
\hline 50693 & $10 / 17 / 89$ & ICP & $7.00 E+00$ \\
\hline 50717 & $10 / 24 / 89$ & ICP & $5.00 E+00$ \\
\hline 50947 & $2 / 16 / 90$ & ICP & 4. $70 E+01$ \\
\hline 50685 & $10 / 13 / 89$ & ISE & 5. $50 \mathrm{E}+01$ \\
\hline 50693 & $10 / 17 / 89$ & ISE & 7. $10 E+01$ \\
\hline 50717 & $10 / 24 / 89$ & ISE & $5.10 E+01$ \\
\hline
\end{tabular}


DATA FOR 244-AR Vault Cooling water

Continued

Constituent
Ammonia
1-Butanol
1-Butanol
1-Butanol
1-Butanol
1-Butanol
2-Butanone
2-Butanone
2-Butanone
2-Butanone
2-Butanone
2-Butanone
2-Butanone
2-Butanone
2-Butanone
2-Butanone
2-Butanone
2-Butanone

Dichloromethane

Dichloromethane

Dichloromethane

Dichloromethane

Dichloromethane

Dichloromethane

Dichloromethane

Dichloromethane

Dichloromethane

Dichloromethane

Dichloromethane

Dichloromethane

Tetrahydrofuran

Tetrahydrofuran

Tetrahydrofuran

Tetrahyarofuran

Tetrahydrofuran

Tetrahydrofuran

Tetrahydrofuran

Tetrahydrofuran

Tetrahydrofuran

Tetrahyarofuran

Tetrahydrofuran

Tetrahydrofuran

Alkalinity (Method B)

Alkalinity (Method B)

Alkalinity (Method B)

Alkalinity (Method B)

Alpha Activity ( $\mathrm{pCi} / \mathrm{L}$ )

Alpha Activity (pCi/L)

Alpha Activity (pCi/L)

Conductivity (us)

Conductivity (us)

Conductivity (us)

Conductivity (uS)

\begin{tabular}{|c|c|c|c|}
\hline Sample & Date & Method & Result \\
\hline 50947 & $2 / 16 / 90$ & ISE & $\div 5.00 \mathrm{E}+01$ \\
\hline 50685 & $10 / 13 / 89$ & DIGC & $<1.00 E+04$ \\
\hline 50693 & $10 / 17 / 89$ & DIGC & $<1.00 E+04$ \\
\hline 50717 & $10 / 24 / 89$ & DIGC & $<1.00 E+04$ \\
\hline 50947 & $2 / 16 / 90$ & VOA & $5.00 \mathrm{E}+00$ \\
\hline 50947 & $2 / 16 / 90$ & DIGC & $<1.00 \mathrm{E}+04$ \\
\hline 50685 & $10 / 13 / 89$ & VOA & $<1.00 E+01$ \\
\hline $50685 B$ & $10 / 13 / 89$ & VOA & $<1.00 \mathrm{E}+01$ \\
\hline $50685 T$ & $10 / 13 / 89$ & VOA & $<1.00 E+01$ \\
\hline 50693 & $10 / 17 / 89$ & VOA & $<1.00 \mathrm{E}+01$ \\
\hline $50693 B$ & $10 / 17 / 89$ & VOA & $<1.00 \mathrm{E}+01$ \\
\hline $50693 \mathrm{~T}$ & $10 / 17 / 89$ & VOA & $<5.00 \mathrm{E}+00$ \\
\hline 50717 & $10 / 24 / 89$ & VOA & $<1.00 \mathrm{E}+01$ \\
\hline $50717 \mathrm{~B}$ & $10 / 24 / 89$ & VOA & $1.30 \mathrm{E}+01$ \\
\hline $50717 \mathrm{~T}$ & $10 / 24 / 89$ & VOA & $<1.00 E+01$ \\
\hline 50947 & $2 / 16 / 90$ & VOA & $<1.00 \mathrm{E}+01$ \\
\hline $50947 \mathrm{~B}$ & $2 / 16 / 90$ & VOA & $<1.00 E+01$ \\
\hline $50947 \mathrm{~T}$ & $2 / 16 / 90$ & VOA & $<5.00 \mathrm{E}+00$ \\
\hline 50685 & $10 / 13 / 89$ & VOA & $<5.00 \mathrm{E}+00$ \\
\hline $50685 B$ & $10 / 13 / 89$ & VOA & $3.90 \mathrm{E}+\mathrm{U} 2$ \\
\hline $50685 \mathrm{~T}$ & $10 / 13 / 89$ & VOA & $3.70 \mathrm{E}+02$ \\
\hline 50693 & $10 / 17 / 89$ & VOA & $<5.00 \mathrm{E}+00$ \\
\hline $50693 B$ & $10 / 17 / 89$ & VOA & $<3.00 E+00$ \\
\hline $50693 \mathrm{~T}$ & $10 / 17 / 89$ & VOA & $1.40 \mathrm{E}+01$ \\
\hline 50717 & $10 / 24 / 89$ & VOA & $<5.00 E+00$ \\
\hline $50717 \mathrm{~B}$ & $10 / 24 / 89$ & VOA & $5.00 E+00$ \\
\hline $50717 \mathrm{~T}$ & $10 / 24 / 89$ & VOA & $6.10 \mathrm{E}+01$ \\
\hline 50947 & $2 / 16 / 90$ & VOA & $<5.00 \mathrm{E}+00$ \\
\hline $50947 B$ & $2 / 16 / 90$ & VOA & $<5.00 \mathrm{E}+00$ \\
\hline $50947 \mathrm{~T}$ & $2 / 16 / 90$ & VOA & $<5.00 \mathrm{E}+00$ \\
\hline 50685 & $10 / 13 / 89$ & VOA & $<1.00 E+01$ \\
\hline $50685 B$ & $10 / 13 / 89$ & VOA & $<1.00 E+01$ \\
\hline $50685 T$ & $10 / 13 / 89$ & VOA & $<1.00 E+01$ \\
\hline 50693 & $10 / 17 / 89$ & VOA & $<1.00 E+01$ \\
\hline $50693 B$ & $10 / 17 / 89$ & VOA & $1.30 \mathrm{E}+01$ \\
\hline $50693 \mathrm{~T}$ & $10 / 17 / 89$ & VOA & $1.30 \mathrm{E}+01$ \\
\hline 50717 & $10 / 24 / 89$ & VOA & $<1.00 E+01$ \\
\hline $50717 \mathrm{~B}$ & $10 / 24 / 89$ & VOA & $2.10 \mathrm{E}+01$ \\
\hline $50717 \mathrm{~T}$ & $10 / 24 / 89$ & VOA & $<7.00 \mathrm{E}+00$ \\
\hline 50947 & $2 / 16 / 90$ & VOA & $=1.00 \mathrm{E}+01$ \\
\hline $50947 B$ & $2 / 16 / 90$ & VOA & $<1.00 E+01$ \\
\hline $50947 \mathrm{~T}$ & $2 / 16 / 90$ & VOA & $<5.00 E+00$ \\
\hline 50685 & $10 / 13 / 89$ & TITRA & $5.80 \mathrm{E}+04$ \\
\hline 50693 & $10 / 17 / 89$ & TITRA & $5.90 \mathrm{E}+04$ \\
\hline 50717 & $10 / 24 / 89$ & TITRA & $5.80 E+04$ \\
\hline 50947 & $2 / 16 / 90$ & TITRA & $5.90 E+04$ \\
\hline 50685 & $10 / 13 / 89$ & Alpha & $1.02 \mathrm{E}+00$ \\
\hline 50693 & $10 / 17 / 89$ & Alpha & $<2.34 \mathrm{E}-01$ \\
\hline 50717 & $10 / 24 / 89$ & Alpha & $7.71 \mathrm{E}-01$ \\
\hline 50685 & $10 / 13 / 89$ & COND-FId & $1.40 \mathrm{E}+02$ \\
\hline 50693 & $10 / 17 / 89$ & COND-FId & $1.27 \mathrm{E}+02$ \\
\hline 50717 & $10 / 24 / 89$ & COND $-F I d$ & $1.17 \mathrm{E}+02$ \\
\hline 50947 & $2 / 16 / 90$ & COND -FId & $1.60 E+02$ \\
\hline
\end{tabular}

D -126 
DATA FOR 244-AR vault Cooling water

Continued

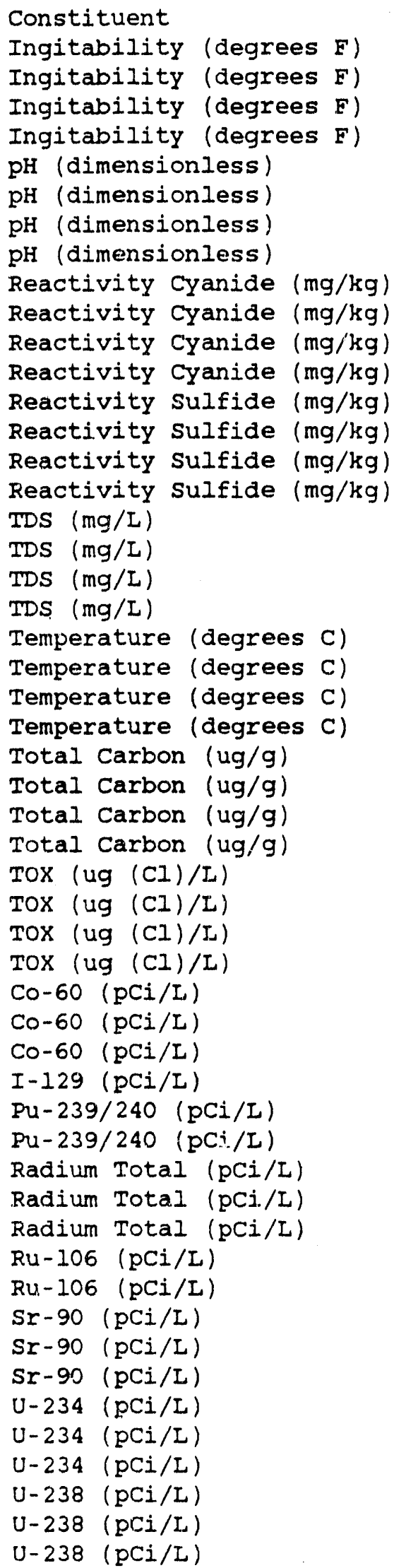

\begin{tabular}{|c|c|c|c|}
\hline ample \# & & Method & Result \\
\hline $50685 \mathrm{E}$ & $10 / 13 / 89$ & IGNIT & $2.12 \mathrm{E}+02$ \\
\hline $50693 E$ & $10 / 17 / 89$ & IGNIT & 2. $10 E+02$ \\
\hline $50717 \mathrm{E}$ & $10 / 24 / 89$ & IGNIT & 2. $10 \mathrm{E}+02$ \\
\hline $50947 \mathrm{E}$ & $2 / 16 / 90$ & IGNIT & 2. $10 \mathrm{E}+02$ \\
\hline 50685 & $10 / 13 / 89$ & PH-FId & $7.00 \mathrm{E}+00$ \\
\hline 50693 & $10 / 17 / 89$ & $\mathrm{PH}-\mathrm{F} I \mathrm{~d}$ & 8. $18 E+00$ \\
\hline 50717 & $10 / 24 / 89$ & PH-FId & 8. $40 \mathrm{E}+00$ \\
\hline 50947 & $2 / 16 / 90$ & PH-FId & $7.00 \mathrm{E}+00$ \\
\hline $50685 \mathrm{E}$ & $10 / 13 / 89$ & DSPEC & $<1.00 \mathrm{E}+02$ \\
\hline $50693 \mathrm{E}$ & $10 / .17 / 89$ & DSPEC & $<1.00 \mathrm{E}+02$ \\
\hline $50717 \mathrm{E}$ & $10 / 24 / 89$ & DSPEC & $<1.00 \mathrm{E}+02$ \\
\hline $50947 \mathrm{E}$ & $2 / 16 / 90$ & DSPEC & $<1.00 E+02$ \\
\hline $50685 \mathrm{E}$ & $10 / 13 / 89$ & DTITRA & $<1.00 \mathrm{E}+02$ \\
\hline $50693 E$ & $10 / 17 / 89$ & DTITRA & $<1.00 \mathrm{E}+02$ \\
\hline $50717 \mathrm{E}$ & $10 / 24 / 89$ & DTITRA & $<1.00 \mathrm{E}+02$ \\
\hline $50947 \mathrm{E}$ & $2 / 16 / 90$ & DTITRA & $<1.00 \mathrm{E}+02$ \\
\hline 50685 & $10 / 13 / 89$ & TDS & $7.10 \mathrm{E}+04$ \\
\hline 50693 & $10 / 17 / 89$ & TDS & $6.50 \mathrm{E}+04$ \\
\hline 50717 & $10 / 24 / 89$ & TDS & $7.10 \mathrm{E}+04$ \\
\hline 50947 & $2 / 16 / 90$ & TDS & $5.60 E+04$ \\
\hline 0685 & $10 / 13 / 89$ & TEMP-FId & $1.92 \mathrm{E}+01$ \\
\hline 50693 & $10 / 17 / 89$ & TEMP - FId & $1.75 \mathrm{E}+01$ \\
\hline 50717 & $10 / 24 / 89$ & TEMP-EId & $1.54 \mathrm{E}+01$ \\
\hline 50947 & $2 / 16 / 90$ & TEMP - FId & $2.26 \mathrm{E}+01$ \\
\hline 50685 & $10 / 13 / 89$ & $\mathrm{TC}$ & $1.60 \mathrm{E}+04$ \\
\hline 50693 & $10 / 17 / 89$ & TC & $1.60 \mathrm{E}+04$ \\
\hline 50717 & $10 / 24 / 89$ & TC & $1.58 \mathrm{E}+04$ \\
\hline 50947 & $2 / 16 / 90$ & $\mathrm{TC}$ & 1. $53 \mathrm{E}+04$ \\
\hline 50685 & $10 / 13 / 89$ & ITOX & $<8.00 E+00$ \\
\hline 50693 & $10 / 17 / 89$ & LTOX & $<5.00 \mathrm{E}+00$ \\
\hline 50717 & $10 / 24 / 89$ & LTOX & $1.00 \mathrm{E}+01$ \\
\hline 50947 & $2 / 16 / 90$ & LTOX & $<8.00 E+00$ \\
\hline 50685 & $10 / 13 / 89$ & GEA & $<2.89 \mathrm{E}-01$ \\
\hline 50693 & $10 / 17 / 89$ & GEA & $<1.01 E-01$ \\
\hline 50717 & $10 / 24 / 89$ & GEA & $1.59 \mathrm{E}+00$ \\
\hline 50685 & $10 / 13 / 89$ & LEPD & $9.08 E-02$ \\
\hline 50685 & $10 / 13 / 89$ & AEA & $7.13 \mathrm{E}-03$ \\
\hline 50693 & $10 / 17 / 89$ & $A E A$ & 8.01E-03 \\
\hline 50685 & $10 / 13 / 89$ & Alpha-Ra & $<1.16 \mathrm{E}-01$ \\
\hline 50693 & $10 / 17 / 89$ & Alpha-Ra & $2.08 E-01$ \\
\hline 50717 & $10 / 24 / 89$ & Alpha-Ra & $1.77 \mathrm{E}-01$ \\
\hline 50685 & $10 / 13 / 89$ & GEA & $<1.06 \mathrm{E}+00$ \\
\hline 50693 & $1.0 / 17 / 89$ & GEA & $5.07 \mathrm{E}+00$ \\
\hline 50685 & $10 / 13 / 89$ & Beta & $<5.20 \mathrm{E}-02$ \\
\hline 50693 & $10 / 17 / 89$ & Beta & $2.02 \mathrm{E}-01$ \\
\hline 50717 & $10 / 24 / 89$ & Beta & $2.70 E-01$ \\
\hline 50685 & $10 / 13 / 89$ & $\mathrm{AEA}$ & $1.99 E-01$ \\
\hline 50693 & $10 / 17 / 89$ & AEA & $2.04 E-01$ \\
\hline 50717 & $10 / 24 / 89$ & AEA & $2.61 E-01$ \\
\hline 50685 & $10 / 13 / 89$ & AEA & $1.53 \mathrm{E}-01$ \\
\hline 50693 & $10 / 17 / 89$ & $A E A$ & $1.62 \mathrm{E}-01$ \\
\hline 50717 & $10 / 24 / 89$ & $A E A$ & $1.82 \mathrm{E}-01$ \\
\hline
\end{tabular}


DATA FOR 284-W Powerhouse Wastewater-Routine Operation

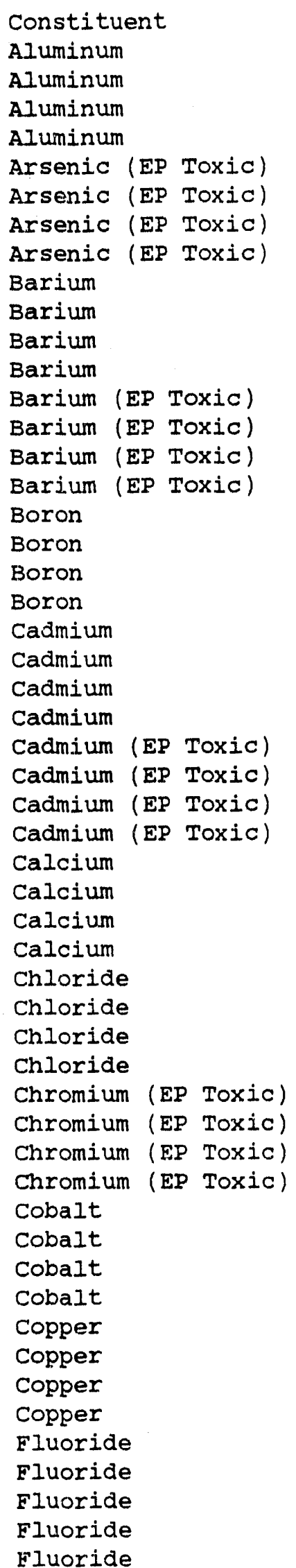

\begin{tabular}{|c|c|c|c|}
\hline Sample \# & Date & Method & Result \\
\hline 50760 & $11 / 13 / 89$ & ICP & $3.61 \mathrm{E}+02$ \\
\hline 50865 & $1 / 02 / 90$ & ICP & $<1.50 E+02$ \\
\hline 50996 & $2 / 28 / 90$ & ICP & $1.68 \mathrm{E}+02$ \\
\hline 51056 & $3 / 16 / 90$ & ICP & $1.54 \mathrm{E}+02$ \\
\hline $50760 \mathrm{E}$ & $11 / 13 / 89$ & ICP & $<5.00 \mathrm{E}+02$ \\
\hline $50865 E$ & $1 / 02 / 90$ & ICP & $<5.00 \mathrm{E}+02$ \\
\hline $50996 \mathrm{E}$ & $2 / 28 / 90$ & ICP & $<5.00 E+02$ \\
\hline $51056 \mathrm{E}$ & $3 / 16 / 90$ & ICP & $<5.00 E+02$ \\
\hline 50760 & $11 / 13 / 89$ & ICP & $6.20 E+01$ \\
\hline 50865 & $1 / 02 / 90$ & ICP & 3. $30 \mathrm{E}+01$ \\
\hline 50996 & $2 / 28 / 90$ & ICP & 4. $30 \mathrm{E}+01$ \\
\hline 51056 & $3 / 16 / 90$ & ICP & $8.33 E+02$ \\
\hline $50760 \mathrm{E}$ & $11 / 13 / 89$ & ICP & $<1.00 \mathrm{E}+03$ \\
\hline $50865 \mathrm{E}$ & $1 / 02 / 90$ & ICP & $<1.00 E+03$ \\
\hline 50996E & $2 / 28 / 90$ & ICP & $<1.00 \mathrm{E}+03$ \\
\hline $51056 \mathrm{E}$ & $3 / 16 / 90$ & ICP & $1.04 \mathrm{E}+03$ \\
\hline 50760 & $11 / 13 / 89$ & ICPP & $1.50 \mathrm{E}+02$ \\
\hline 50865 & $1 / 02 / 90$ & $I C P$ & 1. $20 \mathrm{E}+01$ \\
\hline 50996 & $2 / 28 / 90$ & ICP & $4.40 E+01$ \\
\hline 51056 & $3 / 16 / 90$ & ICP & $3.50 \mathrm{E}+01$ \\
\hline 50760 & $11 / 13 / 89$ & ICP & $<2.00 \mathrm{E}+00$ \\
\hline 50865 & $1 / 02 / 90$ & ICP & $<2.00 \mathrm{E}+00$ \\
\hline 50996 & $2 / 28 / 90$ & ICP & $<2.00 \mathrm{E}+00$ \\
\hline 51056 & $3 / 16 / 90$ & ICP & 1. $10 \mathrm{E}+01$ \\
\hline 50760E & $11 / 13 / 89$ & ICP & $<1.00 \mathrm{E}+02$ \\
\hline $50865 \mathrm{E}$ & $1 / 02 / 90$ & ICP & $<1.00 \mathrm{E}+02$ \\
\hline $50996 \mathrm{E}$ & $2 / 28 / 90$ & ICP & $<1.00 E+02$ \\
\hline $51056 \mathbb{2}$ & $3 / 16 / 90$ & $I C P$ & $<1.00 \mathrm{E}+02$ \\
\hline 50760 & $11 / 13 / 89$ & ICP & $1.35 \mathrm{E}+04$ \\
\hline 50865 & $1 / 02 / 90$ & $I C P$ & $1.96 \mathrm{~s}+04$ \\
\hline 50996 & $2 / 28 / 90$ & ICP & $1.88 \mathrm{E}+04$ \\
\hline 51056 & $3 / 16 / 90$ & $I C P$ & $7.39 E+05$ \\
\hline 50760 & $11 / 13 / 89$ & $I C$ & $4.60 E+03$ \\
\hline 50865 & $1 / 02 / 90$ & IC & $8.40 E+03$ \\
\hline 50996 & $2 / 28 / 90$ & IC & $2.70 E+03$ \\
\hline 51056 & $3 / 16 / 90$ & $I C$ & $2.91 E+06$ \\
\hline $50760 E$ & $11 / 13 / 89$ & $I C P$ & $<5.00 \mathrm{E}+02$ \\
\hline $50865 \mathrm{E}$ & $1 / 02 / 90$ & ICP & $<5.00 \mathrm{E}+02$ \\
\hline $50996 \mathrm{E}$ & $2 / 28 / 90$ & ICP & $<5.00 E+02$ \\
\hline $51056 \mathrm{E}$ & $3 / 16 / 90$ & ICP & $<5.00 \mathrm{E}+02$ \\
\hline 50760 & $11 / 13 / 89$ & ICP & $<2.00 \mathrm{E}+01$ \\
\hline 50865 & $1 / 02 / 90$ & ICP & $<2.00 E+01$ \\
\hline 50996 & $2 / 28 / 90$ & ICP & $<2.00 \mathrm{E}+01$ \\
\hline 51056 & $3 / 16 / 90$ & ICP & $4.60 E+0 I$ \\
\hline 50760 & $11 / 13 / 89$ & ICP & $1.00 E+01$ \\
\hline 50865 & $1 / 02 / 90$ & ICP & $<1.00 \mathrm{E}+01$ \\
\hline 50996 & $2 / 28 / 90$ & ICP & $<1.00 \mathrm{E}+01$ \\
\hline 51056 & $3 / 16 / 90$ & ICP & $1.50 \mathrm{E}+01$ \\
\hline 50760 & $11 / 13 / 89$ & IC & $<5.00 E+02$ \\
\hline 50760 & $11 / 13 / 89$ & ISE & $1.56 \mathrm{E}+02$ \\
\hline 50865 & $1 / 02 / 90$ & IC & $<5.00 \mathrm{E}+02$ \\
\hline 50865 & $1 / 02 / 90$ & ISE & $1.74 E+02$ \\
\hline 50996 & $2 / 28 / 90$ & IC & $<5.00 E+02$ \\
\hline
\end{tabular}


DATA FOR 284-W Powerhouse Wastewater-Routine Operation Continued

Constituent
Fluoride
Fluoride
Fluoride
Ixon
Iron
Iron
Iron
Lead (EP TOXic)
Lead (EP TOXic)
Lead (EP TOxic)
Lead (EP TOXic)
Lithium
Lithium
Lithium
Lithium
Magnesium
Magnesium
Magnesium
Magnesium
Manganese
Manganese
Manganese
Manganese
Mercury
Mercury
Mercury
Mercury
Mercury (EP Toxic)
Mercury (EP Toxic)
Mercury (EP Toxic)
Mercury (EP Toxic)
Nitrate
Nitrate
Nitrate
Nitrate
Potassium
Potassium
Potassium
Potassium
Selenium (EP Toxic)
Selenium (EP Toxic)
Selenium (EP Toxic)
Selenium (EP Toxic)
Silicon
Silicon
Silicon
Silicon
Silver (EP Toxic)
Silver (EP Toxic)
Silver (EP Toxic)
Sodium
Sodium

\begin{tabular}{|c|c|c|c|}
\hline Sample \# & Date & Method & Result \\
\hline 50996 & $2 / 28 / 90$ & ISE & $1.59 \mathrm{E}+02$ \\
\hline 51056 & $3 / 16 / 90$ & IC & $<5.00 \mathrm{E}+02$ \\
\hline 51056 & $3 / 16 / 90$ & ISE & $1.68 \mathrm{E}+02$ \\
\hline 50760 & $11 / 13 / 89$ & ICP & $1.71 \mathrm{E}+02$ \\
\hline 50865 & $1 / 02 / 90$ & ICP & 4. 10E+O1 \\
\hline 50996 & $2 / 28 / 90$ & ICP & $1.49 \mathrm{E}+02$ \\
\hline 51056 & $3 / 16 / 90$ & ICP & $5.50 \mathrm{E}+01$ \\
\hline 50760E & $11 / 13 / 89$ & ICP & $<5.0 O E+02$ \\
\hline $50865 \mathrm{E}$ & $1 / 02 / 90$ & ICP & $<5.00 \mathrm{E}+02$ \\
\hline $50996 \mathrm{E}$ & $2 / 28 / 90$ & ICP & $<5.00 \mathrm{E}+02$ \\
\hline $51056 \mathrm{E}$ & $3 / 16 / 90$ & ICP & $<5.00 \mathrm{E}+02$ \\
\hline 50760 & $11 / 13 / 89$ & ICP & $<1.00 \mathrm{E}+01$ \\
\hline 50865 & $1 / 02 / 90$ & ICP & $<1.00 E+01$ \\
\hline 50996 & $2 / 28 / 90$ & ICP & $<1.00 \mathrm{E}+01$ \\
\hline 51056 & $3 / 16 / 90$ & ICP & $3.90 E+01$ \\
\hline 50760 & $11 / 13 / 89$ & ICP & $2.46 \mathrm{E}+03$ \\
\hline 50865 & $1 / 02 / 90$ & ICP & 4. $32 E+03$ \\
\hline 50996 & $2 / 28 / 90$ & ICP & 4. $33 E+03$ \\
\hline 51056 & $3 / 16 / 90$ & ICP & $2.78 \mathrm{E}+05$ \\
\hline 50760 & $11 / 13 / 89$ & ICP & $<5.00 \mathrm{E}+00$ \\
\hline 50865 & $1 / 02 / 90$ & ICP & $<5.00 E+00$ \\
\hline 50996 & $2 / 28 / 90$ & ICP & $<5.0 O E+00$ \\
\hline 51056 & $3 / 16 / 90$ & ICP & $2.30 \mathrm{E}+01$ \\
\hline 50760 & $11 / 13 / 89$ & CVAA & $<1.00 \mathrm{E}-01$ \\
\hline 50865 & $1 / 02 / 90$ & CVAA & $<1.00 E-01$ \\
\hline 50996 & $2 / 28 / 90$ & CVAA & $<1.00 \mathrm{E}-01$ \\
\hline 51056 & $3 / 16 / 90$ & CVAA & $1.34 \mathrm{E}+00$ \\
\hline $50760 \mathrm{E}$ & $11 / 13 / 89$ & $\mathrm{CVAA} / \mathrm{M}$ & $<2.00 \mathrm{E}+01$ \\
\hline $50865 \mathrm{E}$ & $1 / 02 / 90$ & $C V A A / M$ & $<2.00 E+01$ \\
\hline $50996 \mathrm{E}$ & $2 / 28 / 90$ & CVAA/M & $<2.00 E+01$ \\
\hline $51056 \mathrm{E}$ & $3 / 16 / 90$ & $\mathrm{CVAA} / \mathrm{M}$ & $<2.00 \mathrm{E}+01$ \\
\hline 50760 & $11 / 13 / 89$ & IC & $<5.00 \mathrm{E}+02$ \\
\hline 50865 & $1 / 02 / 90$ & IC & $8.00 \mathrm{E}+02$ \\
\hline 50996 & $2 / 28 / 90$ & IC & $<5.00 \mathrm{E}+02$ \\
\hline 51056 & $3 / 16 / 90$ & IC & $<5.00 \mathrm{E}+02$ \\
\hline 50760 & $11 / 13 / 89$ & ICP & $8.70 \mathrm{E}+02$ \\
\hline 50865 & $1 / 02 / 90$ & ICP & $1.04 \mathrm{E}+03$ \\
\hline 50996 & $2 / 28 / 90$ & ICP & $8.67 \mathrm{E}+02$ \\
\hline 51056 & $3 / 16 / 90$ & ICP & 3. $22 \mathrm{E}+04$ \\
\hline $50760 \mathrm{E}$ & $11 / 13 / 89$ & ICP & $<5.00 \mathrm{E}+02$ \\
\hline $50865 \mathrm{E}$ & $1 / 02 / 90$ & ICP & $<5.00 \mathrm{E}+02$ \\
\hline $50996 \mathrm{E}$ & $2 / 28 / 90$ & ICP & $<5.00 E+02$ \\
\hline $51056 \mathrm{E}$ & $3 / 16 / 90$ & ICP & $<5.00 E+02$ \\
\hline 50760 & $11 / 13 / 89$ & ICP & $3.22 \mathrm{E}+03$ \\
\hline 50865 & $1 / 02 / 90$ & ICP & $3.06 \mathrm{E}+03$ \\
\hline 50996 & $2 / 28 / 90$ & ICP & $2.92 \mathrm{E}+0.3$ \\
\hline 51056 & $3 / 16 / 90$ & ICP & $2.27 \mathrm{E}+03$ \\
\hline 50760E & $11 / 13 / 89$ & ICP & $<5.00 \mathrm{E}+02$ \\
\hline $50865 \mathrm{E}$ & $1 / 02 / 90$ & ICP & $<5.00 \mathrm{E}+02$ \\
\hline 50996E & $2 / 28 / 90$ & ICP & $<5.00 \mathrm{E}+02$ \\
\hline $51056 \mathrm{E}$ & $3 / 16 / 90$ & ICP & $<5.00 E+02$ \\
\hline 50760 & $11 / 13 / 89$ & ICP & $3.27 E+04$ \\
\hline 50865 & $1 / 02 / 90$ & ICP & $1.42 \mathrm{E}+04$ \\
\hline
\end{tabular}


DATA FOR 284-W POwerhouse Wastewater-Routine Operation Continued

\begin{tabular}{|c|c|c|c|c|}
\hline Constituent & Sample \# & Date & Method & Result \\
\hline Sodium & 50996 & $2 / 28 / 90$ & ICP & 1. $30 \mathrm{E}+04$ \\
\hline Sodium & 51056 & $3 / 16 / 90$ & ICP & 1. 11E+06 \\
\hline strontium & 50760 & $11 / 13 / 89$ & ICP & $5.73 E+02$ \\
\hline strontium & 50865 & $1 / 02 / 90$ & ICP & $1.01 \mathrm{E}+02$ \\
\hline strontium & 50996 & $2 / 28 / 90$ & ICP & $2.22 \mathrm{E}+\mathrm{O} 2$ \\
\hline strontium & 51056 & $3 / 16 / 90$ & ICP & 3. $16 \mathrm{E}+03$ \\
\hline Sulfate & 50760 & $11 / 13 / 89$ & IC & 4.17E+04 \\
\hline Sulfate & 50865 & $1 / 02 / 90$ & IC & $2.18 \mathrm{E}+04$ \\
\hline Sulfate & 50996 & $2 / 28 / 90$ & IC & $1.88 \mathrm{E}+04$ \\
\hline sulfate & 51056 & $3 / 16 / 90$ & IC & $2.49 E+04$ \\
\hline Uranium & 50760 & $11 / 13 / 89$ & GEA & $3.73 E-01$ \\
\hline Uranium & 50865 & $1 / 02 / 90$ & GEA & $5.67 \mathrm{E}-01$ \\
\hline Uranium & 51056 & $3 / 16 / 90$ & GEA & $6.15 \mathrm{E} \cdots 01$ \\
\hline Vanadium & 50760 & $11 / 13 / 89$ & ICP & $5.00 \mathrm{E}+00$ \\
\hline Vanadium & 50865 & $1 / 02 / 90$ & $I C P$ & $<5.00 \mathrm{E}+00$ \\
\hline Vanadium & 50996 & $2 / 28 / 90$ & ICP & $<5.00 \mathrm{E}+00$ \\
\hline Vanadium & 51056 & $3 / 16 / 90$ & $I C D$ & $<5.00 E+00$ \\
\hline Zinc & 50760 & $11 / 13 / 89$ & ICP & $1.20 \mathrm{E}+01$ \\
\hline Zinc & 50865 & $1 / 02 / 90$ & ICP & $<5.00 \mathrm{E}+00$ \\
\hline $\operatorname{Zinc}$ & 50996 & $2 / 28 / 90$ & ICP & $<5.00 \mathrm{E}+00$ \\
\hline Zinc & 51056 & $3 / 16 / 90$ & ICP & $6.60 \mathrm{E}+01$ \\
\hline 2-Butanone & 50760 & $11 / 13 / 89$ & VOA & $<1.00 E+01$ \\
\hline 2-Butanone & $50760 \mathrm{~B}$ & $11 / 13 / 89$ & VOA & $<1.00 \mathrm{E}+01$ \\
\hline 2-Butanone & $50760 \mathrm{~T}$ & $11 / 13 / 89$ & VOA & $<1.00 \mathrm{E}+01$ \\
\hline 2-Butanone & 50865 & $1 / 02 / 90$ & VOA & $<1.00 \mathrm{E}+01$ \\
\hline 2-Butanone & $50865 B$ & $1 / 02 / 90$ & VOA & $1.60 \mathrm{E}+01$ \\
\hline 2-Butanone & $50865 \mathrm{~T}$ & $1 / 02 / 90$ & VOA & $1.10 \mathrm{E}+01$ \\
\hline 2-Butanone & 50996 & $2 / 28 / 90$ & VOA & $<1.00 \mathrm{E}+01$ \\
\hline 2-Butanone & $50996 B$ & $2 / 28 / 90$ & VOA & $<1.00 \mathrm{E}+01$ \\
\hline 2-Butanone & $50996 \mathrm{~T}$ & $2 / 28 / 90$ & VOA & $<1.00 \mathrm{E}+01$ \\
\hline 2-Butanone & 51056 & $3 / 16 / 90$ & VOA & $<1.00 \mathrm{E}+01$ \\
\hline 2-Butanone & $51056 \mathrm{~B}$ & $3 / 16 / 90$ & VOA & $<1.00 \mathrm{E}+01$ \\
\hline 2-Butanone & $51056 \mathrm{~T}$ & $3 / 16 / 90$ & VOA & $<1.00 E+01$ \\
\hline Trichloromethane & 50760 & $11 / 13 / 89$ & VOA & $1.00 \mathrm{E}+01$ \\
\hline Trichloromethane & $50760 B$ & $11 / 13 / 89$ & VOA & $<3.00 E+00$ \\
\hline Trichloromethane & $50760 \mathrm{~T}$ & $11 / 13 / 89$ & VOA & $<4.00 E+00$ \\
\hline Trichloromethane & 50865 & $1 / 02 / 90$ & VOA & $5.00 \mathrm{E}+00$ \\
\hline Trichloromethane & $50865 B$ & $1 / 02 / 90$ & VOA & $<5.00 E+00$ \\
\hline Trichloromethane & $50865 \mathrm{~T}$ & $1 / 02 / 90$ & VOA & $<4.00 E+00$ \\
\hline Trichloromethane & 50996 & $2 / 28 / 90$ & VOA & $<5.00 \mathrm{E}+00$ \\
\hline Trichloromethane & $50996 \mathrm{~B}$ & $2 / 28 / 90$ & VOA & $<5.00 E+00$ \\
\hline Trichloromethane & $50996 \mathrm{~T}$ & $2 / 28 / 90$ & VOA & $<5.00 \mathrm{E}+00$ \\
\hline Trichloromethane & 51056 & $3 / 16 / 90$ & VOA & $<5.00 \mathrm{E}+00$ \\
\hline 'Trichloromethane & $51056 \mathrm{~B}$ & $3 / 16 / 90$ & VOA & $<5.00 \mathrm{E}+00$ \\
\hline Trichloromethane & $51.056 \mathrm{~T}$ & $3 / .16 / 90$ & VOA & $<5.00 E+00$ \\
\hline Alkalinity (Method B) & 50760 & $11 / 13 / 89$ & TITRA & $6.80 E+04$ \\
\hline Alkalinity (Method B) & 50865 & $1 / 02 / 90$ & TITRA & $7.80 \mathrm{E}+04$ \\
\hline Alkalinity (Method B) & 50996 & $2 / 28 / 90$ & TITRA & $7.50 E+04$ \\
\hline Alkalinity (Method B) & 51056 & $3 / 16 / 90$ & TITRA & $6.40 E+04$ \\
\hline Beta Activity (pCi/L) & 50760 & $11 / 13 / 89$ & Beta & $2.61 \mathrm{E}+00$ \\
\hline Beta Activity (pci/L) & 50865 & $1 / 02 / 90$ & Beta & $<1.47 \mathrm{E}+00$ \\
\hline Beta Activity (pci/L) & 51056 & $3 / 16 / 90$ & Beta & $5.37 \mathrm{E}+00$ \\
\hline Conductivity (us) & 50760 & $11 / 13 / 89$ & COND-FId & $2.66 \mathrm{E}+02$ \\
\hline
\end{tabular}


DATA FOR 284-W POwerhouse Wastewater-Routine Operation Continued

\begin{tabular}{|c|c|c|c|c|}
\hline Constituent & Sample \# & Date & Method & Result \\
\hline Conductivity & 50865 & $1 / 02 / 90$ & COND-FId & $2.24 E+02$ \\
\hline Conductivity & 50996 & $2 / 28 / 90$ & COND-FId & $1.58 \mathrm{E}+02$ \\
\hline Conductivity & 51056 & $3 / 16 / 90$ & COND-FId & $1.01 \mathrm{E}+03$ \\
\hline Ingitability & $50760 \mathrm{E}$ & $11 / 13 / 89$ & IGNIT & 2. $10 \mathrm{E}+02$ \\
\hline Ingitability & $50865 \mathrm{E}$ & $1 / 02 / 90$ & IGNIT & $2.12 \mathrm{E}+02$ \\
\hline Ingitability (degrees F) & $50996 \mathrm{E}$ & $2 / 28 / 90$ & IGNIT & $2.02 \mathrm{E}+02$ \\
\hline Ingitability (degrees F) & $51056 \mathrm{E}$ & $3 / 16 / 90$ & IGNIT & $1.98 \mathrm{E}+02$ \\
\hline $\mathrm{pH}$ (dimensionless) & 50760 & $11 / 13 / 89$ & PH-FId & $9.35 \mathrm{E}+00$ \\
\hline $\mathrm{pH}$ (dimensionless) & 50865 & $1 / 02 / 90$ & PH-FId & $8.67 \mathrm{E}+00$ \\
\hline $\mathrm{pH}$ (dimensionless) & 50996 & $2 / 28 / 90$ & PH-Fld & $8.96 \mathrm{E}+00$ \\
\hline $\mathrm{pH}$ (dimensionless) & 510.56 & $3 / 16 / 90$ & PH - Fld & $9.20 \mathrm{E}+00$ \\
\hline Reactivity Cyanide (mg/kg) & $50760 \mathrm{E}$ & $11 / 13 / 89$ & DSPEC & $<1.00 \mathrm{E}+02$ \\
\hline Reactivity Cyanide (mg/kg) & $50865 \mathrm{E}$ & $1 / 02 / 90$ & DSPEC & $<1.00 \mathrm{E}+02$ \\
\hline Reactivity Cyanide (mg/kg) & 50996E & $2 / 28 / 90$ & DSPEC & $<1.00 \mathrm{E}+02$ \\
\hline Reactivity Cyanide (mg/kg) & $51056 \mathrm{E}$ & $3 / 16 / 90$ & DSPEC & $<1.00 \mathrm{E}+02$ \\
\hline Reactivity Sulfide (mg/kg) & 50760E & $11 / 13 / 89$ & DTITRA & $<1.00 \mathrm{~L}+02$ \\
\hline Reactivity sulfide (mg/kg) & $50865 \mathrm{E}$ & $1 / 02 / 90$ & DTITRA & $<1.00 \mathrm{E}+02$ \\
\hline Sulfide $(\mathrm{mg} / \mathrm{kg})$ & 50996E & $2 / 28 / 90$ & DTITRA & $<1.00 E+02$ \\
\hline Reactivity Sulfide (mg/kg) & $51056 \mathrm{E}$ & $3 / 16 / 90$ & DTITRA & $<1.00 E+02$ \\
\hline Suspended solids (mg/L) & 50760 & $11 / 13 / 89$ & SSOLID & $1.40 \mathrm{E}+04$ \\
\hline Suspended solids (mg/L) & 50865 & $1 / 02 / 90$ & SSOLID & $<5.00 \mathrm{E}+03$ \\
\hline Suspended solids (mg/L) & 50996 & $2 / 28 / 90$ & SSOLID & $<5.00 \mathrm{E}+03$ \\
\hline Suspended Solids (mg/L) & 51056 & $3 / 16 / 90$ & SSOLID & $<5.00 \mathrm{E}+03$ \\
\hline $\operatorname{TDS}(\mathrm{mg} / \mathrm{L})$ & 50760 & $11 / 13 / 89$ & TDS & $1.18 \mathrm{E}+05$ \\
\hline $\operatorname{TDS}(\mathrm{mg} / \mathrm{I})$ & 50865 & $1 / 02 / 90$ & TDS & $1.26 \mathrm{E}+05$ \\
\hline $\operatorname{TDS}(\mathrm{mg} / \mathrm{L})$ & 50996 & $2 / 28 / 90$ & TDS & $9.30 E+04$ \\
\hline $\operatorname{TDS}(\mathrm{mg} / \mathrm{L})$ & 51056 & $3 / 16 / 90$ & TDS & $3.54 E+06$ \\
\hline Temperature (degrees C) & 50760 & $11 / 13 / 89$ & TEMP-FId & $2.42 \mathrm{E}+01$ \\
\hline Temperature (degrees C) & 50865 & $1 / 02 / 90$ & TEMP-FId & $1.22 \mathbf{E}+01$ \\
\hline Temperature (degrees C) & 50996 & $2 / 28 / 90$ & TEMP-FId & $8.40 E+00$ \\
\hline Temperature (degrees C) & 51056 & $3 / 16 / 90$ & TEMP-FId & $1.07 \mathrm{E}+01$ \\
\hline $\operatorname{TOC}(u g / g)$ & 50760 & $11 / 13 / 89$ & TOC & $<1.40 \mathrm{E}+03$ \\
\hline TOC (ug/g) & 50865 & $1 / 02 / 90$ & TOC & $<1.50 \mathrm{E}+03$ \\
\hline $\operatorname{TOC}(\mathrm{ug} / \mathrm{g})$ & 50996 & $2 / 28 / 90$ & TOC & $1.30 \mathrm{E}+03$ \\
\hline TOC $(u g / g)$ & 51056 & $3 / 16 / 90$ & TOC & $1.30 E+03$ \\
\hline Total Carbon (ug/g) & 50760 & $11 / 13 / 89$ & $\mathrm{TC}$ & $1.42 \mathrm{E}+04$ \\
\hline Total Carbon (ug/g) & 50865 & $1 / 02 / 90$ & $\mathrm{TC}$ & $1.68 \mathrm{E}+04$ \\
\hline Total Carbon (ug/g) & 50996 & $2 / 28 / 90$ & TC & $1.55 E+04$ \\
\hline Total Carbon (ug/g) & 51056 & $3 / 16 / 90$ & $\mathrm{TC}$ & $1.56 \mathrm{E}+04$ \\
\hline $\operatorname{TOX}(\mathrm{ug}(\mathrm{CI}) / \mathrm{I})$ & 50760 & $11 / 13 / 89$ & LTOX & $6.90 E+01$ \\
\hline $\operatorname{TOX}(\mathrm{ug}(\mathrm{CI}) / \mathrm{L})$ & 50865 & $1 / 02 / 90$ & LTOX & $4.40 E+01$. \\
\hline $\operatorname{TOX}(\operatorname{ug}(C I) / L)$ & 50996 & $2 / 28 / 90$ & LTOX & $1.60 \mathrm{E}+01$ \\
\hline $\operatorname{Tox}(u g(C I) / L)$ & 51056 & $3 / 16 / 90$ & LTOX & $7.70 E+01$ \\
\hline
\end{tabular}


DATA FOR Plutonium Finishing Plant wastewater-Not PRF or RMC

Constituent

Arsenic (EP TOxic)

Arsenic (EP TOxic)

Arsenic (EP TOxic)

Arsenic (EP Toxic)

Barium

Barium

Barium

Barium

Barium (EP Toxic)

Barium (EP Toxic)

Barium (EP Toxic)

Barium (EP TOxic)

Boron

Boron

Boron

Boron

Cadmium (EP Toxic)

Cadmium (EP TOXic)

Cadmium (EP Toxic)

cadmium (EP Toxic)

Calcium

Calcium

Calcium

Calcium

Chloride

Chloride

Chloride

Chloride

Chromium (EP Toxic)

Chromium (EP Toxic)

Chromium (EP TOxic)

Chromium (EP Toxic)

Copper

Copper

Copper

Copper

Fluoride

Fluoride

Fluoride

Fluoride

Fluoride

Fluoride

Fluoride

Fluoride

Iron

Iron

Iron

Iron

Lead (EP Toxic)

Lead (EP Toxic)

Lead (EP Toxic)

Lead (EP Toxic)

Magnesium

\begin{tabular}{|c|c|c|c|}
\hline ample \# & Date & Method & Result \\
\hline $50818 E$ & $11 / 30 / 89$ & ICP & $<5.00 \mathrm{E}+02$ \\
\hline $50927 \mathrm{E}$ & $2 / 12 / 90$ & ICP & $<5.00 E+02$ \\
\hline $51068 \mathrm{E}$ & $3 / 19 / 90$ & ICP & $<5.00 \mathrm{E}+02$ \\
\hline $51095 \mathrm{E}$ & $3 / 26 / 90$ & ICP & $<5.00 \mathrm{E}+02$ \\
\hline 50818 & $11 / 30 / 89$ & ICP & $2.80 \mathrm{E}+01$ \\
\hline 50927 & $2 / 12 / 90$ & ICP & $2.90 E+01$ \\
\hline 51068 & $3 / 19 / 90$ & ICP & $2.70 E+01$ \\
\hline 51095 & $3 / 26 / 90$ & ICP & $2.90 E+01$ \\
\hline $50818 \mathrm{E}$ & $11 / 30 / 89$ & ICP & $<1.00 \mathrm{E}+03$ \\
\hline $50927 \mathrm{E}$ & $2 / 12 / 90$ & ICP & $<1.00 E+03$ \\
\hline $51068 \mathrm{E}$ & $3 / 19 / 90$ & ICP & $<1.00 \mathrm{E}+03$ \\
\hline $51095 \mathrm{E}$ & $3 / 26 / 90$ & ICP & $<1.00 \mathrm{E}+03$ \\
\hline 50818 & $11 / 30 / 89$ & ICP & $1.10 \mathrm{E}+\mathrm{O}$ \\
\hline 50927 & $2 / 12 / 90$ & $I C P$ & $2.40 \mathrm{E}+01$ \\
\hline 51068 & $3 / 19 / 90$ & ICP & 1. $20 \mathrm{E}+01$ \\
\hline 51095 & $3 / 26 / 90$ & ICP & $1.30 \mathrm{E}+01$ \\
\hline $50818 \mathrm{E}$ & $11 / 30 / 89$ & ICP & $<1.00 \mathrm{E}+02$ \\
\hline $50927 \mathrm{E}$ & $2 / 12 / 90$ & ICP & $<1.00 \mathrm{E}+02$ \\
\hline $51068 \mathrm{E}$ & $3 / 19 / 90$ & ICP & $<1.00 \mathrm{E}+02$ \\
\hline $51095 \mathrm{E}$ & $3 / 26 / 90$ & ICP & $<1.00 \mathrm{E}+02$ \\
\hline 50818 & $11 / 30 / 89$ & ICP & $1.78 E+04$ \\
\hline 50927 & $2 / 12 / 90$ & ICP & $1.87 \mathrm{E}+04$ \\
\hline 51068 & $3 / 19 / 90$ & ICP & $1.72 \mathrm{E}+04$ \\
\hline 51095 & $3 / 26 / 90$ & ICP & $1.82 \mathrm{E}+04$ \\
\hline 50818 & $11 / 30 / 89$ & $I C$ & $2.70 \mathrm{E}+03$ \\
\hline 50927 & $2 / 12 / 90$ & IC & $2.80 E+03$ \\
\hline 51068 & $3 / 19 / 90$ & IC & $1.90 \mathrm{E}+03$ \\
\hline 51095 & $3 / 26 / 90$ & IC & $2.70 \mathrm{E}+03$ \\
\hline $50818 \mathrm{E}$ & $11 / 30 / 89$ & ICP & $<5.00 \mathrm{E}+02$ \\
\hline $50927 \mathrm{E}$ & $2 / 12 / 90$ & ICP & $<5.00 \mathrm{E}+02$ \\
\hline $51068 \mathrm{E}$ & $3 / 19 / 90$ & ICP & $<5.00 \mathrm{E}+02$ \\
\hline $51095 \mathrm{E}$ & $3 / 26 / 90$ & $I C P$ & $<5.00 \mathrm{E}+02$ \\
\hline 50818 & $11 / 30 / 89$ & ICP & $1.80 \mathrm{E}+01$ \\
\hline 50927 & $2 / 12 / 90$ & ICP & $<1.00 \mathrm{E}+01$ \\
\hline 51068 & $3 / 19 / 90$ & ICP & $1.40 \mathrm{E}+01$ \\
\hline 51095 & $3 / 26 / 90$ & ICP & $<1.00 \mathrm{E}+01$ \\
\hline 50818 & $11 / 30 / 89$ & IC & $<5.00 \mathrm{E}+02$ \\
\hline 50818 & $11 / 30 / 89$ & ISE & 1. $38 \mathrm{E}+02$ \\
\hline 50927 & $2 / 12 / 90$ & IC & $<5.00 \mathrm{E}+02$ \\
\hline 50927 & $2 / 12 / 90$ & ISE & $1.40 \mathrm{E}+02$ \\
\hline 51068 & $3 / 19 / 90$ & IC & $<5.00 \mathrm{E}+02$ \\
\hline 51068 & $3 / 19 / 90$ & ISE & $1.33 \mathrm{E}+02$ \\
\hline 51095 & $3 / 26 / 90$ & IC & $<5.00 \mathrm{E}+02$ \\
\hline 51095 & $3 / 26 / 90$ & ISE & $1.28 \mathrm{E}+02$ \\
\hline 50818 & $11 / 30 / 89$ & ICP & $8.40 E+01$ \\
\hline 50927 & $2 / 12 / 90$ & $I^{\prime} C P$ & $4.40 E+01$ \\
\hline 51068 & $3 / 19 / 90$ & ICP & 1. $33 \mathrm{E}+02$ \\
\hline 51095 & $3 / 26 / 90$ & ICP & $<3.00 E+01$ \\
\hline $50818 \mathrm{E}$ & $11 / 30 / 89$ & ICP & $<5.00 E+02$ \\
\hline $50927 \mathrm{E}$ & $2 / 12 / 90$ & ICP & $<5.00 \mathrm{E}+02$ \\
\hline $51068 \mathrm{E}$ & $3 / 19 / 90$ & ICP & $<5.00 \mathrm{E}+02$ \\
\hline $51095 E$ & $3 / 26 / 90$ & ICP & $<5.00 \mathrm{E}+0 ?$ \\
\hline 50818 & $11 / 30 / 89$ & ICP & $4.00 \mathrm{E}+03$ \\
\hline
\end{tabular}

D -132 
DATA FOR Plutonium Finishing Plant Wastewater-Not PRF or RMC Continued

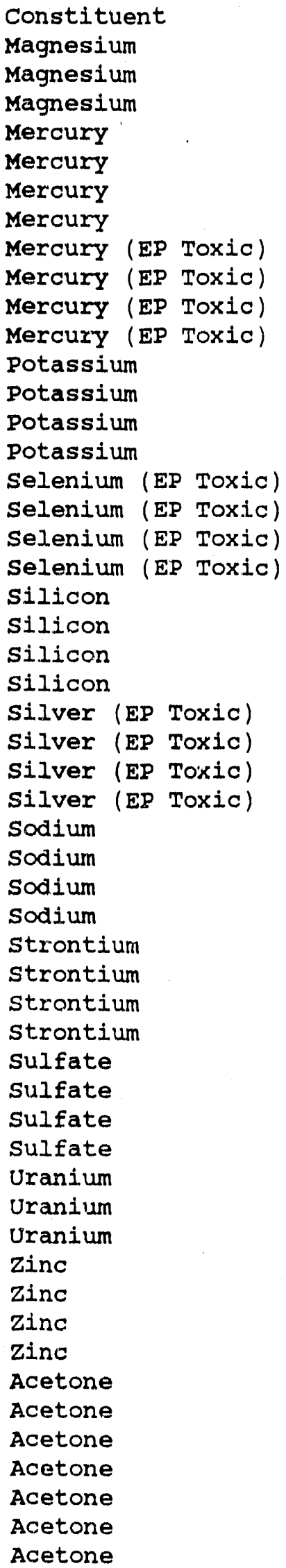

\begin{tabular}{|c|c|c|c|}
\hline Sample \# & Date & Method & Result \\
\hline 50927 & $2 / 12 / 90$ & $I C P$ & $4.32 E+03$ \\
\hline 51068 & $3 / 19 / 90$ & ICP & 4. $24 E+03$ \\
\hline 51095 & $3 / 26 / 90$ & ICP & $4.57 \mathrm{E}+03$ \\
\hline 50818 & $11 / 30 / 89$ & CVAA & $<1.00 E-01$ \\
\hline 50927 & $2 / 12 / 90$ & CVAA & $<1.00 E-01$ \\
\hline 51068 & $3 / 19 / 90$ & CVAA & $4.80 \mathrm{E}-01$ \\
\hline 51095 & $3 / 26 / 90$ & CVAA & $4.20 \mathrm{E}-01$ \\
\hline $50818 \mathrm{E}$ & $11 / 30 / 89$ & CVAA/M & $<2.00 \mathrm{E}+01$ \\
\hline $50927 \mathrm{E}$ & $2 / 12 / 90$ & CVAA/M & $<2.00 E+01$ \\
\hline $51068 \mathrm{E}$ & $3 / 19 / 90$ & CVAA/M & $<2.00 \mathrm{E}+01$ \\
\hline $51095 \mathrm{E}$ & $3 / 26 / 90$ & CVAA $/ M$ & $<2.00 \mathrm{E}+01$ \\
\hline 50818 & $11 / 30 / 89$ & ICP & $7.10 \mathrm{E}+02$ \\
\hline 50927 & $2 / 12 / 90$ & ICP & $6.87 \mathrm{E}+02$ \\
\hline 51068 & $3 / 19 / 90$ & ICP & $7.17 \mathrm{E}+02$ \\
\hline 51095 & $3 / 26 / 90$ & ICP & $7.50 \mathrm{E}+02$ \\
\hline $50818 \mathrm{E}$ & $11 / 30 / 89$ & ICP & $<5.00 E+02$ \\
\hline $50927 \mathrm{E}$ & $2 / 12 / 90$ & ICP & $<5.00 \mathrm{E}+02$ \\
\hline $51.068 \mathrm{E}$ & $3 / 19 / 90$ & ICP & $<5.00 \mathrm{E}+02$ \\
\hline $51.095 \mathrm{E}$ & $3 / 26 / 90$ & ICP & $<5.00 \mathrm{E}+02$ \\
\hline 50818 & $11 / 30 / 89$ & ICP & $2.12 \mathrm{E}+03$ \\
\hline 50927 & $2 / 12 / 90$ & ICP & $2.05 \mathrm{E}+0.3$ \\
\hline 51.068 & $3 / 19 / 90$ & ICP & $1.98 E+03$ \\
\hline 51.095 & $3 / 26 / 90$ & $I C P$ & $1.86 \mathrm{E}+03$ \\
\hline $50818 \mathrm{E}$ & $11 / 30 / 89$ & ICP & $<5.00 \mathrm{E}+02$ \\
\hline $50927 \mathrm{E}$ & $2 / 12 / 90$ & ICP & $<5.00 \mathrm{E}+02$ \\
\hline 51.068E & $3 / 19 / 90$ & ICP & $<5.00 \mathrm{E}+02$ \\
\hline $51.095 \mathrm{E}$ & $3 / 26 / 90$ & ICP & $<5.00 \mathrm{E}+02$ \\
\hline 501818 & $11 / 30 / 89$ & ICP & $1.98 \mathrm{E}+03$ \\
\hline 50927 & $2 / 12 / 90$ & ICP & $2.02 \mathrm{E}+03$ \\
\hline 51.068 & $3 / 19 / 90$ & ICP & $1.96 \mathrm{E}+03$ \\
\hline 51095 & $3 / 26 / 90$ & ICF & $2.03 \mathrm{E}+03$ \\
\hline 50818 & $11 / 30 / 89$ & ICP & $8.90 E+01$ \\
\hline 50,927 & $2 / 12 / 90$ & ICP & $8.50 \mathrm{E}+01$ \\
\hline 51068 & $3 / 19 / 90$ & ICP & $9.10 \mathrm{E}+01$ \\
\hline 51095 & $3 / 26 / 90$ & ICP & $9.80 \mathrm{E}+01$ \\
\hline 50818 & $11 / 30 / 89$ & IC & $1.22 \mathrm{E}+04$ \\
\hline 50927 & $2 / 12 / 90$ & IC & 1. $40 \mathrm{E}+04$ \\
\hline 51068 & $3 / 19 / 90$ & IC & 1.17E+04 \\
\hline 51095 & $3 / 26 / 90$ & $I C$ & $1.44 \mathrm{E}+04$ \\
\hline 50818 & $11 / 30 / 89$ & GEA & $3.64 \mathrm{E}-01$ \\
\hline 51068 & $3 / 19 / 90$ & GEA & $3.70 \mathrm{E}-01$ \\
\hline 51095 & $3 / 26 / 90$ & GEA & 4. 18E-01 \\
\hline 50818 & $11 / 30 / 89$ & ICP & $1.70 E+01$ \\
\hline 50927 & $2 / 12 / 90$ & ICP & $1.60 \mathrm{E}+01$ \\
\hline 5.1068 & $3 / 19 / 90$ & ICP & $1.80 \mathrm{E}+01$ \\
\hline 5.1095 & $3 / 26 / 90$ & ICP & $7.00 E+00$ \\
\hline 50818 & $11 / 30 / 89$ & VOA & $1.50 \mathrm{E}+01$ \\
\hline 50818 & $11 / 30 / 89$ & $A B N$ & $<1.00 E+01$ \\
\hline 50927 & $2 / 12 / 90$ & VOA & $<1.00 \mathrm{E}+01$ \\
\hline 50927 & $2 / 12 / 90$ & $\mathrm{ABN}$ & $<1.00 \mathrm{E}+01$ \\
\hline $50927 \mathrm{~B}$ & $2 / 12 / 90$ & VOA & $<6.00 \mathrm{E}+00$ \\
\hline $50927 \mathrm{~T}$ & $2 / 12 / 90$ & VOA & $<8.00 E+00$ \\
\hline 51068 & $3 / 19 / 90$ & VOA & $<1.00 \mathrm{E}+01$ \\
\hline
\end{tabular}


WHC-EP-0355, Appendix D

DATA FOR Plutonium Finishing Plant wastewater-Not PRF or RMC Continued

\section{Constituent \\ Acetone \\ Acetone \\ Acetone \\ Acetone \\ Acetone \\ Acetone \\ Acetone \\ 1-Butanol \\ 1."Butanol \\ 1-Butanol \\ 1-Butanol \\ 1-Butanol \\ 2-Butanone \\ 2-Butanone \\ 2-Butanone \\ 2-Butanone \\ 2-Butanone \\ 2-Butanone \\ 2-Butanone \\ 2-Butanone \\ 2-Butanone \\ 2-Butanone \\ Caffeine \\ Decane}

Dichloromethane

Dichloromethane

Dichloromethane

Dichloromethane

Dichloromethane

Dichloromethane

Dichloromethane

Dichloromethane

Dichloromethane

Dichloromethane

Tetrach Ioromethane

Tetrach Ioromethane

Tetrachloromethane

Tetrach Ioromethane

Tetrach loromethane

Tetrachloromethane

Tetrach loromethane

Tetrachloromethane

Tetrachloromethane

Tetrachloromethane

Trichloromethane

Trichloromethane

Trichloromethane

Trichloromethane

Trichloromethane

Trichloromethane

Trichloromethane

Trichloromethane

Trichloromethane

\begin{tabular}{|c|c|c|c|}
\hline Sample \# & Date & Method & Result \\
\hline 51068 & $3 / 19 / 90$ & $A B N$ & $<1.00 \mathrm{E}+01$ \\
\hline $51068 B$ & $3 / 19 / 90$ & VOA & $<1.00 E+01$ \\
\hline $51068 \mathrm{~T}$ & $3 / 19 / 90$ & VOA & $<1.00 E+01$ \\
\hline 51095 & $3 / 26 / 90$ & VOA & $<1.00 \mathrm{E}+01$ \\
\hline 51095 & $3 / 26 / 90$ & $A B N$ & $<1.00 \mathrm{E}+01$ \\
\hline $51095 B$ & $3 / 26 / 90$ & VOA & $<5.00 \mathrm{E}+00$ \\
\hline $51095 \mathrm{~T}$ & $3 / 26 / 90$ & VOA & $<6.00 \mathrm{E}+00$ \\
\hline 50818 & $11 / 30 / 89$ & DIGC & $<1.00 \mathrm{E}+04$ \\
\hline 50927 & $2 / 12 / 90$ & DIGC & $<1.00 E+04$ \\
\hline 51068 & $3 / 19 / 90$ & DIGC & $<1.00 \mathrm{E}+04$ \\
\hline $51068 \mathrm{~T}$ & $3 / 19 / 90$ & VOA & $6.00 \mathrm{E}+00$ \\
\hline 51095 & $3 / 26 / 90$ & DIGC & $<1.00 E+04$ \\
\hline 50818 & $11 / 30 / 89$ & VOA & $<8.00 \mathrm{E}+00$ \\
\hline 50927 & $2 / 12 / 90$ & VOA & $<1.00 \mathrm{E}+01$ \\
\hline $50927 \mathrm{~B}$ & $2 / 12 / 90$ & VOA & $2.90 \mathrm{E}+01$ \\
\hline $50927 \mathrm{~T}$ & $2 / 12 / 90$ & VOA & 3. 30E +01 \\
\hline 51068 & $3 / 19 / 90$ & VOA & $<1.00 \mathrm{E}+0.1$ \\
\hline $51068 B$ & $3 / 19 / 90$ & VOA & $<1.00 \mathrm{E}+01$ \\
\hline $51068 \mathrm{~T}$ & $3 / 19 / 90$ & VOA & $<1.00 E+01$ \\
\hline 51095 & $3 / 26 / 90$ & VOA & $<1.00 \mathrm{E}+01$ \\
\hline $51095 B$ & $3 / 26 / 90$ & VOA & $<1.00 E+01$ \\
\hline $51095 \mathrm{~T}$ & $3 / 26 / 90$ & VOA & $<1.00 E+01$ \\
\hline 50927 & $2 / 12 / 90$ & $\mathrm{ABN}$ & $9.00 E+00$ \\
\hline 51095 & $3 / 26 / 90$ & $A B N$ & $8.00 \mathrm{E}+00$ \\
\hline 50818 & $11 / 30 / 89$ & VOA & $<5.00 \mathrm{E}+00$ \\
\hline 50927 & $2 / 12 / 90$ & VOA & $<5.00 E+00$ \\
\hline $50927 B$ & $2 / 12 / 90$ & VOA & $<3.00 \mathrm{E}+00$ \\
\hline $50927 \mathrm{~T}$ & $2 / 12 / 90$ & VOA & $<3.00 \mathrm{E}+00$ \\
\hline 51068 & $3 / 19 / 90$ & VOA & $<5.00 \mathrm{E}+00$ \\
\hline $51068 B$ & $3 / 19 / 90$ & VOA & $<3.00 \mathrm{E}+00$ \\
\hline $51068 T$ & $3 / 19 / 90$ & VOA & $<3.00 \mathrm{E}+00$ \\
\hline 51095 & $3 / 26 / 90$ & VOA & $<5.00 \mathrm{E}+00$ \\
\hline $51095 B$ & $3 / 26 / 90$ & VOA & $2.40 \mathrm{E}+01$ \\
\hline $51095 \mathrm{~T}$ & $3 / 26 / 90$ & VOA & $<5.00 \mathrm{E}+00$ \\
\hline 50818 & $11 / 30 / 89$ & VOA & $<5.00 E+00$ \\
\hline 50927 & $2 / 12 / 90$ & VOA & $5.00 \mathrm{E}+00$ \\
\hline $50927 \mathrm{~B}$ & $2 / 12 / 90$ & VOA & $<5.00 \mathrm{E}+00$ \\
\hline $50927 \mathrm{~T}$ & $2 / 12 / 90$ & VOA & $<5.00 \mathrm{E}+00$ \\
\hline 51068 & $3 / 19 / 90$ & VOA & $<5.00 \mathrm{E}+00$ \\
\hline $51068 B$ & $3 / 19 / 90$ & VOA & $<5.00 \mathrm{E}+00$ \\
\hline 51068'T & $3 / 19 / 90$ & VOA & $<5.00 E+00$ \\
\hline 51095 & $3 / 26 / 90$ & VOA & $<5.00 \mathrm{E}+00$ \\
\hline $51095 \mathrm{~B}$ & $3 / 26 / 90$ & VOA & $<5.00 E+00$ \\
\hline $51095 \mathrm{~T}$ & $3 / 26 / 90$ & VOA & $<5.00 E+00$ \\
\hline 50818 & $11 / 30 / 89$ & VOA & $2.80 \mathrm{E}+01$ \\
\hline 50927 & $2 / 12 / 90$ & VOA & 1. $40 \mathrm{E}+\mathrm{Cl}$ \\
\hline $50927 \mathrm{~B}$ & $2 / 12 / 90$ & VOA & $<4.00 \mathrm{E}+00$ \\
\hline $50927 \mathrm{~T}$ & $2 / 12 / 90$ & VOA & $<4.00 \mathrm{E}+00$ \\
\hline 51.068 & $3 / 19 / 90$ & VOA & $2.60 \mathrm{E}+01$ \\
\hline $51068 B$ & $3 / 19 / 90$ & VOA & $5.00 E+00$ \\
\hline $51068 \mathrm{~T}$ & $3 / 19 / 90$ & VOA & $5.00 \mathrm{E}+00$ \\
\hline 51095 & $3 / 26 / 90$ & VOA & 1. $40 \mathrm{E}+01$ \\
\hline $51095 \mathrm{~B}$ & $3 / 26 / 90$ & VOA & $<4.00 \mathrm{E}+00$ \\
\hline
\end{tabular}


DATA FOR Plutonium Finishing Plant Wastewater-Not PRF or RMC Continued

Constituent

Trichloromethane

Unknown

Unknown

Unknown

Unknown aliphatic HC

Unknown hydrocarbon

Alkalinity (Method B)

Alkalinity (Method B)

Auralinity (Method B)

Alkalinity (Method B)

Alpha Activity ( $\mathrm{pCi} / \mathrm{L}$ )

Alpha Activity (pCi/L)

Alpha Activity (pCi/L)

Conductivity (Lis)

Conductivity (us)

Conductivity (us)

Conductivity (us)

Ingitability (degrees F)

Ingitability (degrees $F$ )

Ingitability (degrees F)

Ingitability (degrees F)

$\mathrm{pH}$ (dimensionless)

pH (dimensionless)

pH (dimensionless)

$\mathrm{pH}$ (dimensionless)

Reactivity Cyanide (mg/kg)

Reactivity Cyanide (mg/kg)

Reactivity Cyanide ( $\mathrm{mg} / \mathrm{kg}$ )

Reactivity Cyanide ( $\mathrm{mg} / \mathrm{kg}$ )

Reactivity sulfide ( $\mathrm{mg} / \mathrm{kg}$ )

Reactivity sulfide (mg/kg)

Reactivity sulfide (mg/kg)

Reactivity sulfide (mg/kg)

TDS (mg/L)

$\operatorname{TDS}(\mathrm{mg} / \mathrm{L})$

$\operatorname{TDS}(\mathrm{mg} / \mathrm{L})$

TDS (mg/L)

Temperature (degrees $C$ )

Temperature (degrees C)

Temperature (degrees C)

Temperature (degrees C)

TOC (ug/g)

$\operatorname{TOC}(u g / g)$

TOC $(u g / g)$

TOC (ug/g)

Total Carbon (ug/g)

Total Carbor. (ug/g)

Total Carbon (ug/g)

Total Carbon (ug/g)

TOX (ug (CI)/L)

$\operatorname{TOX}(u g(C I) / L)$

$\operatorname{TOX}$ iug $(C I) / L)$

$\operatorname{TOX}(u g(C I) / L)$
Sample \# Date Method Result $51095 \mathrm{~T} 3 / 26 / 90$ VOA 5.00E+00

$5081811 / 30 / 89$ ABN $9.90 E+01$

$509272 / 12 / 90$ ABN $3.10 \mathrm{E}+0 \mathrm{~L}$

$51068 \mathrm{~T} 3 / 19 / 90 \mathrm{VOA} \quad 4.60 \mathrm{E}+01$

$510953 / 26 / 90 \mathrm{VOA} \quad 1.00 \mathrm{E}+0 \mathrm{~L}$

$510953 / 26 / 90$ VOA $5.00 E+00$

$5081811 / 30 / 89$ TITRA 5.20E+04

$509272 / 12 / 90$ TITRA $5.60 \mathrm{E}+04$

$510683 / 19 / 90$ TITRA 5.20E+04

$510953 / 26 / 90$ TITRA 5.30E+04

$5081811 / 30 / 89$ Alpha $2.98 \mathrm{E}+00$

$51068 \quad 3 / 19 / 90$ Alpha $5.97 \mathrm{E}+00$

$510953 / 26 / 90$ Alpha 1.61E+0.

$5081811 / 30 / 89$ COND-Fld 1.47E+0:2

$509272 / 12 / 90$ COND-FId 1.46E+0:2

$510683 / 19 / 90$ COND-Fld 1.57E+0.2

$510953 / 26 / 90$ COND-Fl.d 1.44E+0.2

50818E $11 / 30 / 89$ IGNIT $2.08 \mathrm{E}+0.2$

$50927 \mathrm{E} 2 / 12 / 90$ IGNIT $2.08 \mathrm{E}+0.2$

$51068 \mathrm{E} \quad 3 / 19 / 90$ IGNIT $2.10 \mathrm{E}+02$

iI095E $3 / 26 / 90$ IGNIT $2.12 \mathrm{E}+02$

j0818 11/30/89 PH-FId 8.00E+OD

$50927 \quad 2 / 12 / 90 \mathrm{PH}-\mathrm{FId} \quad 7.73 \mathrm{E}+0 \mathrm{O}$

$510683 / 19 / 90 \mathrm{PH}-\mathrm{FId} \quad 5.15 \mathrm{E}+\mathrm{OO}$

$510953 / 26 / 90 \mathrm{PH}-\mathrm{FId} \quad 7.80 \mathrm{E}+\mathrm{OD}$

$50818 \mathrm{E} 11 / 30 / 89$ DSPEC <1.00E+02

$50927 \mathrm{E} 2 / 12 / 90$ DSPEC $<1.00 \mathrm{E}+02$

$51068 \mathrm{E} 3 / 19 / 90$ DSPEC $<1.00 \mathrm{E}+02$

$51095 \mathrm{E} 3 / 26 / 90$ DSPEC $<1.00 \mathrm{E}+02$

50818E 11/30/89 DTITRA <1.00E+02

50927E 2/12/90 DTITRA <1.00E+02

51068E 3/19/90 DTITRA <1.00E+02

51095E 3/26/90 DTITRA <1.00E+02

$5081811 / 30 / 89$ TDS $6.20 \mathrm{E}+0.4$

$509272 / 12 / 90$ TDS $5.80 \mathrm{E}+04$

$510683 / 19 / 90$ TDS $6.70 \mathrm{E}+0.4$

$51095 \quad 3 / 26 / 90$ TDS $8.70 \mathrm{E}+0.4$

$5081811 / 3-189$ TEMP-Fld 1.90E+0.1

$50927 \quad 2 / 12 / 90$ TEMP-FId $1.52 \mathrm{E}+0 . \mathrm{L}$

$510683 / 19 / 90$ TEMP-FId 1.77E+0.L

$510953 / 26 / 90$ TEMP-FId 1.79E+0.L

$5081811 / 30 / 89$ TOC $<1.00 E+0.3$

$509272 / 12 / 90$ TOC $\quad<9.00 E+02$

$51068 \quad 3 / 19 / 90$ TOC $\quad 1.00 \mathrm{E}+0$;

$510953 / 26 / 90$ TOC $\quad 1.10 \mathrm{E}+0.3$

$50818 \quad 11 / 30 / 89$ TC $\quad 1.40 \mathrm{E}+04$

$509272 / 12 / 90 \mathrm{TC} \quad 1.17 \mathrm{E}+04$

$51068 \quad 3 / 19 / 90 \mathrm{TC} \quad 1.42 \mathrm{E}+04$

$510953 / 26 / 90 \mathrm{TC} \quad 1.46 \mathrm{E}+04$

$50818 \quad 12 / 30 / 89$ ITOX $1.72 \mathrm{E}+02$

$509272 / 12 / 90$ I'TOX $1.43 E+02$

$510683 / 19 / 90$ LTOX 1.493+02

$510953 / 26 / 90$ LTOX 1.32E+O2 
DATA FOR Plutonium Finishing plant wastewater-Not PRF or RMC Continued

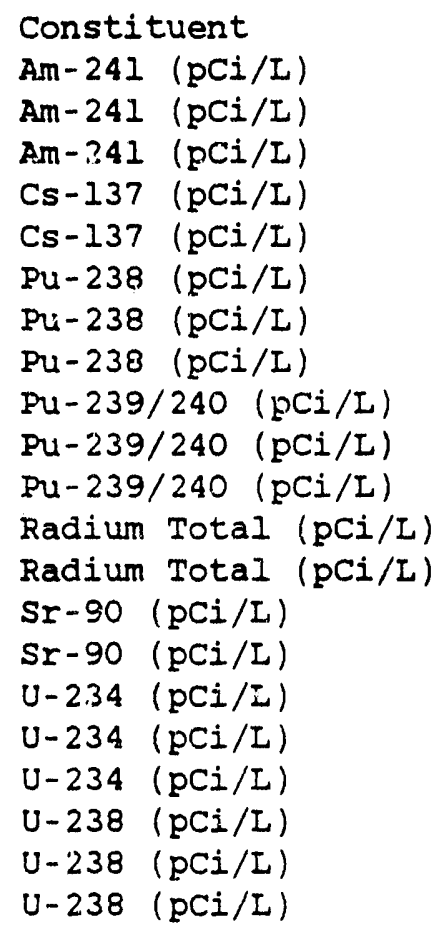

\begin{tabular}{|c|c|c|c|}
\hline Sample \# & Date & Method & Result \\
\hline 50818 & $11 / 30 / 89$ & $A E A$ & $3.71 E-01$ \\
\hline 51068 & $3 / 19 / 50$ & AEA & $1.94 E+00$ \\
\hline 51095 & $3 / 26 / 90$ & AEA & $1.55 \mathrm{E}+00$ \\
\hline 51068 & $3 / 19 / 90$ & GEA & $<5.37 \mathrm{E}-01$ \\
\hline 51095 & $3 / 26 / 90$ & GEA & $1.01 E+00$ \\
\hline 50818 & $11 / 30 / 89$ & $A E A$ & $2.51 \mathrm{E}-01$ \\
\hline 51068 & $3 / 19 / 90$ & $A E A$ & $2.90 E-01$ \\
\hline 51095 & $3 / 26 / 90$ & $A E A$ & 4. $24 E-01$ \\
\hline 50818 & $11 / 30 / 89$ & $A E A$ & $2.52 E+00$ \\
\hline 51068 & $3 / 19 / 90$ & $A E A$ & $8.95 E+00$ \\
\hline 51095 & $3 / 26 / 90$ & $A E A$ & $1.45 \mathrm{E}+01$ \\
\hline 50818 & $11 / 30 / 89$ & Alpha-Ra & $<8.18 \mathrm{E}-02$ \\
\hline 51068 & $3 / 19 / 90$ & Alpha-Ra & $3.49 \mathrm{E}-01$ \\
\hline 50818 & $11 / 30 / 89$ & Beta & $2.05 \mathrm{E}-01$ \\
\hline 51095 & $3 / 26 / 90$ & Beta & $4.65 \mathrm{E}-01$ \\
\hline 50818 & $11 / 30 / 89$ & $\mathrm{AEA}$ & $9.80 \mathrm{E}-02$ \\
\hline 51068 & $3 / 19 / 90$ & $A E A$ & $2.21 \mathrm{E}-01$ \\
\hline 51095 & $3 / 26 / 90$ & $A E A$ & $1.52 \mathrm{E}-01$ \\
\hline 50818 & $11 / 30 / 89$ & $A E A$ & $1.27 \mathrm{E}-01$ \\
\hline 51068 & $3 / 19 / 90$ & $A E A$ & $1.86 \mathrm{E}-01$ \\
\hline 51095 & $3 / 26 / 90$ & $A E A$ & $1.31 \mathrm{E}-01$ \\
\hline
\end{tabular}


WHC-EP-0355

\section{APPENDIX E}

ANALYTICAL RESULTS (MICROFICHE) 
WHC-EP-0355

Appendix E--Analytical Results (Microfiche). 
WHC-EP-0355

APPENDIX $F$

LIST OF COMMON NAMES 
Appendix F--List of Common Names. (sheet 1 of 8 )

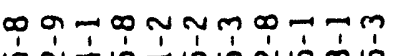

ஆ்

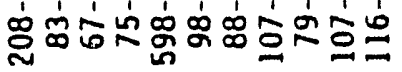

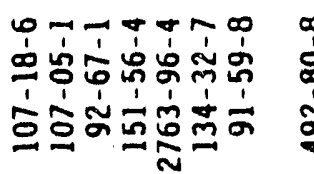

- -m nmunmon

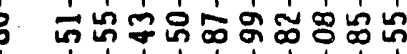
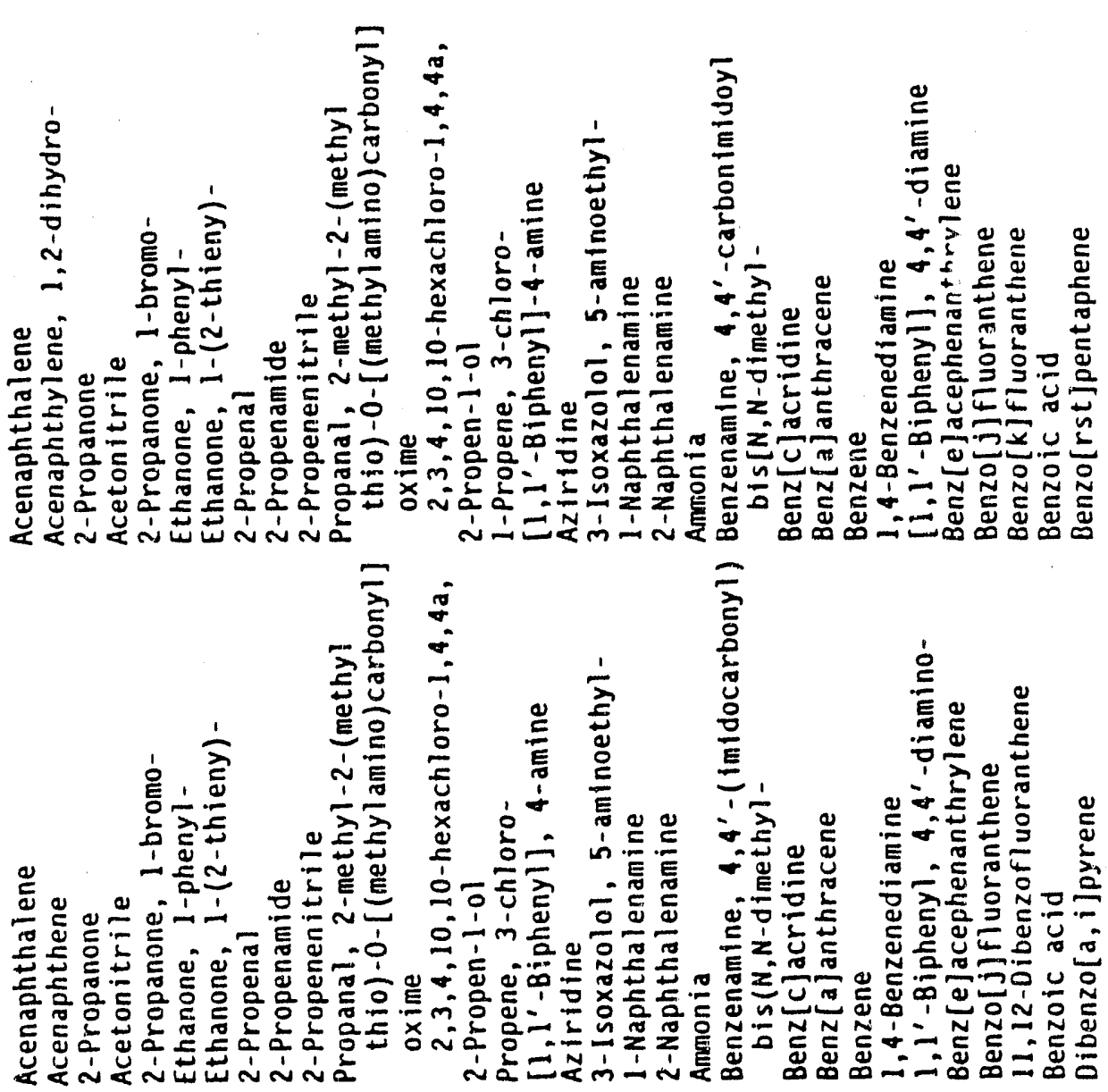
Appendix F--List of Common Names. (sheet 2 of 8 )

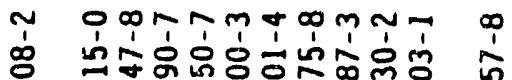

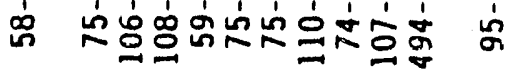
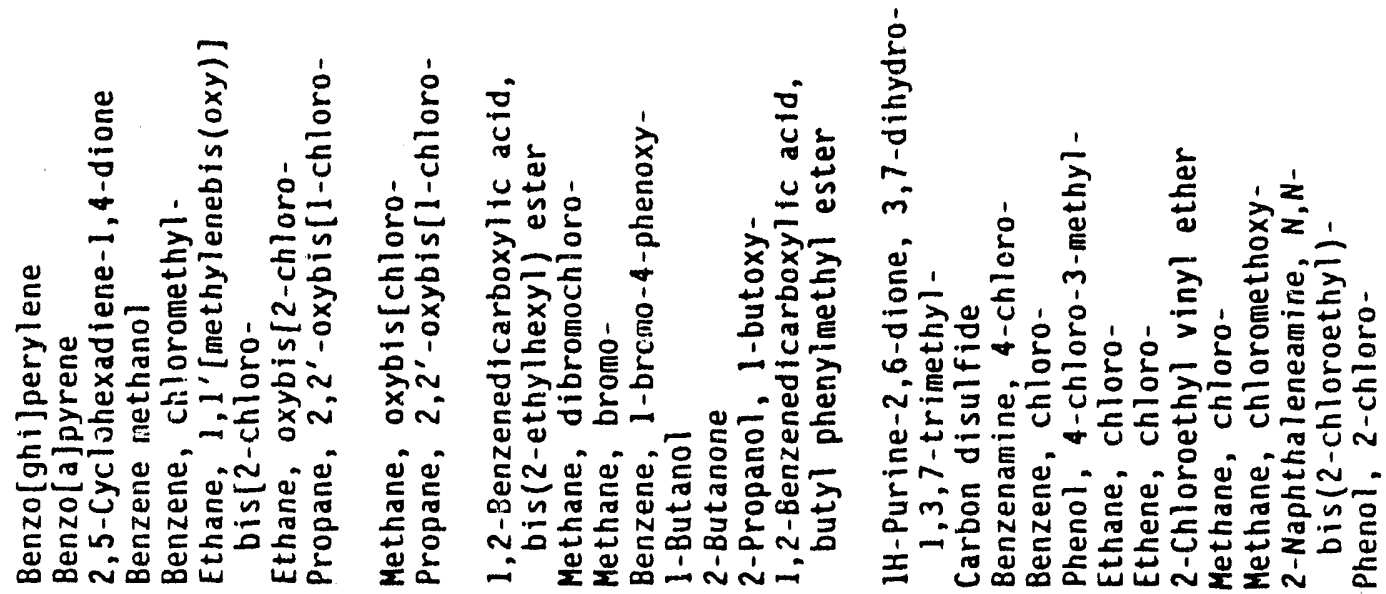

خิ

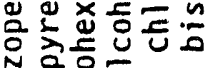

스릉

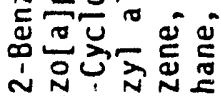

Nㅗㄴ

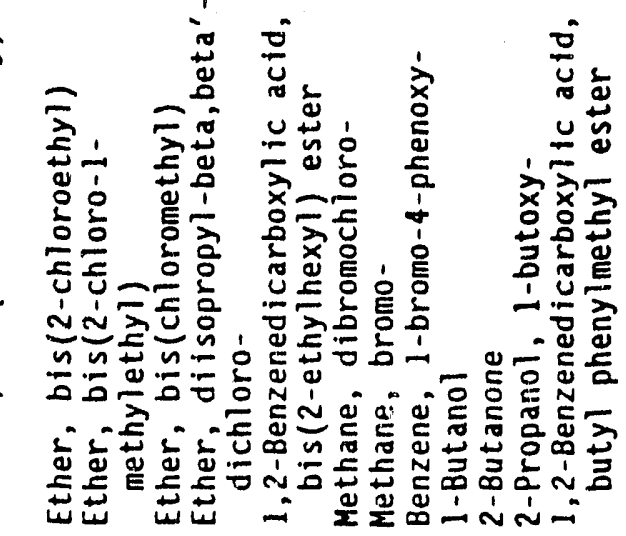

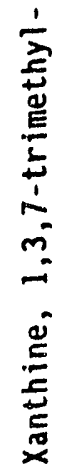

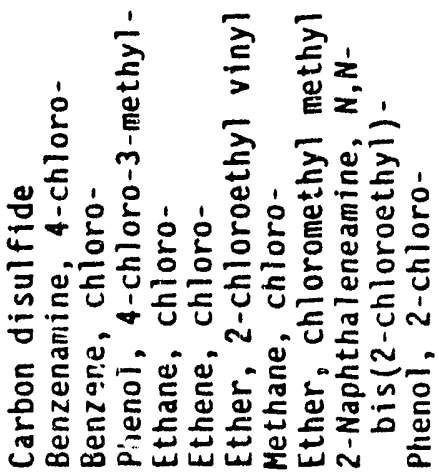

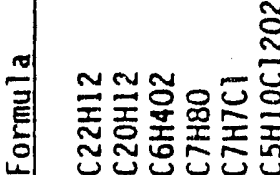

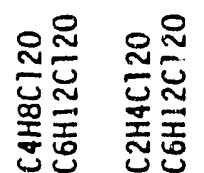

品

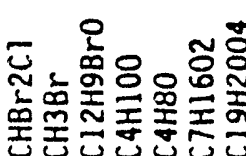

용

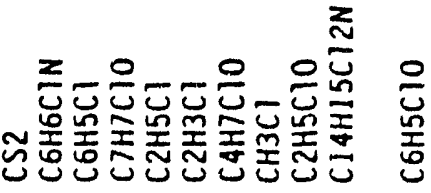

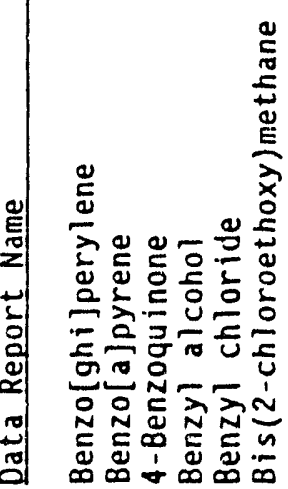

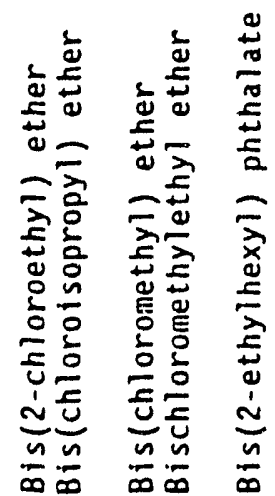

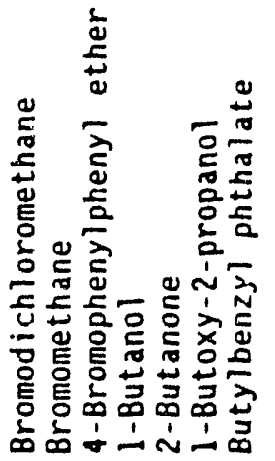

立先

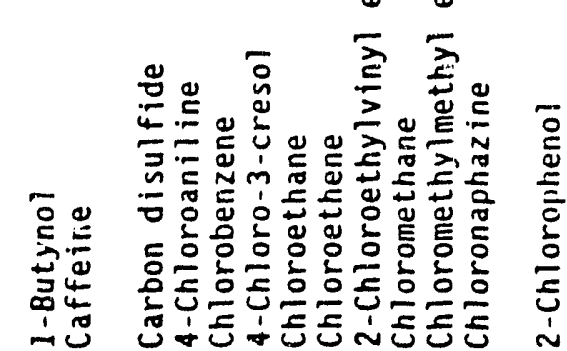


Appendix F--List of Common Names. (sheet 3 of 8 )

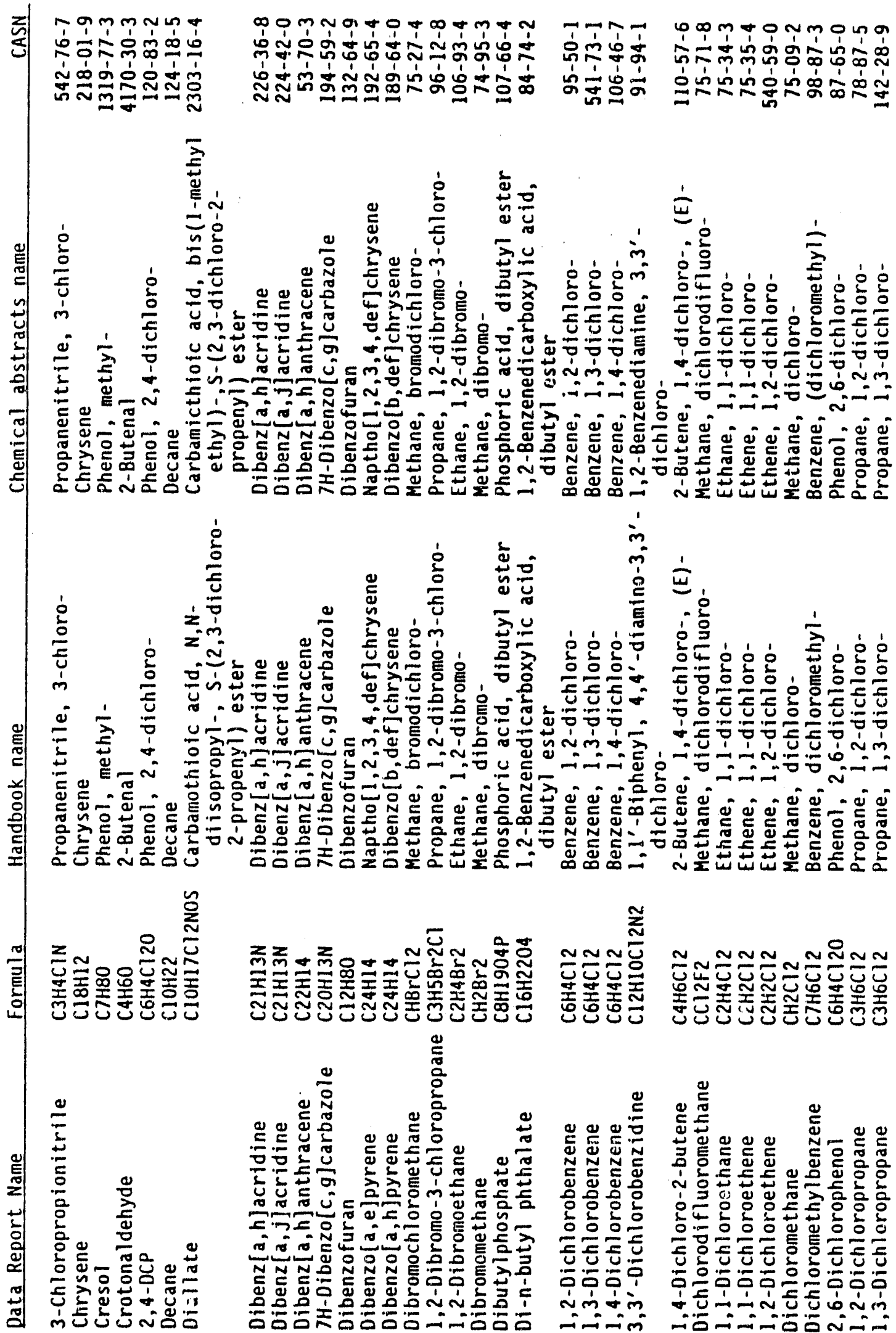


Appendix F--List of Common Names. (sheet 4 of 8)

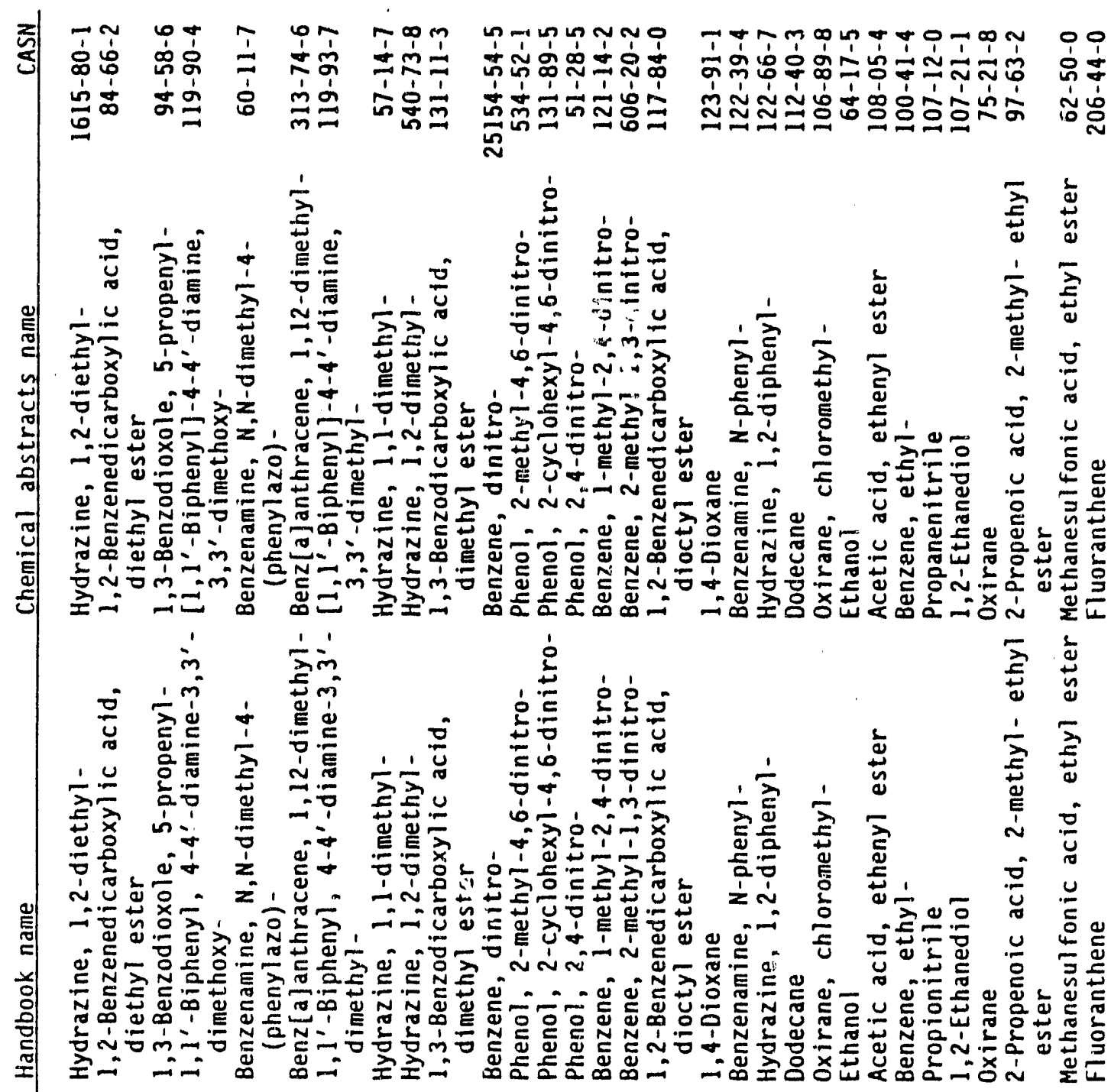

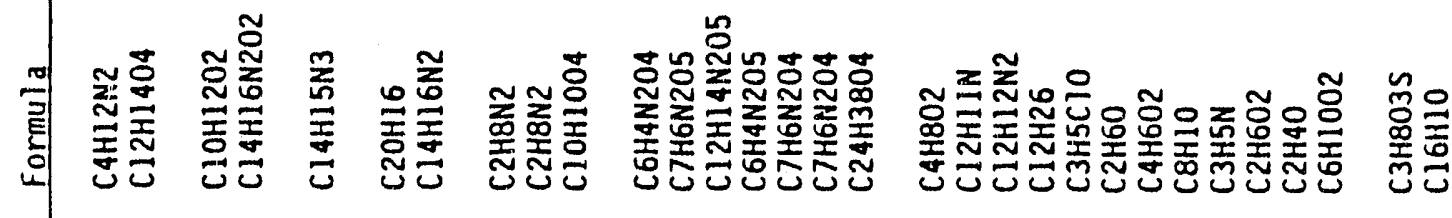

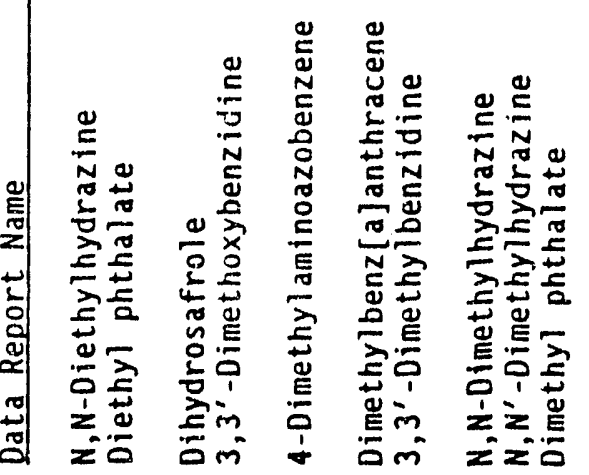
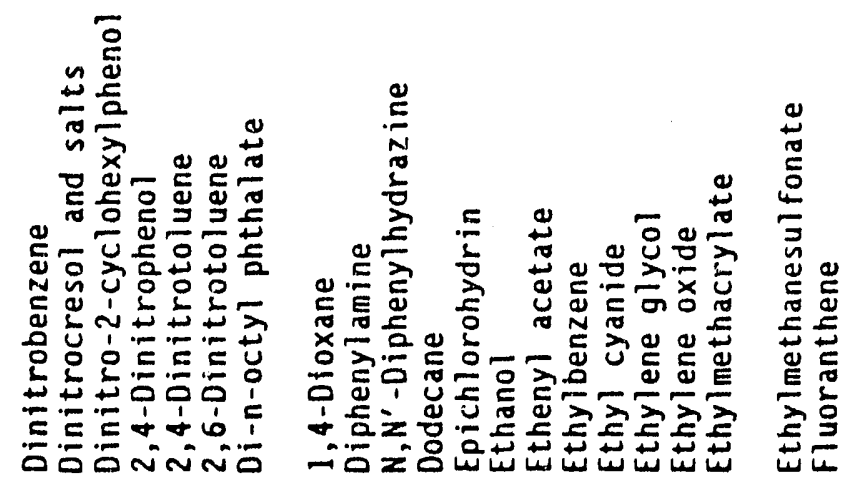


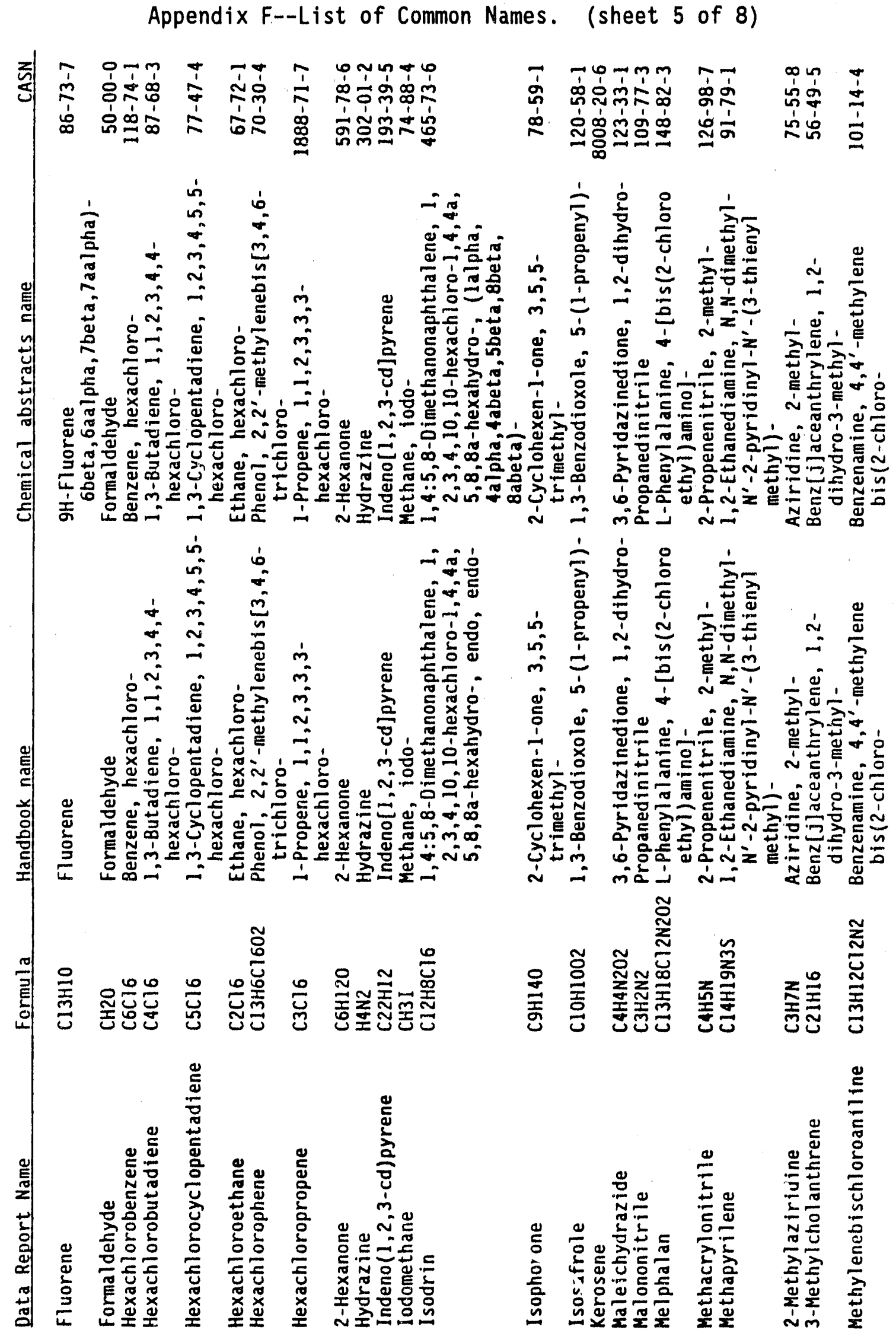


Appendix F--List of Common Names. (sheet 6 of 8)

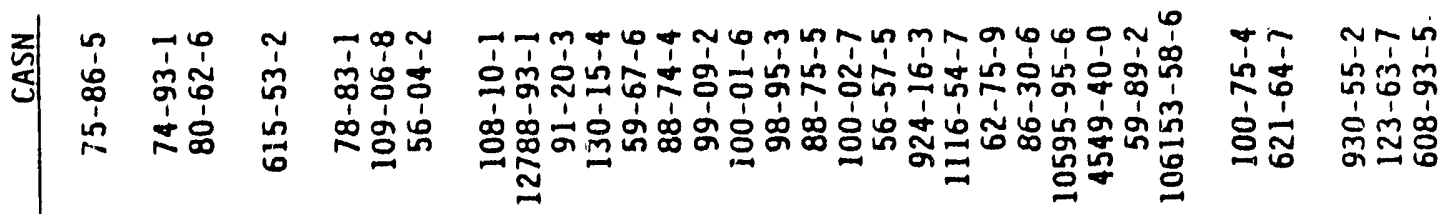

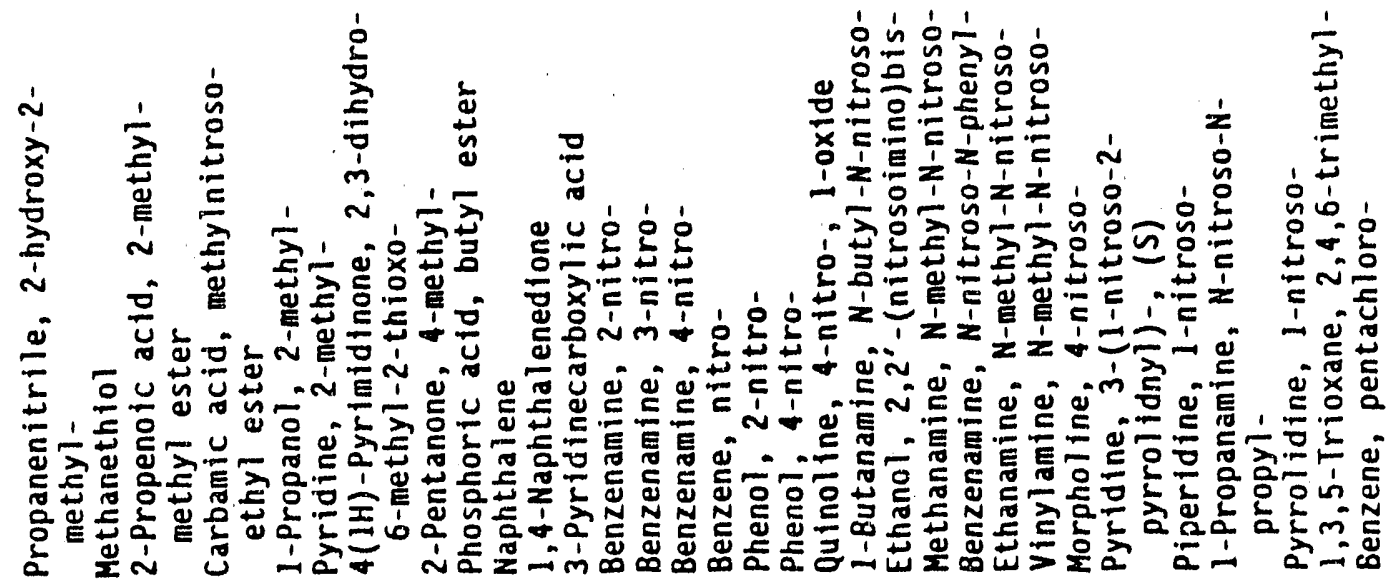

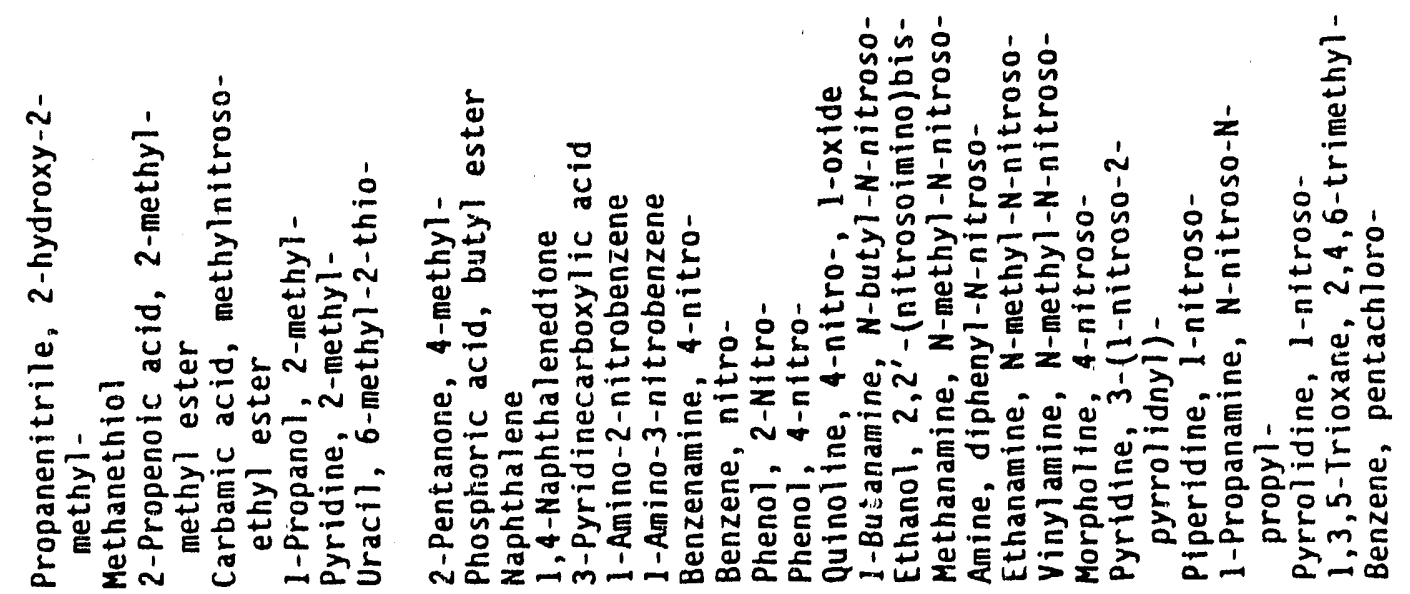

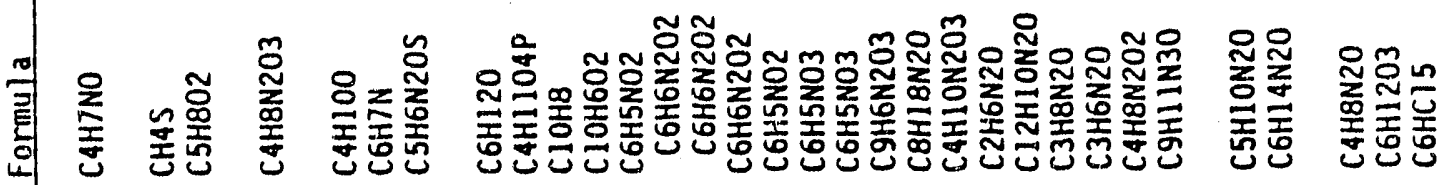

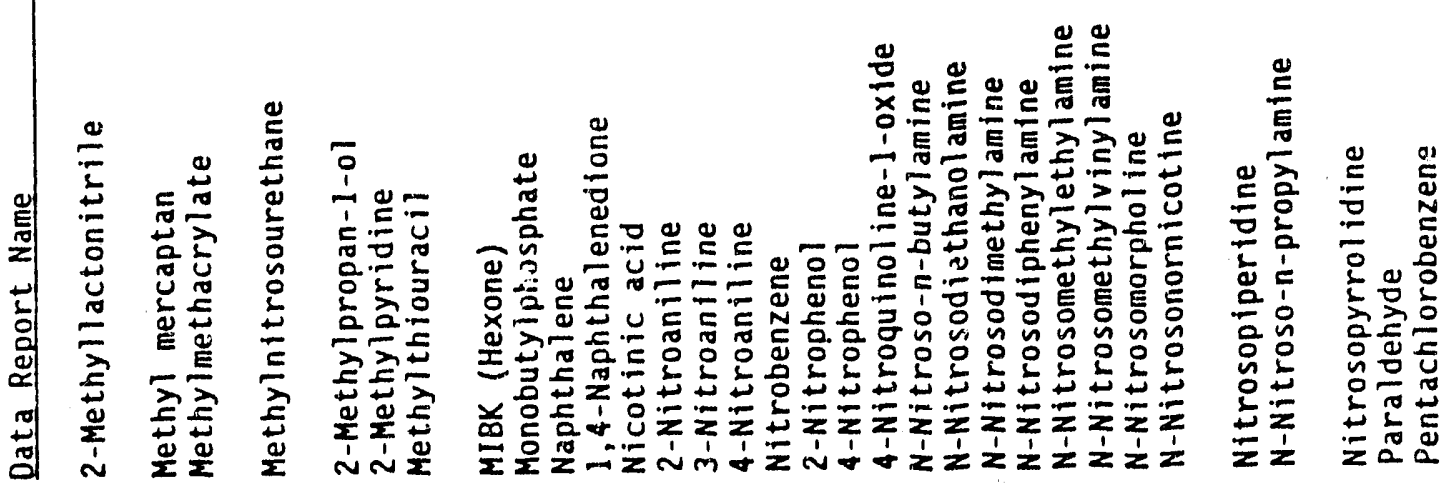


Appendix F--List of Common Names. (sheet 7 of 8)

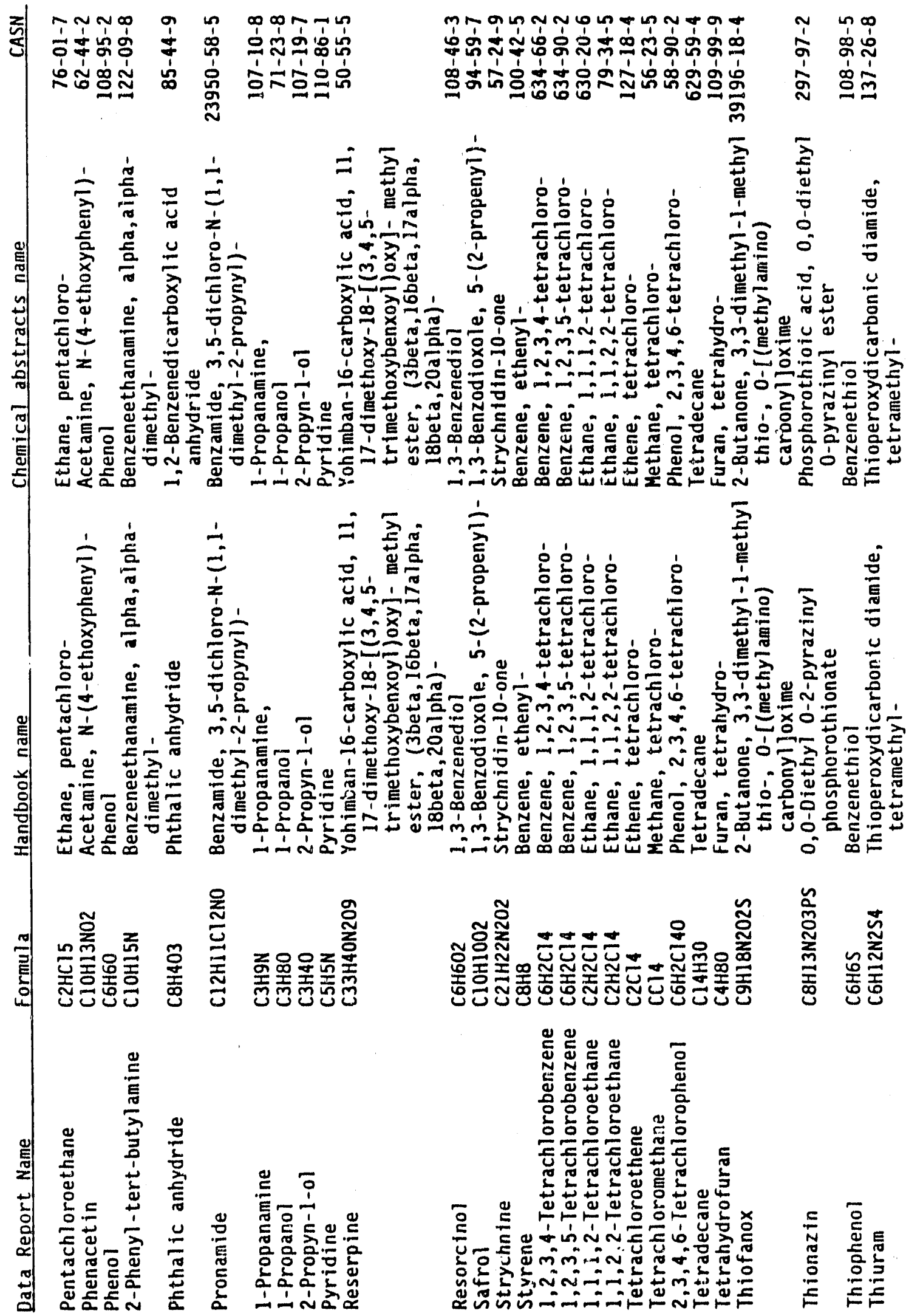


Appendix F--List of Common Names. (sheet 8 of 8)

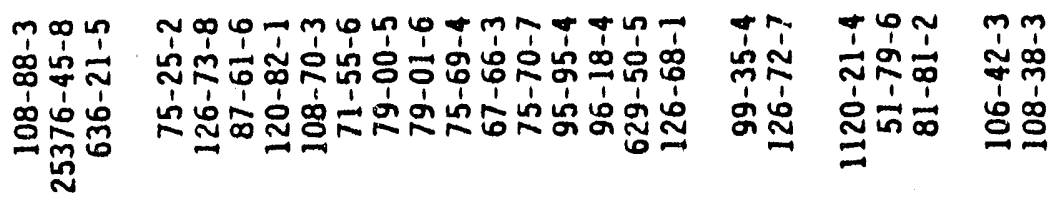
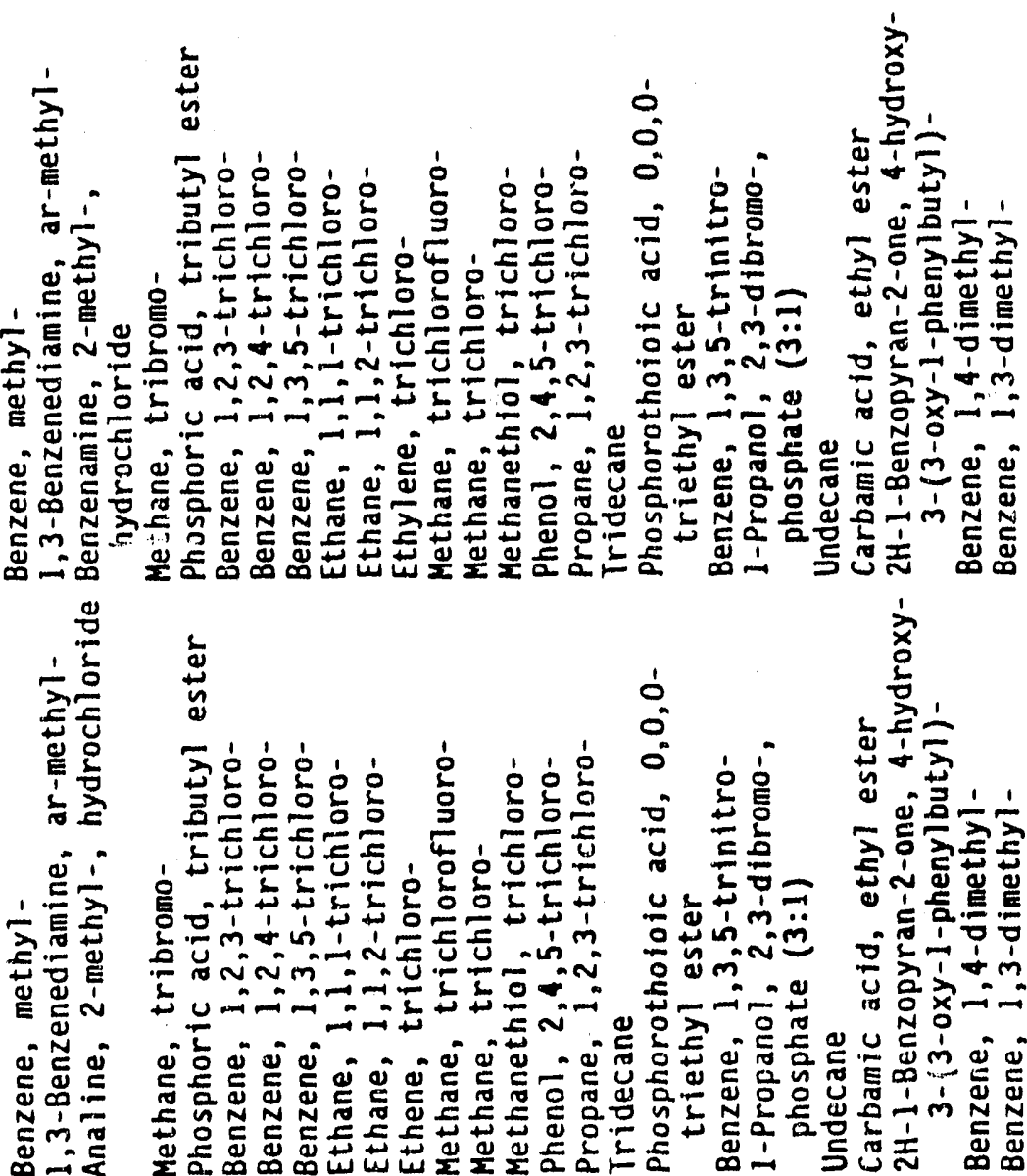

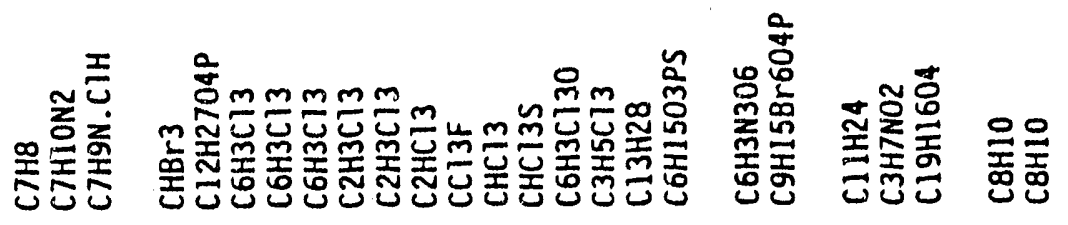

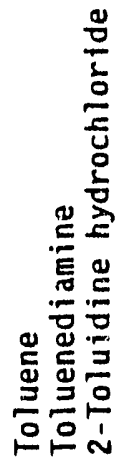
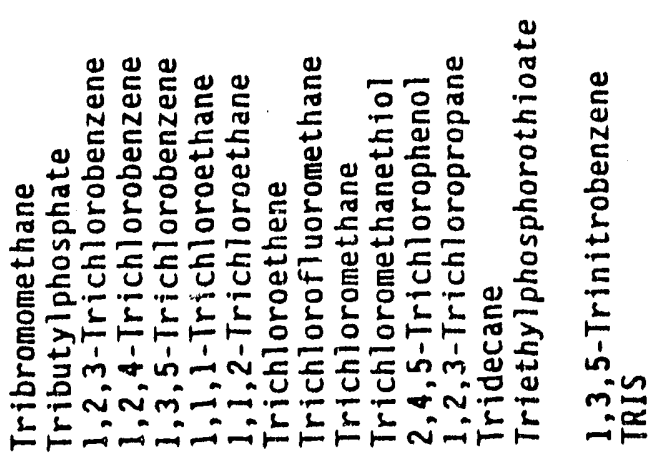

$\frac{\frac{0}{0}}{\frac{1}{2}}$

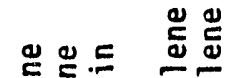

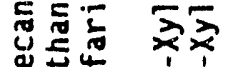

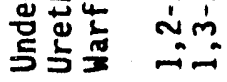



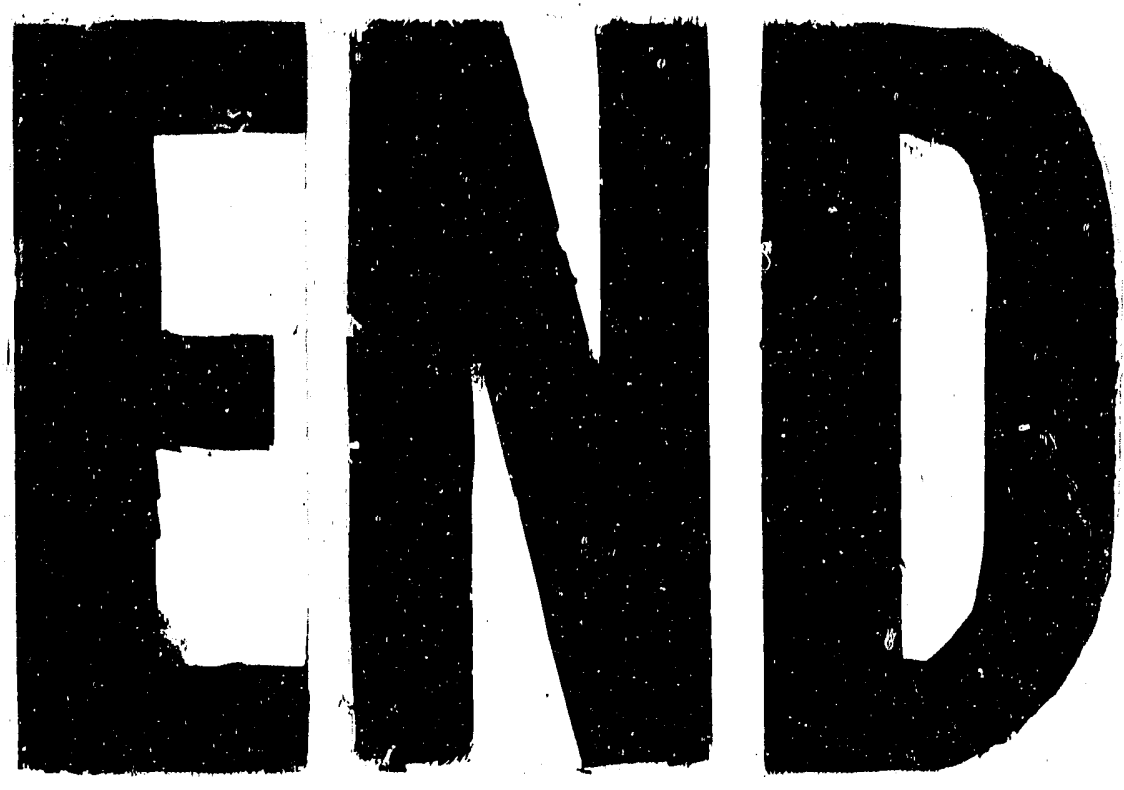

4
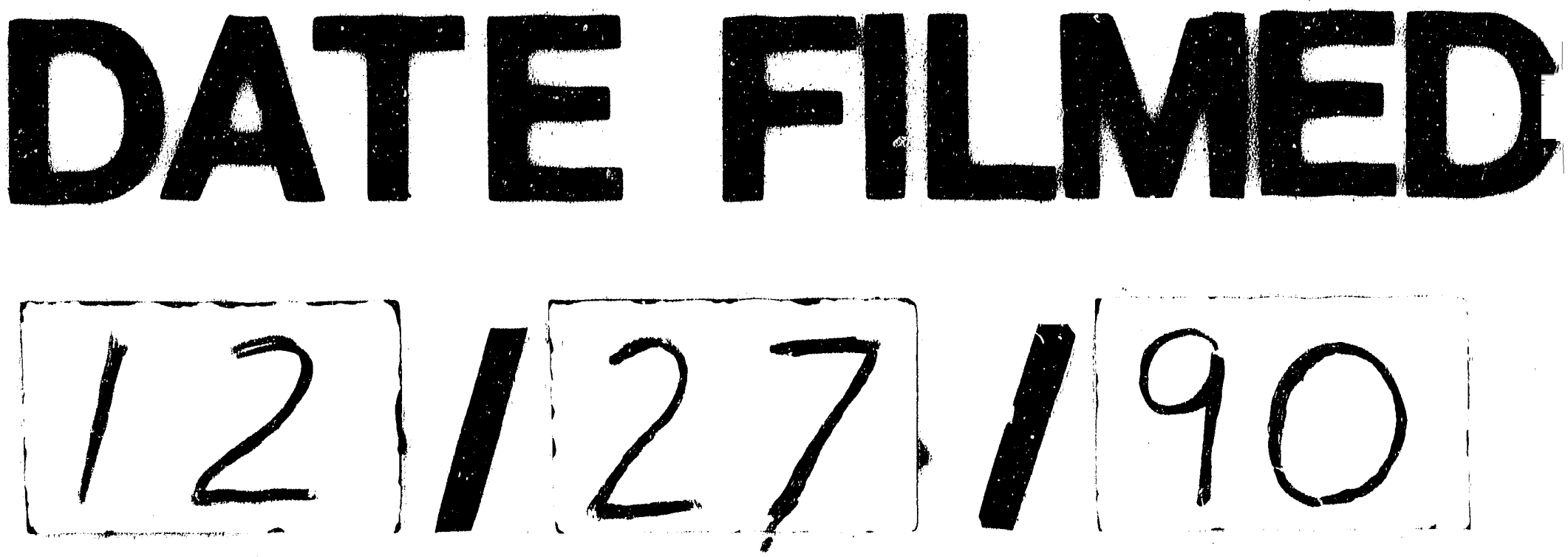